\title{
HUALAPAI WIND PROJECT FEASIBILITY REPORT
}

\author{
PREPARED FOR
}

HUALAPAI DEAPRTMIENT OF PLANNING AND

ECONOMIC DEVELOPMIENT

DECEMBER 20, 2012

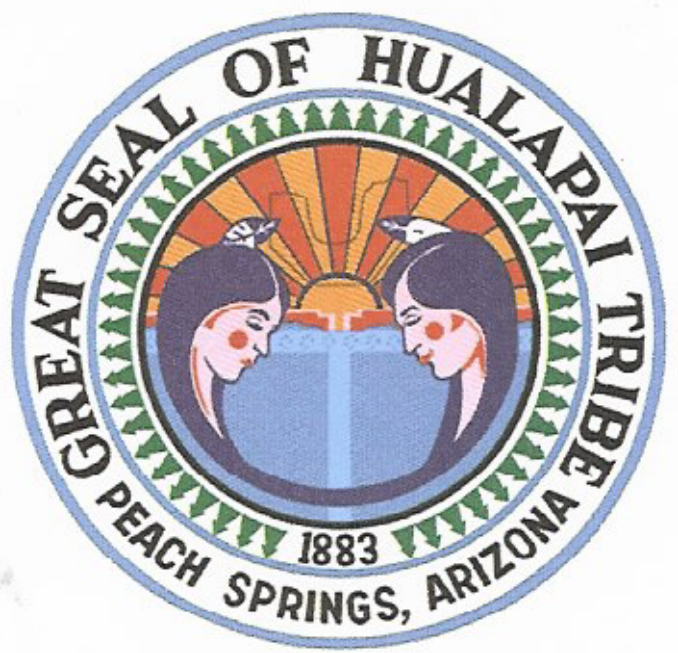




\section{Feasibility Report for Hualapai Tribe Wind Project}

Prepared for

Hualapai Tribal Nation Planning Department

Contributing Consultants:

MJH Power Consulting LLC

Daystar Consulting, LLC

V-Bar, LLC

Power Engineers, Inc.

SWCA Environmental, Inc.

Squire, Sanders, Dempsey, LLP

Ater-Wynne, LLP

First American Financial Advisors, Inc

December 15, 2012, revised June 30, 2013 


\section{Feasibility Report for Hualapai Tribe Wind Project \\ Table of Contents}

Executive Summary

1. Identification of the Site Area and Feasibility for Construction

2. Wind Resource Potential Summarized for the Hualapai Reservation

3. Results of Studies for the Best Turbine Locations in the Identified Site Area

a. Turbine arrays for identified site area on Hualapai land

b. Energy projections for these arrays using two recommended turbine models

4. Transmission and Interconnection Feasibility Summarized
a. Existing near term options for transmission and interconnection
b. Potential with Centennial Transmission Project completion in 2018
c. Preliminary engineering design and estimated costs

5. Initial Permitting \& Environmental Assessment Summarized

6. Preliminary Estimates for Project Costs Summarized
a. Development costs
b. Construction costs

7. Preliminary Project Proforma Summarized

8. Preliminary Project Business Plan

9. Findings and Recommendations 


\title{
List of Exhibits
}

\author{
Exhibit A Hualapai Wind Project Data Check Sheets \\ Exhibit B Project Site Evaluation for Construction ( MJH Consulting) \\ Exhibit C Geotechnical Report for 6" Water Pipeline (Foree \& Vann) \\ Exhibit D Wind Resource Report (Final -August 2012) (V-Bar) \\ Exhibit E Monthly/ Diurnal ( 12 x 24) Energy Pattern Table (V-Bar ) \\ Exhibit $\mathrm{F} \quad$ Wind Farm Development Analysis (V-Bar) \\ Exhibit G Transmission and Interconnection Feasibility Report ( Power Engineers) \\ Exhibit $\mathrm{H} \quad$ WAPA Mead-Peacock 345kV Interconnection Application (Power Engineers) \\ Exhibit I Map showing available transmission lines, proposed POI and turbines (HDP) \\ Exhibit J Critical Issues Analysis (SWCA) \\ Avian Use Assessment - Raptor Nest Survey (SWCA) \\ Fall Raptor Migration Study (SWCA) \\ Exhibit K Typical Development Costs Spreadsheet (MJH) \\ Exhibit L Preliminary Estimates for Project Costs (MJH) \\ Exhibit M Preliminary Project Pro Forma (MJH) \\ Exhibit N Introduction to Financing for Project (Squire Sanders) \\ Exhibit O Refined Financing Model (Ater-Wynne \& First American)
}

Attachment -1

Renewable Energy Development in Indian Country: A Handbook for Tribes (Ater,Whnne, LLP) 


\section{Executive Summary}

The first phase in the development process for utility-scale wind turbine projects is to complete the necessary feasibility studies. The studies needed are:

1. Identify the site area for development and its suitability for construction.

2. Determine the wind resource potential for the identified site area.

3. Determine the electrical transmission and interconnection feasibility to get the electrical power produced to the marketplace.

4. Complete an initial permitting and environmental assessment to determine the feasibility for getting the project permitted.

5. Determine where the best power sale opportunities are and the feasibility for obtaining a Purchase Power Agreement (PPA) that will make the project viable for financing.

The Hualapai Department of Planning and Economic Development, with funding assistance from the U.S. Department of Energy, Tribal Energy Program, and hired consultants has completed the first four key prerequisites listed above. Those studies indicated a suitable wind resource and favorable conditions for permitting and construction. The permitting and environmental study did not reveal any fatal flaws. A review of the best power sale opportunities indicate southern California has the highest potential for obtaining a PPA that may make the project viable. Based on these results, the recommendation is for the Hualapai Tribal Nation to move forward with attracting a qualified wind developer to work with the Tribe to move the project into the second phase - determining the reality factors for developing a wind project. a qualified developer will bid to a utility or negotiate a PPA to make the project viable for financing.

The results of the studies fall within the parameters for wind projects that been constructed near the identified site area for the Hualapai Wind Project. The proximity of the Perrin Ranch Wind Project north of Williams and the White Hills Wind Farm Project being developed to the west of the Reservation make a strong case for the Hualapai Wind Project falling within the parameters studied for constructability, wind resource, permitting and environmental and overall economic feasibility.

\section{Identification of the Site Area and Feasibility for Construction}

The genesis for the development of this potential wind project started about seven years ago. Five areas on the Hualapai Reservation, Peach Springs, Blue Mountain, Nelson, Grand Canyon West and Clay Springs were considered along with the inclusion of adjacent BLM lands along the western boundary of the Hualapai Reservation. With completion of the initial permitting and environmental feasibility studies in 2012 and progress with the Western Area Power Administration (WAPA) for 
potential interconnection access, the Grand Canyon West and Clay Springs areas were identified as the best areas for developing a single, large wind project. Originally Peach Springs and Blue Mountain were considered as these areas have decent winds and good road access, however they were removed from consideration based on visual impact and the presence of important cultural resources, respectively. The Nelson area was abandoned because its wind speeds were too low. The BLM lands were abandoned primarily for concerns by the Tribe over the presence of eagles and migrating raptors and less so by WAPA site control requirements and the expected increased time and costs for permitting.

Having identified the site and completed a site visit, maps of the terrain and available geotech studies were used to complete a feasibility assessment and determine preliminary estimates for the construction costs. While terrain on the tribal lands is complex, with relatively smooth rolling hills, ridgelines, and canyons, its does not appear too difficult for construction equipment to access the proposed wind turbine locations from Buck and Doe Road and improved existing side roads. This work did not show any fatal flaws for access or constructability with any of the proposed turbine sites on the Hualapai Reservation. It was concluded that the initial assessment did not identify any unusual issues specific to the site that may prevent or significantly hinder the construction of the Project.

A report was prepared for the initial site visit and construction feasibility assessment along with a nine-page listing of completed check sheets (Exhibit A) that MJH Power Consulting LLC has used for conducting wind project site evaluations for construction. Access to the wind farm site from the nearest BNSF Railroad siding in Nelson is good; allowing access via paved roadway on Hwy 66 for about 12 miles and BIA Route 1. Access from other BNSF rail sidings near Kingman is discussed further in Exhibit B. The site has excellent availability from existing tribal locations for the crushed rock for the roads and aggregate for the concrete for the turbine foundations. Availability of the geotech report (Exhibit C) for the six-inch water pipeline construction allowed confirmation of the need for rock socket construction techniques for an estimated $75 \%$ of the turbine foundations.

The Hualapai Tribe will need to confirm that water from the 6 inch pipeline or the storage tanks on site would be available as a source of water for the concrete batch plant and other construction water needs such as dust control, etc. The Tribe would typically be reimbursed for the roadbed and crushed stone aggregate and water used. Typically this would be covered in the project lease agreement or partnering agreement between the developer and the Tribe.

\section{Wind Resource Potential Summarized for Hualapai Reservation}

Wind data gathering started in December 2005 and V-Bar has done the analysis and reporting for the wind resource potential. This final update (Exhibit D) analyzes wind data collected on the Hualapai Reservation in northwestern Arizona from December 2005 through August 2012 for five towers, of which four were still operating at the end of August 2012. 
Information for the two towers located in the identified project area is summarized below:

Station Site Number Height $(\mathrm{m}) \quad$ Month Installed

$\begin{array}{rrrr}\text { Grand Canyon West } & 7054 & 50 & \text { Oct } 2006 \\ \text { Clay Springs } & 7055 & 50 & \text { May } 2011\end{array}$

Here are V-bar's resulting long-term mean annual wind speed estimates in meters per second, with extrapolations to $80 \mathrm{~m}$ using site-specific shears for the two sites in the project area:

$\begin{array}{lcc}\text { Level }(\mathrm{m}) & \text { Grand Canyon West } & \text { Clay Springs } \\ 10 & 4.83 & 3.88 \\ 30 & 6.02 & 5.18 \\ 40 / 44 & \ldots & 5.53 \\ 49 / 50 & 6.30 & 5.76 \\ 80 & 6.70 & 6.41\end{array}$

\section{Results of Studies for the Best Turbine Locations in the Identified Site Area}

\section{a. Turbine arrays for identified site area on Hualapai Lands}

This report (Exhibit D) presents four prospective wind farm array plans, developed over the course of many years from meteorological tower measurements, site visits, setback criteria and other factors. Four specific arrays are presented here by V-Bar. All of the prospective array plans are located on the west side of the Hualapai Reservation.

\section{On Hualapai lands only (identified site area):}

200-MW array for GE-1.7/100 turbines with 80-m hub height

200-MW array for Acciona AW-116/3000 turbines with 92-m hub height

\section{On Hualapai and adjacent BLM lands (for potential future developer interest only)}

284-MW array for GE-1.7/100 turbines with 80-m hub height

456-MW array for Acciona AW-116/3000 turbines with 92-m hub height

The 200-MW arrays use more restrictive criteria (tribal lands only and at least 4 miles from identified eagle nests), and these arrays are designed consistent with applications being submitted to the Western Area Power Administration (WAPA) for access to their $345 \mathrm{KV}$ transmission line near 
the southern end of the project Exhibit $\mathrm{H}$. The larger arrays include BLM lands, have reduced (2mile) setbacks from eagle nests, and also have a number of turbines close to the Grand Wash Cliffs, which appears to an active flyway for raptors during their fall migration season. The larger arrays are provided for possible future interest by developers. These arrays have a higher risk for being developed with BLM land content and the reduced setbacks from eagle nests which will increase the permitting time and permitting costs for a wind project.

Detailed wind resource assessments for the 200-MW arrays are provided by V-Bar with a focus on long-term mean annual gross energy projections. Estimated long-term mean annual hub-height wind speeds and gross capacity factors are given for each individual turbine in the 200-MW arrays. The wind speeds are derived from:

- $\quad$ wind speed data from the Grand Canyon West and Clay Springs met towers at the northern and southern ends of the project footprint, respectively

- $\quad$ wind direction data from these towers, to identify prevailing directions, zones where winds can be enhanced by the terrain, and proper spacing between turbines

- topographic and terrain variations across and outside the subject footprint

- $\quad$ surface conditions (vegetation type, height and density, soil and rockiness, etc.)

- observations made during field trips

Aggregate long-term mean annual hub-height wind speeds and gross annual capacity factors are summarized below:

Turbine \#Turbines Aggregate Speed (mps) Gross Capacity Factor(\%)

$\begin{array}{lccc}\text { GE-1.7/100 } & 118 & 6.16 & 33.73 \\ \text { AW-116/3000 } & 67 & 6.35 & 27.74\end{array}$

To convert gross to net output projections, V-bar presents a table showing the various discount factors (losses) that are normally considered in this type of exercise. Some of these discounts are meteorological in nature such as high wind cutouts, icing on the blades and lightning. V-bar has calculated these losses from the on-site data or estimated them based on their experience in the region. Other discounts are non-meteorological in nature such as grid outages and electrical losses. V-Bar has provided typical industry values as placeholders. These non-meteorological discounts can be refined with appropriate contractual and engineering inputs. Power Engineers has completed preliminary engineering for the turbine collector system and transmission to the interconnection point and has estimated the electrical losses at 3.8\% which V-bar has used. V-bar also completed an analysis of the monthly/diurnal (hourly average -12 months $\times 24$ hours ( $12 \times 24$ Table below) energy pattern for the 200MW GE-1.7/100 array. Key findings from the $12 \times 24$ Table shows: 
1. March-June are far the windiest months, the only months to consistently average greater than a $30 \%$ net capacity factor.

2. Diurnal peak winds are strongest in late afternoon through late evening in the warmer months and in general the diurnal range is fairly small the rest of the year.

\begin{tabular}{|c|c|c|c|c|c|c|c|c|c|c|c|c|}
\hline $\begin{array}{l}\text { Hour } \\
\text { (MST) }\end{array}$ & Jan & Feb & Mar & Apr & May & Jun & Jul & Aug & Sep & Oct & Nov & Dec \\
\hline 0 & 28.4 & 30.4 & 32.6 & 36.1 & 34.3 & 36.9 & 25.2 & 25.6 & 23.1 & 26.0 & 24.1 & 24.9 \\
\hline 1 & 26.5 & 26.9 & 33.4 & 35.8 & 33.0 & 35.2 & 24.8 & 25.2 & 22.7 & 24.9 & 24.3 & 25.3 \\
\hline 2 & 27.1 & 26.1 & 34.2 & 35.8 & 31.6 & 33.8 & 24.4 & 23.0 & 21.5 & 25.9 & 24.2 & 26.3 \\
\hline 3 & 26.6 & 25.9 & 31.9 & 32.9 & 28.3 & 30.9 & 20.9 & 21.5 & 19.9 & 26.2 & 24.3 & 25.4 \\
\hline 4 & 26.8 & 26.9 & 32.1 & 32.2 & 27.2 & 28.2 & 17.0 & 19.1 & 20.9 & 27.8 & 24.9 & 25.9 \\
\hline 5 & 27.2 & 27.4 & 33.3 & 31.6 & 26.0 & 25.6 & 15.7 & 18.1 & 21.7 & 28.2 & 24.7 & 26.2 \\
\hline 6 & 28.8 & 26.6 & 33.5 & 31.8 & 23.8 & 24.8 & 13.5 & 18.6 & 20.3 & 27.3 & 26.5 & 27.8 \\
\hline 7 & 30.0 & 26.0 & 33.6 & 28.6 & 20.5 & 22.9 & 11.4 & 15.1 & 20.4 & 25.1 & 27.4 & 28.4 \\
\hline 8 & 30.1 & 25.3 & 29.8 & 26.9 & 21.1 & 25.8 & 10.8 & 14.0 & 18.8 & 21.4 & 25.6 & 28.7 \\
\hline 9 & 24.8 & 22.5 & 29.0 & 27.5 & 23.7 & 28.5 & 12.7 & 16.1 & 20.4 & 20.9 & 24.9 & 27.6 \\
\hline 10 & 21.5 & 22.9 & 29.1 & 31.4 & 27.0 & 32.3 & 15.4 & 17.9 & 21.4 & 22.9 & 23.3 & 25.7 \\
\hline 11 & 20.8 & 24.2 & 29.6 & 34.0 & 30.0 & 32.3 & 17.8 & 21.4 & 21.5 & 23.5 & 25.1 & 26.1 \\
\hline 12 & 21.5 & 25.8 & 32.0 & 37.4 & 32.0 & 33.5 & 19.6 & 23.1 & 24.2 & 25.5 & 27.9 & 25.2 \\
\hline 13 & 21.2 & 27.8 & 33.7 & 36.2 & 32.3 & 33.8 & 22.2 & 22.7 & 24.8 & 28.3 & 28.5 & 25.5 \\
\hline 14 & 22.5 & 29.0 & 35.5 & 36.2 & 33.4 & 34.3 & 23.0 & 24.8 & 26.3 & 28.2 & 28.1 & 25.9 \\
\hline 15 & 23.6 & 28.7 & 36.9 & 37.0 & 33.0 & 33.9 & 24.9 & 25.5 & 24.8 & 28.0 & 29.1 & 26.7 \\
\hline 16 & 24.3 & 27.5 & 36.2 & 38.2 & 34.1 & 35.8 & 23.6 & 24.9 & 24.4 & 27.9 & 27.4 & 26.4 \\
\hline 17 & 24.2 & 25.7 & 33.2 & 36.9 & 35.5 & 39.0 & 24.7 & 25.6 & 24.0 & 26.7 & 25.4 & 25.5 \\
\hline 18 & 25.6 & 25.3 & 32.4 & 37.3 & 35.6 & 40.5 & 26.0 & 24.5 & 22.8 & 25.0 & 24.9 & 25.8 \\
\hline 19 & 26.3 & 27.3 & 31.1 & 35.7 & 34.1 & 39.0 & 25.5 & 23.0 & 20.7 & 25.3 & 24.8 & 25.0 \\
\hline 20 & 26.5 & 27.8 & 30.2 & 33.9 & 32.3 & 34.9 & 24.2 & 22.3 & 19.8 & 26.7 & 26.0 & 24.6 \\
\hline 21 & 26.7 & 28.9 & 31.2 & 34.2 & 32.3 & 34.6 & 21.8 & 24.4 & 20.3 & 26.5 & 25.1 & 26.1 \\
\hline 22 & 28.3 & 29.4 & 31.5 & 37.0 & 32.9 & 35.1 & 24.7 & 26.2 & 20.5 & 27.0 & 25.3 & 24.6 \\
\hline 23 & 29.0 & 31.2 & 31.4 & 36.6 & 33.5 & 36.8 & 24.4 & 26.3 & 21.7 & 27.1 & 25.5 & 25.2 \\
\hline \multirow[t]{2}{*}{ Month } & 25.8 & 26.9 & $\bar{~} 32.4$ & 34.2 & 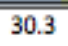 & 32.8 & 20.6 & 222.0 & $\overline{202.0}$ & 25.9 & 25.7 & $\overline{20.0}$ \\
\hline & & & & & & & & & & & Year: & 27.06 \\
\hline
\end{tabular}

\section{b. Energy projections for the two 200MW arrays using 2 recommended turbine models}

V-bar's resulting long-term mean annual net energy projections for the 200MW array plans on only Hualapai Tribe Land and with the 4 mile buffer from eagle nests is summarized below:

Turbine Gross Cap\% Losses Net Cap\% Net MWh/Turbine \#Turbines ArrayNet MWh

$\begin{array}{lllllll}\text { GE-1.7/100 } & 33.73 & 19.77 & 27.06 & 4,030 & 118 & 475,510 \\ \text { AW-116/3000 } & 27.74 & 20.29 & 22.11 & 5,810 & 67 & 389,281\end{array}$




\section{Transmission and Interconnection Feasibility}

Power Engineers has performed a high-level transmission and interconnection feasibility analysis (Exhibit G) of the local Extra High Voltage (EHV) transmission system, existing and planned, adjacent to the Hualapai Renewable Energy Project. The analysis is detailed below.

This task was to evaluate existing EHV transmission options that may be available for interconnecting the proposed Hualapai Renewable Energy Project and provide a preliminary engineering design and estimated costs. The evaluation included potential Points of Interconnection (POI), distances from the project to the POIs and a brief discussion of each POI. Attachment I is a map showing the Hualapai area and the three EHV transmission lines in the immediate vicinity of the $\mathrm{POI}$ and the location of the recommended wind turbine arrays. Three lines exist near the POI. The first two are parallel. One of the parallel lines is the Mead-Perkins 500kV line. The Perkins to Mead 500kV line has 4 owners: APS, WAPA, SRP and the City of Vernon. The second and currently the preferred POI, is the Mead-Peacock $345 \mathrm{kV}$ line. It has one owner, WAPA.

Power Engineer's summary of the proposed preliminary Hualapai Wind and Solar Project design included a preliminary one-line, cost analysis of the required transformers, estimated losses at peak wind and solar output to the point of interconnection. The preliminary one-line diagram of the wind farm to the POI into the Mead-Peacock 345kV line shows a cluster of 100 turbines approximately 23 miles from the point of interconnection. There is an additional cluster of 18 turbines approximately 12 miles from the point of interconnection. The generated wind energy is transmitted to the point of interconnection using bundled double circuit $69 \mathrm{kV}$ transmission lines. The solar generation (which is a being studied as a separate project on fee lands owned by the Tribe at Clay Springs) is approximately 8.4 miles from the point of interconnection and utilizes a single circuit $69 \mathrm{kV}$ transmission line. The generation at each cluster, both wind and solar, is represented as a single aggregate generator collected utilizing a $34.5 \mathrm{kV}$ collector system. The wind and solar $69 \mathrm{kV}$ transmission lines go to a single combined substation where the power is stepped up to $345 \mathrm{kV}$ at the POI with WAPA.

An Interconnection Request has been filed with WAPA in their Phoenix area office (Exhibit $H$ ). The date for the application is 1/12/2012 and the WAPA queue generation number position is 2012G28. WAPA is conducting the initial phase of the interconnection process, the Feasibility Study, at this time.

The third line in the area is the Moenkopi-Eldorado 500kV line which is owned and operated by Arizona Public Service (APS). This line travels across the Hualapai Reservation from east to west and passes by Peach Springs. This line also passes through the project site and therefore the length of the gen-tie would be short to the POI. The scheduling rights on the Moenkopi-Eldorado line are currently owned by Southern California Edison Company (SCE). Those rights however, are set to revert back to APS by the end of 2012 if all regulatory approvals are obtained. APS has created a transmission service request queue for the future available transmission capability for when APS finally acquires the scheduling rights to the line. A recent check of this APS queue (which is available on the APS OASIS) shows that approximately 2000MW of reservations have been posted. 
Power Engineers also looked at a potential interconnection to the Centennial West Clean Line Project (Clean Line Project), which is scheduled for completion in 2018. They evaluated a proposed interconnection near the WAPA Peacock $345 \mathrm{kV}$ substation, southwest of the project area. The Clean Line Project is a high voltage direct current (HVDC) transmission line whereby wind energy produced in New Mexico and solar energy produced in Arizona will be transmitted to the Lugo substation in the SCE service area in California. The purpose of the Arizona interconnection of the Clean Line Project was to allow 500MW of Arizona produced solar generation to be shipped to California. The site of the proposed Arizona substation was to be near the WAPA Peacock 345kV substation. In addition, the Clean Line Project is considered a gen-tie, which means that only generation can be connected to it. No other transmission interconnections to the Arizona transmission system would be allowed. As such, the Hualapai Project may not be able to use the existing Mead to Peacock 345kV line to wheel its power to Peacock to be considered as part of the $500 \mathrm{MW}$ transfer to California. This would need further clarification from Centennial West as to if this would be allowed or not. If not allowed to wheel to Peacock, then the Hualapai Project would have to build its own line, approximately 30 miles, to interconnect into the Clean Line Project substation near Peacock. This line would not have to be $345 \mathrm{kV}$ construction. For $200 \mathrm{MW}$, it could be a double circuit $69 \mathrm{kV}$ line. If the Hualapai Project built its own line, the project would not have to pay WAPA wheeling charges. For additional detail, see the Power Engineers Hualapai Renewable Energy Project Transmission and Interconnection Feasibility Report (Exhibit G)

\section{Initial Permitting \& Environmental Assessment Summarized}

Toward the end of July 2012 SWCA Environmental Consultants completed a Critical Issues Analysis (CIA) (Exhibit J) for six project areas on a combination of Hualapai Tribal lands, Bureau of Land Management (BLM), State, and private lands in Mohave and Coconino Counties, Arizona, having potential for renewable energy development (four are being considered for wind energy development, and two are being considered for solar energy development). The CIA completed by SWCA provides a broad, yet comprehensive overview of key resources identified during preliminary project planning, including recommendations and additional work required to complete the environmental evaluation. The initial findings contained in the CIA did not indicate any fatal flaws present that could affect the viability of renewable energy development. Although each of the six sites has varying levels of risks, including high risk issues, no issues were identified that affect the overall viability of renewable energy development for those sites.

From 25 September to 4 October 2012, SWCA conducted fall raptor migration studies in the project area. Field methods followed Arizona Game and Fish Department protocol. To evaluate use of the project area by migrating raptors, SWCA first conducted a literature and data search and initiated communications with HawkWatch International (HWI), which has been conducting raptor migration studies in the Grand Canyon since 1997. The HWI Grand Canyon study location lies approximately 100 miles east of the project area. All pertinent HWI data were summarized and data collected 
during the SWCA study were compared to those of HWI to in order to ascertain the degree to which migrating raptors use the project area.

A comparison of raptor migration magnitude of the greater project area with the HWI data had shown the greater project area is an area that concentrates raptors during fall migration, with migration magnitude moderate to low across the project area. Importantly, results had shown that the majority of migrating raptors (79\%) were recorded directly on major headwalls and ridgelines of the Grand Wash Cliffs proper. Therefore, although the greater project area is an area that concentrates raptors during fall migration, during the SWCA study the core migration flyway appears to be on the western boundaries of the project area along the Grand Wash Cliffs proper.

\section{Preliminary Estimates for Project Costs Summarized}

\section{a. Development Costs}

Typical development Costs for Utility-Scale Wind Power Projects run from about $1 \%$ to $3 \%$ of the total wind project cost. Utility-Scale wind projects are typically from about 50MW to 300MW in size with larger projects having lower percentages of the total wind project cost. So typically a 300MW project will have development costs running at about $1 \%$ of the total. For the Hualapai Wind Project of 200MW capacity my preliminary estimate of the development costs is about $1.1 \%$ of the project cost for an estimated cost of about $\$ 3.4$ million. A typical breakdown of this estimated costs for the 200MW Hualapai Wind Project are provided in a spreadsheet format (Exhibit K). Note that development costs can vary significantly by wind project depending on the location and many other factors.

\section{b. Construction Costs}

A schedule for the high-level calculation of the various components of total installed costs per $\mathrm{kW}$ of capacity for the Hualapai 200MW Wind Project has been provided (Exhibit L). The wind turbine cost assumptions are the largest component of the project's total cost, typically representing about 75$80 \%$ of the total cost of a wind project. The assumption for turbines below is based on a recent non-binding quote from the manufacturer of the GE-1.6xle/82.5. The GE 1.7/100 meter rotor diameter turbine is the best performing turbine for the Hualapai site. The report uses the quote on the GE- 1.6/82.5 meter turbine as the basis for the calculated assumed cost for the GE 1.7/100 meter turbine. Most of the Balance of Plant (BOP) costs are assessed as a percentage of total project costs with the exception of the foundations/access roads and the transmission/interconnection. Foundation/access road construction and the transmission/interconnection costs for the Hualapai Wind Project are the more costly components of the project and higher than typical. Therefore, MJH Power has estimated the likely cost of foundations and the roads for 80 meter hub height GE-1.7/100 turbines based on a site visit and the existing geotech investigation of the site for a 31 mile 6 inch water line and used preliminary Power Engineers estimates for the transmission/ interconnection as incremental costs over typical . 
MJH power has estimated the costs for environmental and cultural monitoring during construction and avian \& bat mitigations costs based on the recently completed Perrin Ranch Wind Project. The preliminary total installed cost thru financing is estimated at about $\$ 325,821,914$ or about $\$ 1,629 / \mathrm{kW}$.

\section{c. Decommissioning Costs}

Typically $5 \%$ to $10 \%$ of construction costs. If owned by the Tribe, these costs will fall upon the Tribe. Ideally, an escrow account is set up to receive monies, usually a portion of the annual profits, over the life of the wind farm ( 20 to 30 years) to ensure funds are available to decommission the project

\section{Preliminary Project Proforma Summarized}

A preliminary project proforma model (Exhibit M) has been prepared inputting specific available information applicable for the Hualapai Wind Project Where specific information was not available estimates were based on MJH Power's experience in developing and operating wind projects over the past thirteen and $1 / 2$ years. This preliminary proforma model fairly represents the project and can be used to determine the Purchase Power Agreement (PPA) needed pricing levels for various scenarios. The scenario attached reflects current lower market estimates of PPA pricing influenced by low natural gas prices with an extension of the Production Tax Credits (PTCs) at their current $\$ 22 / \mathrm{MWh}$ rate. This PPA pricing does not reflect what may be possible with a Minority Business Enterprise (MBE) Southern California PPA or with creative partnering between potential developers and the Tribe. The industry at large also would consider the attached proforma as only marginally attractive to potential developers unless they can obtain higher PPA pricing like with a MBE incentive and/or through creative financial partnering with the Tribe. If the Government Grant Program (GGP) was to be extended that would make the project more attractive than using the PTCs. GGP provides a better incentive to developers than using the PTC for wind projects below about 33\% Net Capacity Factor.

\section{Preliminary Project Business Plan}

Squire Sanders has prepared an introduction to types of financing commonly used for developing renewable energy projects and provided information on potential deal structures commonly used with renewable energy developers (Exhibit N). Squire and Sanders has also provided a chart titled Energy Development Resources for Tribal Lands for DOE and other federal funding for tribes developing renewable energy. This information may be helpful to the Hualapai Tribe in understanding financing options and working with potential wind developers for possible partnering arrangements for moving the project development ahead.

Given the changes in the market place, notably the continued lowering of PPA prices during the feasibility study caused by increasingly plentiful natural gas in the marketplace as noted in Section 7 , a revised financial model was developed by First American Financial Investors and appears in 
Appendix N. The wind farm appears be financially feasible with PPAs priced in the $\$ 50$ to $\$ 60$ per megawatt range.

Under the primary authorship of Doug C. MacCourt, Ater Wynne, LLP, the National Renewable Energy Laboratory (NREL) and the Alliance for Sustainable Energy, LLC prepared in June of 2010 a handbook for Tribes titled "Renewable Energy Development in Indian Country: A Handbook for Tribes." This handbook provides an introduction to Tribes for energy development on their lands as well as creative partnering arrangements that can be pursued with interested potential developers. It is recommended that a workshop for the Tribe be conducted using this "Handbook" along with discussions on the work and feasibility studies that have been completed to date. The Handbook is Attached to this report ( Attachment -1)

\section{Next Steps}

A major next step needed for the development of this project is to make progress on power sale opportunities for the electrical power projections for this $200 \mathrm{MW}$ Hualapai Wind Project. The recommended major next step is to get a qualified developer on board who would bid to a utility or negotiate a PPA to make the project viable for financing. Qualified developers that have access to Southern California utilities such as San Diego Gas \& Electric, Southern California Edison and Los Angeles Department of Water and Power (LADWP) may be capable of brokering a PPA using Minority Business Enterprise (MBE) opportunities or other creative partnering agreements with the Tribe. It is recommended that the Tribe use its available resources to focus on getting a qualified developer on board who would bid to a utility or negotiate a PPA to make the project viable for financing and potential creative partnering with the Tribe as discussed in Exhibit $\mathrm{N}$.

On June 20, 2012, the Hualapai Renewable Energy Program was selected for inclusion into the Strategic Technical Assistance Response Team (START) Program. START is comprised of DOE and National Renewable Energy Laboratory (NREL) experts in renewable energy project development to help Tribe's achieve their goals of energy self-sufficiency, sustainability, and economic competitiveness. START will work directly with the tribal community-based project team and tribal legal/and finance specialists to further develop market feasibility, due diligence research, resource analysis and documentation; pre-development work to prepare site control, pre-qualify purchase power agreements, and produce a permitting plan. The end result of this assistance will be a prepared pre-development package that can be used in support of the request for proposals (RFP) process and/or similar and appropriate due diligence effort, financing, and construction of the renewable energy project on tribal lands and/or with tribal participation. 


\title{
Exhibit A
}

\section{Hualapai Wind Project Data Check Sheets}

\author{
(MJH Consulting \& Hualapai Planning Department)
}




\section{Hualapai Wind Project Site Evaluation Data Check Sheets Index}

Location - Mohave County Arizona on Hualapai Tribe Reservation

$$
\text { Client - } \text { Hualapai Tribal Nation }
$$

1.0 GENERAL GEOGRAPHICAL DATA

2.0 METEOROLOGICAL DATA

3.0 ENVIRONMENTAL CONSIDERATIONS

3.1 PROPERTY HISTORY

3.2 SITE LAND CONTROL

3.3 ENVIRONMENTAL RESTRICTIONS

4.0 PERMITTING REQUIREMENTS

4.1 GOVERNMENTAL RESTRICTIONS

4.2 ZONING ISSUES

5.0 SPECIFIC CONSTRUCTION CONSIDERATIONS

5.1 SITE PREPARATION

5.2 SITE ACCESS PROVISIONS

5.3 UNLOADING AND LAYDOWN STORAGE AREAS

5.4 UTILITIES AND TEMPORARY FACILITIES

5.5 LOCAL MATERIALS, SERVICES AND

SUBCONTRACTORS

5.6 LOCAL CONDITIONS AND REGULATIONS

5.7 MISCELLANEOUS FACILITIES

6.0 ELECTRICAL INTERCONNECTION - PPA OFF TAKE

6.1 INTERCONNECTION POINT

6.2 PPA OFF TAKE REQUIREMENTS

7.0 PRELIMINARY SITE LAYOUT

7.1 WTG LOCATIONS

7.2 ROADS

7.3 ELECTRICAL COLLECTOR SYSTEM 


\subsection{GENERAL GEOGRAPHICAL DATA}

1.1 Describe the location of the site in relation to the nearest city, main highway, nearest railroad station, nearest airport and nearest harbor. Provide directions, distances and city population.

The nearest city is Peach Springs, AZ 86434, the Hualapai tribal capital, population is 1,090 . The nearest large city is Kingman, $A Z$ 86401 \& 86402 , population is 28,068 . The nearest Interstate is $1-40$ about 30 miles to the south.

1.2 Describe site accessibility, existing roads if any, is it accessible for heavy equipment?

The Buck and Doe Road, Tribal Route 1, runs generally north to south through the overall site areas from the north at County Hwy 261 (Diamond Bar Road) to Route 66 to the south about 3 miles to the west of Peach Springs. Tribal Route 1 is accessible for heavy equipment. Will need some additional gravel and minor grading work in some areas. About 55 miles of roads will need to be constructed to access the rolling hills and ridgelines where the turbines are located.

1.3 Where is the nearest railroad siding in relation to the site?

There are private railroad sidings in Kingman and Nelson with spur access to BNSF's main east-west rail lines.

The Nelson siding is closest to the project area. The route to the project area would be westerly via Hwy 66 for about 8 miles and then north on BIA Route 1(Buck and Doe Road) for 25 miles to reach the lower array and another 19 miles to reach the Grand Canyon West MET Tower at the northern end of the upper array. About 52 miles in total.

From the Kingman Airport Rail Siding to Buck and Doe Road in Peach Springs is about 40 miles; from there north on BIA Route 1 for 25 miles to reach the lower array and another 19 miles to reach the GCW MET Tower at the northern end of the upper array. About 84 miles in total.

From the Kingman Airport Rail Siding to Antares Road 14 miles to the northeast, then 32 miles north on Antares Road to Pierce Ferry Road, 6 miles north on Pierce Ferry and 18 miles east on Diamond Bar Road to the Grand Canyon West MET Tower at the northern end of 
the upper array. Some 9.5 miles of Diamond Bar is currently unpaved. The Hualapai Tribe will be contracting for road improvements in 2013 to provide a continuous paved access to Grand Canyon West. About 70 miles in total. Antares Road is unpaved for 31 of the 32 miles; however, Mohave County does maintain the road to a native material standard. This route has the best quality roads unless they are wet.

An alternate route would be from the airport west through the City of Kingman via Stockton Hill Road approximately 45 miles to Pierce Ferry Road. There are side streets available such as Gordon Avenue, that could be used to avoid moving large components on trucks through the central business district of Kingman. This route would provide the first 55 miles on paved roads.

1.1.1 What are the weight and size limitations on this spur and mainline? 100 tons per standard railcars with option for special heavy duty cars for over 150 tons. BNSF mainline can carry the weight and size limitations are not expected to be an issue.

\subsubsection{Is it a private siding?}

Both are private sidings. The siding in Kingman has excellent facilities and sizeable areas for temporary storage and is idle. The siding in Nelson is heavily used for shipments out of limestone and its lower usage period is in the winter which may be an option for further investigation.

1.2 Is the site level and clear? If not, describe terrain and surface, brush, wooded, forest?

Complex terrain with rolling hills, ridgelines and canyons. Covered with low desert shrubs, sage and pinyon-juniper trees 5-15 feet in height.

1.3 Is a detailed topographic map available covering the boundaries of the site? The 8-12 Wind Resource contains topographic maps of the project areas. However, those maps are not high resolution. Higher resolution maps will be needed for further development.

1.4 Will the general topography of the site present drainage problem, flooding potential?

Not expected to be a problem for this site with turbines located on the rolling hills and ridgelines. 
1.5 What soils does the site have? Sand, sandy, loam, clay, hard rock, marsh, boulders?

Surface soil to 2-3 feet varies from clayey gravel with sand and some cobbles to weathered bedrock and limestone as you go deeper.

Geotech report available. See Exhibit C.

1.6 Location of any existing wells, any previous excavations, any mines, etc? The main water source for the project will be from the well at Westwater that feeds the 6-inch pipeline to Grand Canyon West. There are some other developed wells along the pipeline route that are no longer in use for the pipeline and dedicated to cattle and wildlife use. The Hualapai Natural Resources Department will have information on all wells on the Reservation. There is a gravel pit near the GCW MET Tower on Buck and Doe Road.

1.7 Is a soil report available, soil borings taken, if so obtain copies.

Yes, a geotech investigation report dated 5/29/09 for 31-mile long 6inch water pipeline recently constructed mostly along the Buck and Doe Road. 66 test soil borings taken down to 12 feet along the water pipeline route.

1.8 What is the elevation of the water table?

Not an issue for this site as most of the turbine locations are on the rolling hills and ridgelines.

1.9 What is the elevation of the plant above sea level? Varies from 4,800-6,100 feet.

\subsection{METEOROLOGICAL DATA}

Rich Simon's V-Bar team have been conducting a wind resource assessment for the Project Sites since December 2005 and have over 6 1/2 years of wind data from 2 active met towers in the project area. Average wind speeds for the two met towers at GCW and Clay Springs at a projected hub height of $80 \mathrm{~m}$ is from 6.4 to 6.8 meters/sec. V-Bar has prepared a GE-1.7/100 turbine array consisting of 118 turbines for a 200.6 MW name plate capacity wind project. This wind array has an annual gross capacity factor of $33.73 \%$

2.1 If no meteorological assessment of the site answer 2.2 thru 2.12. See Wind Resource Report 8-12, Exhibit D. 
2.2 What is the yearly rainfall?

Less than 20 inches

2.3 What is the severity and frequency of storms?

The area experiences violent thunderstorms during the "Monsoon

Season" from July through September. These storms can be extremely violent.

2.4 Is there a rainy season? What months?

July through September. Also, winter rains from the Pacific provide more steady late Fall and Winter rain with snow fall occurring several times per year

2.5 During the past 20 years what were the minimum and maximum temperatures?

Maximum of about 100 degrees F, Minimum Near 10 degrees F

2.6 What is the depth which frost is to be used for any building design? 4 feet

2.7 Prevailing wind direction?

Southwest

2.8 Maximum Wind velocity

Gusts of 39 Meters per Second at 30 meters above grade were recorded at the Grand Canyon West MET Tower in January of 2012. The GCW site has consistently recorded the highest gusts compared to the other sites. Peak gusts are typically recorded in November through January. Peak 2-second gust data is available form all sites since 2005 under the MaxV Tab of the Hualapai 8-12 Wind Resource Report.

2.9 Are there any unusual atmospheric conditions which would require extraordinary precautions? (Examples, salt spray corrosive air requiring galvanizing or blowing sand for coatings.)

$\mathrm{NO}$

2.10 What wind resource assessment has been done? If any, obtain results. The Hualapai Tribe has been collecting wind data and V-Bar has been performing the analysis for the Project Sites since December 2005. We have over $61 / 2$ years of wind data from 2 active met Hualapai Wind Project Data Sheets Page 5 of 17 
towers in the project area. Average wind speeds for the four active met towers at a projected hub height of $80 \mathrm{~m}$ is from 6.4 to 6.8 meters/sec. The Hualapai wind resource is summarized in the Hualapai 8-12 Wind Resource Report (Exhibit D). The Tribe has raw data files available, however interpretation of the data is best performed by an analyst that is familiar with the local conditions.

2.11 Is this a complex wind site or fairly uniform wind flow across the site, no obstructions?

The wind is fairly uniform with dominant flows from the Southwest. The surface is scrub brush and low growing pinon and juniper trees.

2.12 Any existing or planned met towers for the site? If existing get GPS coordinates or plans for installing and GPS coordinates for each of the planned locations.

There are two 50-meter met towers in the project area on the west side of the Hualapai Reservation.

The Grand Canyon West MET Tower has been in place since October 2006. Lat - 35deg 54.484' N, Long - 113deg 53.768' W, Elev 1764'

The Clay Springs MET Tower has been recording since May of 2011. Lat - 35deg 44.827' N, Long - 113deg 50.637' W., Elev 1642'

\subsection{ENVIRONMENTAL CONSIDERATIONS}

SWCA Environmental Consultants have completed an initial environmental and permitting Critical Issue Analysis for renewable energy development on the Hualapai Reservation. SWCA also conducted an Avian Use Assessment (eagle and raptor nest survey) and the first year of a multi-year Fall Raptor Migration Study.

\subsection{Property History}

3.1.1 Has an environmental review or assessment been conducted for these properties?

A Phase 1 NEPA survey (Critical Issues Analysis and Permitting Assessment) was completed in 2012. Exhibit J. 
3.1.2 Who are the current owners and occupant, or operator of the properties.

The project area is entirely within the boundaries of the Hualapai Indian Reservation. The wind turbine arrays are mostly within an area designated as Cattle District 1 with a very small area in Cattle District 2

3.1.3 What are the current and previous uses of the properties? Cattle grazing and open range

3.1.4 Who are the past owners and occupants or operator of the properties?

The Hualapai Reservation is held in trust for the Hualapai Tribe by the federal government. The Reservation was established in 1883. Ancestral use of land is from the preColumbian period.

3.1.5 What are the current and previous uses of the adjacent properties? There are 10 Sections of land owned in fee simple by the Hualapai Tribe that are used by Hualapai tribal members for cattle grazing. Other adjacent lands to the west of the project area are controlled by the Bureau of Land Management and private owners. Federal uses are generally grazing leases.

3.1.6 Has the site or any adjacent property been used for industrial, manufacturing, oil or gas production, or mined?

Not aware of any occurrences

3.1.7 Are there, or have there been any disposal facilities, landfills, dump sites or facilities involving hazardous waste within two miles of the site?

Not aware of any occurrences

3.1.8 Any federal, state, or local agency investigated or cited or involved on the property for violation of environmental law?

Not aware of any occurrences

3.1.9 Any public agency listed the property requiring environmental cleanup?

Not aware of any occurrences

Hualapai Wind Project Data Sheets Page 7 of 17 


\subsection{Land Control for Site Properties}

3.2.1 Is the site land leased, tied up with options, or has it been purchased?

Active grazing leases to tribal members. However, District Number One is not currently in use.

3.2.2 Obtain copies of land control agreements.

This needs to be researched and documents produced at some point in the future. (See attached grazing lease).

3.2.3 Specifics on royalty payments if leased, terms, and any landowner requirements or restrictions (i.e., no hunting or fire arms, speed limits, etc.)

Consult with Hualapai Natural Resources Dept. to identify restrictions

3.2.4 Egress and ingress issues with property owners, locked gates for cattle, etc.

There are no ingress or egress restrictions on the main access road (Buck and Doe) other that weather or maintenance related closures. There is a boundary gate across Clay Springs Road at the Reservation boundary that is kept locked to prevent cattle from leaving the grazing area. However, non-Hualapai members must obtain permits to travel this and other roads on the Reservation.

\subsection{Environmental Restrictions}

3.3.1 Have any environmental studies been done?

Yes - Critical Issues Analysis and Permitting Assessment (CIA), Avian Use Study, Fall Raptor Migration Study Exhibit J.

3.3.2 Coastal wet lands - Is there evidence of: estuaries, salt marshes, sloughs, lagoons, or freshwater marshes (tidal zones) on subject properties?

$\mathrm{NO}$ - see CIA 
3.3.3 Inland wetlands - Is there evidence of: marshes, swamps, lakes, ponds, potholes, riparian habitats, or bottom land forests on subject properties?

$\mathrm{NO}$ - see CIA

3.3.4 If wetlands are present, is there any indication the wetlands may be a habitat for threatened or endangered species of wildlife or plants? $\mathrm{NO}$ - see CIA

3.3.5 Flora - any rare or endangered plants on subject properties? Refer to CIA for detail

Fauna, any endangered wildlife or migratory wildlife restrictions on subject properties Refer to CIA for detail

3.3.6 Avian issues (any raptors, sage grouse, seasonal mating or flyway areas where construction is restricted?) on subject properties? Refer to CIA. Avian Use Study and Fall Raptor Migration Study

3.3.7 Any bat issues or restrictions on subject properties? Refer to CIA for detail

3.3.8 Any other environmental restrictions on subject properties Refer to CIA for detail

\subsection{PERMITTING REQUIREMENTS}

\subsection{What are the Governmental Restrictions?}

4.1.1 US Forest Service - No

4.1.2 National Parks/Monuments - Grand Canyon National Park

4.1.3 BLM - refer to section 1.4, page 7 of CIA

4.1.4 State Lands - refer to section 1.4, page 7 of $\mathrm{ClA}$

4.1.5 Military Exclusion Zones - No

4.1.6 Military height restriction zones or radar interference issues - No

4.1.7 FAA/Airport issues with landing patterns, etc - refer to section 1.4, page 19 of $\mathrm{ClA}$

\subsection{What are the local zoning issues for these properties?}

4.2.1 What is the existing permitted use for these properties? Cattle Grazing and Open Range 
4.2.2 Will a zoning change be necessary? Procedure?

There are no Zoning Ordinances on the Hualapai Reservation.

\subsubsection{Any wind farm visibility issues?}

There are tribal members that have expressed that they would not like to see the large wind turbines.

Excerpt from CIA Executive Summary:

Visual resources are considered a high risk for the Grand Canyon West and BLM Wind North wind project areas where the BLM manages lands as Visual Resource Management (VRM) Class II, the objective of which is to retain the existing character of the landscape. The level of change to the characteristic landscape for VRM Class II should be low. The effect of wind energy facilities on the landscape is a known issue of concern and VRM Class II is not considered compatible with wind energy development. Although not considered a fatal flaw, a Resource Management Plan amendment would be required to change the VRM class before completing the permitting process, which can lengthen the permitting process and add additional complexities.

\subsubsection{Any NIMBY issues, local opposition groups, etc.?}

There are tribal members that have expressed that they would not like to see the large wind turbines. Will transmission line/s be permitted?

Yes, The Project will need to get rights of way recorded with the Bureau of Indian Affairs. Once off of the Reservation, rights of way will be required for any BLM crossings.

\subsubsection{Any noise, ice flicker or ice throw restrictions?} $\mathrm{NO}$

\subsubsection{Any setback requirements from wind turbines?}

The Hualapai Tribe has adopted the International Building Code for the Reservation. Otherwise, here are no setback requirements in the Hualapai building ordinances.

\subsubsection{Are public hearings required?}

Public hearings and notice are required. . The Hualapai Tribal Constitution requires that leases of more than $\$ 50,000$ value annually must be submitted to a referendum vote. 
4.2.8 Any archaeological issues for these properties?

Excerpt from CIA Executive Summary:

Cultural resources are also considered a medium risk for all

six proposed project areas. Although the presence of cultural

resources is not expected to affect the permitting and

implementation of renewable energy facilities, inventories, reports, consultation, and agency review time can add considerable cost and time to the process.

Need to perform detailed site investigation (Level 3) based upon more precise locations of the wind turbines.

\subsection{SPECIFIC CONSTRUCTION CONSIDERATIONS}

\subsection{Site Preparation, Roads and Foundations}

5.1.1 What WTG supplier, MW size and number of WTGs are being considered?

Initial plan is for 118 GE-1.7/100 turbines

5.1.2 What are the WTG supplier's construction and transport requirements?

Initial assessment done with GE's specs in mind and rail transport.

5.1.3 What are the estimated miles of new roads that will have to be built? Initial construction assessment estimate is for about 55 miles of new roads.

5.1.4 What is the estimated percentage of the roads that will require cut and fill?

Estimate about $50 \%$

5.1.5 What estimated percentage of the WTG foundation excavations will require cutting and slopes for access?

Estimate about 50\%

5.1.6 What estimated percentage of the WTG foundations if any will require rock socket and blasting techniques?

Estimate about $70 \%$

Hualapai Wind Project Data Sheets Page 11 of 17 
5.1.7 Any special WTG foundation requirements for high ground water such as the need for piles for the foundations?

None expected

5.1.8 How far must excess dirt be hauled for disposal?

It is anticipated that cut and fill quantities will roughly balance in the project area roads and foundation work negating the need for export.

5.1.9 If fill material is required, where is the nearest source and type of material?

Fill material is available in the Project area. There is a gravel quarry on Buck and Doe Road near the northern extent of the turbine array.

5.1.10 Are there any overhead or underground obstructions such as wires or pipes, etc? If so, who and how will these obstructions be removed?

There is a 6-inch water pipeline running south from Grand Canyon West near the project area. Turbine infrastructure will have to avoid this pipeline. There are no electrical or gas lines in the project area, however, regional electrical and gas transmission organizations may have lines in the area that should be identified and mapped.

5.1.11 What right-of-ways exist with the site properties or easements for existing or future power lines, pipelines or access roads for adjacent owners.

Buck and Doe Road runs north and south through the project area. There is a 6-inch water pipeline running north and south from Grand Canyon West near the project area. There are existing access roads branching off of Buck and Doe Road that will need to maintained for access hunting, cattle grazing, and other tribal uses.

5.1.12 Will temporary or permanent fencing be required? If so what areas? Will need to be addressed with the Tribe, not expected to be a problem, contractor may want storage and some other areas fenced for security. 
5.1.13 Where will the materials for the temporary and permanent roadways be obtained (crushed rock)? Fill material is available in the Project area. There is a gravel quarry on Buck and Doe Road near the northern extent of the turbine array.

5.1.14 Where will the aggregate be obtained for the concrete batch plant that may be needed, depending on size of the project and number of large turbine foundations?

There is a gravel quarry on Buck and Doe Road near the northern extent of the turbine array. Access to water for the batch plant will need to be arranged with Tribe from the six inch pipeline or storage tank location near the northern end of the turbine array.

5.1.15 Rank from 1-10 the construction difficulty this site poses ( 1 best, 10 is worst)

Rank this site as a 6 for construction difficulty.

\subsection{Access and Transportation Plan for WTGs to Site}

5.2.1 Has the developer or WTG supplier prepared a transportation plan or done any preliminary transportation planning? WTG supplier not yet selected.

5.2.2 Has a delivery point for hand off of the WTG components to the construction contractor been worked out? TBD

5.2.3 What are the loads and clearance limits on bridges, culverts, tunnels, power lines, etc. been determined for the access route to the site? TBD

5.2.4 Do any of the roads, bridges, etc, on the access route to the site require reworking? Whose responsibility will this be? Any governmental agency? TBD 


\subsection{Unloading, Lay Down and Storage}

5.3.1 Has a plan for material receiving been developed? If so, obtain a copy.

TBD

5.3.2 What facilities are planned for unloading and loading WTG components?

TBD

5.3.3 If rail transport is planned to the site and a railroad siding that can be used is there area around the railroad siding that can be used for the offloading, lay down and storage of WTG components? Yes, this is not expected to be a problem. See 1.1 above and Project Site Evaluation for Construction; Exhibit B, for more specifics on railroad siding availability to project.

5.3.4 What areas of the site properties can be used for lay down and storage?

Lay down area should be limited to minimum numbers of acres required around the turbine base.

\subsection{Utilities and Temporary Facilities}

5.4.1 Are there any existing buildings available for temporary construction use?

Not in the project area. There may be something available at Grand Canyon Resort 10 miles north of the project area.

5.4.2 Is land on site or adjacent land available for construction facilities? Yes, will need to discuss with Tribe

5.4.3 List recommended telephone service, telex and internet for construction?

There is cellular phone service in the project area. There is a cell phone tower at the GCW MET tower site. 
5.4.4 Is electricity available for construction purposes? If so where do we tie in? When will it be available? KW available, voltage, 3 phase? NO. May require portable power or for transmission interconnection and $34.5 \mathrm{KV}$ collector system to be installed first.

\subsection{Local Materials, Services and Subcontractors}

Are local contractors available for the following services?

All listed construction services and contractors are available regionally. Kingman has most, if not all required services available locally (less than 100 miles from site). NextEra built the Perrin Ranch Wind Farm at Williams Arizona, approximately 120 miles east of the project area. The Kingman wind farm was constructed by RMT Energy approximately 70 miles southwest of the project

\subsubsection{Earthwork}

5.5.2 Roads

5.5.3 Concrete Batching

5.5.4 Large cranes and qualified crews for WTG component erection

5.5.5 Industrial buildings

5.5.6 Electrical works, including collector system and transmission lines

5.5.7 Fencing

5.5.8 Capability of large ready-mix concrete, if any, in close proximity to site.

\subsection{Miscellaneous Construction Support}

5.6.1 Are machine-shop facilities available near the site?

Machine Shop services are available in Kingman 70 miles away

5.6.2 Are there first-aid facilities near by, ambulance, doctor, hospital?

First Aid Facilities are available at Grand Canyon Resort, 10 miles

from the north end of the Project area and in Peach Springs.

5.6.3 Is there suitable housing available for construction staff and crews?

Temporary housing should/could be available at Grand Canyon Resort

5.6.4 What local public transportation services are available, if any? Remote area, none are available 
5.6.5 Where will contractors be required to dispose of unburnable trash? Construction debris and Trash must be removed from the project area and disposed of at regional facilities near Kingman.

5.6.6 What provisions for contractors to dispose of burnable trash?

$\mathrm{TBD}$

5.6.7 Is there any item or characteristic of these properties or the surrounding property that may create an unusual construction problem?

None anticipated

5.6.8 Are there any environmental, regulatory or zoning restrictions on the hours that construction work can take place on this site? There are construction hour restrictions, however those may be negotiated due to remote location.

\subsection{ELECTRICAL INTERCONNECTION - PPA OFF TAKE}

\subsection{Electrical Interconnection Point}

6.1.1 Has the electrical interconnect point been established?

Yes, queue position number 2012-G30 with WAPA for POI on the Mead-Peacock 345KV transmission line west of the Reservation near Clay Springs at Antares Road.

6.1.2 What will be the interconnection tie-in voltage $(230 \mathrm{KV}$ or $500 \mathrm{KV})$ ? $345 \mathrm{KV}$

\subsection{PPA -Off Take Requirements}

6.2.1 Have the PPA-Off Take requirements been established? NO

6.2.2 What requirements are levied on the construction contractor? No PPA based requirements at this time. Note that all construction contracts are subject to a the Tribal Employment Rights Ordinance (TERO) tax. 
6.2.3 Any unique contractual requirements in the PPA-Off Take Agreement?

TBD

\subsection{PRELIMINARY SITE LAYOUT}

7.1 Has a preliminary layout been developed for the WTG locations?

Yes A turbine array has be developed by $\mathrm{V}$-Bar for 4 different scenarios.

On Hualapai lands only:

200-MW array for GE-1.7/100 turbines with 80-m hub height

200-MW array for Acciona AW-116/3000 turbines with 92-m hub height

\section{On Hualapai and adjacent BLM lands:}

284-MW array for GE-1.7/100 turbines with 80-m hub height

456-MW array for Acciona AW-116/3000 turbines with 92-m hub height

See the Hualapai Wind Farm Development Analysis, Exhibit F for detailed turbine GIS location data.

7.2 Has a preliminary road layout been developed for the preliminary WTG locations? If so obtain a copy.

No

7.3 Has a preliminary design and routing of the Collector System been integrated with the preliminary WTG layout and preliminary road layout. If so, obtain a copy.

No

7.4 Has preliminary design of the Interconnection \& Substation Facilities been completed and integrated with the collection system preliminary design and preliminary collector system design? If so, obtain a copy.

Yes. See Transmission and Interconnection Feasibility Report ( Power Engineers) Exhibit G and WAPA Mead-Peacock 345kV Interconnection Application, Exhibit $\mathrm{H}$. 


\title{
Exhibit B
}

\section{Project Site Evaluation for Construction}

\author{
(MJH Consulting)
}




\section{Initial Site Visit and Constructability Report for the Hualapai Wind Project}

\section{by Marion J. Horna, PE and Principal of MJH Power Consulting LLC}

The initial site visit was conducted on August 20, 2012 with Rich Simon Director of V-Bar doing the driving (4-WD) and leading the tour of the site. We came in from the west down US 93 from Las Vegas taking a left up Pierce Ferry Road (County Route 25) to Diamond Bar Road (County Route 291) going northeast into the Hualapai Reservation. We turned right on Tribal Buck and Doe Road (Indian Route 1) heading generally south toward Peach Springs through the wind project area.

Our route driving generally south down the Buck and Doe Road allowed us to take advantage of this road along with a few choice side roads to get good vantage points for viewing essentially all the prospective 118 turbine sites that V-Bar has incorporated into their October 7, 2012 turbine array. While terrain on the tribal lands is complex with relatively smooth rolling hills, ridgelines and canyons its does not appear too difficult for construction equipment to access the ridges where the turbines would be located from the Buck and Doe Road and improved existing side roads. I did not see any fatal flaws for access or constructability issues with any of the turbine sites on Hualapai Tribal Lands.

For the BLM sites to the west of the Hualapai tribal lands, there are not really any decent roads to access those areas, and the few roads that do exist require long, slow miles of driving off Buck and Doe Road so we did not get any closer in views. We got a reasonably good look from the distance, though, but not the detailed analysis that we were able to do for the tribal lands. While the constructability on the BLMs looked to be promising I could not confirm it from distance that we were from these areas and I did not do any further desktop investigation as I did for the turbine sites on tribal lands.

The amount of required new roads, I am estimating at about 55 miles all total, and the amount of cut and fill requirements for the roads and for the turbine foundations will increase the overall balance of plant construction costs. However, these costs will be somewhat offset by the excellent availability of onsite crushed rock for the roads and aggregate for the concrete batch plant that will be needed. I am estimating that about $50 \%$ of the roads up to the turbine locations and $50 \%$ of the turbine foundations may require cut and fill earthwork. I am estimating at this early stage that about $75 \%$ of the turbine foundations will require rock socket construction techniques with blasting.

During our site visit we observed the relatively recent installation of a 31 mile long 6 inch water pipeline along portions of the Buck and Doe Road. In subsequent follow up with Mark Randall I learned that a complete geotech investigation report was done for planning the construction 
work for this 31 mile long 6 inch water pipeline. I obtained a copy of that report and after reviewing it was able to determine that it would serve as an initial geotech report for the wind project. While the boring depths (down to 12 feet) are not deep enough for the turbine foundations and most will not be where turbines would be located, the information will be excellent for the road work and electrical collection system design. The report included a map of the regional geology and information on the subsoil conditions that allowed me to confirm along with the observance of several gravel pits that the site has excellent availability for the crushed rock needed for the roads and aggregate for the concrete for the turbine foundations. This report also allowed me to confirm the need for rock socket construction techniques for I estimate about $75 \%$ of the turbine foundations.

The Hualapai Tribe will need to confirm that water from the 6 inch pipeline or the storage tanks on site would be available for a source of water for the concrete batch plant and other construction water needs such as dust control, etc. The Tribe would be reimbursed typically for the roadbed and crushed stone aggregate and water used. This would all be covered in the project lease agreement or partnering agreement between the developer and the Tribe.

I have attached a completed 9 page listing of the check sheets that I use in my work for conducting wind project site evaluations. I have included references to the pipeline geotech report, to the environmental report/s, electrical interconnection, wind resource and array plans and reports. These check sheets include a lot of constructability and other needed information for the projects' construction work. Input from the Tribe is needed for some of the information as well as their confirmation to what I have stated.

BNSF has advised the use of two potential private railroad sidings. One is in Kingman near the airport, which BNSF is recommending as an excellent offloading location for the wind turbine and other large project components most economically shipped by rail. The other is located closer to the Project in Nelson for the Chemical Lime Plant's lime shipping. This siding is quite busy and tied up with lime shipments though something may possibly be arranged for off loading during winter when their lime shipments are reduced. Travel from the Nelson siding is about 10 miles to the southern boundary of the Project and 35 miles for the Kingman siding.

In summary after conducting a site visit and using maps of the terrain and available geotech studies I was able to conclude as follows. The majority of wind power projects requiring construction along rolling hills and ridgelines is challenging and will result in difficult earthwork conditions. However, these conditions are manageable provided that proper engineering work is performed and contractors experienced with this type of construction are utilized. In summary, this initial assessment did not identify any unusual issues specific to the site that may prevent or significantly hinder the construction of the Project. 


\title{
Exhibit D
}

\section{Wind Resource Report - Final - August 2012}

\author{
(V-Bar)
}




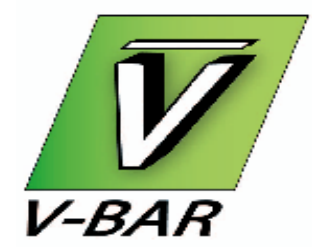

\section{Summary of Wind Measurements at the Hualapai Reservation Final Report with Data from 2005-2012}

Prepared by Richard Simon and Peter Stamus

V-Bar, LLC, 29 September 2012

This report analyses wind data collected on the Hualapai Reservation in northwestern Arizona. The wind monitoring program commenced in December 2005, and this final update analyzes data through August 2012. A companion report presents and evaluates wind farm array possibilities in Grand Canyon West/Clay Springs areas.

Our analyses are presented as a series of tabs, discussed below in sequential order.

Tower Info. Documentation is furnished for the five Hualapai meteorological towers: their location, exposure, logger and sensor types, boom lengths and orientations, and calibration data. This information was assembled from Echelon Environmental Energy and Harness Energy installation logs with on-site verification by R. Simon.

Here is a summary of the five towers, of which four were still operating at the end of August 2012:

\begin{tabular}{llcll}
\multicolumn{1}{c}{ Site } & Station & & Month & \\
Peach Springs & Number & Height $(\mathrm{m})$ & Installed & Removed \\
Blue Mountain & 7052 & 44 & Dec 2005 & $\ldots$ \\
Nelson & 7051 & 50 & Mar 2006 & Sep 2007 \\
Grand Cyn West & 7053 & 50 & Dec 2006 & $\ldots$ \\
Clay Springs & 7054 & 50 & Oct 2006 & $\ldots$ \\
& 7055 & 50 & May 2011 & $\ldots$
\end{tabular}

Maps. There is a regional map showing the Hualapai Reservation and reference anemometers. There are also topographic maps showing the detailed locations and exposures of the Hualapai met towers.

Ref V. Three long-term reference stations (Flagstaff, Grand Canyon, and Kingman airports ) are used to evaluate long-term winds at the Hualapai met towers. These airports measure winds at $10 \mathrm{~m}$ above ground, and they have 10-15 years of data.

All three airports were converted from cup to sonic anemometry in February 2007. On average, mean speeds with sonic anemometers are 4\% less than with cups. 
Page 1 of the "Ref V" tab gives the observed (cup or sonic) monthly mean wind speeds for the reference stations. Page 2 gives their "sonic-equivalent" mean speeds, wherein the cup-period means are decreased by $4 \%$.

Daily V. Daily mean wind speeds have been tabulated for the reference stations and Hualapai met towers. Daily correlation coefficients between the two categories of stations are also given. Grand Canyon airport is the best correlated to the four active Hualapai met towers, with an average daily correlation coefficient of 0.83 , which is considered good. Daily correlation coefficients average 0.77 to Kingman and 0.73 to Flagstaff, which is considered modest.

Mo Vbar. Monthly mean wind speeds are given for all Hualapai and reference stations. Composite monthly and annual mean wind speeds are computed at the bottom of this tab for the entire periods of record for Peach Springs, Grand Canyon West, Nelson, and their concurrent periods at the Grand Canyon airport, as well as Clay Springs and its concurrent period with Grand Canyon West.

Data recovery has been inconsistent over the course of the monitoring program, although it has been very good since May 2011. However, we do note that Nelson has a failed top anemometer, and both top anemometers at Peach Springs are now out of service (the 2nd failed in early March 2012).

L-T Vbar. Long-term mean annual wind speeds for the Hualapai sites are estimated with different methods appropriate to their data sets, as described individually for each tower in the tab. The underlying reference station was the Grand Canyon airport.

Here are the resulting long-term mean annual wind speed estimates in meters per second, with extrapolations to $80 \mathrm{~m}$ using site-specific shears:

\begin{tabular}{|c|c|c|c|c|c|}
\hline & Blue & Peach & & $\begin{array}{l}\text { Grand } \\
\text { Canyon }\end{array}$ & Clay \\
\hline Level (m) & Mountain & Springs & Nelson & West & Springs \\
\hline 10 & 4.19 & 4.60 & 3.95 & 4.83 & 3.88 \\
\hline 30 & 5.69 & 5.68 & 4.56 & 6.02 & 5.18 \\
\hline $40 / 44$ & 5.93 & 5.93 & $\ldots$ & $\ldots$ & 5.53 \\
\hline $49 / 50$ & 6.25 & $\ldots$ & 5.23 & 6.30 & 5.76 \\
\hline 80 & 6.79 & 6.57 & 5.68 & 6.70 & 6.41 \\
\hline
\end{tabular}

Long-term $80-\mathrm{m}$ winds decreased $0.01 \mathrm{mps}$ on average at the four active stations with the addition of May-August 2012 wind data.

Past reports have discussed some discontinuities in predicted long-term winds at Peach Springs. The reader is referred to our May 2011 report for details.

En Sims. Hourly mean wind speeds for the Hualapai met towers were extrapolated to $80 \mathrm{~m}$ using hourly-specific wind shears and compiled into separate wind speed frequency distributions. These distributions were scaled to integer annual mean wind speeds of 5-8 mps and normalized to single, 8760-hour years. Gross annual energy potential was simulated for the GE-1.7/100 and 
Acciona AW-116/3000 turbines using power curves with annual mean air densities of 1.01-1.02 $\mathrm{kg} / \mathrm{m}^{3}$, depending on site elevation.

Relationships between annual gross capacity factors in percent and annual mean wind speeds are summarized below:

for the GE-1.7/100...

\begin{tabular}{lllll}
\multicolumn{1}{c}{ Site } & $5 \mathrm{mps}$ & $6 \mathrm{mps}$ & $7 \mathrm{mps}$ & $8 \mathrm{mps}$ \\
Peach Springs & 21.61 & 32.93 & 43.92 & 53.52 \\
Nelson & 21.65 & 32.65 & 43.31 & 52.75 \\
Grand Cyn West & 21.46 & 32.06 & 42.41 & 51.59 \\
Clay Springs & 22.09 & 32.24 & 42.06 & 50.90
\end{tabular}

for the Acciona AW-116/3000

\begin{tabular}{lllll}
\multicolumn{1}{c}{ Site } & $5 \mathrm{mps}$ & $6 \mathrm{mps}$ & $7 \mathrm{mps}$ & $8 \mathrm{mps}$ \\
Peach Springs & 15.43 & 24.85 & 34.85 & 44.36 \\
Nelson & 15.61 & 24.84 & 34.51 & 43.69 \\
Grand Cyn West & 15.65 & 24.49 & 33.84 & 42.75 \\
Clay Springs & 16.09 & 24.67 & 33.56 & 41.99
\end{tabular}

Rose. Prevailing winds are south to southwest at all stations.

Off-Axis. Wake losses in annual energy between two turbines are shown for the Hualapai met towers as a function of their azimuth orientation and spacing. These tables can be used to help optimize turbine arrays.

Max V. Monthly maximum hourly mean wind speeds and peak gusts are given for all Hualapai meteorological towers. Through September 2012, the observed maximum hourly mean wind speed has been $29.2 \mathrm{mps}$; the peak gust $39 \mathrm{mps}$.

TI. Turbulence statistics are summarized for the 44-50 m levels of the Hualapai met towers. Mean turbulence intensities are roughly $0.11-0.14$ at all towers for wind speeds greater than 5 mps, except Clay Springs which is more turbulent.

Due to lessening effects of friction with height, turbulence levels decrease as one goes higher above ground. Hub-height turbulence will be less than that measured at approximately $50 \mathrm{~m}$. 
Meteorological Tower Information

Hualapai Reservation, Arizona

\begin{tabular}{|c|c|c|c|c|c|c|c|c|c|c|c|}
\hline \multirow[t]{2}{*}{$\begin{array}{l}\text { Time Zone } \\
\text { Magnetic Declination }\end{array}$} & \multicolumn{7}{|c|}{$\begin{array}{l}\text { Mountain Standard Time } \\
12^{\circ} \text { East (true north }=348^{\circ} \text { magnetic) }\end{array}$} & \multirow{2}{*}{$\begin{array}{l}\text { Boom } \\
\text { Orientation } \\
\text { Relative to } \\
\text { True North }\end{array}$} & \multicolumn{2}{|c|}{ Calibration Constants* } & \multirow[b]{2}{*}{ Units } \\
\hline & & Sensor Type & Model & $\begin{array}{l}\text { Serial } \\
\text { Number }\end{array}$ & Channel & $\begin{array}{c}\text { Sensor } \\
\text { Height }(m)\end{array}$ & $\begin{array}{l}\text { Boom } \\
\text { Length (in) }\end{array}$ & & Slope & Offset & \\
\hline Site Number & 7051 & Anemometer & NRG \#40 & --- & 1 & 49 & 43 & SSW & 0.765 & 0.35 & $\mathrm{mps}$ \\
\hline Site Name & Blue Mountain & Anemometer & NRG \#40 & --- & 2 & 49 & 43 & WNW & 0.765 & 0.35 & $\mathrm{mps}$ \\
\hline Installation Date & effectively March 2006 & Anemometer & NRG \#40 & --- & 3 & 40 & 43 & SSW & 0.765 & 0.35 & $\mathrm{mps}$ \\
\hline Logger Type & NRG Symphonie & Anemometer & NRG \#40 & --- & 4 & 30 & 43 & SSW & 0.765 & 0.35 & $\mathrm{mps}$ \\
\hline Logger Serial Number & 7550 & & & & & & & & & & \\
\hline Tower Height (m) & 50 & Wind Vane & NRG 200P & --- & 7 & 49 & 43 & $016^{\circ}$ & 0.351 & 16 & degrees \\
\hline Tower Diameter (in) & 6 & Wind Vane & NRG 200P & --- & 8 & 29 & 43 & $016^{\circ}$ & 0.351 & 16 & degrees \\
\hline Closest Town & Peach Springs, AZ & & & & & & & & & & \\
\hline Latitude (WGS84) & $35^{\circ} 35.914^{\prime} \mathrm{N}$ & Thermometer & NRG $110 \mathrm{~S}$ & --- & 9 & 3 & --- & North & 0.136 & -86.38 & ${ }^{\circ} \mathrm{C}$ \\
\hline Longitude (WGS84) & $113^{\circ} 12.942^{\prime} \mathrm{W}$ & & & & & & & & & & \\
\hline Elevation (m) & 1840 & & & & & & & & & & \\
\hline Site Exposure & near top of mountain, veg & tion consists of $\mathrm{s}$ & tered juniper & and shrubs & ess than 3 & tall & & & & & \\
\hline \multicolumn{12}{|c|}{ Site reconfigured September 2006} \\
\hline Site Number & 7051 & Anemometer & NRG \#40 & --- & 1 & 49 & 43 & SSE & 0.765 & 0.35 & $\mathrm{mps}$ \\
\hline Site Name & Blue Mountain & Anemometer & NRG \#40 & --- & 2 & 49 & 43 & WSW & 0.765 & 0.35 & $\mathrm{mps}$ \\
\hline Maintenance Date & 1 September 2006 & Anemometer & NRG \#40 & --- & 3 & 30 & 43 & SSE & 0.765 & 0.35 & $\mathrm{mps}$ \\
\hline Logger Type & NRG Symphonie & Anemometer & NRG \#40 & --- & 4 & 10 & 43 & SSE & 0.765 & 0.35 & mps \\
\hline Logger Serial Number & 1222 & & & & & & & & & & \\
\hline Tower Height (m) & 50 & Wind Vane & NRG 200P & --- & 7 & 49 & 43 & $016^{\circ}$ & 0.351 & 16 & degrees \\
\hline Tower Diameter (in) & 6 & Wind Vane & NRG 200P & --- & 8 & 29 & 43 & $016^{\circ}$ & 0.351 & 16 & degrees \\
\hline Closest Town & Peach Springs, AZ & & & & & & & & & & \\
\hline Latitude (WGS84) & $35^{\circ} 35.914^{\prime} \mathrm{N}$ & Thermometer & NRG $110 \mathrm{~S}$ & --- & 9 & 3 & --- & side & 0.136 & -86.38 & ${ }^{\circ} \mathrm{C}$ \\
\hline Longitude (WGS84) & $113^{\circ} 12.942^{\prime} \mathrm{W}$ & & & & & & & & & & \\
\hline Elevation (m) & 1840 & & & & & & & & & & \\
\hline Site Exposure & \multicolumn{11}{|c|}{ near top of hill, vegetation consists of scattered junipers and shrubs less than $3 \mathrm{~m}$ tall } \\
\hline Site Number & 7052 & Anemometer & NRG \#40 & --- & 1 & 44 & $65 \pm$ & South & 0.765 & 0.35 & $\mathrm{mps}$ \\
\hline Site Name & Peach Springs & Anemometer & NRG \#40 & --- & 2 & 30 & $65 \pm$ & South & 0.765 & 0.35 & mps \\
\hline Installation Date & 14 December 2005 & Anemometer & NRG \#40 & --- & 3 & 10 & $65 \pm$ & South & 0.765 & 0.35 & $\mathrm{mps}$ \\
\hline Logger Type & NRG Symphonie & Anemometer* & NRG \#40 & --- & $4^{*}$ & 44 & 65 & West & 0.765 & 0.35 & $\mathrm{mps}$ \\
\hline Logger Serial Number & 7789 & & & & & & & & & & \\
\hline Tower Height (m) & 44 & Wind Vane & NRG 200P & --- & 7 & 44 & $65 \pm$ & $360^{\circ}$ & 0.351 & 0 & degrees \\
\hline Tower Diameter (in) & 42 (triangular lattice) & Wind Vane & NRG 200P & --- & 8 & 30 & $65 \pm$ & $360^{\circ}$ & 0.351 & 0 & degrees \\
\hline Closest Town & Peach Springs, AZ & & & & & & & & & & \\
\hline Latitude (WGS84) & $35^{\circ} 33.281^{\prime} \mathrm{N}$ & Thermometer & NRG $110 \mathrm{~S}$ & --- & 9 & 4 & --- & North & 0.136 & -86.38 & ${ }^{\circ} \mathrm{C}$ \\
\hline Longitude (WGS84) & $113^{\circ} 23.714^{\prime} \mathrm{W}$ & Pyranometer & --- & --- & 10 & 4 & --- & North & --- & -- & $\mathrm{W} / \mathrm{m}^{2}$ \\
\hline Elevation $(\mathrm{m})$ & 1671 & & & & data start & December 2 & & & & & \\
\hline Site Exposure & \multicolumn{11}{|l|}{ munications to } \\
\hline
\end{tabular}


Regional Map of the Hualapai Met Towers and Reference Anemometers

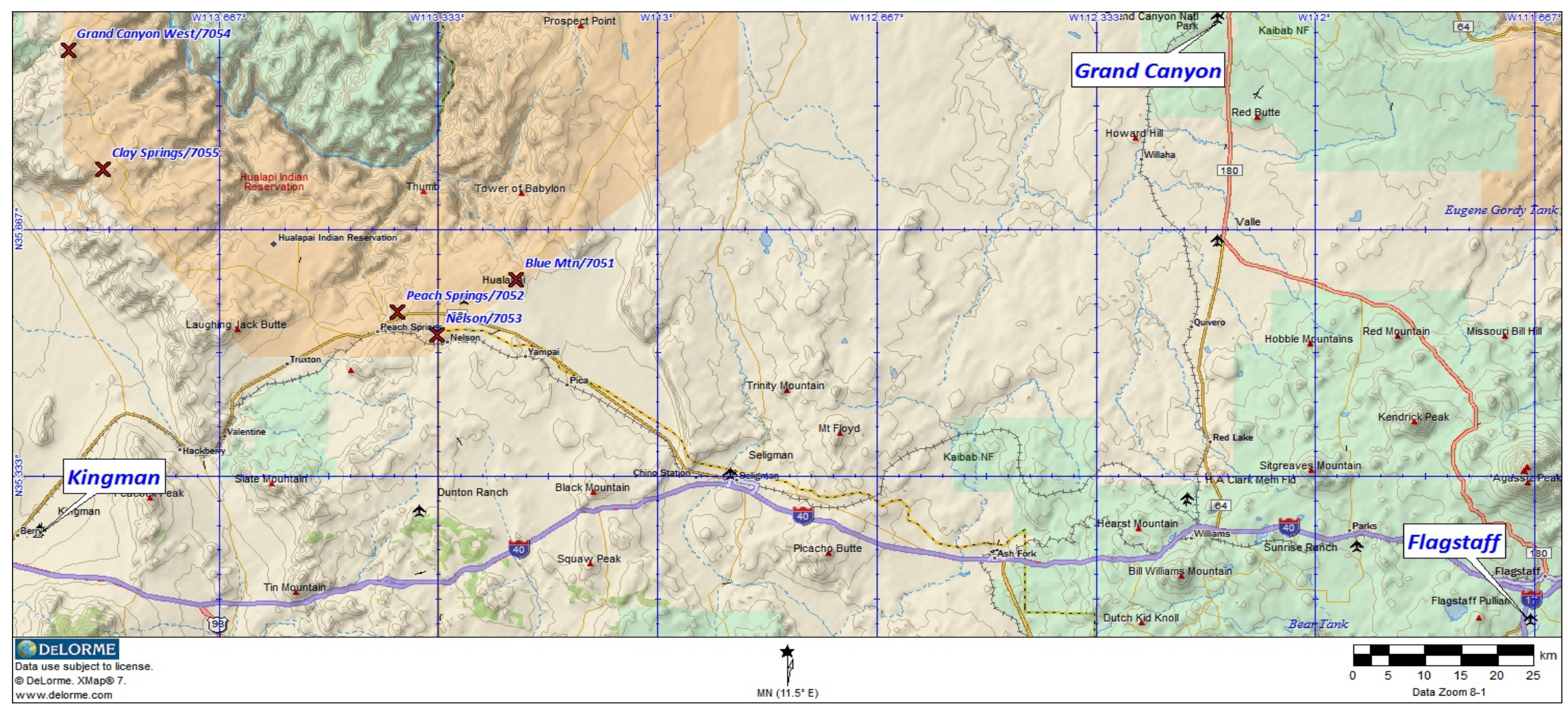




\section{Hualapai Meteorological Towers near Peach Springs}

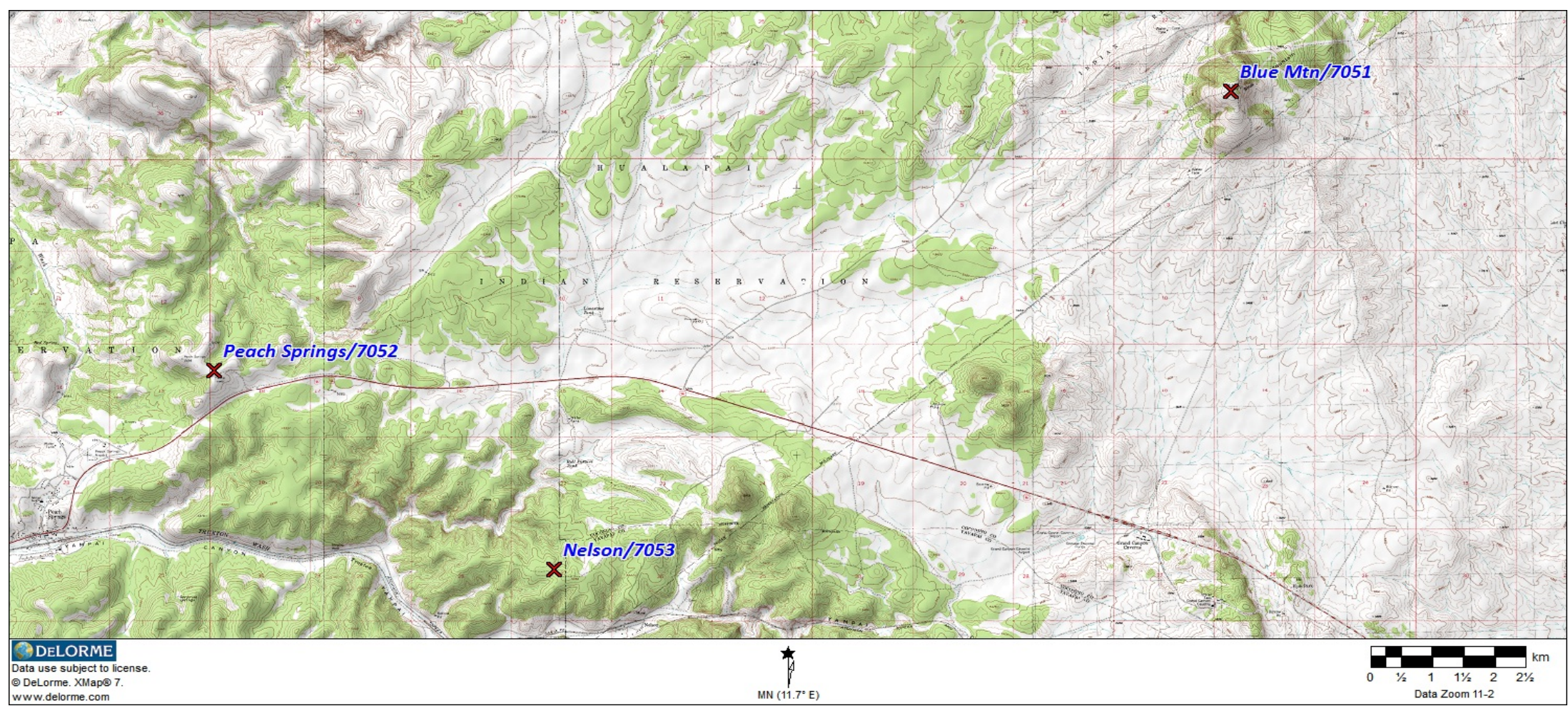




\section{Grand Canyon West Met Tower}

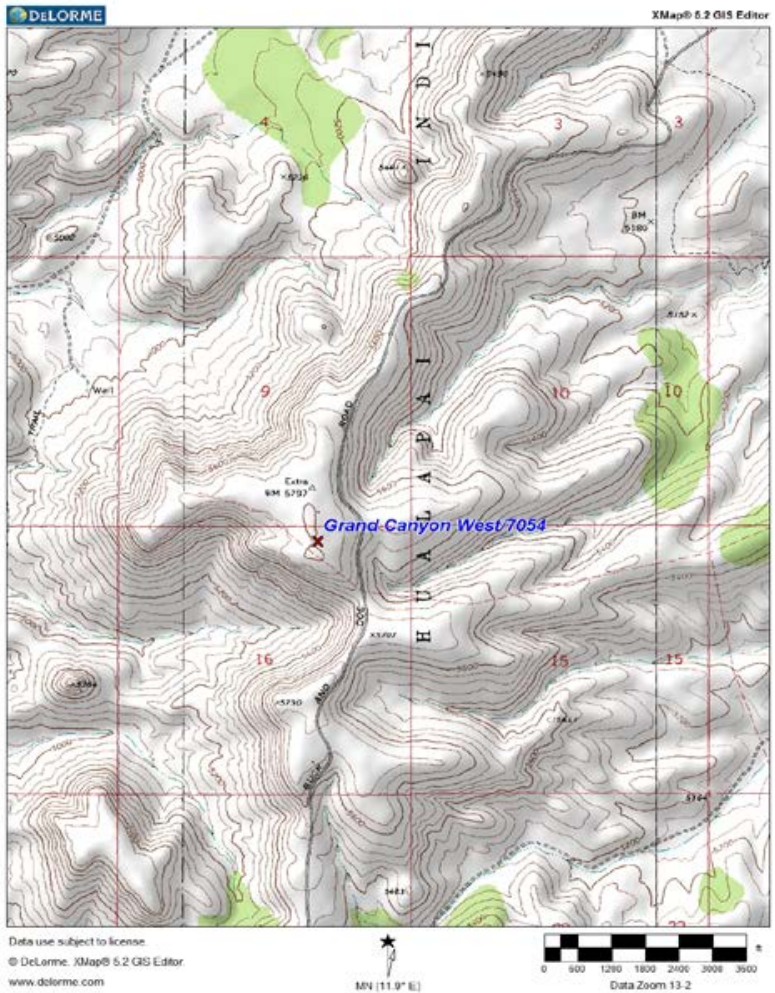


Clay Springs Met Tower

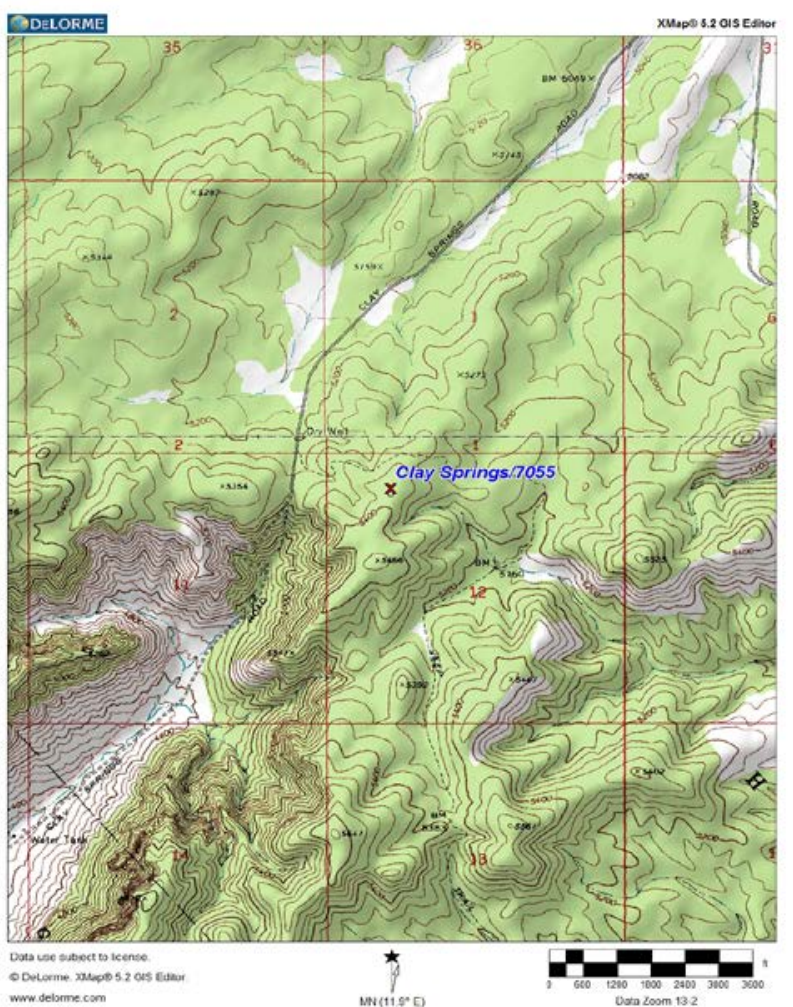


Observed Monthly Mean 10-m Wind Speeds (mps)

Flagstaff Airport, Arizona

ASOS Period Only

Latitude: $35^{\circ} 09^{\prime} \mathrm{N}$

Longitude: $111^{\circ} 40^{\prime} \mathrm{W}$

Sonic Conversion Date: 8 February 2007

Elevation: $2135 \mathrm{~m}$

\begin{tabular}{crrrrrrrrrrrrr} 
Year & Jan & Feb & Mar & Apr & May & Jun & Jul & Aug & Sep & Oct & Nov & Dec & Year \\
\hline \hline 2002 & & & & & & & & & & & 3.3 & 2.4 & \\
2003 & 2.2 & 3.2 & 3.3 & 4.9 & 3.6 & 3.6 & 2.4 & 1.9 & 2.3 & 2.7 & 3.4 & 3.0 & 3.05 \\
2004 & 2.7 & 3.1 & 2.7 & 3.8 & 4.3 & 3.5 & 2.5 & 2.4 & 3.0 & 3.5 & 2.8 & 3.2 & 3.13 \\
2005 & 2.6 & 3.2 & 3.7 & 4.1 & 3.5 & 3.7 & 2.6 & 1.7 & 2.7 & 2.7 & 2.8 & 2.8 & 3.02 \\
2006 & 3.3 & 3.5 & 4.3 & 3.9 & 3.4 & 3.0 & 2.5 & 1.9 & 2.7 & 2.6 & 2.9 & 2.9 & 3.06 \\
2007 & 4.0 & 3.1 & 3.1 & 3.6 & 3.4 & 3.5 & 2.1 & 2.1 & 2.8 & 3.5 & 2.4 & 2.9 & 3.05 \\
2008 & 3.7 & 3.0 & 3.6 & 4.7 & 3.9 & 3.5 & 2.1 & 1.9 & 1.8 & 3.1 & 2.9 & 3.5 & 3.14 \\
2009 & 3.0 & 3.3 & 3.8 & 4.5 & 2.8 & 3.3 & 2.5 & 2.6 & 2.7 & 3.4 & 2.8 & 3.1 & 3.14 \\
2010 & 2.4 & 2.9 & 3.6 & 4.1 & 4.7 & 3.5 & 2.1 & 2.5 & 2.1 & 2.6 & 3.5 & 3.2 & 3.09 \\
2011 & 2.5 & 3.9 & 3.8 & 4.8 & 4.7 & 4.2 & 2.1 & 1.9 & 1.7 & 2.2 & 3.3 & 2.5 & 3.12 \\
2012 & 3.3 & 3.1 & 3.8 & 3.4 & 4.1 & 3.7 & 2.2 & 1.6 & & & & & \\
\hline \hline Overall & 2.97 & 3.23 & 3.56 & 4.18 & 3.84 & 3.55 & 2.30 & 2.04 & 2.42 & 2.92 & 3.02 & 2.95 & 3.08
\end{tabular}

Observed Monthly Mean 10-m Wind Speeds (mps)

Latitude: $35^{\circ} 57^{\prime} \mathrm{N}$

Grand Canyon Airport, Arizona

Longitude: $112^{\circ} 09^{\prime} \mathrm{W}$

ASOS Period Only

Elevation: $2014 \mathrm{~m}$

Sonic Conversion Date: 12 February 2007

\begin{tabular}{cccccccccccccc} 
Year & Jan & Feb & Mar & Apr & May & Jun & Jul & Aug & Sep & Oct & Nov & Dec & Year \\
\hline \hline 1997 & 3.3 & 2.7 & 3.0 & 2.8 & 2.6 & 3.5 & 2.5 & 2.2 & 2.5 & 2.7 & 2.0 & 2.8 & 2.70 \\
1998 & 2.2 & 2.7 & 3.2 & 3.4 & 4.0 & 3.8 & 2.3 & 2.3 & 2.8 & 2.9 & 2.3 & 3.2 & 2.92 \\
1999 & 2.6 & 3.0 & 3.4 & 3.5 & 3.7 & 3.6 & 2.3 & 2.1 & 2.2 & 2.1 & 1.9 & 2.6 & 2.75 \\
2000 & 2.2 & 3.3 & 2.7 & 3.1 & 4.0 & 3.1 & 2.8 & 2.2 & 3.2 & 2.6 & 2.6 & 2.1 & 2.81 \\
2001 & 2.6 & 3.4 & 2.2 & 4.2 & 2.6 & 3.2 & 2.8 & 1.9 & 2.2 & 2.2 & 2.8 & 2.2 & 2.68 \\
2002 & 2.4 & 2.8 & 3.0 & 4.0 & 3.2 & 3.4 & 2.6 & 2.9 & 2.6 & 2.4 & 3.1 & 2.2 & 2.88 \\
2003 & 1.9 & 2.8 & 2.7 & 4.2 & 3.1 & 3.2 & 2.5 & 2.0 & 2.5 & 2.7 & 2.8 & 3.0 & 2.78 \\
2004 & 2.8 & 2.6 & 2.8 & 3.4 & 3.9 & 3.2 & 2.3 & 2.4 & 2.7 & 3.3 & 2.6 & 2.8 & 2.89 \\
2005 & 2.9 & 3.1 & 3.1 & 3.6 & 3.0 & 3.4 & 2.3 & 1.7 & 2.8 & 3.0 & 2.6 & 2.4 & 2.81 \\
2006 & 3.1 & 3.0 & 3.8 & 3.8 & 3.2 & 3.0 & 2.4 & 2.0 & 2.6 & 2.4 & 2.6 & 2.6 & 2.85 \\
2007 & 3.3 & 3.0 & 2.3 & 3.0 & 2.9 & 2.9 & 1.9 & 1.9 & 2.6 & 2.9 & 2.1 & 2.5 & 2.61 \\
2008 & 3.2 & 2.5 & 2.7 & 3.7 & 3.1 & 3.1 & 1.8 & 1.7 & 2.0 & 2.5 & 2.2 & 2.5 & 2.58 \\
2009 & 2.5 & 2.7 & 3.2 & 3.9 & 2.4 & 3.0 & 2.4 & 2.3 & 2.4 & 2.8 & 2.4 & 2.5 & 2.71 \\
2010 & 2.3 & 2.3 & 3.2 & 3.4 & 4.0 & 3.2 & 2.3 & 2.5 & 2.3 & 2.5 & 2.7 & 2.9 & 2.79 \\
2011 & 1.9 & 3.2 & 3.1 & 3.7 & 3.9 & 3.7 & 2.0 & 1.9 & 1.8 & 1.9 & 2.7 & 2.2 & 2.66 \\
2012 & 2.2 & 2.8 & 3.3 & 3.1 & 3.5 & 3.2 & 2.0 & 1.7 & & & & & \\
\hline \hline Overall & 2.57 & 2.87 & 2.98 & 3.53 & 3.31 & 3.29 & 2.31 & 2.10 & 2.46 & 2.58 & 2.49 & 2.56 & 2.76
\end{tabular}

Observed Monthly Mean 10-m Wind Speeds (mps)

Kingman Airport, Arizona

Latitude: $35^{\circ} 15^{\prime} \mathrm{N}$

ASOS Period Only

Longitude: $113^{\circ} 56^{\prime} \mathrm{W}$

Sonic Conversion Date: 20 February 2007

Elevation: $1042 \mathrm{~m}$

\begin{tabular}{|c|c|c|c|c|c|c|c|c|c|c|c|c|c|}
\hline Year & Jan & Feb & Mar & Apr & May & Jun & Jul & Aug & Sep & Oct & Nov & Dec & Year \\
\hline 1997 & 4.3 & 4.2 & 4.1 & 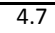 & $\bar{~} 4.7$ & $\overline{c 5.5}$ & 4.8 & 4.8 & 3.7 & 3.8 & 3.2 & 3.6 & 4.28 \\
\hline 1998 & 2.7 & 4.1 & 4.4 & 4.3 & 5.5 & 5.3 & 4.7 & 4.3 & 3.8 & 3.9 & 3.6 & 3.8 & 4.20 \\
\hline 1999 & 4.1 & 3.9 & 4.9 & 5.1 & 5.2 & 5.5 & 4.5 & 4.8 & 3.7 & 3.5 & 3.0 & 3.9 & 4.35 \\
\hline 2000 & 3.4 & 4.4 & 4.3 & 4.7 & 5.6 & 5.2 & 5.4 & 4.3 & 4.3 & 4.0 & 3.4 & 2.8 & 4.31 \\
\hline 2001 & 3.3 & 4.0 & 3.7 & 5.1 & 4.6 & 5.4 & 5.3 & 4.3 & 3.9 & 3.4 & 3.4 & 3.0 & 4.10 \\
\hline 2002 & 3.7 & 3.9 & 4.7 & 5.3 & 4.8 & 5.5 & 4.7 & 5.1 & 4.0 & 3.4 & 3.8 & 2.7 & 4.31 \\
\hline 2003 & 2.9 & 3.7 & 3.9 & 5.4 & 5.0 & 5.4 & 4.3 & 4.1 & 3.8 & 3.7 & 3.2 & 3.3 & 4.06 \\
\hline 2004 & 3.0 & 4.0 & 4.2 & 5.0 & 5.4 & 5.4 & 4.9 & 4.5 & 4.7 & 4.1 & 3.4 & 3.7 & 4.34 \\
\hline 2005 & 3.6 & 3.2 & 4.0 & 4.2 & 4.3 & 5.3 & 4.3 & 3.7 & 4.1 & 3.7 & 3.0 & 3.2 & 3.88 \\
\hline 2006 & 3.8 & 4.2 & 4.8 & 5.2 & 4.4 & 4.7 & 4.4 & 4.4 & 3.7 & 3.8 & 3.5 & 3.7 & 4.20 \\
\hline 2007 & 4.2 & 4.3 & 3.9 & 4.9 & 5.1 & 5.1 & 4.6 & 4.3 & 4.5 & 4.3 & 3.0 & 3.8 & 4.33 \\
\hline 2008 & 4.1 & 3.7 & 4.2 & 5.2 & 4.9 & 4.7 & 4.2 & 4.4 & 3.6 & 3.7 & 3.4 & 3.3 & 4.10 \\
\hline 2009 & 3.3 & 3.8 & 4.1 & 4.9 & 4.3 & 5.0 & 4.8 & 4.3 & 4.2 & 4.4 & 3.5 & 3.3 & 4.15 \\
\hline 2010 & 3.4 & 3.3 & 4.4 & 4.7 & 5.2 & 5.2 & 4.8 & 5.1 & 3.8 & 3.6 & 4.5 & 3.6 & 4.29 \\
\hline 2011 & 3.3 & 4.5 & 4.5 & 5.3 & 5.4 & 5.4 & 4.7 & 4.6 & 3.4 & 3.3 & 3.2 & 3.0 & 4.21 \\
\hline 2012 & 3.4 & 4.2 & 5.0 & 4.4 & 4.9 & 5.2 & 4.4 & 3.4 & & & & & \\
\hline 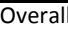 & 52 & .95 & 4.32 & 4.89 & 4.96 & 5.23 & 4.67 & 4.38 & 3.95 & 3.76 & 3.4 & 3.39 & 4.2 \\
\hline
\end{tabular}


Sonic-Equivalent Monthly Mean 10-m Wind Speeds (mps)

Flagstaff Airport, Arizona

ASOS Period Only

Sonic Conversion Date: 8 February 2007

Cup mean speeds decreased by $4.0 \%$

\begin{tabular}{cccccccccccccr} 
Year & Jan & Feb & Mar & Apr & May & Jun & Jul & Aug & Sep & Oct & Nov & Dec & Year \\
\hline \hline 2002 & & & & & & & & & & & 3.2 & 2.3 & \\
2003 & 2.1 & 3.1 & 3.2 & 4.7 & 3.5 & 3.5 & 2.3 & 1.8 & 2.2 & 2.6 & 3.3 & 2.9 & 2.93 \\
2004 & 2.6 & 3.0 & 2.6 & 3.6 & 4.2 & 3.4 & 2.4 & 2.3 & 2.8 & 3.4 & 2.7 & 3.0 & 3.00 \\
2005 & 2.5 & 3.0 & 3.5 & 3.9 & 3.4 & 3.6 & 2.5 & 1.7 & 2.6 & 2.6 & 2.7 & 2.7 & 2.90 \\
2006 & 3.1 & 3.3 & 4.1 & 3.8 & 3.3 & 2.9 & 2.4 & 1.8 & 2.6 & 2.5 & 2.7 & 2.7 & 2.94 \\
2007 & 3.9 & 3.0 & 3.1 & 3.6 & 3.4 & 3.5 & 2.1 & 2.1 & 2.8 & 3.5 & 2.4 & 2.9 & 3.03 \\
2008 & 3.7 & 3.0 & 3.6 & 4.7 & 3.9 & 3.5 & 2.1 & 1.9 & 1.8 & 3.1 & 2.9 & 3.5 & 3.14 \\
2009 & 3.0 & 3.3 & 3.8 & 4.5 & 2.8 & 3.3 & 2.5 & 2.6 & 2.7 & 3.4 & 2.8 & 3.1 & 3.14 \\
2010 & 2.4 & 2.9 & 3.6 & 4.1 & 4.7 & 3.5 & 2.1 & 2.5 & 2.1 & 2.6 & 3.5 & 3.2 & 3.09 \\
2011 & 2.5 & 3.9 & 3.8 & 4.8 & 4.7 & 4.2 & 2.1 & 1.9 & 1.7 & 2.2 & 3.3 & 2.5 & 3.12 \\
2012 & 3.3 & 3.1 & 3.8 & 3.4 & 4.1 & 3.7 & 2.2 & 1.6 & & & & & \\
\hline \hline Overall & 2.91 & 3.17 & 3.50 & 4.11 & 3.78 & 3.49 & 2.26 & 2.01 & 2.37 & 2.87 & 2.96 & 2.89 & 3.03
\end{tabular}

Sonic-Equivalent Monthly Mean 10-m Wind Speeds (mps)

Grand Canyon Airport, Arizona

ASOS Period Only

Sonic Conversion Date: 12 February 2007

Cup mean speeds decreased by $4.0 \%$

\begin{tabular}{|c|c|c|c|c|c|c|c|c|c|c|c|c|c|}
\hline Year & Jan & Feb & Mar & Apr & May & Jun & Jul & Aug & Sep & Oct & Nov & Dec & Year \\
\hline 1997 & 3.1 & 2.6 & 2.8 & 2.7 & 2.5 & 3.4 & 2.4 & 2.1 & 2.4 & 2.6 & 1.9 & 2.7 & 2.60 \\
\hline 1998 & 2.1 & 2.6 & 3.1 & 3.3 & 3.8 & 3.6 & 2.2 & 2.2 & 2.7 & 2.7 & 2.2 & 3.1 & 2.81 \\
\hline 1999 & 2.5 & 2.8 & 3.3 & 3.4 & 3.6 & 3.5 & 2.2 & 2.0 & 2.1 & 2.0 & 1.8 & 2.5 & 2.64 \\
\hline 2000 & 2.1 & 3.1 & 2.6 & 3.0 & 3.9 & 3.0 & 2.7 & 2.1 & 3.0 & 2.5 & 2.4 & 2.0 & 2.70 \\
\hline 2001 & 2.5 & 3.2 & 2.2 & 4.0 & 2.4 & 3.0 & 2.7 & 1.8 & 2.2 & 2.1 & 2.7 & 2.2 & 2.58 \\
\hline 2002 & 2.3 & 2.7 & 2.9 & 3.8 & 3.0 & 3.3 & 2.4 & 2.8 & 2.5 & 2.3 & 3.0 & 2.2 & 2.77 \\
\hline 2003 & 1.8 & 2.7 & 2.6 & 4.0 & 3.0 & 3.1 & 2.4 & 1.9 & 2.4 & 2.6 & 2.7 & 2.9 & 2.67 \\
\hline 2004 & 2.7 & 2.4 & 2.7 & 3.3 & 3.7 & 3.1 & 2.2 & 2.3 & 2.6 & 3.1 & 2.5 & 2.7 & 2.78 \\
\hline 2005 & 2.7 & 3.0 & 3.0 & 3.4 & 2.8 & 3.3 & 2.2 & 1.7 & 2.7 & 2.8 & 2.4 & 2.3 & 2.70 \\
\hline 2006 & 3.0 & 2.9 & 3.6 & 3.6 & 3.0 & 2.9 & 2.3 & 1.9 & 2.5 & 2.3 & 2.4 & 2.5 & 2.74 \\
\hline 2007 & 3.2 & 3.0 & 2.3 & 3.0 & 2.9 & 2.9 & 1.9 & 1.9 & 2.6 & 2.9 & 2.1 & 2.5 & 2.59 \\
\hline 2008 & 3.2 & 2.5 & 2.7 & 3.7 & 3.1 & 3.1 & 1.8 & 1.7 & 2.0 & 2.5 & 2.2 & 2.5 & 2.58 \\
\hline 2009 & 2.5 & 2.7 & 3.2 & 3.9 & 2.4 & 3.0 & 2.4 & 2.3 & 2.4 & 2.8 & 2.4 & 2.5 & 2.71 \\
\hline 2010 & 2.3 & 2.3 & 3.2 & 3.4 & 4.0 & 3.2 & 2.3 & 2.5 & 2.3 & 2.5 & 2.7 & 2.9 & 2.79 \\
\hline 2011 & 1.9 & 3.2 & 3.1 & 3.7 & 3.9 & 3.7 & 2.0 & 1.9 & 1.8 & 1.9 & 2.7 & 2.2 & 2.66 \\
\hline 2012 & 2.2 & 2.8 & 3.3 & 3.1 & 3.5 & 3.2 & 2.0 & 1.7 & & & & & \\
\hline Overall & 2.50 & 2.79 & 2.91 & 3.44 & 3.23 & 3.21 & 2.25 & 2.05 & 2.39 & 2.51 & 2.43 & 2.50 & 2.68 \\
\hline
\end{tabular}

Sonic-Equivalent Monthly Mean 10-m Wind Speeds (mps)

Kingman Airport, Arizona

ASOS Period Only

Sonic Conversion Date: 20 February 2007

Cup mean speeds decreased by $4.0 \%$

\begin{tabular}{|c|c|c|c|c|c|c|c|c|c|c|c|c|c|}
\hline Year & Jan & Feb & Mar & Apr & May & Jun & Jul & Aug & Sep & Oct & Nov & Dec & Year \\
\hline $\begin{array}{l}1997 \\
\end{array}$ & 4.1 & "4.0 & 3.9 & 4.6 & 4.6 & $\overline{5.2}$ & 4.6 & 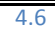 & 3.5 & 3.6 & 3.1 & 3.4 & 4.11 \\
\hline 1998 & 2.6 & 3.9 & 4.3 & 4.2 & 5.2 & 5.1 & 4.5 & 4.1 & 3.7 & 3.7 & 3.4 & 3.7 & 4.03 \\
\hline 1999 & 3.9 & 3.7 & 4.7 & 4.9 & 5.0 & 5.3 & 4.3 & 4.6 & 3.5 & 3.4 & 2.9 & 3.8 & 4.18 \\
\hline 2000 & 3.2 & 4.2 & 4.1 & 4.6 & 5.4 & 5.0 & 5.1 & 4.1 & 4.1 & 3.8 & 3.3 & 2.7 & 4.13 \\
\hline 2001 & 3.1 & 3.9 & 3.6 & 4.8 & 4.4 & 5.1 & 5.1 & 4.1 & 3.7 & 3.3 & 3.3 & 2.8 & 3.94 \\
\hline 2002 & 3.5 & 3.8 & 4.5 & 5.1 & 4.6 & 5.3 & 4.6 & 4.9 & 3.8 & 3.3 & 3.6 & 2.6 & 4.14 \\
\hline 2003 & 2.7 & 3.5 & 3.8 & 5.1 & 4.8 & 5.1 & 4.2 & 3.9 & 3.7 & 3.5 & 3.1 & 3.2 & 3.89 \\
\hline 2004 & 2.9 & 3.8 & 4.0 & 4.8 & 5.2 & 5.1 & 4.7 & 4.3 & 4.5 & 3.9 & 3.2 & 3.6 & 4.17 \\
\hline 2005 & 3.5 & 3.0 & 3.8 & 4.0 & 4.1 & 5.1 & 4.1 & 3.5 & 3.9 & 3.6 & 2.9 & 3.1 & 3.72 \\
\hline 2006 & 3.7 & 4.0 & 4.6 & 5.0 & 4.2 & 4.5 & 4.2 & 4.2 & 3.6 & 3.6 & 3.4 & 3.5 & 4.04 \\
\hline 2007 & 4.0 & 4.1 & 3.9 & 4.9 & 5.1 & 5.1 & 4.6 & 4.3 & 4.5 & 4.3 & 3.0 & 3.8 & 4.30 \\
\hline 2008 & 4.1 & 3.7 & 4.2 & 5.2 & 4.9 & 4.7 & 4.2 & 4.4 & 3.6 & 3.7 & 3.4 & 3.3 & 4.10 \\
\hline 2009 & 3.3 & 3.8 & 4.1 & 4.9 & 4.3 & 5.0 & 4.8 & 4.3 & 4.2 & 4.4 & 3.5 & 3.3 & 4.15 \\
\hline 2010 & 3.4 & 3.3 & 4.4 & 4.7 & 5.2 & 5.2 & 4.8 & 5.1 & 3.8 & 3.6 & 4.5 & 3.6 & 4.29 \\
\hline 2011 & 3.3 & 4.5 & 4.5 & 5.3 & 5.4 & 5.4 & 4.7 & 4.6 & 3.4 & 3.3 & 3.2 & 3.0 & 4.21 \\
\hline 2012 & 3.4 & 4.2 & 5.0 & 4.4 & 4.9 & 5.2 & 4.4 & 3.4 & & & & & \\
\hline & 3.42 & 84 & .21 & .77 & 4.83 & 5.10 & 4.55 & 4.27 & 3.84 & 3.66 & 3.32 & 3.30 & 40 \\
\hline
\end{tabular}


Monthly Mean Wind Speeds (mps) Hualapai Reservation, Arizona

YEAR: 2005

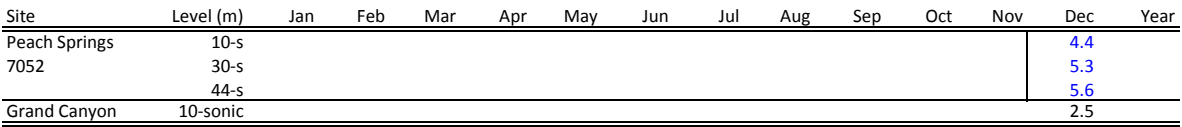

YEAR: 2006

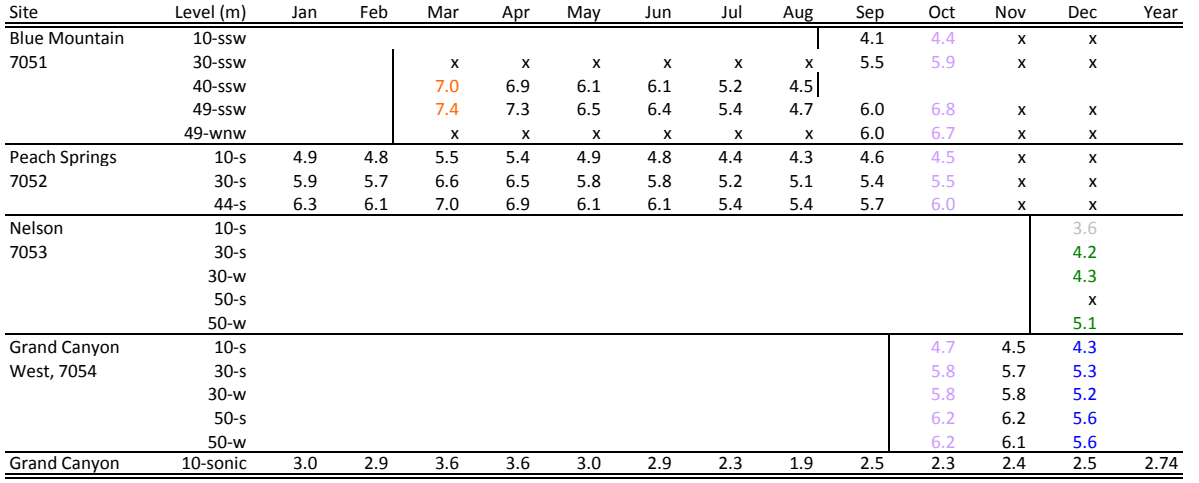

YEAR: 2007

\begin{tabular}{|c|c|c|c|c|c|c|c|c|c|c|c|c|c|c|}
\hline Site & Level $(\mathrm{m})$ & Jan & $\mathrm{Feb}$ & Mar & Apr & May & Jun & Jul & Aug & Sep & Oct & Nov & Dec & Year \\
\hline Blue Mountain & 10 -sse & $\bar{x}$ & 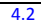 & $\begin{array}{c}4.0 \\
\end{array}$ & $\begin{array}{l}4.6 \\
\end{array}$ & 4.5 & 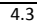 & 3.6 & 3.5 & \begin{tabular}{ll|}
4.5 \\
\end{tabular} & & & & \\
\hline \multirow[t]{3}{*}{7051} & 30-sse & $\mathrm{x}$ & 5.8 & 5.5 & 6.4 & 6.2 & 5.8 & 4.8 & 4.8 & 6.1 & & & & \\
\hline & 49-sse & $\mathrm{x}$ & 6.3 & 6.0 & 7.0 & 6.7 & 6.4 & 5.2 & 5.2 & 6.6 & & & & \\
\hline & 49-wsw & $\mathrm{x}$ & 6.3 & 6.0 & 7.0 & 6.7 & 6.4 & 5.2 & 5.2 & 6.6 & & & & \\
\hline Peach Springs & $10-s$ & $\mathrm{x}$ & $\mathrm{x}$ & $\mathrm{x}$ & $\mathrm{x}$ & $\mathrm{x}$ & $\mathrm{x}$ & $\mathrm{x}$ & $\mathrm{x}$ & $x$ & $\bar{x}$ & 4.2 & 4.5 & \\
\hline \multirow[t]{3}{*}{7052} & $30-\mathrm{s}$ & $\mathrm{x}$ & $\mathrm{x}$ & $\mathrm{x}$ & $\mathrm{x}$ & $\mathrm{x}$ & $\mathrm{x}$ & $\mathrm{x}$ & $\mathrm{x}$ & $\mathrm{x}$ & $\mathrm{x}$ & 5.1 & 5.4 & \\
\hline & 44-s & $\mathrm{x}$ & $x$ & $\mathrm{x}$ & $\mathrm{x}$ & $x$ & $\mathrm{x}$ & $\mathrm{x}$ & $\mathrm{x}$ & $x$ & $\mathrm{x}$ & 5.1 & 5.5 & \\
\hline & $44-w$ & & & & & & & & & & & & 5.5 & \\
\hline Nelson & $10-s$ & 4.0 & 3.8 & 3.6 & 4.1 & 4.3 & 4.2 & 3.3 & 3.6 & 4.3 & 4.2 & 3.4 & 3.7 & 3.88 \\
\hline \multirow[t]{4}{*}{7053} & $30-\mathrm{s}$ & 4.7 & 4.4 & 4.1 & 4.8 & 5.0 & 4.7 & 3.9 & 4.2 & 5.0 & 4.8 & 3.9 & 4.3 & 4.48 \\
\hline & $30-w$ & 4.7 & 4.5 & 4.1 & 4.8 & 4.9 & 4.6 & 3.8 & 4.1 & 4.9 & 4.7 & 3.8 & 4.3 & 4.44 \\
\hline & $50-\mathrm{s}$ & $\mathrm{x}$ & $\mathrm{x}$ & $\mathrm{x}$ & $\mathrm{x}$ & $\mathrm{x}$ & $\mathrm{x}$ & $\mathrm{x}$ & $x$ & $x$ & $\mathrm{x}$ & $\mathrm{x}$ & $\mathrm{x}$ & $x$ \\
\hline & $50-w$ & 5.6 & 5.3 & 4.8 & 5.7 & 5.7 & 5.4 & 4.4 & 4.9 & 5.8 & 5.7 & 4.6 & 5.1 & 5.25 \\
\hline Grand Canyon & $10-\mathrm{s}$ & $\mathrm{x}$ & $x$ & $x$ & $x$ & 5.0 & 5.2 & 4.0 & 4.4 & 5.3 & 4.9 & 4.0 & 4.6 & \\
\hline \multirow[t]{4}{*}{ West, 7054} & $30-s$ & $\mathrm{x}$ & $\mathrm{x}$ & $x$ & $x$ & 6.2 & 6.4 & 4.9 & 5.4 & 6.3 & 6.3 & 5.0 & 5.8 & \\
\hline & 30-w & $\mathrm{x}$ & $\mathrm{x}$ & $\mathrm{x}$ & $x$ & & & & 5.5 & 6.5 & 6.4 & 5.1 & 5.9 & \\
\hline & $50-s$ & $\mathrm{x}$ & $\mathrm{x}$ & $\mathrm{x}$ & $\mathrm{x}$ & 6.5 & 6.6 & 5.2 & 5.7 & 6.8 & 6.8 & 5.4 & 6.3 & \\
\hline & 50-w & $\mathrm{x}$ & $x$ & $\mathrm{x}$ & $\mathrm{x}$ & $\mathrm{x}$ & $\mathrm{x}$ & $\mathrm{x}$ & $\mathrm{x}$ & $x$ & $\mathrm{x}$ & $x$ & $\mathrm{x}$ & \\
\hline Grand Canyon & 10-sonic & 3.2 & 3.0 & 2.3 & 3.0 & 2.9 & 2.9 & 1.9 & 1.9 & 2.6 & 2.9 & 2.1 & 2.5 & 2.59 \\
\hline
\end{tabular}

YEAR: 2008

\begin{tabular}{|c|c|c|c|c|c|c|c|c|c|c|c|c|c|c|}
\hline Site & Level $(\mathrm{m})$ & Jan & Feb & Mar & Apr & May & Jun & Jul & Aug & Sep & Oct & Nov & Dec & Year \\
\hline Peach Springs & 10-s & $\overline{c 5.2}$ & $\begin{array}{l}4.4 \\
\end{array}$ & 4 & 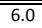 & "5.3 & 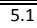 & "4.1 & $\begin{array}{l}4.1 \\
\end{array}$ & $=4.2$ & (24.6 & (4.3 & (4.3 & (4.70 \\
\hline \multirow[t]{3}{*}{7052} & $30-s$ & 6.4 & 5.3 & 5.9 & 7.2 & 6.3 & 6.0 & 4.8 & 4.9 & 5.0 & 5.6 & 5.2 & 5.1 & 5.64 \\
\hline & 44-s & 6.5 & 5.2 & 5.8 & 7.1 & 6.2 & 5.9 & 4.8 & 4.9 & 4.8 & 5.4 & 5.3 & 5.1 & 5.59 \\
\hline & 44-w & 6.4 & 5.3 & 6.0 & 7.4 & 6.4 & 6.1 & 4.8 & 4.9 & 4.9 & 5.4 & 5.3 & 5.2 & 5.68 \\
\hline Nelson & $10-s$ & 4.4 & 3.5 & 4.0 & 4.9 & 4.6 & 4.2 & 3.4 & 3.4 & 3.6 & 3.9 & 3.6 & 3.5 & 3.90 \\
\hline \multirow[t]{4}{*}{7053} & $30-\mathrm{s}$ & 5.0 & 4.0 & 4.5 & 5.5 & 5.1 & 4.7 & 3.8 & 3.8 & 4.0 & 4.4 & 4.2 & 4.0 & 4.39 \\
\hline & $30-w$ & 5.1 & 4.0 & 4.5 & 5.5 & 5.1 & 4.7 & 3.9 & 3.9 & 4.1 & 4.3 & 4.3 & 4.1 & 4.44 \\
\hline & $50-\mathrm{s}$ & $\mathrm{x}$ & $x$ & $\mathrm{x}$ & $\mathrm{x}$ & $\mathrm{x}$ & $\mathrm{x}$ & $\mathrm{x}$ & $\mathrm{x}$ & $\mathrm{x}$ & $\mathrm{x}$ & $\mathrm{x}$ & $\mathrm{x}$ & $x$ \\
\hline & 50-w & 6.0 & 4.8 & 5.3 & 6.5 & 5.9 & 5.5 & 4.3 & 4.3 & 4.6 & 5.0 & 4.9 & 4.7 & 5.14 \\
\hline Grand Canyon & $10-\mathrm{s}$ & 5.6 & 4.5 & 4.8 & 5.7 & 5.1 & 4.9 & 3.9 & 4.3 & 4.0 & 4.4 & 4.3 & 4.7 & 4.68 \\
\hline \multirow{4}{*}{ West, 7054} & $30-\mathrm{s}$ & 7.0 & 5.7 & 6.1 & 7.3 & 6.4 & 6.1 & 4.8 & 5.4 & 4.9 & 5.5 & 5.4 & 5.7 & 5.85 \\
\hline & $30-w$ & 7.0 & 5.7 & 6.1 & 7.3 & 6.5 & 6.2 & 4.9 & 5.4 & 4.9 & 5.5 & 5.5 & 5.8 & 5.89 \\
\hline & $50-\mathrm{s}$ & & & & 7.8 & 6.9 & 6.6 & 5.1 & 5.8 & 5.3 & 5.8 & 6.0 & 6.2 & 6.26 \\
\hline & 50-w & & & & 7.9 & 6.9 & 6.7 & 5.2 & 5.7 & 5.2 & 5.8 & 6.0 & 6.1 & 6.25 \\
\hline Grand Canyon & 10 & 3.2 & 2.5 & 2.7 & 3.7 & 3.1 & 3.1 & 1.8 & 1.7 & 2.0 & 2.5 & 2.2 & 2.5 & 2.58 \\
\hline
\end{tabular}




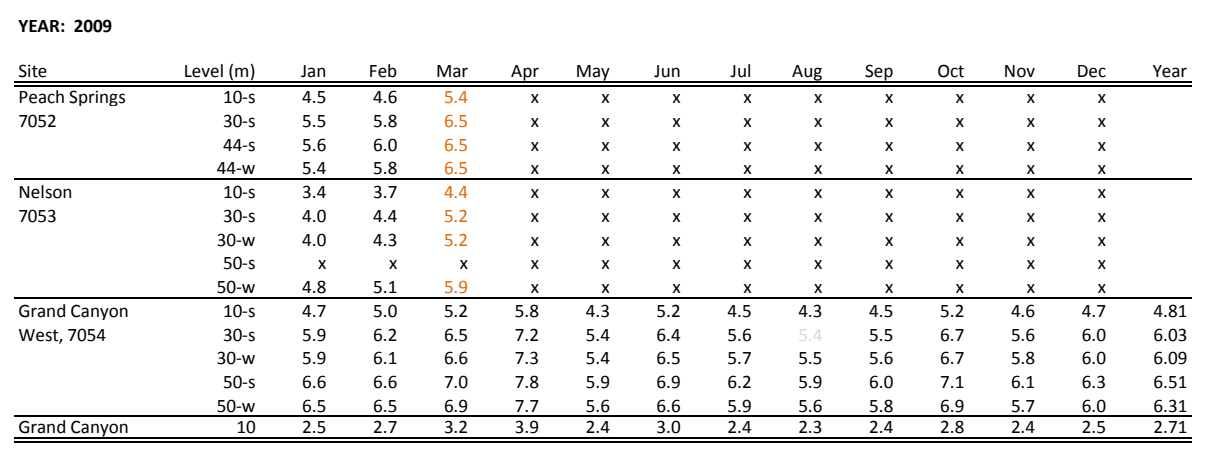

\begin{tabular}{|c|c|c|c|c|c|c|c|c|c|c|c|c|c|c|}
\hline Site & Level $(\mathrm{m})$ & Jan & $\mathrm{Feb}$ & Mar & Apr & May & Jun & Jul & Aug & Sep & Oct & Nov & Dec & Year \\
\hline $\begin{array}{l}\text { Peach Springs } \\
\end{array}$ & $10-\mathrm{s}$ & 3.8 & 3.6 & 4.6 & $\begin{array}{ll}4.9 \\
\end{array}$ & 5.3 & 5.0 & 4.3 & 4.7 & 4.3 & 3.9 & 4.5 & 4.5 & 4.45 \\
\hline \multirow[t]{3}{*}{7052} & $30-5$ & 5.0 & 4.7 & 5.9 & 6.2 & 6.5 & 6.2 & 5.4 & 5.9 & 5.4 & 5.1 & 6.0 & 6.0 & 5.69 \\
\hline & 44-s & 5.3 & 5.0 & 6.3 & 6.5 & 6.9 & 6.5 & 5.6 & 6.2 & 5.7 & 5.4 & 6.4 & 6.4 & 6.01 \\
\hline & $44-w$ & 5.2 & 5.1 & $x$ & $x$ & $x$ & $\mathrm{x}$ & $\mathrm{x}$ & $\mathrm{x}$ & $x$ & $x$ & $x$ & $\mathrm{x}$ & \\
\hline Nelson & $10-\mathrm{s}$ & 3.3 & 3.1 & 4.2 & 4.6 & 4.7 & 4.5 & 3.8 & 4.1 & 3.9 & 3.5 & 4.0 & 3.9 & 3.96 \\
\hline \multirow[t]{4}{*}{7053} & $30-\mathrm{s}$ & 4.0 & 3.7 & 4.8 & 5.3 & 5.3 & 5.0 & 4.2 & 4.8 & 4.5 & 4.1 & 4.9 & 4.6 & 4.61 \\
\hline & $30-w$ & 4.0 & 3.8 & 4.8 & 5.3 & 5.3 & 5.0 & 4.2 & 4.8 & 4.4 & 4.2 & 4.8 & 4.7 & 4.59 \\
\hline & $50-\mathrm{s}$ & $\mathrm{x}$ & $\mathrm{x}$ & $x$ & $x$ & $x$ & $\mathrm{x}$ & $\mathrm{x}$ & $\mathrm{x}$ & $\mathrm{x}$ & $x$ & $x$ & $x$ & \\
\hline & $50-w$ & 4.5 & 4.3 & 5.3 & 5.7 & 5.9 & 5.5 & 4.6 & 5.3 & 4.8 & 4.8 & 5.6 & 5.4 & 5.12 \\
\hline Grand Canyon & $10-\mathrm{s}$ & 4.3 & 4.1 & 5.1 & 5.3 & 5.3 & 5.3 & 4.5 & $\mathrm{x}$ & $\mathrm{x}$ & $\mathrm{x}$ & $\mathrm{x}$ & 5.3 & \\
\hline \multirow[t]{4}{*}{ West, 7054} & $30-\mathrm{s}$ & 5.3 & 5.2 & 6.4 & 6.8 & 6.9 & 6.8 & 5.5 & $\mathrm{x}$ & $\mathrm{x}$ & $\mathrm{x}$ & $x$ & 6.6 & \\
\hline & 30-w & 5.2 & 5.1 & 6.4 & 6.8 & 7.0 & 6.8 & 5.7 & $\mathrm{x}$ & $\mathrm{x}$ & $\mathrm{x}$ & $\mathrm{x}$ & 6.6 & \\
\hline & $50-\mathrm{s}$ & 5.5 & 5.5 & 6.7 & 7.1 & 7.2 & 7.0 & 5.8 & $\mathrm{x}$ & $\mathrm{x}$ & $\mathrm{x}$ & $\mathrm{x}$ & 6.9 & \\
\hline & $50-w$ & 5.3 & 5.3 & 6.5 & 6.9 & 7.1 & 7.0 & 5.8 & $\mathrm{x}$ & $\mathrm{x}$ & $\mathrm{x}$ & $\mathrm{x}$ & 6.9 & \\
\hline $\begin{array}{l}\text { Grand Canyon } \\
\end{array}$ & 10 & 2.3 & 2.3 & 3.2 & 3.4 & 4.0 & 3.2 & 2.3 & 2.5 & 2.3 & 2.5 & 2.7 & $\begin{array}{l}2.9 \\
\end{array}$ & 2.79 \\
\hline
\end{tabular}

\begin{tabular}{|c|c|c|c|c|c|c|c|c|c|c|c|c|c|c|}
\hline Site & Level $(\mathrm{m})$ & Jan & Feb & Mar & Apr & May & Jun & Jul & Aug & Sep & Oct & Nov & Dec & Year \\
\hline Peach Springs & (10-s & "3.6 & $=4.9$ & (4.7 & 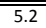 & "5.5 & 5.6 & "4.2 & (24.5 & $=3.9$ & 3.8 & " 4.5 & "3.4 & " \\
\hline \multirow[t]{3}{*}{7052} & $30-\mathrm{s}$ & 4.8 & 6.3 & 6.0 & 6.5 & 6.6 & 6.9 & 5.3 & 5.4 & 4.8 & 4.9 & 5.7 & 4.4 & 5.64 \\
\hline & 44-s & 5.1 & 6.7 & 6.5 & 7.0 & 7.0 & 7.3 & 5.6 & 5.7 & 5.0 & 5.2 & 6.2 & 4.9 & 6.02 \\
\hline & $44-w$ & $\mathrm{x}$ & $\mathrm{x}$ & $x$ & $\mathrm{x}$ & $\mathrm{x}$ & $\mathrm{x}$ & $\mathrm{x}$ & $\mathrm{x}$ & $\mathrm{x}$ & $\mathrm{x}$ & $\mathrm{x}$ & $x$ & \\
\hline Nelson & $10-s$ & 3.0 & 4.3 & 4.1 & $\begin{array}{llll}4.7 & \end{array}$ & 4.8 & 4.9 & 3.6 & 3.7 & 3.4 & 3.5 & 3.8 & 3.2 & 3.91 \\
\hline \multirow[t]{4}{*}{7053} & $30-5$ & 3.4 & 5.0 & 4.8 & 5.4 & 5.5 & 5.6 & 4.1 & 4.4 & 3.9 & 4.1 & 4.7 & 3.8 & 4.55 \\
\hline & $30-w$ & 3.4 & 5.1 & 4.9 & 5.4 & 5.5 & 5.6 & 4.1 & 4.3 & 3.8 & 4.0 & 4.6 & 3.8 & 4.52 \\
\hline & $50-\mathrm{s}$ & $\mathrm{x}$ & $\mathrm{x}$ & $\mathrm{x}$ & $\mathrm{x}$ & $\mathrm{x}$ & $\mathrm{x}$ & $\mathrm{x}$ & $\mathrm{x}$ & $\mathrm{x}$ & $\mathrm{x}$ & $\mathrm{x}$ & $\mathrm{x}$ & \\
\hline & $50-w$ & 4.1 & 5.9 & 5.6 & 6.1 & 6.2 & 6.5 & 4.6 & 4.9 & 4.3 & 4.7 & 5.4 & 4.4 & 5.22 \\
\hline Grand Canyon & $10-5$ & 3.9 & 5.5 & 5.3 & 5.4 & 5.9 & 6.0 & 4.6 & 4.6 & 4.0 & 4.1 & 4.9 & 3.6 & 4.82 \\
\hline \multirow[t]{4}{*}{ West, 7054} & $30-\mathrm{s}$ & 5.0 & 6.9 & 6.7 & 7.0 & 7.4 & 7.5 & 5.7 & 5.7 & 4.8 & 5.1 & 6.1 & 4.5 & 6.03 \\
\hline & $30-w$ & 5.0 & 7.0 & 6.7 & 6.9 & 7.3 & 7.5 & 5.5 & 5.6 & 4.6 & 4.8 & 5.9 & 4.5 & 5.94 \\
\hline & $50-\mathrm{s}$ & 5.2 & 6.9 & 6.8 & 7.2 & 7.5 & 7.5 & 5.8 & 5.9 & 5.1 & 5.3 & 6.4 & 4.9 & 6.21 \\
\hline & $50-w$ & 5.3 & 7.2 & 6.9 & 7.2 & 7.5 & 7.8 & 5.8 & 5.9 & 4.9 & 5.1 & 6.2 & 4.7 & 6.21 \\
\hline Clay Springs & $10-\mathrm{s}$ & & & & & 4.9 & 4.5 & 3.5 & 3.4 & 3.2 & 3.1 & 3.8 & 3.1 & 3.75 \\
\hline \multirow[t]{5}{*}{7055} & $30-\mathrm{s}$ & & & & & 6.6 & 6.0 & 4.6 & 4.6 & 4.1 & 4.1 & 5.1 & 4.2 & 5.06 \\
\hline & 30-nw & & & & & 6.6 & 6.0 & 4.6 & 4.6 & 4.2 & 4.1 & 5.1 & 4.2 & 5.13 \\
\hline & $40-\mathrm{s}$ & & & & & 7.1 & 6.5 & 4.9 & 5.0 & 4.4 & 4.4 & 5.4 & 4.4 & 5.39 \\
\hline & $49-\mathrm{s}$ & & & & & 7.5 & 6.7 & 5.0 & 5.3 & 4.6 & 4.6 & 5.7 & 4.4 & 5.62 \\
\hline & 49-nw & & & & & 7.4 & 6.8 & 5.1 & 5.2 & 4.5 & 4.5 & 5.6 & 4.3 & 5.61 \\
\hline Grand Canyon & 10 & 1.9 & 3.2 & 3.1 & 3.7 & 3.9 & 3.7 & 2.0 & 1.9 & 1.8 & 1.9 & 2.7 & 2.2 & 2.66 \\
\hline
\end{tabular}

\begin{tabular}{|c|c|c|c|c|c|c|c|c|c|c|c|c|c|c|}
\hline Site & Level $(\mathrm{m})$ & Jan & Feb & Mar & Apr & May & Jun & Jul & Aug & Sep & Oct & Nov & Dec & Year \\
\hline 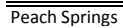 & 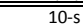 & 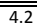 & 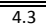 & $=5.0$ & $=4.8$ & 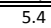 & 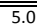 & 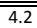 & $=3.5$ & & & & & \\
\hline \multirow[t]{3}{*}{7052} & $30-s$ & 5.4 & 5.6 & 6.3 & 5.9 & 6.6 & 6.0 & 5.2 & 4.3 & & & & & \\
\hline & 44-s & 5.8 & 6.1 & 6.8 & 6.3 & 6.9 & 6.4 & 5.5 & 4.6 & & & & & \\
\hline & 44-w & $\mathrm{x}$ & $\mathrm{x}$ & $\mathrm{x}$ & $\mathrm{x}$ & $\mathrm{x}$ & $\mathrm{x}$ & $\mathrm{x}$ & $\mathrm{x}$ & & & & & \\
\hline Nelson & $10-s$ & 3.6 & 3.6 & 4.3 & 4.2 & 4.5 & 4.4 & 3.3 & 3.0 & & & & & \\
\hline \multirow[t]{4}{*}{7053} & $30-\mathrm{s}$ & 4.3 & 4.3 & 5.2 & 5.1 & 5.3 & 5.0 & 3.9 & 3.6 & & & & & \\
\hline & $30-w$ & 4.3 & 4.3 & 5.2 & 5.0 & 5.1 & 5.0 & 3.9 & 3.5 & & & & & \\
\hline & $50-\mathrm{s}$ & $\mathrm{x}$ & $x$ & $x$ & $\mathrm{x}$ & $\mathrm{x}$ & $\mathrm{x}$ & $\mathrm{x}$ & $\mathrm{x}$ & & & & & \\
\hline & $50-w$ & 5.0 & 5.1 & 6.1 & 5.8 & 5.6 & 5.4 & 4.2 & 3.6 & & & & & \\
\hline Grand Canyon & 10-s & 4.5 & 4.7 & 5.8 & 5.1 & 5.5 & 5.6 & 4.3 & 3.4 & & & & & \\
\hline \multirow[t]{4}{*}{ West, 7054} & $30-\mathrm{s}$ & 5.6 & 6.0 & 7.3 & 6.4 & 6.9 & 6.9 & 5.3 & 4.2 & & & & & \\
\hline & $30-w$ & 5.5 & 5.9 & 7.0 & 6.4 & 6.8 & 7.0 & 5.2 & 4.1 & & & & & \\
\hline & 50-s & 5.7 & 6.0 & 7.1 & 6.5 & 7.0 & 7.1 & 5.5 & 4.5 & & & & & \\
\hline & $50-w$ & 5.8 & 6.2 & 7.3 & 6.7 & 7.1 & 7.4 & 5.5 & 4.2 & & & & & \\
\hline Clay Springs & $10-\mathrm{s}$ & 3.5 & 3.9 & 4.4 & $\begin{array}{lll}4.1 & \end{array}$ & 4.4 & 4.2 & 3.4 & 2.9 & & & & & \\
\hline \multirow[t]{5}{*}{7055} & $30-\mathrm{s}$ & 4.6 & 5.1 & 6.0 & 5.4 & 6.0 & 5.6 & 4.5 & 3.8 & & & & & \\
\hline & $30-n w$ & 4.6 & 5.2 & 6.0 & 5.4 & 5.9 & 5.7 & 4.5 & 3.8 & & & & & \\
\hline & $40-\mathrm{s}$ & 4.9 & 5.5 & 6.4 & 5.7 & 6.3 & 6.1 & 4.8 & 4.0 & & & & & \\
\hline & 49-s & 5.3 & 5.9 & 6.8 & 6.0 & 6.7 & 6.5 & 5.0 & 4.2 & & & & & \\
\hline & 49-nw & 5.1 & 5.7 & 6.6 & 6.0 & 6.6 & 6.4 & 4.9 & 4.1 & & & & & \\
\hline Grand Canyon & 10 & 2.2 & 2.8 & 3.3 & 3.1 & 3.5 & 3.2 & 2.0 & 1.7 & & & & & \\
\hline
\end{tabular}

Data Recovery Key: black $=90-100 \%$; green $=75-90 \%$; blue $=50-75 \%$; orange $=25-50 \%$; purple $=10-25 \%$; $" x "=0-10 \%$. All values represent the estimate for the full month when data recovery is less than $100 \%$. 
Composite Mean Wind Speeds (mps) for Concurrent Data, December 2005-August 2012

\begin{tabular}{|c|c|c|c|c|c|c|c|c|c|c|c|c|c|c|}
\hline Site & Level (m) & Jan & Feb & Mar & Apr & May & Jun & Jul & Aug & Sep & Oct & Nov & Dec & Year \\
\hline 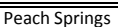 & 10-s & 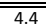 & $\bar{~} 4.4$ & $\bar{~} 5.0$ & 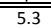 & 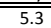 & 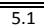 & 4.2 & 4.2 & 4.2 & 4.2 & $\overline{4.4}$ & 40.1 & 4.57 \\
\hline \multirow[t]{2}{*}{7052} & $30-5$ & 5.5 & 5.6 & 6.2 & 6.5 & 6.4 & 6.2 & 5.2 & 5.1 & 5.2 & 5.3 & 5.5 & 5.2 & 5.64 \\
\hline & 44-s & 5.8 & 5.8 & 6.5 & 6.8 & 6.6 & 6.4 & 5.4 & 5.3 & 5.3 & 5.5 & 5.7 & 5.5 & 5.89 \\
\hline$\overline{\text { Grand Canyon }}$ & 10 & 2.5 & 2.7 & 3.2 & 3.5 & 3.5 & 3.2 & $\frac{0.4}{2.1}$ & 2.0 & 2.1 & 2.3 & 2.4 & 2.5 & 2.66 \\
\hline
\end{tabular}

Composite Mean Wind Speeds (mps) for Concurrent Data, November 2006-August 2012

\begin{tabular}{|c|c|c|c|c|c|c|c|c|c|c|c|c|c|c|}
\hline Site & Level (m) & Jan & Feb & Mar & Apr & May & Jun & Jul & Aug & Sep & Oct & Nov & Dec & Year \\
\hline Grand Canyon & 10-s & 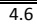 & $\begin{array}{ll}4.8 \\
\end{array}$ & $\overline{5.2}$ & $\overline{c 5.5}$ & $\overline{5.2}$ & 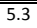 & 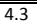 & $\begin{array}{ll}4.2 \\
\end{array}$ & 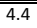 & 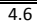 & $\begin{array}{ll}4.5 \\
\end{array}$ & $\begin{array}{ll}4.5 \\
\end{array}$ & $4 \quad 4.76$ \\
\hline \multirow[t]{5}{*}{ West, 7054} & $30-s$ & 5.7 & 6.0 & 6.6 & 6.9 & 6.5 & 6.7 & 5.3 & 5.2 & 5.4 & 5.9 & 5.3 & 5.7 & 5.93 \\
\hline & $30-w$ & 5.7 & 6.0 & 6.6 & 6.9 & 6.5 & 6.7 & 5.3 & 5.2 & 5.4 & 5.9 & 5.3 & 5.7 & 5.94 \\
\hline & $50-\mathrm{s}$ & 6.1 & 6.2 & 6.8 & 7.3 & 6.8 & 7.0 & 5.6 & 5.6 & 5.8 & 6.2 & 5.7 & 6.0 & 6.25 \\
\hline & 50-w & 6.0 & 6.2 & 6.8 & 7.3 & 6.8 & 7.0 & 5.5 & 5.4 & 5.6 & 6.1 & 5.5 & 5.9 & 6.18 \\
\hline & 10 & 2.4 & 2.7 & 3.1 & 3.5 & 3.3 & 3.2 & 2.1 & 1.9 & 2.2 & 2.5 & 2.4 & 2.5 & 2.64 \\
\hline
\end{tabular}

Composite Mean Wind Speeds (mps) for Concurrent Data, December 2006-August 2012

\begin{tabular}{|c|c|c|c|c|c|c|c|c|c|c|c|c|c|c|}
\hline Site & Level $(\mathrm{m})$ & Jan & Feb & Mar & Apr & May & Jun & Jul & Aug & Sep & Oct & Nov & Dec & Year \\
\hline Nelson & $\begin{array}{ll}10-s \\
\end{array}$ & \begin{tabular}{c|}
3.6 \\
\end{tabular} & $\begin{array}{l}3.7 \\
\end{array}$ & 44.1 & 40.5 & 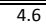 & 40.4 & $\begin{array}{l}3.5 \\
\end{array}$ & 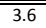 & $\begin{array}{l}3.8 \\
\end{array}$ & 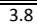 & $\begin{array}{l}.7 \\
\end{array}$ & 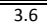 & 3.89 \\
\hline \multirow[t]{4}{*}{7053} & $30-s$ & 4.2 & 4.3 & 4.8 & 5.2 & 5.2 & 5.0 & 4.0 & 4.2 & 4.3 & 4.4 & 4.4 & 4.2 & 4.51 \\
\hline & $30-w$ & 4.3 & 4.3 & 4.8 & 5.2 & 5.2 & 5.0 & 4.0 & 4.1 & 4.3 & 4.3 & 4.4 & 4.2 & 4.49 \\
\hline & $50-w$ & 5.0 & 5.1 & 5.5 & 6.0 & 5.9 & 5.7 & 4.4 & 4.6 & 4.9 & 5.0 & 5.1 & 4.9 & 5.16 \\
\hline & 10 & 2.5 & 2.8 & 3.0 & 3.4 & 3.5 & 3.2 & 2.0 & 1.9 & 2.1 & 2.4 & 2.4 & 2.5 & 2.65 \\
\hline
\end{tabular}

Composite Mean Wind Speeds (mps) for Concurrent Data, May 2011-August 2012

\begin{tabular}{lrrrrrrrrrrrrrr} 
Site & Level $(\mathrm{m})$ & Jan & Feb & Mar & Apr & May & Jun & Jul & Aug & Sep & Oct & Nov & Dec & Year \\
\hline \hline Clay Springs & $10-\mathrm{s}$ & 3.5 & 3.9 & 4.4 & 4.1 & 4.6 & 4.3 & 3.4 & 3.1 & 3.2 & 3.1 & 3.8 & 3.1 & 3.71 \\
7055 & $30-\mathrm{s}$ & 4.6 & 5.1 & 6.0 & 5.4 & 6.3 & 5.8 & 4.6 & 4.2 & 4.1 & 4.1 & 5.1 & 4.2 & 4.95 \\
& $30-\mathrm{nw}$ & 4.6 & 5.2 & 6.0 & 5.4 & 6.2 & 5.9 & 4.6 & 4.2 & 4.2 & 4.1 & 5.1 & 4.2 & 4.96 \\
& $40-\mathrm{s}$ & 4.9 & 5.5 & 6.4 & 5.7 & 6.7 & 6.3 & 4.9 & 4.5 & 4.4 & 4.4 & 5.4 & 4.4 & 5.29 \\
& $49-\mathrm{s}$ & 5.3 & 5.9 & 6.8 & 6.0 & 7.1 & 6.6 & 5.0 & 4.7 & 4.6 & 4.6 & 5.7 & 4.4 & 5.56 \\
& $49-\mathrm{nw}$ & 5.1 & 5.7 & 6.6 & 6.0 & 7.0 & 6.6 & 5.0 & 4.6 & 4.5 & 4.5 & 5.6 & 4.3 & 5.46 \\
\hline Grand Canyon & & & & & & & & & & & & & & \\
West, 7054 & $50-\mathrm{s} / \mathrm{w}$ & 5.7 & 6.1 & 7.2 & 6.6 & 7.3 & 7.4 & 5.6 & 5.1 & 5.0 & 5.2 & 6.3 & 4.8 & 6.03 \\
\hline \hline
\end{tabular}


Estimated Long-Term Mean Annual Wind Speeds (mps)

Hualapai Reservation, Arizona

\begin{tabular}{|c|c|c|c|c|c|c|}
\hline Site & Level $(\mathrm{m})$ & $\begin{array}{r}\text { Composite Mean } \\
\text { Dec 2005 } \\
\text { to Aug 2012* } \\
\end{array}$ & $\begin{array}{r}\text { Estimated } \\
\text { Long Term }\end{array}$ & $\begin{array}{l}\text { Shears } \\
\text { Levels (m) }\end{array}$ & Exponent & $\begin{array}{r}\text { Extrapolated } \\
\text { to } 80 \mathrm{~m} \\
\end{array}$ \\
\hline Peach Springs & 10-s & 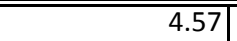 & 4.60 & $10-30$ & 0.192 & \\
\hline \multirow[t]{2}{*}{7052} & $30-s$ & 5.64 & 5.68 & $30-44$ & 0.112 & \\
\hline & 44-s & 5.89 & 5.93 & $10-44$ & 0.171 & 6.57 \\
\hline $\begin{array}{l}\text { Grand Canyon } \\
\end{array}$ & 10 & 2.66 & 2.68 & & & \\
\hline
\end{tabular}

* there were three separate measurement periods with different composite mean annual wind speeds and unexplainable different shears. The data here represent the full data period.

\begin{tabular}{|c|c|c|c|c|c|c|c|}
\hline Site & Level (m) & $\begin{array}{r}\text { Composite Mean } \\
\text { Oct 2006 } \\
\text { to Aug 2012 }\end{array}$ & $\begin{array}{l}\text { Estimated } \\
\text { Long Term }\end{array}$ & $\begin{array}{r}\text { Averaged } \\
\text { by Level }\end{array}$ & $\begin{array}{l}\text { Shears } \\
\text { Levels }(\mathrm{m})\end{array}$ & Exponent & $\begin{array}{r}\text { Extrapolated } \\
\text { to } 80 \mathrm{~m}\end{array}$ \\
\hline 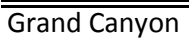 & 10-s & "4.76 & 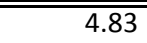 & 4.83 & $10-30$ & "0.201 & \\
\hline \multirow[t]{4}{*}{ West, 7054} & $30-s$ & 5.93 & 6.01 & 6.02 & $30-50$ & 0.091 & \\
\hline & $30-w$ & 5.94 & 6.02 & & & & \\
\hline & $50-s$ & 6.25 & 6.34 & 6.30 & $10-50$ & 0.166 & 6.82 \\
\hline & 50-w & 6.18 & 6.27 & & & & 6.58 \\
\hline Grand Canyon & 10 & 2.64 & 2.68 & & & & \\
\hline
\end{tabular}

\begin{tabular}{|c|c|c|c|c|c|c|c|}
\hline Site & Level (m) & $\begin{array}{r}\text { Composite Mean } \\
\text { Dec } 2006 \\
\text { to Aug } 2012\end{array}$ & $\begin{array}{r}\text { Estimated } \\
\text { Long Term }\end{array}$ & $\begin{array}{r}\text { Averaged } \\
\text { by Level }\end{array}$ & $\begin{array}{l}\text { Shears } \\
\text { Levels }(\mathrm{m})\end{array}$ & Exponent & $\begin{array}{r}\text { Extrapolated } \\
\text { to } 80 \mathrm{~m} \\
\end{array}$ \\
\hline "Nelson & 10-s & 3.89 & 3.95 & 3.95 & "10-30 & 0.132 & \\
\hline \multirow[t]{3}{*}{7053} & $30-\mathrm{s}$ & 4.51 & 4.57 & 4.56 & $30-50$ & 0.268 & \\
\hline & $30-w$ & 4.49 & 4.55 & & & & \\
\hline & 50-w & 5.16 & 5.23 & 5.23 & $10-50$ & 0.175 & 5.68 \\
\hline
\end{tabular}

\begin{tabular}{|c|c|c|c|c|c|c|}
\hline Site & Level (m) & $\begin{array}{r}\text { Overall Mean } \\
\text { Mar } 2006 \\
\text { to Sep 2007 }\end{array}$ & $\begin{array}{r}\text { Estimated } \\
\text { Long Term }\end{array}$ & $\begin{array}{l}\text { Shears } \\
\text { Levels (m) }\end{array}$ & Exponent & $\begin{array}{r}\text { Extrapolated } \\
\text { to } 80 \mathrm{~m} \\
\end{array}$ \\
\hline Blue Mountain & 10 & "4.19 & 4.19 & "10-30 & 0.278 & \multirow{4}{*}{6.79} \\
\hline \multirow[t]{3}{*}{7051} & 30 & 5.69 & 5.69 & $30-40$ & 0.142 & \\
\hline & 40 & 5.93 & 5.93 & $40-49$ & 0.255 & \\
\hline & 49 & 6.24 & 6.25 & $10-49$ & 0.251 & \\
\hline Grand Canyon & 10 & 2.68 & 2.68 & & & \\
\hline
\end{tabular}

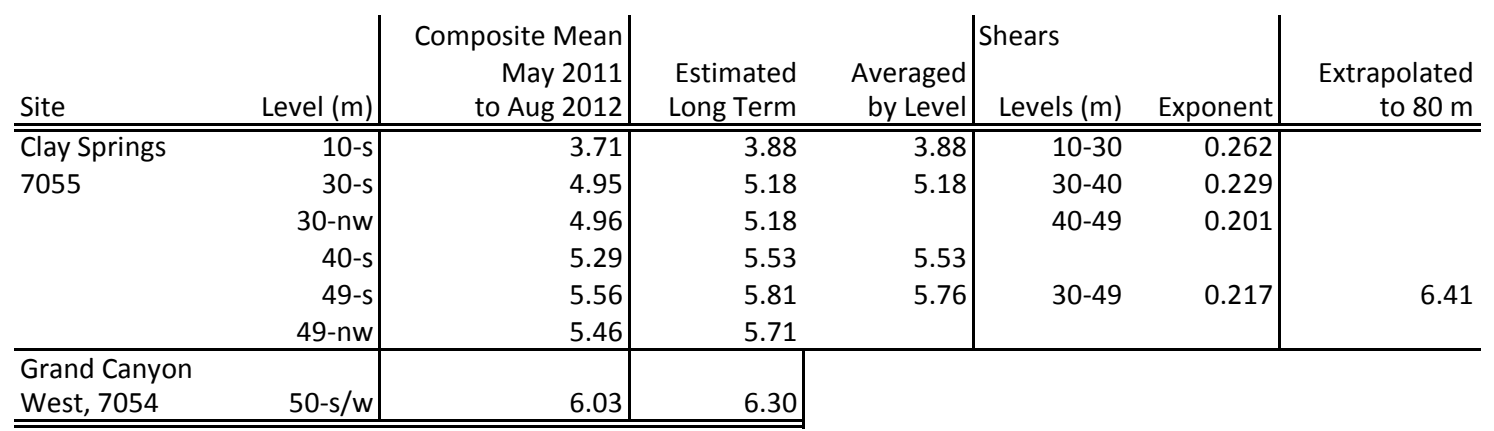


Gross Annual Energy Simulations

Peach Springs, Site 7052

Using Extrapolated 80-m Data, December 2005-September 2012

Turbine: GE-1.7/100 (power curve for moderate turbulence)

Air Density: $1.02 \mathrm{~kg} / \mathrm{m}^{3}$

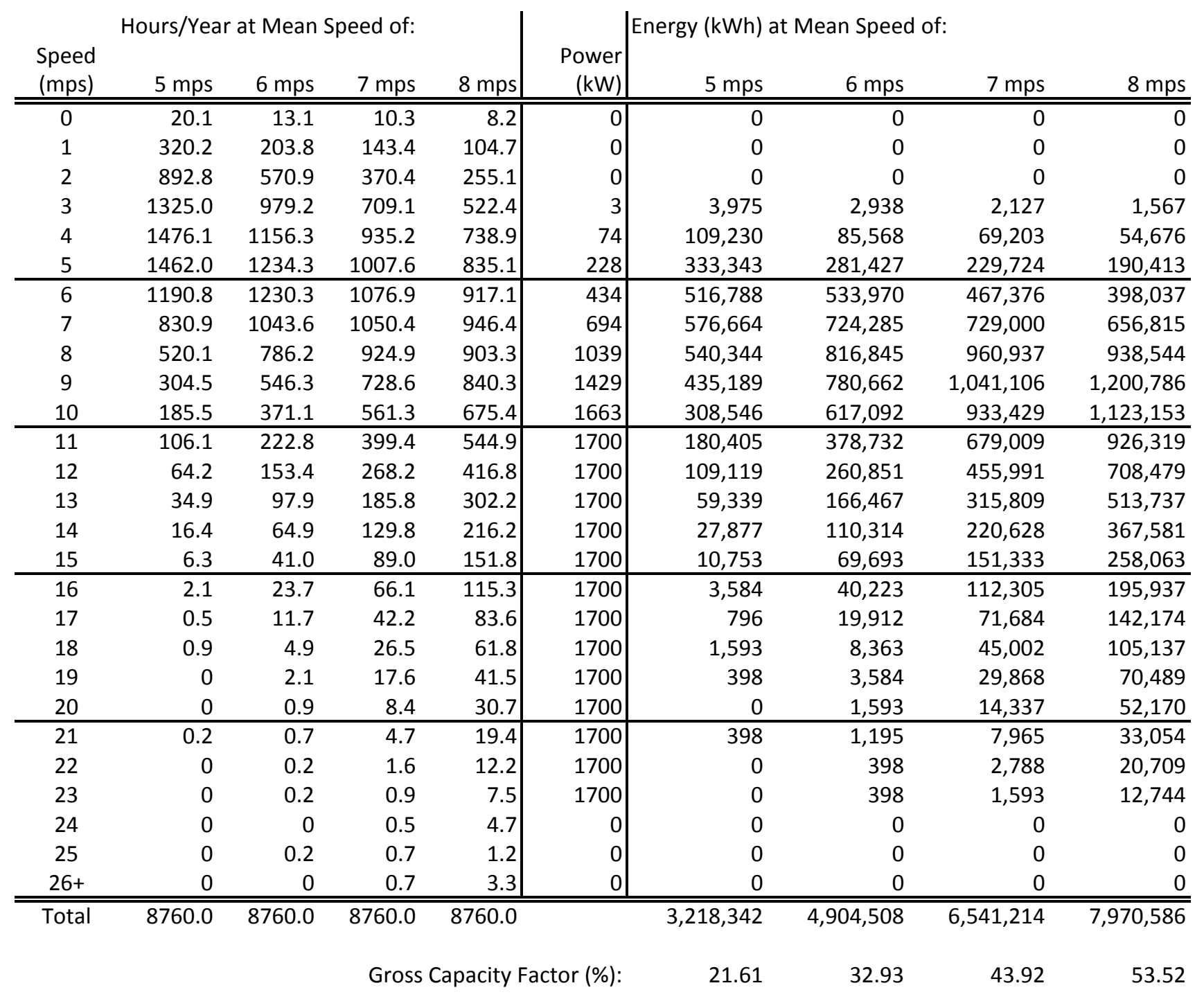


Gross Annual Energy Simulations

Nelson, Site 7053

Using Extrapolated 80-m Data, December 2006-September 2011

Turbine: GE-1.7/100 (power curve for moderate turbulence)

Air Density: $1.02 \mathrm{~kg} / \mathrm{m}^{3}$

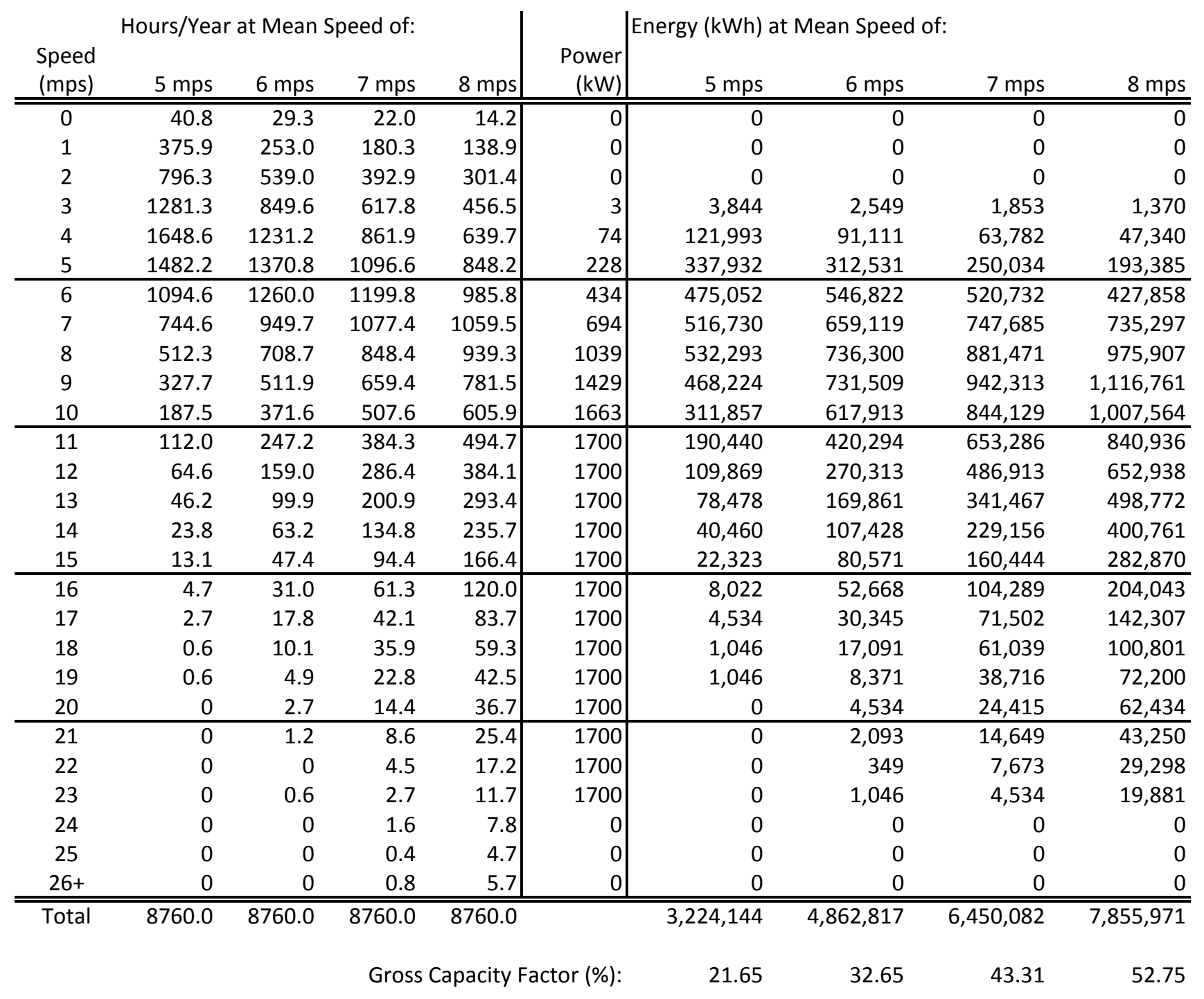


Gross Annual Energy Simulations

Grand Canyon West, Site 7054

Using Extrapolated 80-m Data, October 2006-September 2011

Turbine: GE-1.7/100 (power curve for moderate turbulence)

Air Density: $1.01 \mathrm{~kg} / \mathrm{m}^{3}$

\begin{tabular}{|c|c|c|c|c|c|c|c|c|c|}
\hline & ours/Yea & at Mean & seed of: & & & ergy (kWh) & Mean Speec & & \\
\hline (mps) & $5 \mathrm{mps}$ & $6 \mathrm{mps}$ & $7 \mathrm{mps}$ & $8 \mathrm{mps}$ & $(\mathrm{kW})$ & $5 \mathrm{mps}$ & $6 \mathrm{mps}$ & $7 \mathrm{mps}$ & $8 \mathrm{mps}$ \\
\hline 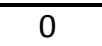 & 29.6 & 18.9 & 12.3 & 8.5 & 0 & 0 & 0 & $\overline{0}$ & $\overline{0}$ \\
\hline 1 & 343.2 & 238.0 & 173.5 & 128.4 & 0 & 0 & 0 & 0 & 0 \\
\hline 2 & 900.9 & 555.8 & 372.4 & 273.9 & 0 & 0 & 0 & 0 & 0 \\
\hline 3 & 1430.3 & 1011.6 & 715.4 & 508.2 & 3 & 4,291 & 3,035 & 2,146 & 1,525 \\
\hline 4 & 1510.3 & 1248.3 & 988.0 & 759.9 & 73 & 110,251 & 91,122 & 72,124 & 55,473 \\
\hline 5 & 1408.5 & 1273.1 & 1078.7 & 920.7 & 226 & 317,606 & 287,076 & 243,252 & 207,619 \\
\hline 6 & 1122.4 & 1169.0 & 1089.4 & 951.7 & 430 & 482,069 & 502,105 & 467,915 & 408,744 \\
\hline 7 & 726.2 & 975.7 & 1008.6 & 940.4 & 687 & 498,873 & 670,301 & 692,940 & 646,026 \\
\hline 8 & 473.9 & 726.2 & 866.7 & 879.8 & 1029 & 487,593 & 747,221 & 891,844 & 905,326 \\
\hline 9 & 291.0 & 483.6 & 681.3 & 783.7 & 1419 & 412,958 & 686,198 & 966,762 & $1,112,114$ \\
\hline 10 & 193.4 & 333.3 & 484.8 & 638.8 & 1660 & 320,868 & 553,118 & 804,475 & $1,060,115$ \\
\hline 11 & 120.3 & 221.7 & 356.9 & 488.7 & 1700 & 204,508 & 376,957 & 606,776 & 830,858 \\
\hline 12 & 89.3 & 160.8 & 262.0 & 362.7 & 1700 & 151,863 & 273,353 & 445,464 & 616,563 \\
\hline 13 & 51.8 & 109.6 & 176.9 & 276.7 & 1700 & 88,080 & 186,285 & 300,688 & 470,437 \\
\hline 14 & 33.5 & 79.6 & 138.6 & 203.5 & 1700 & 57,033 & 135,327 & 235,556 & 345,910 \\
\hline 15 & 13.5 & 60.7 & 98.1 & 151.5 & 1700 & 22,948 & 103,267 & 166,712 & 257,492 \\
\hline 16 & 10.7 & 36.1 & 78.0 & 121.1 & 1700 & 18,223 & 61,420 & 132,627 & 205,858 \\
\hline 17 & 5.4 & 23.6 & 57.4 & 90.1 & 1700 & 9,112 & 40,159 & 97,530 & 153,213 \\
\hline 18 & 3.2 & 12.1 & 40.9 & 68.5 & 1700 & 5,400 & 20,586 & 69,519 & 116,428 \\
\hline 19 & 1.6 & 7.7 & 27.6 & 58.8 & 1700 & 2,700 & 13,161 & 46,909 & 99,892 \\
\hline 20 & 0.8 & 6.4 & 18.7 & 44.5 & 1700 & 1,350 & 10,799 & 31,723 & 75,594 \\
\hline 21 & 0.2 & 4.8 & 11.1 & 28.4 & 1700 & 337 & 8,099 & 18,899 & 48,259 \\
\hline 22 & 0 & 0.8 & 5.6 & 22.0 & 1700 & 0 & 1,350 & 9,449 & 37,460 \\
\hline 23 & 0 & 1.6 & 7.3 & 16.3 & 1700 & 337 & 2,700 & 12,487 & 27,673 \\
\hline 24 & 0 & 0.4 & 3.8 & 10.1 & 0 & 0 & 0 & 0 & 0 \\
\hline 25 & 0 & 0 & 3.0 & 4.6 & 0 & 0 & 0 & 0 & 0 \\
\hline $26+$ & 0 & 0.2 & 3.0 & 18.5 & 0 & 0 & 0 & 0 & 0 \\
\hline \multirow[t]{2}{*}{ Total } & 8760.0 & 8760.0 & 8760.0 & 8760.0 & & $3,196,402$ & $4,773,640$ & $6,315,797$ & $7,682,578$ \\
\hline & \multicolumn{5}{|c|}{ Gross Capacity Factor (\%): } & 21.46 & 32.06 & 42.41 & 51.59 \\
\hline
\end{tabular}


Gross Annual Energy Simulations

Clay Springs, Site 7055

Using Extrapolated 80-m Data, May 2011-September 2012 (expanded to two full years)

Turbine: GE-1.7/100 (power curve for hihg turbulence)

Air Density: $1.02 \mathrm{~kg} / \mathrm{m}^{3}$

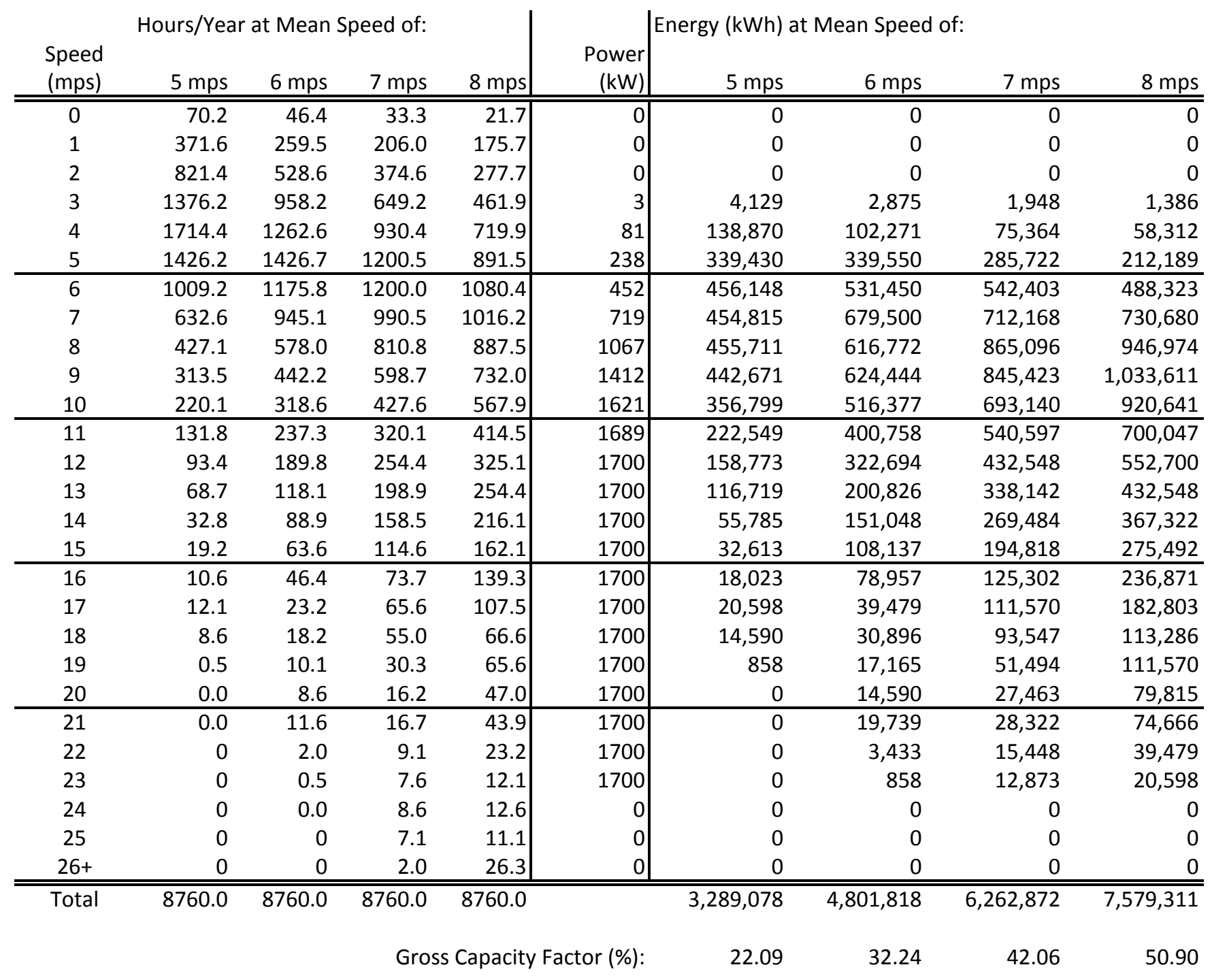


Gross Annual Energy Simulations

Peach Springs, Site 7052

Using Extrapolated 80-m Data, December 2005-September 2012

Turbine: Acciona AW-116/3000

Air Density: $1.02 \mathrm{~kg} / \mathrm{m}^{3}$

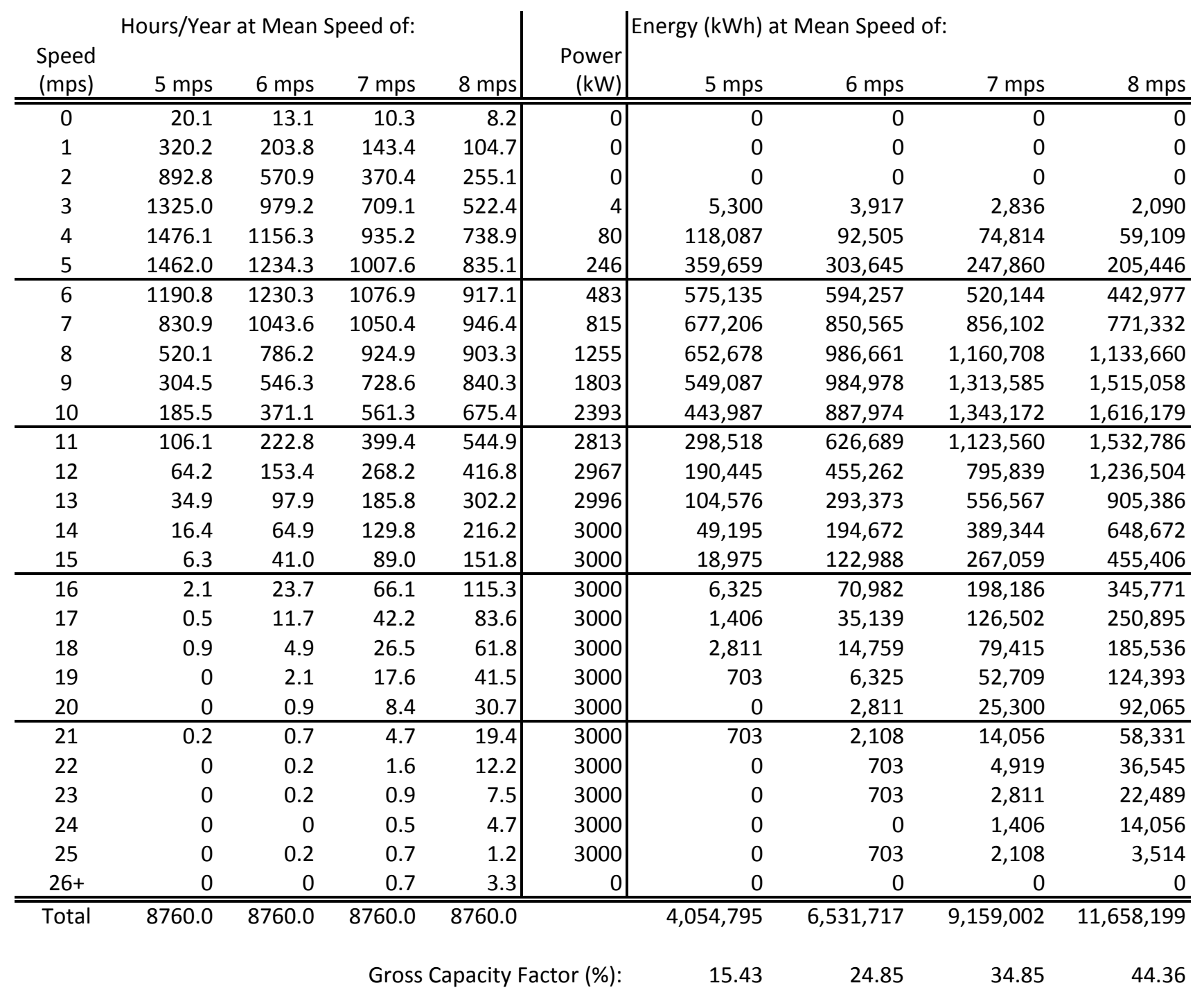


Gross Annual Energy Simulations

Nelson, Site 7053

Using Extrapolated 80-m Data, December 2006-September 2011

Turbine: Acciona AW-116/3000

Air Density: $1.02 \mathrm{~kg} / \mathrm{m}^{3}$

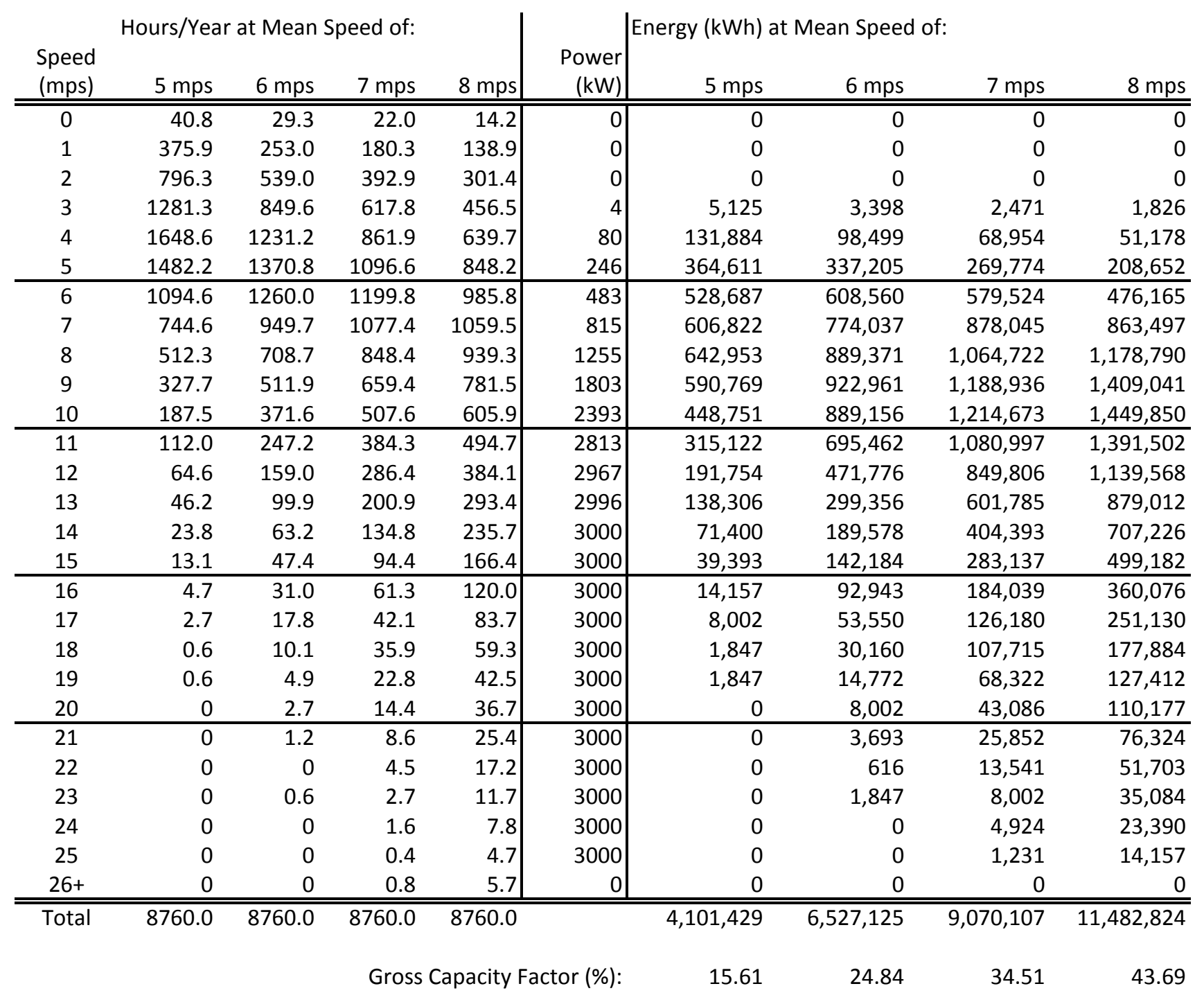


Gross Annual Energy Simulations

Grand Canyon West, Site 7054

Using Extrapolated 80-m Data, October 2006-September 2011

Turbine: Acciona AW-116/3000

Air Density: $1.01 \mathrm{~kg} / \mathrm{m}^{3}$

\begin{tabular}{|c|c|c|c|c|c|c|c|c|c|}
\hline \multirow{2}{*}{$\begin{array}{l}\text { Speed } \\
\text { (mps) }\end{array}$} & \multicolumn{4}{|c|}{ Hours/Year at Mean Speed of: } & \multirow{2}{*}{$\begin{array}{r}\text { Power } \\
(\mathrm{kW}) \\
\end{array}$} & \multicolumn{4}{|c|}{ Energy (kWh) at Mean Speed of: } \\
\hline & $5 \mathrm{mps}$ & $6 \mathrm{mps}$ & $7 \mathrm{mps}$ & $8 \mathrm{mps}$ & & $5 \mathrm{mps}$ & $6 \mathrm{mps}$ & $7 \mathrm{mps}$ & $8 \mathrm{mps}$ \\
\hline 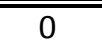 & 29.6 & 18.9 & 12.3 & 8.5 & 0 & $\overline{0}$ & $\overline{0}$ & $\overline{0}$ & $\overline{0}$ \\
\hline 1 & 343.2 & 238.0 & 173.5 & 128.4 & 0 & 0 & 0 & 0 & 0 \\
\hline 2 & 900.9 & 555.8 & 372.4 & 273.9 & 0 & 0 & 0 & 0 & 0 \\
\hline 3 & 1430.3 & 1011.6 & 715.4 & 508.2 & 3 & 4,291 & 3,035 & 2,146 & 1,525 \\
\hline 4 & 1510.3 & 1248.3 & 988.0 & 759.9 & 76 & 114,782 & 94,867 & 75,088 & 57,753 \\
\hline 5 & 1408.5 & 1273.1 & 1078.7 & 920.7 & 243 & 342,254 & 309,355 & 262,129 & 223,731 \\
\hline 6 & 1122.4 & 1169.0 & 1089.4 & 951.7 & 478 & 536,505 & 558,804 & 520,753 & 454,900 \\
\hline 7 & 726.2 & 975.7 & 1008.6 & 940.4 & 806 & 585,287 & 786,409 & 812,969 & 757,929 \\
\hline 8 & 473.9 & 726.2 & 866.7 & 879.8 & 1241 & 588,050 & 901,167 & $1,075,587$ & $1,091,846$ \\
\hline 9 & 291.0 & 483.6 & 681.3 & 783.7 & 1784 & 519,181 & 862,704 & $1,215,436$ & $1,398,176$ \\
\hline 10 & 193.4 & 333.3 & 484.8 & 638.8 & 2374 & 459,018 & 791,264 & $1,150,844$ & $1,516,550$ \\
\hline 11 & 120.3 & 221.7 & 356.9 & 488.7 & 2804 & 337,319 & 621,758 & $1,000,824$ & $1,370,427$ \\
\hline 12 & 89.3 & 160.8 & 262.0 & 362.7 & 2964 & 264,777 & 476,599 & 776,680 & $1,074,995$ \\
\hline 13 & 51.8 & 109.6 & 176.9 & 276.7 & 2995 & 155,177 & 328,190 & 529,742 & 828,799 \\
\hline 14 & 33.5 & 79.6 & 138.6 & 203.5 & 3000 & 100,646 & 238,812 & 415,687 & 610,429 \\
\hline 15 & 13.5 & 60.7 & 98.1 & 151.5 & 3000 & 40,497 & 182,235 & 294,197 & 454,397 \\
\hline 16 & 10.7 & 36.1 & 78.0 & 121.1 & 3000 & 32,159 & 108,388 & 234,047 & 363,280 \\
\hline 17 & 5.4 & 23.6 & 57.4 & 90.1 & 3000 & 16,080 & 70,869 & 172,111 & 270,375 \\
\hline 18 & 3.2 & 12.1 & 40.9 & 68.5 & 3000 & 9,529 & 36,328 & 122,681 & 205,461 \\
\hline 19 & 1.6 & 7.7 & 27.6 & 58.8 & 3000 & 4,764 & 23,226 & 82,780 & 176,280 \\
\hline 20 & 0.8 & 6.4 & 18.7 & 44.5 & 3000 & 2,382 & 19,057 & 55,981 & 133,401 \\
\hline 21 & 0.2 & 4.8 & 11.1 & 28.4 & 3000 & 596 & 14,293 & 33,350 & 85,162 \\
\hline 22 & 0 & 0.8 & 5.6 & 22.0 & 3000 & 0 & 2,382 & 16,675 & 66,105 \\
\hline 23 & 0 & 1.6 & 7.3 & 16.3 & 3000 & 596 & 4,764 & 22,035 & 48,834 \\
\hline 24 & 0 & 0.4 & 3.8 & 10.1 & 3000 & 0 & 1,191 & 11,315 & 30,373 \\
\hline 25 & 0 & 0 & 3.0 & 4.6 & 3000 & 0 & 1,191 & 8,933 & 13,697 \\
\hline $26+$ & 0 & 0.2 & 3.0 & 18.5 & 0 & 0 & 0 & 0 & 0 \\
\hline \multirow[t]{2}{*}{ 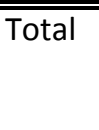 } & 8760.0 & 8760.0 & 8760.0 & 8760.0 & & $4,113,888$ & $6,436,889$ & $8,891,992$ & $11,234,426$ \\
\hline & \multicolumn{5}{|c|}{ Gross Capacity Factor (\%): } & 15.65 & 24.49 & 33.84 & 42.75 \\
\hline
\end{tabular}


Gross Annual Energy Simulations

Clay Springs, Site 7055

Using Extrapolated 80-m Data, May 2011-September 2012 (expanded to two full years)

Turbine: Acciona AW-116/3000

Air Density: $1.02 \mathrm{~kg} / \mathrm{m}^{3}$

\begin{tabular}{|c|c|c|c|c|c|c|c|c|c|}
\hline \multirow{2}{*}{$\begin{array}{l}\text { Speed } \\
\text { (mps) }\end{array}$} & \multicolumn{4}{|c|}{ Hours/Year at Mean Speed of: } & \multirow{2}{*}{$\begin{array}{r}\text { Power } \\
(\mathrm{kW})\end{array}$} & \multicolumn{4}{|c|}{ Energy (kWh) at Mean Speed of: } \\
\hline & $5 \mathrm{mps}$ & $6 \mathrm{mps}$ & $7 \mathrm{mps}$ & $8 \mathrm{mps}$ & & $5 \mathrm{mps}$ & $6 \mathrm{mps}$ & $7 \mathrm{mps}$ & $8 \mathrm{mps}$ \\
\hline 0 & 70.2 & $\overline{46.4}$ & 33.3 & 21.7 & \begin{tabular}{l|l}
0 \\
\end{tabular} & $\overline{0}$ & 0 & $\overline{0}$ & $\overline{0}$ \\
\hline 1 & 371.6 & 259.5 & 206.0 & 175.7 & 0 & 0 & 0 & 0 & 0 \\
\hline 2 & 821.4 & 528.6 & 374.6 & 277.7 & 0 & 0 & 0 & 0 & 0 \\
\hline 3 & 1376.2 & 958.2 & 649.2 & 461.9 & 4 & 5,505 & 3,833 & 2,597 & 1,848 \\
\hline 4 & 1714.4 & 1262.6 & 930.4 & 719.9 & 80 & 137,155 & 101,009 & 74,434 & 57,592 \\
\hline 5 & 1426.2 & 1426.7 & 1200.5 & 891.5 & 246 & 350,839 & 350,963 & 295,326 & 219,321 \\
\hline 6 & 1009.2 & 1175.8 & 1200.0 & 1080.4 & 483 & 487,432 & 567,899 & 579,603 & 521,814 \\
\hline 7 & 632.6 & 945.1 & 990.5 & 1016.2 & 815 & 515,541 & 770,226 & 807,256 & 828,240 \\
\hline 8 & 427.1 & 578.0 & 810.8 & 887.5 & 1255 & 536,005 & 725,444 & $1,017,522$ & $1,113,826$ \\
\hline 9 & 313.5 & 442.2 & 598.7 & 732.0 & 1803 & 565,252 & 797,360 & $1,079,531$ & $1,319,831$ \\
\hline 10 & 220.1 & 318.6 & 427.6 & 567.9 & 2393 & 526,725 & 762,301 & $1,023,248$ & $1,359,095$ \\
\hline 11 & 131.8 & 237.3 & 320.1 & 414.5 & 2813 & 370,651 & 667,455 & 900,355 & $1,165,916$ \\
\hline 12 & 93.4 & 189.8 & 254.4 & 325.1 & 2967 & 277,105 & 563,197 & 754,923 & 964,624 \\
\hline 13 & 68.7 & 118.1 & 198.9 & 254.4 & 2996 & 205,701 & 353,926 & 595,926 & 762,302 \\
\hline 14 & 32.8 & 88.9 & 158.5 & 216.1 & 3000 & 98,444 & 266,556 & 475,560 & 648,216 \\
\hline 15 & 19.2 & 63.6 & 114.6 & 162.1 & 3000 & 57,552 & 190,830 & 343,797 & 486,162 \\
\hline 16 & 10.6 & 46.4 & 73.7 & 139.3 & 3000 & 31,805 & 139,336 & 221,120 & 418,008 \\
\hline 17 & 12.1 & 23.2 & 65.6 & 107.5 & 3000 & 36,349 & 69,668 & 196,888 & 322,593 \\
\hline 18 & 8.6 & 18.2 & 55.0 & 66.6 & 3000 & 25,747 & 54,523 & 165,083 & 199,917 \\
\hline 19 & 0.5 & 10.1 & 30.3 & 65.6 & 3000 & 1,514 & 30,290 & 90,872 & 196,888 \\
\hline 20 & 0.0 & 8.6 & 16.2 & 47.0 & 3000 & 0 & 25,747 & 48,465 & 140,851 \\
\hline 21 & 0.0 & 11.6 & 16.7 & 43.9 & 3000 & 0 & 34,834 & 49,979 & 131,764 \\
\hline 22 & 0 & 2.0 & 9.1 & 23.2 & 3000 & 0 & 6,058 & 27,261 & 69,668 \\
\hline 23 & 0 & 0.5 & 7.6 & 12.1 & 3000 & 0 & 1,514 & 22,718 & 36,349 \\
\hline 24 & 0 & 0.0 & 8.6 & 12.6 & 3000 & 0 & 0 & 25,747 & 37,863 \\
\hline 25 & 0 & 0 & 7.1 & 11.1 & 3000 & 0 & 0 & 21,203 & 33,320 \\
\hline $26+$ & 0 & 0 & 2.0 & 26.3 & 0 & 0 & 0 & 0 & 0 \\
\hline \multirow[t]{2}{*}{ Total } & 8760.0 & 8760.0 & 8760.0 & 8760.0 & & $4,229,321$ & $6,482,969$ & $8,819,413$ & $11,036,005$ \\
\hline & \multicolumn{5}{|c|}{ Gross Capacity Factor (\%): } & 16.09 & 24.67 & 33.56 & 41.99 \\
\hline
\end{tabular}


80-m Wind Rose

Peach Springs, Site 7052

S-September 2012

Hours of Occurrence

\begin{tabular}{|c|c|c|c|c|c|c|c|c|c|c|c|}
\hline Direction & $\begin{array}{r}d \text { Spee } \\
0-3\end{array}$ & $\begin{array}{r}n p s) \ldots \\
3-6\end{array}$ & $6-9$ & $9-12$ & $12-15$ & $15-18$ & $18-21$ & 21-24 & $24+$ & $\begin{array}{l}\text { Row } \\
\text { Total }\end{array}$ & $\begin{array}{l}\text { Meaad } \\
\text { Speed }\end{array}$ \\
\hline 0 & $\begin{array}{l}137 \\
\end{array}$ & 4662 & 291 & 77 & 5 & 1 & 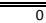 & 0 & 0 & 972 & (5.5 \\
\hline 10 & 111 & 448 & 480 & 106 & 7 & 0 & 0 & 0 & 0 & 1152 & 6.0 \\
\hline 20 & 112 & 411 & 353 & 38 & 1 & 0 & 0 & 0 & 0 & 914 & 5.5 \\
\hline 30 & 112 & 423 & 293 & 18 & 0 & 0 & 0 & 0 & 0 & 846 & 5.2 \\
\hline 40 & 103 & 349 & 220 & 26 & 5 & 2 & 0 & 0 & 0 & 705 & 5.3 \\
\hline 50 & 113 & 224 & 202 & 98 & 19 & 0 & 0 & 0 & 0 & 657 & 6.1 \\
\hline 60 & 79 & 188 & 188 & 178 & 41 & 2 & 0 & 0 & 0 & 676 & 7.2 \\
\hline 70 & 84 & 190 & 271 & 238 & 81 & 4 & 0 & 0 & 0 & 868 & 7.7 \\
\hline 80 & 79 & 181 & 189 & 182 & 118 & 13 & 1 & 0 & 0 & 764 & 8.0 \\
\hline 90 & 86 & 196 & 224 & 177 & 52 & 3 & 1 & 0 & 0 & 738 & $\frac{7.2}{7.2}$ \\
\hline 100 & 97 & 236 & 305 & 130 & 12 & 2 & 0 & 0 & 0 & 782 & 6.5 \\
\hline 110 & 118 & 298 & 319 & 167 & 19 & 4 & 1 & 0 & 0 & 924 & 6.4 \\
\hline 120 & 130 & 374 & 394 & 136 & 17 & 6 & 0 & 0 & 0 & $\begin{array}{r}1057 \\
105\end{array}$ & 6.2 \\
\hline 130 & 141 & 453 & 473 & 171 & 40 & 10 & 4 & 1 & 0 & 1292 & 6.5 \\
\hline 140 & 129 & 471 & 484 & 183 & 63 & 20 & 1 & 0 & 0 & 1351 & 6.7 \\
\hline 150 & 132 & 451 & 598 & 220 & 81 & 32 & 4 & 1 & 0 & 1518 & 7.0 \\
\hline 160 & 145 & 472 & 537 & 252 & 143 & 47 & 8 & 1 & 0 & 1605 & 7.4 \\
\hline 170 & 121 & 427 & 554 & 316 & 124 & 62 & 11 & 1 & 0 & 1615 & 7.7 \\
\hline 180 & 109 & 381 & 559 & 337 & 164 & 67 & 12 & 2 & 2 & 1633 & 8.1 \\
\hline 190 & 106 & 359 & 562 & 367 & 151 & 60 & 8 & 2 & 0 & 1615 & 8.1 \\
\hline 200 & 107 & 413 & 540 & 368 & 140 & 43 & 11 & 1 & 0 & 1623 & 7.9 \\
\hline 210 & 120 & 481 & 620 & 371 & 106 & 34 & 8 & 1 & 0 & 1741 & 7.5 \\
\hline 220 & 126 & 589 & 767 & 328 & 78 & 28 & 8 & 0 & 1 & 1924 & 7.1 \\
\hline 230 & 154 & 583 & 731 & 247 & 67 & 26 & 9 & 2 & 1 & 1819 & 6.9 \\
\hline 240 & 147 & 497 & 524 & 202 & 65 & 16 & 5 & 0 & 0 & 1456 & 6.7 \\
\hline 250 & 130 & 333 & 281 & 125 & 26 & 10 & 2 & 0 & 0 & 906 & 6.2 \\
\hline 260 & 109 & 238 & 129 & 47 & 8 & 0 & 0 & 0 & 0 & 532 & 5.3 \\
\hline 270 & 125 & 189 & 88 & 29 & 2 & 1 & 0 & 0 & 0 & 434 & 4.7 \\
\hline 280 & 114 & 225 & 91 & 25 & 3 & 0 & 0 & 0 & 0 & 457 & 4.7 \\
\hline 290 & 122 & 266 & 99 & 24 & 6 & 0 & 0 & 0 & 0 & 517 & 4.7 \\
\hline 300 & 119 & 280 & 102 & 33 & 3 & 0 & 0 & 0 & 0 & 537 & 4.8 \\
\hline 310 & 126 & 293 & 115 & 33 & 5 & 0 & 0 & 0 & 0 & 572 & 4.9 \\
\hline 320 & 118 & 355 & 126 & 28 & 1 & 0 & 0 & 0 & 0 & 628 & 4.8 \\
\hline 330 & 154 & 428 & 134 & 17 & 1 & 0 & 0 & 0 & 0 & 733 & 4.5 \\
\hline 340 & 148 & 462 & 122 & 20 & 0 & 0 & 0 & 0 & 0 & 752 & 4.5 \\
\hline 350 & 132 & 445 & 189 & 36 & 3 & 0 & 0 & 0 & 0 & 805 & 5.0 \\
\hline Calms & $\begin{array}{r}152 \\
\end{array}$ & & & & & & & & & $\begin{array}{r}1 \\
\end{array}$ & \\
\hline$\overline{T \text { Totals }}$ & "4296 & 13072 & 154 & "5351 & $\begin{array}{ll}1653 \\
\end{array}$ & 4491 & $\overline{72}$ & $\overline{10}$ & 3 & 37121 & $\overline{\overline{6.6}}$ \\
\hline
\end{tabular}

GE-1.7/100 Energy...

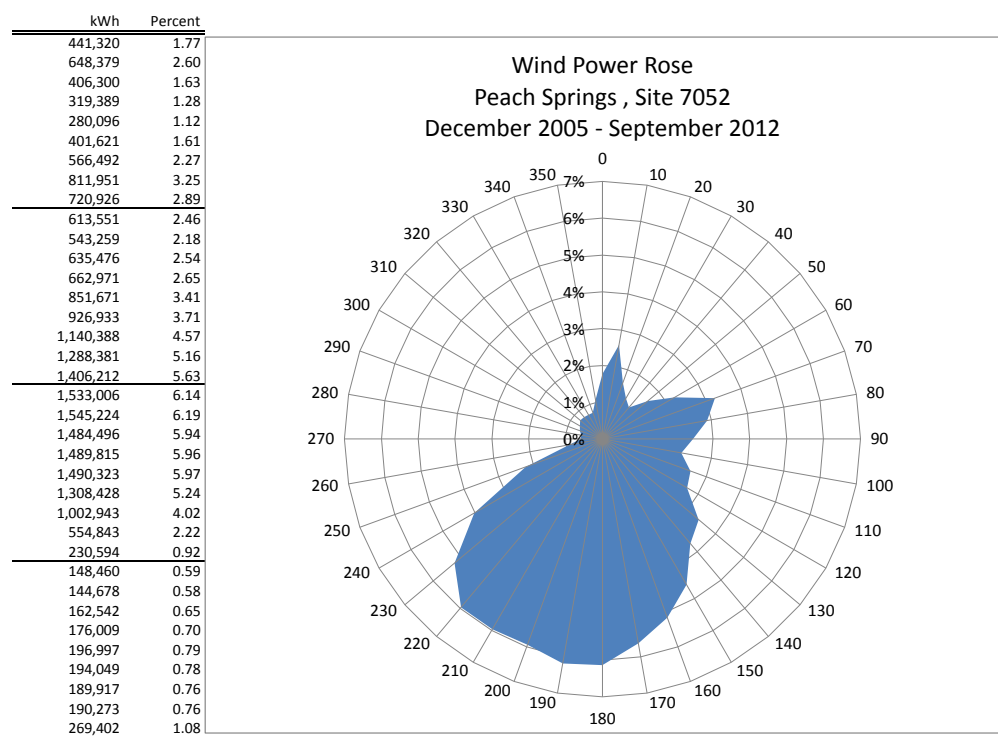

62.9\% Data Recovery 
80-m Wind Rose

Nelson, Site 7053
December 2006-September 2012

Hours of Occurrence

\begin{tabular}{|c|c|c|c|c|c|c|c|c|c|c|c|c|c|}
\hline \multicolumn{4}{|c|}{ Wind Speed (mps)... } & \multirow[b]{2}{*}{$9-12$} & \multirow[b]{2}{*}{$12-15$} & \multirow[b]{2}{*}{$15-18$} & \multirow[b]{2}{*}{$18-21$} & \multirow[b]{2}{*}{ 21-24 } & \multirow[b]{2}{*}{$24+$} & \multirow{2}{*}{$\begin{array}{l}\text { Row } \\
\text { Total }\end{array}$} & \multirow{2}{*}{$\begin{array}{l}\text { Mean } \\
\text { Speed }\end{array}$} & \multicolumn{2}{|c|}{ GE-1.7/100 Energy... } \\
\hline Direction & $0-3$ & $3-6$ & $6-9$ & & & & & & & & & kWh & Percent \\
\hline 0 & "111 & 377 & 345 & 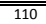 & 15 & 3 & 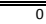 & 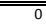 & 0 & 961 & "6.1 & 567,748 & 2.70 \\
\hline 10 & 110 & 415 & 352 & 125 & 19 & 1 & 0 & 0 & 0 & 1022 & 6.1 & 601,210 & 2.86 \\
\hline 20 & 149 & 362 & 193 & 38 & 2 & 0 & 0 & 0 & 0 & 744 & 5.0 & 271,785 & 1.29 \\
\hline 30 & 121 & 286 & 124 & 16 & 1 & 0 & 0 & 0 & 0 & 548 & 4.7 & 162,396 & 0.77 \\
\hline 40 & 127 & 291 & 109 & 15 & 0 & 0 & 0 & 0 & 0 & 542 & 4.6 & 152,163 & 0.72 \\
\hline 50 & 135 & 299 & 162 & 25 & 1 & 1 & 0 & 0 & 0 & 622 & 4.9 & 213,226 & 1.01 \\
\hline 60 & 138 & 297 & 220 & 38 & 4 & 1 & 0 & 0 & 0 & 698 & 5.3 & 297,110 & 1.41 \\
\hline 70 & 168 & 301 & 219 & 100 & 26 & 1 & 0 & 0 & 0 & 814 & 5.7 & 437,189 & 2.08 \\
\hline 80 & 175 & 289 & 239 & 106 & 11 & 1 & 0 & 0 & 0 & 821 & 5.6 & 438,032 & 2.08 \\
\hline 90 & 228 & 342 & 267 & 54 & 4 & 0 & 0 & 0 & 0 & 895 & 5.0 & 361,908 & 1.72 \\
\hline 100 & 271 & 453 & 287 & 50 & 2 & 0 & 0 & 0 & 0 & 1064 & 4.8 & 385,966 & 1.83 \\
\hline 110 & 428 & 960 & 553 & 95 & 4 & 1 & 0 & 0 & 0 & 2040 & 5.0 & 749,107 & 3.56 \\
\hline 120 & 492 & 1463 & 499 & 42 & 3 & 0 & 0 & 0 & 0 & 2499 & 4.6 & 665,918 & 3.16 \\
\hline 130 & 410 & 1291 & 264 & 18 & 2 & 0 & 0 & 0 & 0 & 1985 & 4.3 & 400,342 & 1.90 \\
\hline 140 & 376 & 1073 & 331 & 37 & 1 & 0 & 0 & 0 & 0 & 1817 & 4.5 & 457,738 & 2.17 \\
\hline 150 & 357 & 880 & 394 & 68 & 10 & 1 & 0 & 0 & 0 & 1708 & 4.9 & 566,982 & 2.69 \\
\hline 160 & 317 & 695 & 390 & 107 & 9 & 0 & 0 & 0 & 0 & 1517 & 5.1 & 609,592 & 2.90 \\
\hline 170 & 226 & 576 & 382 & 110 & 23 & 1 & 0 & 0 & 0 & 1317 & 5.5 & 623,169 & 2.96 \\
\hline 180 & 220 & 491 & 389 & 149 & 45 & 9 & 0 & 0 & 0 & 1302 & 6.0 & 737,754 & 3.50 \\
\hline 190 & 190 & 434 & 454 & $\begin{array}{l}309 \\
309\end{array}$ & 117 & 28 & 3 & 0 & 0 & 1535 & 7.1 & $\begin{array}{l}1,227,145 \\
\end{array}$ & 5.83 \\
\hline 200 & 178 & 452 & 568 & 448 & 183 & 47 & 3 & 1 & 0 & 1881 & 7.8 & $1,723,765$ & 8.19 \\
\hline 210 & 134 & 422 & 546 & 425 & 203 & 76 & 14 & 0 & 0 & 1820 & 8.2 & $1,756,660$ & 8.35 \\
\hline 220 & 155 & 461 & 554 & 412 & 137 & 41 & 5 & 2 & 0 & 1767 & 7.6 & $1,564,859$ & 7.43 \\
\hline 230 & 139 & 618 & 729 & 329 & 62 & 18 & 3 & 0 & 0 & 1898 & 6.9 & $1,422,293$ & 6.76 \\
\hline 240 & 120 & 616 & 565 & 180 & 33 & 9 & 0 & 0 & 0 & 1522 & 6.4 & 940,515 & 4.47 \\
\hline 250 & 157 & 605 & 388 & 127 & 38 & 9 & 1 & 0 & 0 & 1325 & 5.9 & 704,767 & 3.35 \\
\hline 260 & 114 & 564 & 475 & 121 & 14 & 4 & 0 & 1 & 0 & 1293 & 6.0 & 716,892 & 3.41 \\
\hline 270 & 118 & 642 & 379 & 44 & 5 & 0 & 0 & 0 & 0 & 1189 & 5.4 & 475,135 & 2.26 \\
\hline 280 & 116 & 513 & 166 & 17 & 4 & 0 & 0 & 0 & 0 & 815 & 4.8 & 231,424 & 1.10 \\
\hline 290 & 113 & 330 & 96 & 17 & 2 & 0 & 0 & 0 & 0 & 557 & 4.6 & 147,283 & 0.70 \\
\hline 300 & 135 & 294 & 109 & 23 & 2 & 0 & 0 & 0 & 0 & 563 & 4.6 & 163,460 & 0.78 \\
\hline 310 & 111 & 302 & 128 & 35 & 1 & 0 & 0 & 0 & 0 & 577 & 5.0 & 202,302 & 0.96 \\
\hline 320 & 112 & 373 & 153 & 32 & 3 & 0 & 0 & 0 & 0 & 673 & 5.0 & 229,491 & 1.09 \\
\hline 330 & 105 & 436 & 158 & 31 & 7 & 0 & 0 & 0 & 0 & 738 & 5.0 & 246,359 & 1.17 \\
\hline 340 & 111 & 417 & 172 & 34 & 5 & 1 & 0 & 0 & 0 & 739 & 5.1 & 258,614 & 1.23 \\
\hline $\begin{array}{l}350 \\
\text { Calms }\end{array}$ & $\begin{array}{r}110 \\
38\end{array}$ & 372 & 240 & 46 & 5 & 0 & 1 & 0 & 0 & $\begin{array}{r}774 \\
38\end{array}$ & 5.4 & 339,146 & 1.61 \\
\hline \begin{tabular}{|l|l|l} 
Totals \\
\end{tabular} & $\overline{6813}$ & 18992 & 11600 & 3932 & 1000 & 249 & $\overline{30}$ & $\overline{4}$ & $\overline{0}$ & 42620 & $\overline{5.7}$ & $\overline{21,049,445}$ & $\overline{100.00}$ \\
\hline
\end{tabular}

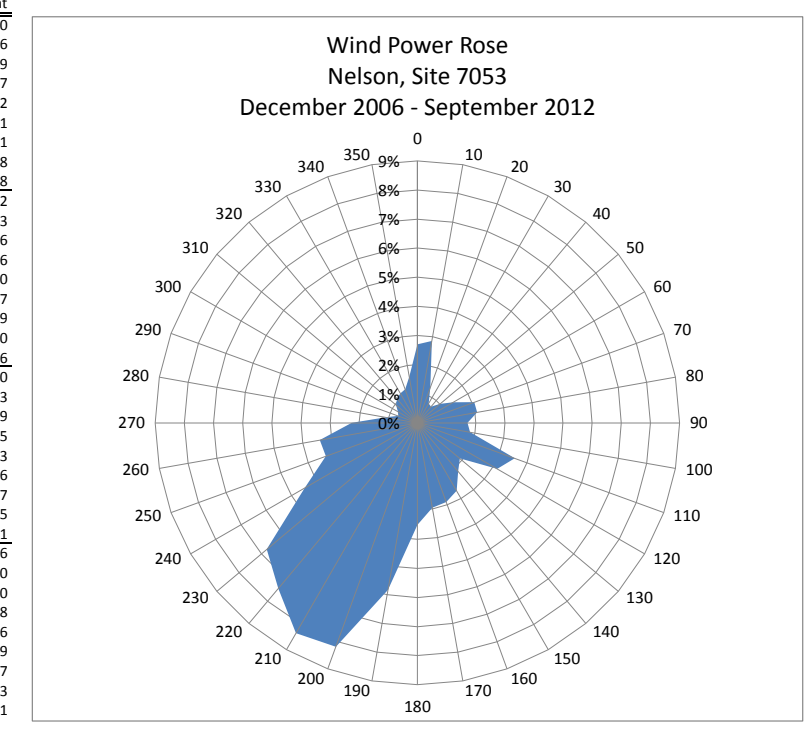

84.5\% Data Recovery 
80-m Wind Rose

Grand Canyon West, Site 7054

Hours of Occurrence

\begin{tabular}{|c|c|c|c|c|c|c|c|c|c|c|c|c|c|}
\hline \multicolumn{4}{|c|}{ Wind Speed (mps)... } & \multirow[b]{2}{*}{ 9-12 } & \multirow[b]{2}{*}{$12-15$} & \multirow[b]{2}{*}{$15-18$} & \multirow[b]{2}{*}{$18-21$} & \multirow[b]{2}{*}{ 21-24 } & \multirow[b]{2}{*}{$24+$} & \multirow[b]{2}{*}{$\begin{array}{l}\text { Row } \\
\text { Total }\end{array}$} & \multicolumn{3}{|c|}{ GE-1.7/100 Energy... } \\
\hline Direction & $0-3$ & 3-6 & 6-9 & & & & & & & & $\begin{array}{l}\text { Mean } \\
\text { Speed }\end{array}$ & $\mathrm{kWh}$ & Percent \\
\hline 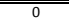 & (94 & 302 & $\begin{array}{ll}328 \\
\end{array}$ & 178 & 22 & 0 & 0 & $\overline{0}$ & 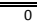 & 925 & $\overline{c 6.6}$ & 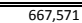 & 2.58 \\
\hline 10 & 102 & 255 & 251 & 129 & 14 & 0 & 0 & 0 & 0 & 750 & 6.3 & 491,396 & 1.90 \\
\hline 20 & 120 & 246 & 186 & 70 & 8 & 0 & 0 & 0 & 0 & 629 & 5.6 & 316,877 & 1.22 \\
\hline 30 & 114 & 325 & 196 & 54 & 7 & 0 & 0 & 0 & 0 & 696 & 5.4 & 308,802 & 1.19 \\
\hline 40 & 133 & 309 & 178 & 35 & 1 & 0 & 0 & 0 & 0 & 656 & 5.0 & 246,567 & 0.95 \\
\hline 50 & 126 & 317 & 184 & 31 & 2 & 0 & 0 & 0 & 0 & 659 & 5.1 & 246,239 & 0.95 \\
\hline 60 & 114 & 338 & 199 & 32 & 4 & 1 & 0 & 0 & 0 & 687 & 5.2 & 266,366 & 1.03 \\
\hline 70 & 84 & 297 & 220 & 34 & 5 & 1 & 2 & 0 & 0 & 643 & 5.6 & 291,139 & 1.12 \\
\hline 80 & 103 & 325 & 269 & 62 & 4 & 2 & 0 & 0 & 0 & 765 & 5.7 & 382,524 & 1.48 \\
\hline 90 & 102 & 361 & 300 & 77 & 12 & 4 & 1 & 0 & 0 & 855 & 5.9 & 456,953 & 1.76 \\
\hline 100 & 105 & 428 & 365 & 101 & 23 & 8 & 0 & 0 & 0 & 1028 & 6.1 & 586,072 & 2.26 \\
\hline 110 & 99 & 394 & 413 & 158 & 34 & 13 & 1 & 0 & 0 & 1110 & 6.6 & 759,274 & 2.93 \\
\hline 120 & 86 & 398 & 440 & 201 & 52 & 28 & 6 & 0 & 0 & 1211 & 7.1 & 925,104 & 3.57 \\
\hline 130 & 94 & 349 & 484 & 211 & 96 & 30 & 10 & 3 & 2 & 1277 & 7.5 & $1,059,062$ & 4.09 \\
\hline 140 & 83 & 355 & 467 & 256 & 108 & 47 & 13 & 4 & 2 & 1336 & 7.9 & $1,178,010$ & 4.55 \\
\hline 150 & 86 & 337 & 456 & 275 & 112 & 46 & 20 & 5 & 0 & 1336 & 8.0 & $1,214,056$ & 4.69 \\
\hline 160 & 77 & 288 & 495 & 380 & 147 & 55 & 16 & 11 & 3 & 1471 & 8.6 & $1,507,587$ & 5.82 \\
\hline 170 & 100 & 293 & 594 & 369 & 163 & 80 & 19 & 8 & 4 & 1631 & 8.6 & $1,646,627$ & 6.36 \\
\hline 180 & 81 & 326 & 582 & 403 & 198 & 83 & 30 & 18 & 2 & 1723 & 8.8 & $1,780,914$ & 6.88 \\
\hline 190 & 110 & 371 & 659 & 451 & 285 & 133 & 57 & 9 & 4 & 2079 & 9.1 & $2,212,999$ & 8.54 \\
\hline 200 & 110 & 438 & 644 & 471 & 309 & 155 & 49 & 12 & 5 & 2193 & 9.1 & $2,308,241$ & 8.91 \\
\hline 210 & 122 & 496 & 517 & 321 & 150 & 73 & 28 & 8 & 6 & 1720 & 8.0 & $1,499,029$ & 5.79 \\
\hline 220 & 145 & 600 & 453 & 182 & 88 & 58 & 20 & 7 & 1 & 1553 & 7.0 & $1,061,845$ & 4.10 \\
\hline 230 & 152 & 584 & 360 & 149 & 63 & 26 & 11 & 1 & 4 & 1350 & 6.5 & 808,613 & 3.12 \\
\hline 240 & 158 & 535 & 273 & 93 & 34 & 17 & 8 & 0 & 1 & 1119 & 5.9 & 549,852 & 2.12 \\
\hline 250 & 128 & 387 & 199 & 54 & 12 & 6 & 1 & 0 & 0 & 787 & 5.4 & 340,139 & 1.31 \\
\hline 260 & 113 & 333 & 126 & 15 & 3 & 1 & 0 & 0 & 0 & 591 & 4.8 & 177,544 & 0.69 \\
\hline 270 & 104 & 275 & 95 & 8 & 2 & 1 & 0 & 0 & 0 & 484 & 4.6 & 125,630 & 0.49 \\
\hline 280 & 90 & 226 & 63 & 8 & 1 & 0 & 0 & 0 & 0 & 388 & 4.4 & 93,497 & 0.36 \\
\hline 290 & 87 & 213 & 58 & 20 & 3 & 0 & 0 & 0 & 0 & 381 & 4.7 & 111,747 & 0.43 \\
\hline 300 & 94 & 225 & 79 & 28 & 4 & 0 & 0 & 0 & 0 & 430 & 4.9 & 146,475 & 0.57 \\
\hline 310 & 82 & 244 & 89 & 33 & 5 & 1 & 0 & 0 & 0 & 455 & 5.1 & 174,730 & 0.67 \\
\hline 320 & 95 & 258 & 134 & 46 & 9 & 2 & 0 & 0 & 0 & 544 & 5.4 & 238,340 & 0.92 \\
\hline 330 & 89 & 287 & 221 & 89 & 23 & 5 & 2 & 0 & 0 & 716 & 6.1 & 424,294 & 1.64 \\
\hline 340 & 112 & 359 & 322 & 154 & 30 & 4 & 1 & 0 & 0 & 980 & 6.4 & 640,529 & 2.47 \\
\hline 350 & 84 & 304 & 338 & 160 & 32 & 1 & 0 & 0 & 0 & 919 & 6.6 & 655,451 & 2.53 \\
\hline Calms & $\begin{array}{r}54 \\
3 \\
\end{array}$ & & & & & & & & & 3 & & & \\
\hline Totals & $\begin{array}{ll}3781 \\
\end{array}$ & 2377 & 238 & 334 & 064 & 878 & 291 & 86 & 32 & 6080 & 7.0 & 00,095 & $\overline{00.00}$ \\
\hline
\end{tabular}

Wind Power Rose

Grand Canyon West, Site 7054

October 2006 - August 2012
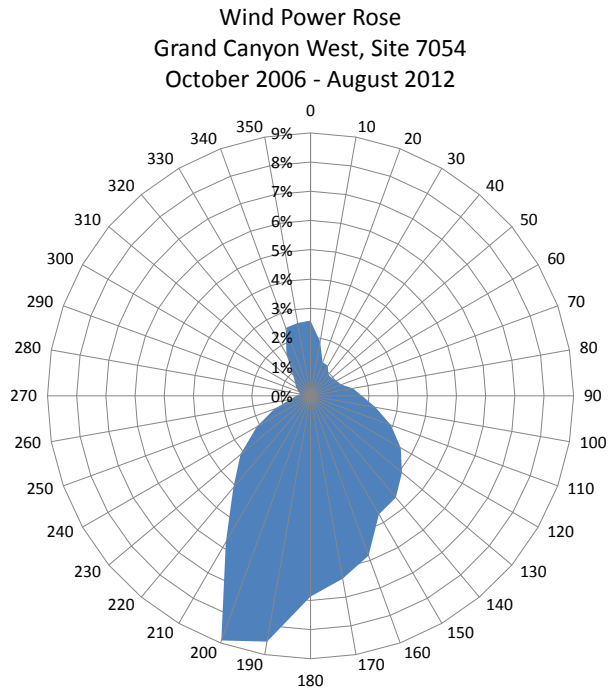

70.8\% Data Recovery 
80-m Wind Rose

Clay Springs, Site 7055

2012 (expanded to two full years)

Hours of Occurrence

\begin{tabular}{|c|c|c|c|c|c|c|c|c|c|c|c|}
\hline Direction & $\begin{array}{c}\text { d Spee } \\
0-3\end{array}$ & $\begin{array}{r}m p s) \ldots \\
3-6\end{array}$ & $6-9$ & $9-12$ & $12-15$ & $15-18$ & $18-21$ & 21-24 & $24+$ & $\begin{array}{l}\text { Row } \\
\text { Total }\end{array}$ & $\begin{array}{l}\text { Mean } \\
\text { Speed }\end{array}$ \\
\hline 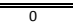 & 47 & 204 & $\begin{array}{lll}158 \\
\end{array}$ & 26 & 0 & 0 & 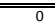 & 0 & 0 & 2435 & 5.6 \\
\hline 10 & 45 & 188 & 119 & 26 & 0 & 0 & 0 & 0 & 0 & 377 & 5.5 \\
\hline 20 & 56 & 165 & 104 & 16 & 2 & 0 & 0 & 0 & 0 & 343 & 5.2 \\
\hline 30 & 59 & 146 & 83 & 27 & 2 & 0 & 0 & 0 & 0 & 317 & 5.2 \\
\hline 40 & 63 & 136 & 68 & 13 & 1 & 0 & 0 & 0 & 0 & 280 & 4.9 \\
\hline 50 & 84 & 152 & 68 & 7 & 1 & 0 & 0 & 0 & 0 & 312 & 4.5 \\
\hline 60 & 58 & 169 & 74 & 5 & 2 & 0 & 0 & 0 & 0 & 308 & 4.8 \\
\hline 70 & 84 & 180 & 34 & 3 & 0 & 0 & 0 & 0 & 0 & 302 & 4.0 \\
\hline 80 & 108 & 231 & 37 & 5 & 1 & 0 & 0 & 0 & 0 & 381 & 3.9 \\
\hline 90 & 100 & 214 & 76 & 9 & 3 & 0 & 0 & 0 & 0 & 402 & 4.5 \\
\hline 100 & 107 & 254 & 143 & 22 & 2 & 0 & 0 & 0 & 0 & 528 & 5.0 \\
\hline 110 & 89 & 274 & 159 & 23 & 1 & 0 & 0 & 0 & 0 & 546 & 5.1 \\
\hline 120 & 96 & 155 & 65 & 5 & 1 & 0 & 0 & 0 & 0 & 321 & 4.3 \\
\hline 130 & 56 & 103 & 31 & 3 & 0 & 0 & 0 & 0 & 0 & 193 & 4.2 \\
\hline 140 & 62 & 51 & 25 & 1 & 0 & 0 & 0 & 0 & 0 & 139 & 3.8 \\
\hline 150 & 46 & 47 & 26 & 13 & 1 & 0 & 0 & 0 & 0 & 133 & 4.7 \\
\hline 160 & 44 & 81 & 59 & 33 & 10 & 7 & 0 & 0 & 0 & 234 & 6.3 \\
\hline 170 & 55 & 87 & 115 & 31 & 8 & 0 & 0 & 0 & 0 & 295 & 5.9 \\
\hline 180 & 63 & 183 & 203 & 64 & 26 & 9 & 2 & 0 & 0 & 550 & 6.6 \\
\hline 190 & 97 & 295 & 351 & 227 & 101 & 23 & 6 & 0 & 0 & 1100 & 7.6 \\
\hline 200 & 122 & 454 & 555 & 571 & 318 & 147 & 46 & 21 & 0 & 2234 & 9.2 \\
\hline 210 & 140 & 1108 & 820 & 333 & 183 & 73 & 16 & 6 & 1 & 2681 & 7.1 \\
\hline 220 & 103 & 710 & 349 & 61 & 15 & 3 & 0 & 0 & 0 & 1240 & 5.5 \\
\hline 230 & 96 & 274 & 104 & 21 & 5 & 0 & 4 & 2 & 0 & 505 & 5.1 \\
\hline 240 & 66 & 112 & 50 & 12 & 7 & 0 & 0 & 0 & 0 & 247 & 4.9 \\
\hline 250 & 46 & 82 & 38 & 8 & 2 & 0 & 0 & 0 & 0 & 176 & 4.6 \\
\hline 260 & 32 & 44 & 17 & 3 & 1 & 0 & 0 & 0 & 0 & 96 & 4.3 \\
\hline 270 & 41 & 61 & 20 & 3 & 0 & 0 & 0 & 0 & 0 & 124 & 4.0 \\
\hline 280 & 29 & 50 & 36 & 8 & 0 & 0 & 0 & 0 & 0 & 122 & 4.9 \\
\hline 290 & 41 & 61 & 38 & 8 & 2 & 0 & 0 & 0 & 0 & 150 & 4.9 \\
\hline 300 & 40 & 99 & 109 & 12 & 0 & 0 & 0 & 0 & 0 & 260 & 5.6 \\
\hline 310 & 49 & 122 & 120 & 25 & 2 & 0 & 0 & 0 & 0 & 318 & 5.6 \\
\hline 320 & 34 & 119 & 87 & 39 & 4 & 0 & 0 & 0 & 0 & 283 & 6.0 \\
\hline 330 & 47 & 124 & 110 & 59 & 3 & 0 & 0 & 0 & 0 & 343 & 6.2 \\
\hline 340 & 38 & 160 & 167 & 62 & 12 & 2 & 0 & 0 & 0 & 441 & 6.5 \\
\hline 350 & 63 & 240 & 155 & 35 & 10 & 1 & 0 & 0 & 0 & 504 & 5.7 \\
\hline $\begin{array}{c}\text { Calms } \\
\end{array}$ & 17 & & & & & & & & & 17 & \\
\hline Totals & 2424 & $\begin{array}{l}7133 \\
\end{array}$ & 4773 & 1816 & $\begin{array}{l}724 \\
\end{array}$ & 264 & $\begin{array}{l}74 \\
\end{array}$ & 29 & 1 & 17237 & $\overline{6.2}$ \\
\hline
\end{tabular}

GE-1.7/100 Energy...

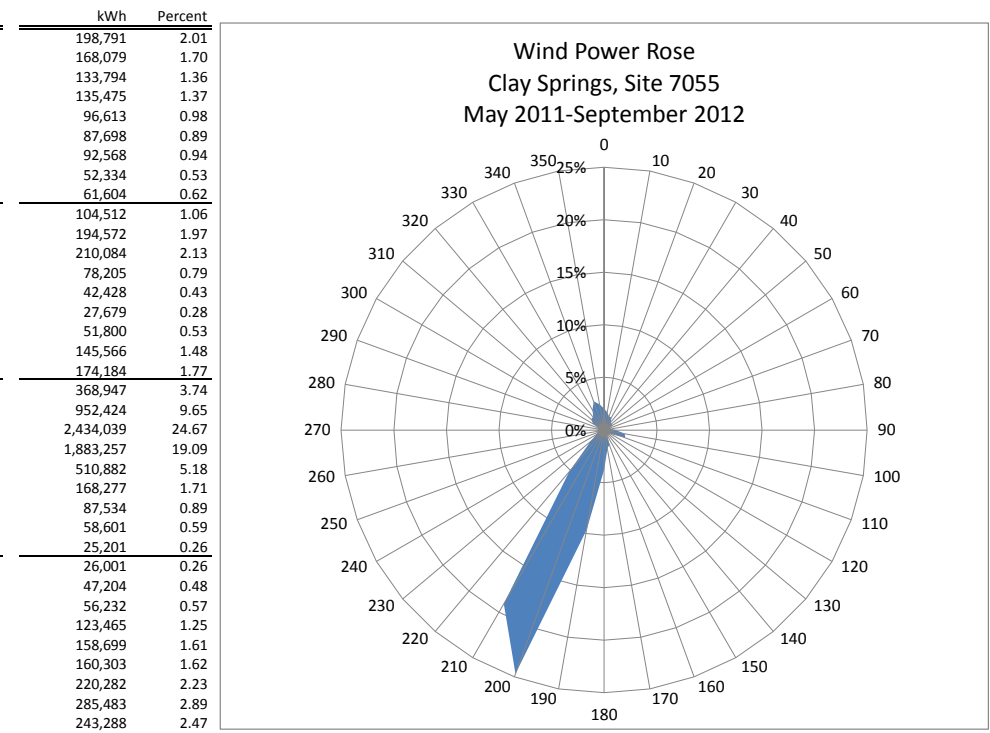

98.1\% Data Recovery 
Off-Axis Wake Losses (\%)

Peach Springs, Site 7052

Using Data from December 2005-September 2012

Turbine: GE-1.7/100

\begin{tabular}{crrrrrr}
$\begin{array}{c}\text { Sow } \\
\text { Roacing (Rotor Diameters) .. } \\
\text { Orientation }\end{array}$ & 1.5 RD & 2.0 RD & 2.5 RD & 3.0 RD & 3.5 RD & 4.0 RD \\
\hline \hline $360 / 180$ & 22.83 & 14.18 & 9.72 & 7.30 & 5.69 & 4.66 \\
$010 / 190$ & 23.70 & 14.74 & 10.13 & 7.62 & 5.95 & 4.88 \\
$020 / 200$ & 23.75 & 14.64 & 9.95 & 7.42 & 5.75 & 4.68 \\
$030 / 210$ & 23.11 & 14.09 & 9.47 & 7.02 & 5.41 & 4.39 \\
$040 / 220$ & 21.97 & 13.37 & 9.01 & 6.72 & 5.20 & 4.24 \\
$050 / 230$ & 20.31 & 12.53 & 8.54 & 6.39 & 4.96 & 4.05 \\
$060 / 240$ & 18.30 & 11.32 & 7.75 & 5.81 & 4.53 & 3.70 \\
$070 / 250$ & 15.95 & 9.72 & 6.59 & 4.93 & 3.82 & 3.12 \\
$080 / 260$ & 13.61 & 8.04 & 5.32 & 3.93 & 3.02 & 2.44 \\
\hline $090 / 270$ & 11.74 & 6.74 & 4.36 & 3.18 & 2.41 & 1.93 \\
$100 / 280$ & 10.77 & 6.13 & 3.96 & 2.91 & 2.22 & 1.79 \\
$110 / 290$ & 10.74 & 6.25 & 4.11 & 3.04 & 2.33 & 1.88 \\
$120 / 300$ & 11.61 & 6.88 & 4.59 & 3.41 & 2.63 & 2.14 \\
$130 / 310$ & 13.01 & 7.81 & 5.23 & 3.88 & 3.00 & 2.44 \\
$140 / 320$ & 14.79 & 8.90 & 5.97 & 4.45 & 3.43 & 2.79 \\
$150 / 330$ & 16.85 & 10.13 & 6.79 & 5.05 & 3.89 & 3.17 \\
$160 / 340$ & 19.11 & 11.53 & 7.72 & 5.73 & 4.42 & 3.60 \\
$170 / 350$ & 21.18 & 13.01 & 8.80 & 6.54 & 5.04 & 4.10 \\
\hline \hline
\end{tabular}


Off-Axis Wake Losses (\%)

Nelson, Site 7053

Using Data from December 2005-September 2012

Turbine: GE-1.7/100

\begin{tabular}{crrrrrr}
$\begin{array}{c}\text { Row } \\
\text { Spacing (Rotor Diameters) .. } \\
\text { Orientation }\end{array}$ & 1.5 RD & 2.0 RD & 2.5 RD & 3.0 RD & 3.5 RD & 4.0 RD \\
\hline \hline $360 / 180$ & 20.43 & 12.41 & 8.33 & 6.18 & 4.76 & 3.86 \\
$010 / 190$ & 23.36 & 14.61 & 10.07 & 7.58 & 5.93 & 4.86 \\
$020 / 200$ & 25.33 & 16.02 & 11.11 & 8.39 & 6.58 & 5.40 \\
$030 / 210$ & 25.75 & 16.23 & 11.17 & 8.36 & 6.50 & 5.31 \\
$040 / 220$ & 24.74 & 15.31 & 10.43 & 7.80 & 6.05 & 4.94 \\
$050 / 230$ & 22.73 & 13.81 & 9.30 & 6.94 & 5.38 & 4.39 \\
$060 / 240$ & 20.26 & 12.24 & 8.19 & 6.07 & 4.66 & 3.78 \\
$070 / 250$ & 17.78 & 10.74 & 7.22 & 5.35 & 4.13 & 3.35 \\
$080 / 260$ & 15.70 & 9.34 & 6.27 & 4.71 & 3.68 & 3.01 \\
\hline $090 / 270$ & 13.97 & 8.28 & 5.48 & 4.04 & 3.09 & 2.50 \\
$100 / 280$ & 12.72 & 7.57 & 4.99 & 3.64 & 2.76 & 2.21 \\
$110 / 290$ & 11.91 & 7.11 & 4.78 & 3.59 & 2.80 & 2.29 \\
$120 / 300$ & 11.42 & 6.87 & 4.66 & 3.51 & 2.74 & 2.25 \\
$130 / 310$ & 11.31 & 6.77 & 4.49 & 3.29 & 2.51 & 2.02 \\
$140 / 320$ & 11.66 & 6.84 & 4.49 & 3.30 & 2.52 & 2.04 \\
$150 / 330$ & 12.58 & 7.32 & 4.85 & 3.62 & 2.80 & 2.28 \\
$160 / 340$ & 14.45 & 8.40 & 5.52 & 4.08 & 3.13 & 2.53 \\
$170 / 350$ & 17.23 & 10.15 & 6.65 & 4.87 & 3.71 & 2.98 \\
\hline \hline
\end{tabular}


Off-Axis Wake Losses (\%)

Grand Canyon West, Site 7054

Using Data from October 2006-August 2012

Turbine: GE-1.7/100

\begin{tabular}{crrrrrr}
$\begin{array}{c}\text { Spacing (Rotor Diameters) ... } \\
\text { Row } \\
\text { Orientation }\end{array}$ & 1.5 RD & 2.0 RD & 2.5 RD & 3.0 RD & 3.5 RD & 4.0 RD \\
\hline \hline $360 / 180$ & 28.12 & 17.69 & 12.13 & 9.05 & 7.01 & 5.71 \\
$010 / 190$ & 27.94 & 17.71 & 12.29 & 9.29 & 7.27 & 5.97 \\
$020 / 200$ & 25.89 & 16.33 & 11.33 & 8.57 & 6.73 & 5.53 \\
$030 / 210$ & 22.42 & 13.82 & 9.38 & 6.99 & 5.40 & 4.39 \\
$040 / 220$ & 18.30 & 10.88 & 7.17 & 5.26 & 4.01 & 3.23 \\
$050 / 230$ & 14.38 & 8.30 & 5.42 & 4.00 & 3.07 & 2.48 \\
$060 / 240$ & 11.26 & 6.48 & 4.25 & 3.14 & 2.41 & 1.95 \\
$070 / 250$ & 9.32 & 5.34 & 3.47 & 2.55 & 1.94 & 1.57 \\
$080 / 260$ & 8.46 & 4.80 & 3.09 & 2.27 & 1.73 & 1.39 \\
\hline $090 / 270$ & 8.54 & 4.88 & 3.16 & 2.32 & 1.77 & 1.43 \\
$100 / 280$ & 9.44 & 5.51 & 3.63 & 2.68 & 2.05 & 1.66 \\
$110 / 290$ & 10.97 & 6.54 & 4.37 & 3.25 & 2.50 & 2.03 \\
$120 / 300$ & 12.96 & 7.78 & 5.24 & 3.90 & 3.02 & 2.46 \\
$130 / 310$ & 15.39 & 9.19 & 6.14 & 4.57 & 3.53 & 2.87 \\
$140 / 320$ & 18.11 & 10.85 & 7.22 & 5.34 & 4.10 & 3.32 \\
$150 / 330$ & 21.07 & 12.73 & 8.55 & 6.35 & 4.90 & 3.98 \\
$160 / 340$ & 24.08 & 14.69 & 9.95 & 7.44 & 5.78 & 4.72 \\
$170 / 350$ & 26.69 & 16.48 & 11.20 & 8.37 & 6.50 & 5.30 \\
\hline \hline
\end{tabular}


Off-Axis Wake Losses (\%)

Clay Springs, Site 7055

Using Data from May 2011-September 2012

Turbine: GE-1.7/100

Spacing (Rotor Diameters)...

\begin{tabular}{crrrrrr}
$\begin{array}{c}\text { Row } \\
\text { Orientation }\end{array}$ & $1.5 \mathrm{RD}$ & $2.0 \mathrm{RD}$ & $2.5 \mathrm{RD}$ & $3.0 \mathrm{RD}$ & $3.5 \mathrm{RD}$ & $4.0 \mathrm{RD}$ \\
\hline \hline $360 / 180$ & 30.16 & 17.52 & 10.96 & 7.67 & 5.58 & 4.35 \\
$010 / 190$ & 38.42 & 25.12 & 17.56 & 13.12 & 10.16 & 8.26 \\
$020 / 200$ & 42.43 & 29.34 & 21.84 & 17.19 & 13.97 & 11.76 \\
$030 / 210$ & 39.92 & 27.26 & 20.03 & 15.60 & 12.55 & 10.49 \\
$040 / 220$ & 31.60 & 19.79 & 13.21 & 9.52 & 7.12 & 5.65 \\
$050 / 230$ & 21.15 & 11.01 & 6.28 & 4.24 & 2.96 & 2.24 \\
$060 / 240$ & 11.86 & 5.23 & 2.81 & 2.00 & 1.47 & 1.16 \\
$070 / 250$ & 6.58 & 3.07 & 1.82 & 1.31 & 0.98 & 0.78 \\
$080 / 260$ & 5.19 & 2.75 & 1.68 & 1.19 & 0.87 & 0.68 \\
\hline $090 / 270$ & 5.41 & 3.17 & 2.08 & 1.52 & 1.16 & 0.93 \\
$100 / 280$ & 6.04 & 3.79 & 2.64 & 2.02 & 1.59 & 1.32 \\
$110 / 290$ & 6.58 & 4.20 & 2.95 & 2.25 & 1.77 & 1.46 \\
$120 / 300$ & 6.99 & 4.26 & 2.88 & 2.15 & 1.66 & 1.35 \\
$130 / 310$ & 7.53 & 4.30 & 2.76 & 2.01 & 1.53 & 1.23 \\
$140 / 320$ & 8.41 & 4.80 & 3.08 & 2.23 & 1.68 & 1.34 \\
$150 / 330$ & 10.17 & 5.85 & 3.86 & 2.86 & 2.20 & 1.78 \\
$160 / 340$ & 13.86 & 7.55 & 4.91 & 3.67 & 2.84 & 2.32 \\
$170 / 350$ & 21.03 & 11.00 & 6.64 & 4.81 & 3.61 & 2.88 \\
\hline \hline
\end{tabular}


Maximum Hourly Wind Speeds (mps) Hualapai Reservation, Arizona

YEAR: 2005

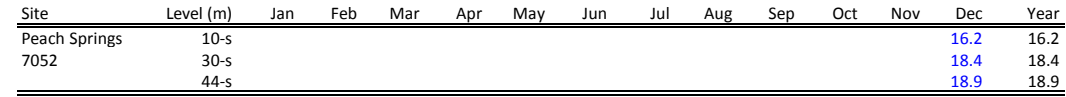

YEAR: 2006

\begin{tabular}{|c|c|c|c|c|c|c|c|c|c|c|c|c|c|c|}
\hline Site & Level $(\mathrm{m})$ & Jan & Feb & Mar & Apr & May & Jun & Jul & Aug & Sep & Oct & Nov & Dec & Year \\
\hline 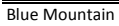 & $\begin{array}{l}10-\text { ssw } \\
\end{array}$ & & & & & & & & & 1313.2 & 9.5 & $\bar{x}$ & $\bar{x}$ & 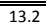 \\
\hline \multirow[t]{4}{*}{7051} & 30-ssw & $\mathrm{x}$ & $\mathrm{x}$ & $\mathrm{x}$ & $\mathrm{x}$ & $\mathrm{x}$ & $\mathrm{x}$ & $\mathrm{x}$ & $x$ & 17.4 & 12.4 & $\mathrm{x}$ & $\mathrm{x}$ & 17.4 \\
\hline & 40-ssw & $\mathrm{x}$ & $\mathrm{x}$ & 15.1 & 19.8 & 18.3 & 16.3 & 14.5 & 10.6 & $\mathrm{x}$ & $\mathrm{x}$ & $\mathrm{x}$ & $\mathrm{x}$ & 19.8 \\
\hline & 49-ssw & $\mathrm{x}$ & $\mathrm{x}$ & 15.5 & 21.0 & 18.8 & 16.8 & 14.9 & 11.0 & 18.6 & 13.4 & $\mathrm{x}$ & $\mathrm{x}$ & 21.0 \\
\hline & 49-wnw & $\mathrm{x}$ & $x$ & 15.5 & 21.3 & $x$ & $x$ & $x$ & $x$ & 18.8 & 13.5 & $\mathrm{x}$ & $x$ & 21.3 \\
\hline Peach Springs & $10-5$ & 13.1 & 19.1 & 15.4 & 13.2 & 16.1 & 14.6 & 12.4 & 10.4 & 16.2 & 10.6 & $\mathrm{x}$ & $x$ & 19.1 \\
\hline \multirow[t]{2}{*}{7052} & $30-5$ & 14.8 & 21.6 & 17.8 & 17.1 & 17.7 & 16.7 & 14.1 & 13.0 & 18.1 & 11.7 & $\mathrm{x}$ & $\mathrm{x}$ & 21.6 \\
\hline & $44-s$ & 16.2 & 22.2 & 18.5 & 18.5 & 18.1 & 17.2 & 14.5 & 13.7 & 18.4 & 12.0 & $\mathrm{x}$ & $\mathrm{x}$ & 22.2 \\
\hline Nelson & $10-\mathrm{s}$ & & & & & & & & & & & & $x$ & \\
\hline \multirow[t]{4}{*}{7053} & $30-5$ & & & & & & & & & & & & 14.7 & 14.7 \\
\hline & $30-w$ & & & & & & & & & & & & 14.5 & 14.5 \\
\hline & $50-\mathrm{s}$ & & & & & & & & & & & & 15.6 & 15.6 \\
\hline & 50-w & & & & & & & & & & & & 15.6 & 15.6 \\
\hline Grand Canyon & $10-\mathrm{s}$ & & & & & & & & & & 7.6 & 12.5 & 13.6 & 13.6 \\
\hline \multirow{4}{*}{ West, 7054} & $30-5$ & & & & & & & & & & 8.8 & 15.9 & 16.9 & 16.9 \\
\hline & 30-w & & & & & & & & & & 8.9 & 15.9 & 17.0 & 17.0 \\
\hline & $50-\mathrm{s}$ & & & & & & & & & & 9.4 & 15.9 & 16.9 & 16.9 \\
\hline & 50-w & & & & & & & & & & 9.4 & 16.2 & 17.3 & 17.3 \\
\hline
\end{tabular}

YEAR: 2007

\begin{tabular}{|c|c|c|c|c|c|c|c|c|c|c|c|c|c|c|}
\hline Site & Level $(\mathrm{m})$ & Jan & Feb & Mar & Apr & May & Jun & Jul & Aug & Sep & Oct & Nov & Dec & Year \\
\hline Blue Mountain & 10-sse & $\bar{x}$ & $\begin{array}{ll}10.7 \\
\end{array}$ & 12.5 & 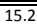 & $\begin{array}{l}13.4 \\
\end{array}$ & $\overline{13.2}$ & 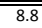 & 9.3 & \begin{tabular}{ll|}
11.4 \\
\end{tabular} & & & & $\overline{15.2}$ \\
\hline \multirow[t]{3}{*}{7051} & 30-sse & $\mathrm{x}$ & 14.1 & 16.1 & 19.2 & 17.2 & 17.4 & 11.0 & 12.0 & 14.5 & & & & 19.2 \\
\hline & 49-sse & $\mathrm{x}$ & 15.3 & 17.2 & 20.1 & 17.9 & 18.5 & 11.4 & 13.2 & 10.5 & & & & 20.1 \\
\hline & 49-wsw & $\mathrm{x}$ & 15.3 & 17.2 & 19.8 & 17.7 & 18.7 & 11.3 & 13.1 & 15.1 & & & & 19.8 \\
\hline & $10-s$ & $x$ & $x$ & $x$ & $\mathrm{x}$ & $\mathrm{x}$ & $\mathrm{x}$ & $\bar{x}$ & $x$ & $\mathrm{x}$ & $x$ & 12.3 & 16.9 & 16.9 \\
\hline Peach Springs & $30-s$ & $\mathrm{x}$ & $\mathrm{x}$ & $\mathrm{x}$ & $\mathrm{x}$ & $\mathrm{x}$ & $\mathrm{x}$ & $\mathrm{x}$ & $\mathrm{x}$ & $x$ & $\mathrm{x}$ & 14.0 & 19.4 & 19.4 \\
\hline \multirow[t]{2}{*}{7052} & 44-s & $\mathrm{x}$ & $x$ & $x$ & $\mathrm{x}$ & $\mathrm{x}$ & $\mathrm{x}$ & $\mathrm{x}$ & $\mathrm{x}$ & $\mathrm{x}$ & $\mathrm{x}$ & 14.5 & 20.5 & 20.5 \\
\hline & 44-w & & & & & & & & & & & & 20.6 & 20.6 \\
\hline Nelson & $10-5$ & $x$ & $x$ & $x$ & 12.9 & 11.9 & 12.2 & 9.4 & 9.7 & 11.2 & 13.5 & 8.2 & 13.1 & 13.5 \\
\hline \multirow[t]{4}{*}{7053} & $30-\mathrm{s}$ & 13.4 & 12.1 & 12.4 & 15.3 & 14.1 & 14.7 & 11.4 & 11.8 & 13.0 & 15.7 & 9.7 & 15.4 & 15.7 \\
\hline & $30-w$ & 13.4 & 12.0 & 12.0 & 14.9 & 13.6 & 14.3 & 11.2 & 11.4 & 12.9 & 15.4 & 10.2 & 15.4 & 15.4 \\
\hline & $50-s$ & 14.6 & 13.6 & 13.1 & 16.7 & 14.9 & 16.4 & 12.9 & 12.9 & 14.0 & 17.3 & 10.6 & 17.4 & 17.4 \\
\hline & 50-w & 14.9 & 13.8 & 13.1 & 17.0 & 14.9 & 16.4 & 12.8 & 12.9 & 14.3 & 17.5 & 11.1 & 17.6 & 17.6 \\
\hline Grand Canyon & $10-5$ & & & & & 11.3 & 13.5 & 10.5 & 10.9 & 15.5 & 12.5 & 11.4 & 15.0 & 15.5 \\
\hline \multirow[t]{3}{*}{ West, 7054} & $30-\mathrm{s}$ & & & & & 13.6 & 18.9 & 11.9 & 12.5 & 19.8 & 15.0 & 13.3 & 18.5 & 19.8 \\
\hline & $30-w$ & & & & & $\mathrm{x}$ & $\mathrm{x}$ & $\mathrm{x}$ & 12.2 & 19.9 & 15.1 & 13.4 & 18.6 & 19.9 \\
\hline & $50-\mathrm{s}$ & & & & & 13.8 & 21.7 & 12.8 & 13.7 & 19.6 & 15.6 & 13.6 & 18.3 & 21.7 \\
\hline
\end{tabular}

YEAR: 2008

\begin{tabular}{lrrrrrrrrrrrrrr} 
Site & Level $(\mathrm{m})$ & Jan & Feb & Mar & Apr & May & Jun & Jul & Aug & Sep & Oct & Nov & Dec & Year \\
\hline \hline Peach Springs & $10-\mathrm{s}$ & 16.5 & 16.1 & 14.8 & 16.6 & 14.2 & 15.1 & 11.6 & 10.0 & 11.2 & 14.3 & 14.6 & 22.0 & 22.0 \\
7052 & $30-\mathrm{s}$ & 19.9 & 18.1 & 16.2 & 18.8 & 15.8 & 17.6 & 13.0 & 11.8 & 12.8 & 16.3 & 16.5 & 24.5 & 24.5 \\
& $44-\mathrm{s}$ & 21.3 & 19.2 & 16.4 & 18.9 & 16.0 & 17.7 & 13.0 & 12.1 & 12.8 & 16.4 & 16.8 & 25.6 & 25.6 \\
& $44-\mathrm{w}$ & 21.5 & 19.4 & 16.2 & 18.7 & 16.2 & 17.7 & 12.2 & 11.4 & 12.5 & 16.7 & 16.6 & 25.0 & 25.0 \\
\hline Nelson & $10-\mathrm{s}$ & 13.5 & 12.0 & 10.6 & 12.5 & 10.3 & 10.8 & 9.8 & 8.7 & 9.0 & 11.8 & 10.5 & 15.9 & 15.9 \\
7053 & $30-\mathrm{s}$ & 16.0 & 13.9 & 12.5 & 14.5 & 11.8 & 12.6 & 11.7 & 10.2 & 10.4 & 13.5 & 12.4 & 18.7 & 18.7 \\
& $30-\mathrm{w}$ & 16.1 & 14.1 & 13.1 & 14.3 & 11.7 & 12.2 & 10.0 & 10.2 & 10.3 & 13.5 & 12.4 & 18.6 & 18.6 \\
& $50-\mathrm{s}$ & 18.0 & 15.6 & 13.4 & 16.0 & 13.8 & 13.6 & 13.1 & 11.3 & $\mathrm{x}$ & $\mathrm{x}$ & 10.2 & $\mathrm{x}$ & 18.0 \\
& $50-\mathrm{w}$ & 18.1 & 15.8 & 14.5 & 16.0 & 13.9 & 13.6 & 12.5 & 11.7 & 11.6 & 15.4 & 13.7 & 20.5 & 20.5 \\
\hline Grand Canyon & $10-\mathrm{s}$ & 19.2 & 16.6 & 11.9 & 13.6 & 15.6 & 12.4 & 10.5 & 12.4 & 13.0 & 12.7 & 12.6 & 20.0 & 20.0 \\
West, 7054 & $30-\mathrm{s}$ & 24.3 & 22.6 & 15.9 & 17.1 & 20.2 & 15.0 & 12.6 & 14.5 & 15.9 & 16.4 & 14.8 & 24.1 & 24.3 \\
& $30-\mathrm{w}$ & 24.1 & 22.3 & 15.7 & 17.1 & 20.0 & 15.1 & 12.7 & 14.6 & 16.0 & 17.0 & 14.9 & 24.4 & 24.4 \\
& $50-\mathrm{s}$ & $\mathrm{x}$ & $\mathrm{x}$ & $\mathrm{x}$ & 19.2 & 21.1 & 15.9 & 13.3 & 15.1 & 16.3 & 17.4 & 15.6 & 24.7 & 24.7 \\
& $50-\mathrm{w}$ & $\mathrm{x}$ & $\mathrm{X}$ & $\mathrm{x}$ & 19.3 & 21.1 & 15.9 & 13.4 & 15.2 & 16.3 & 17.4 & 15.6 & 24.8 & 24.8 \\
\hline \hline
\end{tabular}



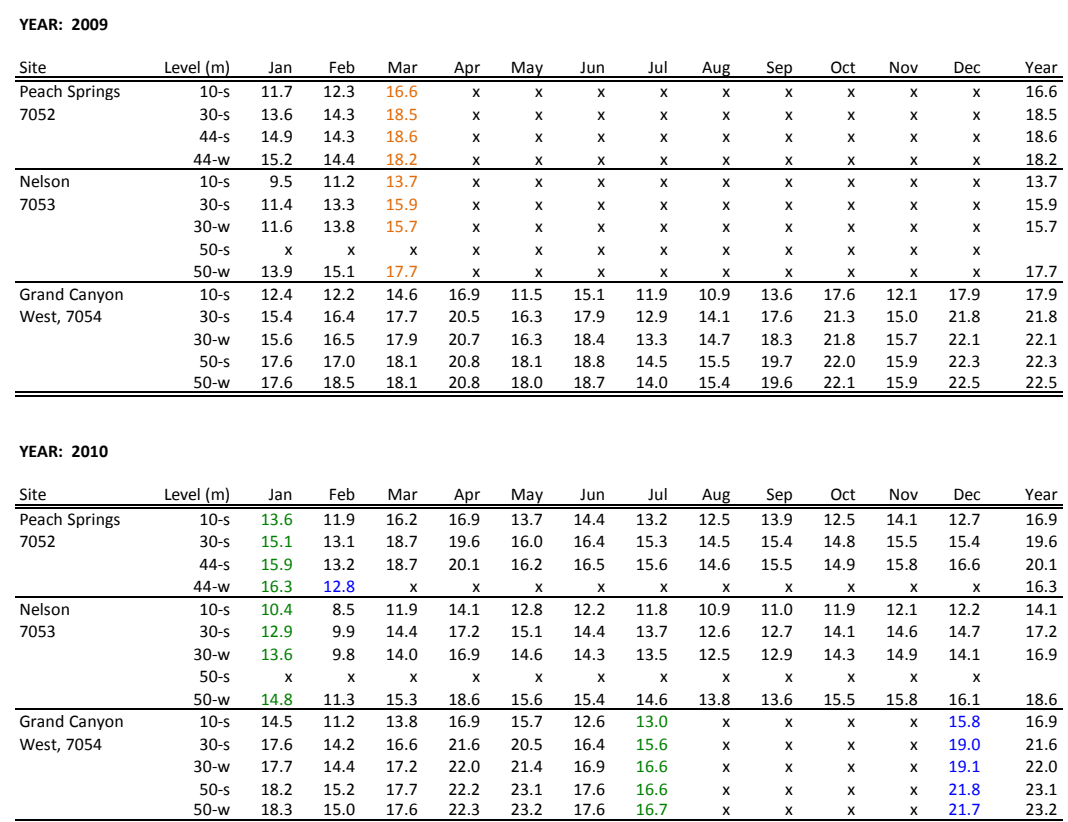

YEAR: 2011

\begin{tabular}{|c|c|c|c|c|c|c|c|c|c|c|c|c|c|c|}
\hline Site & Level (m) & Jan & Feb & Mar & Apr & May & Jun & Jul & Aug & Sep & Oct & Nov & Dec & Year \\
\hline Peach Springs & 10-s & 8.7 & $\begin{array}{ll}14.8 \\
\end{array}$ & 14.4 & 15.0 & 15.4 & 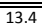 & 12.8 & 12.3 & 13.0 & 13.6 & 12.5 & 12.1 & 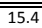 \\
\hline \multirow[t]{3}{*}{7052} & $30-5$ & 11.3 & 16.5 & 16.9 & 17.4 & 17.7 & 15.2 & 15.7 & 13.9 & 15.6 & 15.3 & 14.5 & 15.0 & 17.7 \\
\hline & $44-5$ & 11.7 & 16.3 & 17.3 & 17.6 & 17.8 & 15.4 & 16.9 & 14.7 & 16.5 & 16.3 & 14.8 & 15.4 & 17.8 \\
\hline & 44-w & $\mathrm{x}$ & $x$ & $\mathrm{x}$ & $x$ & $x$ & $\mathrm{x}$ & $x$ & $x$ & $x$ & $x$ & $x$ & $x$ & \\
\hline Nelson & $10-5$ & 8.7 & 12.1 & 11.5 & 13.8 & 12.7 & 11.8 & 10.0 & 10.0 & 9.9 & 11.4 & 11.3 & 12.2 & 13.8 \\
\hline \multirow[t]{4}{*}{7053} & $30-\mathrm{s}$ & 10.1 & 14.1 & 13.8 & 16.3 & 14.8 & 13.7 & 11.9 & 11.6 & 12.5 & 13.8 & 13.3 & 14.5 & 16.3 \\
\hline & $30-w$ & 10.3 & 13.9 & 14.1 & 16.2 & 14.7 & 13.5 & 12.0 & 11.3 & 11.6 & 14.3 & 13.3 & 14.1 & 16.2 \\
\hline & 50-s & $\mathrm{x}$ & $\mathrm{x}$ & $\mathrm{x}$ & $\mathrm{x}$ & $\mathrm{x}$ & $\mathrm{x}$ & $\mathrm{x}$ & $\mathrm{x}$ & $\mathrm{x}$ & $\mathrm{x}$ & $\mathrm{x}$ & $\mathrm{x}$ & \\
\hline & 50-w & 11.9 & 15.7 & 15.3 & 17.9 & 16.0 & 14.8 & 13.8 & 12.3 & 13.8 & 15.4 & 14.6 & 15.1 & 17.9 \\
\hline Grand Canyon & $10-\mathrm{s}$ & 10.8 & 14.8 & 14.5 & 12.8 & 14.8 & 14.7 & 13.5 & 11.8 & 10.5 & 15.5 & 17.0 & 9.5 & 17.0 \\
\hline \multirow[t]{4}{*}{ West, 7054} & $30-\mathrm{s}$ & 13.1 & 19.1 & 17.4 & 18.0 & 18.5 & 18.4 & 14.8 & 14.8 & 13.7 & 19.1 & 21.4 & 11.0 & 21.4 \\
\hline & $30-w$ & 13.2 & 19.4 & 17.5 & 18.1 & 19.0 & 18.9 & 13.1 & 14.9 & 13.8 & 19.5 & 21.8 & 11.0 & 21.8 \\
\hline & 50-s & 13.5 & 19.7 & 19.7 & 20.0 & 18.8 & 18.6 & 16.1 & 15.0 & 14.2 & 19.6 & 21.4 & 11.8 & 21.4 \\
\hline & $50-w$ & 13.5 & 19.7 & 19.7 & 20.0 & 18.9 & 18.7 & 15.8 & 15.1 & 14.3 & 19.8 & 21.7 & 12.0 & 21.7 \\
\hline Clay Springs & $10-\mathrm{s}$ & & & & & 11.9 & 9.8 & 10.5 & 8.3 & 9.9 & 10.3 & 13.2 & 8.6 & 13.2 \\
\hline \multirow[t]{5}{*}{7055} & $30-5$ & & & & & 16.8 & 13.9 & 11.8 & 12.2 & 11.0 & 13.4 & 18.2 & 11.6 & 18.2 \\
\hline & 30-nw & & & & & 16.4 & 13.8 & 12.0 & 12.1 & 11.1 & 13.3 & 17.9 & 10.7 & 17.9 \\
\hline & $40-\mathrm{s}$ & & & & & 18.3 & 15.2 & 12.3 & 13.6 & 11.3 & 14.2 & 19.0 & 11.1 & 19.0 \\
\hline & $49-\mathrm{s}$ & & & & & 19.6 & 16.4 & 12.0 & 14.7 & 12.2 & 15.0 & 19.8 & 12.5 & 19.8 \\
\hline & 49-nw & & & & & 19.4 & 16.2 & 12.3 & 14.5 & 12.1 & 14.9 & 19.6 & 11.5 & 19.6 \\
\hline
\end{tabular}

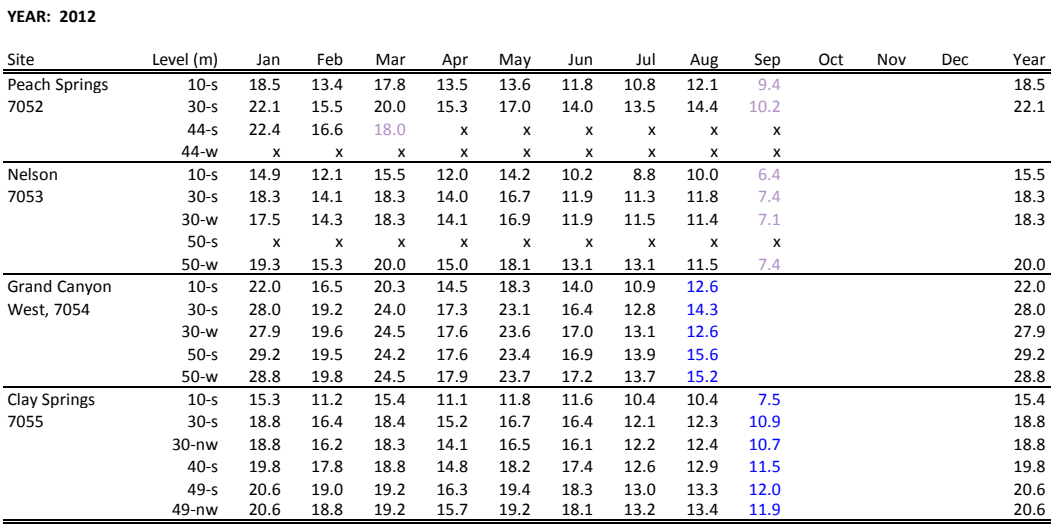

Overall Maximum: 29.2

Data Recovery Key: black $=90-100 \% ;$ green $=75-90 \% ;$ blue $=50-75 \%$; orange $=25-50 \% ;$ purple $=10-25 \% ; " x^{\prime \prime}=0-10 \%$. 
Peak 2-Second Gusts (mps)

Hualapai Reservation, Arizon

YEAR: 2005
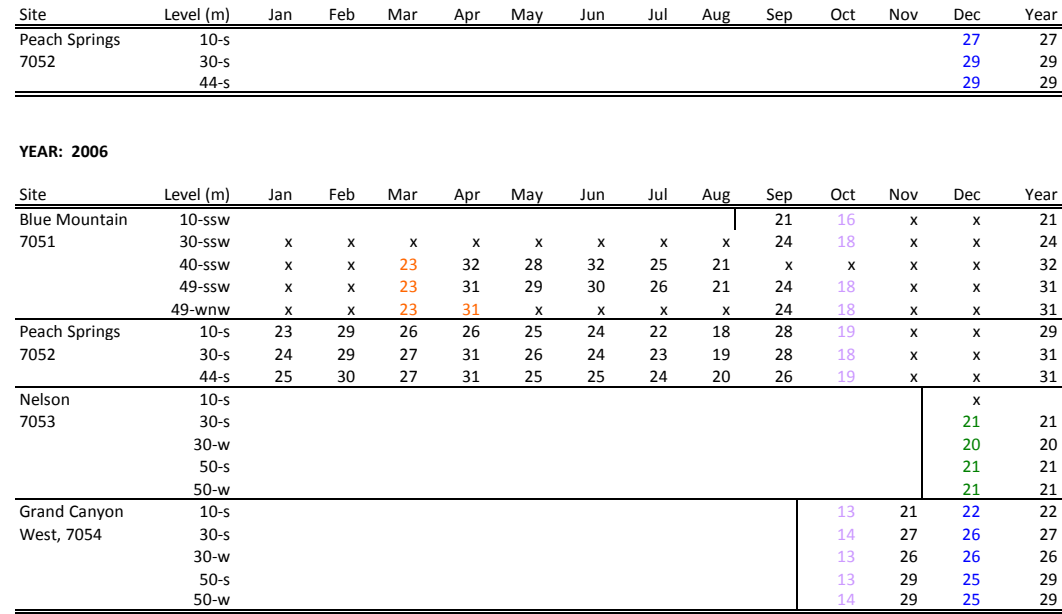

\begin{tabular}{|c|c|c|c|c|c|c|c|c|c|c|c|c|c|c|}
\hline Site & Level $(\mathrm{m})$ & Jan & Feb & Mar & Apr & May & Jun & Jul & Aug & Sep & Oct & Nov & Dec & Year \\
\hline Blue Mountain & 10-sse & $\bar{x}$ & 19 & 21 & 24 & 23 & 21 & 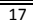 & 21 & 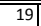 & & & & 24 \\
\hline \multirow{3}{*}{7051} & 30 -sse & $\mathrm{x}$ & 21 & 25 & 26 & 25 & 24 & 21 & 23 & 21 & & & & 26 \\
\hline & 49-sse & $\mathrm{x}$ & 22 & 26 & 27 & 26 & 24 & 22 & 25 & 20 & & & & 27 \\
\hline & 49-wsw & $\mathrm{x}$ & 22 & 25 & 26 & 26 & 24 & 22 & 25 & 22 & & & & 26 \\
\hline & $10-5$ & $x$ & $x$ & $x$ & $\mathrm{x}$ & $x$ & $x$ & $x$ & $x$ & $x$ & $x$ & 22 & 27 & 27 \\
\hline Peach Springs & $30-\mathrm{s}$ & $\mathrm{x}$ & $\mathrm{x}$ & $\mathrm{x}$ & $\mathrm{x}$ & $x$ & $\mathrm{x}$ & $\mathrm{x}$ & $x$ & $\mathrm{x}$ & $\mathrm{x}$ & 20 & 27 & 27 \\
\hline 7052 & $\begin{array}{r}44-\mathrm{s} \\
44-\mathrm{w}\end{array}$ & $x$ & $x$ & $x$ & $\mathrm{x}$ & $x$ & $x$ & $x$ & $x$ & $x$ & $x$ & 22 & $\begin{array}{l}29 \\
29 \\
\end{array}$ & $\begin{array}{l}29 \\
29 \\
\end{array}$ \\
\hline $\begin{array}{l}\text { Nelson } \\
\end{array}$ & $10-5$ & $x$ & $\mathrm{x}$ & $\mathrm{x}$ & 22 & 21 & 24 & 20 & 19 & 19 & 22 & 15 & 21 & 24 \\
\hline \multirow[t]{4}{*}{7053} & $30-\mathrm{s}$ & 22 & 18 & 23 & 23 & 22 & 24 & 22 & 23 & 21 & 24 & 16 & 23 & 24 \\
\hline & $30-w$ & 22 & 19 & 23 & 23 & 21 & 23 & 22 & 23 & 21 & 24 & 17 & 23 & 24 \\
\hline & $50-\mathrm{s}$ & 23 & 20 & 24 & 23 & 23 & 24 & 23 & 23 & 21 & 24 & 18 & 25 & 25 \\
\hline & 50-w & 23 & 20 & 25 & 24 & 23 & 24 & 22 & 23 & 21 & 25 & 18 & 25 & 25 \\
\hline Grand Canyon & $10-\mathrm{s}$ & & & & & 19 & 27 & 29 & 21 & 26 & 21 & 21 & 26 & 29 \\
\hline \multirow[t]{4}{*}{ West, 7054} & $30-\mathrm{s}$ & & & & & 24 & 31 & 30 & 23 & 27 & 23 & 23 & 28 & 31 \\
\hline & 30-w & & & & & $x$ & $\mathrm{x}$ & $x$ & 23 & 27 & 23 & 23 & 28 & 28 \\
\hline & $50-\mathrm{s}$ & & & & & 23 & 31 & 26 & 23 & 27 & 23 & 23 & 28 & 31 \\
\hline & 50-w & & & & & $\mathrm{x}$ & $\mathrm{x}$ & $\mathrm{x}$ & $\mathrm{x}$ & $\mathrm{x}$ & $\mathrm{x}$ & $\mathrm{x}$ & $\mathrm{x}$ & \\
\hline
\end{tabular}

\begin{tabular}{lrrrrrrrrrrrrrr} 
YEAR: 2008 & & & & & & & & & & & & \\
Site & Level $(\mathrm{m})$ & Jan & Feb & Mar & Apr & May & Jun & Jul & Aug & Sep & Oct & Nov & Dec & Year \\
\hline \hline Peach Springs & $10-\mathrm{s}$ & 28 & 26 & 23 & 28 & 23 & 27 & 25 & 19 & 20 & 24 & 23 & 32 & 32 \\
7052 & $30-\mathrm{s}$ & 28 & 26 & 23 & 27 & 25 & 27 & 25 & 22 & 20 & 26 & 23 & 34 & 34 \\
& $44-\mathrm{s}$ & 28 & 27 & 23 & 27 & 26 & 24 & 26 & 23 & 21 & 25 & 24 & 35 & 35 \\
& $44-\mathrm{w}$ & 28 & 26 & 23 & 26 & 26 & 24 & 26 & 23 & 21 & 24 & 24 & 34 & 34 \\
\hline Nelson & $10-\mathrm{s}$ & 23 & 21 & 20 & 21 & 19 & 21 & 18 & 18 & 19 & 21 & 20 & 26 & 26 \\
7053 & $30-\mathrm{s}$ & 26 & 21 & 20 & 22 & 20 & 23 & 19 & 20 & 20 & 21 & 23 & 30 & 30 \\
& $30-\mathrm{w}$ & 25 & 22 & 20 & 22 & 20 & 24 & 19 & 22 & 20 & 21 & 23 & 30 & 30 \\
& $50-\mathrm{s}$ & 26 & 23 & 23 & 23 & 22 & 24 & 20 & 21 & $\mathrm{x}$ & $\mathrm{x}$ & 16 & $\mathrm{x}$ & 26 \\
& $50-\mathrm{w}$ & 26 & 23 & 23 & 23 & 23 & 23 & 20 & 20 & 21 & 22 & 24 & 30 & 30 \\
\hline Grand Canyon & $10-\mathrm{s}$ & 30 & 31 & 22 & 24 & 25 & 25 & 19 & 20 & 24 & 22 & 23 & 34 & 34 \\
West, 7054 & $30-\mathrm{s}$ & 34 & 34 & 24 & 26 & 28 & 24 & 20 & 22 & 23 & 24 & 24 & 34 & 34 \\
& $30-\mathrm{w}$ & 33 & 33 & 24 & 26 & 28 & 24 & 20 & 22 & 23 & 24 & 24 & 36 & 36 \\
& $50-\mathrm{s}$ & $\mathrm{x}$ & $\mathrm{x}$ & $\mathrm{x}$ & 27 & 28 & 26 & 20 & 21 & 22 & 25 & 24 & 38 & 38 \\
& $50-\mathrm{w}$ & $\mathrm{x}$ & $\mathrm{x}$ & $\mathrm{x}$ & 27 & 28 & 26 & 21 & 21 & 22 & 25 & 24 & 38 & 38 \\
\hline \hline
\end{tabular}



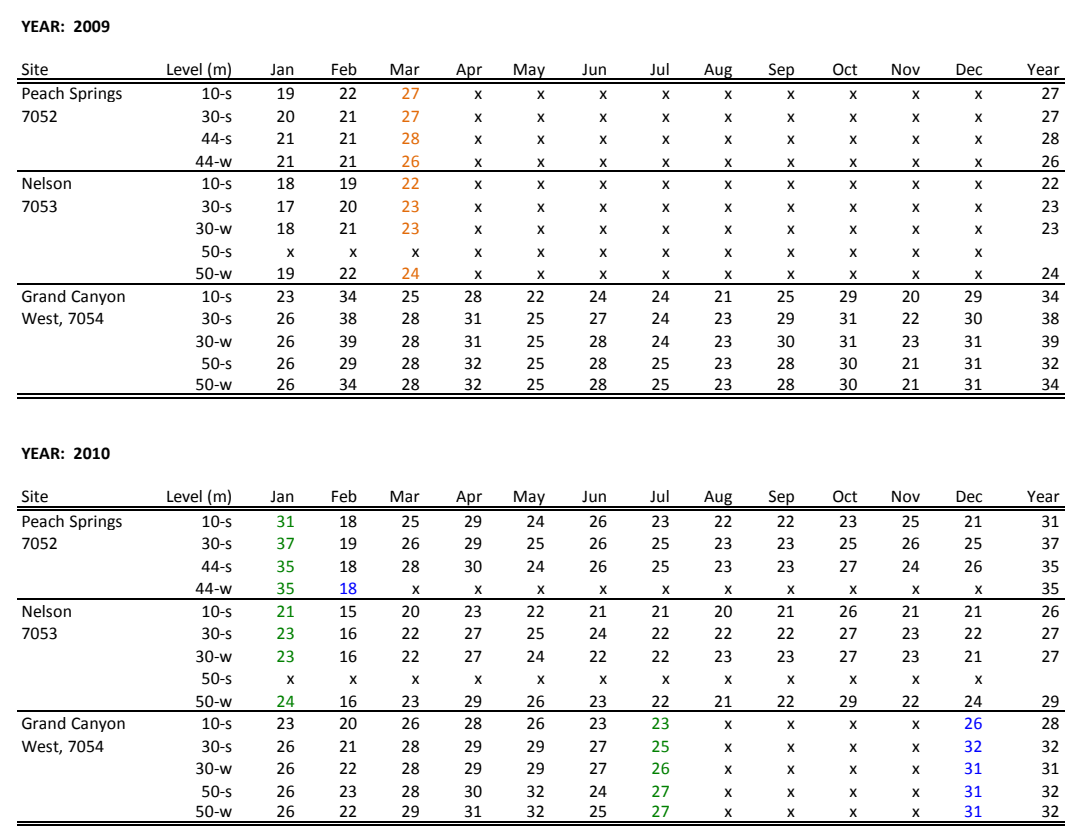

YEAR: 2011

\begin{tabular}{|c|c|c|c|c|c|c|c|c|c|c|c|c|c|c|}
\hline Site & Level (m) & Jan & Feb & Mar & Apr & May & Jun & Jul & Aug & Sep & Oct & Nov & Dec & Year \\
\hline Peach Springs & $10-5$ & 17 & 23 & 23 & 26 & 24 & 23 & 23 & 20 & 23 & 22 & 21 & 19 & 26 \\
\hline \multirow{3}{*}{7052} & $30-\mathrm{s}$ & 19 & 25 & 24 & 26 & 24 & 23 & 26 & 22 & 24 & 24 & 23 & 21 & 26 \\
\hline & $44-5$ & 20 & 24 & 24 & 25 & 24 & 23 & 25 & 21 & 24 & 24 & 23 & 21 & 25 \\
\hline & 44-w & $x$ & $x$ & $x$ & $x$ & $x$ & $x$ & $x$ & $x$ & $x$ & $x$ & $x$ & $\mathrm{x}$ & \\
\hline Nelson & $10-5$ & 17 & 20 & 22 & 23 & 21 & 22 & 24 & 18 & 17 & 20 & 20 & 19 & 24 \\
\hline \multirow[t]{4}{*}{7053} & $30-\mathrm{s}$ & 17 & 21 & 24 & 25 & 23 & 22 & 25 & 19 & 20 & 23 & 23 & 21 & 25 \\
\hline & $30-w$ & 18 & 21 & 24 & 24 & 24 & 22 & 24 & 18 & 18 & 22 & 23 & 20 & 24 \\
\hline & $50-5$ & $x$ & $\mathrm{x}$ & $x$ & $x$ & $x$ & $\mathrm{x}$ & $\mathrm{x}$ & $\mathrm{x}$ & $x$ & $x$ & $x$ & $\mathrm{x}$ & \\
\hline & 50-w & $\hat{19}$ & 22 & 27 & 26 & 24 & $\hat{23}$ & 23 & $\hat{19}$ & 20 & $\hat{23}$ & 23 & 21 & 27 \\
\hline Grand Canyon & $10-5$ & 18 & 24 & 26 & 26 & 26 & 25 & 25 & 21 & 21 & 26 & 29 & 14 & 29 \\
\hline \multirow[t]{4}{*}{ West, 7054} & $30-5$ & 18 & 26 & 29 & 29 & 28 & 27 & 26 & 23 & 25 & 29 & 30 & 17 & 30 \\
\hline & $30-w$ & 20 & 27 & 29 & 29 & 29 & 27 & 26 & 24 & 26 & 29 & 30 & 17 & 30 \\
\hline & $50-\mathrm{s}$ & 19 & 27 & 31 & 28 & 30 & 29 & 27 & 25 & 26 & 28 & 29 & 16 & 31 \\
\hline & $50-w$ & 18 & 28 & 30 & 28 & 29 & 28 & 26 & 25 & 26 & 28 & 30 & 18 & 30 \\
\hline Clay Springs & $10-5$ & & & & & 23 & 22 & 23 & 17 & 19 & 20 & 23 & 16 & 23 \\
\hline \multirow[t]{5}{*}{7055} & $30-5$ & & & & & 28 & 26 & 25 & 20 & 20 & 24 & 26 & 16 & 28 \\
\hline & 30-nw & & & & & 27 & 26 & 26 & 20 & 20 & 24 & 26 & 16 & 27 \\
\hline & $40-\mathrm{s}$ & & & & & 29 & 26 & 26 & 21 & 20 & 24 & 27 & 17 & 29 \\
\hline & 49-s & & & & & 31 & 26 & 25 & 22 & 20 & 25 & 29 & 16 & 31 \\
\hline & 49-nw & & & & & 31 & 25 & 25 & 21 & 20 & 25 & 29 & 17 & 31 \\
\hline
\end{tabular}

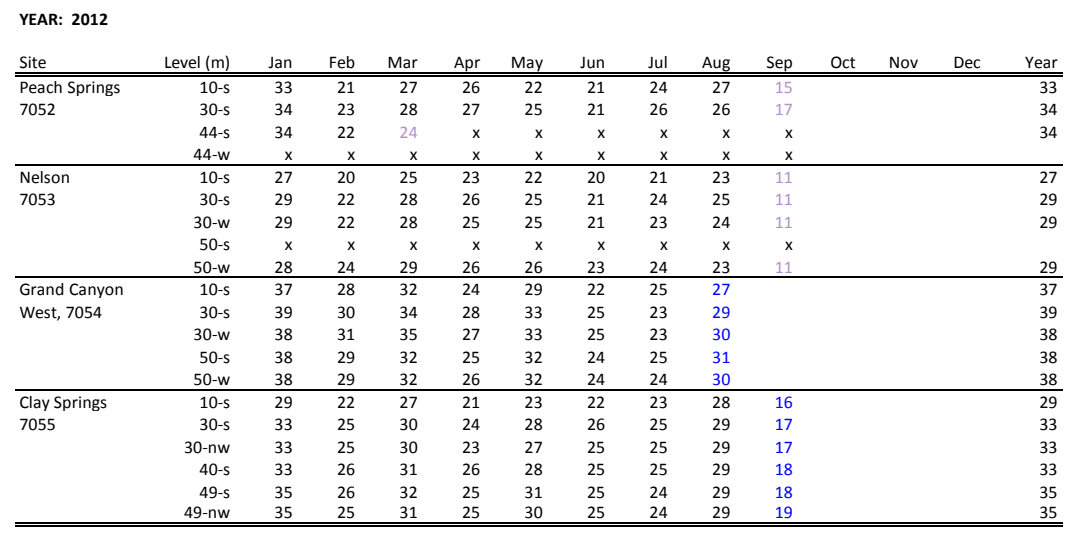

\begin{tabular}{ll}
\hline Overall Maximum: $\quad 39$ \\
\hline
\end{tabular}

Data Recovery Key: black $=90-100 \% ;$ green $=75-90 \% ;$ blue $=50-75 \%$; orange $=25-50 \% ;$ purple $=10-25 \% ; " x^{\prime \prime}=0-10 \%$. 
Turbulence Intensity Statistics

Blue Mountain, Site 7051, 49-m Level

Using Hourly Data, March 2006-July 2007*

\begin{tabular}{|c|c|c|c|c|c|}
\hline $\begin{array}{r}\text { Speed } \\
\text { (mps) } \\
\end{array}$ & $\begin{array}{l}\text { Number } \\
\text { of Hours }\end{array}$ & Mean T. I. & $\begin{array}{c}\text { Standard } \\
\text { Deviation } \\
\text { of T. I. } \\
\end{array}$ & $\begin{array}{r}\text { Mean + } \\
1 \text { Std. Dev. }\end{array}$ & $\begin{array}{r}\text { Maximum } \\
\text { T. I. } \\
\end{array}$ \\
\hline$\overline{\overline{0}}$ & $\overline{20}$ & $\overline{0.414}$ & $\overline{c 0.091}$ & "0.505 & "0.660 \\
\hline 1 & 228 & 0.422 & 0.193 & 0.615 & 0.946 \\
\hline 2 & 422 & 0.269 & 0.134 & 0.403 & 0.814 \\
\hline 3 & 830 & 0.217 & 0.118 & 0.335 & 0.577 \\
\hline 4 & 1140 & 0.172 & 0.098 & 0.270 & 0.575 \\
\hline 5 & 1210 & 0.148 & 0.084 & 0.232 & 0.423 \\
\hline 6 & 1140 & 0.135 & 0.075 & 0.211 & 0.413 \\
\hline 7 & 1032 & 0.126 & 0.071 & 0.197 & 0.417 \\
\hline 8 & 927 & 0.113 & 0.063 & 0.176 & 0.296 \\
\hline 9 & 724 & 0.105 & 0.054 & 0.159 & 0.350 \\
\hline 10 & 445 & 0.104 & 0.050 & 0.154 & 0.246 \\
\hline 11 & 283 & 0.110 & 0.041 & 0.152 & 0.262 \\
\hline 12 & 147 & 0.117 & 0.040 & 0.158 & 0.326 \\
\hline 13 & 81 & 0.113 & 0.026 & 0.139 & 0.167 \\
\hline 14 & 59 & 0.111 & 0.031 & 0.141 & 0.168 \\
\hline 15 & 36 & 0.119 & 0.027 & 0.146 & 0.181 \\
\hline 16 & 27 & 0.117 & 0.022 & 0.139 & 0.156 \\
\hline 17 & 24 & 0.115 & 0.018 & 0.133 & 0.163 \\
\hline 18 & 10 & 0.115 & 0.020 & 0.136 & 0.161 \\
\hline 19 & 9 & 0.118 & 0.018 & 0.136 & 0.158 \\
\hline 20 & 1 & 0.110 & & & 0.110 \\
\hline 21 & 1 & 0.090 & & & 0.090 \\
\hline 22 & 0 & & & & \\
\hline 23 & 0 & & & & \\
\hline 24 & 0 & & & & \\
\hline 25 & 0 & & & & \\
\hline $26+$ & 0 & & & & \\
\hline Total & $\overline{8796}$ & & & & \\
\hline
\end{tabular}

* values reflect actual 10-minute turbulence, due to the processing algorithm of the Symphonie Data Retriever software 
Turbulence Intensity Statistics

Peach Springs, Site 7052, 44-m Level

Using Hourly Data, December 2005-September 2012*

\begin{tabular}{crrrrr}
$\begin{array}{r}\text { Speed } \\
\text { (mps) }\end{array}$ & $\begin{array}{r}\text { Number } \\
\text { of Hours }\end{array}$ & Mean T. I. & $\begin{array}{r}\text { Standard } \\
\text { Deviation } \\
\text { of T. I. }\end{array}$ & $\begin{array}{r}\text { Mean + } \\
\text { 1 Std. Dev. }\end{array}$ & $\begin{array}{r}\text { Maximum } \\
\text { T. I. }\end{array}$ \\
\hline \hline 0 & 68 & 0.416 & 0.092 & 0.508 & 0.750 \\
1 & 902 & 0.358 & 0.162 & 0.520 & 0.843 \\
2 & 2435 & 0.240 & 0.111 & 0.351 & 0.620 \\
3 & 4272 & 0.191 & 0.095 & 0.286 & 0.864 \\
4 & 5384 & 0.156 & 0.081 & 0.238 & 0.543 \\
5 & 5799 & 0.133 & 0.071 & 0.204 & 0.579 \\
\hline 6 & 5341 & 0.120 & 0.065 & 0.184 & 0.398 \\
7 & 4119 & 0.120 & 0.060 & 0.179 & 0.385 \\
8 & 2948 & 0.122 & 0.057 & 0.179 & 0.330 \\
9 & 2027 & 0.127 & 0.052 & 0.179 & 0.309 \\
10 & 1465 & 0.126 & 0.047 & 0.173 & 0.317 \\
\hline 11 & 964 & 0.125 & 0.041 & 0.166 & 0.252 \\
12 & 626 & 0.124 & 0.037 & 0.161 & 0.241 \\
13 & 413 & 0.127 & 0.031 & 0.158 & 0.206 \\
14 & 274 & 0.131 & 0.023 & 0.154 & 0.192 \\
15 & 176 & 0.132 & 0.020 & 0.152 & 0.184 \\
\hline 16 & 101 & 0.129 & 0.017 & 0.146 & 0.177 \\
17 & 57 & 0.126 & 0.019 & 0.145 & 0.184 \\
18 & 27 & 0.129 & 0.017 & 0.146 & 0.165 \\
19 & 18 & 0.122 & 0.016 & 0.137 & 0.157 \\
20 & 5 & 0.120 & 0.015 & 0.135 & 0.138 \\
\hline 21 & 1 & 0.149 & & & 0.149 \\
22 & 4 & 0.112 & 0.007 & 0.119 & 0.120 \\
23 & 0 & & & & \\
24 & 1 & 0.112 & & & 0.112 \\
25 & 0 & & & & \\
$26+$ & 1 & 0.104 & & & \\
\hline \hline
\end{tabular}

Total 37428

* values reflect actual 10-minute turbulence, due to the processing algorithm of the Symphonie Data Retriever software 
Turbulence Intensity Statistics

Grand Canyon West, Site 7054, 50-m Level

Using Hourly Data, October 2006-August 2012*

\begin{tabular}{crrrrr}
$\begin{array}{c}\text { Speed } \\
\text { (mps) }\end{array}$ & $\begin{array}{r}\text { Number } \\
\text { of Hours }\end{array}$ & Mean T. I. & $\begin{array}{r}\text { Standard } \\
\text { Deviation } \\
\text { of T. I. }\end{array}$ & $\begin{array}{r}\text { Mean + } \\
\text { 1 Std. Dev. }\end{array}$ & $\begin{array}{r}\text { Maximum } \\
\text { T. I. }\end{array}$ \\
\hline \hline 0 & 89 & 0.356 & 0.149 & 0.504 & 0.918 \\
1 & 1070 & 0.349 & 0.166 & 0.515 & 0.864 \\
2 & 2544 & 0.231 & 0.121 & 0.352 & 0.810 \\
3 & 4425 & 0.179 & 0.100 & 0.279 & 0.602 \\
4 & 5807 & 0.156 & 0.086 & 0.242 & 0.476 \\
5 & 6034 & 0.146 & 0.079 & 0.225 & 0.468 \\
\hline 6 & 5690 & 0.133 & 0.071 & 0.204 & 0.427 \\
7 & 4948 & 0.126 & 0.063 & 0.189 & 0.436 \\
8 & 3726 & 0.124 & 0.058 & 0.181 & 0.338 \\
9 & 2575 & 0.125 & 0.053 & 0.178 & 0.303 \\
10 & 1823 & 0.132 & 0.046 & 0.178 & 0.312 \\
\hline 11 & 1262 & 0.135 & 0.039 & 0.174 & 0.274 \\
12 & 836 & 0.135 & 0.034 & 0.169 & 0.262 \\
13 & 615 & 0.127 & 0.029 & 0.156 & 0.262 \\
14 & 429 & 0.126 & 0.026 & 0.151 & 0.235 \\
15 & 314 & 0.123 & 0.024 & 0.147 & 0.235 \\
\hline 16 & 208 & 0.118 & 0.024 & 0.142 & 0.222 \\
17 & 138 & 0.118 & 0.022 & 0.141 & 0.202 \\
18 & 78 & 0.114 & 0.020 & 0.134 & 0.161 \\
19 & 49 & 0.115 & 0.019 & 0.135 & 0.151 \\
20 & 35 & 0.111 & 0.014 & 0.126 & 0.133 \\
\hline 21 & 24 & 0.115 & 0.023 & 0.138 & 0.168 \\
22 & 19 & 0.112 & 0.012 & 0.124 & 0.132 \\
23 & 9 & 0.111 & 0.009 & 0.120 & 0.127 \\
24 & 5 & 0.119 & 0.016 & 0.134 & 0.145 \\
25 & 4 & 0.108 & 0.020 & 0.129 & 0.139 \\
$26+$ & 1 & 0.086 & & & 0.086 \\
\hline \hline Total & 42757 & & & & \\
& & & & &
\end{tabular}

* values reflect actual 10-minute turbulence, due to the processing algorithm of the Symphonie Data Retriever software 
Turbulence Intensity Statistics

Clay Springs, Site 7055, 49-m Level

Using Hourly Data, May 2011-September 2012*

\begin{tabular}{|c|c|c|c|c|c|}
\hline $\begin{array}{l}\text { Speed } \\
\text { (mps) }\end{array}$ & $\begin{array}{l}\text { Number } \\
\text { of Hours }\end{array}$ & Mean T. I. & $\begin{array}{c}\text { Standard } \\
\text { Deviation } \\
\text { of T. I. }\end{array}$ & $\begin{array}{r}\text { Mean + } \\
1 \text { Std. Dev. }\end{array}$ & $\begin{array}{c}\text { Maximum } \\
\text { T. I. } \\
\end{array}$ \\
\hline$\overline{0} 0$ & $\overline{237}$ & 0.356 & $\overline{0.111}$ & 0.467 & $\overline{0.617}$ \\
\hline 1 & 451 & 0.352 & 0.169 & 0.521 & 0.753 \\
\hline 2 & 921 & 0.226 & 0.104 & 0.331 & 0.538 \\
\hline 3 & 1454 & 0.172 & 0.090 & 0.262 & 0.491 \\
\hline 4 & 1915 & 0.152 & 0.081 & 0.233 & 0.523 \\
\hline 5 & 1911 & 0.150 & 0.077 & 0.227 & 0.518 \\
\hline 6 & 1665 & 0.153 & 0.078 & 0.231 & 0.412 \\
\hline 7 & 1129 & 0.148 & 0.068 & 0.216 & 0.373 \\
\hline 8 & 734 & 0.153 & 0.064 & 0.217 & 0.327 \\
\hline 9 & 542 & 0.163 & 0.051 & 0.215 & 0.334 \\
\hline 10 & 380 & 0.167 & 0.043 & 0.210 & 0.274 \\
\hline 11 & 272 & 0.162 & 0.038 & 0.200 & 0.266 \\
\hline 12 & 178 & 0.168 & 0.030 & 0.197 & 0.236 \\
\hline 13 & 121 & 0.164 & 0.028 & 0.192 & 0.226 \\
\hline 14 & 93 & 0.158 & 0.027 & 0.185 & 0.223 \\
\hline 15 & 42 & 0.159 & 0.032 & 0.191 & 0.213 \\
\hline 16 & 30 & 0.139 & 0.027 & 0.166 & 0.215 \\
\hline 17 & 13 & 0.140 & 0.026 & 0.165 & 0.196 \\
\hline 18 & 17 & 0.150 & 0.016 & 0.166 & 0.191 \\
\hline 19 & 19 & 0.137 & 0.018 & 0.155 & 0.168 \\
\hline 20 & 2 & 0.110 & 0.008 & 0.117 & 0.118 \\
\hline 21 & 1 & 0.174 & & & 0.174 \\
\hline 22 & 0 & & & & \\
\hline 23 & 0 & & & & \\
\hline 24 & 0 & & & & \\
\hline 25 & 0 & & & & \\
\hline $26+$ & 0 & & & & \\
\hline
\end{tabular}

* values reflect actual 10-minute turbulence, due to the processing algorithm of the Symphonie Data Retriever software 
Turbulence Intensity Statistics

Nelson, Site 7053, 50-m Level

Using Hourly Data, December 2006-September 2012*

\begin{tabular}{|c|c|c|c|c|c|}
\hline $\begin{array}{l}\text { Speed } \\
\text { (mps) }\end{array}$ & $\begin{array}{l}\text { Number } \\
\text { of Hours }\end{array}$ & Mean T. I. & $\begin{array}{c}\text { Standard } \\
\text { Deviation } \\
\text { of T. I. }\end{array}$ & $\begin{array}{r}\text { Mean + } \\
1 \text { Std. Dev. }\end{array}$ & $\begin{array}{r}\text { Maximum } \\
\text { T. I. }\end{array}$ \\
\hline 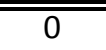 & 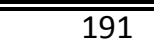 & 0.360 & 0.113 & $\overline{0.474}$ & 1.109 \\
\hline 1 & 1664 & 0.366 & 0.149 & 0.515 & 0.871 \\
\hline 2 & 3624 & 0.249 & 0.115 & 0.363 & 0.689 \\
\hline 3 & 5874 & 0.185 & 0.093 & 0.278 & 0.636 \\
\hline 4 & 7755 & 0.152 & 0.080 & 0.231 & 0.491 \\
\hline 5 & 7580 & 0.138 & 0.071 & 0.209 & 0.421 \\
\hline 6 & 5315 & 0.139 & 0.064 & 0.202 & 0.412 \\
\hline 7 & 3647 & 0.141 & 0.056 & 0.198 & 0.379 \\
\hline 8 & 2506 & 0.143 & 0.049 & 0.192 & 0.310 \\
\hline 9 & 1739 & 0.143 & 0.039 & 0.182 & 0.311 \\
\hline 10 & 1049 & 0.144 & 0.033 & 0.176 & 0.302 \\
\hline 11 & 698 & 0.146 & 0.025 & 0.170 & 0.217 \\
\hline 12 & 416 & 0.142 & 0.022 & 0.165 & 0.220 \\
\hline 13 & 252 & 0.143 & 0.019 & 0.162 & 0.216 \\
\hline 14 & 180 & 0.139 & 0.016 & 0.155 & 0.194 \\
\hline 15 & 98 & 0.140 & 0.017 & 0.157 & 0.176 \\
\hline 16 & 48 & 0.141 & 0.015 & 0.156 & 0.169 \\
\hline 17 & 17 & 0.135 & 0.011 & 0.146 & 0.163 \\
\hline 18 & 10 & 0.132 & 0.015 & 0.148 & 0.154 \\
\hline 19 & 3 & 0.153 & 0.009 & 0.161 & 0.165 \\
\hline 20 & 3 & 0.127 & 0.011 & 0.138 & 0.135 \\
\hline 21 & 0 & & & & \\
\hline 22 & 0 & & & & \\
\hline 23 & 0 & & & & \\
\hline 24 & 0 & & & & \\
\hline 25 & 0 & & & & \\
\hline $26+$ & 0 & & & & \\
\hline
\end{tabular}

* values reflect actual 10-minute turbulence, due to the processing algorithm of the Symphonie Data Retriever software 


\title{
Exhibit F
}

\section{Wind Farm Development Analysis}

\author{
(V-Bar)
}




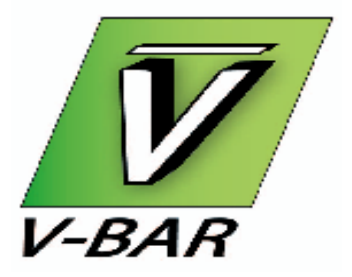

\section{Prospective Wind Farm Development Report \\ for Hualapai Tribal Lands}

Prepared by Richard Simon and David Matson

V-Bar, LLC,

7 October 2012

This report presents four prospective wind farm array plans, developed over the course of many years from meteorological tower measurements, site visits, setback criteria and other factors. Four specific arrays are presented herein:

On Hualapai lands only: 200-MW array for GE-1.7/100 turbines with 80-m hub height 200-MW array for Acciona AW-116/3000 turbines with 92-m hub height

On Hualapai and adjacent BLM lands...

284-MW array for GE-1.7/100 turbines with 80 -m hub height

456-MW array for Acciona AW-116/3000 turbines with 92-m hub height

The 200-MW arrays use more restrictive criteria (tribal lands only, at least 4 miles from eagle nests), and these arrays are designed consistent with applications being submitted to WAPA for access to their transmission line near the southern end of the projects. The larger arrays include BLM lands, have reduced (2-mile) setbacks from eagle nests, and also have a number of turbines close to the edge of the Grand Wash Cliffs, which, according to recent field studies, appears to be an active travel route for migrating raptors.

These arrays are shown in map and tabular form on the Maps and Coords tabs of this report.

WERA. Detailed wind resource assessments for the 200-MW arrays are given in this tab. We focus on long-term mean annual gross energy projections. Estimated long-term mean annual hub-height wind speeds and gross capacity factors are given for each individual turbine in the 200-MW arrays. The wind speeds are derived from:

- wind speed data from the Grand Canyon West and Clay Springs MET towers at the northern and southern ends of the project footprint, respectively

- wind direction data from these towers, to identify prevailing directions, zones where winds can be enhanced by the terrain, and proper spacing between turbines

- topographic and terrain variations across and outside the project footprint 
- surface conditions (vegetation type, height and density, soil and rockiness, etc.)

- observations made during field trips

Aggregate long-term mean annual hub-height wind speeds and gross annual capacity factors are summarized below:

$\begin{array}{lccc} & \text { Turbine } & \text { Aggregate } & \text { Gross Cap. } \\ \text { Turbine } & \text { Count } & \text { Speed (mps) } & \text { Factor }(\%) \\ \text { GE-1.7/100 } & 118 & 6.16 & 33.73 \\ \text { AW-116/3000 } & 67 & 6.35 & 27.74\end{array}$

Losses. To convert gross to net projections, we present a table showing the various discount factors that are normally considered in this type of exercise. Some of these discounts are meteorological in nature, and we have calculated them from the on-site data or estimated them based on our experience in the region. Other discounts are non-meteorological in nature, and we have provided typical industry values as placeholders. These non-meteorological discounts can be refined with appropriate contractual and engineering inputs.

Here is a summary of the resulting long-term mean annual net energy projections for the two 200-MW array plans:

\begin{tabular}{|c|c|c|c|c|c|c|}
\hline & Iross Cap. & Total \% & Net Cap. & Net MWh/ & Turbine & Net M \\
\hline urbin & Factor $(\%)$ & Discount & Factor $(\%)$ & Turbine & Count & for $\mathrm{Ar}$ \\
\hline$/ 100$ & 33.73 & 18.69 & 27.43 & 4,084 & 118 & 481,93 \\
\hline AW-116/3000 & 27.74 & 19.21 & 22.41 & 5,889 & 67 & 394, \\
\hline
\end{tabular}

These wind resource projections are approximate, given that the terrain is very complicated, wind data have only been measured at two locations, and the wind measurement towers are only 50 meters tall. To reduce uncertainties, we recommend that the existing met towers be refurbished with new sensors, the old sensors sent for post-calibration, additional met towers be installed with a minimum $60-\mathrm{m}$ height, and the use of SODAR or LIDAR is recommended to further document site conditions.

No detailed projections are provided for the larger arrays, since it is unclear whether they can even be permitted, let alone feed the extra energy into the WAPA transmission lines. In general, the additional turbine are likely to have a slightly higher resource than the 200-MW arrays examined in detail. 


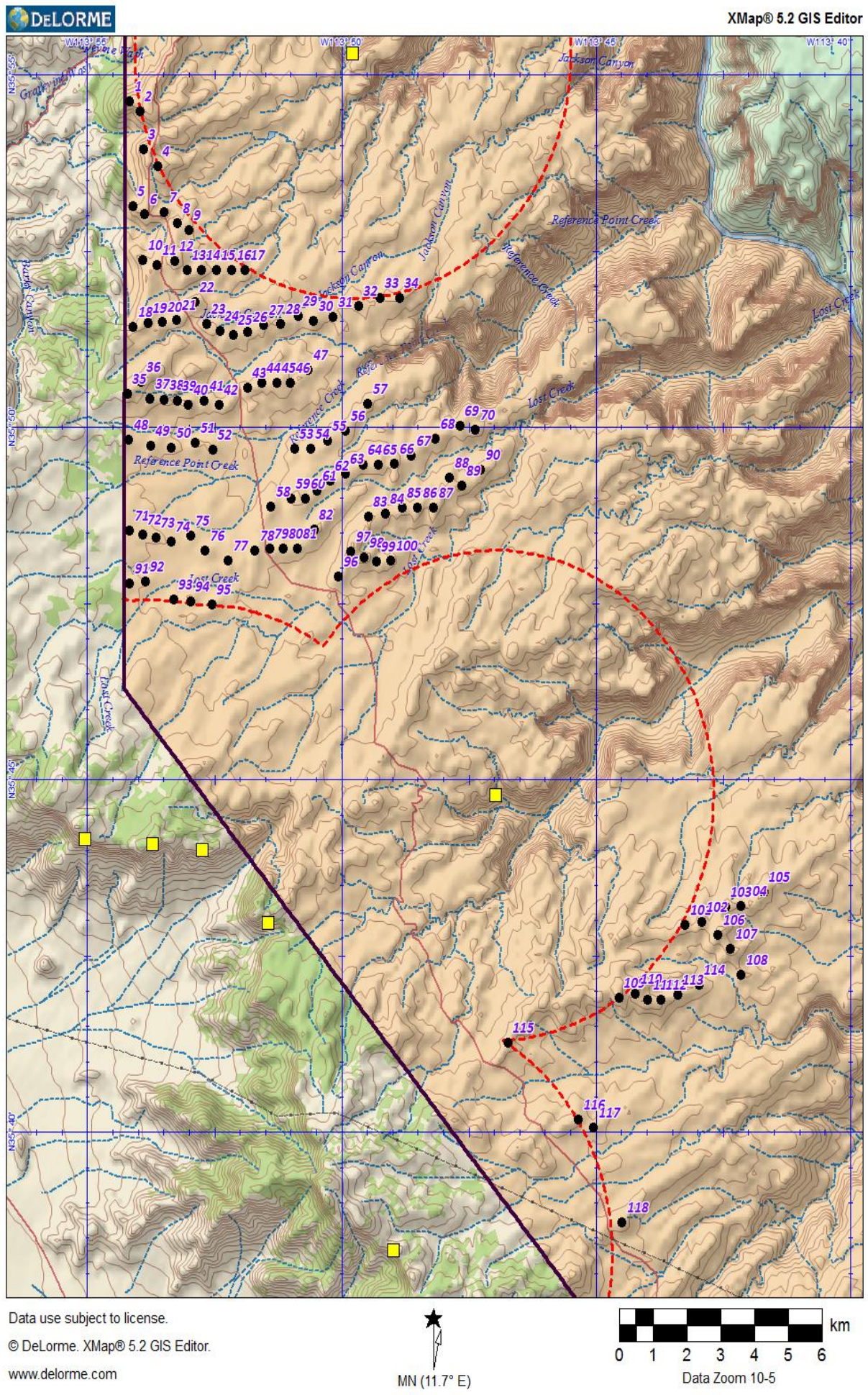




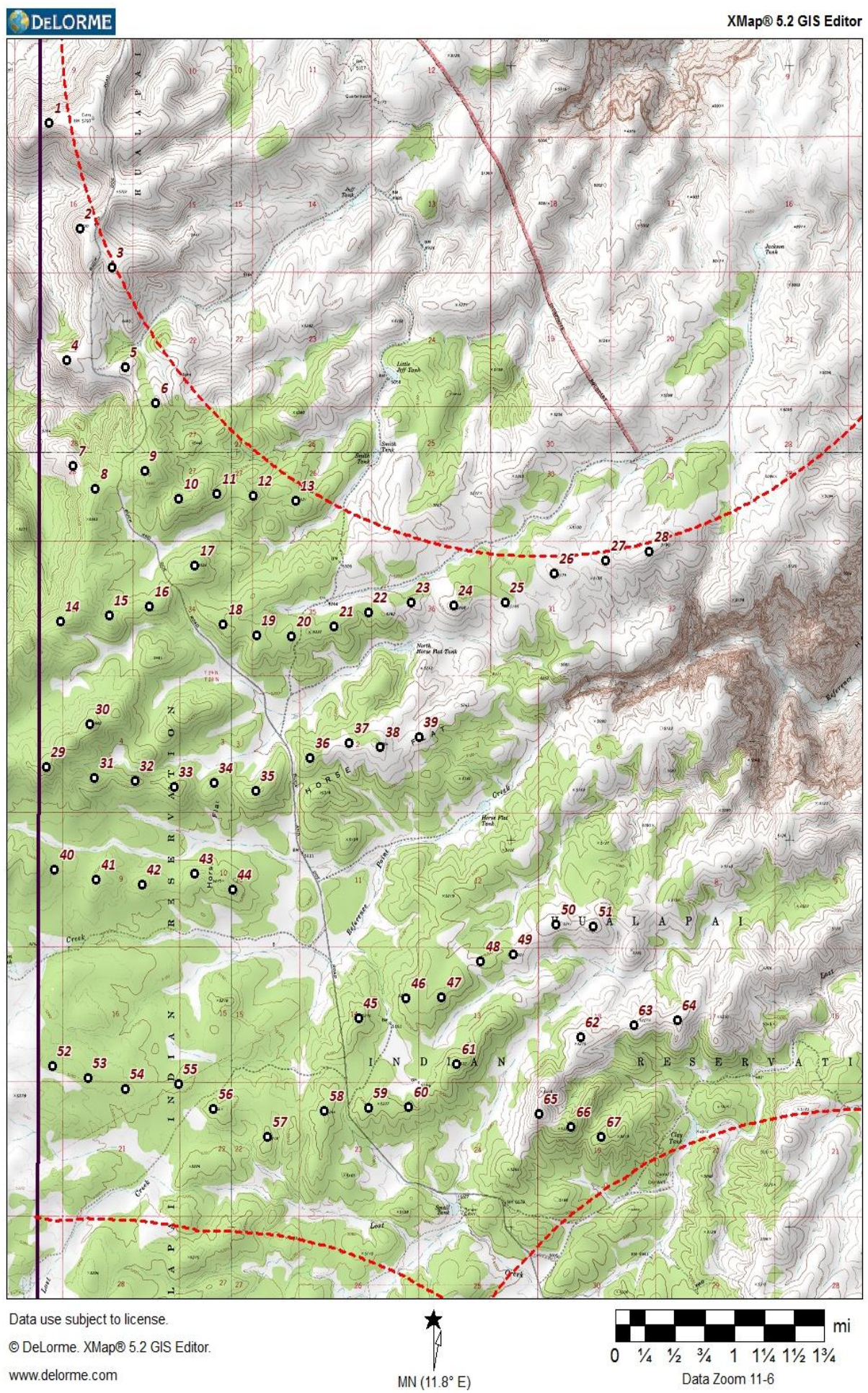


Full Development Array Plan for GE-1.7/100 Turbines on Tribal and BLM Lands

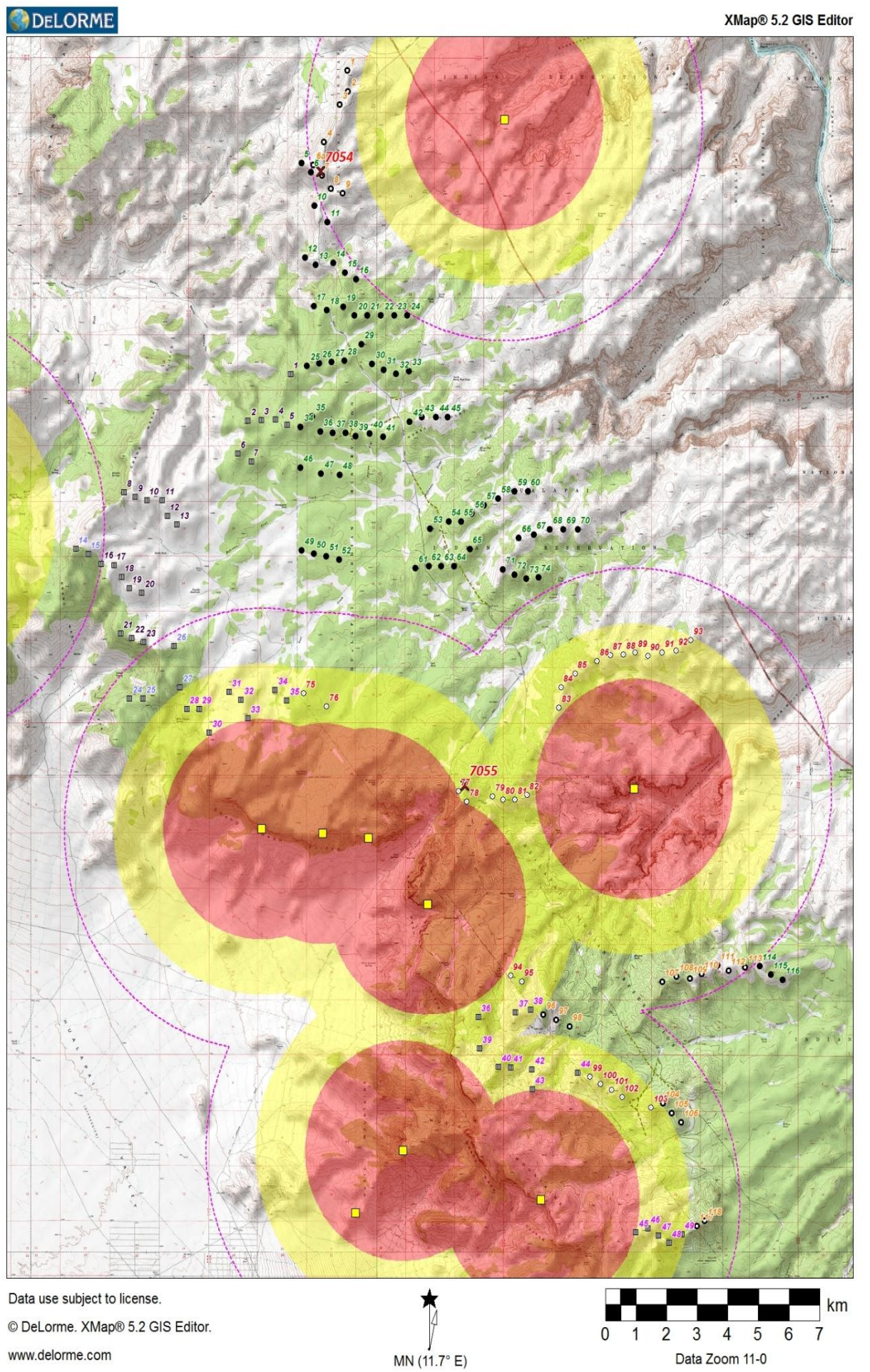




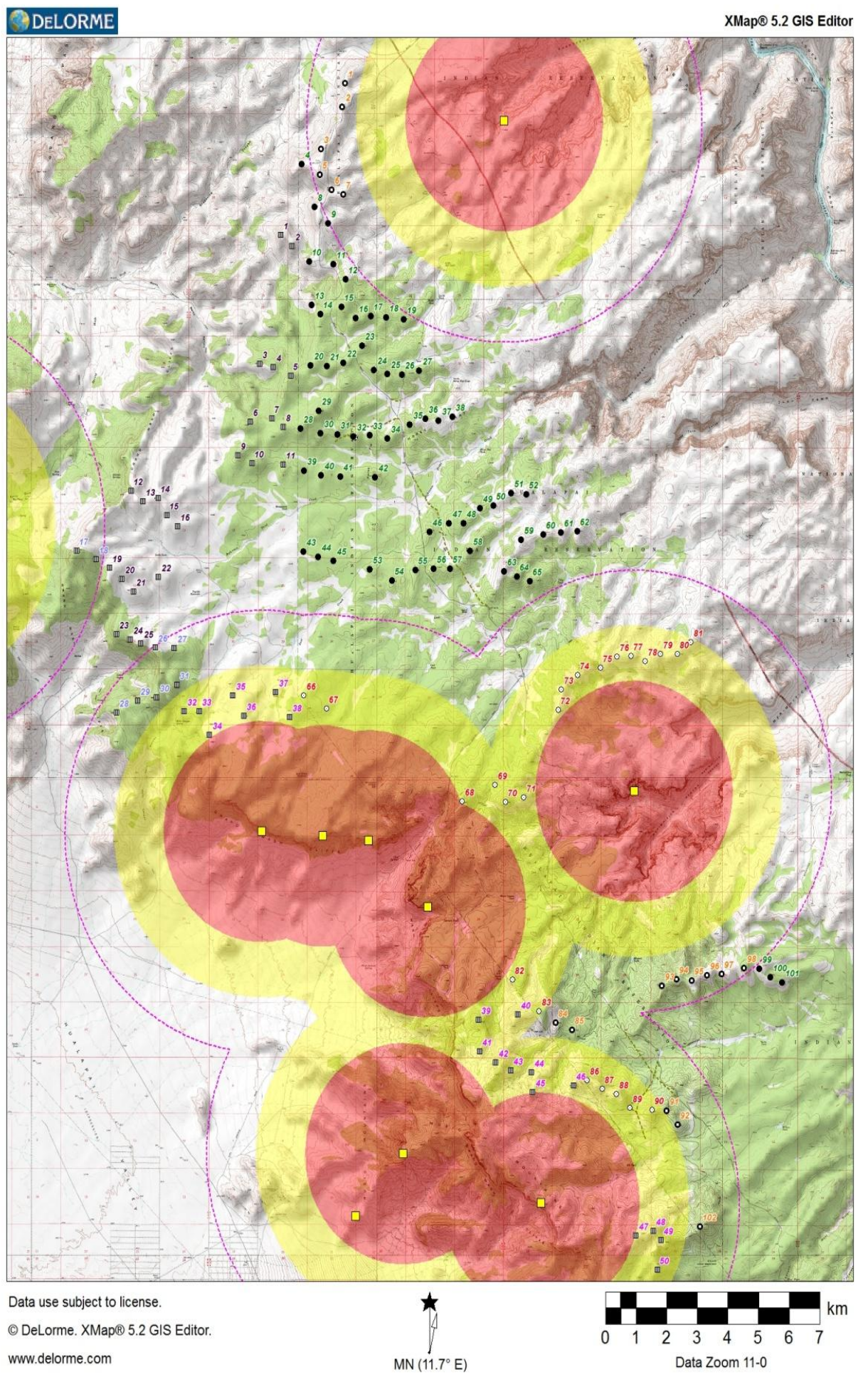


Prospective 200-MW Array Plan for GE-1.7/100 Turbines

Hualapai Reservation, Arizona

Prepared by Richard Simon, V-Bar, LLC

WGS84 Datum, Partially Field Checked

\begin{tabular}{|c|c|c|c|c|c|}
\hline Turbine & Latitude & Longitude & Turbine & Latitude & Longitude \\
\hline 1 & 35.910401 & -113.902949 & 60 & 35.816288 & -113.845253 \\
\hline 2 & 35.907955 & -113.899452 & 61 & 35.818348 & -113.841443 \\
\hline 3 & 35.899072 & -113.898283 & 62 & 35.820622 & -113.836944 \\
\hline 4 & 35.894866 & -113.893515 & 63 & 35.822339 & -113.831969 \\
\hline 5 & 35.885596 & -113.901723 & 64 & 35.824313 & -113.826095 \\
\hline 6 & 35.883708 & -113.897910 & 65 & 35.824313 & -113.821226 \\
\hline 7 & 35.884094 & -113.891486 & 66 & 35.824614 & -113.816166 \\
\hline 8 & 35.881605 & -113.887196 & 67 & 35.826502 & -113.810450 \\
\hline 9 & 35.879846 & -113.883291 & 68 & 35.830484 & -113.802636 \\
\hline 10 & 35.872765 & -113.898438 & 69 & 35.833497 & -113.794414 \\
\hline 11 & 35.871692 & -113.893777 & 70 & 35.832767 & -113.789598 \\
\hline 12 & 35.872550 & -113.887899 & 71 & 35.808821 & -113.902931 \\
\hline 13 & 35.870361 & -113.883821 & 72 & 35.807920 & -113.898486 \\
\hline 14 & 35.870361 & -113.879266 & 73 & 35.807276 & -113.893987 \\
\hline 15 & 35.870361 & -113.874235 & 74 & 35.806332 & -113.889277 \\
\hline 16 & 35.870361 & -113.869415 & 75 & 35.807619 & -113.882792 \\
\hline 17 & 35.870361 & -113.864861 & 76 & 35.804057 & -113.877977 \\
\hline 18 & 35.856886 & -113.901662 & 77 & 35.801654 & -113.870357 \\
\hline 19 & 35.857873 & -113.896583 & 78 & 35.804100 & -113.861871 \\
\hline 20 & 35.858173 & -113.892081 & 79 & 35.804572 & -113.856948 \\
\hline 21 & 35.858560 & -113.887422 & 80 & 35.804615 & -113.852397 \\
\hline 22 & 35.862765 & -113.881214 & 81 & 35.804572 & -113.847793 \\
\hline 23 & 35.857573 & -113.877520 & 82 & 35.809164 & -113.842184 \\
\hline 24 & 35.856113 & -113.873178 & 83 & 35.812061 & -113.824610 \\
\hline 25 & 35.855126 & -113.868783 & 84 & 35.812898 & -113.819053 \\
\hline 26 & 35.855727 & -113.864230 & 85 & 35.814271 & -113.813390 \\
\hline 27 & 35.857412 & -113.858701 & 86 & 35.814250 & -113.808495 \\
\hline 28 & 35.857701 & -113.853261 & 87 & 35.814250 & -113.803177 \\
\hline 29 & 35.859632 & -113.847505 & 88 & 35.821309 & -113.798023 \\
\hline 30 & 35.858474 & -113.842581 & 89 & 35.819335 & -113.793789 \\
\hline 31 & 35.859418 & -113.836227 & 90 & 35.823283 & -113.787757 \\
\hline 32 & 35.861907 & -113.827543 & 91 & 35.796418 & -113.902883 \\
\hline 33 & 35.863752 & -113.820818 & 92 & 35.796719 & -113.897592 \\
\hline 34 & 35.863752 & -113.814252 & 93 & 35.792642 & -113.888228 \\
\hline 35 & 35.841136 & -113.903357 & 94 & 35.792084 & -113.882672 \\
\hline 36 & 35.843883 & -113.899175 & 95 & 35.791440 & -113.875688 \\
\hline 37 & 35.839934 & -113.896157 & 96 & 35.797856 & -113.834509 \\
\hline 38 & 35.839634 & -113.891605 & 97 & 35.803778 & -113.830164 \\
\hline 39 & 35.839548 & -113.887105 & 98 & 35.802362 & -113.826064 \\
\hline 40 & 35.838647 & -113.883505 & 99 & 35.801396 & -113.821751 \\
\hline 41 & 35.839462 & -113.878317 & 100 & 35.801761 & -113.817253 \\
\hline 42 & 35.838518 & -113.873446 & 101 & 35.715609 & -113.720901 \\
\hline 43 & 35.842552 & -113.864023 & 102 & 35.716317 & -113.715246 \\
\hline 44 & 35.843754 & -113.859470 & 103 & 35.719728 & -113.707317 \\
\hline 45 & 35.843754 & -113.854494 & 104 & 35.720158 & -113.702454 \\
\hline 46 & 35.843711 & -113.850100 & 105 & 35.723376 & -113.694843 \\
\hline 47 & 35.846801 & -113.844302 & 106 & 35.713248 & -113.710013 \\
\hline 48 & 35.830407 & -113.903250 & 107 & 35.709944 & -113.706049 \\
\hline 49 & 35.828991 & -113.895945 & 108 & 35.703903 & -113.702475 \\
\hline 50 & 35.828519 & -113.889064 & 109 & 35.698389 & -113.742314 \\
\hline 51 & 35.829678 & -113.881415 & 110 & 35.699252 & -113.737112 \\
\hline 52 & 35.827918 & -113.875486 & 111 & 35.697919 & -113.733112 \\
\hline 53 & 35.828218 & -113.848544 & 112 & 35.697885 & -113.728620 \\
\hline 54 & 35.828218 & -113.843463 & 113 & 35.699172 & -113.723072 \\
\hline 55 & 35.830150 & -113.837905 & 114 & 35.701543 & -113.716152 \\
\hline 56 & 35.832381 & -113.831977 & 115 & 35.687799 & -113.778601 \\
\hline 57 & 35.838690 & -113.824845 & 116 & 35.669518 & -113.755830 \\
\hline 58 & 35.814516 & -113.856625 & 117 & 35.667801 & -113.750863 \\
\hline 59 & 35.816288 & -113.849751 & 118 & 35.645206 & -113.741578 \\
\hline
\end{tabular}


Discount Factors to Convert Gross to Net Energy Projections

Hualapai 200-MW Wind Farm

Mesa Power/Wind Tex Energy

Total Discount

GE-1.7/100 (Rev 2$)$

\begin{tabular}{r|r|}
19.77 & 0.8023 \\
\hline
\end{tabular}

20.29)

Detail by Category

Loss (\%) Efficiency

Loss (\%) Efficiency Description

High-wind downtime correlation

Electrical

\section{Wake Effects}

\begin{tabular}{rr}
4.00 & 0.9600 \\
\hline
\end{tabular}

Row-to-row wake losses

Off-axis wake losses

Wakes from pre-existing turbines

Wakes from future turbines

Turbine Performance

Turbulence

Inclined flow, high shear

Sub-optimal operations

Power curve inaccuracy

Environmenta

Blade degradation and soiling

Icing

High/low temperature shutdowns

Other meteorological events

Site access, force majeure

Tree growth or felling

Curtailment

Wind direction sector management

Off-taker curtails power delivery

Other causes

Balance of Plant

\begin{tabular}{|r|r|}
\hline 4 & 0.960 \\
0 & 0.96 \\
\hline 3.80 & 0.9620 \\
\hline .52 & 0.9248 \\
\hline 6.3 & 0.937 \\
1.3 & 0.987 \\
0 & 1 \\
0 & 1 \\
\hline 3.10 & 0.9690 \\
\hline 1 & 0.99 \\
0 & 1 \\
0.13 & 0.9987 \\
1 & 0.99 \\
1 & 0.99 \\
\hline .09 & 0.9791 \\
\hline 1 & 0.99 \\
0.8 & 0.992 \\
0 & 1 \\
0.2 & 0.998 \\
0.1 & 0.999 \\
0 & 1 \\
\hline 00 & 1.0000 \\
\hline 0 & 1 \\
0 & 1 \\
0 & 1 \\
\hline .00 & 0.9900 \\
\hline
\end{tabular}

\begin{tabular}{|c|c|c|}
\hline 4.00 & 0.9600 & \\
\hline 4 & 0.96 & Assumes typical O\&M downtime \\
\hline 0 & 1 & Downtime correlated with high-wind events, assumed zero for this project \\
\hline 3.80 & 0.9620 & from turbines to high-side of interconnect point, rovided by POWER Engineers \\
\hline 8.01 & 0.9199 & \\
\hline 6.7 & 0.933 & Based on V-Bar wake model \\
\hline 1.4 & 0.986 & Calculated with Off-Axis tab \\
\hline 0 & 1 & No pre-existing turbines \\
\hline 0 & 1 & No future turbines anticipated \\
\hline 3.21 & 0.9679 & \\
\hline 1 & 0.99 & Standard value, aerodynamic inefficiency due to high turbulence or yaw error \\
\hline 0 & & None expected \\
\hline 0.25 & 0.9975 & Calculated from "En Sims" tab and control algorithm near cut-out speed \\
\hline 1 & 0.99 & Control software settings, hardware performance, O\&M inefficiency \\
\hline 1 & 0.99 & based on presentations at AWEA 2012 wind resource assessment workshop \\
\hline & & \\
\hline 1 & 0.99 & Industry standard value \\
\hline 0.8 & 0.992 & Based on climate and observed anemometer icing \\
\hline 0 & & Assumes cold or hot weather packages if needed \\
\hline 0.2 & 0.998 & Includes lightning, severe weather, etc. \\
\hline 0.1 & 0.999 & Non-meteorological events that affect site access \\
\hline 0 & 1 & Not applicable \\
\hline 0.00 & 1.0000 & \\
\hline 0 & 1 & Assumed zero, pending turbine vendor's site suitability assessment \\
\hline 0 & 1 & Site-specific, need client or off-taker input \\
\hline 0 & 1 & Noise, shadow flicker, animal activity/migration, military operations, etc. \\
\hline 1.00 & 0.9900 & Plant downtime (substation, collection, etc., not turbine-related) \\
\hline
\end{tabular}




\title{
Exhibit E
}

\section{Monthly/ Diurnal 12 x 24 Energy Pattern Table}

\author{
(V-Bar)
}


Simulated Monthly/Diurnal Mean Net Capacity Factor (\%)

200-MW Array Using GE-1.7/100 Turbines on Tribal Lands

Hualapai Reservation, Arizona

Based on Data from Grand Canyon West Site 7054, October 2006-August 2012

\begin{tabular}{|c|c|c|c|c|c|c|c|c|c|c|c|c|}
\hline $\begin{array}{l}\text { Hour } \\
\text { (MST) }\end{array}$ & Jan & Feb & Mar & Apr & May & Jun & Jul & Aug & Sep & Oct & Nov & Dec \\
\hline 0 & 28.4 & 30.4 & 32.6 & 36.1 & 34.3 & 36.9 & 25.2 & 25.6 & 23.1 & 26.0 & 24.1 & 24.9 \\
\hline 1 & 26.5 & 26.9 & 33.4 & 35.8 & 33.0 & 35.2 & 24.8 & 25.2 & 22.7 & 24.9 & 24.3 & 25.3 \\
\hline 2 & 27.1 & 26.1 & 34.2 & 35.8 & 31.6 & 33.8 & 24.4 & 23.0 & 21.5 & 25.9 & 24.2 & 26.3 \\
\hline 3 & 26.6 & 25.9 & 31.9 & 32.9 & 28.3 & 30.9 & 20.9 & 21.5 & 19.9 & 26.2 & 24.3 & 25.4 \\
\hline 4 & 26.8 & 26.9 & 32.1 & 32.2 & 27.2 & 28.2 & 17.0 & 19.1 & 20.9 & 27.8 & 24.9 & 25.9 \\
\hline 5 & 27.2 & 27.4 & 33.3 & 31.6 & 26.0 & 25.6 & 15.7 & 18.1 & 21.7 & 28.2 & 24.7 & 26.2 \\
\hline 6 & 28.8 & 26.6 & 33.5 & 31.8 & 23.8 & 24.8 & 13.5 & 18.6 & 20.3 & 27.3 & 26.5 & 27.8 \\
\hline 7 & 30.0 & 26.0 & 33.6 & 28.6 & 20.5 & 22.9 & 11.4 & 15.1 & 20.4 & 25.1 & 27.4 & 28.4 \\
\hline 8 & 30.1 & 25.3 & 29.8 & 26.9 & 21.1 & 25.8 & 10.8 & 14.0 & 18.8 & 21.4 & 25.6 & 28.7 \\
\hline 9 & 24.8 & 22.5 & 29.0 & 27.5 & 23.7 & 28.5 & 12.7 & 16.1 & 20.4 & 20.9 & 24.9 & 27.6 \\
\hline 10 & 21.5 & 22.9 & 29.1 & 31.4 & 27.0 & 32.3 & 15.4 & 17.9 & 21.4 & 22.9 & 23.3 & 25.7 \\
\hline 11 & 20.8 & 24.2 & 29.6 & 34.0 & 30.0 & 32.3 & 17.8 & 21.4 & 21.5 & 23.5 & 25.1 & 26.1 \\
\hline 12 & 21.5 & 25.8 & 32.0 & 37.4 & 32.0 & 33.5 & 19.6 & 23.1 & 24.2 & 25.5 & 27.9 & 25.2 \\
\hline 13 & 21.2 & 27.8 & 33.7 & 36.2 & 32.3 & 33.8 & 22.2 & 22.7 & 24.8 & 28.3 & 28.5 & 25.5 \\
\hline 14 & 22.5 & 29.0 & 35.5 & 36.2 & 33.4 & 34.3 & 23.0 & 24.8 & 26.3 & 28.2 & 28.1 & 25.9 \\
\hline 15 & 23.6 & 28.7 & 36.9 & 37.0 & 33.0 & 33.9 & 24.9 & 25.5 & 24.8 & 28.0 & 29.1 & 26.7 \\
\hline 16 & 24.3 & 27.5 & 36.2 & 38.2 & 34.1 & 35.8 & 23.6 & 24.9 & 24.4 & 27.9 & 27.4 & 26.4 \\
\hline 17 & 24.2 & 25.7 & 33.2 & 36.9 & 35.5 & 39.0 & 24.7 & 25.6 & 24.0 & 26.7 & 25.4 & 25.5 \\
\hline 18 & 25.6 & 25.3 & 32.4 & 37.3 & 35.6 & 40.5 & 26.0 & 24.5 & 22.8 & 25.0 & 24.9 & 25.8 \\
\hline 19 & 26.3 & 27.3 & 31.1 & 35.7 & 34.1 & 39.0 & 25.5 & 23.0 & 20.7 & 25.3 & 24.8 & 25.0 \\
\hline 20 & 26.5 & 27.8 & 30.2 & 33.9 & 32.3 & 34.9 & 24.2 & 22.3 & 19.8 & 26.7 & 26.0 & 24.6 \\
\hline 21 & 26.7 & 28.9 & 31.2 & 34.2 & 32.3 & 34.6 & 21.8 & 24.4 & 20.3 & 26.5 & 25.1 & 26.1 \\
\hline 22 & 28.3 & 29.4 & 31.5 & 37.0 & 32.9 & 35.1 & 24.7 & 26.2 & 20.5 & 27.0 & 25.3 & 24.6 \\
\hline 23 & 29.0 & 31.2 & 31.4 & 36.6 & 33.5 & 36.8 & 24.4 & 26.3 & 21.7 & 27.1 & 25.5 & 25.2 \\
\hline Month & 25.8 & 26.9 & 32.4 & 34.2 & 30.3 & 32.8 & 20.6 & 22.0 & 22.0 & 25.9 & 25.7 & 26.0 \\
\hline
\end{tabular}




\title{
Exhibit G
}

Transmission and Interconnection Feasibility Report

\author{
(Power Engineers)
}


November 16, 2012

\section{Subject: 124957 Hualapai Renewable Energy Project Transmission And Interconnection Feasibility Report}

Power Engineers has performed a high level transmission and interconnection feasibility analysis of the local EHV transmission system, existing and planned, adjacent to the Hualapai Renewable Energy Project. The analysis is detailed below.

\section{Task 1: Transmission Analysis of Existing Transmission System}

This task was to evaluate existing EHV transmission options that may be available for interconnecting the proposed Hualapai Renewable Energy Project. The evaluation included potential points of interconnection (POI), distances from the project to the POIs and a brief discussion of each POI. Figure 1 below shows the Hualapai area and the three EHV transmission lines in the immediate vicinity of the POI. Three lines exist near the POI. The first two are parallel. One of the parallel lines is the Mead-Perkins $500 \mathrm{kV}$ line. The Perkins to Mead 500kV line has 4 owners: APS, WAPA, SRP and the City of Vernon. The second and currently the preferred POI, is the Mead-Peacock $345 \mathrm{kV}$ line. It has one owner, WAPA. Companies that are interconnected into WAPA at their Mead 230kV bus include SCE, NPC, SRP and the City of Vernon. Wheeling through WAPA can get you to the Marketplace and McCullough 500kV substations. SCE has a tie to the McCullough 500kV

Figure 1

Google Earth View of the EHV Transmission Systen in the Hualapai Area

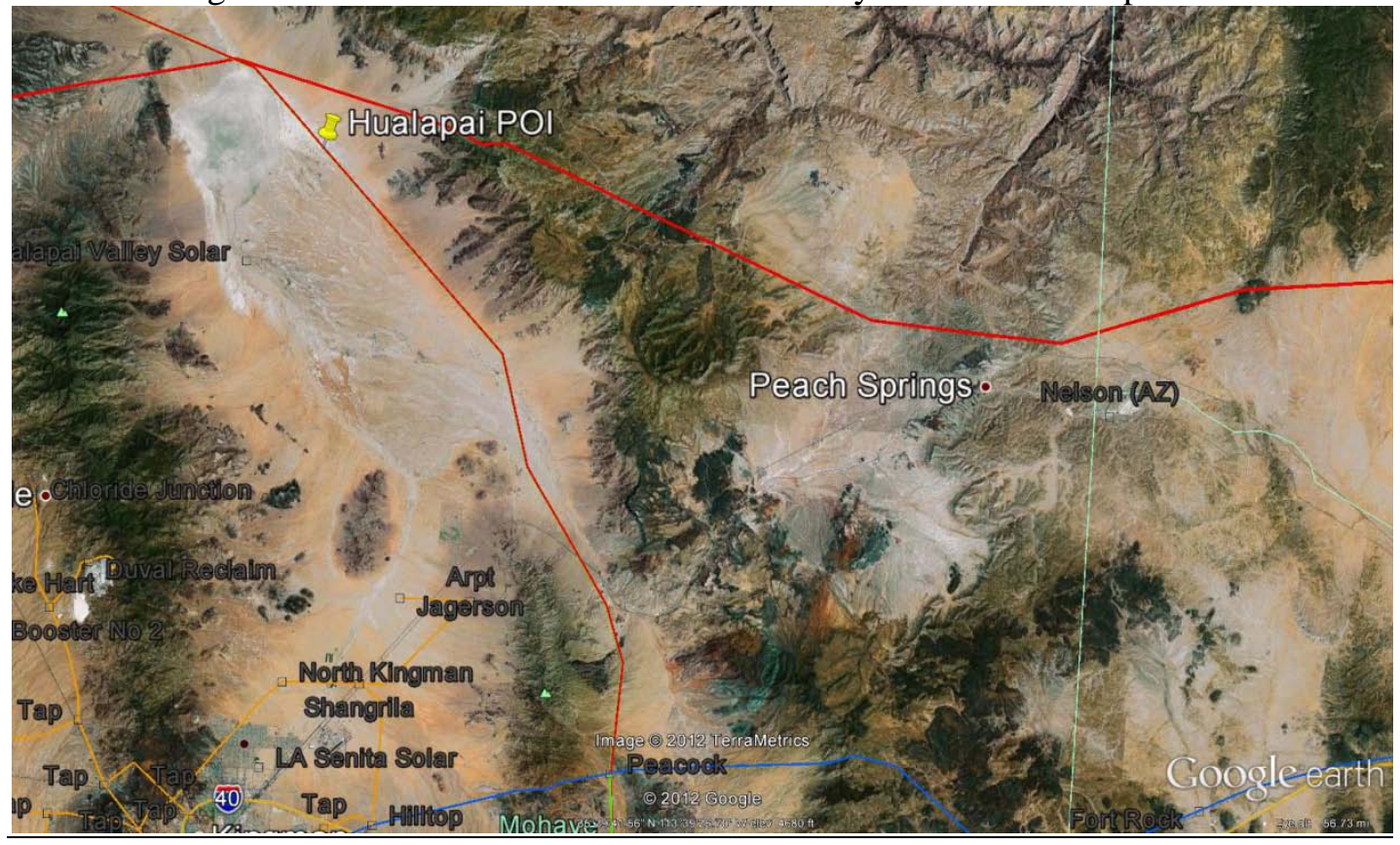

substation from the Eldorado $500 \mathrm{kV}$ substation. LADWP is a participant in the McCullough substation. By getting to the Eldorado substation, you can schedule to any of the CAISO participants. The two parallel lines primarily run north to south and both pass by Peacock at the bottom center of the figure. Both of the parallel lines are operated by 
Hualapai

November 16, 2012

Page 2

Western Area Power Administration (WAPA). The length of the gen-ties would range from approximately 8 miles to 18 miles long to the point of interconnection (POI). An Interconnection Request has been filed with WAPA LC in their Phoenix area office. The date for the application is $1 / 12 / 2012$ and the WAPA queue generation number position is 2012-G28. The Feasibility Study (FeS) is ongoing at this time.

The third line is the Moenkopi-Eldorado $500 \mathrm{kV}$ line which is owned and operated by Arizona Public Service (APS). This line travels across from east to west on the figure and passes by Peach Springs. This line does pass through the property and therefore the length of the gen-tie would be short to the POI. The scheduling rights, however, are currently owned by Southern California Edison Company. Those rights however, are set to revert back to APS by the end of this year if all regulatory approvals are obtained. APS has created a transmission service request queue for the future available transmission capability for when APS finally acquires the scheduling rights to the line. A recent check of this APS queue (which is available on the APS OASIS) shows that approximately 2000MW of reservations have been posted.

The substation interconnection cost for either of the $500 \mathrm{kV}$ 3-breaker ring substation is approximately \$20 million. This doesn't include the $500 \mathrm{kV}$ step-up transformer either. The approximate cost for a $345 \mathrm{kV}$ 3-breaker ring substation is approximately $\$ 15$ million. This also doesn't include the $345 \mathrm{kV}$ step-up transformer either.

\section{Task 2: Transmission Analysis of Proposed Transmission System}

The task is to specifically evaluate the proposed Centennial West Clean Line Project (Project) interconnection near the WAPA Peacock $345 \mathrm{kV}$ substation. Centennial West is a high voltage direct current (HVDC) terminal whereby wind energy produced in New Mexico and solar energy produced in Arizona will be transmitted via an overhead HVDC line and will interconnect to the Lugo substation in the SCE footprint. The purpose of the Arizona interconnection of the Clean Line Project was to allow 500MW of Arizona produced solar generation to be shipped to California on the Project. The purpose that we were told at the time we were preparing the CAISO interconnection request in 2011 of only solar was for the increased diversity combining solar and wind would provide. The site of the proposed Arizona substation was to be near the WAPA Peacock $345 \mathrm{kV}$ substation. In addition, the Project, for CAISO renewable generation consideration purposes, is considered a gen-tie which means that only generation can be tied to the Project. No other transmission interconnections to the Arizona transmission system would be allowed. As such, the Hualapai Project may not be able to use the existing Mead to Peacock $345 \mathrm{kV}$ line to wheel its power to Peacock to be considered as part of the 500MW transfer to California. This would need further clarification from Centennial West as to if this would be allowed or not. If not allowed to wheel to Peacock, then the Hualapai Project would have to build its own line, approximately 30 miles, to interconnect into the Centennial West collector substation. This line would not have to be $345 \mathrm{kV}$ construction. For $150 \mathrm{MW}$, it could be a double circuit $69 \mathrm{kV}$ line. If the Hualapai Project built its own line, the project would not have to pay WAPA wheeling. 
Hualapai

November 16, 2012

Page 3

\section{Additional Task: Summary of Proposed Preliminary Hualapai Wind and Solar Project Design}

An additional task has been included to summarize the proposed preliminay design of the wind and solar projects assuming an aggragate model for the generation. This includes the preliminay one-line, cost analysis of the required transformers, estimated losses at peak wind and solar output to the point of interconnection.

Figure 2 below is the preliminary one-line of the project to the point of interconnection into the Mead-Peacock $345 \mathrm{kV}$ line. There is a cluster of 100 turbines approximately 23 miles from the point of interconnection. There is an additional cluster of 18 turbines approximately 12 miles from the point of interconnection. The generation is transmitted to the point of interconnection using bundled double circuit $69 \mathrm{kV}$ transmission lines. The solar generation is approximately 8.4 miles from the point of interconnection and utilitizes a single circuit $69 \mathrm{kV}$ transmission line. The generation at each cluster, both wind and solar, is represented as a single aggregate generator collected utilizing a $34.5 \mathrm{kV}$ collector system. Figure 3 summarizes the individual component losses in MW. Total losses for the entire project are 9.6MW. Losses for the wind is approximately 7.7 MW and for the solar the losses are 1.9MW.

Because of the number of transformers required for this project, a high level cost estimate for the transformers was provided. The following values are installed costs.

$1-345 / 69 \mathrm{kV} 132 / 176 / 220 \mathrm{MVA}=\$ 4,000,000.00$ (located at the POI for the wind) $1-345 / 69 \mathrm{kV} 30 / 40 / 50 \mathrm{MVA}=\$ 2,500,000$ (located at the POI for the solar) $1-69 / 34.5 \mathrm{kV} 108 / 144 / 180 \mathrm{MVA}=\$ 2,100,000$ (located at the large wind turbine cluster) $1-69 / 34.5 \mathrm{kV} 20 / 26 / 33 \mathrm{MVA}=\$ 650,000$ (located at the small wind turbine cluster) $1-69 / 34.5 \mathrm{kV} 33 / 44 / 55 \mathrm{MVA}=\$ 900,000.00$ (located at the solar farm)

To meet the power factor requirements at the point of interconnection of \pm .95 power factor, several shunt capacitor banks were required. To minimize the cost, a 40MVAR 69kV shunt capacitor located at the POI and a $30 \mathrm{MVAR} 34.5 \mathrm{kV}$ shunt capacitor located at the $34.5 \mathrm{kV}$ side of the larger $69 / 34.5 \mathrm{kV}$ transformer minimized the reactive flow at the point of interconnection for the wind generation. A 15MVAR $69 \mathrm{kV}$ shunt capacitor bank is required for the solar plant to meet the power factor requirement and to minimize the reactive at the point of interconnection. 


\section{FIGURE 2}

\section{PRELIMINARY PROJECT ONE-LINE DIAGRAM}

\section{Hualapai Wind and Solar Project}
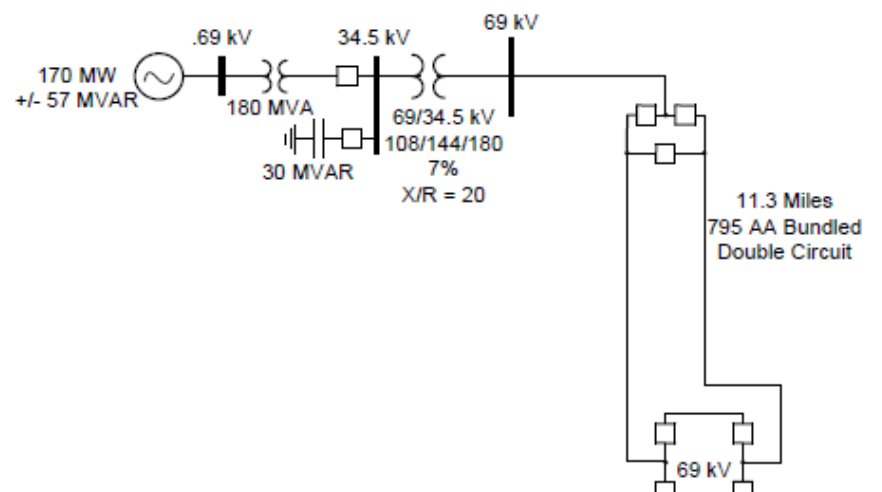

$69 / 34.5 \mathrm{kV}$

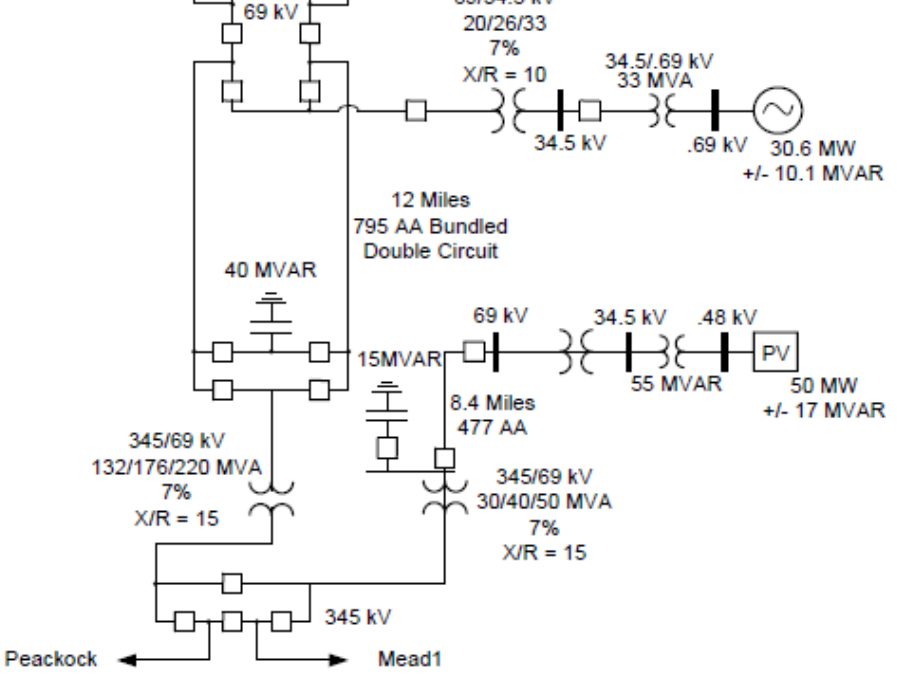




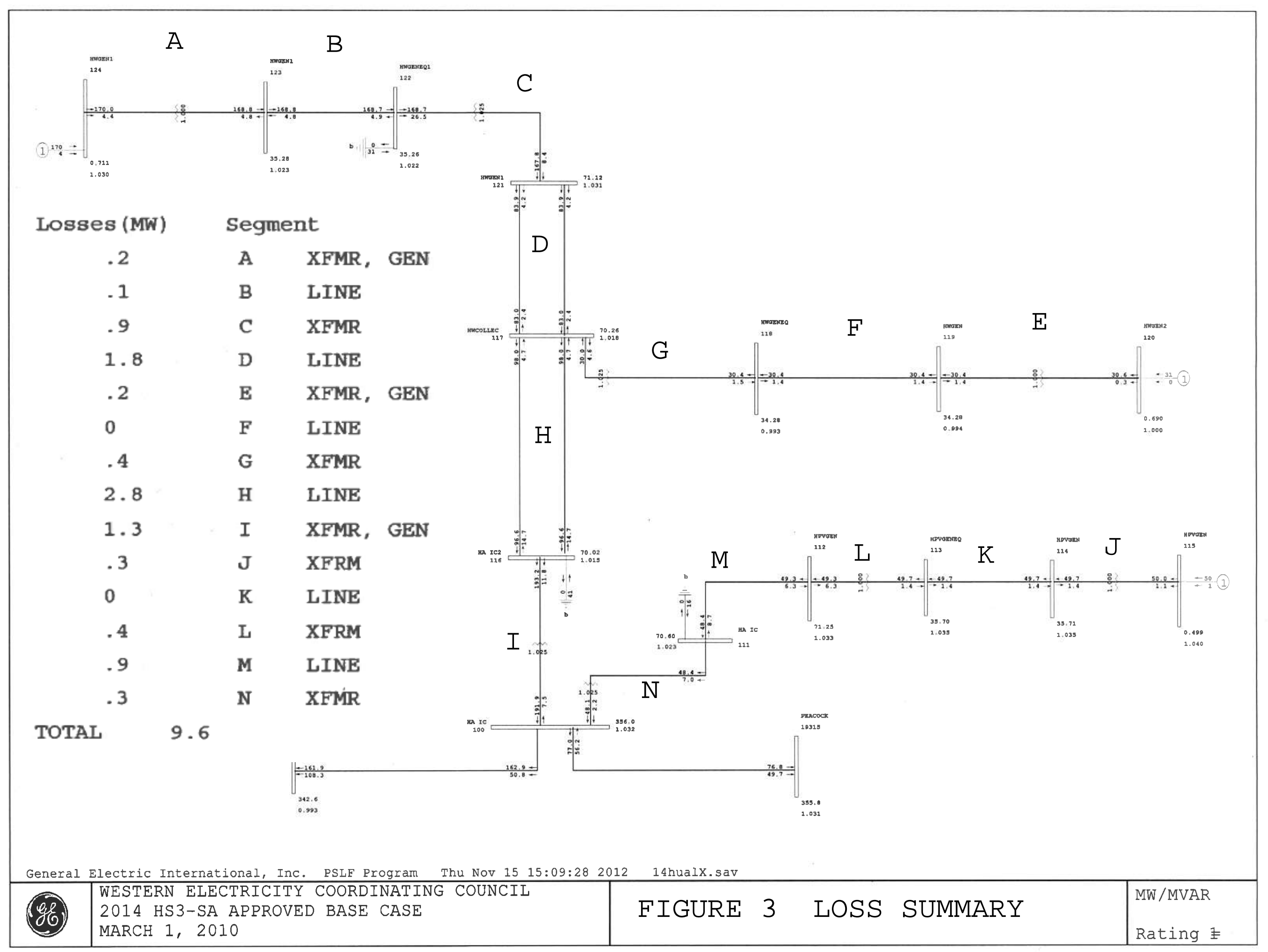




\title{
Exhibit $\mathrm{H}$
}

WAPA 345kV Interconnection Application

\author{
(Power Engineers)
}


The Great Spirit created Man and Woman in his own image. In doing so, both were created as equals. Both depending on each other in order to survive. Great respect was shown for each other; in doing so, happiness and contentment was achieved then, as it should be now.

The connecting of the Hair makes them one person; for happiness or contentment cannot be achieved without each other.

The Canyons are represented by the purples in the middle ground, where the people were created. These canyons are Sacred, and should be so treated at all times

The Reservation is pictured to represent the land that is ours, treat it well.

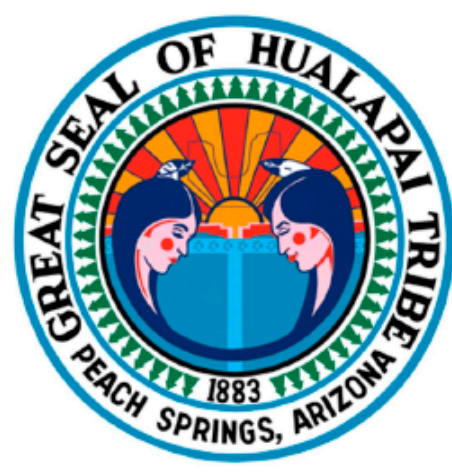

The Reservation is our heritage and the heritage of our children yet unborn. Be good to our land and it will continue to be good to us.

The Sun is the symbol of life, without it nothing is possible - plants don't grow - there will be no life - nothing. The Sun also represents the dawn of the Hualapai people. Through hard work, determination and education, everything is possible and we are assured bigger and brighter days ahead.

The Tracks in the middle represent the coyote and other animals which were here before us.

The Green around the symbol are pine trees, representing our name Hualapai - PEOPLE OF THE TALL PINES -

\section{Hualapai Tribal Nation Department of Planning \& Economic Development}

September 30, 2011

Mr. John Steward

Western Area Power Administration

615 S. $43^{\text {rd }}$ Avenue

Phoenix, AZ 85005

The Hualapai Tribe respectively submits this interconnection request for a $150 \mathrm{MW}$ net wind facility and a $100 \mathrm{MW}$ Solar facility in Mohave County, Arizona. Attached to this request please find,

(i) a check in the amount of $\$ 10,000$

(ii) a completed application in the form of WALC's LGIC IR, and

(iii) site control documentation

Thank you in advance for your consideration of this request for interconnection. If you have any questions regarding this request or the designations, please don't hesitate to call me at (928) 7691310 or to contact our transmission and interconnection consultant, Mr. Mark Etherton at PDS Consulting (480) 838-1427. We look forward to working with Western on the vital and exciting project for the future of the Hualapai Tribe.

Sincerely,

Jack Ehrhardt, Director

Hualapai Tribe Planning \& Economic Development

P.O. Box 179/887 W. Highway 66

Peach Springs, AZ. 86434

Enclosure 


\section{APPENDIX 1 to LGIP \\ INTERCONNECTION REQUEST FOR A \\ LARGE GENERATING FACILITY}

1. The undersigned Interconnection Customer submits this request to interconnect its Large Generating Facility with Transmission Provider's Transmission System pursuant to a Tariff.

2. This Interconnection Request is for (check one):

_ xx_ A proposed new Large Generating Facility.

An increase in the generating capacity or a Material Modification of an existing Generating Facility.

3. The type of interconnection service requested (check one):

__xx_ Energy Resource Interconnection Service

Network Resource Interconnection Service

4. _ C C C C _ neck here only if Interconnection Customer requesting Network Resource Interconnection Service also seeks to have its Generating Facility studied for Energy Resource Interconnection Service

5. Interconnection Customer provides the following information:

a. Address or location or the proposed new Large Generating Facility site (to the extent known) or, in the case of an existing Generating Facility, the name and specific location of the existing Generating Facility;

The location of the Hualapai Solar and Wind Development Project is on the Hualapai Indian Reservation located near Peach Springs in north-west Arizona.

b. Maximum summer at_37_degrees $\mathrm{C}$ and winter at _ 3 _ _ degrees $\mathrm{C}$ megawatt electrical output of the proposed new Large Generating Facility or the amount of megawatt increase in the generating capacity of an existing Generating Facility;

200.6 MW of wind generating facilities with 50MW of PV solar. (118 turbines at 1.7 MW nameplate each; 100 inverters at 0.5 MW AC nameplate each)

c. General description of the equipment configuration;

The wind side of the Hualapai Project is proposed to have $\mathbf{2}$ arrangements of $\mathbf{1 0 0}$ and $18 \mathrm{GE}$ wind turbines (1.7MW per turbine) each collected via a $34.5 \mathrm{kV}$ collector system. The solar side is proposed to be arranged with 1 arrangements of 1000.5 MW AE Solaron $500 \mathrm{~kW}$ inverters with a $34.5 \mathrm{kV}$ collector system also. These 3 collector systems feed into three $34.5 / 69 \mathrm{kV}$ transformers from which the power flows to the main Hualapai Project substation on $69 \mathrm{kV}$ transmission lines to the point of interconnection. The Hualapai Project substation would be arranged with 2 step-up transformers from $69 \mathrm{kV}$ to $345 \mathrm{kV}$, one for the wind and one for the solar generation. The project will be constructed in 2 phases. The first phase will be with 200.6 MW of wind located at the Clay Springs site. The second phase will be with $50 \mathrm{MW}$ of solar also near the Clay Springs site. 
d. Commercial Operation Date (Day, Month, and Year);

Phase 1 date is 6/1/2015; Phase 2 date is 6/1/2016 these may have to be moved back a year given the NEPA process.

e. Name, address, telephone number, and e-mail address of Interconnection Customer's contact person;

\begin{tabular}{|l|l|}
\hline Primary Contact & Alternate Contact \\
Mr. Kevin Davidson, Director & Mr. Mark Etherton, P.E. \\
Hualapai Department of Planning & Director, Electrical Engineering \\
P.O. Box 179 & 3231 S. Country Club Way, Suite 103 \\
887 W. Hwy 66 & Tempe, AZ 85282 \\
Peach Springs, AZ 86434 & Phone: 602-809-0707 \\
928-624-1310 ext.22 & Fax: 713-655-1711 \\
Email: kdavidson@hualapai-nsn.com & E-Mail: mark.etherton@powereng.com \\
& \\
\hline
\end{tabular}

f. Approximate location of the proposed Point of Interconnection (optional); and

The interconnection for the Hualapai Project is into the existing WALC PeacockMead 345kV line at the following coordinates: 35.659507 LAT, $-\mathbf{- 1 1 3 . 9 8 7 1 7 6}$ LONG.

g. Interconnection Customer Data (set forth in Attachment A)

(Attached to this Interconnection Request)

6. Applicable deposit amount as specified in the LGIP.

$\$ 10,000$ check is attached.

7. Evidence of Site Control as specified in the LGIP (check one)

_ $\mathrm{xx}_{\text {_ }} \quad$ Is attached to this Interconnection Request

- Will be provided at a later date in accordance with this LGIP

8. This Interconnection Request shall be submitted to the representative indicated below:

Mr. John Steward

615 S. $43^{\text {rd }}$ Avenue

Phoenix, AZ 85005

9. Representative of Interconnection Customer to contact:

Mr. Kevin Davidson

E-Mail: KDavidson@Hualapai-nsn.gov 
10. This Interconnection Request is submitted by:

Name of Interconnection Customer:

By (signature):

Name (type or print):

Title:

Date: 


\section{LARGE GENERATING FACILITY DATA}

UNIT RATINGS

$\mathrm{kVA}$

${ }^{\circ} \mathrm{F}$ Voltage

Power Factor

Speed (RPM)

Short Circuit Ratio

Stator Amperes at Rated kVA

Max Turbine MW
Connection (e.g. Wye)

Frequency, Hertz

Field Volts

\section{COMBINED TURBINE-GENERATOR-EXCITER INERTIA DATA}

Inertia Constant, $\mathrm{H}=$

Moment-of-Inertia, $\mathrm{WR}^{2}=$
$\mathrm{kW}$ sec/kVA

lb. ft. ${ }^{2}$

\section{REACTANCE DATA (PER UNIT-RATED KVA)}

DIRECT AXIS

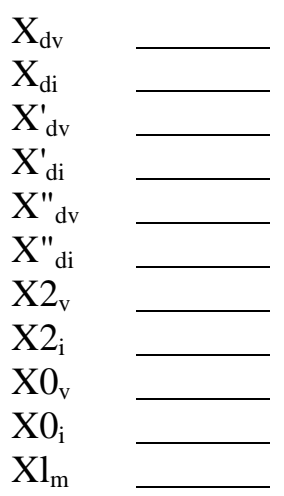

QUADRATURE AXIS

Synchronous - saturated Synchronous - unsaturated

Transient - saturated

Transient - unsaturated

Subtransient - saturated

Subtransient - unsaturated

Negative Sequence - saturated

Negative Sequence - unsaturated

Zero Sequence - saturated

Zero Sequence - unsaturated

Leakage Reactance

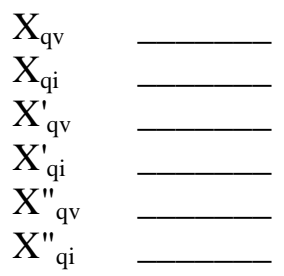


FIELD TIME CONSTANT DATA (SEC)

Open Circuit

Three-Phase Short Circuit Transient

Line to Line Short Circuit Transient

Line to Neutral Short Circuit Transient

Short Circuit Subtransient

Open Circuit Subtransient
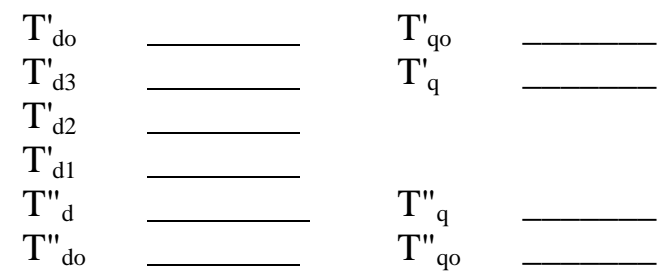

ARMATURE TIME CONSTANT DATA (SEC)

Three Phase Short Circuit

Line to Line Short Circuit

$\mathrm{T}_{\mathrm{a} 3}$

Line to Neutral Short Circuit

$\mathrm{T}_{\mathrm{a} 2}$

$\mathrm{T}_{\mathrm{a} 1}$

NOTE: If requested information is not applicable, indicate by marking "N/A."

\section{MW CAPABILITY AND PLANT CONFIGURATION \\ LARGE GENERATING FACILITY DATA}

\section{ARMATURE WINDING RESISTANCE DATA (PER UNIT)}

Positive

Negative

Zero

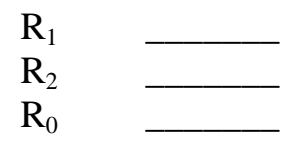

Rotor Short Time Thermal Capacity $\mathrm{I}_{2}{ }^{2} \mathrm{t}=$

Field Current at Rated kVA, Armature Voltage and PF $=$

Field Current at Rated kVA and Armature Voltage, 0 PF = amps

Three Phase Armature Winding Capacitance =

Field Winding Resistance $=$ ohms microfarad amps

Armature Winding Resistance (Per Phase) = ${ }^{\circ} \mathrm{C}$ ohms ${ }^{\circ} \mathrm{C}$ 


\section{CURVES}

Provide Saturation, Vee, Reactive Capability, Capacity Temperature Correction curves.

Designate normal and emergency Hydrogen Pressure operating range for multiple curves.

\section{WIND GENERATOR STEP-UP TRANSFORMER DATA RATINGS}

Capacity

Self-cooled/Maximum Nameplate

$1800 / 1800$ kVA

Voltage Ratio(Generator Side/System side/Tertiary)

$\underline{0.69 / 34.5 / N / A ~} \mathrm{kV}$

Winding Connections (Low V/High V/Tertiary V (Delta or Wye))

Wye (Ungrounded) / Delta / N/A

Fixed Taps Available $\pm 5 \%$ ( \pm 2 taps from nominal)

Present Tap Setting _ Neutral

IMPEDANCE

Positive

$\mathrm{Z}_{1}$ (on self-cooled $\mathrm{kVA}$ rating) $\quad \mathbf{7}^{\prime} \% \quad \mathbf{1 0} \mathrm{X} / \mathrm{R}$

Zero

$\mathrm{Z}_{0}$ (on self-cooled $\mathrm{kVA}$ rating) $\quad \mathbf{7}^{\prime} \% \quad \mathbf{1 0} \mathrm{X} / \mathrm{R}$

SOLAR GENERATOR STEP-UP TRANSFORMER DATA RATINGS

Capacity

Self-cooled/Maximum Nameplate

$1000 / 1000 \mathrm{kVA}$

Voltage Ratio(Generator Side/System side/Tertiary)

$\underline{0.48 / 34.5 / N / A ~} \mathrm{kV}$

Winding Connections (Low V/High V/Tertiary V (Delta or Wye))

Wye (Ungrounded) / Delta / N/A

Fixed Taps Available $\pm \underline{ \pm 5 \%( \pm 2 \text { taps from nominal) }}$

Present Tap Setting _ Neutral

\section{IMPEDANCE}

Positive $\quad Z_{1}$ (on self-cooled kVA rating) $\quad \mathbf{6}^{\%}{ }^{\circ} \mathbf{1 0} X / \mathrm{R}$

Zero $\quad Z_{0}$ (on self-cooled kVA rating) $\quad \mathbf{6}^{\%} \% \quad \mathbf{1 0} \mathrm{X} / \mathrm{R}$ 


\section{EXCITATION SYSTEM DATA}

Identify appropriate IEEE model block diagram of excitation system and power system stabilizer (PSS) for computer representation in power system stability simulations and the corresponding excitation system and PSS constants for use in the model.

\section{GOVERNOR SYSTEM DATA}

Identify appropriate IEEE model block diagram of governor system for computer representation in power system stability simulations and the corresponding governor system constants for use in the model.

\section{WIND GENERATORS}

Number of generators to be interconnected pursuant to this Interconnection Request:

118 turbines $(1.7 \mathrm{MW}$ per turbine $)=200.6 \mathrm{MW}$

Elevation: Approx. 3500 -4000 feet __ Single Phase _ XX Three Phase

Inverter manufacturer, model name, number, and version:

GE 1.7-100 Wind Turbine

List of adjustable set points for the protective equipment or software:

Note: A completed General Electric Company Power Systems Load Flow (PSLF) data sheet or other compatible formats, such as IEEE and PTI power flow models, must be supplied with the Interconnection Request. If other data sheets are more appropriate to the proposed device, then they shall be provided and discussed at Scoping Meeting. See attached.

\section{SOLAR INVERTERS}

Number of generators to be interconnected pursuant to this Interconnection Request:

100 inverters $(0.5 \mathrm{MW}$ per inverter $)=50 \mathrm{MW}$

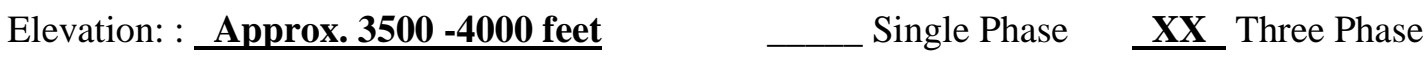

Inverter manufacturer, model name, number, and version:

AE Solaron 500kW inverters

List of adjustable set points for the protective equipment or software:

Note: A completed General Electric Company Power Systems Load Flow (PSLF) data sheet or other compatible formats, such as IEEE and PTI power flow models, must be supplied with the 
Interconnection Request. If other data sheets are more appropriate to the proposed device, then they shall be provided and discussed at Scoping Meeting. See attached.

\section{INDUCTION GENERATORS}

(*) Field Volts:

(*) Field Amperes:

(*) Motoring Power $(\mathrm{kW})$ :

(*) Neutral Grounding Resistor (If Applicable):

$\left(^{*}\right) \mathrm{I}_{2}{ }^{2} \mathrm{t}$ or K (Heating Time Constant):

(*) Rotor Resistance:

(*) Stator Resistance:

(*) Stator Reactance:

(*) Rotor Reactance:

(*) Magnetizing Reactance:

(*) Short Circuit Reactance:

(*) Exciting Current:

(*) Temperature Rise:

$\left(^{*}\right)$ Frame Size:

$(*)$ Design Letter:

(*) Reactive Power Required In Vars (No Load):

(*) Reactive Power Required In Vars (Full Load):

(*) Total Rotating Inertia, H: Per Unit on KVA Base

Note: Please consult Transmission Provider prior to submitting the Interconnection Request to determine if the information designated by $(*)$ is required. 


\title{
Exhibit I
}

\author{
Project Area Map
}

(Hualapai Planning Department) 


\section{Draft Turbine Array Plan Depicting 118, GE 1.7 MW turbines for 200 MW}

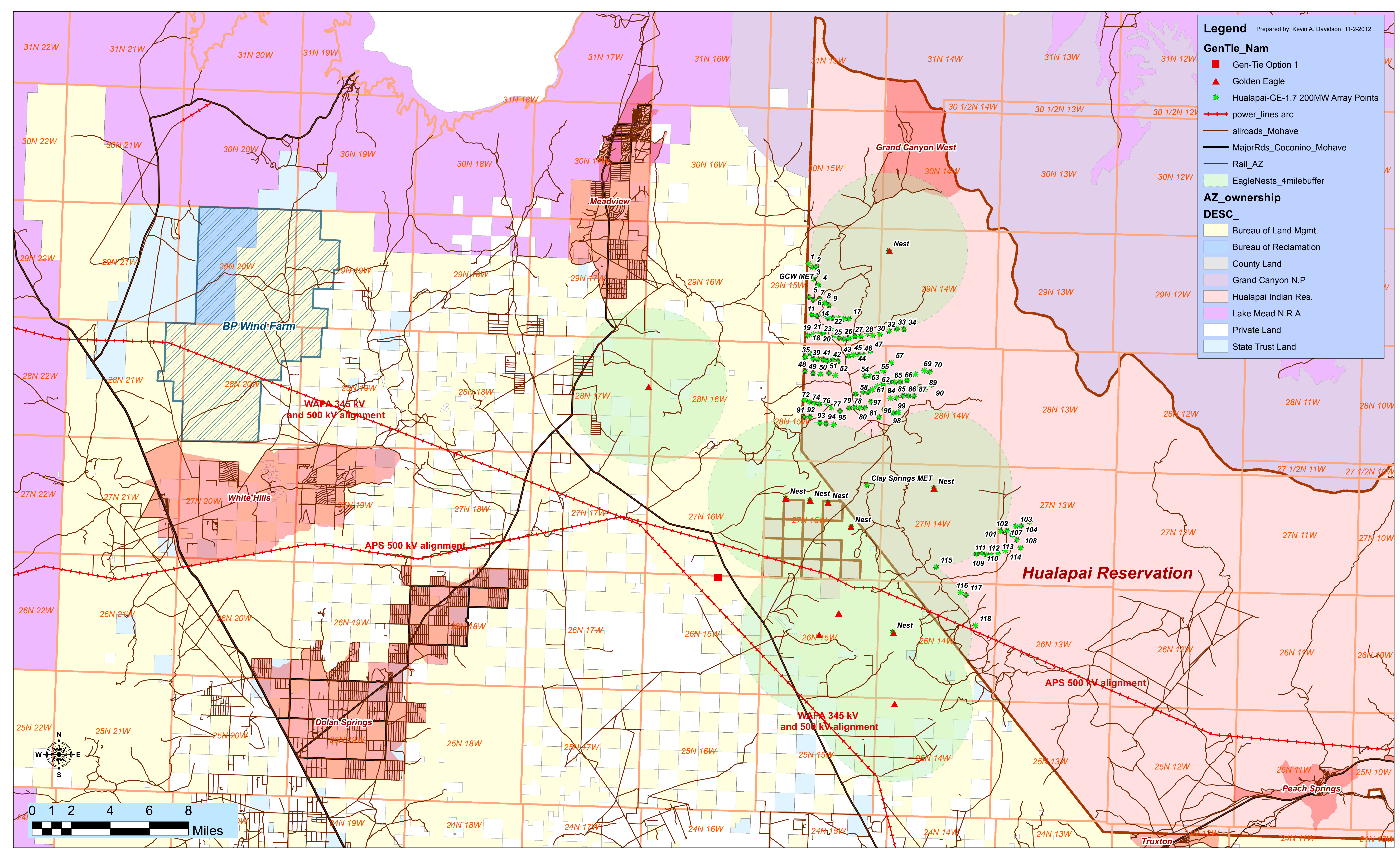




\section{Exhibit J}

Critical Issues Analysis

(SWCA Environmental) 


\section{Critical Issues Analysis of Renewable Energy Development Project Areas}

Prepared for

Hualapai Tribal Nation Planning Department

Prepared by

SWCA Environmental Consultants

July 2012 



\title{
CRITICAL ISSUES ANALYSIS OF RENEWABLE ENERGY DEVELOPMENT PROJECT AREAS
}

\author{
Prepared for \\ Hualapai Tribal Nation Planning Department
}

Prepared by

SWCA Environmental Consultants

7373 Peak Drive, Suite 170

Las Vegas, Nevada 89128

(702) 248-3880

www.swca.com

SWCA Project No. 23049

July 2012 



\section{CONTENTS}

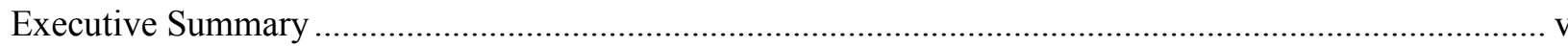

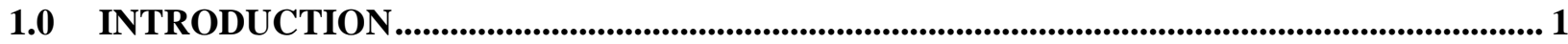

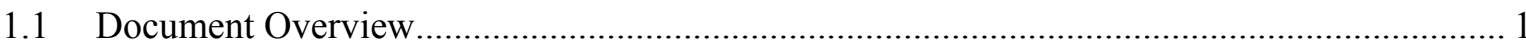

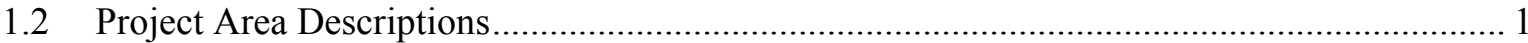

1.2.1 Grand Canyon West Wind Energy Project Area ...................................................... 1

1.2.2 Clay Springs Wind Energy Project Area.................................................................. 1

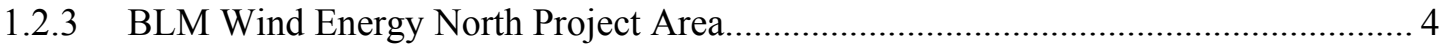

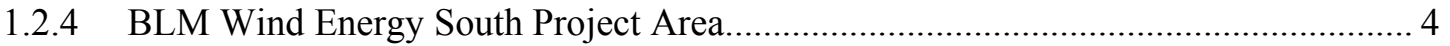

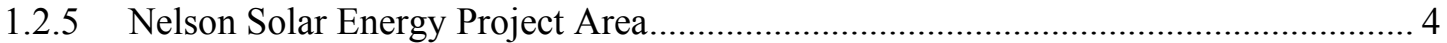

1.2.6 Hualapai Solar Energy Project Area ….................................................................... 4

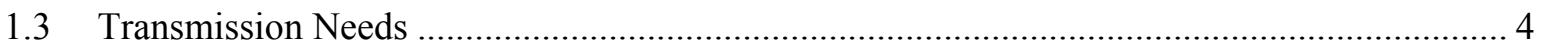

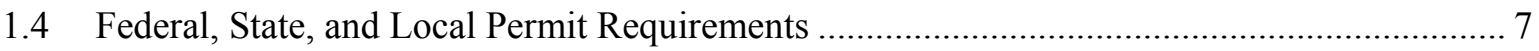

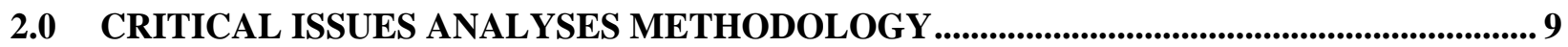

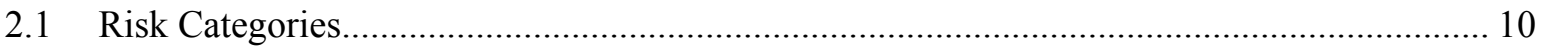

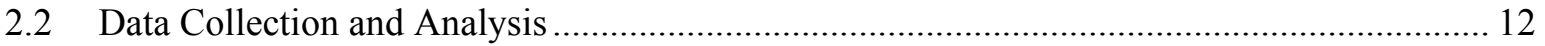

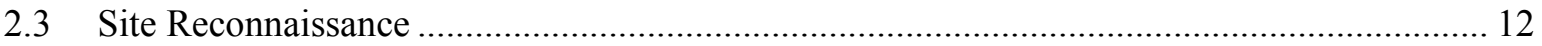

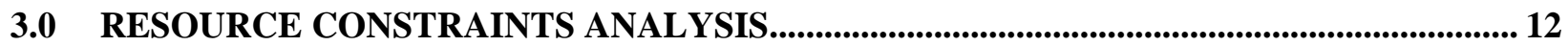

3.1 Land Use (including BLM, Private, Towns, Areas of Critical Environmental Concern,

National Preserves, Wilderness, Other Federal and State Lands) ........................................ 12

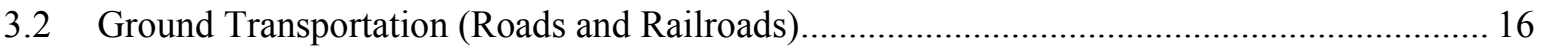

3.3 Airports and Aviation (Private, Commercial, Federal Aviation Administration, and

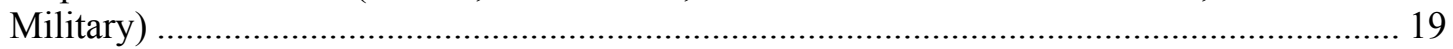

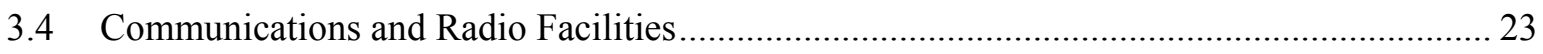

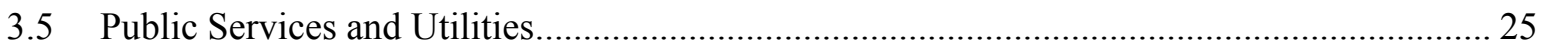

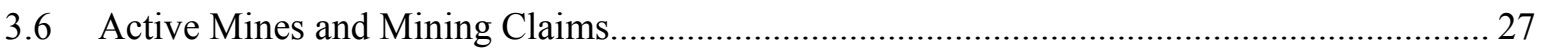

3.7 Visual Resources and Visual Resource Management Classifications................................... 29

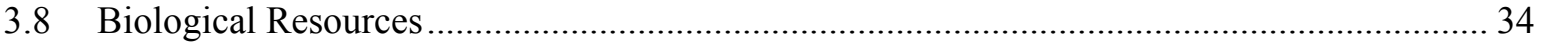

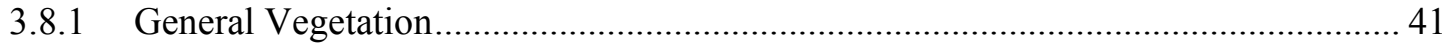

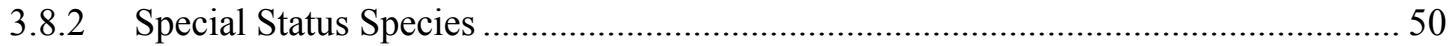

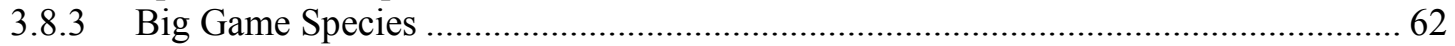

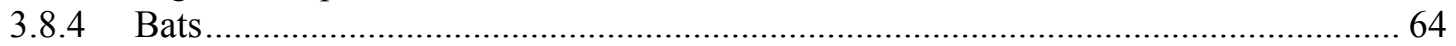

3.8.5 Birds, including Bald and Golden Eagles, Raptor Migration Flyways, and

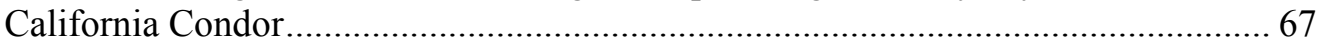

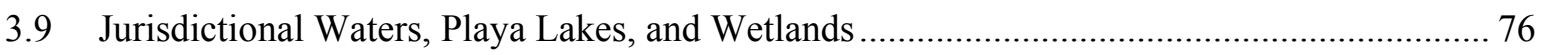

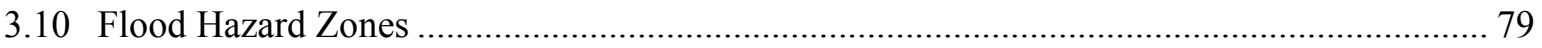

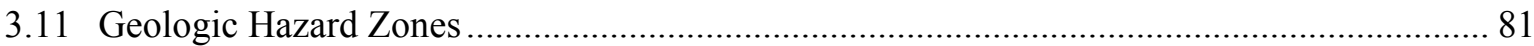

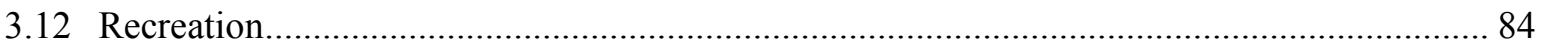

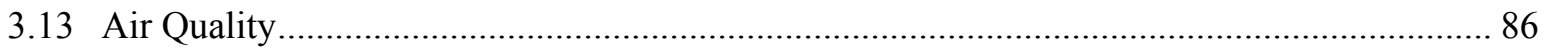

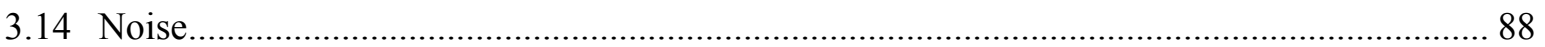

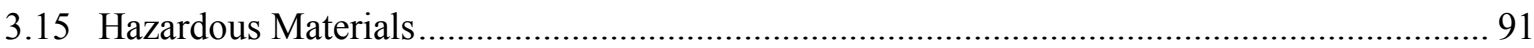

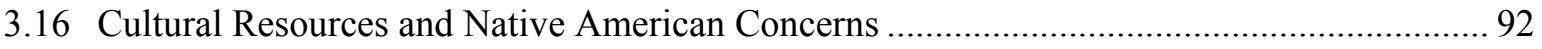

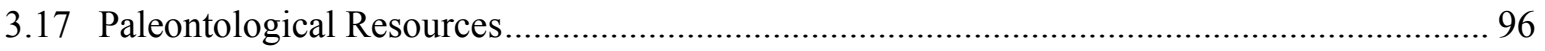




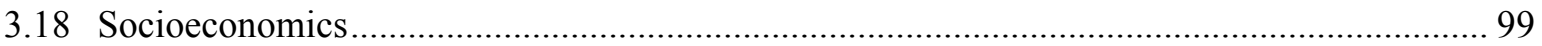

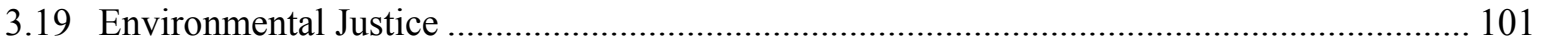

3.19.1 Environmental Justice Communities In or Near the Study Area.............................. 102

3.19.2 Disproportionately High and Adverse Effects ....................................................... 103

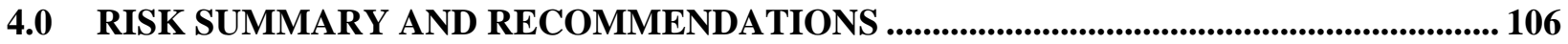

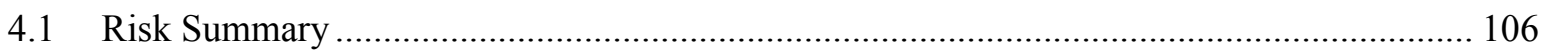

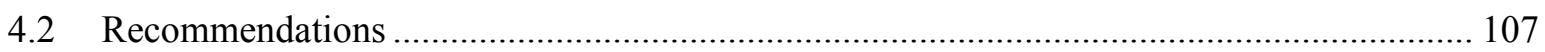

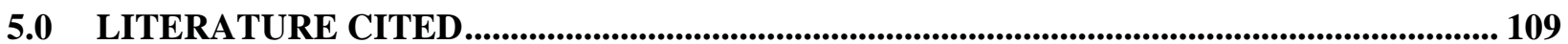

\section{Figures}

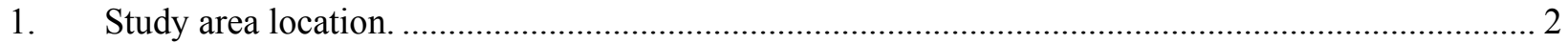

2. Grand Canyon West Wind Energy project area. …................................................................ 3

3. Clay Springs Wind Energy project area. ................................................................................ 3

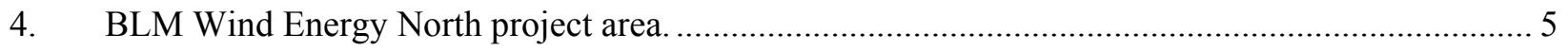

5. BLM Wind Energy South project area, view from Antares Road............................................... 5

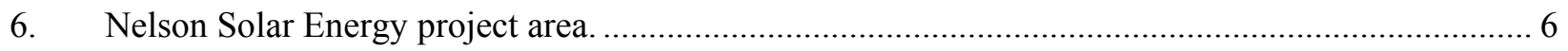

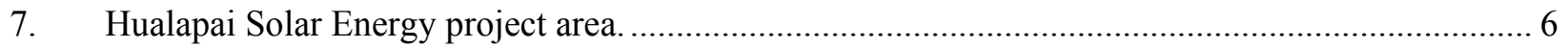

8. Four Corners-Moenkopi-Eldorado 500-kV line crossing the southern tip of the Clay Springs

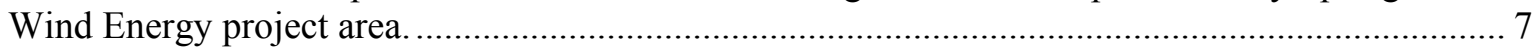

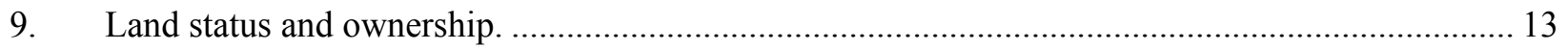

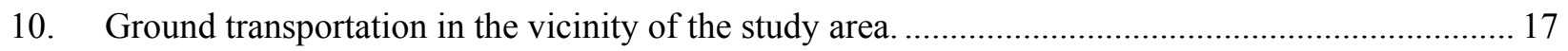

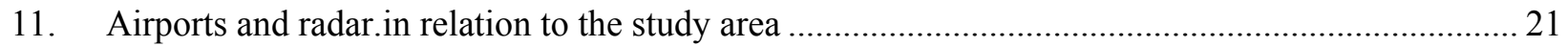

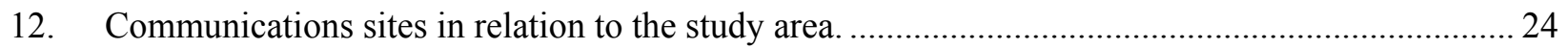

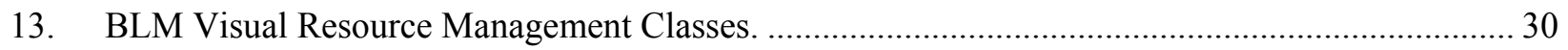

14. Grand Canyon West Wind Energy project area viewshed delineation.......................................... 31

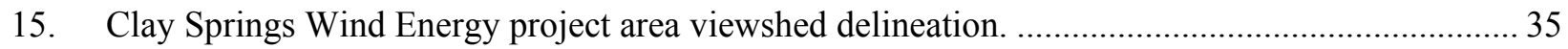

16. BLM Wind Energy North project area viewshed delineation. .................................................... 37

17. BLM Wind Energy South project area viewshed delineation. ..................................................... 39

18. Vegetation communities within the Grand Canyon West Wind Energy and BLM Wind

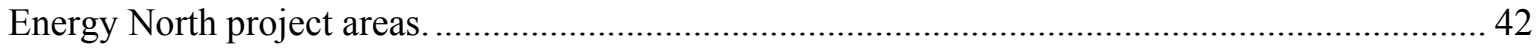

19. Vegetation communities within the Clay Springs Wind Energy, BLM Wind Energy South, and Hualapai Solar Energy project areas................................................................................. 43

20. Vegetation communities within the Nelson Solar Energy project area.......................................... 44

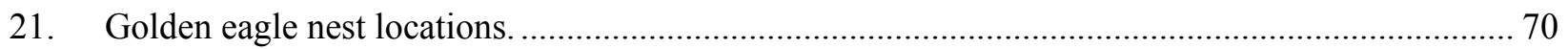

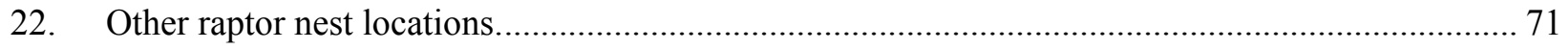

23. Draft 2009 California condor movement/location data obtained via satellite telemetry studies...... 73

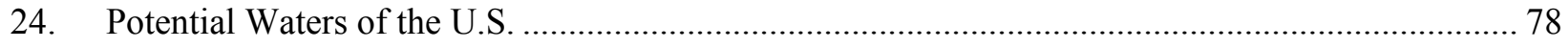

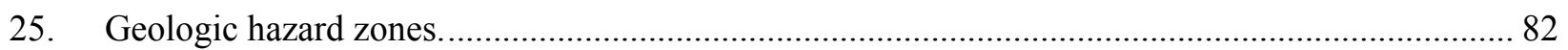




\section{Tables}

1. Permits, Certifications, and Authorizations Possibly Required for the Six Project Areas ................ 7

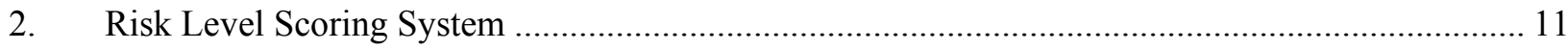

3. Vegetation Communities in the Grand Canyon West Wind Energy Project Area ........................ 47

4. Vegetation Communities in the Clay Springs Wind Energy Project Area ..................................... 48

5. Vegetation Communities in the BLM Wind Energy North Project Area .......................................... 49

6. Vegetation Communities in the BLM Wind Energy South Project Area ........................................ 49

7. Vegetation Communities in the Nelson Solar Energy Project Area ................................................ 50

8. Vegetation Communities in the Hualapai Solar Energy Project Area........................................... 50

9. Special Status Species Known to Occur in Mohave and Coconino Counties ................................. 51

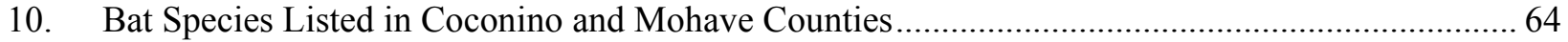

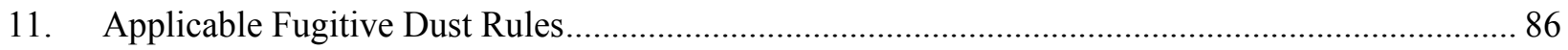

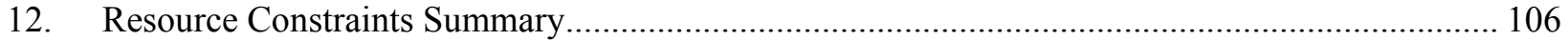


This page intentionally left blank. 


\section{EXECUTIVE SUMMARY}

The Hualapai Tribe has identified six project areas on a combination of Hualapai Tribal lands, Bureau of Land Management (BLM), State, and private lands in Mohave and Coconino Counties, Arizona, as having potential for renewable energy development (four are being considered for wind energy development, and two are being considered for solar energy development). To assess the environmental constraints associated with these projects areas, information was collected from a variety of sources, including a site reconnaissance visit and aerial raptor nest survey conducted by SWCA Environmental Consultants, published literature, reports, maps, aerial photography, databases, public records, and available geographic information system (GIS) datasets. Using this information, a risk level was assigned based on the potential for each issue to negatively affect the implementation, cost, schedule, or permitting for the renewable energy developments. Because the risk levels are based entirely on the evaluation of existing data, there is a potential that detailed site-specific studies would reveal additional risks, or elevate or diminish currently identified risk levels.

The following Critical Issues Analysis (CIA) provides a broad, yet comprehensive overview of key resources identified during preliminary project planning, including recommendations and additional work required to complete the environmental evaluation. The initial findings contained in the CIA did not indicate any fatal flaws present that could affect the viability of renewable energy development. Although each of the six sites has varying levels of risks, including high risk issues, no issues were identified that affect the overall viability of renewable energy development for those sites.

Visual resources are considered a high risk for the Grand Canyon West and BLM Wind North wind project areas where the BLM manages lands as Visual Resource Management (VRM) Class II, the objective of which is to retain the existing character of the landscape. The level of change to the characteristic landscape for VRM Class II should be low. The effect of wind energy facilities on the landscape is a known issue of concern and VRM Class II is not considered compatible with wind energy development. Although not considered a fatal flaw, a Resource Management Plan amendment would be required to change the VRM class before completing the permitting process, which can lengthen the permitting process and add additional complexities.

Cultural resources are also considered a medium risk for all six proposed project areas. Although the presence of cultural resources is not expected to affect the permitting and implementation of renewable energy facilities, inventories, reports, consultation, and agency review time can add considerable cost and time to the process.

Finally, bird and bat mortality is an issue known to be high profile in relation to wind energy facility siting in the four wind project areas. Additional site-specific data are necessary to demonstrate that wind energy facilities located within these sites would not significantly interfere with behavior, flight patterns, or migration movements. 
This page intentionally left blank. 


\section{$1.0 \quad$ INTRODUCTION}

\subsection{Document Overview}

The objective of this document is to identify the potential for any significant risks, fatal flaws, or "red flags" to project viability resulting from conflicts with environmental, physical, or social conditions present within six project areas (study area) identified by the Hualapai Planning Department for renewable energy project development (four are being considered for wind energy development, and two are being considered for solar energy development). This document also serves to present a comprehensive characterization of the six project areas and provide recommendations to assist the Hualapai Planning Department in ranking the project location(s) according to any identified conflicts, with areas identified as having fewer risks being ranked higher for potential project development. To accomplish this, SWCA Environmental Consultants (SWCA) has performed a desktop review of reasonably available information for the project areas, including published literature, reports, maps, aerial photography, databases, public records, and available geographic information system (GIS) datasets, and has conducted a field reconnaissance visit, to complete this analysis. An aerial raptor nest survey was also completed for all six sites, because of the potential avian risks associated with energy development.

\subsection{Project Area Descriptions}

The study area (four wind project areas and two solar project areas) are located in Mohave and Coconino Counties, Arizona, approximately 30 miles east of Kingman, Arizona (Figure 1). Based on their proximity to energy markets, existing transmission infrastructure, and wind and solar resources, the study area has been identified by the Hualapai Tribe as having potential for development and distribution of renewable energy.

\subsubsection{Grand Canyon West Wind Energy Project Area}

The Grand Canyon West Wind Energy project area is located entirely on Hualapai Tribal lands in Mohave County, Arizona, with the northern project area boundary located approximately 8.5 miles south of the Colorado River within Grand Canyon National Park. The vegetation within the project area is characterized primarily by low desert shrubs and pinyon-juniper woodland. The Grand Wash Cliffs and associated headwalls and cliff faces border the western side of the project area; the project area encompasses rolling hills and canyons of varying size. Figure 2 provides an overview photograph of the eastern portion of the project area.

\subsubsection{Clay Springs Wind Energy Project Area}

The Clay Springs Wind Energy project area is located south of the Grand Canyon West Wind Energy site and is located entirely on Hualapai Tribal lands in Mojave County, Arizona. The vegetation within the project area is characterized primarily by low desert shrubs and pinyon-juniper woodland. The Grand Wash Cliffs and associated headwalls and cliff faces border the western side of the project area; the project area encompasses rolling terrain and canyons of varying size. Figure 3 provides an overview photograph of the project area. 


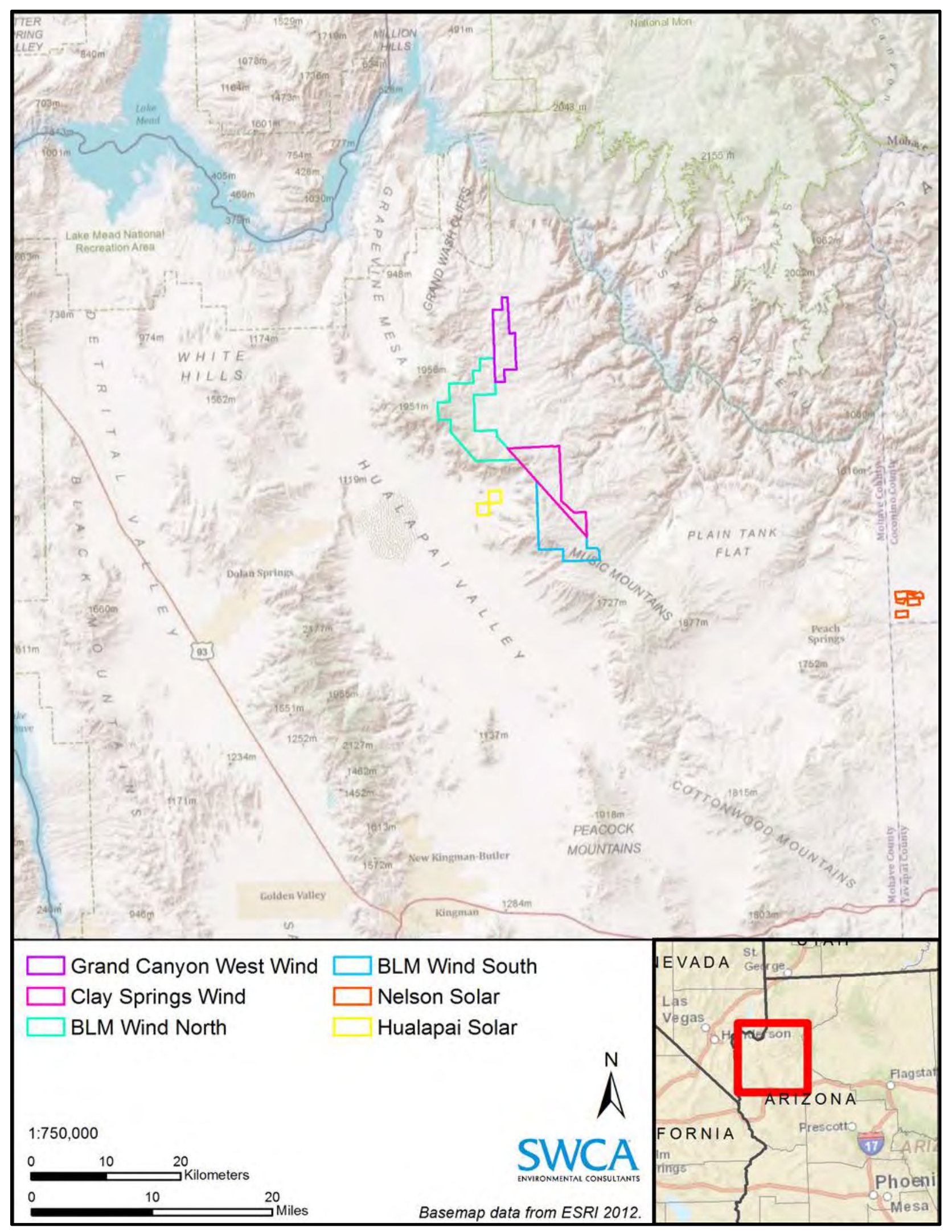

Figure 1. Study area location. 


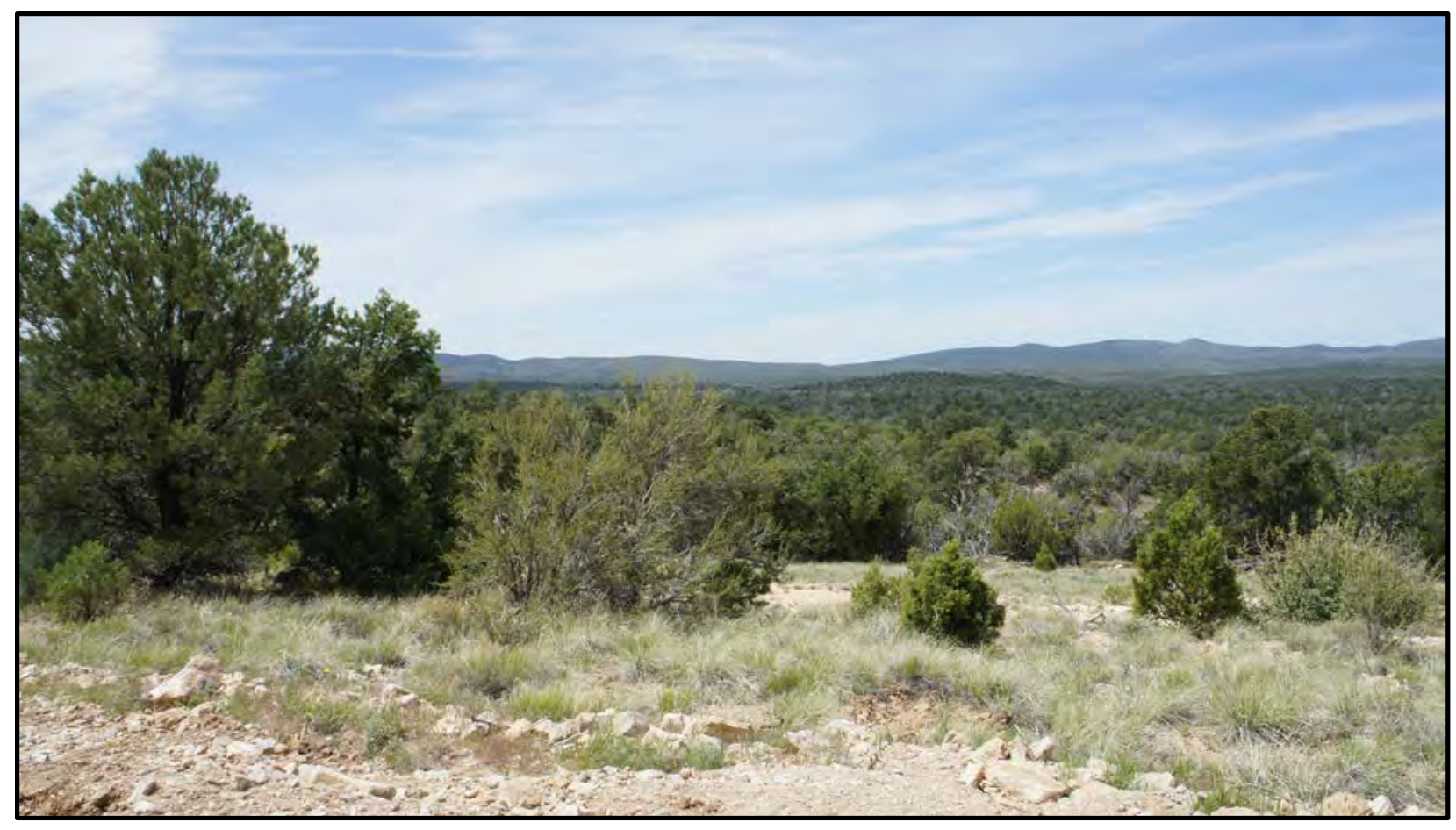

Figure 2. Grand Canyon West Wind Energy project area.

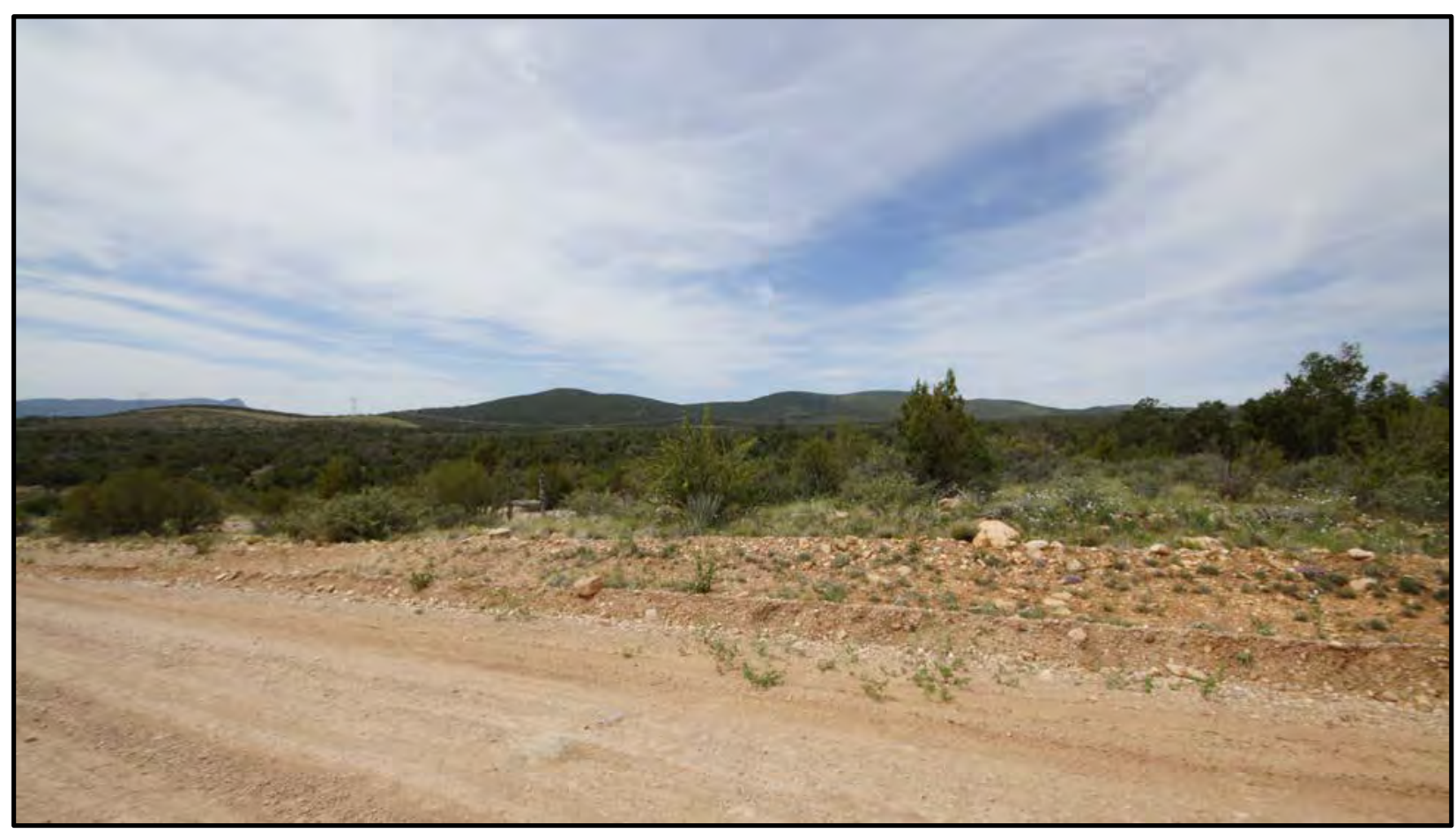

Figure 3. Clay Springs Wind Energy project area. 


\subsubsection{BLM Wind Energy North Project Area}

The Bureau of Land Management (BLM) Wind Energy North project area is located southwest and adjacent to the southwestern portion of the Grand Canyon West Wind Energy project area in Mojave County, Arizona. The project area is located mostly on BLM lands, with portions of four sections located on private lands. The vegetation within the project area is characterized primarily by low desert shrubs and pinyon-juniper woodland. The Grand Wash Cliffs and associated headwalls and cliff faces border the western side of the project area; the project area encompasses rolling terrain and canyons of varying size. Figure 4 provides an overview photograph of the project area.

\subsubsection{BLM Wind Energy South Project Area}

The BLM Wind Energy South project area is located south and adjacent to the southwestern portion of the Clay Springs Wind Energy project area and is located primarily on BLM lands in Mohave County, Arizona. The project area includes a parcel of State land along the southern edge. The project area straddles the Four Corners-Moenkopi-Eldorado transmission line and BLM Section 368 Energy Corridor. The vegetation within the project area is characterized primarily by low desert shrubs and pinyon-juniper woodland. The Grand Wash Cliffs and associated cliff faces border the western side of the project area; the project area encompasses rolling terrain and canyons of varying size. Figure 5 provides an overview photograph of the project area.

\subsubsection{Nelson Solar Energy Project Area}

The Nelson Solar Energy project area is located entirely on Hualapai Tribal lands in the southwestern corner of Coconino County, Arizona. The Nelson Solar Energy project area is made up of six separate parcels on five sections of land: five north of Route 66 and one south of Route 66. Terrain within the Nelson Solar Energy site parcels is primarily gently rolling hills and grassland, covered in low-growing shrubs, scattered pinyon-juniper woodland, and grasses typical of semi-desert grasslands common throughout the Arizona Mojave Desert. Figure 6 provides an overview photograph of the project area.

\subsubsection{Hualapai Solar Energy Project Area}

The Hualapai Solar Energy project area is on Hualapai Tribal lands in Mojave County, Arizona, south of the BLM Wind Energy North project area and west of the BLM Wind Energy South project area. The project area is located on two sections of Hualapai Tribal land that straddle the Four CornersMoenkopi-Eldorado transmission line and BLM Section 368 Energy Corridor, north and east of Route 66 and County Road 149. Terrain within the project area is gently rolling, covered in low-growing shrubs and grasses typical of semi-desert grasslands common throughout the Arizona Mojave Desert. Figure 7 provides an overview photograph of the project area.

\section{$1.3 \quad$ Transmission Needs}

The nearest transmission lines to all six of the project areas include the Four Corners-Moenkopi-Eldorado 500-kilovolt $(\mathrm{kV})$ line which crosses the Hualapai Solar Energy, BLM Wind Energy South, and southern tip of the Clay Springs Wind Energy project areas, and the Mead Phoenix Project 500-kV line that lies southwest of the Four Corners-Moenkopi-Eldorado 500-kV line (Figure 8). It is assumed that new transmission lines would be required for the remaining five project areas and that portions of those new transmission lines would cross BLM-managed lands. 


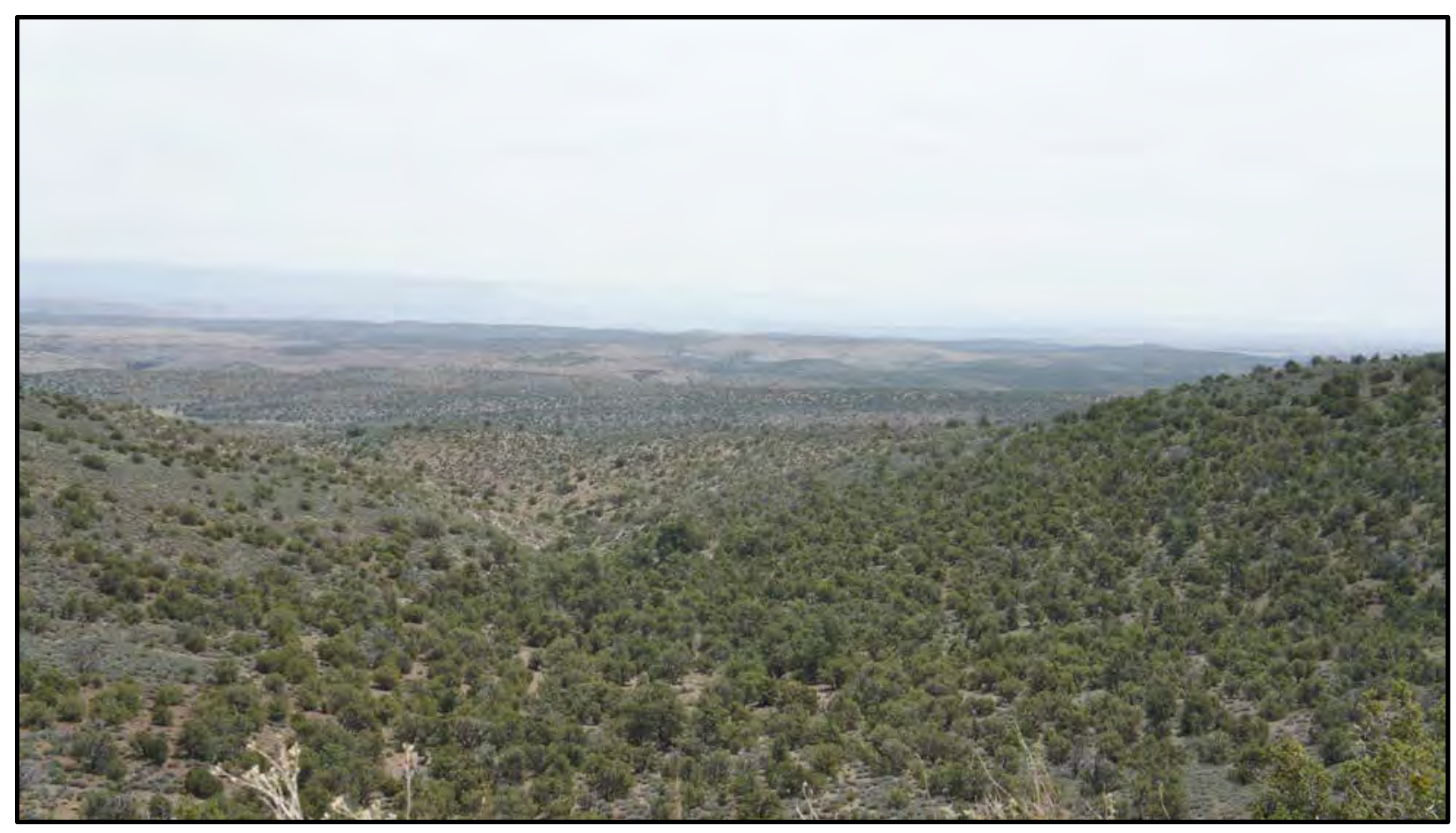

Figure 4. BLM Wind Energy North project area.

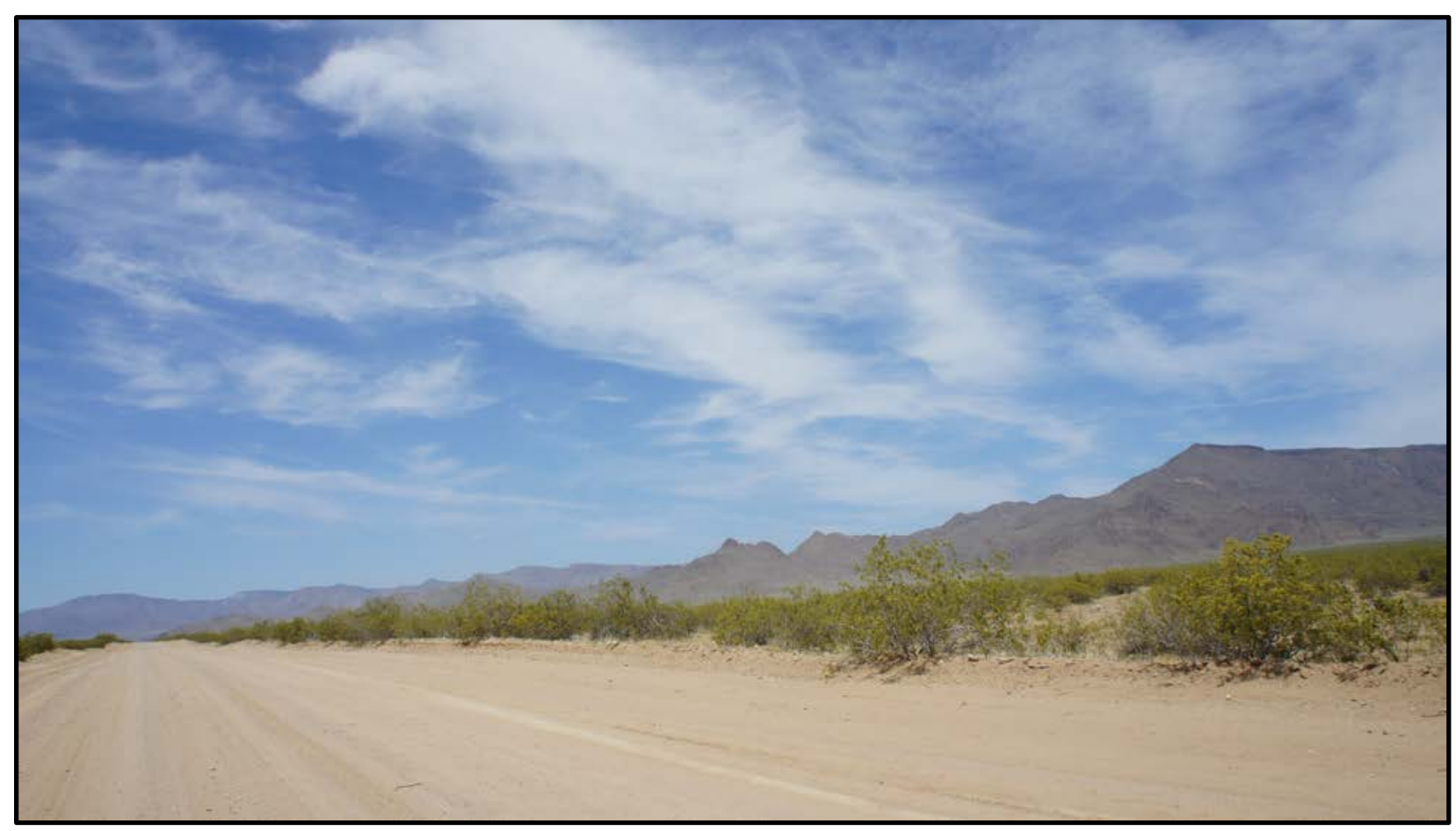

Figure 5. BLM Wind Energy South project area, view from Antares Road. 


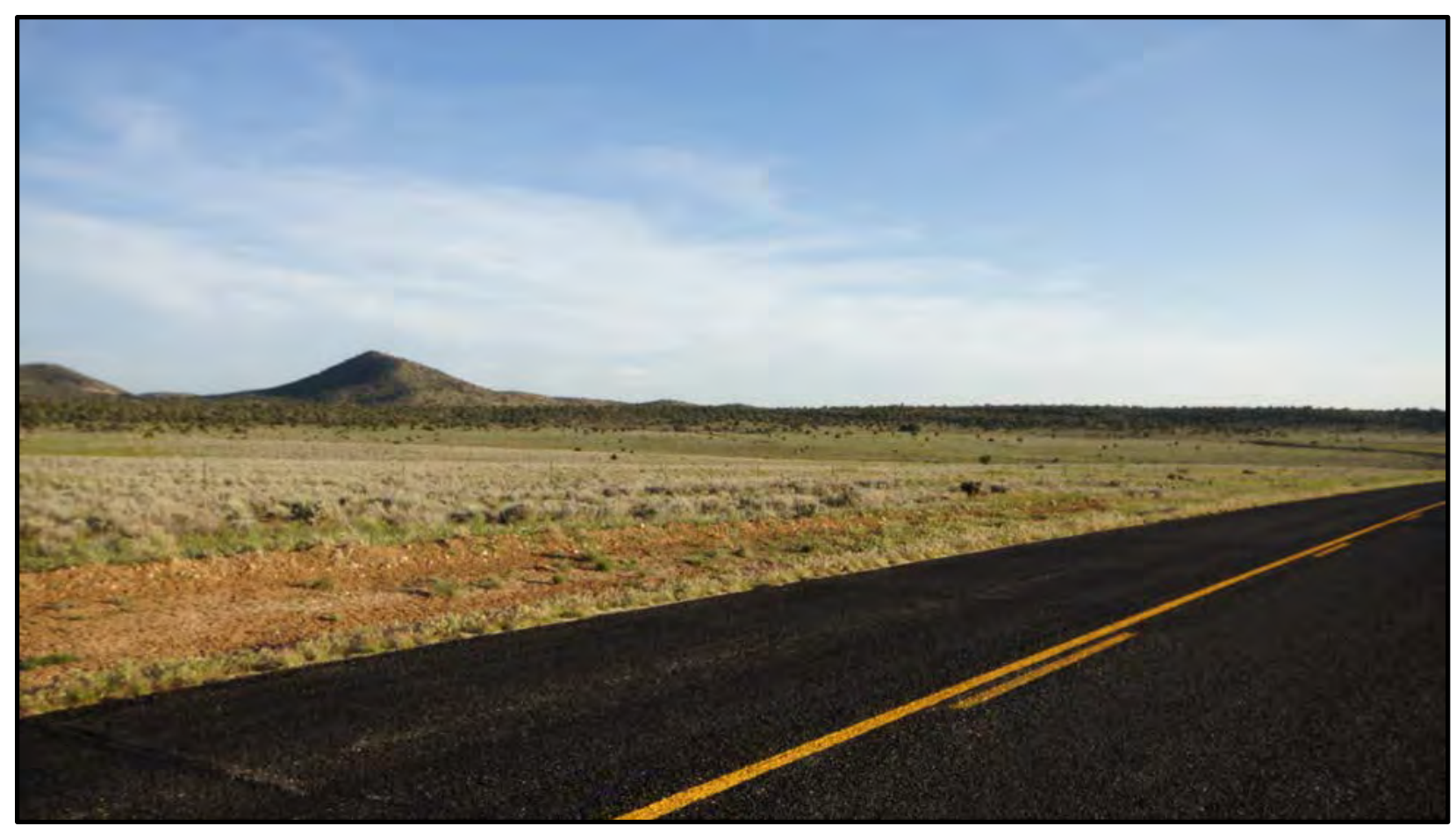

Figure 6. Nelson Solar Energy project area.

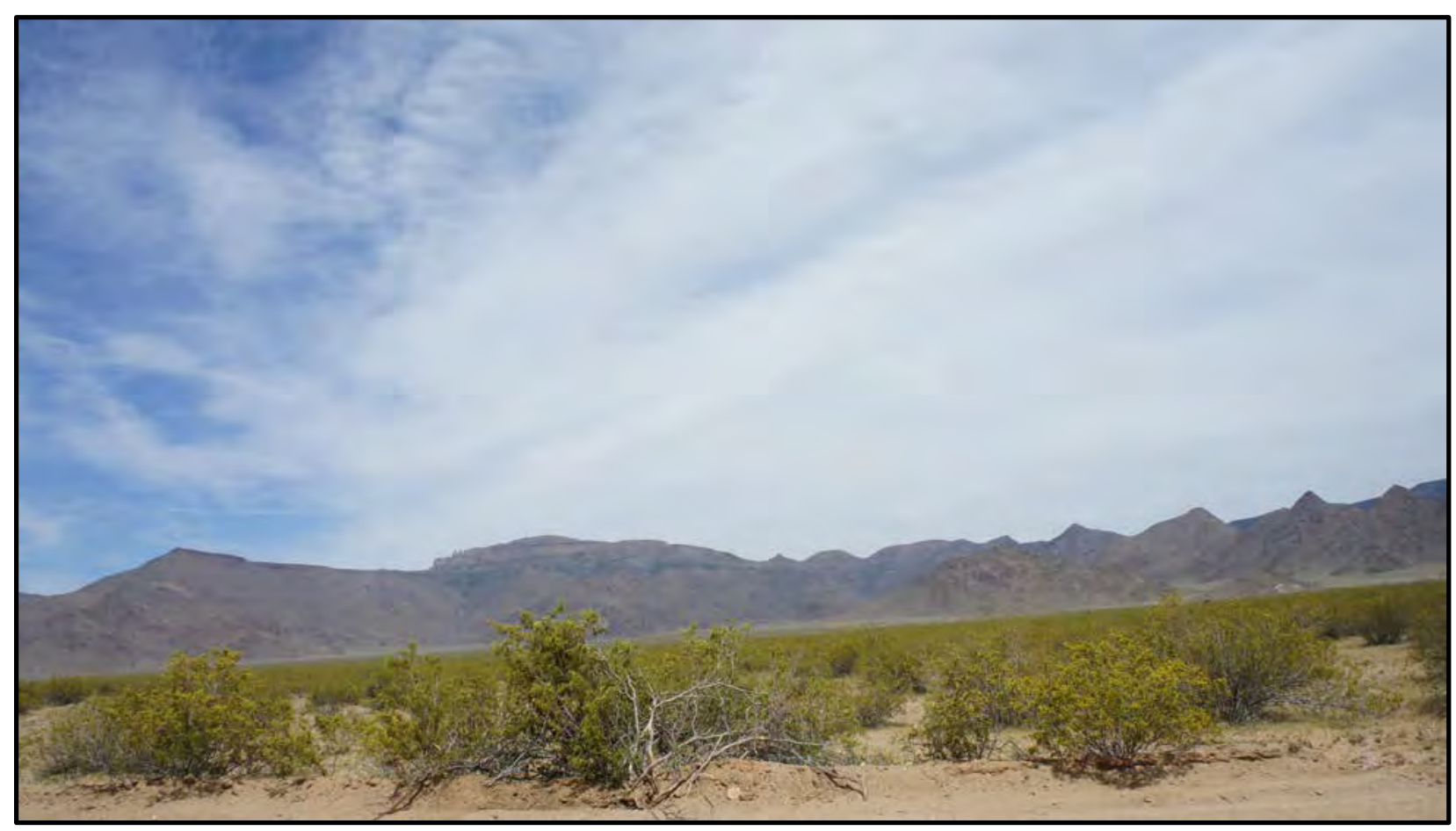

Figure 7. Hualapai Solar Energy project area. 


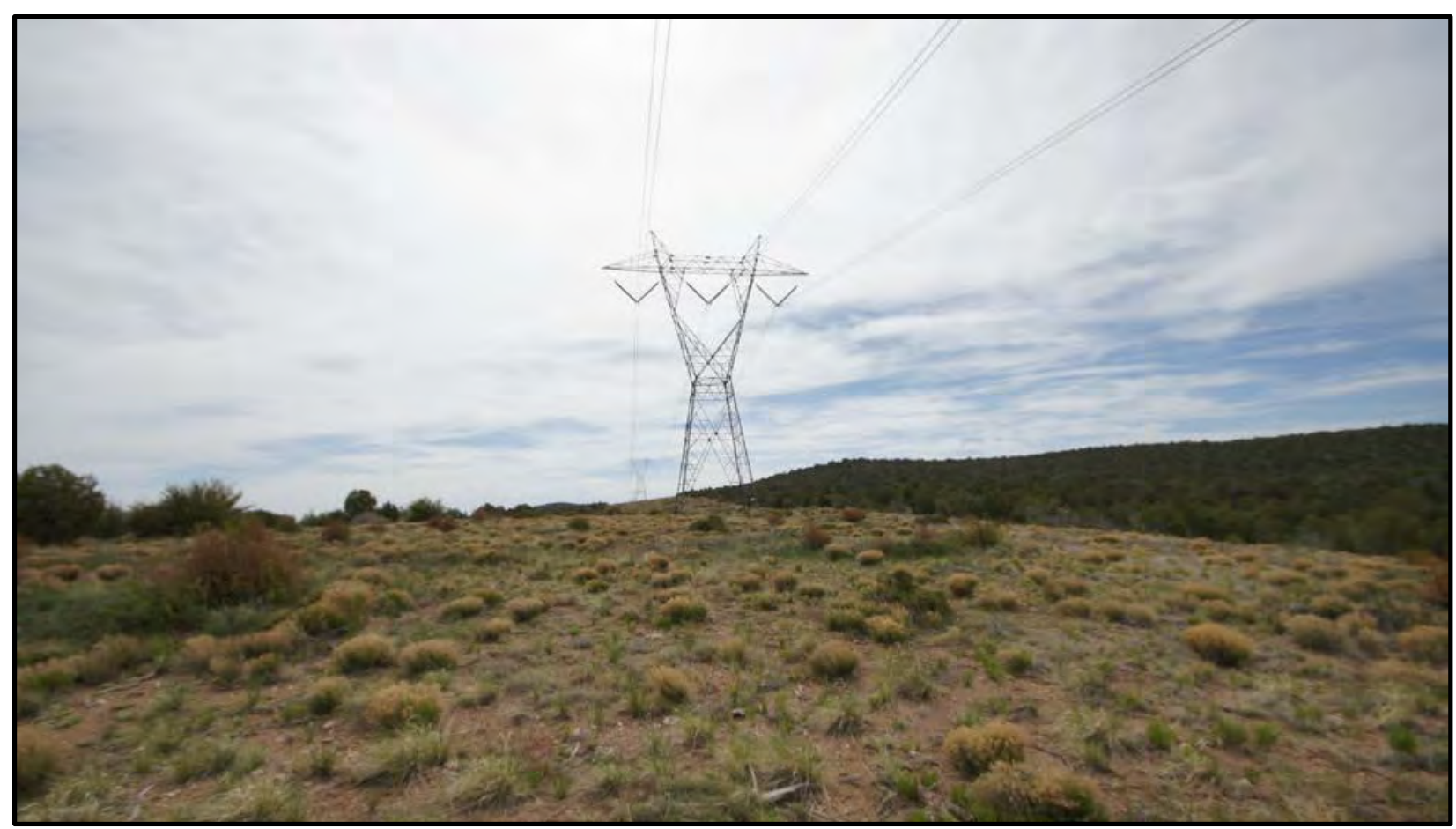

Figure 8. Four Corners-Moenkopi-Eldorado 500-kV line crossing the southern tip of the Clay Springs Wind Energy project area.

\section{$1.4 \quad$ Federal, State, and Local Permit Requirements}

Because proposed infrastructure for all six project areas would cross or be located on BLM-administered lands, private lands, and State lands, the primary federal environmental laws that would require consideration are the National Environmental Policy Act (NEPA), Endangered Species Act (ESA), Migratory Bird Treaty Act of 1918 (MBTA), Bald and Golden Eagle Protection Act (BGEPA), National Historic Preservation Act (NHPA), and Clean Water Act (CWA). These Acts apply to projects on both private and public lands.

A comprehensive list of the potential permits, certificates, and authorizations that may be required prior to the start of construction for any of the project areas are listed in Table 1.

Table 1. Permits, Certifications, and Authorizations Possibly Required for the Six Project Areas

\begin{tabular}{lll}
\hline Authorization & Agency Authority & Statutory Reference \\
\hline $\begin{array}{l}\text { Federal } \\
\begin{array}{l}\text { Right-of-way for land under federal } \\
\text { management }\end{array}\end{array}$ & BLM & \\
& & $\begin{array}{l}\text { Federal Land Policy and Management Act } \\
\text { (FLPMA) of 1976 (Public Law [PL] 94-579); } \\
\text { 43 United States Code (USC) 1761-1771; } \\
\text { 43 Code of Federal Regulations (CFR) 2800 }\end{array}$ \\
$\begin{array}{l}\text { National Environmental Policy Act } \\
\text { compliance }\end{array}$ & Bureau of Indian Affairs or BLM & NEPA (PL 91-190, 42 USC 4321-4347, \\
& & $\begin{array}{l}\text { January 1, 1970, as amended by PL 94-52, } \\
\text { July 3, 1975; PL 94-83, August 9, 1975; and }\end{array}$ \\
\hline Endangered Species Act & & PL 97-258, 4[b], September 13, 1982) \\
\hline
\end{tabular}


Table 1. Permits, Certifications, and Authorizations Possibly Required for the Six Project Areas (Continued)

\begin{tabular}{|c|c|c|}
\hline Authorization & Agency Authority & Statutory Reference \\
\hline \multicolumn{3}{|l|}{ Federal, cont'd. } \\
\hline Migratory Bird Treaty Act & USFWS & 16 USC 703-711; 50 CFR Subchapter B \\
\hline Bald and Golden Eagle Protection Act & USFWS & 16 USC 668-668(d) \\
\hline $\begin{array}{l}\text { National Historic Preservation Act } \\
\text { compliance }\end{array}$ & $\begin{array}{l}\text { Arizona State Historic } \\
\text { Preservation Office (SHPO) }\end{array}$ & NHPA 106 (PL 89-665; 16 USC 470 et seq.) \\
\hline $\begin{array}{l}\text { Notice of Proposed Construction or } \\
\text { Alteration (Form 7460.1) }\end{array}$ & $\begin{array}{l}\text { Federal Aviation Administration } \\
\text { (FAA) }\end{array}$ & $\begin{array}{l}49 \text { USC } 44718 \text { and, if applicable, } 14 \text { CFR } 77 \\
\text { (2005) to determine whether the structure } \\
\text { exceeds obstruction standards or would be } \\
\text { a hazard to air navigation }\end{array}$ \\
\hline Notice of Actual Construction (Form 7460-2) & FAA & 14 CFR Part 77 (2005) \\
\hline $\begin{array}{l}\text { Clean Water Act Section } 404 \text { Dredge and Fill } \\
\text { Permit }\end{array}$ & U.S. Army Corps of Engineers & 33 USC 1344 \\
\hline Consultation regarding military radar & Department of Homeland Security & $\mathrm{N} / \mathrm{A}$ \\
\hline $\begin{array}{l}\text { Clean Water Act Section } 402 \text { National } \\
\text { Pollutant Discharge Elimination System } \\
\text { (NPDES) during operation }\end{array}$ & $\begin{array}{l}\text { Environmental Protection Agency } \\
\text { (EPA) and Arizona Department of } \\
\text { Environmental Quality (ADEQ) for } \\
\text { portions not on Tribal lands }\end{array}$ & 33 USC 1251 et seq. \\
\hline $\begin{array}{l}\text { Clean Water Act Section } 402 \text { NPDES } \\
\text { Notification for Stormwater Management } \\
\text { during construction }\end{array}$ & $\begin{array}{l}\text { EPA (ADEQ for portions not on } \\
\text { Tribal lands) }\end{array}$ & 73 CFR 40338 \\
\hline \multicolumn{3}{|l|}{ State } \\
\hline $\begin{array}{l}\text { National Historic Preservation Act Section } \\
106 \text { Determination of Effect Concurrence }\end{array}$ & Arizona SHPO & $\begin{array}{l}16 \text { USC } 470 \text { et seq., Arizona Revised } \\
\text { Statutes (ARS) } 41\end{array}$ \\
\hline $\begin{array}{l}\text { Application for Arizona Protected Native } \\
\text { Plants And Wood Removal }\end{array}$ & Arizona Department of Agriculture & $\begin{array}{l}\text { ARS 3-904; Arizona Administrative Code } \\
\text { (AAC) 11 R3-1101-1111 }\end{array}$ \\
\hline $\begin{array}{l}\text { Guidelines for Reducing Impacts to Wildlife } \\
\text { from Wind Energy Development in Arizona }\end{array}$ & $\begin{array}{l}\text { Arizona Game and Fish } \\
\text { Department }\end{array}$ & No statutory requirement \\
\hline Operating Permit (Clean Air Act, Title V) & ADEQ & $\begin{array}{l}18 \text { AAC Chapter 2, } 49 \text { ARS Chapter } 3 \\
\text { Article } 2\end{array}$ \\
\hline Clean Water Act, Section 401 Certification & ADEQ & $\begin{array}{l}18 \text { AAC Chapter 11, } 49 \text { ARS Chapter } 2 \\
\text { Article } 2\end{array}$ \\
\hline Groundwater Discharge Permit & ADEQ & 49 ARS Chapter 2 Article 3.1 \\
\hline $\begin{array}{l}\text { Clean Water Act Section } 402 \text { AZPDES } \\
\text { Notification for Stormwater Management } \\
\text { during construction }\end{array}$ & ADEQ & 49 ARS Chapter 2 Article 3.1 \\
\hline Notice of Intent to Clear Land & Arizona Department of Agriculture & ARS 3-904 \\
\hline ROW Encroachment Permit & $\begin{array}{l}\text { Arizona Department of } \\
\text { Transportation (ADOT) }\end{array}$ & AAC R17-3-501-509 \\
\hline Over Legal Size/Load Permit & ADOT & ARS 28-1103 Article 18 \\
\hline $\begin{array}{l}\text { Uniform Permit (for transportation of } \\
\text { hazardous materials) }\end{array}$ & $\begin{array}{l}\text { Arizona Department of Public } \\
\text { Safety }\end{array}$ & AAC R17-5-209 \\
\hline Assignment of Water Rights & $\begin{array}{l}\text { Arizona Department of Water } \\
\text { Resources (ADWR) }\end{array}$ & AAC R12-15-104 \\
\hline Permit to Drill a non-exempt well & ADWR & ARS 41-1008 and 1079 \\
\hline Phase I Environmental Site Assessment & ADEQ & $\begin{array}{l}\text { Comprehensive Environmental Response, } \\
\text { Compensation, and Liability Act, as } \\
\text { amended, } 42 \text { USC 9601, et seq. }\end{array}$ \\
\hline
\end{tabular}


Table 1. Permits, Certifications, and Authorizations Possibly Required for the Six Project Areas (Continued)

\begin{tabular}{lll}
\hline Authorization & Agency Authority & Statutory Reference \\
\hline $\begin{array}{l}\text { Mohave County / Local } \\
\text { Zoning Use Permit }\end{array}$ & $\begin{array}{l}\text { Mohave County Development } \\
\text { Services Department }\end{array}$ & $\begin{array}{l}\text { Mohave County Zoning Ordinance } \\
\text { Section 28 }\end{array}$ \\
\hline Building Permit & $\begin{array}{l}\text { Mohave County Development } \\
\text { Services Department }\end{array}$ & Mohave County Ordinance 2009-02 \\
\hline Grading Permit & $\begin{array}{l}\text { Mohave County Planning and } \\
\text { Zoning Department }\end{array}$ & $\begin{array}{l}\text { Mohave County Zoning Ordinance } \\
\text { Section 28 }\end{array}$ \\
\hline Commercial Septic Holding Tank Permit & $\begin{array}{l}\text { Mohave County Environmental } \\
\text { Health Division }\end{array}$ & \\
\hline
\end{tabular}

\subsection{CRITICAL ISSUES ANALYSES METHODOLOGY}

The key resources listed below were analyzed and a risk category was assigned for each resource by project area. Because of the expectation that a BLM right-of-way (ROW) will be required for each of the six project areas, whether for development of the two BLM Wind Energy North and South areas, or for associated transmission and other ancillary facilities, risk categories were based on BLM Instruction Memorandum (IM) 2011-061, Solar and Wind Energy Applications - Pre-Application and Screening (BLM 2011). Risk categories were used to assess the potential of each resource to affect project implementation, and additional work needed related to permitting requirements. Using these standardized risk categories ensures consistency with other alternative-energy development projects in Arizona with a federal nexus. Following the resource analysis, a summary of risks has been prepared by project area, which includes a project risk matrix that considers the cumulative risk of all resources for each project area, to assist in comparing the overall project constraints and risks.

\section{Resources Analyzed:}

- Land Use

- Ground Transportation

- Airports and Aviation

- Communications and Radio Facilities

- Public Services and Utilities

- Active Mines and Mining Claims

- Visual Resources

- Biological Resources

o Vegetation

o Special Status Species

o Big Game Species

o Bats

o Birds, including Bald and Golden Eagles

- Jurisdictional Waters 
- Flood Hazard Zones

- Geologic Hazard Zones

- Recreation

- Air Quality

- Noise

- Hazardous Materials

- Cultural Resources

- Paleontological Resources

- Socioeconomics

- Environmental Justice

\section{$2.1 \quad$ Risk Categories}

The following risk analysis consists of a scoring system designed to evaluate risk levels associated with each resource considered (Table 2). These resource risk levels are based on those identified in BLM IM 2011-061 (BLM 2011), and are provided below.

Low Potential for Conflict-timely or expedited authorizations possible:

- Lands specifically identified for solar or wind energy development

- Previously disturbed sites or areas adjacent to previously disturbed or developed sites

- Locations that minimize construction of new roads and/or transmission lines

- Lands adjacent to designated transmission corridors

- Lands currently designated as Visual Resource Management (VRM) Class IV (refer to Section 3.7 for a definition of VRM classes)

- Lands identified as suitable for disposal (sale into private ownership) in BLM land use plans

Medium Potential for Conflict-projects that have resource conflicts that can potentially be resolved:

- Designated BLM special management areas, including Areas of Critical Environmental Concern (ACECs), that provide for some limited development

- Lands with wilderness characteristics outside Wilderness and Wilderness Study Areas that have been identified in an updated wilderness characteristics inventory

- ROW avoidance areas

- Areas where project development may adversely affect properties listed in the National Register of Historic Places or areas with sensitive cultural and/or historical resource values and other designated areas such as National Natural Landmarks and National Historic Landmarks

- Areas where project development may adversely affect National Historic and Scenic Trails and National Recreation Trails

- Riparian areas, or areas of importance for federal or State sensitive species 
- Lands currently designated as VRM Class III

- Department of Defense operating areas, including areas with significant radar, airspace, or land use conflicts

- Areas where project development may adversely affect lands acquired for conservation purposes

- Developed recreation sites and/or facilities

- Projects with proposed groundwater uses within groundwater basins that have been overappropriated by State water resource agencies

\section{High Potential for Conflict-more complex projects that will require a greater level of consultation, analysis, and mitigation to resolve issues, or may not be feasible to authorize:}

- Lands near or adjacent to lands designated by Congress, the President, or the Secretary of the Interior for the protection of sensitive viewsheds, resources, and values (e.g., units of the National Park System, U.S. Fish and Wildlife Service [USFWS] Refuge System, National Forest System, and the BLM National Landscape Conservation System), which may be adversely affected by development

- Lands adjacent to Wild, Scenic and Recreational Rivers and river segments determined eligible/suitable for Wild and Scenic River status if project development may have significant adverse effects on sensitive viewsheds, resources, and values

- Designated critical habitat for federally threatened and/or endangered species if project development is likely to result in the destruction or adverse modification of that critical habitat

- Sensitive habitat areas, including important eagle use areas, priority sage-grouse habitat, and projects requiring an Bird and Bat Conservation Strategy/Eagle Conservation Strategy (BBCS/ECS)

- Lands currently designated as VRM Class 1 or Class II

- ROW exclusion areas

- Lands currently designated as no surface occupancy (NSO) in BLM land use plan prescriptions

Table 2. Risk Level Scoring System

\begin{tabular}{llc}
\hline Category & Description & Score \\
\hline None & Resource is not present within a 1-mile buffer of the project area. & 0 \\
\hline Low & $\begin{array}{l}\text { Resource is present within 1 mile of the project area, but impacts are unlikely and would } \\
\text { require minimal permitting and agency coordination. Timely or expedited authorizations } \\
\text { possible. }\end{array}$ & 1 \\
\hline Medium & $\begin{array}{l}\text { Resource is present within 1 mile of the project area, and resource conflicts are likely but } \\
\text { could be easily resolved. These issues would require permitting and/or agency } \\
\text { coordination. }\end{array}$ & 2 \\
\hline High & $\begin{array}{l}\text { Resource is present within 1 mile of the project area, and impacts are likely and difficult to } \\
\text { address. These are generally more complex projects that would require a greater level of } \\
\text { consultation, analysis, and mitigation to resolve issues, or may not be feasible to authorize. }\end{array}$ & 3 \\
\hline Fatal Flaw & $\begin{array}{l}\text { Resource is present within 1 mile of the project site, and impacts will occur and may } \\
\text { exceed limitations described above. These issues will require extensive permitting and } \\
\text { agency coordination that could lead to denial of the project. }\end{array}$ & 4 \\
\hline
\end{tabular}


It should be noted that risk categories assigned in this document are based on currently available data as presented. As new data become available, such as from the completion of formal, site-specific surveys, risk categories may change.

\subsection{Data Collection and Analysis}

Data were collected from multiple sources that provided relevant information about the study area, including federal, state, and local issues. These data were then compiled, analyzed, and used in the evaluation of risk categories. Sources for data collection included:

- Existing literature

- Online literature searches

- Existing GIS data

- Mohave and Coconino County data and documents

- U.S. Geological Survey (USGS) GIS data

- Desktop analysis of possibly suitable golden eagle nesting habitat, and aerial eagle/raptor nest surveys

\section{$2.3 \quad$ Site Reconnaissance}

A reconnaissance-level site visit was conducted on May 2, 2012, to evaluate land use and resource conditions within the study area and to help determine if any areas that support critical resources were present. A biologist and environmental planner drove through the sites on existing roads and reviewed existing data on field maps and identified unique features not identified on maps. Representative photographs were taken at each site for reference.

\subsection{RESOURCE CONSTRAINTS ANALYSIS}

\subsection{Land Use (including BLM, Private, Towns, Areas of Critical Environmental Concern, National Preserves, Wilderness, Other Federal and State Lands)}

The study area is located in rural areas with little residential or commercial uses. There are no cities, towns, or villages within or immediately adjacent to the project sites. No rural residential clusters or homes are located within or immediately adjacent to the project sites. Private lands are present only within the BLM Wind Energy North project area and are minimal (less than four sections), and include undeveloped, checkerboard parcels interspersed with BLM lands. Lands within the study area are primarily undeveloped; however there are existing roads that enable access to the project areas (Figure 9). There would be a federal nexus associated with the development of renewable energy projects for each of the six project areas.

The Mohave County General Plan, as adopted by the Mohave County Planning and Zoning Department, identifies lands in the project area as Indian Reservation and Public Lands. The intent of the Public Lands designation is to indicate land in rural areas that is owned by a public agency, but is not primarily devoted to parks and recreation use. 


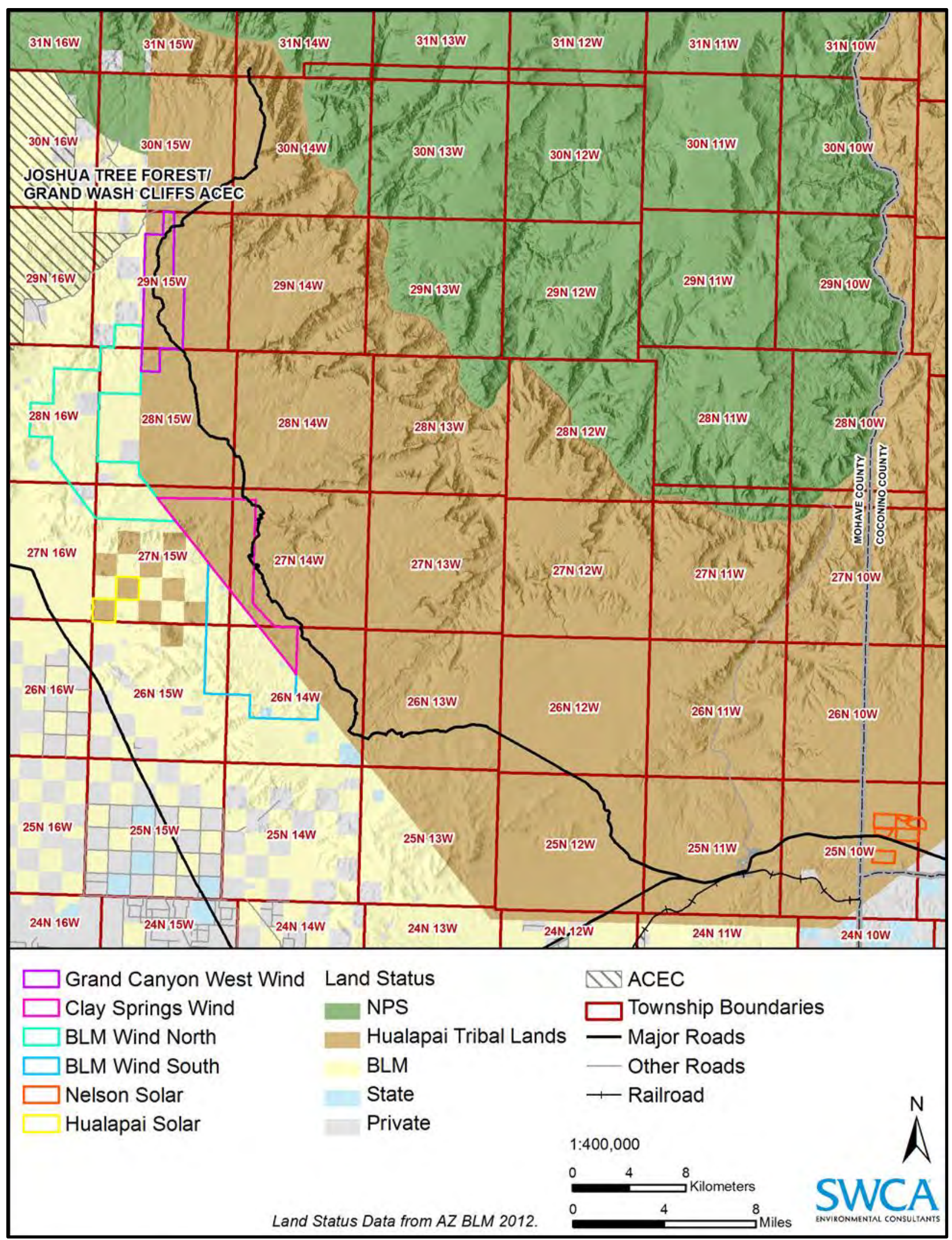

Figure 9. Land status and ownership. 


\section{GRAND CANYON WEST WIND ENERGY PROJECT AREA}

The project area is located on Hualapai Tribal lands in Mohave County, Arizona. There are no BLM, private lands, cities, towns, or villages, ACECs, national preserves, wilderness, or other federal and state lands within or surrounding the project area. There are a number of parcels in proximity to the project area with private development or the potential for future private development.

Risk Category. Low. Because the site is wholly on Hualapai Tribal lands, establishment of land uses that would be in conflict with a wind energy project would be at the discretion of the Hualapai Tribal Council.

Additional Work Required. An in-depth land use analysis would be conducted as part of the environmental analysis per NEPA including consideration of the wind energy site as a connected action. The detailed NEPA analysis would identify specific impacts or benefits that would result if the project were implemented. Additionally, public scoping and outreach would identify any land use concerns.

BLM will require the submittal of an SF-299 ROW application and plan of development (POD) for any transmission facilities crossing BLM-managed land.

\section{CLAY SPRINGS WIND ENERGY PROJECT AREA}

The project area is located on Hualapai Tribal lands in Mohave County, Arizona. There are no BLM, private lands, cities, towns, or villages, ACECs, national preserves, wilderness, or other federal and state lands within or surrounding the project area. There are a number of parcels in proximity to the project area with private development or the potential for future private development.

Risk Category. Low. Because the site is wholly on Hualapai Tribal lands, establishment of land uses that would be in conflict with a wind energy project would be at the discretion of the Hualapai Tribal Council.

Additional Work Required. Additional work required would be the same as that described under the Grand Canyon West project area.

\section{BLM WIND ENERGY NORTH PROJECT AREA}

Public lands within and surrounding the project area are administered by the BLM Kingman Field Office (KFO). The KFO manages public land for multiple uses and provides opportunities for utility ROWs, mining, wildlife habitat, conservation, and recreation in addition to other resource values and activities. The primary legal basis for granting a ROW on BLM land is Section 302 of the Federal Land Policy and Management Act of 1976 (FLPMA). FLPMA provides the BLM with authority to issue leases and permits for the use, occupancy, and development of public lands. The regulations establishing procedures for the processing of these leases and permits are found in 43 Code of Federal Regulations (CFR) 2920. In addition, the KFO Resource Management Plans (RMPs) provide guidance for management of public lands within the project area.

The BLM special designation Joshua Tree Forest/Grand Wash Cliffs ACEC is located approximately 0.5 mile from the northwest corner of the project area. The Joshua Tree Forest/Grand Wash Cliffs ACEC is approximately 44,255 acres and is primarily designated to protect the vegetation, scenic, and cultural resources of the area (BLM 1993). No other ACECs or special designations would be potentially affected by the project.

Risk Category. Low. There are four parcels of private land located within the project area. There are no state lands, cities, towns, or villages, ACECs, national preserves, or wilderness within the BLM Wind Energy North site. 
Additional Work Required. BLM will require the submittal of an SF-299 ROW application and POD. An in-depth land use analysis will be conducted as part of the environmental analysis per NEPA. The detailed NEPA analysis would identify specific impacts or benefits that would result if the project were implemented. Any changes to livestock improvements (fences, tanks, corrals) or decrease in the available allotment acreage would require a 2-year notification to the allotment permittee. Additionally, public scoping and outreach would identify any land use concerns.

The proximity of the Joshua Tree Forest/Grand Wash Cliffs ACEC may require engineering design and location considerations to decrease the impact to the scenic resources which the ACEC was designated to protect. In December 2008, the BLM issued IM 2009-043, Right-of-Way Management, Wind Energy, which updated the previous IM 2006-216. The updated IM provides guidance on issues related to ROWs for wind energy testing, monitoring, and development as well as clarifies BLM wind energy development polices and best management practices. IM 2009-043 states that ACECs will not be universally excluded from wind energy as long as it is consistent with the protection of resource values and the specific special management prescriptions for the individual ACEC.

\section{BLM WIND ENERGY SOUTH PROJECT AREA}

The BLM KFO has established grazing allotments for the purposes of livestock grazing on public lands. There are six existing grazing allotments within the project area. These grazing allotments are identified by the BLM KFO as Diamond Bar Unit A, Upper Music Mountain, Clay Springs, Music Mountain, Middle Water, and Cedar Canyon allotments (BLM 1993).

There is a small parcel of state land along the southern edge of the project area.

Risk Category. Low. There are no private lands, cities, towns, or villages, ACECs, national preserves, or wilderness within the BLM Wind Energy South site.

Additional Work Required. BLM will require the submittal of an SF-299 ROW application and POD. An in-depth land use analysis will be conducted as part of the environmental analysis per NEPA. The detailed NEPA analysis would identify specific impacts or benefits that would result if the project were implemented. Additionally, public scoping and outreach would identify any land use concerns.

A special land use permit and ROW from the Arizona State Land Department (ASLD) would be necessary to use state lands. The process for obtaining an ASLD ROW is available online (see ASLD 2008). The processing time for an ASLD ROW is typically between 12 and 16 months. Similar to NEPA, the process for obtaining a state land use permit and a ROW on Arizona State lands requires clearances for archaeological resources, native plants, any necessary permits related to waters of the U.S., and other resources as determined necessary by additional State agencies, including the Arizona Department of Environmental Quality (ADEQ). The other resource needs are addressed throughout the CIA in their respective resource sections.

\section{NELSON SOLAR ENERGY PROJECT AREA}

The project area parcels are located on Hualapai Tribal lands within the southwest corner of Coconino County. There are no BLM, private lands, cities, towns, or villages, ACECs, national preserves, wilderness, or other federal and state lands within or surrounding the project area.

Risk Category. Low. There are no BLM, private lands, cities, towns, or villages, ACECs, national preserves, wilderness, or other federal and state lands within or surrounding the project area. Because the site is wholly on Hualapai Tribal lands, establishment of land uses that would be in conflict with a solar energy project would be at the discretion of the Hualapai Tribal Council. 
Additional Work Required. An in-depth land use analysis will be conducted as part of the environmental analysis per NEPA. The detailed NEPA analysis would identify specific impacts or benefits that would result if the project were implemented. Additionally, public scoping and outreach would identify any site-specific land use concerns.

In addition, BLM will require the submittal of an SF-299 RIW application and POD for any associated transmission facilities crossing BLM lands.

\section{HUALAPAI SOLAR ENERGY PROJECT AREA}

The project area is located on Hualapai Tribal lands in Mohave County, Arizona. The project area is two 40-acre parcels interspersed with BLM checkerboard parcels.

Risk Category. Low. There are no BLM, private lands, cities, towns, or villages, ACECs, national preserves, wilderness, or other federal and state lands within or surrounding the project area. Because the site is wholly on Hualapai Tribal lands, establishment of land uses that would be in conflict with a solar energy project would be at the discretion of the Hualapai Tribal Council.

Additional Work Required. Additional work required would be the same as that described for the Nelson Solar Energy project area.

\subsection{Ground Transportation (Roads and Railroads)}

The study area is dominated by undeveloped terrain; however, existing roads provide access to each of the project areas. Existing roads through each of the project areas include both maintained dirt surface roads and unmaintained dirt roads. Mohave County Public Works Road Division maintains 751 miles of dirt surface and gravel roadways throughout the rural areas of Mohave County. The BNSF and Amtrak passenger train is located approximately 14 miles south of the Clay Springs portion of the study area and approximately 1.5 miles south of the Nelson portion of the study area (Figure 10).

\section{GRAND CANYON WEST WIND ENERGY PROJECT AREA}

Access to the project area is from Buck and Doe Road from the south and Diamond Bar Road to the north/northwest. Buck and Doe road is a primitive, dirt surface road. Diamond Bar Road includes portions of paved and graded dirt surface road.

Risk Category. Low. No federal or state roadways traverse the project area. No existing or future activities would prevent project-related use of the Mohave County or Tribal Routes that provide access to the project areas.

Additional Work Required. A route study and transportation plan to manage traffic flow and reduce delays during construction activities associated with wind energy and solar facilities is recommended, particularly along the Diamond Bar Road. These studies and plans should be prepared in coordination with the Arizona Department of Transportation (ADOT), the Hualapai Tribe Public Works, and Mohave County. Easements across private property may be required in instances in which ground transportation (roads) into the project area cross private property.

Coordination with the Hualapai Tribe Public Works will be required on all road improvements, construction, and maintenance activities that are to occur on Hualapai Tribal lands. 


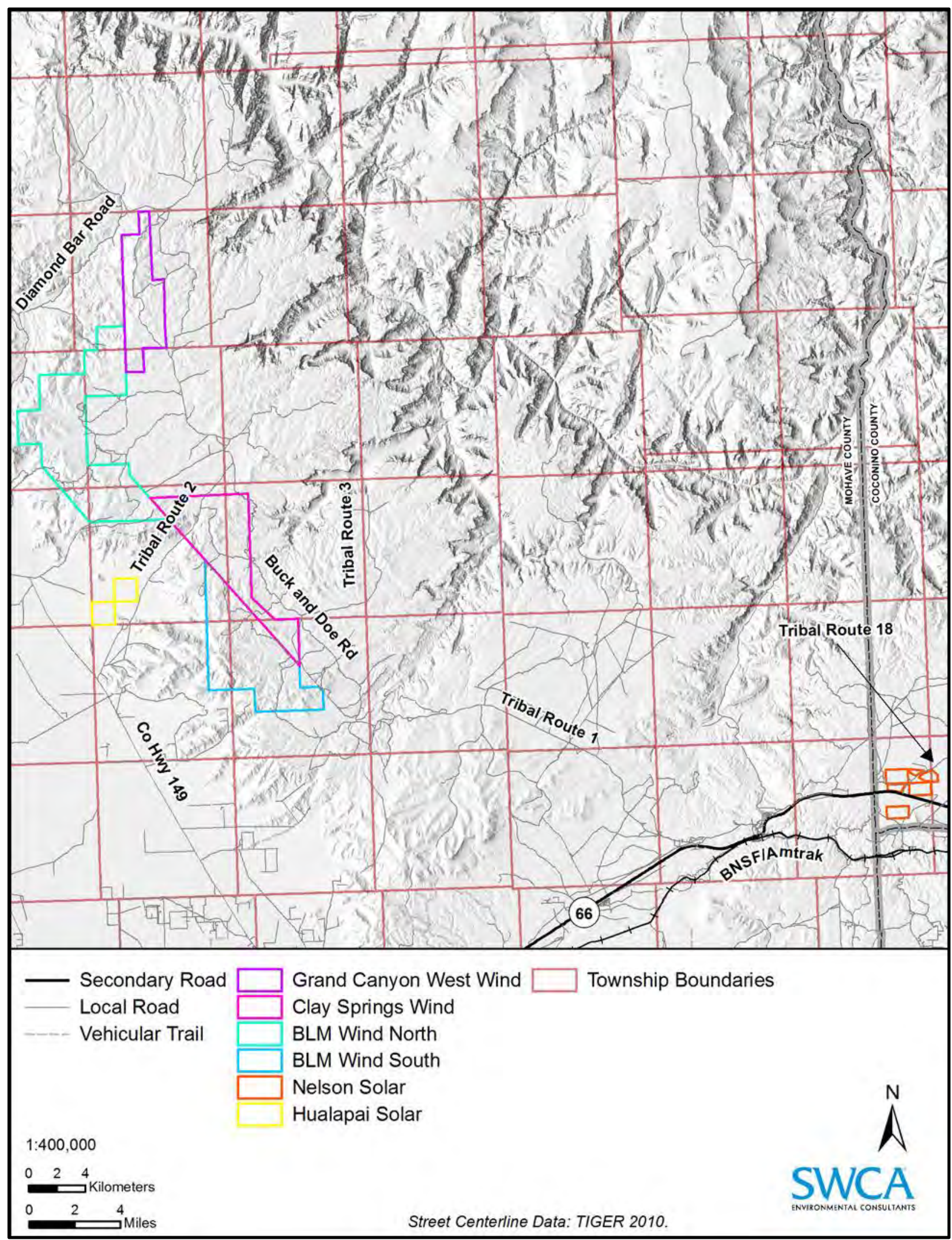

Figure 10. Ground transportation in the vicinity of the study area. 


\section{CLAY SPRINGS WIND ENERGY PROJECT AREA}

Access to the project area is from Buck and Doe Road. The Clay Springs Road goes from Antares Road northeast through the Clay Springs project area.

Risk Category. Low. No paved federal or state roadways traverse the project area. No existing or future activities would prevent project-related use of the BLM, Mohave County, or Tribal Routes that provide access to the project area.

Additional Work Required. Additional work required would be the same as that described under the Grand Canyon West Wind Energy project area.

\section{BLM WIND ENERGY NORTH PROJECT AREA}

Access to the project area is from Antares Road.

Risk Category. Low. No paved federal or state roadways traverse the project area. No existing or future activities would prevent project-related use of the BLM, Mohave County, or Tribal Routes that provide access to the project area.

Additional Work Required. A route study and transportation plan to manage traffic flow and reduce delays during construction activities associated with wind energy facilities is recommended. These studies and plans should be prepared in coordination with ADOT, the Hualapai Tribe Public Works, and Mohave County. Easements across private property may be required in instances in which ground transportation (roads) into the project area cross private property.

If the project includes any plans to widen existing ADOT ROWs, improve intersections, or alter existing drainage systems, an ADOT encroachment permit and/or fulfillment of stormwater requirements may be required. In addition, a Mohave County Public Works-Road Division road maintenance petition may be required.

\section{BLM WIND ENERGY SOUTH PROJECT AREA}

The limited primitive-road system in the remote canyons and plateaus of BLM Wind Energy South project area are rough, dirt roads.

Risk Category. Low. No paved federal or state roadways traverse the project area. No existing or future activities would prevent project-related use of the BLM, Mohave County, or Tribal Routes that provide access to the project area.

Additional Work Required. Additional work required would be the same as that described under the BLM Wind Energy North project area.

\section{NELSON SOLAR PROJECT AREA}

The nearest paved road to the Nelson Solar project area is Historic Route 66, located to the south. Access to the project area would be from the dirt surface Indian Route 18 which bisects the project area.

Risk Category. Low. No federal or state roadways traverse the project area. No existing or future activities would prevent project-related use of the Mohave County or Tribal Routes that provide access to the project areas. 
Additional Work Required. If the project includes any plans to widen existing ADOT ROWS, improve intersections, or alter existing drainage systems, an ADOT encroachment permit and/or fulfillment of stormwater requirements may be required. In addition, a Mohave County Public Works-Road Division road maintenance petition may be required. Coordination of all road improvements, construction, and maintenance activities that are likely to occur on Hualapai Tribal lands will need to be approved by the Hualapai Tribe Public Works.

\section{HUALAPAI SOLAR PROJECT AREA}

Access currently exists along the transmission line dirt surface access road that bisects the project area.

Risk Category. Low. No federal or state roadways traverse the project area. No existing or future activities would prevent project-related use of the Mohave County or Tribal Routes that provide access to the project area.

Additional Work Required. If the project includes any plans to widen existing ADOT ROWs, improve intersections, or alter existing drainage systems, an ADOT encroachment permit and/or fulfillment of stormwater requirements may be required. In addition, a Mohave County Public Works-Road Division road maintenance petition may be required. Coordination of all road improvements, construction, and maintenance activities that are likely to occur on Hualapai Tribal lands will need to be approved by the Hualapai Tribe Public Works.

\subsection{Airports and Aviation (Private, Commercial, Federal Aviation Administration, and Military)}

The Federal Aviation Administration (FAA) is an agency of the U.S. Department of Transportation that regulates civil aviation in the United States. Because tall structures can have an effect on general air navigation, the FAA must be contacted for any proposed construction or alteration of objects within navigable airspace under the following categories:

- proposed objects more than 200 feet above ground level at the structure's proposed location;

- within 20,000 feet of an airport that has at least one runway longer than 3,200 feet, and the proposed object would exceed a slope of 100:1 horizontally from the closest point of the nearest runway;

- within 10,000 feet of an airport that does not have a runway longer than 3,200 feet, and the proposed object would exceed a 50:1 horizontal slope from the closest point of the nearest runway; and/or

- within 5,000 feet of a heliport and the proposed object would exceed a 25:1 horizontal slope from the nearest landing and takeoff area of that heliport (14 CFR 77; FAA 2007).

Grand Canyon National Park is a popular destination for both helicopter and fixed-wind flight tours, and the FAA along with the National Park Service has identified both fixed-wing and helicopter air tour routes to and over the National Park airspace for tourism purposes. Annual overflights of the park are currently estimated to be 130,000 with activity at Grand Canyon National Park Airport running between 70 and 90 flights per hour during the busy summer season. Over 20 different companies with flights originating out of five different states offer aerial tours of the Grand Canyon. The FAA and National Park Service have also established the Grand Canyon National Park Special Flight Rules Area. The Special Flight Rules Area includes an area much larger than the National Park boundary and does include 
Hualapai Tribal lands in addition to private and state lands south of the Grand Canyon; however it does not overlap with any of the proposed project areas (FAA 2003).

Air navigation concerns also exist within military airspace. The Department of Defense (DoD) must be consulted regarding potential conflicts with military airspace, which consists of the following: military operations areas (MOAs), military training routes (MTRs), or activities next to military testing and training ranges. An MOA is airspace designated for military training activities. An MTR is a series of airspace segments that are linked together where training activities are conducted. The floor and ceiling for both MOA and MTR airspace are defined, and, in either type of space, the floor may extend all the way down to the ground level (BLM 2005). On November 8, 2011, the Natural Resources Defense Council released Version 1.0 of the GIS dataset "Military Installations, Ranges, and Training Areas (point locations and boundaries)." This dataset contains locations and available boundaries for 480 DoD sites (FAA 2011).

Large, metropolitan airports are located within the city of Las Vegas, Nevada, and include McCarran International Airport and North Las Vegas Airport, approximately 75 miles northwest of the study area boundary. Laughlin/Bullhead International Airport is located approximately 60 miles southwest of the study area boundary in Bullhead City, Arizona. There are also airports in Prescott, Arizona, and Flagstaff, Arizona, to the south and east of the project area boundary. Kingman Airport is located approximately 33 miles south of the project area boundary in Kingman, Arizona, in Mohave County and has paved airstrips that are 6,827 feet and 6,725 feet long. The Grand Canyon West Airport has a 5,058-foot-long paved airstrip and is located 4 miles northeast of the study area boundary. The airports identified above all have airstrips longer than 3,200 feet and are all located more than 20,000 feet from the proposed study area boundary (FAA 2011).

There are also a number of dirt-surface airstrips near the project area boundary. All of these airstrips are located more than 10,000 feet from any of the proposed project area boundaries.

Nellis Air Force Base, located in Las Vegas, Nevada, is the closest military airport, located approximately 75 miles to the northwest of the study area boundary. The Gila Bend Air Force Auxiliary Field is located approximately 200 miles south. The Luke Air Force Base outside Glendale, Arizona, is approximately 180 miles south of the study area boundary. No MOAs or MTRs have been identified over the study area. The nearest special use airspace MOAs are the Gladden/Bagdad MOA south of Kingman, Arizona, which is approximately 100 miles south of the study area, and the Sunny MOA north of Phoenix, Arizona, which is approximately 150 miles east of the study area (Luke Air Force Base 2009). The Desert and Turtle MOAs in Nevada and California are approximately 100 miles to the west and northwest. The nearest MTRs are associated with the Barry M. Goldwater Range and are located approximately 50 miles to the south and east of the study area (Figure 11).

\section{GRAND CANYON WEST WIND ENERGY PROJECT AREA}

The Grand Canyon West airstrip and helipads are approximately 5 miles east of the project area boundary. The Pearce Ferry Airport has a 2,900-foot-long airstrip and is located approximately 14 miles north of the project area boundary.

Risk Category. Low. Aviation facilities are not located within proximity to the project area. Because of the distance from the project area boundaries to the surrounding airstrips, airports, and regulated airspace, risks would be associated with turbine height and potential radar interference. It is anticipated these concerns can be addressed in a timely manner.

Additional Work Required. Further consultation with the FAA and DoD would be necessary to determine conflicts with structure heights or facility lighting. 


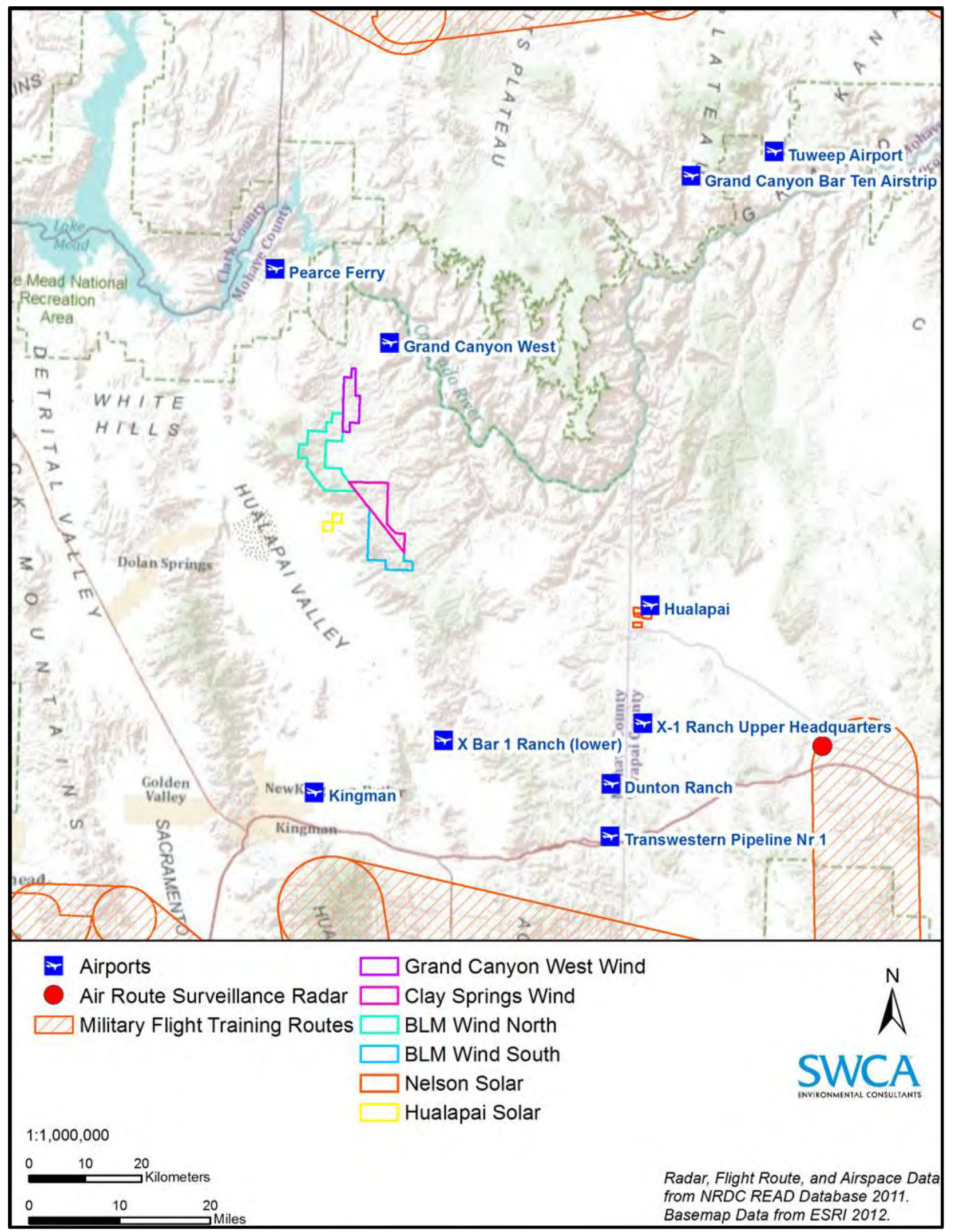

Figure 11. Airports and radar.in relation to the study area 


\section{CLAY SPRINGS WIND ENERGY PROJECT AREA}

No airports, MOAs, or MTRs were identified within 20,000 feet of the Clay Springs Wind Energy site. The 4,250-foot-long X Bar 1 Ranch (Lower) airstrip is approximately 21 miles west of the project area boundary.

Risk Category. Low. Aviation facilities are not located within proximity to the project area. Because of the distance from the project area boundaries to the surrounding airstrips, airports, and regulated airspace, risks would be associated with turbine height and potential radar interference. It is anticipated these concerns can be addressed in a timely manner

Additional Work Required. Further consultation with the FAA and DoD would be necessary to determine conflicts with structure heights or facility lighting.

\section{BLM WIND ENERGY NORTH PROJECT AREA}

No airports, MOAs, or MTRs were identified within 20,000 feet of the BLM Wind Energy North site.

Risk Category. Low. Aviation facilities are not located within proximity to the project area. Because of the distance from the project area boundaries to the surrounding airstrips, airports, and regulated airspace, risks would be associated with turbine height and potential radar interference. It is anticipated these concerns can be addressed in a timely manner

Additional Work Required. Further consultation with the FAA and DoD would be necessary to determine conflicts with structure heights or facility lighting.

\section{BLM WIND ENERGY SOUTH PROJECT AREA}

No airports, MOAs, or MTRs were identified within 20,000 feet of the BLM Wind Energy South site.

Risk Category. Low. Aviation facilities are not located within proximity to the project area. Because of the distance from the project area boundaries to the surrounding airstrips, airports, and regulated airspace, risks would be associated with turbine height and potential radar interference. It is anticipated these concerns can be addressed in a timely manner

Additional Work Required. Further consultation with the FAA and DoD would be necessary to determine conflicts with structure heights or facility lighting.

\section{NELSON SOLAR ENERGY PROJECT AREA}

Two airports were identified within 20,000 feet of the Nelson Solar Site. Hualapai Airport has a 4,790-foot-long paved airstrip and is located approximately 1,400 feet northeast of the Nelson Solar site boundary. Grand Canyon Caverns Airport consists of a 5,100-foot-long gravel airstrip and is located 2.5 miles southeast of the Nelson Solar project area boundary.

Risk Category. Medium. Although two airports exist within 20,000 feet of the site, because the Nelson Solar site is being considered for solar energy development, any concerns are expected to be easily resolved.

Additional Work Required. Further consultation with the FAA and DoD would be necessary to determine conflicts with structure heights or facility lighting. 


\section{HUALAPAI SOLAR ENERGY PROJECT AREA}

No airports, MOAs, or MTRs were identified within 20,000 feet of the Hualapai Solar Energy site.

Risk Category. Low. Aviation facilities are not located within proximity to the project area. Because of the distance from the project area boundaries to the surrounding airstrips, airports, and regulated airspace, risks would be associated with turbine height and potential radar interference. It is anticipated these concerns can be addressed in a timely manner

Additional Work Required. Further consultation with the FAA and DoD would be necessary to determine conflicts with structure heights or facility lighting.

\subsection{Communications and Radio Facilities}

Installation and operation of wind turbines has the potential to interfere with the electromagnetic signals that compose modern communication networks. The interference results when a turbine is placed between a radio, television, or microwave transmitter and a receiver. Figure 12 shows communication sites and radio tower in relation to the study area.

\section{GRAND CANYON WEST WIND ENERGY PROJECT AREA}

No communication sites were identified within the project area. Based on site reconnaissance and review of KFO RMP GIS data, the closest communications facility, Patterson Slope, is located within 10.5 miles of the project area (see Figure 12).

Risk Category. Low. No communication sites were identified within the project area.

Additional Work Required. Microwave beam path analysis and coordination with the facility owner/operator would be needed to avoid conflicts with facility operation.

\section{CLAY SPRINGS WIND ENERGY PROJECT AREA}

No communication sites were identified within the project area. Based on site reconnaissance and review of KFO RMP GIS data, the closest communications facility, Patterson Slope, is located within 13.5 miles of the project area.

Risk Category. Low. No communication sites were identified within the project area.

Additional Work Required. Microwave beam path analysis and coordination with the facility owner/operator would be needed to avoid conflicts with facility operation.

\section{BLM WIND ENERGY NORTH PROJECT AREA}

No communication sites were identified within the project area. Based on site reconnaissance and review of KFO RMP GIS data, the closest communications facility, Patterson Slope, is located within 6.5 miles of the project area.

Risk Category. Low. No communication sites were identified within the project area.

Additional Work Required. Microwave beam path analysis and coordination with the facility owner/operator would be needed to avoid conflicts with facility operation. 


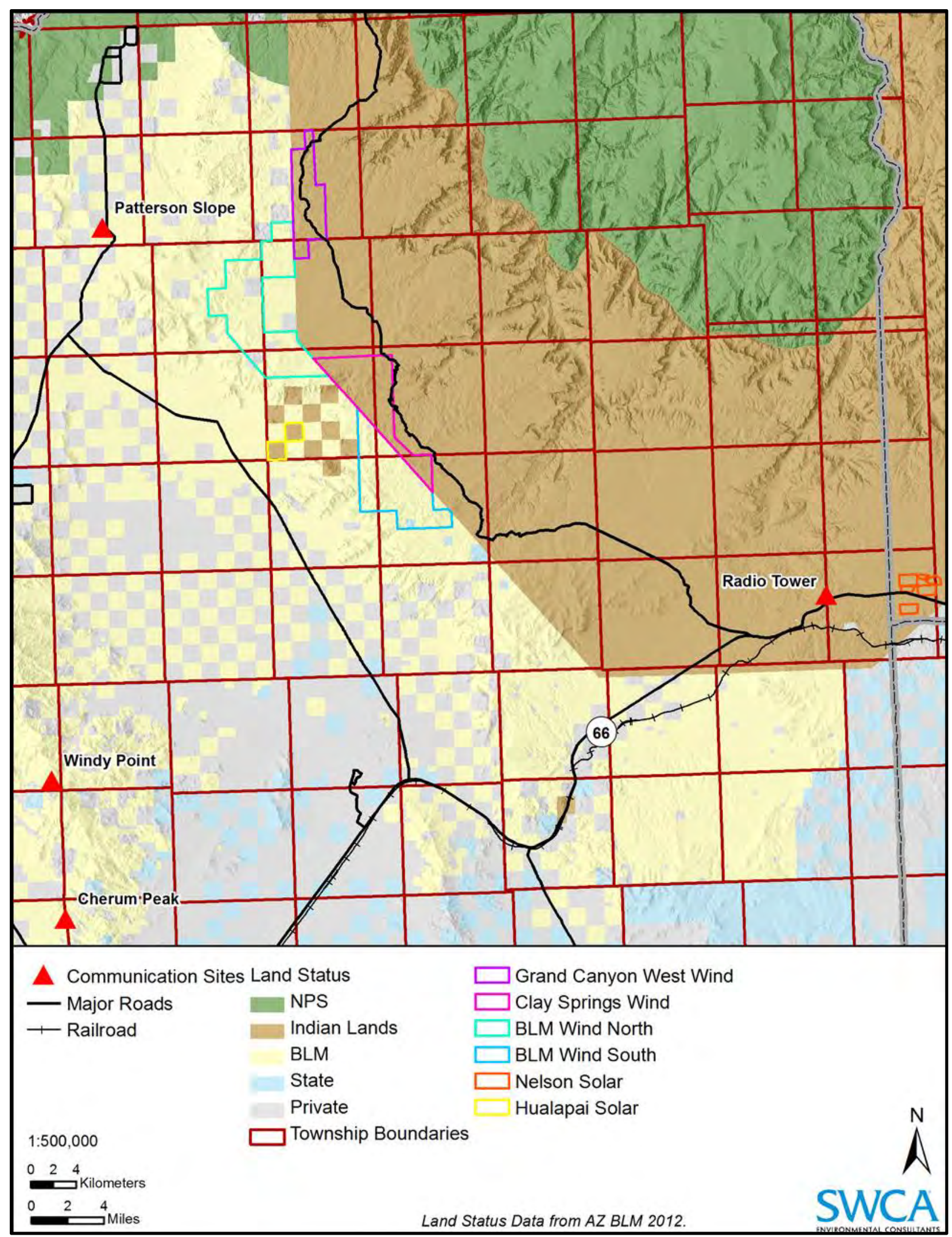

Figure 12. Communications sites in relation to the study area. 


\section{BLM WIND ENERGY SOUTH PROJECT AREA}

No communication sites were identified within the project area. Based on site reconnaissance and review of KFO RMP GIS data, the Patterson Slope communications facility is located within 17 miles of the project area.

Risk Category. Low. No communication sites were identified within the project area.

Additional Work Required. Microwave beam path analysis and coordination with the facility owner/operator would be needed to avoid conflicts with facility operation.

\section{NELSON SOLAR ENERGY PROJECT AREA}

No communication sites were identified within the project area. Based on site reconnaissance and review of KFO RMP GIS data, an unnamed radio tower is located within 4 miles of the project area.

Risk Category. Low. No communication sites were identified within the project area.

Additional Work Required. Microwave beam path analysis and coordination with the facility owner/operator would be needed to avoid conflicts with facility operation.

\section{HUALAPAI SOLAR ENERGY PROJECT AREA}

No communication sites were identified within the project area. Based on site reconnaissance and review of KFO RMP GIS data, the Windy Point communications facility is located within 14 miles of the project area.

Risk Category. Low. No communication sites were identified within the project area.

Additional Work Required. Microwave beam path analysis and coordination with the facility owner/operator would be needed to avoid conflicts with facility operation.

\subsection{Public Services and Utilities}

Services and utilities on public lands typically consist of power transmission lines, gas pipelines, water lines, and fiber optics. Utilities on public lands require a ROW permit from the BLM and compliance with the Utility Environmental Protection Act.

\section{GRAND CANYON WEST WIND ENERGY PROJECT AREA}

Services and utilities typically consist of power transmission lines, gas pipelines, water lines, and fiber optics. High-voltage power lines $(345 \mathrm{kV}$ and $500 \mathrm{kV})$ traverse roughly east and south of the project area. The Four Corners-Moenkopi-Eldorado 500-kV power line is located 15 miles from the project area. Truxton Canyon Water Company is the local water provider; however water, sewer, and waste within the Hualapai Nation are managed by Hualapai Public Works Department. A water line is currently under construction on Buck and Doe Road. No compressed natural gas lines are located within 50 miles of the project area. Most of the public services and utilities are located over 10 miles from the project area within the adjacent communities of Peach Springs, Meadview, and Dolan Springs, Arizona.

Risk Category. Low. No conflicts with public services and utilities were identified in the project area. 
Additional Work Required. Interconnection Feasibility and Interconnection System Impact studies would be required to tie-in to the existing transmission line. Interconnection studies would identify thermal overloads, voltage limitations, capacity exceedance, cost responsibility, and timeframe for construction and operation. The existing transmission line in closest proximity to the site (Four CornersMoenkopi-Eldorado $500 \mathrm{kV}$ ) is currently operated by Arizona Public Service.

\section{CLAY SPRINGS WIND ENERGY PROJECT AREA}

Services and utilities typically consist of power transmission lines, gas pipelines, water lines, and fiber optics. High-voltage power lines $(345 \mathrm{kV}$ and $500 \mathrm{kV})$ traverse roughly east and south of the project area. The Four Corners-Moenkopi-Eldorado $500-\mathrm{kV}$ power line is located approximately 4 miles from the project area. Truxton Canyon Water Company is the local water provider; however water, sewer, and waste within the Hualapai Nation are managed by Hualapai Public Works Department. A water line is currently under construction on Buck and Doe Road. No compressed natural gas lines are located within 50 miles of the project area. Most of the public services and utilities are located over 10 miles from the project areas within the adjacent communities of Peach Springs, Meadview, and Dolan Springs, Arizona.

Risk Category. Low. No conflicts with public services and utilities were identified in the project area.

Additional Work Required. Additional work required would be the same as that described under the Grand Canyon West Wind Energy project area.

\section{BLM WIND ENERGY NORTH PROJECT AREA}

Services and utilities typically consist of power transmission lines, gas pipelines, water lines, and fiber optics. High-voltage power lines $(345 \mathrm{kV}$ and $500 \mathrm{kV})$ traverse roughly east and south of the project area. The Four Corners-Moenkopi-Eldorado 500-kV power line is located 15 miles from the project area. Truxton Canyon Water Company is the local water provider; however water, sewer, and waste within the Hualapai Nation are managed by Hualapai Public Works Department. A water line is currently under construction on Buck and Doe Road. No compressed natural gas lines are located within 50 miles of the project area. Most of the public services and utilities are located over 10 miles from the project area within the adjacent communities of Peach Springs, Meadview, and Dolan Springs, Arizona.

Risk Category. Low. No conflicts with public services and utilities were identified in the project area.

Additional Work Required. Additional work required would be the same as that described under the Grand Canyon West Wind Energy project area.

\section{BLM WIND ENERGY SOUTH PROJECT AREA}

Services and utilities typically consist of power transmission lines, gas pipelines, water lines, and fiber optics. High-voltage power lines $(345 \mathrm{kV}$ and $500 \mathrm{kV})$ traverse roughly east and south of the project area. The Four Corners-Moenkopi-Eldorado $500-\mathrm{kV}$ power line is located approximately 4 miles from the project area. Truxton Canyon Water Company is the local water provider; however water, sewer, and waste within the Hualapai Nation are managed by Hualapai Public Works Department. A water line is currently under construction on Buck and Doe Road. No compressed natural gas lines are located within 50 miles of the project area. Most of the public services and utilities are located over 10 miles from the project areas within the adjacent communities of Peach Springs, Meadview, and Dolan Springs, Arizona.

Risk Category. Low. No conflicts with public services and utilities were identified in the project area. 
Additional Work Required. Additional work required would be the same as that described under the Grand Canyon West Wind Energy project area.

\section{NELSON SOLAR ENERGY PROJECT AREA}

Services and utilities typically consist of power transmission lines, gas pipelines, water lines, and fiber optics. High-voltage power lines $(345 \mathrm{kV}$ and $500 \mathrm{kV})$ traverse roughly east-west just north of the Nelson Solar Energy project area. The Four Corners-Moenkopi-El Dorado 500-kV power line is located approximately 0.5 mile north of the northern boundary of the project area. Truxton Canyon Water Company is the local water provider; however water, sewer, and waste within the Hualapai Nation are managed by Hualapai Public Works Department. No compressed natural gas lines are located within 50 miles of the project area. Most of the public services and utilities are located over 10 miles from the project area within the nearby community of Peach Springs, Arizona.

Risk Category. Low. No conflicts with public services and utilities were identified in the project area.

Additional Work Required. Additional work required would be the same as that described under the Grand Canyon West Wind Energy project area.

\section{HUALAPAI SOLAR ENERGY PROJECT AREA}

Services and utilities typically consist of power transmission lines, gas pipelines, water lines, and fiber optics. High-voltage power lines $(345 \mathrm{kV}$ and $500 \mathrm{kV})$ traverse roughly east and south of the project area. The Four Corners-Moenkopi-Eldorado $500-\mathrm{kV}$ power line is located approximately 4 miles from the project area. Truxton Canyon Water Company is the local water provider; however water, sewer, and waste within the Hualapai Nation are managed by Hualapai Public Works Department. A water line is currently under construction on Buck and Doe Road. No compressed natural gas lines are located within 50 miles of the project area. Most of the public services and utilities are located over 10 miles from the project areas within the adjacent communities of Peach Springs, Meadview, and Dolan Springs, Arizona.

Risk Category. Low. No conflicts with public services and utilities were identified in the project area.

Additional Work Required. Additional work required would be the same as that described under the Grand Canyon West Wind Energy project area.

\subsection{Active Mines and Mining Claims}

The General Mining Law of 1872 states all valuable mineral deposits belonging to the United States are free and open to exploration and purchase. The General Mining Law provides citizens of the United States the opportunity to explore, discover, and purchase mineral deposits on BLM lands. A mining claim is a parcel of land where a claimant has asserted the right of possession and the right to develop and extract a discovered and valuable mineral deposit. Mine claims may not be located in areas withdrawn from mineral entry such as National Monuments, National Parks, and designated Wilderness Areas.

\section{GRAND CANYON WEST WIND ENERGY PROJECT AREA}

There are no active mine claims located within the proposed project area. According to the BLM database, there are 14 abandoned mines over 8 miles to the west of the project area.

Risk Category. Low. There are no active mine claims in the project area. 
Additional Work Required. Because no active mine claims have been identified, no additional work is recommended at this time.

\section{CLAY SPRINGS WIND ENERGY PROJECT AREA}

There are no active mine claims located within the proposed project area. According to the BLM database, there are 14 abandoned mines over 8 miles to the west of the project area.

Risk Category. Low. There are no active mine claims in the project area.

Additional Work Required. Additional work required would be the same as described under the Grand Canyon West Wind Energy site.

\section{BLM WIND ENERGY NORTH AREA}

There are no active mine claims located within the proposed project area. According to the BLM database, there are 14 abandoned mines over 8 miles to the west of the project area.

Risk Category. Low. There are no active mine claims in the project area.

Additional Work Required. Additional work required would be the same as described under the Grand Canyon West Wind Energy site.

\section{BLM WIND ENERGY SOUTH PROJECT AREA}

There are no active mine claims located within the proposed project area. According to the BLM database, there are 14 abandoned mines over 8 miles to the west of the project area.

Risk Category. Low. There are no active mine claims in the project area.

Additional Work Required. Additional work required would be the same as described under the Grand Canyon West Wind Energy site.

\section{NELSON SOLAR ENERGY PROJECT AREA}

There are no active mine claims located within the proposed project area. The Nelson Quarry and Plant is located just south of the Nelson Solar Energy project area and is the only active mine adjacent to the project area. The Nelson Quarry and Plant is operated by Chemical Lime Company of Arizona and its primary production is lime.

Risk Category. Low. There are no active mine claims in the project area.

Additional Work Required. Additional work required would be the same as described under the Grand Canyon West Wind Energy site.

\section{HUALAPAI SOLAR ENERGY PROJECT AREA}

There are no active mine claims located within the proposed project area. Based on data from the BLM's Legacy Rehost 2000 system, nine abandoned mining claims are located over 2 miles south of the Hualapai Solar Energy project area (BLM 2012). According to the BLM database, there are 14 abandoned mines over 8 miles to the west of the project area.

Risk Category. Low. There are no active mine claims in the project area. 
Additional Work Required. Additional work required would be the same as described under the Grand Canyon West Wind Energy site.

\subsection{Visual Resources and Visual Resource Management Classifications}

Visual resources (the landscape) consist of landform (topography and soils), vegetation, bodies of water (lakes, streams, and rivers), and human-made structures (roads, buildings, and modifications of the land, vegetation, and water). These elements of the landscape can be described in terms of their form, line, color, and texture.

Visual impacts are often raised as an issue during project development for wind facilities, and to a lesser extent for solar facilities. The risk is determined by evaluating the existing landscape, including current structures and developments, the proximity to viewers with high sensitivity, and how the proposed project would contrast with existing conditions. Although Mohave County has not yet passed a wind energy ordinance that would define minimum setbacks for development, other Counties in Arizona (as seen in recent 2011 Coconino County conditional use permit stipulations) have identified increased setbacks to address concerns regarding impacts to aesthetic resources such as landscapes and community viewsheds. Mohave County has passed an outdoor light control ordinance (Mohave County Ordinance No. 87-1) that restricts the types of outdoor lighting that may be used and requires shielding and filtering outdoor light emissions to minimize impacts to dark night skies. No ordinances are currently in place for solar projects. BLM lands within and surrounding the study area are managed as VRM Class II and IV (Figure 13).

\section{GRAND CANYON WEST WIND ENERGY PROJECT AREA}

Preliminary visibility mapping (Figure 14) indicates that views of the wind turbines are largely dependent upon topography and siting of the turbine (along ridgelines and higher elevations). In the northern portion of the project area, the majority of lands would reveal between one and 20 turbines, whereas along ridgelines and higher elevations, over 50 turbines may be visible from surrounding areas with a line of sight to the ridgelines.

The existing unpaved Buck and Doe Road bisects the Grand Canyon West project area and views to the east reveal long-distance overlooks of canyons and plateaus (e.g., Grand Canyon, Horse Flat Canyon, Quartermaster Canyon, Burnt Canyon, and Sanup Plateau); these landscape features are located in the borderlands of Grand Canyon National Park. Visibility of turbines would be evident within a 5- to 7-mile radius of the proposed site, and beyond 10 miles the turbines would be significantly less visible (mostly visible from higher elevations). Easterly views would reveal more facilities, and views of turbines from Peach Springs along Route 66 may be vaguely evident in the far background (beyond 25 miles).

Risk Category. High/Medium. The project area is located entirely on Hualapai Tribal land. This landscape is largely uninhabited. Access includes an unpaved road (Buck and Doe Road) that is not heavily traveled. This area is within 5 miles of the Grand Canyon Skywalk access which is very well traveled by tourists.

Additional Work Required. Photographic simulation of visual impacts from the proposed wind turbines may be required to ensure that critical or culturally significant views are evaluated. Siting of wind turbines to reduce obstruction of critical views may be required. Collaboration with interested stakeholders and community members may be necessary to identify cultural, tribal, or community interest in preserving views in this area. 


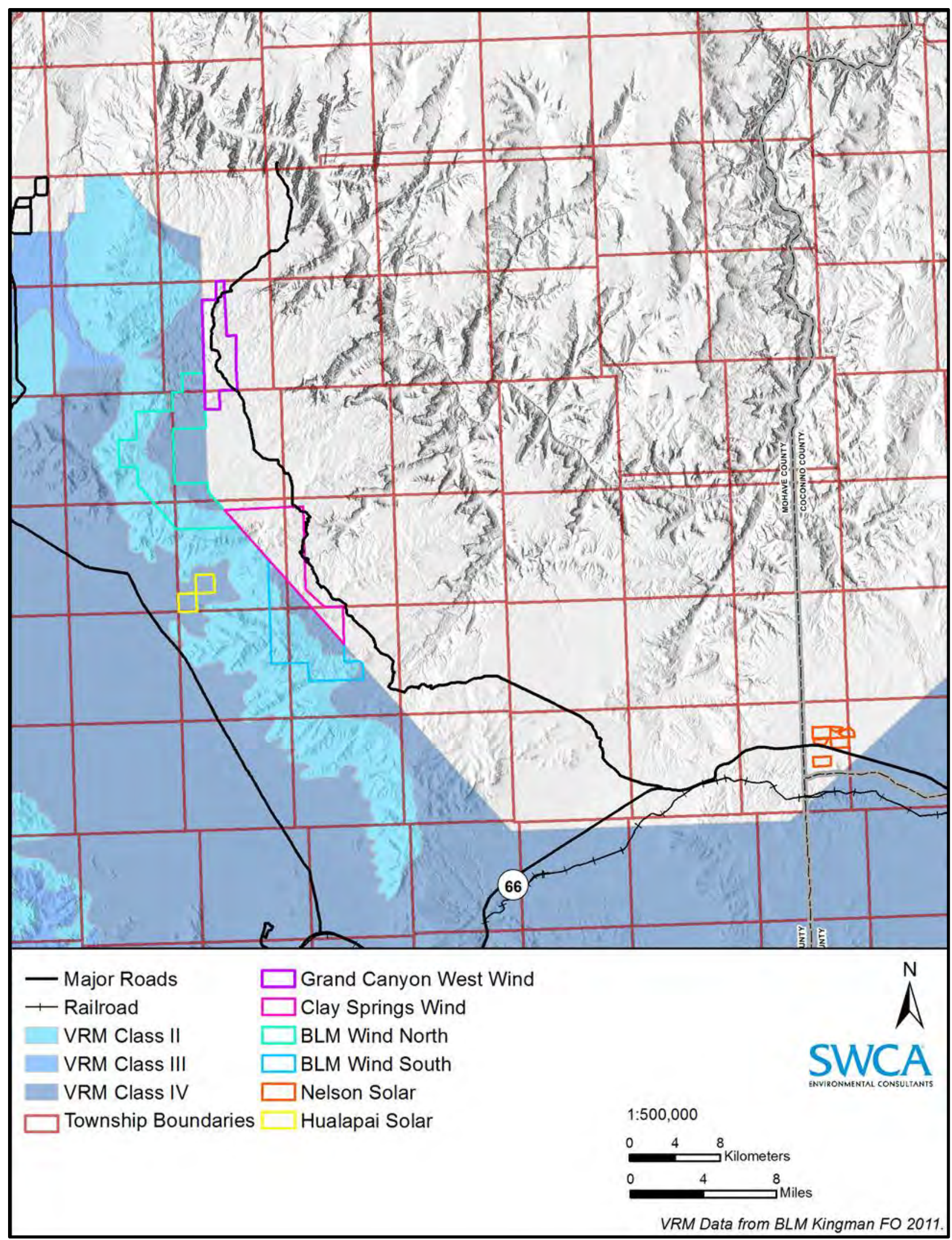

Figure 13. BLM Visual Resource Management Classes. 


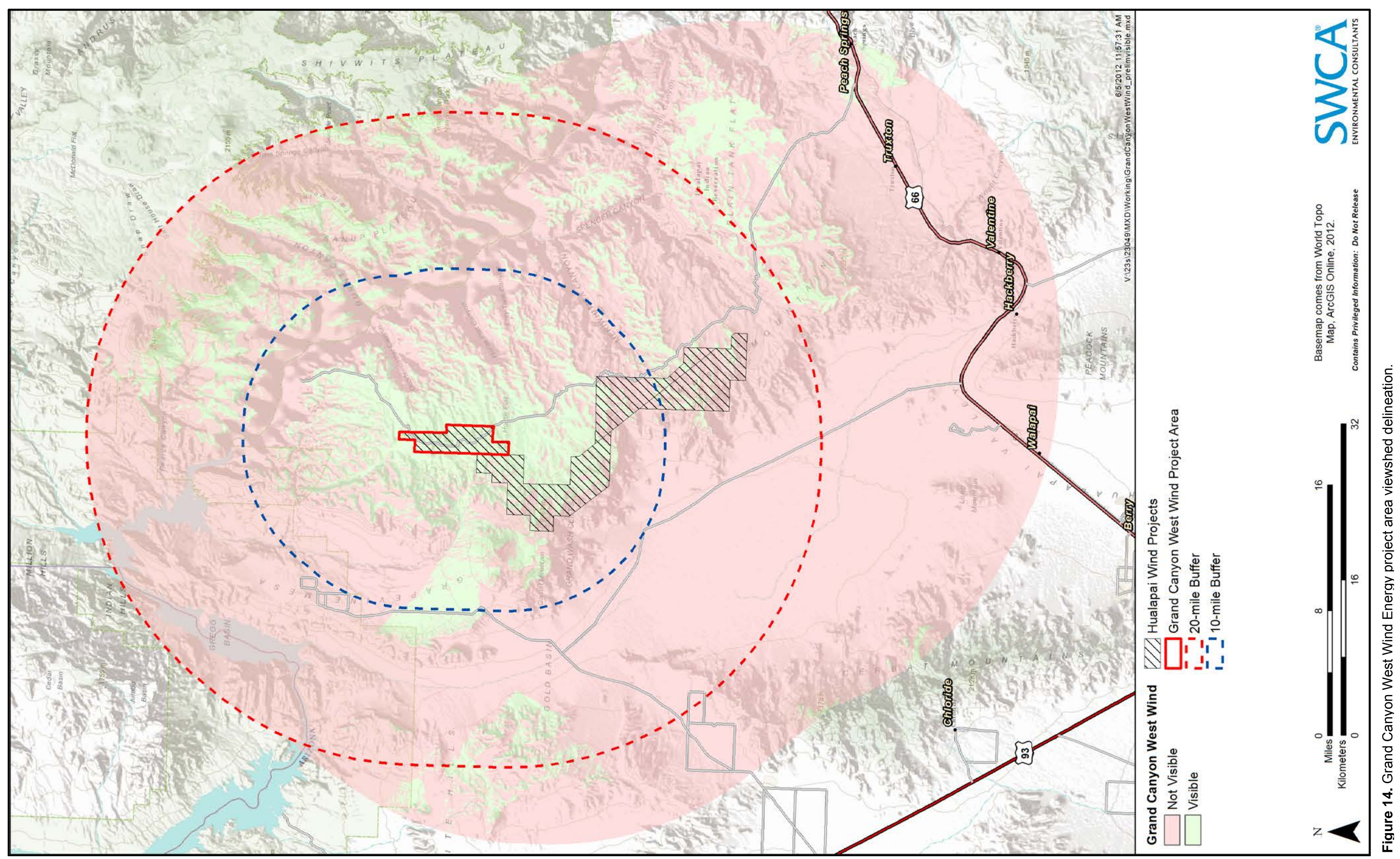


This page intentionally left blank. 


\section{CLAY SPRINGS WIND ENERGY PROJECT AREA}

Preliminary visibility mapping indicates views of the wind turbines area largely to the west of the Clay Springs project area (Figure 15). The Buck and Doe Road follows the eastern border of the project area with views of Meriwhitica Canyon to the east. Turbines within the Clay Springs Wind Energy site may be less visible from surrounding lands as a result of the existing topography and natural visual obstruction.

Risk Category. Medium. Located entirely on Hualapai Tribal land, this landscape is largely uninhabited. Access includes an unpaved road (Buck and Doe Road).

Additional Work Required. Photographic simulation of visual impacts from the proposed wind turbines may be required to ensure that critical or culturally significant views are evaluated.

\section{BLM WIND ENERGY NORTH PROJECT AREA}

Preliminary visibility mapping (Figure 16) indicates that views of the wind turbines are largely to the west and south. Views of turbines from the far background distances along Route 66 would be evident at distances beyond 20 miles. Most evident views are to the east of the site along Buck and Doe Road.

Landscape features to the west include the Grand Wash Cliffs, Garnet Mountain, and Grapevine Mesa. Further to the west is the Gold Basin which lies in lower elevations, allowing views to the east of turbines.

Risk Category. High/Medium. Located within VRM Class II and Class IV land, this area is relatively close to the access road and entrance to the Grand Canyon Skywalk. The objective of VRM Class II is to retain the existing character of the landscape. The level of change to the characteristic landscape should be low. Installation of facilities on lands managed as VRM Class II would elevate the risk category to medium. The objective of VRM Class IV is to provide for management activities that require major modification of the existing character of the landscape (e.g., mining, energy development, wind farms). The level of change to the characteristic landscape can be high.

Additional Work Required. Plan amendments may be required should the proposed facilities be located on BLM land within VRM Class II landscape.

\section{BLM WIND ENERGY SOUTH PROJECT AREA}

Preliminary visibility mapping indicates views of wind turbines west and some from east of the site (Figure 17). For the most part, this site would present the least impact to the viewshed attributable to topographic obstructions and vegetative screening within the site and along viewing areas like Buck and Doe Road. Views to the west from Gold Basin, which lies in lower elevations, would allow views of over 20 to 60 turbines. From populated areas along Route 66, some turbines would be visible in the distance but would not dominate the viewshed due to distance and visual obstructions (i.e., topographic, structural, vegetative).

Risk Category. Medium/Low. Located within VRM Class II and Class IV land, this area is relatively close to populated areas along Route 66. The objective of VRM Class II is to retain the existing character of the landscape. The level of change to the characteristic landscape should be low. Installation of facilities on lands managed as VRM Class II would elevate the risk category to medium. The objective of VRM Class IV is to provide for management activities that require major modification of the existing character of the landscape (e.g., mining, energy development, wind farms). The level of change to the characteristic landscape can be high. 
Additional Work Required. Plan amendments may be required should the proposed facilities be located on BLM land within VRM Class II landscape. This site is the closest in proximity to Route 66 and populated areas and may require coordination with communities and photographic simulations showing the potential visual change to views from these areas.

\section{NELSON SOLAR ENERGY PROJECT AREA}

Views of solar facilities are typically less visually intrusive when compared with wind turbines, because of their lower profile and the absence of moving blades. The Nelson Solar Energy project area is located on relatively flat, open landscape and facilities would likely be visible within foreground and middleground distances (but not likely to be visible beyond 5 miles). Proximity to potential viewers would be from Route 66 to the south.

Risk Category. Low. Located entirely on Hualapai Tribal land and although adjacent to Route 66, because of the local topography and visual obstruction, views of solar facilities would be reduced.

Additional Work Required. Photographic simulation of visual impacts from the proposed solar facilities may be required to ensure that critical or culturally significant views are evaluated.

\section{HUALAPAI SOLAR ENERGY PROJECT AREA}

Views of solar facilities are typically less visually intrusive when compared with wind turbines, because of their lower profile and the absence of moving blades. The proposed Hualapai Solar Energy site is located on relatively flat, open landscape and facilities would likely be visible within foreground and middleground distances (but not likely to be visible beyond 5 miles). Proximity to potential viewers would be from Antares Road to the west.

Risk Category. Low. The project area is surrounded by BLM VRM Class IV land.

Additional Work Required. Photographic simulation of visual impacts from the proposed solar facilities may be required to ensure that critical or culturally significant views are evaluated.

\subsection{Biological Resources}

Impacts to biological resources are typically one of the major concerns for the development of wind and solar energy facilities, especially impacts to birds and bats. In March 2012, the USFWS issued their Final Land-Based Wind Energy Guidelines (USFWS 2012a) to assist wind energy project developers avoid and minimize impacts of land-based wind projects on wildlife and their habitats. These guidelines represent the most current guidance from the USFWS. Additionally, the Arizona Game and Fish Department (AGFD) has released Guidelines for Reducing Impacts to Wildlife from Wind Energy Development in Arizona (AGFD 2009). The guidelines are intended to reduce impacts to both bats and birds from proposed wind energy projects in Arizona.

The federal and state guidelines include recommendations on preliminary screening; preconstruction study design; assessing direct, indirect, and cumulative impacts; developing avoidance and minimization measures; establishing mitigations; and post-construction monitoring, analysis, and reporting methods. The following sections detail the biological resources for the study area and discuss potential issues related to those resources. 


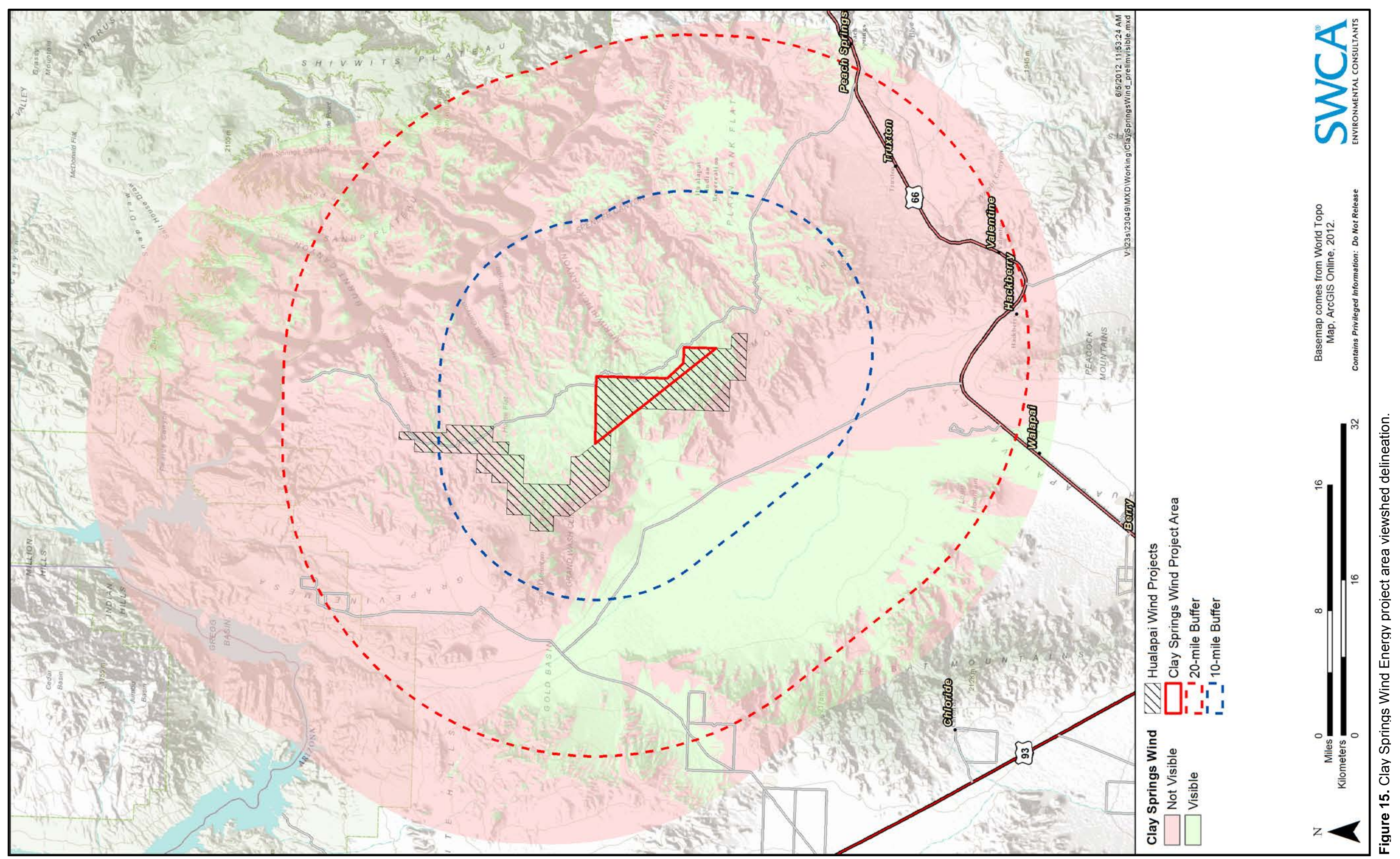


This page intentionally left blank. 


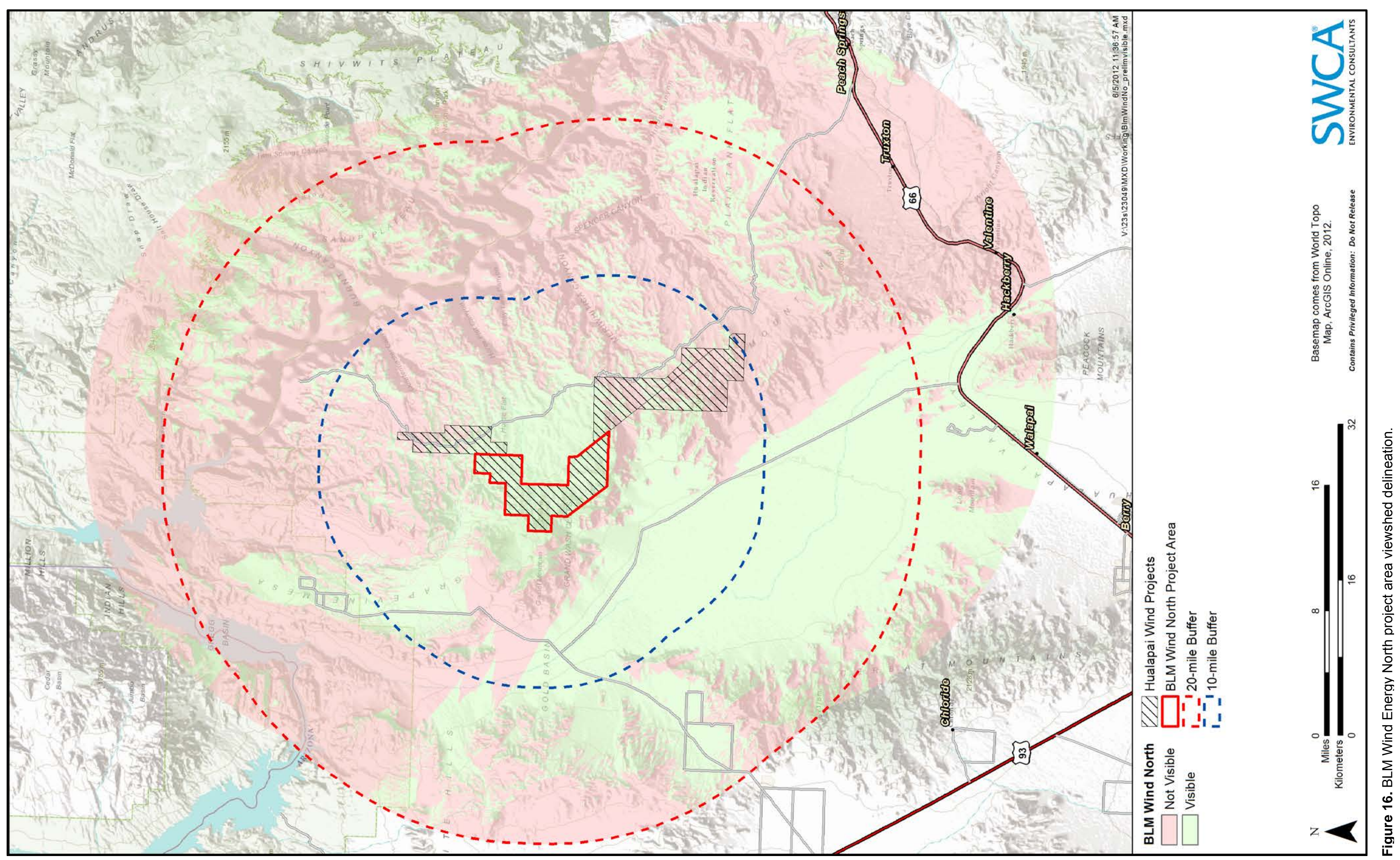


This page intentionally left blank. 


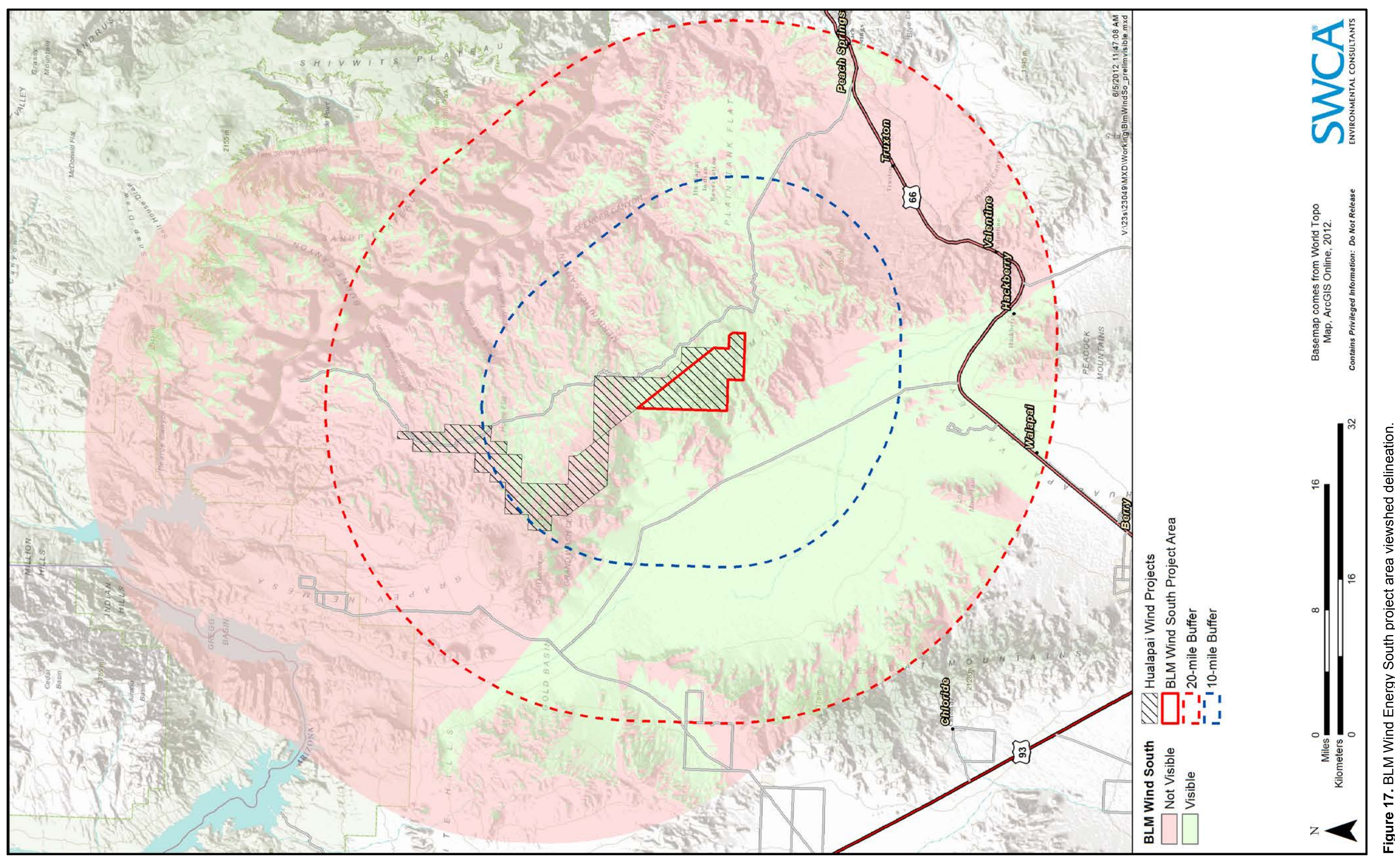


This page intentionally left blank. 


\subsubsection{General Vegetation}

The study area has the potential to contain 14 different general vegetation communities (USGS 2004) associated with western Arizona Mojave Desert Habitat (Figures 18, 19, 20). General descriptions of these vegetation communities are provided below. It should be noted that since Southwest Regional Gap Analysis (SWReGAP) data are coarse, ground-truthing may result in the identification of fewer vegetation communities, especially in the case of communities which comprise a minor percentage of cover within the project area. Generally, the loss of vegetation communities from the proposed project would not be considered a significant impact, unless those vegetation communities support sensitive wildlife or plant species (see Sections 3.8.2-3.8.5) or there are substantial amounts removed (i.e., $>10 \%$ of a species' range). In some cases, the BLM may also provide protection to a vegetation community because it is special or unique for the area.

The Arizona Native Plant Law (Arizona Revised Statutes [ARS] 3-904) states that protected native plants cannot be removed from any lands, including private lands, without permission and a permit from the Arizona Department of Agriculture (ADA). Highly Safeguarded native plants are those species for which removal is not allowed except with an ADA scientific permit. Salvage Restricted native plants are those plants for which a salvage permit is required. No Highly Safeguarded plant species have been identified within the study area.

Finally, the ADA defines noxious weeds as "any species of plant which is, or liable to be, detrimental or destructive and difficult to control or eradicate" (ARS 3-201). The management of weeds on BLM land is also guided by the BLM KFO.

The following sections provide general descriptions of the 14 different vegetation communities that have the potential to occur across the study area.

Sonora-Mojave Creosotebush-White Bursage Desert Scrub: This ecological system occurs in broad valleys, lower bajadas, plains, and low hills in the Mojave and lower Sonoran Deserts. This system ranges from sparse to moderately dense layer $(2 \%-50 \%$ cover). Creosotebush (Larrea tridentata) and white bursage (Ambrosia dumosa) are the typical dominant species, but a variety of shrub, dwarf-shrub, and cacti may be present to codominant. Additional species may include fourwing saltbush (Atriplex canescens), desert holly (Atriplex hymenelytra), brittlebush (Encelia farinosa), ephedra (Ephedra sp.), ocotillo (Fouquieria splendens), wolfberry (Lycium andersonii), and beavertail prickly pear (Opuntia basilaris). The herbaceous layer is sparse but may be seasonally abundant with annual species such as sandmat (Chamaesyce spp.), desert trumpet (Eriogonum inflatum), wooly fluffgrass (Erioneuron pulchella), forget-me-not (Cryptantha sp.), and scorpion weed (Phacelia sp.).

Sonoran Mid-Elevation Desert Scrub: This transitional ecological system has a climate which is too dry for chaparral species to be abundant and freezing temperatures during winter are too frequent and prolonged for many of the frost-sensitive species that are characteristic of the Paloverde Mixed-Cacti Desert Scrub. Substrates are generally rocky soils derived from parent materials such as limestone, granitic rocks, or rhyolite. The vegetation is typically composed of an open shrub layer of creosotebush, narrowleaf goldenbrush (Ericameria linearifolia), or Eastern Mohave buckwheat (Eriogonum fasciculatum) with taller shrub such as ocotillo, canotia (Canotia holacantha) (on limestone or granite), or jojoba (Simmondsia chinensis) (on rhyolite). The herbaceous layer is generally sparse. 


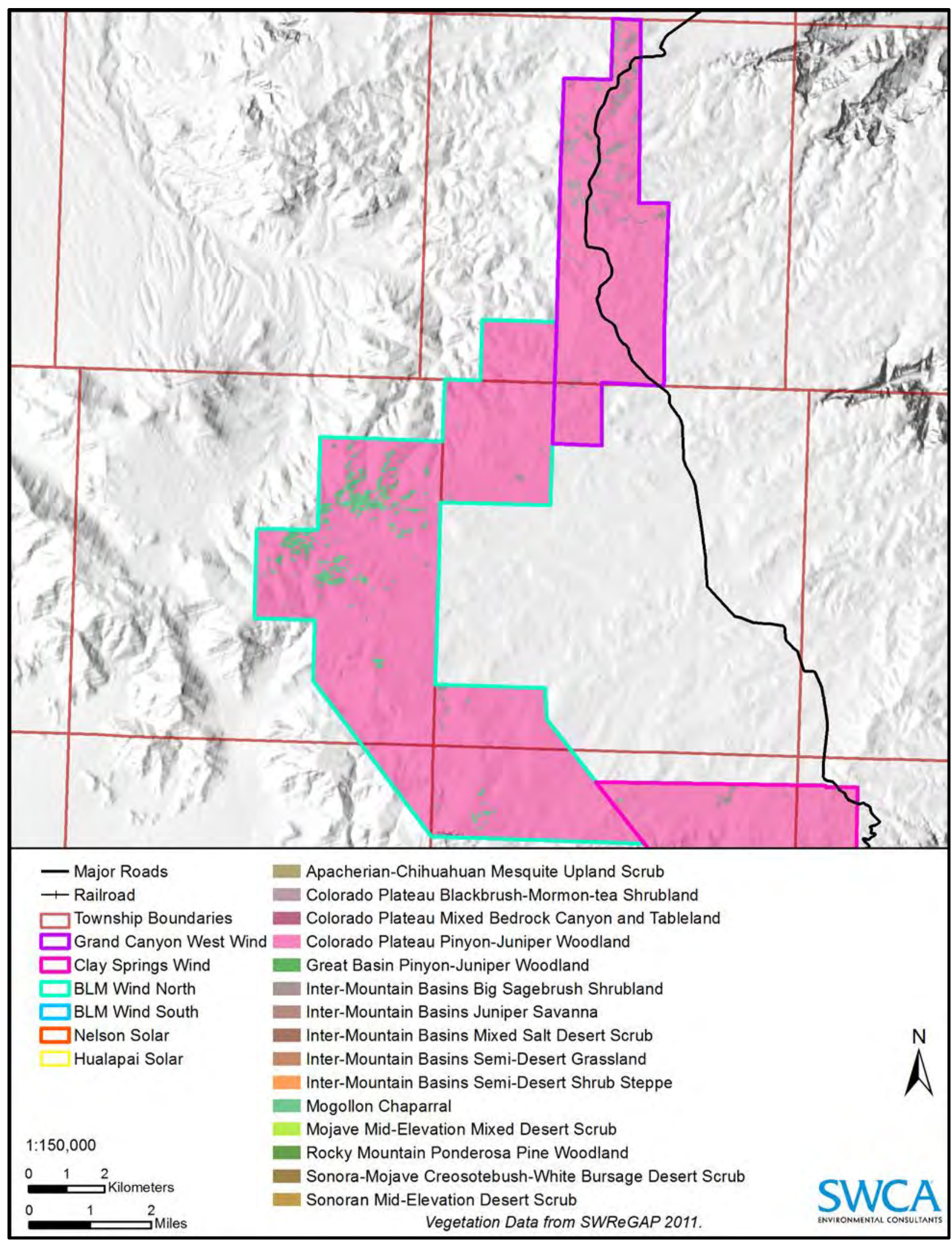

Figure 18. Vegetation communities within the Grand Canyon West Wind Energy and BLM Wind Energy North project areas. 


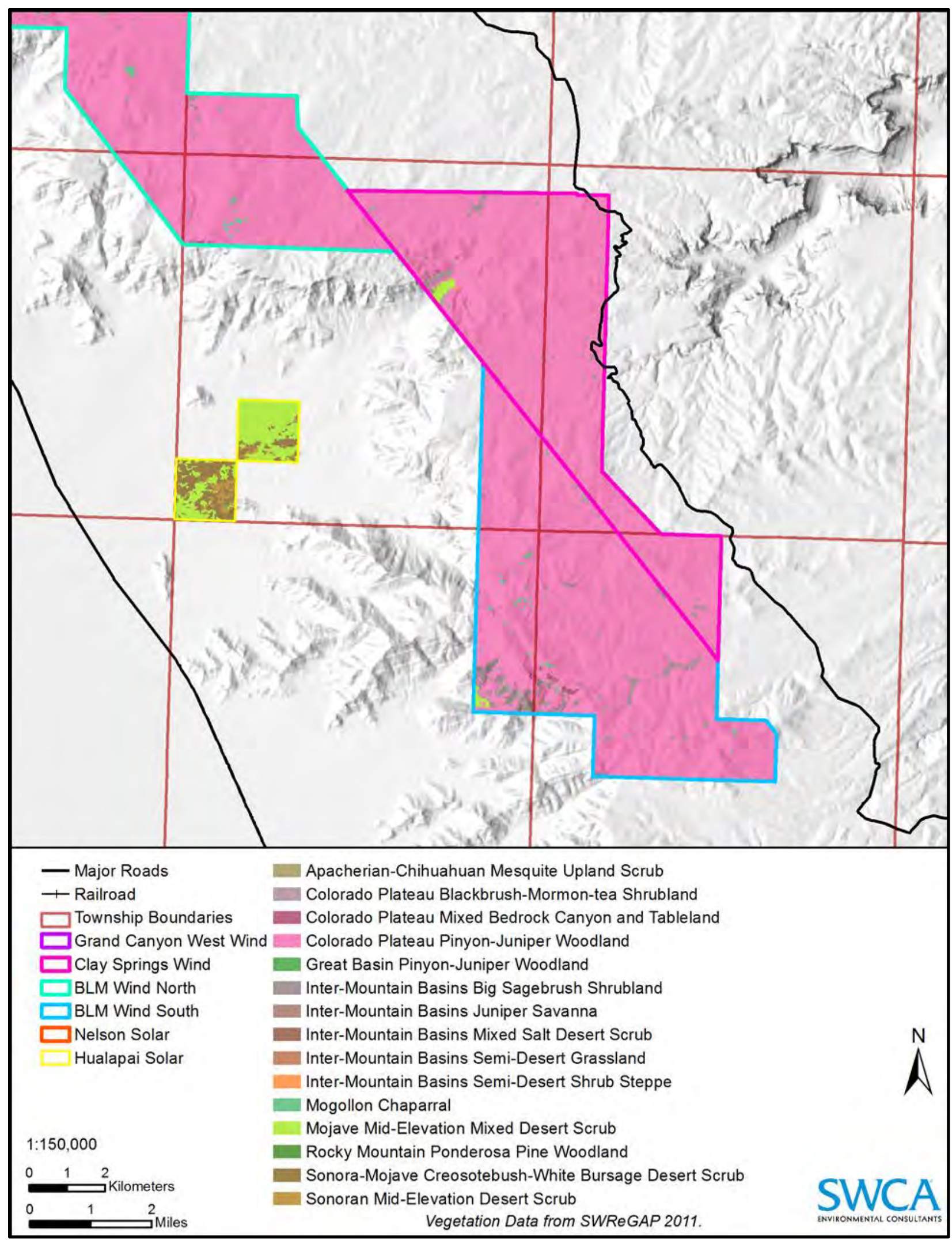

Figure 19. Vegetation communities within the Clay Springs Wind Energy, BLM Wind Energy South, and Hualapai Solar Energy project areas. 


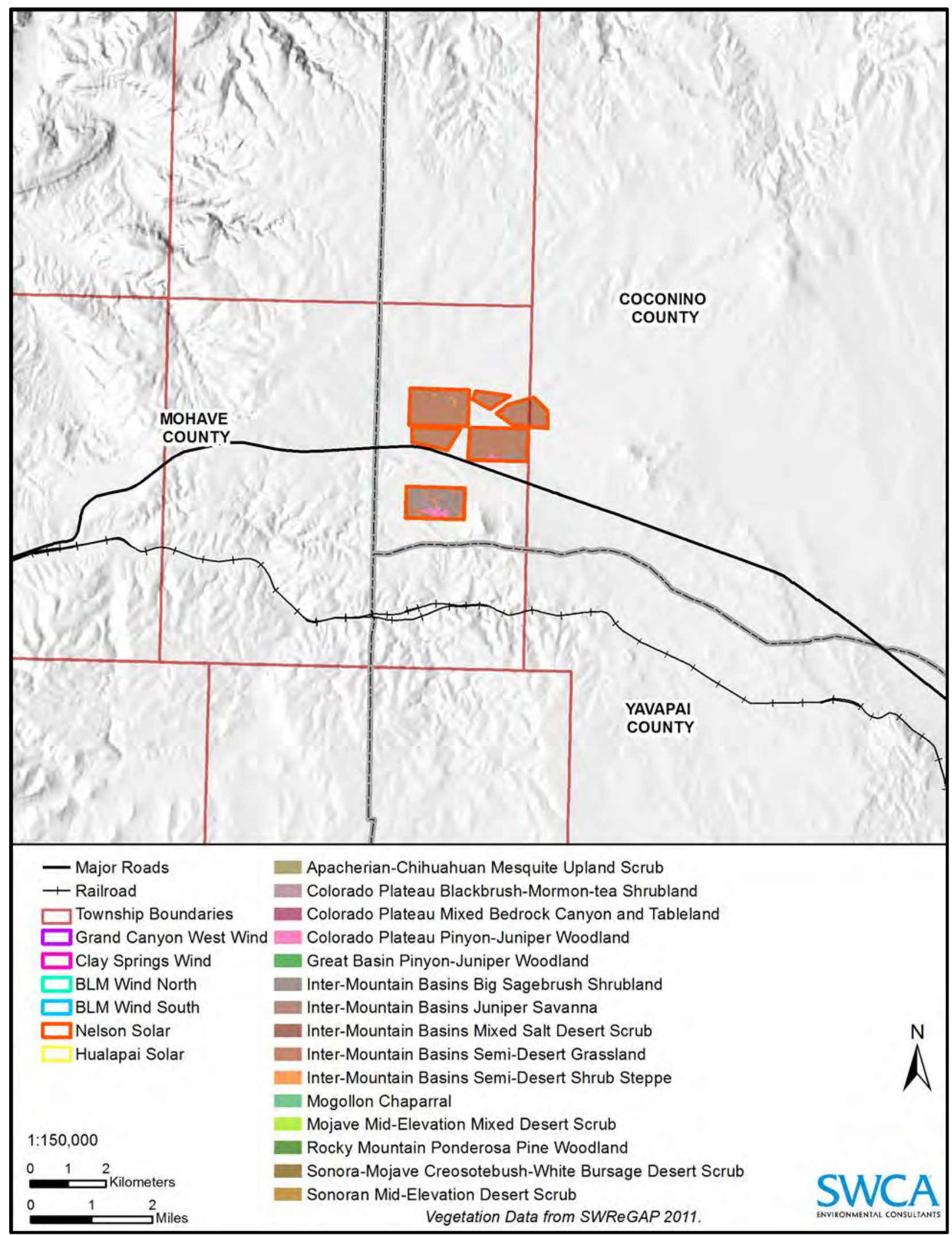

Figure 20. Vegetation communities within the Nelson Solar Energy project area. 
Apacherian-Chihuahuan Mesquite Upland Scrub: This ecological system occurs as upland shrublands with substrates typically derived from alluvium which limits infiltration and storage of winter precipitation in deeper soil layers. Vegetation is typically dominated by Arizona mesquite (Prosopis glandulosa) or velvet mesquite (Prosopis velutina) and succulents. Other desert scrub that may codominate or dominate includes viscid acacia (Acacia neovernicosa), whitethorn acacia (Acacia constricta), one-seed juniper (Juniperus monosperma), or redberry juniper (Juniperus coahuilensis). Grass cover is typically low. During the last century, the area occupied by this system has increased through conversion of desert grasslands as a result of drought, overgrazing by livestock, and/or decreases in fire frequency.

Great Basin Pinyon-Juniper Woodland: This ecological system occurs on dry mountain ranges, plateaus, and ridges of the Great Basin region at lower elevations ranging from 5,249 to 8,530 feet. Vegetation is composed of pure or nearly pure occurrences of Utah juniper (Juniperus osteosperma) and pinyon pine (Pinus monophylla), or woodlands dominated solely by Utah juniper. Curl-leaf mountain mahogany (Cercocarpus ledifolius) is commonly associated, and understory layers are variable.

Mojave Mid-Elevation Mixed Salt Desert Scrub: This ecological system occurs in the eastern central Mojave Desert. Diagnostic species include blackbrush (Coleogyne Ramosissima), Eastern Mojave buckwheat, Nevada ephedra (Ephedra nevadensis), spiny hopsage (Grayia spinosa), spiny menodora (Menodora spinescens), beargrass (Nolina spp.), buckhorn cholla (Opuntia acanthocarpa), Mexican bladdersage (Salazaria mexicana), Parish's goldeneye (Viguiera parishii), Joshua tree (Yucca brevifolia), or Mojave yucca (Yucca schidigera). Desert grasses include Indian ricegrass (Achnatherum hymenoides), bush muhly (Muhlenbergia porter), and big galleta (Pleuraphis rigida), which may form an herbaceous layer. Scattered Utah juniper or desert scrub species may also be found in this ecological system.

Colorado Plateau Blackbrush-Mormon-tea Shrubland: This ecological system occurs in the Colorado Plateau on benchlands, colluvial slopes, pediments, or bajadas. Elevation ranges from 1,837 to 5,413 feet. Substrates are shallow, typically calcareous, non-saline and gravelly or sandy soils over sandstone or limestone bedrock, caliche, or limestone alluvium. It also occurs in deeper soils on sandy plains where it may have invaded desert grasslands. The vegetation is characterized by extensive open shrublands dominated by blackbrush, often with Mormon tea (Ephedra viridis), Torrey's jointfir (Ephedra torreyana), or spiny hopsage. Sandy portions may include sand sagebrush (Artemisia filifolia) as codominant. The herbaceous layer is sparse and composed of graminoids such as Indian ricegrass, James' galleta (Pleuraphis jamesii), or sand dropseed (Sporobolus cryptandrus).

Colorado Plateau Mixed Bedrock Canyon and Tableland: The distribution of this ecological system is centered on the Colorado Plateau where it comprises barren and sparsely vegetated landscapes (generally $<10 \%$ plant cover) of steep cliff faces, narrow canyons, and open tablelands of predominantly sedimentary rocks, such as sandstone, shale, and limestone. Some eroding shale layers similar to Inter-Mountain Basins Shale Badland (CES304.789) may be interbedded between the harder rocks. The vegetation is characterized by very open tree canopy or scattered trees and shrubs with a sparse herbaceous layer. Common species includes twoneedle pinyon (Pinus edulis), ponderosa pine (Pinus ponderosa), juniper (Juniperus spp.), littleleaf mountain mahogany (Cercocarpus intricatus), and other short-shrub and herbaceous species, utilizing moisture from cracks and pockets where soil accumulates.

Colorado Plateau Pinyon-Juniper Woodland: This ecological system occurs in dry mountains and foothills of the Colorado Plateau region including the Western Slope of Colorado to the Wasatch Range, south to the Mogollon Rim, and east into the northwestern corner of New Mexico. It is typically found at lower elevations ranging from 4,920 to 8,005 feet. These woodlands occur on warm, dry sites on mountain slopes, mesas, plateaus, and ridges. Severe climatic events occurring during the growing season, such as frosts and drought, are thought to limit the distribution of pinyon-juniper woodlands to relatively 
narrow altitudinal belts on mountainsides. Soils supporting this system vary in texture ranging from stony, cobbly, gravelly sandy loams to clay loam or clay. Twoneedle pinyon and/or Utah juniper dominate the tree canopy. In the southern portion of the Colorado Plateau in northern Arizona and northwestern New Mexico, one-seed juniper and juniper hybrids of may dominate or codominate the tree canopy. Rocky Mountain juniper (Juniperus scopulorum) may codominate or replace Utah juniper at higher elevations. Understory layers are variable and may be dominated by shrubs, graminoids, or be absent. Associated species include greenleaf manzanita (Arctostaphylos patula), big sagebrush (Artemisia tridentata), littleleaf mountain mahogany, alderleaf mountain mahogany (Cercocarpus montanus), blackbrush, Stansbury cliffrose (Purshia stansburiana), antelope bitterbrush (Purshia tridentata), Gambel oak (Quercus gambelii), blue grama (Bouteloua gracilis), James' galleta, or muttongrass (Poa fendleriana). This system occurs at higher elevations than Great Basin Pinyon-Juniper Woodland and Colorado Plateau shrubland systems where sympatric.

Inter-Mountain Basins Big Sagebrush Shrubland: This ecological system occurs throughout much of the western United States, typically in broad basins between mountain ranges, plains, and foothills between 4,920 and 7,546 feet elevation. Soils are typically deep, well-drained and non-saline. These shrublands are dominated by big sagebrush. Scattered juniper, greasewood (Sarcobatus vermiculatus), and saltbush (Atriplex spp.) may be present in some stands. Rubber rabbitbrush (Ericameria nauseosa), yellow rabbitbrush (Chrysothamnus viscidiflorus), antelope bitterbrush, or snowberry (Symphoricarpos oreophilus) may codominate disturbed stands. Perennial herbaceous components typically contribute less than $25 \%$ vegetative cover. Common graminoid species include Indian ricegrass, blue grama, thickspike wheatgrass (Elymus lanceolatus), Idaho fescue (Festuca idahoensis), needle and thread (Hesperostipa comata), basin wildrye (Leymus cinereus), James' galleta, western wheatgrass (Pascopyrum smithii), Sandberg bluegrass (Poa secunda), or bluebunch wheatgreass (Pseudoroegneria spicata).

Inter-Mountain Basins Juniper Savanna: This widespread ecological system occupies dry foothills and sandsheets of western Colorado, northwestern New Mexico, northern Arizona, Utah, west into the Great Basin of Nevada and southern Idaho. It is typically found at lower elevations ranging from 4,920 to 7,546 feet. This system is generally found at lower elevations and more xeric sites than Great Basin PinyonJuniper Woodland (CES304.773) or Colorado Plateau Pinyon-Juniper Woodland (CES304.767). These occurrences are found on lower mountain slopes, hills, plateaus, basins, and flats, often where juniper is expanding into semi-desert grasslands and steppe. The vegetation is typically open savanna, although there may be inclusions of denser juniper woodlands. This savanna is typically dominated by Utah juniper trees with high cover of perennial bunch grasses and forbs, with blue grama, needle and thread, and James' galleta being most common. In the southern Colorado Plateau, one-seed juniper or juniper hybrids may dominate the tree layer. Pinyon trees are typically not present because sites are outside the ecological or geographic range of twoneedle pinyon and pinyon pine.

Inter-Mountain Basins Mixed Salt Desert Scrub: This extensive ecological system includes opencanopied shrublands of typically saline basins, alluvial slopes and plains across the Intermountain western United States. This type also extends in limited distribution into the southern Great Plains. Substrates are often saline and calcareous, medium- to fine-textured, alkaline soils, but include some coarser-textured soils. The vegetation is characterized by a typically open to moderately dense shrubland composed of one or more Atriplex species. Other shrubs present to codominate may include Wyoming big sagebrush (Artemisia tridentata ssp. wyomingensis), yellow rabbitbrush, rubber rabbitbrush, Nevada jointfir (Ephedra nevadensis), spiny hopsage, winterfat (Krascheninnikovia lanata), desert-thorn (Lycium spp.), bud sagebrush (Picrothamnus desertorum), or horsebrush (Tetradymia spp.). Greasewood is generally absent, but if present does not codominate. The herbaceous layer varies from sparse to moderately dense and is dominated by perennial graminoids such as Indian ricegrass, blue grama, thickspike wheatgrass, western wheatgrass, James' galleta, big galleta (Pleuraphis rigida), Sandberg bluegrass, or alkali sacaton (Sporobolus airoides). Various forbs are also present. 
Inter-Mountain Basins Semi-Desert Grassland: This widespread ecological system occurs throughout the intermountain western United States on dry plains and mesas, at approximately 4,750 to 7,610 feet elevation. These grasslands occur in lowland and upland areas and may occupy swales, playas, mesa tops, plateau parks, alluvial flats, and plains, but sites are typically xeric. Substrates are often well-drained sandy or loamy-textured soils derived from sedimentary parent materials but are quite variable and may include fine-textured soils derived from igneous and metamorphic rocks. When they occur near foothill grasslands they will be at lower elevations. The dominant perennial bunch grasses and shrubs within this system are all very drought-resistant plants. These grasslands are typically dominated or codominated by Indian ricegrass, threeawn (Aristida spp.), blue grama, needle and thread, muhly (Muhlenbergia sp.), or James' galleta and may include scattered shrubs and dwarf-shrubs of species of sagebrush, saltbush, blackbrush, ephedra, snakeweed (Gutierrezia sp.), or winterfat.

Mogollon Chaparral (Chaparral): This ecological system is somewhat limited in its distribution, occurring within central Arizona along the Mogollon Rim and into western New Mexico and southern Utah and Nevada. The Chaparral land cover class occurs in drier habitats and on foothills, mountain slopes, and canyons and is often associated with limestone, basalt, or alluvial substrates (USGS 2005). Developed shrub communities characterize this vegetation community and common species include scrub oaks (Quercus turbinella, Q. toumeyi), alderleaf mountain mahogany, ceanothus (Ceanothus greggii), Stansbury cliffrose, and others.

Rocky Mountain Ponderosa Pine Woodland (Ponderosa Pine Woodland): This widespread land cover class is found scattered throughout the West at elevations ranging from approximately 6,200 to 9,185 feet (USGS 2005). Although this land cover class occurs on all slopes and aspects, it is typically found on moderate to steep slopes and along ridgelines (USGS 2005). Two-needle pinyon pine and juniper may be found growing within this land cover class (USGS 2005). The understory includes a variety of shrub species including sagebrush, manzanita (Arctostaphylos spp.), bitterbrush, and serviceberry (Amelanchier spp.) (USGS 2005). Some grasses may occur and could include needle and thread, needlegrass (Achnatherum spp.), muhly grasses (Muhlenbergia spp.), and grama grasses (Bouteloua eriopoda, B. hirsuta, B. rothrockii, B. curtipendula, and B. gracilis).

\section{GRAND CANYON WEST WIND ENERGY PROJECT AREA}

The Grand Canyon West Wind Energy project area consists primarily of pinyon-juniper woodland (see Figure 18). This vegetation community is widespread throughout northern Arizona. Table 3 summarizes the vegetation communities within the project area.

Table 3. Vegetation Communities in the Grand Canyon West Wind Energy Project Area

\begin{tabular}{lc}
\hline Vegetation Community & Acres in Project Area \\
\hline Colorado Plateau Pinyon-Juniper Woodland & $5,385.54$ \\
\hline Inter-Mountain Basins Big Sagebrush Shrubland & 54.15 \\
\hline Colorado Plateau Blackbrush-Mormon-tea Shrubland & 410.20 \\
\hline Inter-Mountain Basins Juniper Savanna & 0.16 \\
\hline Inter-Mountain Basins Semi-Desert Shrub Steppe & 0.04 \\
\hline Total & $\mathbf{5 , 8 5 0 . 0 9}$ \\
\hline
\end{tabular}

Risk Category. Low. Preliminary data indicate that vegetation communities that support sensitive wildlife or plant species or are unique to the area are not present within the project area. 
Additional Work Required. Additional short-term work is anticipated to complete the evaluation of vegetation within the project area. Salvage permit and plant salvage, a noxious weed risk assessment, weed management plan, and preparation of a vegetation restoration and reclamation plan will likely be required.

\section{CLAY SPRINGS WIND ENERGY PROJECT AREA}

The Clay Springs Wind Energry project area consists primarily of pinyon-juniper woodland (see Figure 19). This vegetation community is widespread throughout northern Arizona. Table 4 summarizes the vegetation communities within the project area.

Table 4. Vegetation Communities in the Clay Springs Wind Energy Project Area

\begin{tabular}{lc}
\hline Vegetation Community & Acres in Project Area \\
\hline Colorado Plateau Mixed Bedrock Canyon and Tableland & 2.21 \\
\hline Colorado Plateau Pinyon-Juniper Woodland & $8,367.87$ \\
\hline Inter-Mountain Basins Big Sagebrush Shrubland & 74.25 \\
\hline Mogollon Chaparral & 4.62 \\
\hline Colorado Plateau Blackbrush-Mormon-tea Shrubland & 60.41 \\
\hline Mojave Mid-Elevation Mixed Desert Scrub & 50.43 \\
\hline Inter-Mountain Basins Juniper Savanna & 0.83 \\
\hline Inter-Mountain Basins Semi-Desert Shrub Steppe & 3.20 \\
\hline Inter-Mountain Basins Semi-Desert Grassland & 0.62 \\
\hline Total & $\mathbf{8 , 5 6 4 . 4 4}$ \\
\hline
\end{tabular}

Risk Category. Low. Preliminary data indicate that vegetation communities that support sensitive wildlife or plant species or are unique to the area are not present within the project area.

Additional Work Required. Additional short-term work is anticipated to complete the evaluation of vegetation within the project area. Salvage permit and plant salvage, a noxious weed risk assessment, weed management plan, and preparation of a vegetation restoration and reclamation plan will likely be required.

\section{BLM WIND ENERGY NORTH PROJECT AREA}

The BLM Wind Energy North project area consists primarily of pinyon-juniper woodland (see Figure 18). This vegetation community is widespread throughout northern Arizona. Table 5 summarizes the vegetation communities within the project area.

Risk Category. Low. Preliminary data indicate that vegetation communities that support sensitive wildlife or plant species or are unique to the area are not present within the project area.

Additional Work Required. Additional short-term work is anticipated to complete the evaluation of vegetation within the project area. Salvage permit and plant salvage, a noxious weed risk assessment, weed management plan, and preparation of a vegetation restoration and reclamation plan will likely be required. 
Table 5. Vegetation Communities in the BLM Wind Energy North Project Area

\begin{tabular}{lc}
\hline Vegetation Community & Acres in Project Site \\
\hline Colorado Plateau Mixed Bedrock Canyon and Tableland & 7.03 \\
\hline Rocky Mountain Ponderosa Pine Woodland & 0.76 \\
\hline Colorado Plateau Pinyon-Juniper Woodland & $14,124.94$ \\
\hline Inter-Mountain Basins Big Sagebrush Shrubland & 46.50 \\
\hline Mogollon Chaparral & 578.68 \\
\hline Colorado Plateau Blackbrush-Mormon-tea Shrubland & 4.49 \\
\hline Inter-Mountain Basins Semi-Desert Shrub Steppe & 0.70 \\
\hline Total & $\mathbf{1 4 , 7 6 3 . 1 0}$ \\
\hline
\end{tabular}

\section{BLM WIND ENERGY SOUTH PROJECT AREA}

The BLM Wind Energy South project area consists primarily of pinyon-juniper woodland (see Figure 19). This vegetation community is widespread throughout northern Arizona. Table 6 summarizes the vegetation communities within the project area.

Table 6. Vegetation Communities in the BLM Wind Energy South Project Area

\begin{tabular}{lc}
\hline Vegetation Community & Acres in Project Area \\
\hline Colorado Plateau Mixed Bedrock Canyon and Tableland & 22.17 \\
\hline Colorado Plateau Pinyon-Juniper Woodland & $10,005.67$ \\
\hline Inter-Mountain Basins Big Sagebrush Shrubland & 315.58 \\
Mogollon Chaparral & 40.81 \\
\hline Colorado Plateau Blackbrush-Mormon-tea Shrubland & 85.63 \\
\hline Mojave Mid-Elevation Mixed Desert Scrub & 30.38 \\
\hline Inter-Mountain Basins Mixed Salt Desert Scrub & 4.12 \\
\hline Inter-Mountain Basins Semi-Desert Shrub Steppe & 3.05 \\
\hline Total & $\mathbf{1 0 , 5 0 7 . 4 1}$ \\
\hline
\end{tabular}

Risk Category. Low. Preliminary data indicate that vegetation communities that support sensitive wildlife or plant species or are unique to the area are not present within the project area.

Additional Work Required. Additional short-term work is anticipated to complete the evaluation of vegetation within the project area. Native plant survey, salvage permit and plant salvage, a noxious weed risk assessment, weed management plan, and preparation of a vegetation restoration and reclamation plan will likely be required.

\section{NELSON SOLAR ENERGY PROJECT AREA}

The Nelson Solar Energy project area consists primarily of semi-desert grassland (see Figure 20). This vegetation community is widespread throughout the Arizona Mojave Desert. Table 7 summarizes the vegetation communities within the project area. 
Table 7. Vegetation Communities in the Nelson Solar Energy Project Area

\begin{tabular}{lc}
\hline Vegetation Community & Acres in Project Area \\
\hline Colorado Plateau Pinyon-Juniper Woodland & 43.08 \\
\hline Inter-Mountain Basins Big Sagebrush Shrubland & 192.46 \\
\hline Inter-Mountain Basins Mixed Salt Desert Scrub & 3.50 \\
\hline Inter-Mountain Basins Semi-Desert Shrub Steppe & 23.18 \\
\hline Inter-Mountain Basins Semi-Desert Grassland & 821.70 \\
\hline Total & $\mathbf{1 , 0 8 3 . 9 2}$ \\
\hline
\end{tabular}

Risk Category. Low. Preliminary data indicate that vegetation communities that support sensitive wildlife or plant species or are unique to the area are not present within the project area.

Additional Work Required. Additional short-term work is anticipated to complete the evaluation of vegetation within the project area. Salvage permit and plant salvage, a noxious weed risk assessment, weed management plan, and preparation of a vegetation restoration and reclamation plan will likely be required.

\section{HUALAPAI SOLAR ENERGY PROJECT AREA}

The Hualapai Solar Energy site consists mainly of mixed desert scrub and creosotebush communities (see Figure 19). These communities are widespread throughout the Arizona Mojave Desert. Table 8 summarizes the vegetation communities within the project area.

Table 8. Vegetation Communities in the Hualapai Solar Energy Project Area

\begin{tabular}{lc}
\hline Vegetation Community & Acres in Project Area \\
\hline Great Basin Pinyon-Juniper Woodland & 5.11 \\
\hline Apacherian-Chihuahuan Mesquite Upland Scrub & 3.03 \\
\hline Mojave Mid-Elevation Mixed Desert Scrub & 743.15 \\
\hline Sonora-Mojave Creosotebush-White Bursage Desert Scrub & 421.38 \\
\hline Sonoran Mid-Elevation Desert Scrub & 104.79 \\
\hline Total & $\mathbf{1 , 2 7 7 . 4 6}$ \\
\hline
\end{tabular}

Risk Category. Low. Preliminary data indicate that vegetation communities that support sensitive wildlife or plant species or are unique to the area are not present within the project area.

Additional Work Required. Additional short-term work is anticipated to complete the evaluation of vegetation within the project area. Salvage permit and plant salvage, a noxious weed risk assessment, weed management plan, and preparation of a vegetation restoration and reclamation plan will likely be required.

\subsubsection{Special Status Species}

Special status species include any species which is listed, or proposed for listing, as threatened or endangered by the USFWS under the provisions of the ESA; any species designated by the USFWS as a candidate or species of concern, and any species listed by the State of Arizona. The Arizona Heritage Data Management System (HDMS) indicates that 160 special status species are known to occur within 
Mohave and Coconino Counties (Table 9). Table 9 also includes an evaluation of occurrence potential within the study area based on habitat requirements from HDMS (AGFD 2012a) and a query of the AGFD HDMS online tool (AZHGIS 2012).

Table 9. Special Status Species Known to Occur in Mohave and Coconino Counties

\begin{tabular}{|c|c|c|c|c|c|c|}
\hline Common Name & Scientific Name & County* & $\begin{array}{l}\text { USFWS } \\
\text { Status }\end{array}$ & $\begin{array}{c}\text { BLM }_{\text {Status }}^{\dagger, \S} \\
\end{array}$ & State Status ${ }^{\dagger, \pi}$ & $\begin{array}{c}\text { Potential for } \\
\text { Occurrence } \\
\text { within the } \\
\text { Study Area }\end{array}$ \\
\hline \multicolumn{7}{|l|}{ Plants } \\
\hline Bigelow onion & Allium bigelovii & M & & & SR & $\mathrm{L}$ \\
\hline $\begin{array}{l}\text { Las Vegas } \\
\text { bearpoppy }\end{array}$ & Arctomecon californica & M & SC & & SR & $\mathrm{L}$ \\
\hline $\begin{array}{l}\text { Roaring springs } \\
\text { prickly- poppy }\end{array}$ & Argemone arizonica & C & SC & & & $\mathrm{L}$ \\
\hline Welsh's milkweed & Asclepias welshii & $\mathrm{C}$ & LT & & SR & L \\
\hline Gumbo milkvetch & Astragalus ampullarius & C, M & SC & & & $\mathrm{L}$ \\
\hline Sentry milkvetch & $\begin{array}{l}\text { Astragalus } \\
\text { cremnophylax var. } \\
\text { cremnophylax }\end{array}$ & C & LE & & HS & L \\
\hline $\begin{array}{l}\text { Marble Canyon } \\
\text { milkvetch }\end{array}$ & $\begin{array}{l}\text { Astragalus } \\
\text { cremnophylax var. } \\
\text { hevronii }\end{array}$ & C & & $S$ & & $\mathrm{~L}$ \\
\hline Cliff milkvetch & $\begin{array}{l}\text { Astragalus } \\
\text { cremnophylax var. } \\
\text { myriorrhaphis }\end{array}$ & C & SC & $S$ & SR & $\mathrm{L}$ \\
\hline $\begin{array}{l}\text { Beaver Dam } \\
\text { milkvetch }\end{array}$ & $\begin{array}{l}\text { Astragalus geyeri var. } \\
\text { triquetrus }\end{array}$ & M & SC & $S$ & & $\mathrm{~L}$ \\
\hline $\begin{array}{l}\text { Holmgren (Paradox) } \\
\text { milkvetch }\end{array}$ & $\begin{array}{l}\text { Astragalus } \\
\text { holmgreniorum }\end{array}$ & M & LE & & HS & L \\
\hline Freckled milkvetch & $\begin{array}{l}\text { Astragalus lentiginosus } \\
\text { var. ambiguus }\end{array}$ & M & SC & & & $\mathrm{L}$ \\
\hline Aquarius milkvetch & $\begin{array}{l}\text { Astragalus newberryi } \\
\text { var. aquarii }\end{array}$ & M & & $S$ & & $\mathrm{~L}$ \\
\hline $\begin{array}{l}\text { Diamond Butte } \\
\text { milkvetch }\end{array}$ & $\begin{array}{l}\text { Astragalus toanus var. } \\
\text { scidulus }\end{array}$ & M & & $S$ & & $\mathrm{~L}$ \\
\hline Gladiator milkvetch & Astragalus xiphoides & C & SC & & SR & $\mathrm{L}$ \\
\hline Dainty moonwort & Botrychium crenulatum & $\mathrm{C}$ & SC & & & $\mathrm{L}$ \\
\hline Golden suncup & Camissonia brevipes & M & SC & & & $\mathrm{L}$ \\
\hline $\begin{array}{l}\text { Slender evening- } \\
\text { primrose }\end{array}$ & Camissonia exilis & $C, M$ & SC & & SR & L \\
\hline $\begin{array}{l}\text { Grand Canyon } \\
\text { evening-primrose }\end{array}$ & $\begin{array}{l}\text { Camissonia specuicola } \\
\text { ssp. hesperia }\end{array}$ & $\mathrm{C}, \mathrm{M}$ & SC & & & L \\
\hline
\end{tabular}


Table 9. Special Status Species Known to Occur in Mohave and Coconino Counties (Continued)

\begin{tabular}{|c|c|c|c|c|c|c|}
\hline Common Name & Scientific Name & County* & $\begin{array}{l}\text { USFWS } \\
\text { Status }^{\dagger, \neq}\end{array}$ & $\begin{array}{l}\text { BLM } \\
\text { Status }\end{array}$ & State Status $s^{\dagger, \pi}$ & $\begin{array}{l}\text { Potential for } \\
\text { Occurrence } \\
\text { within the } \\
\text { Study Area }\end{array}$ \\
\hline \multicolumn{7}{|l|}{ Plants, cont'd. } \\
\hline Navajo sedge & Carex specuicola & $\mathrm{C}$ & LT & & HS & L \\
\hline $\begin{array}{l}\text { Tusayan } \\
\text { rabbitbrush }\end{array}$ & $\begin{array}{l}\text { Chrysothamnus } \\
\text { molestus }\end{array}$ & C & SC & & & L \\
\hline Arizona bugbane & Cimicifuga arizonica & $\mathrm{C}$ & SC & & HS & L \\
\hline Parry's thistle & $\begin{array}{l}\text { Cirsium parryi ssp. } \\
\text { mogollonicum }\end{array}$ & C & SC & & SR & L \\
\hline Missouri corycactus & $\begin{array}{l}\text { Coryphantha } \\
\text { missouriensis }\end{array}$ & M & & & SR & L \\
\hline Smooth catseye & Cryptantha semiglabra & M & & $S$ & & L \\
\hline Jones' cycladenia & $\begin{array}{l}\text { Cycladenia humilis var. } \\
\text { jonesii }\end{array}$ & M & LT & & HS & L \\
\hline $\begin{array}{l}\text { Cameron water- } \\
\text { parsley }\end{array}$ & $\begin{array}{l}\text { Cymopterus } \\
\text { megacephalus }\end{array}$ & $\mathrm{C}$ & SC & & & $\mathrm{L}$ \\
\hline $\begin{array}{l}\text { Clustered barrel } \\
\text { cactus }\end{array}$ & $\begin{array}{l}\text { Echinocactus } \\
\text { polycephalus var. } \\
\text { polycephalus }\end{array}$ & M & & & SR & M \\
\hline $\begin{array}{l}\text { Grand Canyon } \\
\text { cottontop cactus }\end{array}$ & $\begin{array}{l}\text { Echinocactus } \\
\text { polycephalus var. } \\
\text { xeranthemoides }\end{array}$ & M & & & SR & $\mathrm{H}$ \\
\hline Silverleaf sunray & Enceliopsis argophylla & M & & $S$ & & $\mathrm{~L}$ \\
\hline $\begin{array}{l}\text { Morton wild- } \\
\text { buckwheat }\end{array}$ & $\begin{array}{l}\text { Eriogonum } \\
\text { mortonianum }\end{array}$ & M & SC & & SR & $\mathrm{L}$ \\
\hline $\begin{array}{l}\text { Ripley wild- } \\
\text { buckwheat }\end{array}$ & Eriogonum ripleyi & $\mathrm{C}$ & SC & & SR & M \\
\hline $\begin{array}{l}\text { Atwood wild- } \\
\text { buckwheat }\end{array}$ & $\begin{array}{l}\text { Eriogonum } \\
\text { thompsoniae var. } \\
\text { atwoodii }\end{array}$ & M & SC & & SR & $\mathrm{L}$ \\
\hline Sticky buckwheat & Eriogonum viscidulum & M & SC & & & L \\
\hline $\begin{array}{l}\text { Roundleaf } \\
\text { errazurizia }\end{array}$ & Errazurizia rotundata & C & & S & SR & $\mathrm{L}$ \\
\hline $\begin{array}{l}\text { Viviparous foxtail } \\
\text { cactus }\end{array}$ & $\begin{array}{l}\text { Escobaria vivipara var. } \\
\text { rosea }\end{array}$ & M & & & SR & $\mathrm{L}$ \\
\hline Desert barrel cactus & $\begin{array}{l}\text { Ferocactus } \\
\text { cylindraceus }\end{array}$ & C & & & SR & L \\
\hline $\begin{array}{l}\text { Grand Canyon } \\
\text { flaveria }\end{array}$ & Flaveria mcdougallii & $C, M$ & & & SR & $\mathrm{L}$ \\
\hline Flannelbush & $\begin{array}{l}\text { Fremontodendron } \\
\text { californicum }\end{array}$ & M & & S & SR & $\mathrm{L}$ \\
\hline $\begin{array}{l}\text { Flagstaff false } \\
\text { pennyroyal }\end{array}$ & Hedeoma diffusa & C & & & SR & L \\
\hline Kaibab bladderpod & $\begin{array}{l}\text { Lesquerella } \\
\text { kaibabensis }\end{array}$ & C & SC & & & $\mathrm{L}$ \\
\hline $\begin{array}{l}\text { Broadleaf } \\
\text { twayblade }\end{array}$ & Listera convallarioides & C & & & SR & L \\
\hline
\end{tabular}


Table 9. Special Status Species Known to Occur in Mohave and Coconino Counties (Continued)

\begin{tabular}{|c|c|c|c|c|c|c|}
\hline Common Name & Scientific Name & County* & $\begin{array}{l}\text { USFWS } \\
\text { Status }\end{array}$ & $\begin{array}{c}\text { BLM } \\
\text { Status }\end{array}$ & State Status ${ }^{\dagger, \pi}$ & $\begin{array}{c}\text { Potential for } \\
\text { Occurrence } \\
\text { within the } \\
\text { Study Area }\end{array}$ \\
\hline \multicolumn{7}{|l|}{ Plants, cont'd. } \\
\hline $\begin{array}{l}\text { Purple adder's } \\
\text { mouth }\end{array}$ & Malaxis porphyrea & C & & & SR & L \\
\hline $\begin{array}{l}\text { Varied fishhook } \\
\text { cactus }\end{array}$ & Mammillaria viridiflora & M & & & SR & L \\
\hline $\begin{array}{l}\text { September } 11 \\
\text { stickleaf }\end{array}$ & Mentzelia memorabalis & $\mathrm{M}$ & & $S$ & & L \\
\hline Yellow beavertail & $\begin{array}{l}\text { Opuntia basilaris var. } \\
\text { aurea }\end{array}$ & $C, M$ & & & SR & L \\
\hline $\begin{array}{l}\text { Grand Canyon } \\
\text { beavertail cactus }\end{array}$ & $\begin{array}{l}\text { Opuntia basilaris var. } \\
\text { longiareolata }\end{array}$ & $\mathrm{C}, \mathrm{M}$ & & & SR & L \\
\hline Straw-top cholla & Opuntia echinocarpa & M & & & SR & L \\
\hline $\begin{array}{l}\text { Navajo Bridge } \\
\text { cactus }\end{array}$ & Opuntia nicholii & $\mathrm{C}, \mathrm{M}$ & & & SR & L \\
\hline $\begin{array}{l}\text { Kingman's prickly- } \\
\text { pear }\end{array}$ & Opuntia superbospina & $M$ & & & SR & L \\
\hline $\begin{array}{l}\text { Blue Diamond } \\
\text { cholla }\end{array}$ & $\begin{array}{l}\text { Opuntia whipplei var. } \\
\text { multigeniculata }\end{array}$ & M & SC & & SR & L \\
\hline Whipple cholla & $\begin{array}{l}\text { Opuntia whipplei var. } \\
\text { whipplei }\end{array}$ & M & & & SR & L \\
\hline $\begin{array}{l}\text { San Francisco } \\
\text { Peaks ragwort }\end{array}$ & Packera franciscana & C & LT & & HS & L \\
\hline $\begin{array}{l}\text { Brady pincushion } \\
\text { cactus }\end{array}$ & Pediocactus bradyi & $\mathrm{C}$ & LE & & HS & L \\
\hline $\begin{array}{l}\text { Kaibab pincushion } \\
\text { cactus }\end{array}$ & Pediocactus paradinei & C & SC & S & HS & L \\
\hline $\begin{array}{l}\text { Fickeisen Plains } \\
\text { cactus }\end{array}$ & $\begin{array}{l}\text { Pediocactus } \\
\text { peeblesianus var. } \\
\text { fickeiseniae }\end{array}$ & $C, M$ & $\mathrm{C}$ & & HS & L \\
\hline $\begin{array}{l}\text { Siler pincushion } \\
\text { cactus }\end{array}$ & Pediocactus sileri & $C, M$ & LT & $S$ & HS & L \\
\hline $\begin{array}{l}\text { Simpson plains } \\
\text { cactus }\end{array}$ & Pediocactus simpsonii & C & & & SR & L \\
\hline $\begin{array}{l}\text { Beaver Dam scurf- } \\
\text { pea }\end{array}$ & $\begin{array}{l}\text { Pediomelum } \\
\text { castoreum }\end{array}$ & M & SC & & & L \\
\hline Kane scurf-pea & Pediomelum epipsilum & M & SC & & & $\mathrm{L}$ \\
\hline $\begin{array}{l}\text { White-margined } \\
\text { penstemon }\end{array}$ & $\begin{array}{l}\text { Penstemon } \\
\text { albomarginatus }\end{array}$ & $M$ & SC & $S$ & SR & L \\
\hline Cerbat beardtongue & $\begin{array}{l}\text { Penstemon bicolor ssp. } \\
\text { roseus }\end{array}$ & M & SC & $S$ & SR & L \\
\hline $\begin{array}{l}\text { Sunset Crater } \\
\text { beardtongue }\end{array}$ & Penstemon clutei & C & SC & & SR & L \\
\hline $\begin{array}{l}\text { Mt. Trumbull } \\
\text { beardtongue }\end{array}$ & Penstemon distans & M & SC & S & SR & L \\
\hline Parish's phacelia & Phacelia parishii & M & & $S$ & & L \\
\hline Cinder phacelia & Phacelia serrata & C & SC & & & L \\
\hline
\end{tabular}


Table 9. Special Status Species Known to Occur in Mohave and Coconino Counties (Continued)

\begin{tabular}{|c|c|c|c|c|c|c|}
\hline Common Name & Scientific Name & County* & $\begin{array}{l}\text { USFWS } \\
\text { Status }\end{array}$ & $\begin{array}{c}\text { BLM } \\
\text { Status }\end{array}$ & State Status ${ }^{\dagger, \pi}$ & $\begin{array}{c}\text { Potential for } \\
\text { Occurrence } \\
\text { within the } \\
\text { Study Area }\end{array}$ \\
\hline \multicolumn{7}{|l|}{ Plants, cont'd. } \\
\hline Welsh phacelia & Phacelia welshii & C & SC & & & $\mathrm{L}$ \\
\hline $\begin{array}{l}\text { Rocky Mountain } \\
\text { bristlecone pine }\end{array}$ & Pinus aristata & $\mathrm{C}$ & & & SR & L \\
\hline Alcove bog-orchid & Platanthera zothecina & C & SC & & & $\mathrm{L}$ \\
\hline Mohave indigo bush & $\begin{array}{l}\text { Psorothamnus } \\
\text { arborescens var. } \\
\text { pubescens }\end{array}$ & $\mathrm{C}, \mathrm{M}$ & & $S$ & & L \\
\hline Whiting indigo bush & $\begin{array}{l}\text { Psorothamnus } \\
\text { thompsonae var. } \\
\text { whitingii }\end{array}$ & C & SC & & & $\mathrm{L}$ \\
\hline Parish alkali grass & Puccinellia parishii & C & SC & & HS & L \\
\hline Arizona cliffrose & Purshia subintegra & M & LE & & HS & $\mathrm{L}$ \\
\hline Grand Canyon rose & $\begin{array}{l}\text { Rosa stellata ssp. } \\
\text { abyssa }\end{array}$ & $\mathrm{C}, \mathrm{M}$ & SC & $S$ & SR & L \\
\hline Bloomer's dock & Rumex orthoneurus & C & SC & & HS & L \\
\hline $\begin{array}{l}\text { Intermediate } \\
\text { fishhook cactus }\end{array}$ & $\begin{array}{l}\text { Sclerocactus parviflorus } \\
\text { ssp. intermedius }\end{array}$ & $\mathrm{C}, \mathrm{M}$ & & & SR & L \\
\hline $\begin{array}{l}\text { Smallflower } \\
\text { fishhook cactus }\end{array}$ & $\begin{array}{l}\text { Sclerocactus parviflorus } \\
\text { ssp. parviflorus }\end{array}$ & C & & & SR & $\mathrm{L}$ \\
\hline $\begin{array}{l}\text { Siler fishhook } \\
\text { cactus }\end{array}$ & Sclerocactus sileri & C & & $S$ & SR & $\mathrm{L}$ \\
\hline $\begin{array}{l}\text { Grand Canyon } \\
\text { catchfly }\end{array}$ & Silene rectiramea & C & SC & & & L \\
\hline Gierisch mallow & Sphaeralcea gierischii & M & C & & & $\mathrm{L}$ \\
\hline $\begin{array}{l}\text { Tusayan flame } \\
\text { flower }\end{array}$ & Talinum valudulum & C & SC & & SR & M \\
\hline Aravaipa wood fern & $\begin{array}{l}\text { Thelypteris puberula } \\
\text { var. sonorensis }\end{array}$ & $\mathrm{C}, \mathrm{M}$ & & $S$ & & L \\
\hline Mazatzal triteleia & Triteleia lemmoniae & C & & & SR & $\mathrm{L}$ \\
\hline Our Lords candle & Yucca whipplei & $\mathrm{C}, \mathrm{M}$ & & & SR & $\mathrm{L}$ \\
\hline Green death camas & Zigadenus virescens & C & & & SR & L \\
\hline \multicolumn{7}{|l|}{ Invertebrates } \\
\hline California floater & Anodonta californiensis & C & SC & & & $\mathrm{L}$ \\
\hline $\begin{array}{l}\text { Grand Canyon cave } \\
\text { pseudoscorpion }\end{array}$ & Archeolarca cavicola & $\mathrm{C}$ & SC & & & $\mathrm{L}$ \\
\hline $\begin{array}{l}\text { Maricopa tiger } \\
\text { beetle }\end{array}$ & $\begin{array}{l}\text { Cicindela oregona } \\
\text { maricopa }\end{array}$ & $\mathrm{C}, \mathrm{M}$ & SC & & & $\mathrm{L}$ \\
\hline Striate disc & Discus shimekii & C & SC & & & L \\
\hline $\begin{array}{l}\text { Page Spring micro } \\
\text { caddisfly }\end{array}$ & Metrichia nigritta & C & SC & & & L \\
\hline
\end{tabular}


Table 9. Special Status Species Known to Occur in Mohave and Coconino Counties (Continued)

\begin{tabular}{|c|c|c|c|c|c|c|}
\hline Common Name & Scientific Name & County* & $\begin{array}{l}\text { USFWS } \\
\text { Status }\end{array}$ & $\begin{array}{c}\text { BLM } \\
\text { Status }\end{array}$ & State Status ${ }^{\dagger, \pi}$ & $\begin{array}{l}\text { Potential for } \\
\text { Occurrence } \\
\text { within the } \\
\text { Study Area }\end{array}$ \\
\hline \multicolumn{7}{|l|}{$\begin{array}{l}\text { Invertebrates, } \\
\text { cont'd. }\end{array}$} \\
\hline Niobrara ambersnail & $\begin{array}{l}\text { Oxyloma haydeni } \\
\text { haydeni }\end{array}$ & C & & $S$ & & $\mathrm{~L}$ \\
\hline Kanab ambersnail & $\begin{array}{l}\text { Oxyloma haydeni } \\
\text { kanabensis }\end{array}$ & $\mathrm{C}$ & LE & $S$ & & $\mathrm{~L}$ \\
\hline $\begin{array}{l}\text { Grand Wash } \\
\text { springsnail }\end{array}$ & Pyrgulopsis bacchus & M & SC & $S$ & & L \\
\hline Kingman springsnail & Pyrgulopsis conica & M & SC & $S$ & & $\mathrm{~L}$ \\
\hline Desert springsnail & Pyrgulopsis deserta & M & & $S$ & & L \\
\hline $\begin{array}{l}\text { Navajo Jerusalem } \\
\text { cricket }\end{array}$ & Stenopelmatus navajo & $\mathrm{C}$ & SC & & & $\mathrm{L}$ \\
\hline \multicolumn{7}{|l|}{ Fishes } \\
\hline Gila longfin dace & $\begin{array}{l}\text { Agosia chrysogaster } \\
\text { chrysogaster }\end{array}$ & M & SC & $S$ & & L \\
\hline Desert sucker & Catostomus clarkii & $\mathrm{C}, \mathrm{M}$ & SC & $S$ & & L \\
\hline Sonora sucker & Catostomus insignis & $\mathrm{C}, \mathrm{M}$ & SC & $S$ & & $\mathrm{~L}$ \\
\hline $\begin{array}{l}\text { Flannelmouth } \\
\text { sucker }\end{array}$ & Catostomus latipinnis & $\mathrm{C}, \mathrm{M}$ & SC & $S$ & & L \\
\hline $\begin{array}{l}\text { Little Colorado } \\
\text { Sucker }\end{array}$ & Catostomus sp. 3 & $\mathrm{C}$ & $\mathrm{SC}$ & $S$ & WSC & $\mathrm{L}$ \\
\hline Desert pupfish & Cyprinodon macularius & $\mathrm{M}$ & LE & & WSC & $\mathrm{L}$ \\
\hline Humpback chub & Gila cypha & $\mathrm{C}, \mathrm{M}$ & LE & & WSC & L \\
\hline Bonytail & Gila elegans & M & LE & & WSC & L \\
\hline Roundtail chub & Gila robusta & $\mathrm{C}, \mathrm{M}$ & C & $S$ & WSC & $\mathrm{L}$ \\
\hline Virgin River chub & Gila seminuda & M & LE & & WSC & L \\
\hline Virgin spinedace & $\begin{array}{l}\text { Lepidomeda } \\
\text { mollispinis mollispinis }\end{array}$ & M & SC & $S$ & WSC & L \\
\hline $\begin{array}{l}\text { Little Colorado } \\
\text { spinedace }\end{array}$ & Lepidomeda vittata & $\mathrm{C}$ & LT & & WSC & $\mathrm{L}$ \\
\hline Apache trout & Oncorhynchus apache & $\mathrm{C}$ & LT & & WSC & $\mathrm{L}$ \\
\hline Woundfin & $\begin{array}{l}\text { Plagopterus } \\
\text { argentissimus }\end{array}$ & M & LE & & WSC & L \\
\hline Speckled dace & Rhinichthys osculus & $\mathrm{C}, \mathrm{M}$ & SC & $S$ & & L \\
\hline Razorback sucker & Xyrauchen texanus & $\mathrm{C}, \mathrm{M}$ & LE & & WSC & $\mathrm{L}$ \\
\hline \multicolumn{7}{|l|}{ Amphibians } \\
\hline Arizona toad & $\begin{array}{l}\text { Anaxyrus } \\
\text { microscaphus }\end{array}$ & $\mathrm{C}, \mathrm{M}$ & SC & & & $\mathrm{L}$ \\
\hline $\begin{array}{l}\text { Chiricahua leopard } \\
\text { frog }\end{array}$ & $\begin{array}{l}\text { Lithobates } \\
\text { chiricahuensis }\end{array}$ & $\mathrm{C}$ & LT & & WSC & $\mathrm{L}$ \\
\hline Relict leopard frog & Rana onca & $M$ & $\mathrm{C}$ & & WSC & $\mathrm{L}$ \\
\hline
\end{tabular}


Table 9. Special Status Species Known to Occur in Mohave and Coconino Counties (Continued)

\begin{tabular}{|c|c|c|c|c|c|c|}
\hline Common Name & Scientific Name & County* & $\begin{array}{l}\text { USFWS } \\
\text { Status }\end{array}$ & $\begin{array}{c}\text { BLM } \\
\text { Status }\end{array}$ & State Status ${ }^{\dagger, \pi}$ & $\begin{array}{l}\text { Potential for } \\
\text { Occurrence } \\
\text { within the } \\
\text { Study Area }\end{array}$ \\
\hline \multicolumn{7}{|l|}{$\begin{array}{l}\text { Amphibians, } \\
\text { cont'd. }\end{array}$} \\
\hline $\begin{array}{l}\text { Northern leopard } \\
\text { frog }\end{array}$ & Rana pipiens & $\mathrm{C}, \mathrm{M}$ & & $S$ & WSC & L \\
\hline $\begin{array}{l}\text { Lowland leopard } \\
\text { frog }\end{array}$ & Rana yavapaiensis & $\mathrm{C}, \mathrm{M}$ & SC & $S$ & WSC & L \\
\hline \multicolumn{7}{|l|}{ Reptiles } \\
\hline $\begin{array}{l}\text { Desert tortoise, } \\
\text { Mojave population }\end{array}$ & Gopherus agassizii & M & $\mathrm{T}$ & & WSC & L \\
\hline $\begin{array}{l}\text { Desert tortoise, } \\
\text { Sonoran population }\end{array}$ & Gopherus agassizii & M & $\mathrm{C}$ & & WSC & L \\
\hline $\begin{array}{l}\text { Morafka's desert } \\
\text { tortoise }\end{array}$ & Gopherus morafkai & M & SC & $S$ & WSC & L \\
\hline $\begin{array}{l}\text { Banded Gila } \\
\text { monster }\end{array}$ & $\begin{array}{l}\text { Heloderma suspectum } \\
\text { cinctum }\end{array}$ & M & SC & $\begin{array}{c}\mathrm{S} \\
\text { ( } \mathrm{N} \text { and } \mathrm{W} \text { of } \\
\text { Colorado } \\
\text { River) }\end{array}$ & & $\mathrm{L}$ \\
\hline Desert rosy boa & $\begin{array}{l}\text { Lichanura trivirgata } \\
\text { gracia }\end{array}$ & M & SC & $S$ & & L \\
\hline $\begin{array}{l}\text { Northern Mexican } \\
\text { garter snake }\end{array}$ & $\begin{array}{l}\text { Thamnophis eques } \\
\text { megalops }\end{array}$ & $\mathrm{C}$ & $\mathrm{C}$ & & WSC & L \\
\hline $\begin{array}{l}\text { Narrow-headed } \\
\text { garter snake }\end{array}$ & $\begin{array}{l}\text { Thamnophis } \\
\text { rufipunctatus }\end{array}$ & C & SC & & WSC & L \\
\hline \multicolumn{7}{|l|}{ Birds } \\
\hline Northern goshawk & Accipiter gentilis & $\mathrm{C}, \mathrm{M}$ & $\mathrm{SC}$ & $S$ & WSC & $\mathrm{L}$ \\
\hline Clark's grebe & Aechmophorus clarkii & M & & $S$ & WSC & $\mathrm{L}$ \\
\hline Golden eagle & Aquila chrysaetos & $\mathrm{C}, \mathrm{M}$ & & $S$ & & $\mathrm{H}$ \\
\hline $\begin{array}{l}\text { Western burrowing } \\
\text { owl }\end{array}$ & $\begin{array}{l}\text { Athene cunicularia } \\
\text { hypugaea }\end{array}$ & $\mathrm{C}, \mathrm{M}$ & SC & $S$ & & M \\
\hline Ferruginous hawk & Buteo regalis & $\mathrm{C}, \mathrm{M}$ & SC & $S$ & WSC & $\mathrm{H}$ \\
\hline $\begin{array}{l}\text { Common black- } \\
\text { hawk }\end{array}$ & $\begin{array}{l}\text { Buteogallus } \\
\text { anthracinus }\end{array}$ & M & & $S$ & WSC & L \\
\hline $\begin{array}{l}\text { Yellow-billed } \\
\text { cuckoo (Western } \\
\text { U.S. DPS**) }\end{array}$ & Coccyzus americanus & M & $\mathrm{C}$ & & WSC & L \\
\hline $\begin{array}{l}\text { Southwestern } \\
\text { willow flycatcher }\end{array}$ & $\begin{array}{l}\text { Empidonax traillii } \\
\text { extimus }\end{array}$ & $\mathrm{C}, \mathrm{M}$ & LE & & WSC & $\mathrm{L}$ \\
\hline $\begin{array}{l}\text { American peregrine } \\
\text { falcon }\end{array}$ & $\begin{array}{l}\text { Falco peregrinus } \\
\text { anatum }\end{array}$ & $\mathrm{C}, \mathrm{M}$ & SC & $S$ & WSC & $\mathrm{H}$ \\
\hline California condor & $\begin{array}{l}\text { Gymnogyps } \\
\text { californicus }\end{array}$ & $\mathrm{C}, \mathrm{M}$ & $\mathrm{LE}, \mathrm{XN}$ & & WSC & $\mathrm{L}$ \\
\hline Bald eagle & $\begin{array}{l}\text { Haliaeetus } \\
\text { leucocephalus }\end{array}$ & $\mathrm{C}, \mathrm{M}$ & SC & $S$ & WSC & L \\
\hline Least bittern & Ixobrychus exilis & M & & & WSC & $\mathrm{L}$ \\
\hline
\end{tabular}


Table 9. Special Status Species Known to Occur in Mohave and Coconino Counties (Continued)

\begin{tabular}{|c|c|c|c|c|c|c|}
\hline Common Name & Scientific Name & County* & $\begin{array}{l}\text { USFWS } \\
\text { Status }^{\dagger, \ddagger}\end{array}$ & $\begin{array}{c}\text { BLM }_{\text {Status }}^{\dagger, \S} \\
\end{array}$ & State Status $^{\dagger, \pi}$ & $\begin{array}{c}\text { Potential for } \\
\text { Occurrence } \\
\text { within the } \\
\text { Study Area }\end{array}$ \\
\hline \multicolumn{7}{|l|}{ Birds, cont'd. } \\
\hline California black rail & $\begin{array}{l}\text { Laterallus jamaicensis } \\
\text { coturniculus }\end{array}$ & M & SC & $S$ & WSC & L \\
\hline White-faced ibis & Plegadis chihi & $\mathrm{C}$ & SC & & & $\mathrm{L}$ \\
\hline Yuma clapper rail & $\begin{array}{l}\text { Rallus longirostris } \\
\text { yumanensis }\end{array}$ & M & LE & & WSC & L \\
\hline California least tern & Sterna atillarum browni & M & LE & & & $\mathrm{L}$ \\
\hline $\begin{array}{l}\text { Mexican spotted } \\
\text { owl }\end{array}$ & $\begin{array}{l}\text { Strix occidentalis } \\
\text { lucida }\end{array}$ & $\mathrm{C}, \mathrm{M}$ & LT & & WSC & $\mathrm{L}$ \\
\hline \multicolumn{7}{|l|}{ Mammals } \\
\hline $\begin{array}{l}\text { Mexican long- } \\
\text { tongued bat }\end{array}$ & $\begin{array}{l}\text { Choeronycteris } \\
\text { mexicana }\end{array}$ & $\mathrm{C}$ & SC & $S$ & WSC & $\mathrm{L}$ \\
\hline $\begin{array}{l}\text { Pale Townsend's } \\
\text { big-eared bat }\end{array}$ & $\begin{array}{l}\text { Corynorhinus } \\
\text { townsendii pallescens }\end{array}$ & $\mathrm{C}, \mathrm{M}$ & SC & $S$ & & $\mathrm{H}$ \\
\hline $\begin{array}{l}\text { Houserock Valley } \\
\text { chisel-toothed } \\
\text { kangaroo rat }\end{array}$ & $\begin{array}{l}\text { Dipodomys microps } \\
\text { leucotis }\end{array}$ & C & SC & $S$ & WSC & L \\
\hline Spotted bat & Euderma maculatum & $\mathrm{C}, \mathrm{M}$ & SC & $S$ & WSC & M \\
\hline $\begin{array}{l}\text { Greater western } \\
\text { bonneted bat }\end{array}$ & $\begin{array}{l}\text { Eumops perotis } \\
\text { californicus }\end{array}$ & $\mathrm{C}, \mathrm{M}$ & SC & $S$ & & M \\
\hline $\begin{array}{l}\text { Allen's lappet- } \\
\text { browed bat }\end{array}$ & Idionycteris phyllotis & C, M & SC & & & M \\
\hline Western red bat & Lasiurus blossevillii & $\mathrm{C}, \mathrm{M}$ & & $S$ & WSC & L \\
\hline $\begin{array}{l}\text { California leaf- } \\
\text { nosed bat }\end{array}$ & Macrotus californicus & M & SC & $S$ & WSC & M \\
\hline $\begin{array}{l}\text { Hualapai Mexican } \\
\text { vole }\end{array}$ & $\begin{array}{l}\text { Microtus mexicanus } \\
\text { hualpaiensis }\end{array}$ & $\mathrm{C}, \mathrm{M}$ & LE & & WSC & $\mathrm{H}$ \\
\hline $\begin{array}{l}\text { Navajo Mexican } \\
\text { vole }\end{array}$ & $\begin{array}{l}\text { Microtus mexicanus } \\
\text { navaho }\end{array}$ & $\mathrm{C}$ & SC & & WSC & $\mathrm{L}$ \\
\hline Black-footed ferret & Mustela nigripes & C & LE, XN & & WSC & M \\
\hline $\begin{array}{l}\text { Western small- } \\
\text { footed myotis }\end{array}$ & Myotis ciliolabrum & $\mathrm{C}, \mathrm{M}$ & SC & & & $\mathrm{H}$ \\
\hline Long-eared myotis & Myotis evotis & C & SC & & & L \\
\hline Arizona myotis & Myotis occultus & $\mathrm{C}, \mathrm{M}$ & SC & & & M \\
\hline Fringed myotis & Myotis thysanodes & $\mathrm{C}, \mathrm{M}$ & SC & & & M \\
\hline Cave myotis & Myotis velifer & $\mathrm{C}, \mathrm{M}$ & SC & & & L \\
\hline Long-legged myotis & Myotis volans & $C, M$ & SC & & & $\mathrm{H}$ \\
\hline Yuma myotis & Myotis yumanensis & M & SC & & & M \\
\hline Big free-tailed bat & Nyctinomops macrotis & $\mathrm{C}, \mathrm{M}$ & SC & & & $M$ \\
\hline
\end{tabular}


Table 9. Special Status Species Known to Occur in Mohave and Coconino Counties (Continued)

\begin{tabular}{|c|c|c|c|c|c|}
\hline Common Name & Scientific Name & County* & $\begin{array}{l}\text { USFWS } \\
\text { Status }\end{array}$ & $\underset{\text { Status }^{\dagger, \S}}{\text { BLM }}$ State Status ${ }^{\dagger, \pi}$ & $\begin{array}{l}\text { Potential for } \\
\text { Occurrence } \\
\text { within the } \\
\text { Study Area }\end{array}$ \\
\hline \multicolumn{6}{|l|}{ Mammals, cont'd. } \\
\hline $\begin{array}{l}\text { Wupatki Arizona } \\
\text { pocket mouse }\end{array}$ & $\begin{array}{l}\text { Perognathus amplus } \\
\text { cineris }\end{array}$ & C & SC & & L \\
\hline \multicolumn{6}{|c|}{ Source: AGFD (2012b). } \\
\hline \multicolumn{6}{|c|}{ * Status data taken from the Heritage Data Management System (AGFD 2012a, 2012b). } \\
\hline \multicolumn{6}{|c|}{${ }^{\dagger} \mathrm{C}=$ Coconino County; $\mathrm{M}=$ Mojave County } \\
\hline \multicolumn{6}{|c|}{$\begin{array}{l}{ }^{\ddagger} \mathrm{C}=\text { Candidate for Listing; } \mathrm{LE}=\text { Listed Endangered; } \mathrm{LT}=\text { Listed Threatened; } \mathrm{SC}=\text { Species of Concern; } \mathrm{XN}=\text { Experimental Nonessential population. } \\
\S \mathrm{S}=\mathrm{BLM} \text { Special Status (BLM State Office). }\end{array}$} \\
\hline \multicolumn{6}{|c|}{$\begin{array}{l}\pi \text { HS = Highly Safeguarded: no collection allowed; SR = Salvage Restricted: collection only with permit; WSC = Wildlife of Special Concern in } \\
\text { Arizona. }\end{array}$} \\
\hline \multicolumn{6}{|c|}{$\begin{array}{l}\# \mathrm{~L}=\text { Low (unlikely to occur; outside of species range, and/or habitat is not present); } \mathrm{M}=\text { Moderate (habitat is limited, may be used for foraging or } \\
\text { passing through); } \mathrm{H}=\text { High (observed on site or habitat is widespread, likely nests and forages on site). }\end{array}$} \\
\hline
\end{tabular}

\section{THREATENED AND ENDANGERED SPECIES}

The USFWS is responsible for enforcing the ESA, which provides protection for species whose populations are in peril. The USFWS threatened, endangered, and candidate species lists for Mojave and Coconino Counties were reviewed (USFWS 2012b). There are 22 species known to occur in Mojave County and 20 species known to occur in Coconino County. A query of the AGFD Heritage Data Management System online tool indicates that eight USFWS species of concern and one endangered species have been identified within 5 miles of the study area (AZHGIS 2012). With exception to the Hualapai Mexican vole (Microtus mexicanus hualpaiensis), it is unlikely that any species currently designated as threatened or endangered would occur in the study area. This species is known to occur in the nearby Music Mountains, Hualapai Indian Reservation, and Hualapai Mountains.

The study area does not contain critical habitat for any federally listed species. The study area is not located within or does not contain a specially designated state or federal management area, an Important Bird Area, a Western Hemisphere Shorebird Reserve Network site, or a Ramsar Convention site. The study area is located within an experimental California condor (Gymnogyps californianus) population which is established as a "non-essential experimental population" under section 10(j) of the federal ESA. Outside of the designated 10(j) areas this species is considered endangered.

\section{GRAND CANYON WEST WIND ENERGY PROJECT AREA}

Suitable habitat for the Hualapai Mexican vole may be present, based on occurrence records and habitat descriptions provided by the AGFD wildlife abstract (AGFD 2012a) which states that this species prefers grassy areas adjacent to spruce-fir, ponderosa pine, and pinyon-juniper. No other federally listed species are anticipated to occur in the project area; however, it is within the 10(j) non-essential/experimental population for California condor. Section 10(j) provides that a species can be released in an area without impacting current or future land use planning, meaning that the species would require assessment, but should not stop development.

Risk Category. Medium. There is the potential for a federally listed species to be present in the project area, which would require additional survey and mitigation. 
Additional Work Required. Additional surveys and mitigation may be required for Hualapai Mexican vole. Consultation with the USFWS will be needed to determine if surveys or other tasks are required to ascertain the status of Hualapai Mexican vole within the project area. A USFWS conference would be required to address potential impacts to the California condor.

\section{CLAY SPRINGS WIND ENERGY PROJECT AREA}

Suitable habitat for the Hualapai Mexican vole may be present, based on occurrence records and habitat descriptions provided by the AGFD wildlife abstract (AGFD 2012a) which states that this species prefers grassy areas adjacent to spruce-fir, ponderosa pine, and pinyon-juniper. No other federally listed species are anticipated to occur in the project area; however, it is within the 10(j) non-essential experimental population for California condor. Section 10(j) provides that a species can be released in an area without impacting current or future land use planning, meaning that the species would require assessment, but should not stop development.

Risk Category. Medium. There is the potential for a federally listed species to be present in the project area, which would require additional survey and mitigation.

Additional Work Required. Additional surveys and mitigation may be required for Hualapai Mexican vole. Consultation with the USFWS will be needed to determine if surveys or other tasks are required to ascertain the status of Hualapai Mexican vole within the project area. A USFWS conference would be required to address potential impacts to the California condor.

\section{BLM WIND ENERGY NORTH PROJECT AREA}

Suitable habitat for the Hualapai Mexican vole may be present, based on occurrence records and habitat descriptions provided by the AGFD wildlife abstract (AGFD 2012a) which states that this species prefers grassy areas adjacent to spruce-fir, ponderosa pine, and pinyon-juniper. No other federally listed species are anticipated to occur in the project area; however, it is within the 10(j) non-essential experimental population for California condor. Section 10(j) provides that a species can be released in an area without impacting current or future land use planning, meaning that the species would require assessment, but should not stop development.

Risk Category. Medium. There is the potential for a federally listed species to be present in the project area, which would require additional survey and mitigation.

Additional Work Required. Additional surveys and mitigation may be required for Hualapai Mexican vole. Consultation with the USFWS will be needed to determine if surveys or other tasks are required to ascertain the status of Hualapai Mexican vole within the project area. A USFWS conference would be required to address potential impacts to the California condor.

\section{BLM WIND ENERGY SOUTH PROJECT AREA}

Suitable habitat for the Hualapai Mexican vole may be present, based on occurrence records and habitat descriptions provided by the AGFD wildlife abstract (AGFD 2012a) which states that this species prefers grassy areas adjacent to spruce-fir, ponderosa pine, and pinyon-juniper. No other federally listed species are anticipated to occur in the project area; however, it is within the 10(j) non-essential experimental population for California condor. Section 10(j) provides that a species can be released in an area without impacting current or future land use planning, meaning that the species would require assessment, but should not stop development. 
Risk Category. Medium. There is the potential for a federally listed species to be present in the project area, which would require additional survey and mitigation.

Additional Work Required. Additional surveys and mitigation may be required for Hualapai Mexican vole. Consultation with the USFWS will be needed to determine if surveys or other work is required to ascertain the status of Hualapai Mexican vole within the project area. A USFWS conference would be required to address potential impacts to the California condor.

\section{NELSON SOLAR ENERGY PROJECT AREA}

No occurrences for Hualapai Mexican vole have been recorded within 5 miles of this project area. This project area is within 5 miles of the designated non-essential experimental population area of the blackfooted ferret (Mustela nigripes). Section 10(j) provides that the species can be released in an area without impacting current or future land use planning, meaning that the species would require assessment, but should not stop development.

Risk Category. Low. Suitable habitat may be present for federally listed species, but none except for the non-essential experimental population of black-footed ferret have been recorded within 5 miles of the project area.

Additional Work Required. Early coordination with the AGFD and the USFWS, as well as the BLM, regarding protected species is encouraged. An assessment of potential impacts to black-footed ferret may also be required.

\section{HUALAPAI SOLAR ENERGY PROJECT AREA}

Suitable habitat for the Hualapai Mexican vole may be present, based on occurrence records and habitat descriptions provided by the AGFD wildlife abstract (AGFD 2012a) which states that this species prefers grassy areas adjacent to spruce-fir, ponderosa pine, and pinyon-juniper.

Risk Category. Medium. There is the potential for a federally listed species to be present in the project area, which would require additional survey and mitigation.

Additional Work Required. Additional surveys and mitigation may be required for Hualapai Mexican vole. Consultation with the USFWS will be needed to determine if surveys or other tasks are required to ascertain the status of Hualapai Mexican vole within the project area.

\section{SENSITIVE NON-AVIAN AND BAT SPECIES}

The AGFD and the BLM also maintain lists of sensitive species. Sensitive species known to occur within 5 miles of the study area have been identified using the AGFD Heritage Data Management System online tool.

\section{GRAND CANYON WEST WIND ENERGY PROJECT AREA}

The Grand Canyon cottontop cactus (Echinocactus polycephalus var. xeranthemoides) has the potential to occur within the project area, which is a salvage restricted species in the state of Arizona.

Risk Category. Medium. Salvage restricted plant species have potential to occur which would require avoidance of certain areas if identified. 
Additional Work Required. Surveys for sensitive plants would need to be completed for planned disturbance areas.

\section{CLAY SPRINGS WIND ENERGY PROJECT AREA}

The Grand Canyon cottontop cactus (Echinocactus polycephalus var. xeranthemoides) has the potential to occur within the project area, which is a salvage restricted species in the state of Arizona.

Risk Category. Medium. Salvage restricted plant species have potential to occur which would require avoidance of certain areas if identified.

Additional Work Required. No additional studies anticipated.

\section{BLM WIND ENERGY NORTH PROJECT AREA}

Clustered barrel cactus (Echinocactus polycephalus var. polychephalus) and Grand Canyon cottontop cactus have the potential to occur within the project area, both of which are salvage restricted species in the state of Arizona.

Risk Category. Medium. Salvage restricted plant species have potential to occur which would require avoidance of certain areas if identified.

Additional Work Required. Surveys for sensitive plants would need to be completed for planned disturbance areas.

\section{BLM WIND ENERGY SOUTH PROJECT AREA}

Clustered barrel cactus (Echinocactus polycephalus var. polychephalus) and Grand Canyon cottontop cactus have the potential to occur within the project area, both of which are salvage restricted species in the state of Arizona.

Risk Category. Medium. Salvage restricted plant species have potential to occur which would require avoidance of certain areas if identified.

Additional Work Required. Surveys for sensitive plants would need to be completed for planned disturbance areas.

\section{NELSON SOLAR ENERGY PROJECT AREA}

Ripley wild buckwheat (Eriogonum ripleyi) and Tusayan flame thrower (Talanium validulum) are USFWS species of concern and salvage restricted plants in the state of Arizona that also have the potential to occur within the project area.

Risk Category. Medium. Salvage restricted plant species have potential to occur which would require avoidance of certain areas if identified.

Additional Work Required. Surveys for sensitive plants would need to be completed for planned disturbance areas. 


\section{HUALAPAI SOLAR ENERGY PROJECT AREA}

Clustered barrel cactus (Echinocactus polycephalus var. polychephalus) and Grand Canyon cottontop cactus have the potential to occur within the project area, both of which are salvage restricted species in the state of Arizona.

Risk Category. Medium. Salvage restricted plant species have potential to occur which would require avoidance of certain areas if identified.

Additional Work Required. Surveys for sensitive plants would need to be completed for planned disturbance areas.

\subsubsection{Big Game Species}

The desert bighorn sheep (Ovis canadensis nelsoni), mule deer (Odocoileus hemionus), pronghorn antelope (Antilocapra americana), elk (Cervus elaphus), and mountain lion (Puma concolor) are regulated by the AGFD as big game species and have the potential to occur within the study area. Desert bighorn sheep occupy mountains and foothills containing shrub-steppe or open grassland communities and require steep, rocky terrain such as cliffs and talus slopes for suitable habitat. Pronghorn antelope are distributed throughout the grasslands of northern and southern Arizona. Their habitat is generally open grasslands or forest parks. Mule deer are widely distributed across Arizona. This species utilizes a variety of habitats, including coniferous forest, desert shrub, chaparral, grassland, and agricultural fields. In Arizona, elk are found in mixed conifer and pinyon-juniper forests. Mountain lion habitat consists of desert and forested mountains with broken terrain and steep slopes (AGFD 2012c). Potential effects on big game species resulting from wind and solar energy development include: displacement from suitable habitat; interference with behavioral activities such as migration, foraging, or reproduction; and reduction in habitat quality (BLM 2005).

\section{GRAND CANYON WEST WIND ENERGY PROJECT AREA}

No designated crucial habitat for big game species is present within the project area, although suitable habitat for big game is present.

Risk Category. Low. Potential interference with habitat and behavioral activities of big game species may occur within the project area.

Additional Work Required. Short-term and long-term surveys may be required to complete the inventory and evaluation of big game species.

\section{CLAY SPRINGS WIND ENERGY PROJECT AREA}

No designated crucial habitat for big game species is present within the project area, although suitable habitat for big game is present.

Risk Category. Low. Potential interference with habitat and behavioral activities of big game species may occur within the project area.

Additional Work Required. Short-term and long-term surveys may be required to complete the inventory and evaluation of big game species. 


\section{BLM WIND ENERGY NORTH PROJECT AREA}

The project area is located within AGFD Game Management Unit 15A (AGFD 2012d). This unit is regulated by the AGFD for hunting of mule deer, pronghorn antelope (Antilocarpa americana), desert bighorn sheep (Ovis canadensis), elk (Cervus canadensis), and mountain lion (Puma concolor). No designated crucial habitat is present within the project area.

Risk Category. Low. Potential interference with habitat and behavioral activities of big game species may occur within the project area.

Additional Work Required. Short-term and long-term surveys may be required to complete the inventory and evaluation of big game species. Additional survey may be required and could include presence/absence, telemetry, or behavioral effects studies, among others. Early coordination with the AGFD is recommended to determine the survey type and intensity required.

\section{BLM WIND ENERGY SOUTH PROJECT AREA}

The project areas are located within AGFD Game Management Unit 15A (AGFD 2012d). This unit is regulated by the AGFD for hunting of mule deer, pronghorn antelope, desert bighorn sheep, elk, and mountain lion. No designated crucial habitat is present within the project area.

Risk Category. Low. Potential interference with habitat and behavioral activities of big game species may occur within the project area.

Additional Work Required. Short-term and long-term surveys may be required to complete the inventory and evaluation of big game species. Additional survey may be required and could include presence/absence, telemetry, or behavioral effects studies, among others. Early coordination with the AGFD is recommended to determine the survey type and intensity required.

\section{NELSON SOLAR ENERGY PROJECT AREA}

No designated crucial habitat for big game species is present within the project area, although suitable habitat for big game is present.

Risk Category. Low. Interference with habitat and behavioral activities of big game species may occur within the project area.

Additional Work Required. Short-term and long-term surveys may be required to complete the inventory and evaluation of big game species. Additional survey may be required and could include presence/absence, telemetry, or behavioral effects studies, among others. Early coordination with the AGFD is recommended to determine the survey type and intensity required.

\section{HUALAPAI SOLAR ENERGY PROJECT AREA}

No designated crucial habitat for big game species is present within the project area, although suitable habitat for big game is present.

Risk Category. Low. Interference with habitat and behavioral activities of big game species may occur within the project area.

Additional Work Required. Short-term and long-term surveys may be required to complete the inventory and evaluation of big game species. Additional survey may be required and could include 
presence/absence, telemetry, or behavioral effects studies, among others. Early coordination with the AGFD is recommended to determine the survey type and intensity required.

\subsubsection{Bats}

Mortality of bats at wind facilities results from direct strikes from turbine blades (Arnett et al. 2008) and lung hemorrhage (barotrauma [Baerwald et al. 2008]) but does not affect all bats equally. Although there are 45 species of bats that occur in the United States, only a subset of these species have been documented as mortalities at wind energy facilities in the United States (Arnett et al. 2008). Scientific literature identifies six bat species that have comprised nearly all mortalities at wind energy facilities within 11 western states as of 2006 (BLM 2005; Kerlinger et al. 2006): little brown bat (Myotis lucifugus), Brazilian free-tailed bat (Tadarida brasiliensis), big brown bat (Eptesicus fuscus), silver-haired bat (Lasionycteris noctivagans), hoary bat (Lasiurus cinereus), and eastern red bat (Lasiurus borealis).

Many theories on the causal mechanisms driving bat mortality are presented by Kunz et al. (2007), though scientific evaluation of these theories has not been published. Until other species are identified as being "at-risk" from mortality studies at wind energy developments, it is anticipated that impacts will continue to disproportionately affect the six species listed above. However, no bat species should be considered to be without risk, due to their highly mobile nature.

Within Coconino and Mohave Counties there are 13 USFWS species of concern, three of which are also listed as wildlife species of concern by the AGFD, and one species which is listed as an AGFD wildlife species of concern but is not considered a species of concern by the USFWS (Table 10). Although these species are of primary concern due to current regulations, there is potential to identify a number of bat species that disperse through the study area. The study area largely consists of desert scrub and pinyonjuniper habitat. Desert scrub habitat has not been shown to concentrate bat activity (Bradley et al. 2006). However, pinyon-juniper habitat is described by Bradley et al. (2006) as providing foraging and roosting habitat for the following species: long-legged myotis (Myotis volans), long-eared myotis (Myotis evotis), hoary bat, Townsend's big-eared bat, and Allen's lappet-browed bats (Idionycteris phyllotis). The study area is in close proximity to the Colorado River, which may serve as a migratory corridor and attractant feature for bats. Riparian corridors provide roosting resources for a number of tree-roosting species, including western red bat, hoary bat, and silver-haired bat (Bradley et al. 2006), all of which have been recorded as mortalities at other wind energy facilities. There are a number of known caves along the Colorado River corridor, which may provide roosting space for species which require these resources. Bat activity is anticipated to be moderate to high given the type of habitat within the study area and nearby habitat features within the Colorado River corridor.

Table 10. Bat Species Listed in Coconino and Mohave Counties

\begin{tabular}{lll}
\hline Scientific Name & Common Name & Listing Status \\
\hline $\begin{array}{l}\text { Family Vespertilionidae } \\
\text { Choeronycteris mexicana }\end{array}$ & Mexican long-tongued bat & SC, WSC \\
\hline Corynorhinus townsendii & Western big-eared bat & SC \\
\hline Euderma maculatum & Spotted bat & SC, WSC \\
\hline Eumops perotis californicus & Greater western bonneted bat & SC \\
\hline Idionycteris phyllotis & Allen's lappet-browed bat & SC \\
\hline Lasiurus blossevillii & Western red bat & WSC \\
\hline Macrotis californicus & California leaf nosed bat & SC, WSC \\
\hline Myotis ciliolabrum & Western small-footed myotis & $\mathrm{SC}$ \\
\hline
\end{tabular}


Table 10. Bat Species Listed in Coconino and Mohave Counties (Continued)

\begin{tabular}{lll}
\hline Scientific Name & Common Name & Listing Status \\
\hline $\begin{array}{l}\text { Family Vespertilionidae, cont'd. } \\
\text { Myotis occultus }\end{array}$ & Arizona myotis & SC \\
\hline Myotis thysanodes & Fringed myotis & SC \\
\hline Myotis velifer & Cave myotis & SC \\
\hline Myotis volans & Long-legged myotis & SC \\
\hline Myotis yumanensis & Yuma myotis & SC \\
\hline Family Mollosidae & & \\
Nyctinomops macrotis & Big free-tailed bat & SC \\
\hline
\end{tabular}

Source: AGFD (2012b)

Note: SC = USFWS species of concern, WSC = AGFD Wildlife of Special Concern in Arizona

Potentially important resources for bats were observed during the site assessment and included cliff and rock outcrops, and human-made water resources (small human-made stick ponds/tanks). Cliff and rock outcrops can provide roosting resources for some species of bats including: pallid bat (Antrozous pallidus), Townsend's big-eared bat, big brown bat, California myotis (Myotis californicus), western small-footed myotis (M. ciliolabrum), fringed myotis (M. thysanodes), and Brazilian free-tailed bat (Bradley et al. 2006). However, the resources observed during the site assessment are not anticipated to provide roosting resources to large colonies of bats, but do provide numerous dispersed roosting opportunities. Water features generally concentrate bat activity, especially in the arid Southwest. These features provide a source of water and generally attract insects upon which some bat species feed.

The AGFD has issued Guidelines for Reducing Impacts to Wildlife from Wind Energy Development in Arizona (AGFD 2009) which specifies techniques and timing for pre-construction bat studies. These guidelines identify appropriate methods for assessing bat activity and include roost searches, capture surveys, and acoustic studies. All of these methods have limitations and therefore, a combination of methods is beneficial in determining presence/absence, relative abundance, and seasonal and nightly patterns in activity. Additionally, the BLM Programmatic Environmental Impact Statement (BLM 2005) provides specific directives for development of wind energy projects on BLM lands:

- Turbines should not be located near known bat hibernation, breeding, and maternity/nursery colonies, in migration corridors, or in flight paths between colonies and feeding areas.

- Bat use of the project area should be evaluated, and the project should be designed to minimize or mitigate the potential for bat strikes. Both macro- and micro-siting options can be considered to minimize impacts to bats.

\section{GRAND CANYON WEST WIND ENERGY PROJECT AREA}

The Grand Canyon West Wind Energy project area consists primarily of pinyon-juniper woodland. This vegetation community is anticipated to provide habitat for a variety of bat species as described by Bradley et al. (2006). The pale Townsend's big-eared bat (Corynorhinus townsendii pallescens), western smallfooted myotis (Myotis ciliolabrum), and long-legged myotis (Myotis volans) are all BLM-sensitive and USFWS species of concern that have been identified as having high potential to occur within the project area. 
Risk Category. Medium. A moderate diversity of bat species and moderate to high levels of activity are anticipated in this project area because of the type of available habitat and proximity to the Grand Canyon.

Additional Work Required. Long-term acoustic monitoring should be done at meteorological towers and other potential sites for a 1- to 2-year period. Acoustic monitoring equipment should be deployed in the turbine rotor-swept area whenever possible. Capture surveys should be conducted at bat attractant resources throughout the year. Until wildlife agency coordination occurs, it is anticipated that five capture surveys per year should be sufficient to assess presence/absence. Capture surveys should target species that are expected to occur but are unlikely to be detected using acoustic methods (i.e., spotted bat and Townsend's big-eared bat). Roost searches should be conducted to identify any substantial roost locations for bats. Radar surveys may be requested, though this is unlikely unless substantial bat activity is documented. Additionally, a BBCS likely will be required that identifies initial avoidance and minimization measures, mitigations, and post-construction monitoring, analysis, and reporting needs.

\section{CLAY SPRINGS WIND ENERGY PROJECT AREA}

The Clay Springs Wind Energy project area consists primarily of pinyon-juniper woodland. This vegetation community is anticipated to provide habitat for a variety of bat species as described by Bradley et al. (2006). The pale Townsend's big-eared bat (Corynorhinus townsendii pallescens), western smallfooted myotis (Myotis ciliolabrum), and long-legged myotis (Myotis volans) are all BLM-sensitive and USFWS species of concern that have been identified as having high potential to occur within the project area.

Risk Category. Medium. A moderate diversity of bat species and moderate to high levels of activity are anticipated in this project area because of the type of available habitat and proximity to the Grand Canyon.

Additional Work Required. Additional work required is the same as that described for the Grand Canyon West project area.

\section{BLM WIND ENERGY NORTH PROJECT AREA}

The BLM Wind Energy North project area consists primarily of pinyon-juniper woodland. This vegetation community is anticipated to provide habitat for a variety of bat species as described by Bradley et al. (2006). The pale Townsend's big-eared bat (Corynorhinus townsendii pallescens), western smallfooted myotis (Myotis ciliolabrum), and long-legged myotis (Myotis volans) are all BLM-sensitive and USFWS species of concern that have been identified as having high potential to occur within the project area.

Risk Category. Medium. A moderate diversity of bat species and moderate to high levels of activity are anticipated in this project area because of the type of available habitat and proximity to the Grand Canyon.

Additional Work Required. Additional work required is the same as that described for the Grand Canyon West project area.

\section{BLM WIND ENERGY SOUTH PROJECT AREA}

The BLM Wind Energy North project area consists primarily of pinyon-juniper woodland. This vegetation community is anticipated to provide habitat for a variety of bat species as described by Bradley et al. (2006). The pale Townsend's big-eared bat (Corynorhinus townsendii pallescens), western small- 
footed myotis (Myotis ciliolabrum), and long-legged myotis (Myotis volans) are all BLM-sensitive and USFWS species of concern that have been identified as having high potential to occur within the project area.

Risk Category. Medium. A moderate diversity of bat species and moderate to high levels of activity are anticipated in this project area because of the type of available habitat and proximity to the Grand Canyon.

Additional Work Required. Additional work required is the same as that described for the Grand Canyon West project area.

\section{NELSON SOLAR ENERGY PROJECT AREA}

The Nelson Solar Energy project area consists primarily of semi-desert grassland. This desert shrub vegetation community is expected to have a low diversity of bat species and low volume of bat activity (Bradley et al. 2006). Spotted bat (Euderma maculatum), and greater western bonneted bat (Eumops perotis californicus) are BLM-sensitive and USFWS species of concern that have been identified as having the potential to occur within the project area. The spotted bat is also considered wildlife of special concern in Arizona.

Risk Category. Low. A low diversity of bat species and low levels of activity are anticipated in this project area because of the type of available habitat and proximity to the Grand Canyon. Additionally, solar facilities are not anticipated to pose the same risks to bats that are found at wind energy facilities.

Additional Work Required. No formal guidelines identify the appropriate level of surveys for bats at potential solar facilities. Survey guidelines should be determined during wildlife agency coordination for this project. However, based on our experience with other solar projects, it is anticipated that at least 1 year of acoustic monitoring will be requested in order to determine species presence/absence. A BBCS will likely also be required to identify initial avoidance and minimization measures, mitigations, and postconstruction monitoring, analysis, and reporting needs.

\section{HUALAPAI SOLAR ENERGY PROJECT AREA}

The Hualapai Solar Energy site consists mainly of mixed desert scrub and creosotebush communities. Although these desert shrub vegetation communities are expected to have a low diversity of bat species and low volume of bat activity (Bradley et al. 2006), The pale Townsend's big-eared bat (Corynorhinus townsendii pallescens), western small-footed myotis (Myotis ciliolabrum), and long-legged myotis (Myotis volans) are all BLM-sensitive and USFWS species of concern that have been identified as having potential to occur within the project area.

Risk Category. Low. A low diversity of bat species and low levels of activity are anticipated in this project area because of the type of available habitat and proximity to the Grand Canyon. Additionally, solar facilities are not anticipated to pose the same risks to bats that are found at wind energy facilities.

Additional Work Required. Additional work required is the same as that described for the Nelson Solar Energy project area.

\subsubsection{Birds, including Bald and Golden Eagles, Raptor Migration Flyways, and California Condor}

The regulatory framework for protecting birds includes the ESA, the MBTA of 1918 (which includes any part, nest, or egg), BGEPA, and Executive Order (EO) 13186. All of the sensitive birds as well as most 
other bird species that are likely to occur in the project area are protected by the MBTA. The MBTA prohibits the take of migratory birds and does not include provisions for allowing unauthorized take. Although it is not possible for the USFWS to absolve individuals, companies, or agencies from liability, the USFWS and Department of Justice have executed prosecutorial discretion in the past for those who have made good faith efforts to avoid take of migratory birds (USFWS 2003). The BGEPA is similar to the MBTA in that it prohibits the take of bald and golden eagles. The USFWS published a Final Eagle Permit Rule (Eagle Permit Rule) on September 11, 2009, under the BGEPA, authorizing limited issuance of permits to take bald eagles where the take is associated with, but not the purpose of, an otherwise lawful activity. However, they went on to say that golden eagle take permits (herein Take Permit) would not be issued until better data on the species were obtained and a process for issuing permits was put in place. Since that time, the USFWS has issued numerous guidelines, recommendations, and gray literature describing the compliance process, oftentimes contradicting one another. Most recently the USFWS issued their Draft Eagle Conservation Plan (ECP) Guidance in January 2011 (USFWS 2011).

The 2011 Draft ECP Guidance states that project proponents with operating or soon-to-be operating facilities at the time the Draft ECP Guidance was first released should coordinate with the USFWS if interesting in obtaining a programmatic eagle Take Permit (USFWS 2011). Although some elements of the Draft ECP Guidance may be contradictory, the sections that address risk assessment and impact minimization efforts are relevant to the six project areas. Should a Take Permit be obtained once available, it is recommended that a BBCS/ECS be prepared that meets the intent of the Draft ECP Guidance, or final guidance if it has been published.

The BBCS/ECS should outline any additional data collection needs, avoidance and minimization measures, post-construction monitoring, and adaptive management. The BBCS/ECS should be completed in coordination with the USFWS (Ecological Services and Migratory Birds Offices) to ensure it meets their requirements to protect the species.

In addition to the USFWS guidelines, the AGFD has issued Guidelines for Reducing Impacts to Wildlife from Wind Energy Development in Arizona (AGFD 2009). The guidelines specifically discuss techniques and timing for pre-construction studies. Based on those guidelines, this project would likely require at least 2 years of avian point-count and migration studies.

Although little is known about impacts to avian species from operation of wind energy facilities in arid desert habitats, analysis of mortalities at other facilities shows that raptors are especially susceptible where they aggregate along migratory pathways or wintering grounds that provide ample prey opportunities. Raptors are of particular concern because they are slow to recover from anthropogenic impacts as a result of their long lifespan, long time required to reach sexual maturity, and low reproductive rate relative to other bird species. Mortalities of birds in these groups mostly consist of common, ubiquitous species for which little concern is raised. Common mortalities at other wind generating facilities include horned larks (Eremophila alpestris), sparrows (Family Emberizidae), warblers (Family Parulidae), and upland gamebirds (Erickson 2003). Horned larks were observed throughout the project area during the site reconnaissance visit.

Aside from concerns about wintering and migratory habitat for raptors, risk to avian species/communities would likely be highest in areas that provide unique habitats in otherwise homogenous vegetation communities (e.g., the Colorado River in the middle of the Mojave Desert). Additionally, placing a wind energy facility in areas that provide habitat for sensitive avian species could also equate to elevated levels of risk.

The entire study area can be expected to be used as foraging habitat by raptors, including bald and golden eagles. However, given the absence of water bodies that support fish, bald eagle would potentially occur 
primarily during winter, with no potentially suitable bald eagle breeding habitat present within or immediately adjacent to the project area.

\section{Desk Top Analysis of Potentially Suitable Golden Eagle Nesting Habitat and Aerial Eagle/Raptor Nest Surveys}

Based on a desktop analysis using topographical maps and aerial imagery, 125,527 acres of potentially suitable golden eagle nesting habitat was located within a 3-mile radius of the study area (Figure 21). It should be noted, however, that desktop habitat suitability analyses may overestimate potentially suitable golden eagle nesting habitat. In addition, bald eagles have been documented along the Colorado River (AZHGIS 2012), primarily during fall and winter. Through our local golden eagle experience in the area, we anticipate that a study area of this size, including the USFWS recommended 10-mile survey area, can support up to approximately eight pairs of golden eagles, depending upon the availability of prey and suitable nesting habitat. More important than the number of golden eagle nests, though, is the distribution of these nests relative to the project facilities. Two years of helicopter aerial surveys is suggested to ascertain the locations of nests within a 10-mile radius of study area to determine the potential impact that nesting eagles would have on development of a project.

From May 31 to June 2, 2012, SWCA conducted aerial eagle/raptor nest surveys within 3 miles of the study area, totaling 125,527 acres of possibly suitable golden eagle nesting habitat surveyed. Ninetythree unique nest structures were located within the survey area. Two additional nests were located opportunistically just outside of the 3-mile survey area. Including the two nests located opportunistically just outside of the survey area, a total of 30 golden eagle and possible golden eagle nests was located; two nests were occupied with eagle young observed (see Figure 21). The remaining 63 nests were identified as: "ferruginous hawk," "red-tailed hawk," "peregrine falcon or prairie falcon," "common raven," "undetermined: resembles Buteo spp.," "undetermined: resembles Buteo spp. or common raven," "undetermined: resembles common raven," "undetermined: resembles common raven or packrat" (Figure 22).

Generally, USFWS recommends that wind turbines should not be constructed within 4 miles of eagle nests. Figure 21 depicts 4-mile buffers around all golden eagle and possible golden eagle nests located during aerial surveys. However, eagle nest buffers could be less or greater than 4 miles, depending upon eagle use in a specific project area (determined via targeted field studies) and/or the feasibility and/or viability of a wind energy project, depending on nest buffer size. USFWS also typically recommends a 0.5-mile buffer around all non-eagle raptor nests (see Figure 22), and that construction activities near raptor nests be limited outside of the raptor breeding season.

\section{Raptor Migration Flyways}

Raptor migration has been a well-studied phenomenon for decades, and it is well known that migrating raptors concentrate along long-established routes or corridors. Hawkwatch International (HWI) has identified a major raptor migration area at Yaki and Lipan Points, within Grand Canyon National Park, and studies currently being conducted at the nearby Aubrey Cliffs indicate that certain topographic features (e.g., north-south ridgelines) concentrate migrant raptors in northern Arizona. Although southbound migrating raptors do concentrate when crossing the Grand Canyon in fall, the concentration of birds quickly disperses, resulting in a broad migration front (i.e., widely dispersed individuals) as individuals move south (personal communication, M. Neal, HWI, April 2010). The study area is situated on the Grand Wash Cliffs that comprise somewhat prominent north-south-trending ridgelines that would concentrate raptor migration. It is anticipated that AGFD would request 2 years of study to determine migrant raptor passage rates within the study area. 


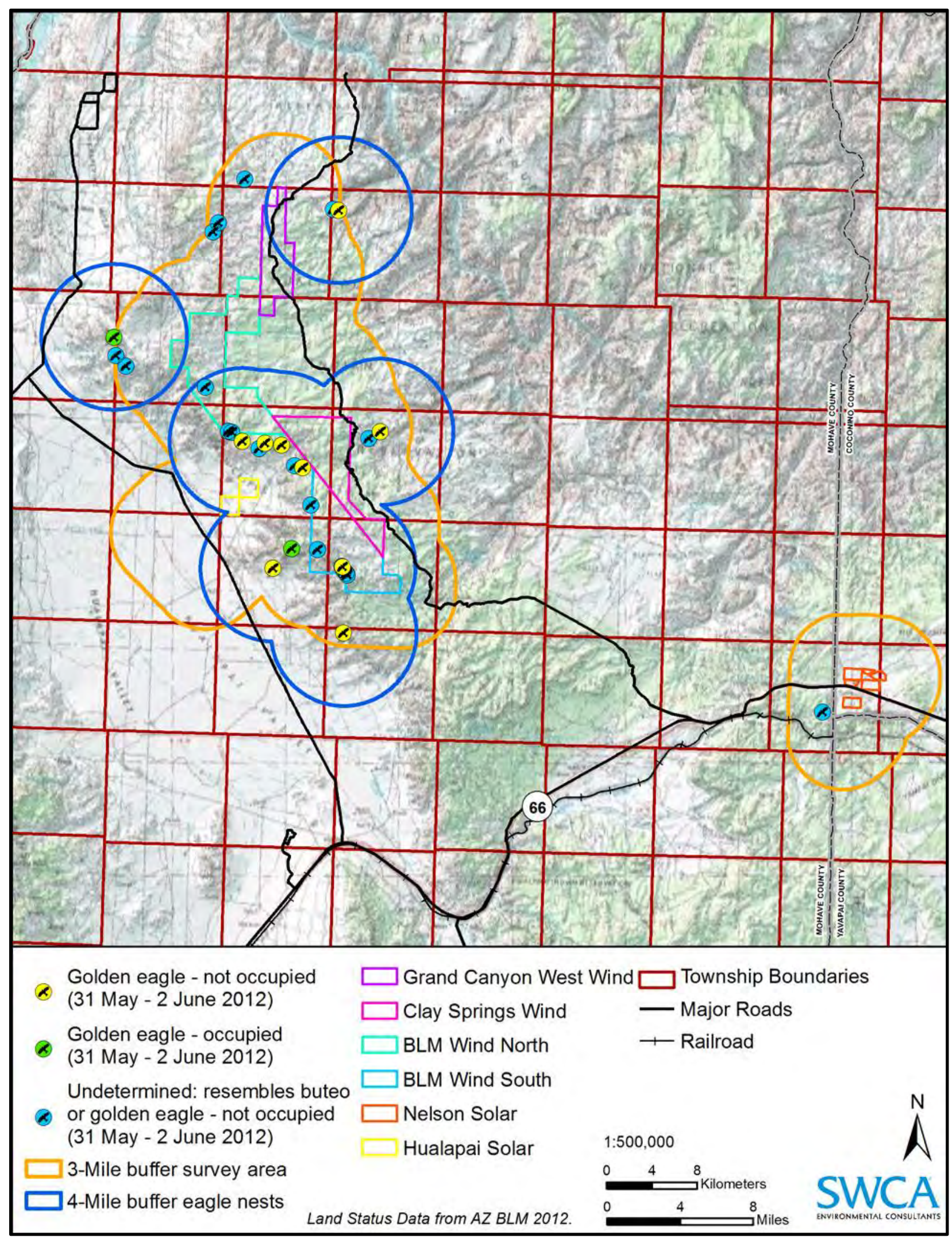

Figure 21. Golden eagle nest locations. 


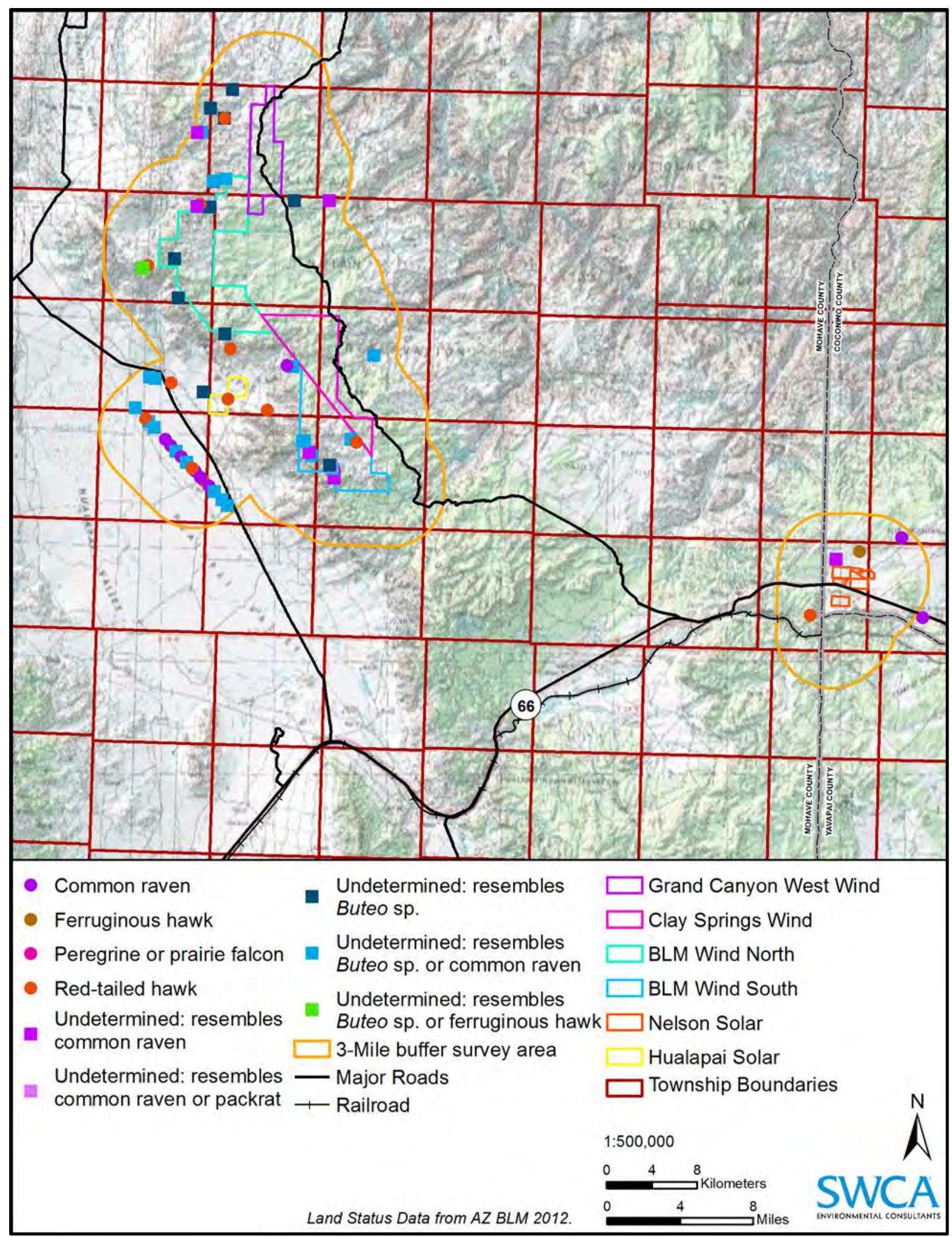

Figure 22. Other raptor nest locations. 


\section{California Condor}

With respect to the California condor, range-wide movement studies conducted from 1996 to 2006 (Hunt et al. 2007; Southwest Condor Review Team 2007) indicate that species occurrence within the study area would be low. Recent (2009) condor movement data collected via satellite telemetry from 12 individuals were obtained from the Peregrine Fund (Figure 23). The 2009 data are consistent with those from 1996 to 2006, with no locations recorded near the study area. Furthermore, 2007 to 2010 movement data indicate that it is unlikely condors will occur near the study area, as the species is using higherquality habitat from Grand Canyon northwest to southern Utah (personal communication, Chris Parrish, September 3, 2010). However, although current telemetry studies show that condors do not use the study area, they are a wide-ranging species that can travel long distances and may expand beyond their current range over time. Therefore, potential exists for condors to occur in the study area in the future.

\section{GRAND CANYON WEST WIND ENERGY PROJECT AREA}

The project area consists of high-quality raptor habitat, including habitats and land features used or potentially used for nesting, foraging, and/or migration. Golden eagle, ferruginous hawk (Buteo regalis), peregrine falcon (Falco peregrinusanatum), bald eagle (Haliaeetus leucocephalus), and western burrowing owl (Athene cunicularia hyugaea) are all BLM-sensitive and USFWS species of concern that have been identified as having the potential or to occur within the project area. Ferruginous hawk is also considered wildlife of special concern in Arizona. At least one golden eagle nest and two red-tailed hawk nests were located outside but within 3 miles of the project area boundary during aerial surveys in May/June 2012 (see Figures 21 and 22). Given the absence of water bodies that support fish, bald eagle would potentially occur primarily during winter. The project area lies immediately east of the Grand Wash Cliffs that comprise somewhat prominent north-south-trending ridgelines that potentially could concentrate raptor migration in fall. Although current telemetry studies show that California condors do not use the project area (see Figure 23), potential exists for condors to occur in the project area in the future.

Risk Category. High. High-quality raptor habitat present in project area. Golden eagle nesting required an ECS and may lead to needing a Golden Eagle Take Permit.

Additional Work Required. Large and small bird use surveys (2 years), raptor nest surveys (2 years), and raptor migration studies (2 years) are typically required. Studies for golden and bald eagles in accordance with the most recently accepted USFWS and AGFD eagle study protocols are recommended. To document any eagle nesting, all potentially suitable eagle nesting habitat (e.g., cliff faces, rocky outcrops, woody snags, and large trees) would need to be surveyed via helicopter within a 10-mile radius of the project area over 2 years. Within a 2-mile radius of the project area, all raptor nests would be recorded via aerial surveys and using GIS technology, per guidelines issued by the AGFD.

Given the lack of formal agency guidance, and changing requirements regarding golden eagle and alternative energy projects, multi-agency agreement on eagle habitat and home-range use studies as related to a wind energy project will be critical in completing the necessary environmental studies. Once the USFWS begins issuing Take Permits for golden eagle, an application can be submitted if deemed necessary. The application must consist of a completed application Form 3-200-71 and all required attachments (to be determined: most likely study reports and BBCS/ECS).

\section{CLAY SPRINGS WIND ENERGY PROJECT AREA}

The project area consists of high-quality raptor habitat, including habitats and land features used or potentially used for nesting, foraging, and/or migration. Golden eagle, ferruginous hawk, peregrine falcon, bald eagle, and western burrowing owl are all BLM-sensitive and USFWS species of concern that 


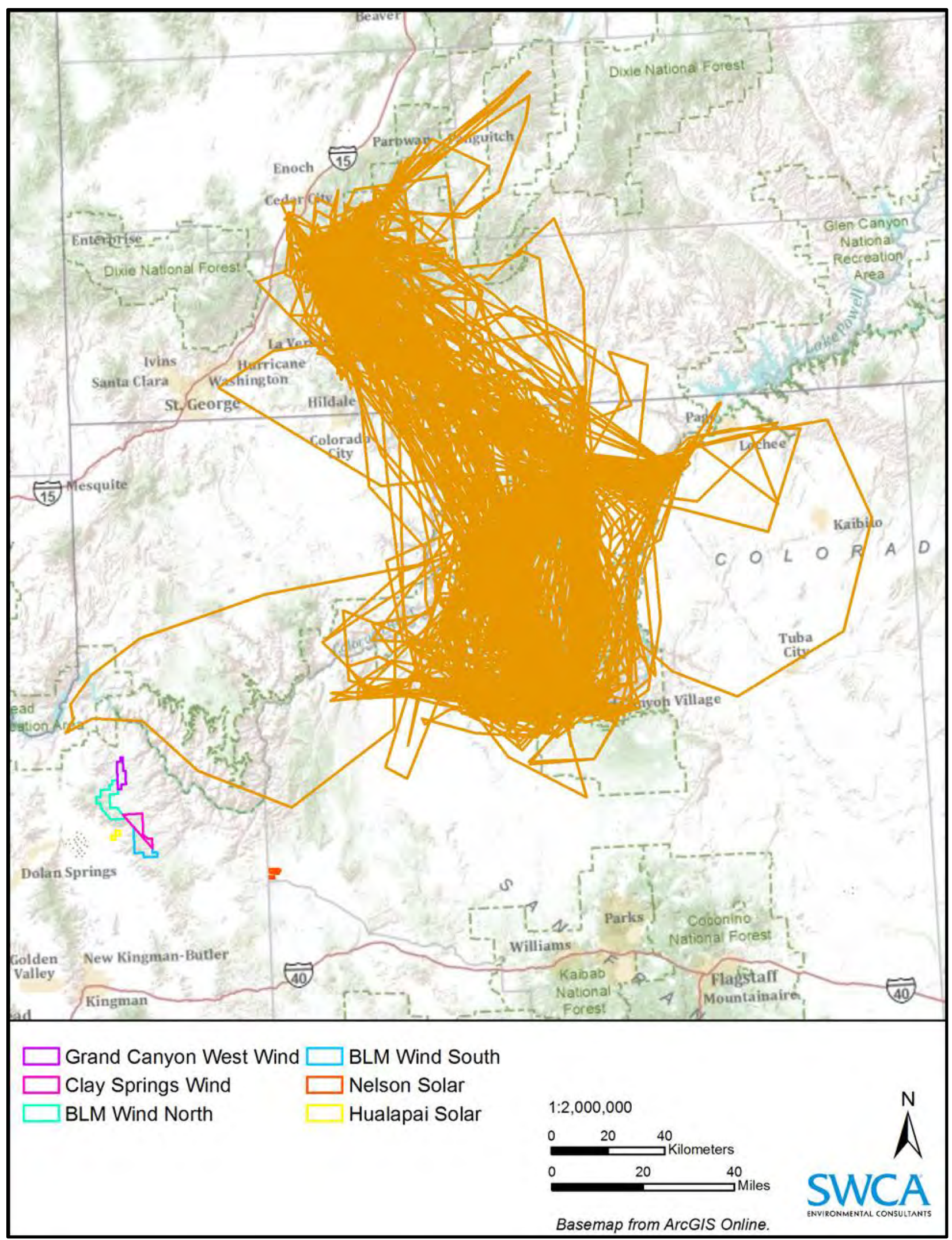

Figure 23. Draft 2009 California condor movement/location data obtained via satellite telemetry studies. 
have been identified as having the potential or to occur within the project area. Ferruginous hawk is also considered wildlife of special concern in Arizona. At least eight golden eagle nests (one occupied with young in nest) and four red-tailed hawk nests were located outside but within 3 miles of the project area boundary during aerial surveys in May/June 2012 (see Figures 21 and 22). Given the absence of water bodies that support fish, bald eagle would potentially occur primarily during winter. The project area lies immediately east of the Grand Wash Cliffs that comprise somewhat prominent north-south-trending ridgelines that potentially could concentrate raptor migration in fall. Although current telemetry studies show that California condors do not use the project area (see Figure 23), potential exists for condors to occur in the project area in the future.

Risk Category. High. High-quality raptor habitat present in project area. Golden eagle nesting required an ECS and may lead to needing a Golden Eagle Take Permit.

Additional Work Required. Large and small bird use surveys (2 years), raptor nest surveys (2 years), and raptor migration studies (2 years) are typically required. Studies for golden and bald eagles in accordance with the most recently accepted USFWS and AGFD eagle study protocols are recommended. To document any eagle nesting, all potentially suitable eagle nesting habitat (e.g., cliff faces, rocky outcrops, woody snags, and large trees) would need to be surveyed via helicopter within a 10-mile radius of the project area over 2 years. Within a 2-mile radius of the project area, all raptor nests would be recorded via aerial surveys and using GIS technology, per guidelines issued by the AGFD.

Given the lack of formal agency guidance, and changing requirements regarding golden eagle and alternative energy projects, multi-agency agreement on eagle habitat and home-range use studies as related to a wind energy project will be critical in completing the necessary environmental studies. Once the USFWS begins issuing Take Permits for golden eagle, an application can be submitted if deemed necessary. The application must consist of a completed application Form 3-200-71 and all required attachments (to be determined: most likely study reports and BBCS/ECS).

\section{BLM WIND ENERGY NORTH PROJECT AREA}

The project area consists of high-quality raptor habitat, including habitats and land features used or potentially used for nesting, foraging, and/or migration. Golden eagle, ferruginous hawk, peregrine falcon, bald eagle, and western burrowing owl are all BLM-sensitive and USFWS species of concern that have been identified as having the potential or to occur within the project area. Ferruginous hawk is also considered wildlife of special concern in Arizona. At least five golden eagle nests (one occupied with young in nest) and five red-tailed hawk nests were located outside but within 3 miles of the project area boundary during aerial surveys in May/June 2012 (see Figures 21 and 22). Based on aerial nest surveys in May 2011, golden eagle has been identified as occurring within and immediately adjacent to the project area.

Given the absence of water bodies that support fish, bald eagle would potentially occur primarily during winter. The project area lies immediately east of the Grand Wash Cliffs that comprise somewhat prominent north-south-trending ridgelines that potentially could concentrate raptor migration in fall. Although current telemetry studies show that California condors do not use the project area (see Figure 23 ), potential exists for condors to occur in the project area in the future.

Risk Category. High. High-quality raptor habitat present in project area. Golden eagle nesting required an ECS and may lead to needing a Golden Eagle Take Permit.

Additional Work Required. Large and small bird use surveys (2 years), raptor nest surveys (2 years), and raptor migration studies (2 years) are typically required. Studies for golden and bald eagles in accordance with the most recently accepted USFWS and AGFD eagle study protocols are recommended. 
To document any eagle nesting, all potentially suitable eagle nesting habitat (e.g., cliff faces, rocky outcrops, woody snags, and large trees) would need to be surveyed via helicopter within a 10-mile radius of the project area over 2 years. Within a 2-mile radius of the project area, all raptor nests would be recorded via aerial surveys and using GIS technology, per guidelines issued by the AGFD.

Given the lack of formal agency guidance, and changing requirements regarding golden eagle and alternative energy projects, multi-agency agreement on eagle habitat and home-range use studies as related to a wind energy project will be critical in completing the necessary environmental studies. Once the USFWS begins issuing Take Permits for golden eagle, an application can be submitted if deemed necessary. The application must consist of a completed application Form 3-200-71 and all required attachments (to be determined: most likely study reports and BBCS/ECS).

\section{BLM WIND ENERGY SOUTH PROJECT AREA}

The project area consists of high-quality raptor habitat, including habitats and land features used or potentially used for nesting, foraging, and/or migration. Golden eagle, ferruginous hawk, and peregrine falcon are BLM-sensitive and USFWS species of concern that have been identified as occurring or having the potential to occur within the project area. Ferruginous hawk is also considered wildlife of special concern in Arizona. At least eight golden eagle nests (one occupied with young in nest) and three redtailed hawk nests were located outside but within 3 miles of the project area boundary during aerial surveys in May/June 2012; one golden eagle and one red-tailed hawk nest were located within the project area (see Figures 21 and 22). Given the absence of water bodies that support fish, bald eagle would potentially occur primarily during winter. The project area lies immediately east of the Grand Wash Cliffs that comprise somewhat prominent north-south-trending ridgelines that potentially could concentrate raptor migration in fall. Although current telemetry studies show that California condors do not use the project area (see Figure 23), potential exists for condors to occur in the project area in the future.

Risk Category. High. High-quality raptor habitat present in project area. Golden eagle nesting required an ECS and may lead to needing a Golden Eagle Take Permit.

Additional Work Required. Large and small bird use surveys (2 years), raptor nest surveys (2 years), and raptor migration studies (2 years) are typically required. Studies for golden and bald eagles in accordance with the most recently accepted USFWS and AGFD eagle study protocols are recommended. To document any eagle nesting, all potentially suitable eagle nesting habitat (e.g., cliff faces, rocky outcrops, woody snags, and large trees) would need to be surveyed via helicopter within a 10-mile radius of the project area over 2 years. Within a 2-mile radius of the project area, all raptor nests would be recorded via aerial surveys and using GIS technology, per guidelines issued by the AGFD.

Given the lack of formal agency guidance, and changing requirements regarding golden eagle and alternative energy projects, multi-agency agreement on eagle habitat and home-range use studies as related to a wind energy project will be critical in completing the necessary environmental studies. Once the USFWS begins issuing Take Permits for golden eagle, an application can be submitted if deemed necessary. The application must consist of a completed application Form 3-200-71 and all required attachments (to be determined: most likely study reports and BBCS/ECS).

\section{NELSON SOLAR ENERGY SITE}

The project area consists of high-quality raptor habitat, including habitats used or potentially used for nesting and foraging. Ferruginous hawk is a BLM-sensitive and USFWS species of concern that have been identified as having the potential to occur within the project area. Ferruginous hawk is also considered wildlife of special concern in Arizona. One potential golden eagle nest, one ferruginous hawk, and one red-tailed hawk nest were located outside but within 3 miles of the project area boundary during 
aerial surveys in May/June 2012 (see Figures 21 and 22). Given the absence of water bodies that support fish, bald eagle would potentially occur primarily during winter. The project area does not contain prominent north-south-trending ridgelines that potentially could concentrate raptor migration in fall. Although current telemetry studies show that California condors do not use the project area (see Figure $23)$, potential exists for condors to occur in the project area in the future.

Risk Category. Low. Although high-quality raptor habitat is present, there is low risk from development of a solar project. Further, no golden eagle nests have been identified within 3 miles of the project area.

Additional Work Required. No formal guidelines identify the appropriate level of avian surveys at potential solar energy facilities. Survey guidelines should be determined during wildlife agency coordination for this project. However, based on our experience with other solar projects, it is anticipated that at least 1 year of surveys will be requested in order to determine species presence/absence.

\section{HUALAPAI SOLAR ENERGY SITE}

The project area consists of high-quality raptor habitat, including habitats and land features used or potentially used for nesting, foraging, and/or migration. Golden eagle, ferruginous hawk, peregrine falcon, bald eagle, and western burrowing owl are all BLM-sensitive and USFWS species of concern that have been identified as having the potential or to occur within the project area. Ferruginous hawk is also considered wildlife of special concern in Arizona. At least six golden eagle nests (one occupied with young in nest) and four red-tailed hawk nests were located outside but within 3 miles of the project area boundary during aerial surveys in May/June 2012 (see Figures 21 and 22). Given the absence of water bodies that support fish, bald eagle would potentially occur primarily during winter. The project area lies immediately west of the Grand Wash Cliffs that comprise somewhat prominent north-south-trending ridgelines that potentially could concentrate raptor migration in fall. Although current telemetry studies show that California condors do not use the project area (see Figure 23), potential exists for condors to occur in the project area in the future.

Risk Category. Moderate. Although high-quality raptor habitat is present, there is low risk from development of a solar project. However, golden eagle nests have been identified within 3 miles of the project area, increasing potential risk and permitting requirements.

Additional Work Required. No formal guidelines identify the appropriate level of avian surveys at potential solar energy facilities. Survey guidelines should be determined during wildlife agency coordination for this project. However, based on our experience with other solar projects, it is anticipated that at least 1 year of surveys will be requested in order to determine species presence/absence.

\subsection{Jurisdictional Waters, Playa Lakes, and Wetlands}

The U.S. Army Corp of Engineers (USACE) has jurisdiction over Waters of the U.S. (WUS), including wetlands and ephemeral channels under Section 404 of the Clean Water Act. In order for an area to be classified as a wetland, it must satisfy three criteria, including vegetation (dominated by hydrophytic vegetation), hydrology (visual evidence of water), and hydric soil (saturated sufficiently to produce soil types characteristic of a wetland).

For the USACE to have jurisdiction over an ephemeral or intermittent wash, the wash must exhibit a distinct ordinary high water mark (OHWM) defined as "the line on the shore established by the fluctuations of water and is indicated by physical characteristics, such as a clear, natural line impressed on the bank, shelving, changes in the character of the soil, destruction of terrestrial vegetation, the presence of litter or debris, or other appropriate means that consider the characteristics of the surrounding area" 
(33 CFR 328.3). In addition to exhibiting a distinct OHWM, an ephemeral wash must convey flow to WUS or provide commerce, such as crossing state lines.

Placement of fill materials into WUS is regulated under CWA Sections 404 and 401 and enforced by the USACE. The USACE can issue several types of permits depending on the type of project and amount of potential disturbance. Due to their linear nature, wind energy projects can generally avoid wetlands and WUS through project design. Solar projects are somewhat more challenging because the disturbed area tends to be less flexible; however, these types of projects can also be designed to avoid wetlands and WUS. If avoidance is possible, no other regulatory processes are required relative to Section 404 of the CWA. If WUS cannot be avoided, most often by roads and underground utility lines, then a permit is required. Placement of fill from land-based renewable energy generation facilities and associated features such as roads are covered under CWA Nationwide Permit 51 if impacts are less than 0.5 acre, or less than 300 linear feet of streambed. Utility lines transferring energy are generally covered under CWA Nationwide Permit 12 if impacts are less than 0.5 acre. If fill exceeds the acre or linear feet threshold, a letter of permission or individual permit would be required. An initial review of aerial photography indicated that a moderate number of ephemeral wash channels are present that may qualify as WUS (Figure 24).

\section{GRAND CANYON WEST WIND ENERGY PROJECT AREA}

An initial review of the National Wetlands Inventory Database revealed there are no online wetlands data within the project area. There are a number of ephemeral washes within the project area that may be jurisdictional if certain criteria are satisfied.

Risk Category. Medium. There may be ephemeral washes or wetlands in the project area that qualify as jurisdictional.

Additional Work Required. Following the identification of infrastructure locations, a detailed examination of potential WUS within the defined project area should be conducted. If WUS cannot be avoided, a jurisdictional determination report should be prepared. In addition, the following permit requirements would need to be met: CWA Section 404 permit issued by the USACE, Water Quality Certification under Section 401 issued by the ADEQ, Surface Water Section, and a Notice of Intent and Stormwater Pollution Prevention Plan to comply with Section 402 of the CWA. The CWA would result in a federal nexus requiring additional NEPA analysis if an Individual Permit is required.

\section{CLAY SPRINGS WIND ENERGY PROJECT AREA}

An initial review of the National Wetlands Inventory Database revealed there are no online wetlands data within the project area. There are a number of ephemeral washes within the project area that may be jurisdictional if certain criteria are satisfied.

Risk Category. Medium. There may be ephemeral washes or wetlands in the project area that qualify as jurisdictional.

Additional Work Required. Additional work required would be the same as that described under the Grand Canyon West Wind Energy site.

\section{BLM WIND ENERGY NORTH PROJECT AREA}

An initial review of the National Wetlands Inventory Database revealed there are no online wetlands data within the project area. There are a number of ephemeral washes within the project area that may be jurisdictional if certain criteria are satisfied. 


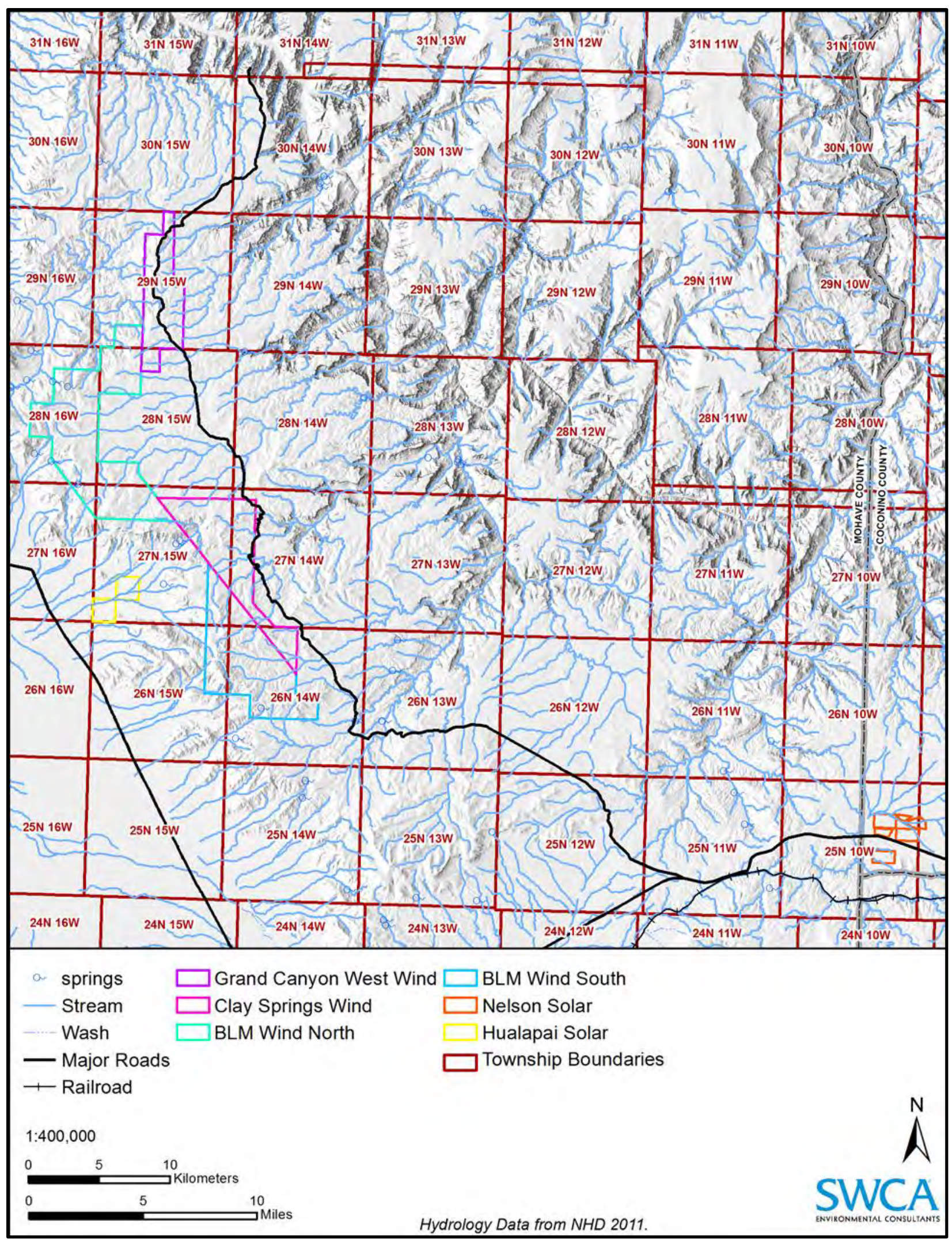

Figure 24. Potential Waters of the U.S. 
Risk Category. Medium. There may be ephemeral washes or wetlands in the project area that qualify as jurisdictional.

Additional Work Required. Additional work required would be the same as that described under the Grand Canyon West Wind Energy site.

\section{BLM WIND ENERGY SOUTH PROJECT AREA}

An initial review of the National Wetlands Inventory Database revealed there are no online wetlands data within the project area. There are a number of ephemeral washes within the project area that may be jurisdictional if certain criteria are satisfied.

Risk Category. Medium. There may be ephemeral washes or wetlands in the project area that qualify as jurisdictional.

Additional Work Required. Additional work required would be the same as that described under the Grand Canyon West Wind Energy site.

\section{NELSON SOLAR ENERGY PROJECT AREA}

An initial review of the National Wetlands Inventory Database revealed there are no online wetlands data within the project area. There are a number of ephemeral washes within the project area that may be jurisdictional if certain criteria are satisfied.

Risk Category. Medium. There may be ephemeral washes or wetlands in the project area that qualify as jurisdictional.

Additional Work Required. Additional work required would be the same as that described under the Grand Canyon West Wind Energy site.

\section{HUALAPAI SOLAR ENERGY PROJECT AREA}

An initial review of the National Wetlands Inventory Database revealed there are no online wetlands data within the project area. There are a number of ephemeral washes within the project area that may be jurisdictional if certain criteria are satisfied.

Risk Category. Medium. There may be ephemeral washes or wetlands in the project area that qualify as jurisdictional.

Additional Work Required. Additional work required would be the same as that described under the Grand Canyon West Wind Energy site.

\subsection{Flood Hazard Zones}

A flood hazard zone is an area that has been identified on a FEMA Flood Insurance Rate Map as being prone to floods. Different flood zones are defined by FEMA according to risk. Each zone reflects the severity or type of flooding in an area. 


\section{GRAND CANYON WEST WIND ENERGY PROJECT AREA}

Insurance Maps for the project area were reviewed, and the project area is categorized as Zone D. Flood Zone D designations indicate that no flood hazard analysis has been conducted. Zone D is an undetermined risk area and has not been subject to an accepted hydraulics study.

Risk Category. Low. No designated FEMA flood zones are present within the project area, and flood hazards are not anticipated.

Additional Work Required. Additional work includes flood zone mapping, potential CWA permitting, micro-siting to avoid construction in flood zones, and county permit acquisition, if necessary.

\section{CLAY SPRINGS WIND ENERGY PROJECT AREA}

Insurance Maps for the project area were reviewed, and the project area is categorized as Zone D. Flood Zone D designations indicate that no flood hazard analysis has been conducted. Zone $\mathrm{D}$ is an undetermined risk area and has not been subject to an accepted hydraulics study.

Risk Category. Low. No designated FEMA flood zones are present within the project area, and flood hazards are not anticipated.

Additional Work Required. Additional work includes flood zone mapping, potential CWA permitting, micro-siting to avoid construction in flood zones, and county permit acquisition, if necessary.

\section{BLM WIND ENERGY NORTH PROJECT AREA}

Insurance Maps for the project area were reviewed and no flood zone areas were identified in the project area. Flood Zone A areas are located north and west of the project area.

Risk Category. Low. No designated FEMA flood zones are present within the project area, and flood hazards are not anticipated.

Additional Work Required. Additional work includes flood zone mapping, potential CWA permitting, micro-siting to avoid construction in flood zones, and county permit acquisition, if necessary.

\section{BLM WIND ENERGY SOUTH PROJECT AREA}

Insurance Maps for the project area were reviewed, and the southern portion of the project area is categorized as Zone D. Flood Zone D designations indicate that no flood hazard analysis has been conducted. Zone D is an undetermined risk area and has not been subject to an accepted hydraulics study. No other flood zones have been identified in the project area.

Risk Category. Low. No designated FEMA flood zones are present within the project area, and flood hazards are not anticipated.

Additional Work Required. Additional work includes flood zone mapping, potential CWA permitting, micro-siting to avoid construction in flood zones, and county permit acquisition, if necessary. 


\section{NELSON SOLAR ENERGY PROJECT AREA}

Insurance Maps for the project area were reviewed, and the project area is categorized as Zone D. Flood Zone D designations indicate that no flood hazard analysis has been conducted. Zone D is an undetermined risk area and has not been subject to an accepted hydraulics study.

Risk Category. Low. No designated FEMA flood zones are present within the project area, and flood hazards are not anticipated.

Additional Work Required. Additional work includes flood zone mapping, potential CWA permitting, micro-siting to avoid construction in flood zones, and county permit acquisition, if necessary.

\section{HUALAPAI SOLAR ENERGY PROJECT AREA}

Insurance Maps for the project area were reviewed, and the project area is categorized as Zone D. Flood Zone D designations indicate that no flood hazard analysis has been conducted. Zone D is an undetermined risk area and has not been subject to an accepted hydraulics study. Flood Zone A areas are located west of the project area.

Risk Category. Low. No designated FEMA flood zones are present within the project area, and flood hazards are not anticipated.

Additional Work Required. Additional work includes flood zone mapping, potential CWA permitting, micro-siting to avoid construction in flood zones, and county permit acquisition, if necessary.

\subsection{Geologic Hazard Zones}

Geologic hazards in northwestern Arizona consist primarily of seismic activity, unstable or corrosive soils, and steep slopes (Figure 25). Faults are fractures between two blocks of rock that allow the blocks to move relative to each other. During an earthquake, one side of the fault suddenly slips, relative to the other side. Seismic activity across the project area is on par with other areas of Arizona (USGS 2012a). Peak ground acceleration is the maximum acceleration experienced by a particle during the course of an earthquake, described in terms of the acceleration due to the force of gravity $(\% \mathrm{~g})$.

\section{GRAND CANYON WEST WIND ENERGY PROJECT AREA}

Peak ground acceleration (pga) within the project area ranges from $10 \% \mathrm{~g}$ to $12 \% \mathrm{~g}$, with a $2 \%$ probability of exceedance in 50 years. The Grand Canyon West Wind Energy site is mapped within an area showing a pga of $12 \% \mathrm{~g}$ to $14 \% \mathrm{~g}$ (USGS 2012c). No areas of landslide susceptibility were identified within the project area. The probability of an earthquake with a magnitude greater than 5.0 within 20 years ranges from 0.06 to 0.10 . The probability of an earthquake with a magnitude greater than 5.0 within 50 years ranges from 0.15 to 0.25 (USGS 2009b).

Risk Category. Low. Potential geologic hazards were not identified within the project area.

Additional Work Required. Soil testing and geotechnical studies to determine suitability to support project infrastructure will be required. 


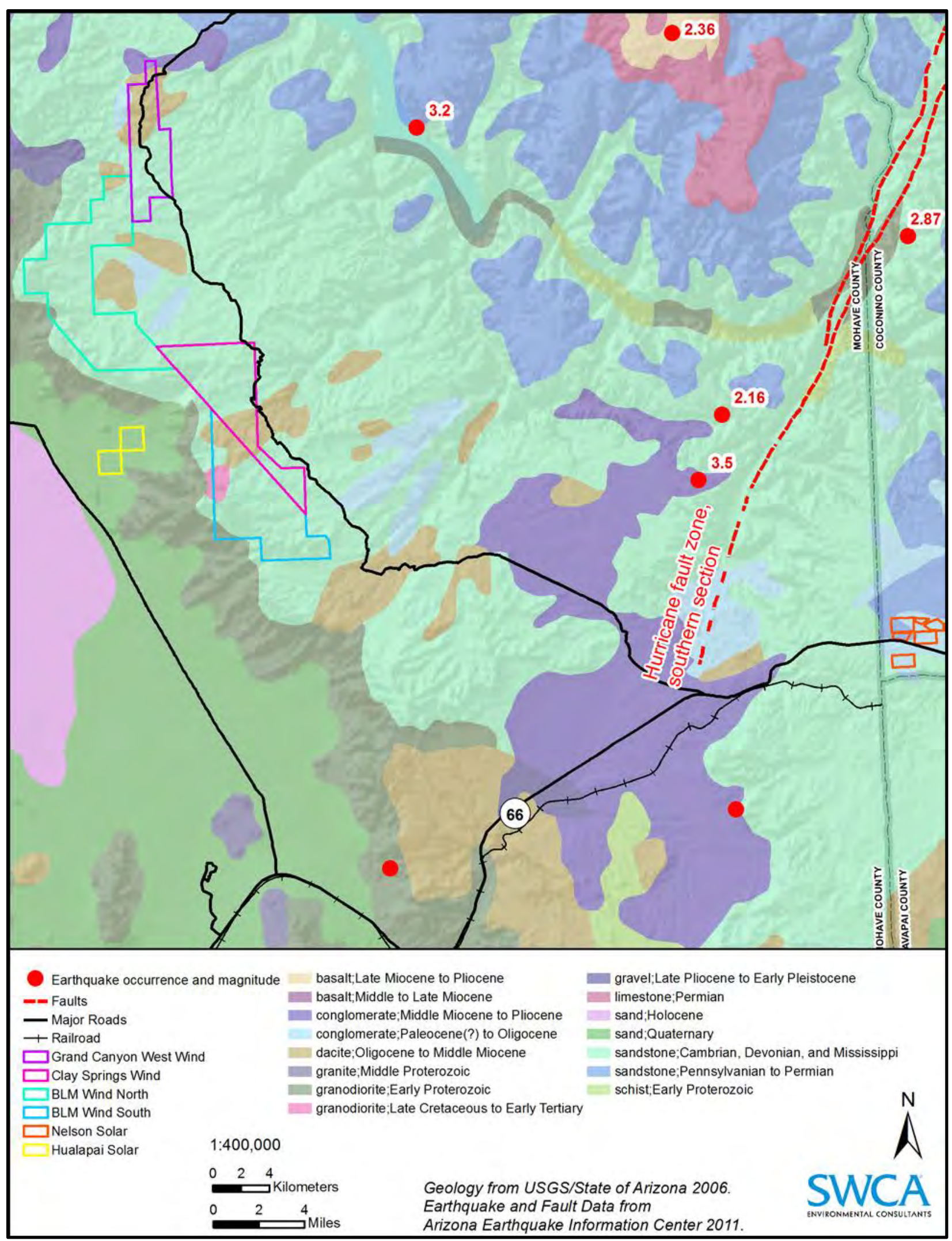

Figure 25. Geologic hazard zones. 


\section{CLAY SPRINGS WIND ENERGY PROJECT AREA}

Mapping from the AZGS Geologic Map of Arizona displays one low-angle fault appearing to run across the Clay Springs Wind Energy project area from southwest to northeast (see Figure 25). A USGS Google Earth overlay of all known Quaternary faults does not display any faults on or in the vicinity of the project area (USGS 2012b).

Risk Category. Low. Potential geologic hazards were not identified within the project area.

Additional Work Required. Additional work required would be the same as that described under the Grand Canyon West Wind Energy site.

\section{BLM WIND ENERGY NORTH PROJECT AREA}

Pga within the project area ranges from $10 \% \mathrm{~g}$ to $12 \% \mathrm{~g}$, with a $2 \%$ probability of exceedance in 50 years. The BLM Wind Energy North project area is mapped within an area showing a pga of $12 \% \mathrm{~g}$ to $14 \% \mathrm{~g}$ (USGS 2012c). No areas of landslide susceptibility were identified within the project area.

The probability of an earthquake with a magnitude greater than 5.0 within 20 years ranges from 0.06 to 0.10 . The probability of an earthquake with a magnitude greater than 5.0 within 50 years ranges from 0.15 to 0.25 (USGS 2009b).

Risk Category. Low. Potential geologic hazards were not identified within the project area.

Additional Work Required. Additional work required would be the same as that described under the Grand Canyon West Wind Energy site.

\section{BLM WIND ENERGY SOUTH PROJECT AREA}

Mapping from the AZGS Geologic Map of Arizona displays one low-angle fault appearing to run across the project area from southwest to northeast (see Figure 25). A USGS Google Earth overlay of all known Quaternary faults does not display any faults on or in the vicinity of the project area (USGS 2012b).

Risk Category. Low. Potential geologic hazards were not identified within the project area.

Additional Work Required. Additional work required would be the same as that described under the Grand Canyon West Wind Energy site.

\section{HUALAPAI SOLAR ENERGY PROJECT AREA}

Pga within the project area ranges from $10 \% \mathrm{~g}$ to $12 \% \mathrm{~g}$, with a $2 \%$ probability of exceedance in 50 years. The Grand Canyon West Wind Energy site is mapped within an area showing a pga of $12 \% \mathrm{~g}$ to $14 \% \mathrm{~g}$ (USGS 2012c). No areas of landslide susceptibility were identified within the project area.

The probability of an earthquake with a magnitude greater than 5.0 within 20 years ranges from 0.06 to 0.10 . The probability of an earthquake with a magnitude greater than 5.0 within 50 years ranges from 0.15 to 0.25 (USGS 2009b). A USGS Google Earth overlay of all known Quaternary faults does not display any faults on or in the vicinity of the project area (USGS 2012b).

Risk Category. Low. Potential geologic hazards were not identified within the project area.

Additional Work Required. Additional work required would be the same as that described under the Grand Canyon West Wind Energy site. 


\section{NELSON SOLAR ENERGY PROJECT AREA}

The Yampai graben Quaternary fault, located approximately 4.25 miles southwest of the proposed Nelson Solar Energy project area (east of Peach Springs) is a narrow, shallow, symmetric graben located on the west side of the Aubrey Valley near the southwestern margin of the Colorado Plateau. Paleozoic rocks are displaced at least 98 feet from the graben shoulders to the valley bottom. The escarpments are fairly steep and quite linear, which suggests Quaternary fault activity, but there is no documented displacement of the scarce Quaternary deposits along the fault. Faulting has generated two steep, linear escarpments on Paleozoic bedrock; these escarpments form the sides of a narrow trough that is filled with young fan deposits that are not faulted (USGS 2009a).

Risk Category. Low. Potential geologic hazards were not identified within the project area.

Additional Work Required. Additional work required would be the same as that described under the Grand Canyon West Wind Energy site.

\subsection{Recreation}

The study area is located on a mixture of Tribal, BLM, state, and privately owned lands. There are no designated recreation sites within or adjacent to the study area, but the areas may be used casually for dispersed recreation such as camping, backpacking, hiking, OHV use, picnicking, hunting, photography, rock hounding, and horseback riding. Much of the land in and surrounding the study area is remote and provides excellent opportunities for solitude and primitive camping and backpacking. No special recreation management areas are present within or adjacent to the study area. There are no designated wilderness areas within or adjacent to the study area.

\section{GRAND CANYON WEST WIND ENERGY PROJECT AREA}

There are no recreation sites, or recreation areas within the project area. The Grand Canyon West Skywalk is located north of the project area, and some visitors are expected to travel through the project area on their way to the Skywalk. Grand Canyon National Park is located approximately 9 miles east of the project area. The project area may be used for dispersed recreation activities.

Risk Category. Low. No designated recreational areas were identified. Tribal routes within or surrounding the area are used to access Grand Canyon West Skywalk.

Additional Work Required. Since the Nelson Solar Energy site is wholly on Tribal lands, additional work to determine the potential impacts on recreation would likely not be required and may be eliminated from detailed analysis in subsequent NEPA analysis.

\section{CLAY SPRINGS WIND ENERGY PROJECT AREA}

There are no recreation sites or recreation areas within the project area. The nearest designated wilderness is Mount Tipton Wilderness, located approximately 13 miles west of the Clay Springs project area. Grand Canyon National Park is located approximately 9 miles to the east of the Clay Springs Wind Energy project area and approximately 14 miles north of the Nelson Solar Energy project area.

Risk Category. Low. No designated recreational areas were identified. Some of the Tribal routes within or surrounding the Clay Springs Wind Energy site are used to access Grand Canyon National Park. Although the BLM lands surrounding the Clay Springs portion of the project area may be used for 
dispersed recreation, these lands are managed as an Extensive Recreation Management Area and would not require a specific management strategy or activity-level planning.

Additional Work Required. NEPA analysis would be required to determine whether the proposed project would result in impacts to recreation settings, experiences, and opportunities.

\section{BLM WIND ENERGY NORTH PROJECT AREA}

The project area is managed as an Extensive Recreation Management Area and would not require a specific management strategy or activity-level planning. Routes within or surrounding the area may be used to access Grand Canyon West Skywalk.

Risk Category. Low. No designated recreational areas were identified.

Additional Work Required. Additional work to determine the potential impacts on recreation would likely not be required and may be eliminated from detailed analysis in subsequent NEPA analysis.

\section{BLM WIND ENERGY SOUTH PROJECT AREA}

The BLM lands in the project area are managed as an Extensive Recreation Management Area and would not require a specific management strategy or activity-level planning. The project area's State land is currently designated open for recreation; however, a recreation permit is required to camp, hike, or travel on State Trust land if it is designated open for recreation. The State land within the project area is not currently authorized for other uses other than recreation and grazing.

Risk Category. Low. No designated recreational areas were identified.

Additional Work Required. Additional work to determine the potential impacts on recreation would likely not be required and may be eliminated from detailed analysis in subsequent NEPA analysis.

\section{NELSON SOLAR ENERGY PROJECT AREA}

There are no recreation sites, or recreation areas within the project area. Grand Canyon National Park is located approximately 14 miles north of the Nelson Solar Energy project area.

Risk Category. Low. No designated recreational areas were identified. Although the BLM lands surrounding the Nelson Solar Energy site may be used for dispersed recreation, these lands are managed as an Extensive Recreation Management Area and would not require a specific management strategy or activity-level planning.

Additional Work Required. NEPA analysis would be required to determine whether the proposed project would result in impacts to recreation settings, experiences, and opportunities.

\section{HUALAPAI SOLAR ENERGY PROJECT AREA}

There are no recreation sites, or recreation areas within the project area. The nearest designated wilderness is Mount Tipton Wilderness, located approximately 10 miles west of the project area. Grand Canyon National Park is located approximately 15 miles to the east of the project area.

Risk Category. Low. No designated recreational areas were identified. Although the BLM lands surrounding the Hualapai Solar Energy site may be used for dispersed recreation, these lands are managed 
as an Extensive Recreation Management Area and would not require a specific management strategy or activity-level planning.

Additional Work Required. NEPA analysis would be required to determine whether the proposed project would result in impacts to recreation settings, experiences, and opportunities.

\subsection{Air Quality}

Air quality is determined by the ambient concentrations of pollutants that are known to have detrimental effects. The U.S. Environmental Protection Agency (EPA) has classified National Ambient Air Quality Standards (NAAQS) for six criteria pollutants: carbon monoxide, nitrogen dioxide, particulate matter with diameter of 10 microns or less $\left(\mathrm{PM}_{10}\right)$, particulate matter with diameter of 2.5 microns or less $\left(\mathrm{PM}_{2.5}\right)$, ozone, sulfur dioxide, and lead. Areas with air quality that do not meet the standards are designated "non-attainment areas" by the EPA.

Areas where the ambient concentrations exceed the NAAQS are considered non-attainment and, as such, are regulated more strictly to reduce emissions in order to meet the NAAQS pollutant levels. Mohave and Coconino Counties are in attainment for all criteria pollutants.

Facilities located on Tribal lands in Arizona, with the exception of the Navajo Nation, are under the jurisdiction of the EPA Region 9. However, sources operating in multiple counties, or outside of the jurisdiction listed above, even for a short amount of time, must obtain permits from the ADEQ. Both the EPA and ADEQ monitor and enforce air quality regulations in the study area.

The state air permit regulations are contained in Arizona Administrative Code (AAC) Title 18, Environmental Quality, Chapter 2, Department of Environmental Quality, Air Pollution Control; however, the projects are not anticipated to involve emissions greater than de minimus thresholds with the exception of mobile source emission from vehicles and construction equipment. Therefore, the projects will not be subject to AAC Title 18, Environmental Quality, Chapter 2, Department of Environmental Quality, Air Pollution Control, Article 3, Permits and Permit Revisions.

Air quality rules for fugitive dust emissions apply to construction activities and clearing of land.

They require reasonable precautions to prevent dust from becoming airborne, including 1) using water or chemicals to control dust where possible, 2) covering open-bodied trucks at all times while transporting materials likely to produce airborne dusts, and 3) promptly removing earth or material from paved streets.

The construction activities associated with the projects will take place in Mohave and Coconino Counties, Arizona. These activities are governed by the applicable rules and regulations promulgated by the EPA and the ADEQ.

Table 11 provides the applicable ADEQ fugitive dust rules with which the project must comply.

Table 11. Applicable Fugitive Dust Rules

\begin{tabular}{lll}
\hline Agency & Rule Number & Rule Description \\
\hline ADEQ & R18-2-604 & Construction on "open areas"' fugitive dust limitations \\
\hline ADEQ & R18-2-605 & Road construction fugitive dust limitations \\
\hline ADEQ & R18-2-606 & Material handling fugitive dust limitations \\
\hline ADEQ & R18-2-607 & Storage pile fugitive dust limitations \\
\hline ADEQ & R18-2-614 & Opacity limitation for non-point sources \\
\hline
\end{tabular}


Table 11. Applicable Fugitive Dust Rules (Continued)

\begin{tabular}{lll}
\hline Agency & Rule Number & Rule Description \\
\hline ADEQ & R18-2-702 & Visible emission limitations \\
\hline ADEQ & R18-2-802 & Off-road machinery opacity limitations \\
\hline ADEQ & R18-2-805 & Roadway and site-clearing opacity limitations \\
\hline
\end{tabular}

The following provide a brief overview of the potentially applicable fugitive dust rules to the projects.

- The Arizona Administrative Code Title 18, Environmental Quality, Chapter 2, Department of Environmental Quality, Air Pollution Control, Section R18-2-614, effective July 18, 2005, prohibits visible dust emissions with opacity greater than $40 \%$ from any non-point source measured in accordance with the Arizona Testing Manual, Reference Method 9.

- $\quad$ EPA Reference Method 9 involves the determination of plume opacity by qualified observers. The method includes procedures for the training and certification of observers, as well as procedures to be used in the field for determination of plume opacity.

- These rules also require control of visible dust from open areas, road construction, material handling, storage piles, roadway, and site clearing. ADEQ rules apply to areas of the project that occur outside of the Hualapai Tribal lands.

\section{GRAND CANYON WEST WIND ENERGY PROJECT AREA}

No EPA-regulated facilities were identified within the project area. The project area is in attainment for all pollutants as regulated by the EPA.

Risk Category. Low. The project area is in attainment for all pollutants as regulated by the EPA.

Additional Work Required. Potential Clean Air Act (CAA) permitting (e.g., temporary stationary sources used during construction activities, such as concrete batch plants, reciprocating internal combustion engines, etc.), along with coordination with the EPA and ADEQ, if necessary.

\section{CLAY SPRINGS WIND ENERGY PROJECT AREA}

No EPA-regulated facilities were identified within the project area. The project area is in attainment for all pollutants as regulated by the EPA.

Risk Category. Low. The project area is in attainment for all pollutants as regulated by the EPA.

Additional Work Required. Additional work required would be similar to that described for the Grand Canyon West Wind Energy project area.

\section{BLM WIND ENERGY NORTH PROJECT AREA}

No EPA-regulated facilities were identified within the project area. The project area is in attainment for all pollutants as regulated by the EPA.

Risk Category. Low. The project area is in attainment for all pollutants as regulated by the EPA.

Additional Work Required. Additional work required would be similar to that described for the Grand Canyon West Wind Energy project area. 


\section{BLM WIND ENERGY SOUTH PROJECT AREA}

No EPA-regulated facilities were identified within the project area. The project area is in attainment for all pollutants as regulated by the EPA.

Risk Category. Low. The project area is in attainment for all pollutants as regulated by the EPA.

Additional Work Required. Additional work required would be similar to that described for the Grand Canyon West Wind Energy project area.

\section{NELSON SOLAR ENERGY PROJECT AREA}

The project area is in attainment for all pollutants as regulated by the EPA.

Risk Category. Low. The project area is in attainment for all pollutants as regulated by the EPA.

Additional Work Required. Additional work required would be similar to that described for the Grand Canyon West Wind Energy project area.

\section{HUALAPAI SOLAR ENERGY PROJECT AREA}

The project area is in attainment for all pollutants as regulated by the EPA.

Risk Category. Low. The project area is in attainment for all pollutants as regulated by the EPA.

Additional Work Required. Additional work required would be similar to that described for the Grand Canyon West Wind Energy project area.

\subsection{Noise}

Noise is defined as unwanted sound because it interferes with speech communication and hearing or is otherwise annoying. Under certain conditions, noise may cause hearing loss, interfere with human activities at home and work, and in various ways affect people's health and well-being. Sensitive noise receptors are those human-occupied locations and uses within an area that are subject to stress, annoyance, and interference from noise and include residences, hospitals, libraries, recreation areas, churches, and other similar venues.

The Noise Control Act of 1972 recognizes that uncontrolled noise can lead to impacts to the health and welfare of the nation's population. The act further declares that it is the policy of the United States to promote an environment free from noise that jeopardizes the health or welfare (EPA 1974). In 1974, the EPA released a document identifying a 24-hour exposure level of 70 day-night equivalent level as the level of environmental noise to prevent measurable hearing loss over a lifetime (EPA 1974). The same document identified levels of 55 decibels $(\mathrm{dB})$ outdoors and $45 \mathrm{~dB}$ indoors to prevent annoyance.

The Grand Canyon National Park Enlargement Act of 1975 established that natural quiet should be protected as a resource and a value to the Park. Natural quiet is defined as the level of all natural sounds in an area, excluding all mechanical, electrical, and other human-caused sounds.

The Quiet Communities Act of 1978 promotes the development of state and local noise control programs. There is no state or local noise control program in the area of analysis; therefore, standards established by the EPA would be applied. 
The Mohave County General Plan (Mohave County Planning and Zoning Department [MCPZD] 2010) addresses the effects of noise on the local community and has established the county-wide goal "to minimize noise levels throughout the County and, whenever possible, mitigate the effects of noise to provide a safe and healthy environment." The County also established standards for noise and land use compatibility (MCPZD 2010; Exhibit V5) and the Board of Supervisors may employ the General Planning process or zoning ordinance to protect noise-sensitive land uses.

Although Mohave and Coconino Counties have not yet passed a wind energy ordinance that would define minimum setbacks for development, other Counties in Arizona have identified increase setbacks to address concerns regarding noise generated by wind turbines. In Navajo County, an ordinance was passed establishing setbacks to existing residences of at least 0.25 mile so that sound standards will not be exceeded (Navajo County Ordinance No. 06-10). Additionally, Bullhead City, Arizona, has an Alternative Energy Systems ordinance (Bullhead City, Arizona, Code of Ordinances, Title 17 - Zoning Chapter 17.39) which states:

Alternative energy systems shall be designed, installed, and operated so that the systems do not make loud, unnecessary, or offensive noise or and noise which may reasonably be anticipated to annoy, disturb, injure or endanger the comfort, slumber, peace, health or safety of any reasonable person or persons of normal sensitivity, whether due to volume or duration or both and shall not exceed 60 $\mathrm{dBA}$, as measured at ground level at the nearest inhabited dwelling unit, except during short-term events such a utility outages and/or sever wind storms.

However, this ordinance does not apply to wind farms, which are prohibited within the Bullhead City limits.

\section{GRAND CANYON WEST WIND ENERGY PROJECT AREA}

Sensitive noise receptors within the project area were identified through analysis of GIS data and during field reconnaissance efforts. Few sensitive noise receptors were identified within or near the project area. Turbine siting to avoid noise impacts, along with standard setback requirements, and operational management and monitoring of the wind farms would decrease the risk of noise-related impacts. Occupied residences adjacent to a project area could potentially be affected by noise associated with construction activities and/or facility operation. Additionally, other Counties and federal agencies are requiring baseline studies to determine existing background noise levels and models that simulate anticipated project noise.

Risk Category. Low. Few sensitive noise receptors are located within the project area.

Additional Work Required. A baseline study to determine existing background noise levels, identify sensitive noise receptors, and a model to simulate anticipated project noise will be included in the environmental evaluation process. However, due to the undeveloped nature of the project area, it is likely that if sensitive receptors or noise-sensitive land uses are identified, additional noise mitigation measures will be required. Occupied residences adjacent to a project area could potentially be affected by noise associated with construction activities and/or facility operation. Additionally, other Counties and federal agencies are requiring baseline studies to determine existing background noise levels and models that simulate anticipated project noise.

\section{CLAY SPRINGS WIND ENERGY PROJECT AREA}

Sensitive noise receptors within the project area were identified through analysis of GIS data and during field reconnaissance efforts. Similar to the Grand Canyon West project area, few sensitive noise receptors were identified within or near the project area. Turbine siting to avoid noise impacts, along with standard 
setback requirements, and operational management and monitoring of the wind farms would decrease the risk of noise-related impacts.

Risk Category. Low. Few sensitive noise receptors are located within the project area.

Additional Work Required. Additional work required would be similar to that described for the Grand Canyon West Wind Energy project area.

\section{BLM WIND ENERGY NORTH PROJECT AREA}

Sensitive noise receptors within the project area were identified through analysis of GIS data and during field reconnaissance efforts. Similar to the Grand Canyon West project area, few sensitive noise receptors were identified within or near the project area. Turbine siting to avoid noise impacts, along with standard setback requirements, and operational management and monitoring of the wind farms would decrease the risk of noise-related impacts.

Risk Category. Low. Few sensitive noise receptors are located within the project area.

Additional Work Required. Additional work required would be similar to that described for the Grand Canyon West Wind Energy project area.

\section{BLM WIND ENERGY SOUTH PROJECT AREA}

Sensitive noise receptors within the project area were identified through analysis of GIS data and during field reconnaissance efforts. Similar to the Grand Canyon West project area, few sensitive noise receptors were identified within or near the project area. Turbine siting to avoid noise impacts, along with standard setback requirements, and operational management and monitoring of the wind farms would decrease the risk of noise-related impacts.

Risk Category. Low. Few sensitive noise receptors are located within the project area.

Additional Work Required. Additional work required would be similar to that described for the Grand Canyon West Wind Energy project area.

\section{NELSON SOLAR ENERGY PROJECT AREA}

Sensitive noise receptors within the project area were identified through analysis of GIS data and during field reconnaissance efforts. Similar to the Grand Canyon West project area, few sensitive noise receptors were identified within or near the project area.

Risk Category. Low. Few sensitive noise receptors are located within the project area.

Additional Work Required. Additional work required would be similar to that described for the Grand Canyon West Wind Energy project area. Because this is solar project area, occupied residences adjacent to the project area could potentially be affected by noise associated with construction activities, and not necessarily from facility operation.

\section{HUALAPAI SOLAR ENERGY PROJECT AREA}

Sensitive noise receptors within the project area were identified through analysis of GIS data and during field reconnaissance efforts. Similar to the Grand Canyon West project area, few sensitive noise receptors were identified within or near the project area. 
Risk Category. Low. Few sensitive noise receptors are located within the project area.

Additional Work Required. Additional work required would be similar to that described for the Nelson Solar Energy project area.

\subsection{Hazardous Materials}

Hazardous materials are defined differently by various regulatory agencies. In general, the definitions include substances that are a "health hazard," "physical hazard," or any substance that could cause harm to people, plants, or animals when released into the environment. For this project, SWCA evaluated for the potential presence of anthropogenic contamination that could affect the purchase, construction, or operation of the proposed use of the property.

The potential for presence of hazardous materials within the study area was identified through analysis and review of aerial photographs dated 2010; 1:24,000 scale topographic maps depicting the sites; the ADEQ interactive GIS eMaps website (ADEQ 2012); and EPA Region 9 databases of Tribal leaking underground storage tank (LUST) and underground storage tank (UST) sites (EPA 2010). This analysis was not intended to replace an American Society of Testing and Materials (ASTM) standard Phase I Environmental Site Assessment (ESA).

Hazardous materials are regulated under a number of laws and agencies, including the EPA, the U.S. Occupational Safety and Health Administration, and the U.S. Nuclear Regulatory Commission. Under the Comprehensive Environmental Response Compensation and Liability Act (CERCLA), persons may be held liable for cleaning up hazardous substances at properties that they either currently own or operate. However, the CERCLA Brownfields Amendments provide liability exemptions including the "innocent landowner" defense. In 1986, the Superfund Amendments and Reauthorization Act created an "innocent landowner" defense to CERCLA liability by adding Section 101(35)(B), which requires the buyer to perform certain due diligence prior to the purchase of a property. To qualify for the innocent landowner defense, the buyer must complete all appropriate inquiries as described in ASTM Standard 1527-05, Standard Practice for Environmental Site Assessments: Phase I Environmental Site Assessment Process (ASTM 2005) or ASTM Standard E 2247-08 (ASTM 2008), Standard Practice for Environmental Site Assessments: Phase I Environmental Site Assessment Process for Forestland or Rural Property. The preliminary review conducted for the study area herein does not meet the ASTM standard for "all appropriate inquiry."

\section{GRAND CANYON WEST WIND ENERGY PROJECT AREA}

Based on the preliminary environmental review and site visit, no potential Recognized Environmental Conditions (RECs) were identified for the project area.

Risk Category. Low. Potential RECs were not identified within the project area.

Additional Work Required. A Phase I ESA may be required, per the prospective lender's requirements. Because the project area is rural and undeveloped, ASTM Standard E 2247-08, Standard Practice for Environmental Site Assessments: Phase I Environmental Site Assessment Process for Forestland or Rural Property would be recommended.

\section{CLAY SPRINGS WIND PROJECT AREA}

Based on the preliminary environmental review and site visit, no potential RECs were identified for the project area. 
Risk Category. Low. Potential RECs were not identified within the project area.

Additional Work Required. Additional work required would be similar to that described for the Grand Canyon West Wind Energy project area.

\section{BLM WIND ENERGY NORTH PROJECT AREA}

Based on the preliminary environmental review and site visit, no potential RECs were identified for the project area.

Risk Category. Low. Potential RECs were not identified within the project area.

Additional Work Required. Additional work required would be similar to that described for the Grand Canyon West Wind Energy project area.

\section{BLM WIND ENERGY SOUTH PROJECT AREA}

Based on the preliminary environmental review and site visit, no potential RECs were identified for the project area.

Risk Category. Low. Potential RECs were not identified within the project area.

Additional Work Required. Additional work required would be similar to that described for the Grand Canyon West Wind Energy project area.

\section{NELSON SOLAR ENERGY PROJECT AREA}

Based on the preliminary environmental review and site visit, no potential RECs were identified for the project area.

Risk Category. Low. Potential RECs were not identified within the project area.

Additional Work Required. Additional work required would be similar to that described for the Grand Canyon West Wind Energy project area.

\section{HUALAPAI SOLAR ENERGY PROJECT AREA}

Based on the preliminary environmental review and site visit, no potential RECs were identified for the project area.

Risk Category. Low. Potential RECs were not identified within the project area.

Additional Work Required. Additional work required would be similar to that described for the Grand Canyon West Wind Energy project area.

\subsection{Cultural Resources and Native American Concerns}

Cultural resources in Arizona are protected by federal and state laws, regulations, and statutes. Section 106 of the National Historic Preservation Act, as amended in 2000, requires government agencies to take into account the effects of their actions on properties listed or eligible for listing in the National Register of Historic Places (NRHP). To determine whether a project would affect NRHP-eligible properties, cultural resources must be inventoried and evaluated for eligibility for inclusion in the NRHP. The BLM, 
in consultation with the Arizona SHPO and in accordance with the regulations set forth in 36 CFR 800, will determine the area of potential effects (APE) and the methods to be used during identification efforts.

Cultural resources also include traditional cultural properties, that is, properties that are important to a community's practices and beliefs and that are necessary for maintaining the community's cultural identity. Cultural resources refer to both human-made and natural physical features associated with human activity and, in most cases, are finite, unique, fragile, and nonrenewable. Cultural resources that meet the eligibility criteria for listing in the NRHP are considered "significant" resources and must be taken into consideration during the planning of federal projects. Federal agencies are also required to consider the effects of their actions on sites, areas, and other resources (e.g., plants) that are of religious significance to Native Americans as established under the American Indian Religious Freedom Act (Public Law [PL] 95-341). Native American graves and burial grounds are protected by the Native American Graves Protection and Repatriation Act (NAGPRA; PL 101-601).

The following cultural resources overview and impact assessment for the Alternative Energy Critical impact analysis was prepared by Peter Bungart, Senior Archaeologist, Hualapai Tribe Department of Cultural Resources:

Because of the similarities between the project areas, this section provides a cultural resources overview of the study area and highlight potential issues that may affect implementation of large-scale undertakings in the area. For the purposes of this section, the discussion delineates two main areas: the Clay SpringsGrand Wash Cliffs area, and the Nelson area. These two main areas are distinctly different from each other in various ways, and the cultural resources issues vary accordingly. The following discussion attempts to address issues of archaeological sites, traditional cultural places (TCPs), including cultural landscapes, and potential NAGPRA concerns.

The information summarized here was obtained from a variety of sources: the Hualapai Tribe's Department of Cultural Resources archaeological database, from a GIS-based atlas of Hualapai places of historical and cultural importance (the Hualapai Atlas), and from the BLM KFO site and project records. What this discussion does not include is ethnohistoric information collected specifically for the two main areas, although legacy information is included that was readily available at the time of this writing (information primarily retrieved from the Hualapai Atlas or other published and unpublished sources).

The part of the analysis area including the Grand Canyon West Wind Energy site, the Clay Springs Wind Energy site, the BLM Wind Energy North, BLM Wind Energy South, and the Hualapai Solar Energy sites together with the Grand Wash Cliffs area is a culturally unique landscape. Clay Springs specifically was long the home base for the Clay Springs Band (Ha Du:ba Pa'a in Hualapai), one of the 14 historical bands of the Hualapai. The area thus holds considerable importance to Hualapai history and culture. Occupying the territory along the Grand Wash Cliffs, the Clay Springs Band had access to a very diverse variety of food and material resources. Aside from crucial water sources at the numerous springs issuing along the cliffs, they hunted and gathered from the upland areas of the western edge of the Colorado Plateau, the canyon country that incised the plateau, and the broad valley bottoms to the west that mark the transition to the Basin and Range physiographic province (e.g., the Hualapai Valley and Red Lake areas). Some areas were favorable for gardening and small-scale agriculture, either in the immediate vicinity of springs or, during moister climatic periods, in upper alluvial washes and valleys where dry farming was sometimes possible. This latter scenario in particular is demonstrated at archaeological sites that contain evidence of maize consumption and the more sedentary lifeway necessary to maintain field areas.

Archaeologically, the Grand Wash Cliffs area is one of the most intensively occupied areas on the Hualapai reservation and in northwestern Arizona in general. It is not uncommon for recorded sites to exceed 10 acres in area or to contain thousands of artifacts, including chipped stone, ceramics, and 
grinding tools. Furthermore, as the Hualapai people hold their ancestral territory sacred and are very protective of ancient sites from outsiders, it is clear that there has been little looting or illicit artifact collecting relative to most other areas in the southwestern United States, particularly when compared with non-reservation lands in general. As an example, a large site recorded by the Hualapai Department of Cultural Resources virtually on the edge of the BLM Wind Energy South project area contained over 35 classifiable projectile points (and many more unclassifiable fragmentary examples), including multiple clusters that were strong evidence of a high degree of contextual integrity. Although this may be a somewhat exceptional example, many other sites exist that demonstrate an abundance of artifacts and high degree of preservation.

The vast majority of sites that have been recorded in the vicinity of the Clay Springs-Grand Wash Cliffs study area are open artifact scatters. These tend to be concentrated in pinyon-juniper woodland areas above 5,500 feet in elevation. In fact, as elevation increases, vegetation diversity also seems to increase, and site density and intensity also generally increases. Sites throughout this area range from camps with diverse artifact types (such as chipped stone, ceramics, and grinding tools found together, interpreted as evidence of seasonal residential use), to hunting-oriented camps (primarily chipped stone, often with several projectile points and cutting tools), gathering and seed-processing loci (with multiple grinding tools, often with relatively low numbers of chipped stone artifacts), to residential agricultural sites (with deep, formalized trough and basin metates, abundant ceramics, and generally more diverse artifact assemblages resulting from more intensive long-term occupation). The BLM KFO archaeologist Timothy Watkins reports investigating small cave and rockshelter sites along the cliffs in the vicinity of Clay Springs on BLM land, and a search of records at the BLM office also suggests numerous extensive sites with thousands of chipped stone artifacts on BLM lands just west of the reservation within the study area.

Notable larger archaeological projects in the Clay Springs-Grand Wash Cliffs area include a survey of Arizona Public Service's 500-kV transmission line (which bisects the southern part of the area and the checkerboard lands west of the contiguous reservation); drought relief/waterline surveys that currently provide water for livestock and Grand Canyon Skywalk (these border much of the eastern margin of the study area); a sample survey of selected parcels across the Hualapai Reservation (one of which lies near the southern study area about 2 miles to the east); the Boston Patch Fuelwood Survey (approximately 6 miles south of the study area, but in a very similar landscape and containing abundant archaeological sites); and a 7-mile fence line survey from the vicinity of Buck and Doe Road to the rim of the Grand Wash Cliffs generally overlooking Clay Springs.

Although the area undoubtedly contains many burial sites, their locations are largely unknown, especially those interred prior to the establishment of family or community cemeteries in the past 100 years or so. Common past burial practices included cremation or placing the deceased in rock crevasses, which would have been particularly more likely along the Grand Wash Cliffs where there are many small caves and rockshelters. Because of the low-visibility, dispersed, and obscure locations of these burial sites, it is extremely difficult to inventory or predict where they might be. Suffice it to say that, considering the general Clay Springs-Grand Wash Cliffs area was a major settlement and use area for many centuries for the Hualapai, there are undoubtedly many burial sites throughout the study area.

In addition, because of the long history of occupation and the importance of the area on many levels, there are numerous landmarks and places of cultural and historical importance (TCPs) throughout the area, some of which have been documented but others that are very likely not yet documented.

\section{GRAND CANYON WEST WIND ENERGY PROJECT AREA}

Risk Category. Medium. Any large-scale development in this area may entail numerous issues. Direct and indirect impacts to an extremely important cultural and historical TCP would raise complicated and 
potentially difficult mitigation challenges. Concerning the abundant archaeological sites in this area, it would also be challenging to avoid direct impacts to sites during infrastructure construction, including access roads and transmission lines. Mitigation would therefore likely involve extensive archaeological excavations, analysis, and curation, all of which would entail considerable expense. Mitigating impacts to TCPs, perhaps most obviously the visual effects of large infrastructure development, should include broad participation by Hualapai tribal members, especially those with direct ancestral ties to the area.

Additional Work Required. A Class I and Class III cultural resources inventory may be needed in order to determine the effect of the proposed project on resources within the APE. Consultation with Native American tribes about concerns they have about the project site may be necessary. There are seven tribes with interests in the project area: Hualapai, Chemehuevi, Fort Mohave, Navajo, Hopi, Quechan, and the Colorado River Indian Tribes. It is expected that the consultation process will be time-consuming. In addition, a comprehensive ethnohistoric study, analogous to an archaeological inventory, should be conducted as part of any planning for large-scale development in the area, as early in the process as possible.

\section{CLAY SPRINGS WIND ENERGY PROJECT AREA}

Risk Category. Medium. Similar to that described under the Grand Canyon West Wind Energy project area.

Additional Work Required. Additional work required would be similar to that described under the Grand Canyon West Wind Energy project area.

\section{BLM WIND ENERGY NORTH PROJECT AREA}

Risk Category. Medium. Similar to that described under the Grand Canyon West Wind Energy project area.

Additional Work Required. Additional work required would be similar to that described under the Grand Canyon West Wind Energy project area.

\section{BLM WIND ENERGY SOUTH PROJECT AREA}

Risk Category. Medium. Similar to that described under the Grand Canyon West Wind Energy project area.

Additional Work Required. Additional work required would be similar to that described under the Grand Canyon West Wind Energy project area.

\section{NELSON SOLAR ENERGY PROJECT AREA}

Risk Category. Medium. Similar to that described under the Grand Canyon West Wind Energy project area.

Additional Work Required. A Class I and Class III cultural resources inventory may be needed in order to determine the effect of the proposed project on resources within the APE. Consultation with Native American tribes about concerns they have about the project site may be necessary. There are seven tribes with interests in the project area: Hualapai, Chemehuevi, Fort Mohave, Navajo, Hopi, Quechan, and the Colorado River Indian Tribes. It is expected that the consultation process will be time-consuming. 


\section{HUALAPAI SOLAR ENERGY PROJECT AREA}

Risk Category. Medium. Similar to that described under the Grand Canyon West Wind Energy project area.

Additional Work Required. Additional work required would be similar to that described under the Grand Canyon West Wind Energy project area.

\subsection{Paleontological Resources}

Paleontological resources, or fossils, are the remains, imprints, or traces of once-living organisms preserved in rocks and sediments. These include mineralized, partially mineralized, or unmineralized bones and teeth, soft tissues, shell, wood, leaf impressions, footprints, burrows, and microscopic remains. Fossils are considered nonrenewable resources because the organisms they represent no longer exist. In the following discussion, the paleontological sensitivity of each geologic unit within the project area is ranked according to the Potential Fossil Yield Classification System (PFYC), a resource management classification system that is widely utilized by the BLM for the western United States (BLM 2008). This system is based on the fact that occurrences of paleontological resources are closely related to the geologic units that contain them. Therefore, the potential for finding important paleontological resources can be broadly predicted by the presence of the pertinent geologic units at or near the surface. Figure 25 provides a map of the geology-based paleontological potential of the study area.

Fossils are classified as nonrenewable scientific resources and are protected by various laws, ordinances, regulations, and standards across the country. Professional standards for the assessment and mitigation of adverse impacts to paleontological resources have been established by the Society of Vertebrate Paleontology $(1995,1996)$. Federal protections for scientifically significant paleontological resources apply to projects if any construction-related or other related project impacts occur on federally owned or managed lands, involve the crossing of state lines, or are federally funded. Federal protections apply to scientifically significant paleontological resources on federally owned/administered lands within the APE for the study area.

\section{GRAND CANYON WEST WIND ENERGY PROJECT AREA}

Based on the mapping of Billingsley et al. (2006) the project area is underlain by seven geologic units, listed in stratigraphic sequence from oldest to youngest: the middle Cambrian Muav limestone $(\mathrm{Cm})$; the upper to middle Devonian Temple Butte Formation (Dtb); upper to lower Mississippian undivided Redwall limestone (Mr); upper Cretaceous-aged quartz monzonite pluton (Tp); middle to lower Mioceneaged andesite and basalt flows (Tv); Pliocene to upper Miocene young gravel (Tg); Pleistocene, and Pliocene and middle Miocene old gravels (QTg). Volcanic rocks and granitic rocks do not typically contain paleontological resources as these rocks are formed at extremely high temperatures, making preservation of fossils extremely rare. Therefore, the andesite, basalt, and quartz pluton underlying the project area is determined to have a low paleontological sensitivity, or a PFYC Class 1 . The northern portion of the project area is predominantly underlain by volcanic and granitic rocks; however the southern portion of the project site consists largely of Paleozoic sedimentary rocks with some Cenozoic sedimentary rocks. The Paleozoic and Cenozoic sediments may have paleontological resource potential. The Cenozoic sedimentary rocks are determined to have a low potential, or PFYC Class 2. The Paleozoic sedimentary rocks also likely have a low potential (PFYC Class 2), but may be locally sensitive with a moderate potential (PFYC Class 3a).

Risk Category. Low-Medium. Geology indicates few sources that contain high fossil potential. 
Additional Work Required. Because of the low to moderate paleontological sensitivity of the Paleozoic (Cm, Dtb, and Mr) and Cenozoic (Tg and QTg) sedimentary rocks in the project area, a paleontological resources museum locality search and field reconnaissance may be required to further evaluate the paleontological potential of these sediments.

\section{CLAY SPRINGS WIND ENERGY PROJECT AREA}

Based on the mapping of Billingsley et al. (2006), the project area is underlain by seven geologic units, listed in stratigraphic sequence from oldest to youngest: the middle Cambrian Muav limestone (Cm); the upper to middle Devonian Temple Butte Formation (Dtb); middle to lower Miocene-aged andesite and basalt flows (Tv); Pliocene to upper Miocene young gravel (Tg); Holocene and Pleistocene valley fill deposits (Qv); Holocene and Pleistocene (?) young alluvial fan deposits (Qay); and Holocene stream channel deposits (Qs). Volcanic, granitic, and other crystalline rocks do not typically contain paleontological resources as these rocks are formed at extremely high temperatures, making preservation of fossils extremely rare. Therefore, the volcanic rocks underlying the project area are determined to have a low paleontological sensitivity, or a PFYC Class 1 . The project area is predominantly underlain by the Cambrian-aged Muav limestone and basalt flows; however, other Paleozoic rocks, and Cenozoic sedimentary rocks are found primarily in the northern portion of project area. The Paleozoic and Cenozoic sediments may have paleontological resource potential. The Cenozoic sedimentary rocks are determined to have a low potential, or PFYC Class 2 . The Paleozoic sedimentary rocks also likely have a low potential (PFYC Class 2), but may be locally sensitive with a moderate potential (PFYC Class 3a).

Risk Category. Low-Medium. Geology indicates few sources that contain high fossil potential.

Additional Work Required. Because of the low to moderate paleontological sensitivity of the Paleozoic (Cm, and Dtb) and Cenozoic (Tg, Qv, and Qay) sedimentary rocks in the project area, a paleontological resources museum locality search and field reconnaissance may be required to further evaluate the paleontological potential of these sediments.

\section{BLM WIND ENERGY NORTH PROJECT AREA}

Based on the mapping of Billingsley et al. (2006), the project area is underlain by 10 geologic units, listed in stratigraphic sequence from oldest to youngest: the middle to lower (?) Cambrian Tapeats Sandstone $(\mathrm{Ct})$; the middle Cambrian Bright Angel shale (Cba); the middle Cambrian Muav limestone $(\mathrm{Cm})$; the upper to middle Devonian Temple Butte Formation (Dtb); middle to lower Miocene-aged andesite and basalt flows (Tv); Pliocene to upper Miocene young gravel (Tg); Pleistocene, and Pliocene and middle Miocene old gravels (QTg); Holocene and Pleistocene valley fill deposits (Qv); and Holocene and Pleistocene landslide deposits $(\mathrm{Q})$. The project is also underlain by undifferentiated crystalline rocks of indeterminate age $(\mathrm{Xu})$. Volcanic, granitic, and other crystalline rocks do not typically contain paleontological resources as these rocks are formed at extremely high temperatures, making preservation of fossils extremely rare. Therefore, the crystalline and volcanic rocks underlying the project area are determined to have a low paleontological sensitivity, or a PFYC Class 1 . The project area is predominantly underlain by the Cambrian-aged Muav limestone; however other Paleozoic rocks, and Cenozoic sedimentary rocks exist primarily along the margins of project area. The Paleozoic and Cenozoic sediments may have paleontological resource potential. The Cenozoic sedimentary rocks are determined to have a low potential, or PFYC Class 2. The Paleozoic sedimentary rocks also likely have a low potential (PFYC Class 2), but may be locally sensitive with a moderate potential (PFYC Class 3a).

Risk Category. Low-Medium. Geology indicates few sources that contain high fossil potential.

Additional Work Required. Because of the low to moderate paleontological sensitivity of the Paleozoic (Ct, Cba, Cm, and Dtb) and Cenozoic (Tg, QTg, Ql, and Qv) sedimentary rocks in the project site, a 
paleontological resources museum locality search and field reconnaissance may be required to further evaluate the paleontological potential of these sediments.

\section{BLM WIND ENERGY SOUTH PROJECT AREA}

Based on the mapping of Billingsley et al. (2006), the project area is underlain by nine geologic units, listed in stratigraphic sequence from oldest to youngest: the middle to lower (?) Cambrian Tapeats Sandstone $(\mathrm{Ct})$; the middle Cambrian Bright Angel shale (Cba); the middle Cambrian Muav limestone $(\mathrm{Cm})$; the upper to middle Devonian Temple Butte Formation (Dtb); middle to lower Miocene-aged andesite and basalt flows (Tv); Holocene and Pleistocene valley fill deposits (Qv); and Holocene and Pleistocene landslide deposits (Q1); and Holocene and Pleistocene (?) young alluvial fan deposits (Qay). The project is also underlain by undifferentiated crystalline rocks of indeterminate age $(\mathrm{Xu})$. Volcanic, granitic, and other crystalline rocks do not typically contain paleontological resources as these rocks are formed at extremely high temperatures, making preservation of fossils extremely rare. Therefore, the crystalline and volcanic rocks underlying the project area are determined to have a low paleontological sensitivity, or a PFYC Class 1 . The project area is predominantly underlain by the Cambrian-aged Muav limestone; however other Paleozoic rocks, and Cenozoic sedimentary rocks occur primarily along the margins of project area. The Paleozoic and Cenozoic sediments may have paleontological resource potential. The Cenozoic sedimentary rocks are determined to have a low potential, or PFYC Class 2. The Paleozoic sedimentary rocks also likely have a low potential (PFYC Class 2), but may be locally sensitive with a moderate potential (PFYC Class 3a).

Risk Category. Low-Medium. Geology indicates few sources that contain high fossil potential.

Additional Work Required. Because of the low to moderate paleontological sensitivity of the Paleozoic (Ct, Cba, Cm, and Dtb) and Cenozoic (Tg, QTg, Q1, and Qv) sedimentary rocks in the project area, a paleontological resources museum locality search and field reconnaissance may be required to further evaluate the paleontological potential of these sediments.

\section{NELSON SOLAR ENERGY PROJECT AREA}

Based on the mapping of Billingsley et al. (2006), the project area is underlain by four geologic units, listed in stratigraphic sequence from oldest to youngest: the upper and lower Mississippian undivided Redwall Limestone (Mr); the undivided upper Pennsylvanian Wescogame Formation, middle Pennsylvanian Manakacha Formation, lower Pennsylvanian - upper Mississippian Watahomigi Formation (PMs); Pleistocene, and Pliocene and middle Miocene old gravels (QTg); and Holocene and Pleistocene valley fill deposits (Qv). Paleozoic sendimentary rocks and Quaternary alluvial deposits may have paleontological resource potential. The Quaternary sedimentary deposits are determined to have a low potential, or PFYC Class 2. The Paleozoic sedimentary rocks also likely have a low potential (PFYC Class 2), but may be locally sensitive with a moderate potential (PFYC Class 3a).

Risk Category. Low-Medium. Geology indicates few sources that contain high fossil potential.

Additional Work Required. Because of the low to moderate potential sedimentary rocks in the project site, a paleontological resources museum locality search and field reconnaissance may be required to further evaluate the paleontological potential of these sediments.

\section{HUALAPAI SOLAR ENERGY PROJECT AREA}

Based on the mapping of Billingsley et al. (2006), the project area is underlain by three geologic units, listed in stratigraphic sequence from oldest to youngest: Holocene and Pleistocene (?) young alluvial fan deposits (Qay); and Holocene stream channel deposits (Qs). The project is also underlain by 
undifferentiated crystalline rocks of indeterminate age $(\mathrm{Xu})$. Volcanic rocks and granitic rocks do not typically contain paleontological resources as these rocks are formed at extremely high temperatures, making preservation of fossils extremely rare. Therefore, the crystalline rocks underlying the project area are determined to have a low paleontological sensitivity, or a PFYC Class 1. Quaternary alluvial deposits may have paleontological resource potential and are determined to have a low potential, or PFYC Class 2.

Risk Category. Low. Holocene and Pleistocene (?) young alluvial fan deposits (Qay) and Holocene stream channel deposits (Qs).

Additional Work Required. Because of the low potential sedimentary rocks in the project area, a paleontological resources museum locality search and field reconnaissance may be required to further evaluate the paleontological potential of these sediments.

\subsection{Socioeconomics}

The study area is located in rural areas with little residential or commercial uses. Population on Tribal lands in the study area decreased 51.4\%, from 1,353 in 2000 to 658 in 2010 (Headwaters Economics 2012). Additionally, unemployment on Hualapai Tribal lands was approximately $25 \%$ in 2010 (Headwaters Economics 2012), compared to 10\% for Arizona during the same year (Arizona Workforce Informer 2012). When unemployment is high, the local economy suffers from underemployment of labor and human capital, translating to lower real incomes and a lower standard of living. The Hualapai Tribe has limited economic opportunities and relatively high unemployment for a variety of reasons including the lack of utility infrastructure and lack of water rights. To combat this, the Tribe has established a vision to supervise the effective, economic, environmental and culturally appropriate utilization of Tribal lands for renewable energy development projects.

Mohave County had a 2010 population of 199,177 , a $28.5 \%$ increase over 2000. Coconino County had a population of 131,824 in 2010, a 13.3\% increase over 2000 counts. Growth in Mohave and Coconino Counties was consistent with Arizona (21.8\% for the same time period), though a little higher.

\section{GRAND CANYON WEST WIND ENERGY PROJECT AREA}

The project area is located in a rural area with few potential conflicts for social and economic considerations. The closest populated communities to the Clay Springs Wind Energy site are Dolan Springs (population 1,655 in 2010) and Kingman (population 27,790 in 2010) in Mohave County. The rates of growth for these communities between 2000 and 2010 was $-11.4 \%$ for Dolan Springs, and $38.5 \%$ for Kingman.

Risk Category. Low. Because the Hualapai Tribe has limited income sources, the economic activity (employment, area income, taxes, etc.) associated with constructing and operating a wind facility could have a beneficial impact on the Tribe and area communities.

Additional Work Required. An in-depth socioeconomic analysis will be conducted as part of the environmental analysis per NEPA. The detailed analysis would identify specific benefits that may be derived from the project. Additionally, public scoping and outreach would identify any social or economic concerns area residents may have.

\section{CLAY SPRINGS WIND ENERGY PROJECT AREA}

The project area is located in a rural area with few potential conflicts for social and economic considerations. The closest populated communities to the Clay Springs Wind Energy site are Dolan 
Springs (population 1,655 in 2010) and Kingman (population 27,790 in 2010) in Mohave County. The rates of growth for these communities between 2000 and 2010 was $-11.4 \%$ for Dolan Springs, and $38.5 \%$ for Kingman.

Risk Category. Medium. The project area is located in a rural area with few potential conflicts for social and economic considerations. As above, because the Hualapai Tribe has limited income sources, the economic activity (employment, area income, taxes, etc.) associated with constructing and operating a wind facility could have a beneficial impact on the Tribe and area communities.

Additional Work Required. As above, an in-depth socioeconomic analysis will be conducted as part of the environmental analysis per NEPA. The detailed analysis would identify specific conflicts (i.e., grazing) or benefits (i.e., economic activity) that may be derived from the project. Additionally, public scoping and outreach would identify any social or economic concerns area residents may have.

\section{BLM WIND ENERGY NORTH PROJECT AREA}

The project area is located in a rural area with few potential conflicts for social and economic considerations. The closest populated communities to the BLM Wind Energy South site are Dolan Springs (population 1,655 in 2010) and Kingman (population 27,790 in 2010) in Mohave County. The rates of growth for these communities between 2000 and 2010 was $-11.4 \%$ for Dolan Springs, and $38.5 \%$ for Kingman. In addition, there are several active grazing allotments (BLM 1993).

Risk Category. Medium. The project area is located in a rural area with few potential conflicts for social and economic considerations. There are several active grazing allotments located on BLM land in the project area. The economic effect of these allotments, and the associated change in land use, may result in conflicts with grazing allottees. Additionally, BLM grazing allottees need to be notified 2 years in advance of the cancellation or change to their grazing lease (per the Taylor Grazing Act of 1916).

Additional Work Required. As above, an in-depth socioeconomic analysis will be conducted as part of the environmental analysis per NEPA. The detailed analysis would identify specific conflicts (i.e., grazing) or benefits (i.e., economic activity) that may be derived from the project. Additionally, public scoping and outreach would identify any social or economic concerns area residents may have. Special attention should be paid to including grazing allottees in public outreach efforts.

\section{BLM WIND ENERGY SOUTH PROJECT AREA}

The closest populated communities to the BLM Wind Energy South site are Dolan Springs (population 1,655 in 2010) and Kingman (population 27,790 in 2010) in Mohave County. The rates of growth for these communities between 2000 and 2010 was $-11.4 \%$ for Dolan Springs, and 38.5\% for Kingman. In addition, there are several active grazing allotments (BLM 1993).

Risk Category. Medium. The project area is located in a rural area with few potential conflicts for social and economic considerations. There are several active grazing allotments in the project area. The economic effect of these allotments, and the associated change in land use, may result in conflicts with grazing allottees. Additionally, BLM grazing allottees need to be notified 2 years in advance of the cancellation or change to their grazing lease (per the Taylor Grazing Act of 1916).

Additional Work Required. As above, an in-depth socioeconomic analysis will be conducted as part of the environmental analysis per NEPA. The detailed analysis would identify specific conflicts (i.e., grazing) or benefits (i.e., economic activity) that may be derived from the project. Additionally, public scoping and outreach would identify any social or economic concerns area residents may have. Special attention should be paid to including grazing allottees in public outreach efforts. 


\section{NELSON SOLAR ENERGY PROJECT AREA}

The Nelson Solar Energy project area is located on Tribal lands within Coconino County, just north of Yavapai County. The nearest populated communities to the Nelson Solar Energy project area are Peach Springs and Nelson, located in Mohave County. Peach Springs and Nelson are Census Designated Places (CDPs). The U.S. Census Bureau defines a CDP as "a geographic entity that serves as the statistical counterpart of an incorporated place for the purpose of presenting census data for an area with a concentration of population, housing, and commercial structures that is identifiable by name, but is not within an incorporated place" (U.S. Census Bureau 2008). Population in Peach Springs was 617 in 2010, a $2.8 \%$ increase over 2000 counts, and population in Nelson was 146 in 2010 (no data available for Nelson in 2000) (Headwaters Economics 2012).

Risk Category. Low. As above, the project area is located in a rural area with few potential conflicts for social and economic considerations. The project would likely to benefit the Tribe in terms of the economic activity (employment, area income, taxes, etc.) associated with constructing and operating a solar facility.

Additional Work Required. As above, an in-depth socioeconomic analysis will be conducted as part of the environmental analysis per NEPA. The detailed analysis would identify specific benefits that may be derived from the project. Additionally, public scoping and outreach would identify any social or economic concerns area residents may have.

\section{HUALAPAI SOLAR ENERGY PROJECT AREA}

The closest populated communities to the Hualapai Solar Energy site are Dolan Springs (population 1,655 in 2010) and Kingman (population 27,790 in 2010) in Mohave County. The rates of growth for these communities between 2000 and 2010 was $-11.4 \%$ for Dolan Springs, and 38.5\% for Kingman.

Risk Category. Medium. The project area is located in a rural area with few potential conflicts for social and economic considerations. As above, because the Hualapai Tribe has limited income sources, the economic activity (employment, area income, taxes, etc.) associated with constructing and operating a wind facility could have a beneficial impact to the Tribe and area communities.

Additional Work Required. As above, an in-depth socioeconomic analysis will be conducted as part of the environmental analysis per NEPA. The detailed analysis would identify specific conflicts (i.e., grazing) or benefits (i.e., economic activity) that may be derived from the project. Additionally, public scoping and outreach would identify any social or economic concerns area residents may have.

\subsection{Environmental Justice}

Executive Order 12898 (February 11, 1994) and its accompanying memorandum require that "each federal agency shall make achieving environmental justice part of its mission by identifying and addressing, as appropriate, disproportionately high and adverse human health or environmental effects of its programs, policies, and activities on minority populations and low-income populations" (Council on Environmental Quality 1997). To comply with EO 12898, the following assessment evaluates the presence or absence of, and potential impacts to (if any), environmental justice communities in and near the proposed project. Criteria to assess environmental justice issues, as defined by the EPA (Council on Environmental Quality 1997), are outlined below. 
Minority populations are generally defined as follows:

1. Minority: Individual(s) classified by Office of Management and Budget Directive No. 15 as Black/African American, Hispanic, Asian and Pacific Islander, American Indian, Eskimo, Aleut, and other non-White persons.

2. A minority population exists where either

- the minority population of the affected area exceeds $50 \%$; or

- the minority population percentage of the affected area is meaningfully greater than the minority population percentage in the general population or other appropriate unit of geographic analysis.

A minority population also exists if there is more than one minority group present and the minority percentage, as calculated by aggregating all minority persons, meets one of the above-stated thresholds.

Low-income populations in an affected area are populations below the annual, statistical poverty thresholds from the U.S. Census Bureau's current population reports on income and poverty. Families and persons are classified by the Census Bureau as "below poverty level" if their total family income or unrelated individual income is less than the poverty threshold specified for the applicable family size, age of householder, and number of related children under 18 that are present.

\subsubsection{Environmental Justice Communities In or Near the Study Area}

Executive Order 12898 establishes the need for federal agencies to ensure "fair treatment and meaningful involvement of all people regardless of race, color, national origin, or income with respect to the development, implementation, and enforcement of environmental laws, regulations, and policies." The order directs those agencies to address any disproportionately high and adverse human health or environmental effects of their actions, programs, or policies on minority and low-income populations.

Minority populations are identified as those belonging to any of the following racial groups: Hispanic, Black or African-American, American Indian or Alaska Native, Asian, Native Hawaiian, or Other Pacific Islander. Kingman, Arizona is the nearest city, and is located 30 miles west of the project area. According to the 2010 U.S. Census, the minority population in Mohave County is $15 \%$ (Headwaters Economics 2012).

Low-income populations are identified as individuals who fall below the poverty line. The poverty line takes into account family size and age of individuals in the family. In 2009, the poverty level for a household (i.e., family of four with two children under 18) was $\$ 21,756$ (Headwaters Economics 2012). In $2010,13.8 \%$ of individuals and $10.1 \%$ of families were living below the poverty level in the United States. At the County level, $18.6 \%$ and $16.1 \%$ of individuals in Coconino and Mohave Counties, respectively, were living below the poverty level (Headwaters Economics 2012).

Based on minority and low-income data for communities in and near the study area, as described above, the Hualapai Tribal Nation overall and the community of Peach Springs in particular, are considered environmental justice communities. 


\subsubsection{Disproportionately High and Adverse Effects}

According to EO 12898, when determining whether human health effects are disproportionately high and adverse, agencies must consider the following three factors to the extent practicable:

1. Whether the health effects, which may be measured in risks and rates, are significant, unacceptable, or above generally accepted norms (adverse health effects may include bodily impairment, infirmity, illness, or death).

2. Whether the risk or rate of hazard exposure by a minority population or low-income population to an environmental hazard is significant and appreciably exceeds or is likely to appreciably exceed the risk or rate to the general population or other appropriate comparison group.

3. Whether health effects occur in a minority or low-income population affected by cumulative or multiple adverse exposures from environmental hazards.

According to EO 12898, when determining whether environmental effects are disproportionately high and adverse, agencies are to consider the following three factors to the extent practicable:

1. Whether there is or will be an impact on the natural or physical environment that significantly and adversely affects a minority population, low-income population, or Indian tribe. Such effects may include ecological, cultural, human health, economic, or social impacts on minority communities, low-income communities, or Indian tribes when those impacts are interrelated to impacts on the natural or physical environment; and

2. Whether environmental effects are significant and are or may be having an adverse impact on minority populations, low-income populations, or Indian tribes that appreciably exceeds or is likely to appreciably exceed those on the general population or other appropriate comparison group; and

3. Whether the environmental effects occur or would occur in a minority population, low-income population, or Indian tribe affected by cumulative or multiple adverse exposures from environmental hazards.

\section{GRAND CANYON WEST WIND ENERGY PROJECT AREA}

The project area is located entirely on Hualapai Tribal lands, and only $3.6 \%$ of the 2010 population was "white alone," meaning $96.4 \%$ of the population is considered a minority population (primarily American Indian [81.2\%]). On Hualapai Tribal lands, $41.2 \%$ of individuals and $45.5 \%$ of families were living below the poverty level. Based on minority and low-income data for communities in and near the project areas, as described above, the Hualapai Tribal Nation overall is considered an environmental justice community.

Risk Category. Medium. Environmental justice communities have been identified in the project vicinity.

Additional Work Required. Given the presence of environmental justice communities in the project vicinity, there may be environmental justice implications for the project area that will need further analysis (i.e., assessment of disproportionate human health and environmental effects, using criteria listed below).

According to EO 12898, when determining whether human health effects are disproportionately high and adverse, agencies must consider the following three factors to the extent practicable:

1. Whether the health effects, which may be measured in risks and rates, are significant, unacceptable, or above generally accepted norms (adverse health effects may include bodily impairment, infirmity, illness, or death). 
2. Whether the risk or rate of hazard exposure by a minority population or low-income population to an environmental hazard is significant and appreciably exceeds or is likely to appreciably exceed the risk or rate to the general population or other appropriate comparison group.

3. Whether health effects occur in a minority or low-income population affected by cumulative or multiple adverse exposures from environmental hazards.

According to EO 12898, when determining whether environmental effects are disproportionately high and adverse, agencies are to consider the following three factors to the extent practicable:

1. Whether there is or will be an impact on the natural or physical environment that significantly and adversely affects a minority population, low-income population, or Indian tribe. Such effects may include ecological, cultural, human health, economic, or social impacts on minority communities, low-income communities, or Indian tribes when those impacts are interrelated to impacts on the natural or physical environment; and

2. Whether environmental effects are significant and are or may be having an adverse impact on minority populations, low-income populations, or Indian tribes that appreciably exceeds or is likely to appreciably exceed those on the general population or other appropriate comparison group; and

3. Whether the environmental effects occur or would occur in a minority population, low-income population, or Indian tribe affected by cumulative or multiple adverse exposures from environmental hazards.

\section{CLAY SPRINGS WIND ENERGY PROJECT AREA}

The project area is located entirely on Hualapai Tribal lands, and only $3.6 \%$ of the 2010 population was "white alone," meaning $96.4 \%$ of the population is considered a minority population (primarily American Indian [81.2\%]). On Hualapai Tribal lands, $41.2 \%$ of individuals and $45.5 \%$ of families were living below the poverty level. Similarly the percent of individuals and families living below the poverty level in Peach Springs was $40.0 \%$ and $46.3 \%$, respectively. Based on minority and low-income data for communities in and near the project areas, as described above, the Hualapai Tribal Nation overall is considered an environmental justice community.

Risk Category. Medium. The project is located on the Hualapai Tribal lands; the Hualapai Tribe is considered an environmental justice community.

Additional Work Required. Additional work required would be the same as described under the Grand Canyon West Wind Energy project area.

\section{BLM WIND ENERGY NORTH PROJECT AREA}

In $2010,13.8 \%$ of individuals and $10.1 \%$ of families were living below the poverty level in the United States. At the County level, $16.1 \%$ of individuals in Mohave County were living below the poverty level (Headwaters Economics 2012).

Risk Category. Low. The project area is located on BLM-managed lands adjacent to Hualapai Tribal lands.

Additional Work Required. Given the proximity of environmental justice communities in the project vicinity, there may be environmental justice implications for the project area that will need further analysis (i.e., assessment of disproportionate human health and environmental effects, using criteria listed below). 


\section{BLM WIND ENERGY SOUTH PROJECT AREA}

In $2010,13.8 \%$ of individuals and $10.1 \%$ of families were living below the poverty level in the United States. At the County level, $16.1 \%$ of individuals in Mohave County were living below the poverty level (Headwaters Economics 2012).

Risk Category. Low. The project area is located on BLM-managed lands adjacent to Hualapai Tribal lands.

Additional Work Required. Given the proximity of environmental justice communities in the project vicinity, there may be environmental justice implications for the project area that will need further analysis (i.e., assessment of disproportionate human health and environmental effects, using criteria listed below).

\section{NELSON SOLAR ENERGY PROJECT AREA}

The project area is located entirely on Hualapai Tribal lands, and only $3.6 \%$ of the 2010 population was "white alone," meaning $96.4 \%$ of the population is considered a minority population (primarily American Indian [81.2\%]). In Peach Springs, $98.1 \%$ of the population is considered a minority population $(81.8 \%$ American Indian).

On Hualapai Tribal lands, $41.2 \%$ of individuals and $45.5 \%$ of families were living below the poverty level. Similarly, the percent of individuals and families living below the poverty level in Peach Springs was $40.0 \%$ and $46.3 \%$, respectively. No data were available for Nelson, likely due to small population size.

Based on minority and low-income data for communities in and near the project areas, as described above, the Hualapai Tribal Nation overall, and the community of Peach Springs located to the west of the Nelson Solar Energy project area, are considered environmental justice communities.

Risk Category. Medium. The project is located on the Hualapai Tribal lands; the Hualapai Tribe is considered an environmental justice community.

Additional Work Required. Additional work required would be the same as described under the Grand Canyon West Wind Energy project area.

A detailed environmental justice analysis will be included in the environmental evaluation process per NEPA and Executive Order 12898. Minority and low-income populations located near the project areas will be included in the scoping process. In addition, the appropriate level of public outreach will be provided to ensure that environmental justice communities are involved early and often in the NEPA process.

\section{HUALAPAI SOLAR ENERGY PROJECT AREA}

The project area is located entirely on Hualapai Tribal lands, and only $3.6 \%$ of the 2010 population was "white alone," meaning $96.4 \%$ of the population is considered a minority population (primarily American Indian [81.2\%]). On Hualapai Tribal lands, $41.2 \%$ of individuals and $45.5 \%$ of families were living below the poverty level. Based on minority and low-income data for communities in and near the project areas, as described above, the Hualapai Tribal Nation overall is considered an environmental justice community.

Risk Category. Medium. The project is located on the Hualapai Tribal lands; the Hualapai Tribe is considered an environmental justice community. 
Additional Work Required. Additional work required would be the same as described under the Grand Canyon West Wind Energy project area.

\subsection{RISK SUMMARY AND RECOMMENDATIONS}

\subsection{Risk Summary}

Table 12 summarizes the resource constraints that are expected for the study area based on current available information. Each project area has been evaluated for all of the considered resources and each resource has been assigned a risk category to help evaluate potential issues. At the bottom of the table, the risks are added for each site and a total risk number is calculated; the higher the number, the greater the environmental constraints on the project. Additionally, the final row of the table discloses if any potential fatal flaws have been identified for each site. It is possible that a site could have a low overall risk number, but a potential fatal flaw. In that case, it would likely be easier to develop a site with a higher overall risk, but no fatal flaws. It is also important to note that policy changes and land use designations, especially for renewable energy, occur fairly regularly and could influence project constraints.

Table 12. Resource Constraints Summary

\begin{tabular}{|c|c|c|c|c|c|c|}
\hline \multirow[b]{2}{*}{ Resource } & \multicolumn{4}{|c|}{ Wind Energy Sites } & \multicolumn{2}{|c|}{ Solar Energy Sites } \\
\hline & $\begin{array}{c}\text { Grand } \\
\text { Canyon } \\
\text { West }\end{array}$ & $\begin{array}{l}\text { Clay } \\
\text { Springs }\end{array}$ & $\begin{array}{l}\text { BLM } \\
\text { North }\end{array}$ & $\begin{array}{l}\text { BLM } \\
\text { South }\end{array}$ & Nelson & Hualapai \\
\hline Land use & 1 & 1 & 1 & 2 & 1 & 1 \\
\hline Ground transportation & 1 & 1 & 1 & 1 & 1 & 1 \\
\hline Airports (private, commercial, FAA, military) & 1 & 1 & 1 & 1 & 2 & 1 \\
\hline Communications and radio facilities & 1 & 1 & 1 & 1 & 1 & 1 \\
\hline Public services and utilities & 1 & 1 & 1 & 1 & 1 & 1 \\
\hline Active mines and mining claims & 1 & 1 & 1 & 1 & 1 & 1 \\
\hline Visual resources and VRM classifications & 3 & 2 & 3 & 2 & 1 & 1 \\
\hline \multicolumn{7}{|l|}{ Biological Resources } \\
\hline General vegetation & 1 & 1 & 1 & 1 & 1 & 1 \\
\hline Threatened and endangered species & 2 & 2 & 2 & 2 & 1 & 2 \\
\hline Special status species & 2 & 2 & 2 & 2 & 2 & 2 \\
\hline Big game species & 2 & 2 & 2 & 2 & 2 & 2 \\
\hline Bats & 2 & 2 & 2 & 2 & 1 & 1 \\
\hline Birds, including migration pathways & 3 & 3 & 3 & 3 & 1 & 2 \\
\hline \multicolumn{7}{|l|}{ Water Resources } \\
\hline $\begin{array}{l}\text { Jurisdictional waters, playa lakes, and } \\
\text { wetlands }\end{array}$ & 2 & 2 & 2 & 2 & 2 & 2 \\
\hline Flood hazard zones & 1 & 1 & 1 & 1 & 1 & 1 \\
\hline Geologic hazard zones & 1 & 1 & 1 & 1 & 1 & 1 \\
\hline Recreation & 1 & 1 & 1 & 1 & 1 & 1 \\
\hline Air quality & 1 & 1 & 1 & 1 & 1 & 1 \\
\hline Noise & 1 & 1 & 1 & 1 & 1 & 1 \\
\hline
\end{tabular}


Table 12. Resource Constraints Summary (Continued)

\begin{tabular}{|c|c|c|c|c|c|c|}
\hline \multirow[b]{2}{*}{ Resource } & \multicolumn{4}{|c|}{ Wind Energy Sites } & \multicolumn{2}{|c|}{ Solar Energy Sites } \\
\hline & $\begin{array}{c}\text { Grand } \\
\text { Canyon } \\
\text { West }\end{array}$ & $\begin{array}{c}\text { Clay } \\
\text { Springs }\end{array}$ & $\begin{array}{l}\text { BLM } \\
\text { North }\end{array}$ & $\begin{array}{l}\text { BLM } \\
\text { South }\end{array}$ & Nelson & Hualapa \\
\hline Hazardous materials & 1 & 1 & 1 & 1 & 1 & 1 \\
\hline $\begin{array}{l}\text { Cultural resources and Native American } \\
\text { concerns }\end{array}$ & 2 & 2 & 2 & 2 & 2 & 2 \\
\hline Paleontological resources & 1 & 1 & 1 & 1 & 1 & 1 \\
\hline Socioeconomics & 1 & 2 & 2 & 2 & 1 & 2 \\
\hline Environmental justice & 2 & 2 & 1 & 1 & 2 & 2 \\
\hline Total & 35 & 35 & 35 & 35 & 30 & 32 \\
\hline Any fatal flaws? & No & No & No & No & No & No \\
\hline
\end{tabular}

\subsection{Recommendations}

Based on review of the environmental constraints for the study area, the two proposed solar energy sites have the fewest environmental constraints. The environmental constraints of the four proposed wind energy sites are very similar, with the same total risk category amounts for each site.

SWCA recommends early coordination with BLM regarding VRM classes in the BLM Wind Energy North and Wind Energy South project areas. Development of projects in areas managed as VRM Class II may require a land use plan amendment to change the VRM class.

SWCA also recommends early coordination with the AGFD and USFWS be completed to determine necessary survey requirements for avian and bat species, as well as other sensitive species. Avian and bat surveys can often take several years to complete and methods vary between regions and managing agencies; therefore, coordination with the agencies is the most effective way of ensuring that the needs and requirements of all agencies are met. Based on our current understanding of USFWS guidelines and AGFD guidelines, in most cases, a BBCS/ECS will need to be prepared that includes all practical up-front avoidance and minimization measures, long-term monitoring, and adaptive management that may include both compensatory and operational (i.e., curtailment) mitigation.

A detailed examination of potential WUS within the study area should be conducted once a project layout has been defined, to determine whether an individual permit would be required.

Early coordination with the BLM, Mohave and Coconino Counties, the State of Arizona, and surrounding landowners regarding the proposed study area should be completed. Early coordination can help identify those timeframes and potential mitigation needs and avoid surprises at the end of a project. 
This page intentionally left blank. 


\subsection{LITERATURE CITED}

American Society for Testing and Materials (ASTM). 2005. Standard Practice for Environmental Site Assessments: Phase I Environmental Site Assessment Process. Document No. E 1527-05. West Conshohocken, Pennsylvania: ASTM International.

2008. Standard Practice for Environmental Site Assessments: Phase I Environmental Site Assessment Process for Forestland or Rural Property. Document No. E 2247-08. West Conshohocken, Pennsylvania: ASTM International.

Arizona Department of Environmental Quality (ADEQ). 2012. Interactive geographic information system eMaps website. Available at: http://www.azdeq.gov/function/programs/gis.html. Accessed May $28,2012$.

Arizona Game and Fish Department (AGFD). 2009. Guidelines for Reducing Impacts to Wildlife from Wind Energy Development in Arizona. Available at: http://www.azgfd.gov/hgis/pdfs/windenergyguidelines.pdf. Accessed May 29, 2012.

_ 2012a. Heritage Data Management System — special status species by county, taxon, scientific name. Available at: http://www.azgfd.gov/w_c/edits/hdms_abstracts.shtml. Accessed May 30, 2012.

- 2012b. Heritage Data Management System - special status species by county, taxon, scientific name. Available at: http://www.azgfd.gov/w_c/edits/documents/allspecies_bycounty_005.pdf. Accessed May 29, 2012.

2012c. Big game species. Available at: http://www.azgfd.gov/h_f/biggame_species.shtml. Accessed June 29, 2012.

2012d. Game Management Unit 15A. Available at:

http://www.azgfd.gov/h_f/hunting_units_15a.shtml. Accessed May 31, 2012.

Arizona Heritage Geographic Information System (AZHGIS). 2012. Arizona Game and Fish Department online environmental review tool. Available at: http://www.AGFD.gov/hgis/. Accessed May 25, 2012.

Arizona State Land Department (ASLD). 2008. Right of Way Instruction Sheet. Available at: http://www.land.state.az.us/programs/realestate/instructions/Right\%20of\%20Way\%20Instruction s.pdf. Accessed May 2012.

Arizona Workforce Informer. 2012. Local unemployment statistics. Available at: http://www.workforce.az.gov/unemployment-data.aspx. Accessed May 23, 2012.

Arnett, E.B., W.K. Brown, W.P. Erickson, J.K. Fiedler, B.L. Hamilton, T.H. Henry, A. Jain, G.D. Johnson, J. Kerns, R.R. Koford, C.P. Nicholson, T.J. O'Connell, M.D. Piorkowski, and R.D. Tankersley, Jr. 2008. Patterns of bat fatalities at wind energy facilities in North America. Journal of Wildlife Management 72(1):61-78.

Baerwald, E.F., G.H. D'Amours, B.J. Klug, and R.M.R. Barclay. 2008. Barotrauma is a significant cause of bat fatalities at wind turbines. Current Biology 18:R695-R696. 
Billingsley, G. H.; Block, D. L.; Dyer, H. C., 2006, Geologic Map of the Peach Springs 30' X 60' Quadrangle, Coconino Counties, Northern Arizona: U.S. Geological Survey Scientific Information Map 2900.

Bradley, P.V., M.J. O'Farrell, J.A. Williams, and J.E. Newmark (eds.). 2006. The Revised Nevada Bat Conservation Plan. Reno, Nevada: Nevada Bat Working Group.

Bureau of Land Management (BLM). 1993. Kingman Field Office Resource Management Plan. Washington, D.C.: U.S. Department of the Interior, Bureau of Land Management.

2005. Final Programmatic Environmental Impact Statement on Wind Energy Development on BLM-Administered Lands in the Western United States. Washington, D.C.: U.S. Department of the Interior, Bureau of Land Management. Available at: http://windeis.anl.gov/documents/fpeis/index.cfm. Accessed June 1, 2012.

2008 (revised). Paleontology Resources Management Manual and Handbook H-8270-1. Washington, D.C.: U.S. Department of the Interior, Bureau of Land Management.

2011. Solar and Wind Energy Applications-Pre-Application and Screening. Instructional Memorandum No. 2011-061. Washington, D.C.: U.S. Department of the Interior, Bureau of Land Management.

2012. GeoCommunicator. Available at:

http://www.geocommunicator.gov/GeoComm/index.shtm. Accessed June 29, 2012.

Council on Environmental Quality. 1997. Environmental Justice, Guidance Under the National Environmental Policy Act. Available at: http://ceq.hss.doe.gov/nepa/regs/ej/justice.pdf. Accessed May 23, 2012.

Headwaters Economics. 2012. Economic Profile System - Human Dimensions Toolkit (EPS HDT). Socioeconomic profiles. Available at: http://headwaterseconomics.org/. Accessed May 23, 2012.

Erickson, W. 2003. Updated Information Regarding Bird and Bat Mortality and Risk at New Generation Wind Projects in the West and Midwest. Cheyenne, Wyoming: Western EcoSystems Technology, Inc.

Federal Aviation Administration (FAA). 2003. Grand Canyon National Park Special Flight Rules Area. Available at: http://www.grandcanyonairspace.iat.gov/index1.html. Accessed May 2012.

—. 2007. Obstruction Marking and Lighting. Advisory Circular Report Number AC 70/7460-1K. Prepared for Air Traffic Airspace Management. U.S. Department of Transportation.

- 2011. Special use airspace and air traffic control assigned airspace. Available at: http://sua.faa.gov/sua/siteFrame.app. Accessed May 29, 2012.

Hunt, G.W., C.N. Parrish, S.C. Farry, T.G. Lord, and R. Seig. 2007. Movements of introduced California Condors in Arizona in relation to lead exposure. In California Condors in the $21^{\text {st }}$ Century, Series in Ornithology, No. 2.

Kerlinger, P., R. Curry, L. Culp, A. Jain, C. Wilkerson, B. Fischer, and A. Hasch. 2006. PostConstruction Avian and Bat Fatality Monitoring Study for the High Winds Wind Power Project, Solano County, California: Two Year Report. Prepared for High Winds, LLC, FPL Energy. McLean, Virginia: Curry and Kerlinger, LLC. 
Kunz, T.H., E.B. Arnett, W.P. Erickson, A.R. Hoar, G.D. Johnson, R.P. Larkin, M.D. Strickland, R.W. Thresher, and M.D. Tuttle. 2007. Ecological impacts of wind energy development on bats: questions, research needs and hypotheses. Frontiers in Ecology and the Environment 5(6): 315-324.

Luke Air Force Base. 2009. Mid Air Collision Avoidance Program. Arizona: Luke Air Force Base. Available at: http://www.luke.af.mil/shared/media/document/AFD-110112-027.pdf. Accessed May 24, 2012.

Mohave County Planning and Zoning Department (MCPZD). 2010. Mohave County, Arizona General Plan. Available at: legacy.co.mohave.az.us/deptfiles/PZ/misc_file/GP_Draft1b.pdf. Accessed August 12, 2011.

Society of Vertebrate Paleontology. 1995. Assessment and mitigation of adverse impacts to nonrenewable paleontologic resources-standard guidelines. Society of Vertebrate Paleontology News Bulletin $163: 22-27$.

- 1996. Conditions of receivership for paleontologic salvage collections. Society of Vertebrate Paleontology News Bulletin 166:31-32.

U.S. Census Bureau. 2008. Census Designated Place (CDP) Program for the 2010 Census-Final Criteria. Available at: http://www.census.gov/geo/www/psap2010/cdp_criteria.html. Accessed May 23, 2012.

U.S. Environmental Protection Agency (EPA). 1974. Information on Levels of Environmental Noise Requisite to Protect Public Health and Welfare with an Adequate Margin of Safety. Available at: http://www.nonoise.org/library/levels74/levels74.htm. Accessed August 3, 2011.

2010. Region 9 Tribal LUST and UST sites Excel spreadsheets. Available at: http://www.epa.gov/region9/foia/frequent/Region9-Tribal-LUST-Sites-FEB2010.xls and http://www.epa.gov/region9/foia/frequent/Region9-Tribal-UST-Sites-FEB2010.xlsx. Accessed October 21, 2011.

U.S. Fish and Wildlife Service (USFWS). 2003. Interim Guidelines to Avoid and Minimize Wildlife Impacts from Wind Turbines. Available at: http://www.fws.gov/habitatconservation/wind.pdf. Accessed August 2, 2011.

2011. Draft Eagle Conservation Plan Guidance. Available at: http://www.fws.gov/windenergy/docs/ECP_draft_guidance_2_10_final_clean_omb.pdf. Accessed August 2, 2011.

2012a. Final Land-Based Wind Energy Guidelines. Available at: http://www.fws.gov/windenergy/docs/WEG_final.pdf. Accessed May 29, 2012.

- 2012b. List of species by county for Arizona. Available at: http://www.fws.gov/southwest/es/EndangeredSpecies/EndangeredSpecies_Lists/EndangeredSpec ies_ListSpecies.cfm. Accessed April 25, 2012.

U.S. Geological Survey (USGS). 2004. Provisional digital land cover map for the Southwestern United States. Version 1.0. Logan, Utah: RS/GIS Laboratory, College of Natural Resources, Utah State University. 
2005. SWReGAP landcover legend description database. Available at:

http://earth.gis.usu.edu/swgap/legenddataquery.php. Accessed June 29, 2012.

. 2009a. Earthquake Hazards Program. Complete Report for Yampai graben (Class A) No. 996. Available at:

http://geohazards.usgs.gov/cfusion/qfault/qf_web_disp.cfm?disp_cd=C\&qfault_or=1869\&ims_cf _cd=cf. Accessed May 28, 2012.

— 2009b. Earthquake Hazards Program. Probabilistic Seismic Hazards Assessment (PSHA) Model, based on national seismic hazard maps. Available at: http://earthquake.usgs.gov/hazards/. Accessed May 28, 2012.

—. 2012a. Earthquake Hazards Program. Arizona seismic hazard map. Available at: http://earthquake.usgs.gov/earthquakes/states/arizona/hazards.php. Accessed May 28, 2012.

- 2012b. Earthquake Hazards Program. Quaternary faults in Google Earth. Available at: http://earthquake.usgs.gov/hazards/qfaults/google.php. Accessed May 28, 2012.

. 2012c. Earthquake Hazards Program. Custom hazard mapping. Available at: http://earthquake.usgs.gov/hazards/apps/. Accessed May 28, 2012. 


\section{Exhibit J}

\section{Avian Use Assessment - Raptor Nest Survey}

(SWCA Environmental) 


\section{Avian Use Assessment Study - \\ Potential Renewable Energy \\ Development for the Hualapai \\ Tribal Nation}

Prepared for

Hualapai Tribal Nation Planning Department

Prepared by

SWCA Environmental Consultants

August 2012 



\title{
AVIAN USE ASSESSMENT STUDY - POTENTIAL RENEWABLE ENERGY DEVELOPMENT FOR THE HUALAPAI TRIBAL NATION
}

\author{
Prepared for \\ Hualapai Tribal Nation Planning Department \\ P.O. Box 179 \\ 941 Hualapai Way \\ Peach Springs, Arizona 86434
}

\author{
Prepared by \\ SWCA Environmental Consultants \\ 114 North San Francisco Street, Suite 100 \\ Flagstaff, Arizona 86001 \\ (928) 774-5500 \\ www.swca.com
}

SWCA Project No. 23377

August 2012 



\section{CONTENTS}

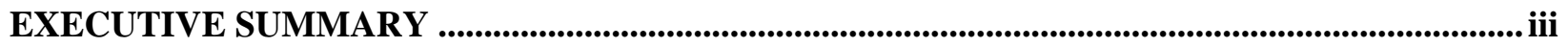

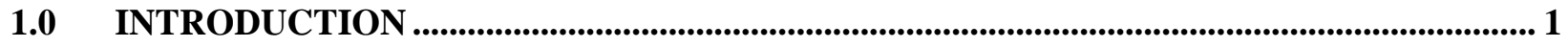

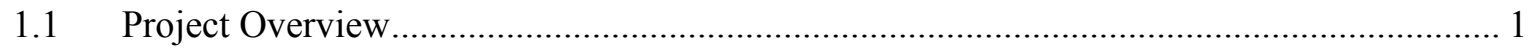

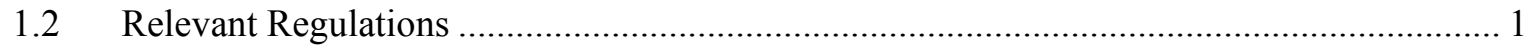

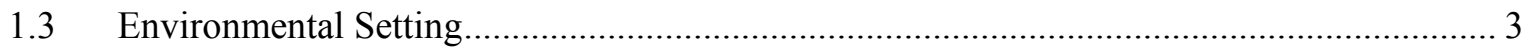

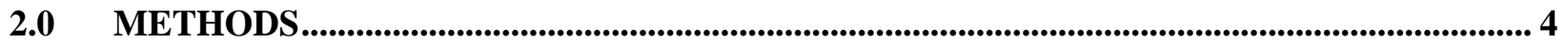

$2.1 \quad$ Listed and Special-Status Avian Species Occurrence ..................................................... 4

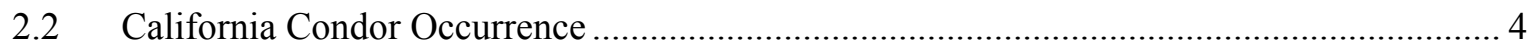

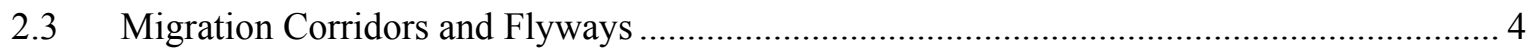

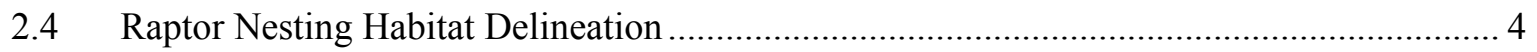

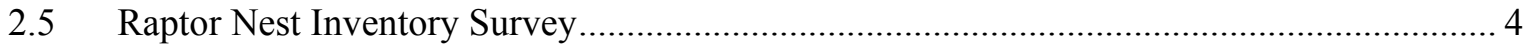

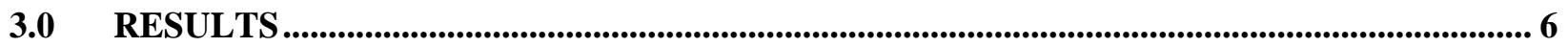

3.1 Listed and Special-Status Avian Species Occurrence ..................................................... 6

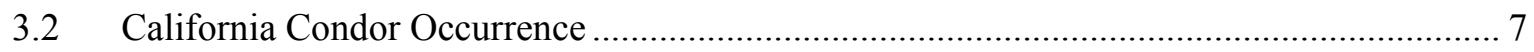

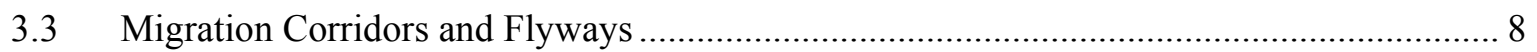

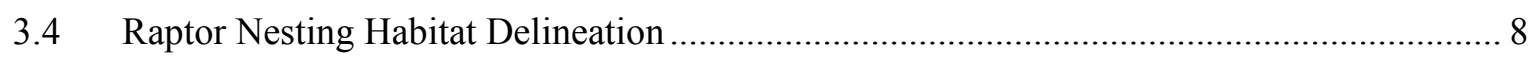

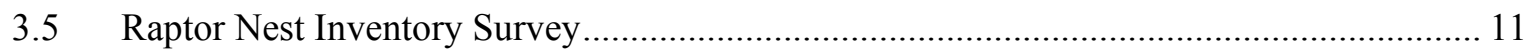

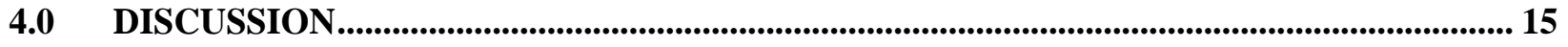

4.1 Potential Renewable Energy Development Impacts to Birds .......................................... 15

4.2 Pre-construction Avian Assessment Summary and Recommendations............................. 16

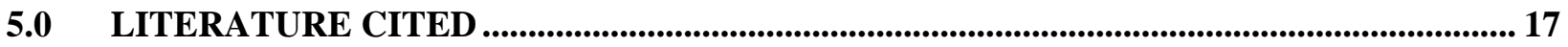

\section{Appendix}

A. Raptor Nest Data 


\section{Figures}

1. The two study areas, including the six project areas and 3-mile-radius aerial raptor nest inventory survey areas

2. Draft 2009 California condor movement/location data obtained via satellite telemetry studies (data obtained from the Peregrine Fund, October 1, 2010).

3. Potential golden eagle nesting habitat delineated for the HREDP survey areas and May- June 2012 raptor nest inventory survey tracks.

4. Golden eagle and possible golden eagle nests found within the HREDP survey areas, May-June 2012.

5. Raptor nests, excluding golden eagle and possible golden eagle, found within Grand Wash Cliffs survey area, 2012.

6. Raptor nests, excluding golden eagle and possible golden eagle, found within the Nelson survey area, May-June 2012

\section{Tables}

1. Special-Status Avian Species Known to Occur in Mohave and Coconino Counties......................... 6

2. Nests Found within the HREDP 3-Mile-Radius Survey Areas, May-June 2012 ......................... 11 


\section{EXECUTIVE SUMMARY}

The Hualapai Tribal Nation's Planning Department has identified six project areas for potential alternative energy development in Mohave and Coconino Counties, Arizona. The six project areas are known collectively in this report as the Hualapai Renewable Energy Development Project. SWCA Environmental Consultants was (SWCA) retained by the Hualapai Tribe to conduct a pre-construction avian risk assessment study for the proposed project. With a focus on golden and bald eagle use/risk related to the proposed project, the report summarizes:

- Listed and special-status avian species occurrence

- California condor (Gymnogyps californianus) occurrence

- Avian migration corridors and flyways

- Raptor nesting habitat

- Raptor nest inventory

This report informs a Critical Issues Analysis of the Hualapai Renewable Energy Development Project, prepared in July 2012 (SWCA 2012).

Three special-status species have a high potential to occur in the proposed project areas: American peregrine falcon (Falco peregrinus anatum), golden eagle (Aquila chrysaetos), and ferruginous hawk (Buteo regalis). Golden eagle and ferruginous hawk individuals were observed during the aerial raptor nest inventory survey, and nests for these species were identified. Western burrowing owl (Athene cunicularia hypugaea) was identified as having a moderate potential for occurrence. No avian species protected by the Endangered Species Act are likely to occur, and no critical habitat is present within the proposed project area.

The project is within an experimental California condor population, which is established as a "nonessential experimental population" under Section 10(j) of the ESA; however, telemetry data obtained from the Peregrine Fund show that telemetered individuals use habitat within the Grand Canyon and point northwest into Utah. It is possible that individuals could use the project in the future, as condors are wideranging and the species has the potential to expand with recovery efforts continuing.

With the exception of the Nelson Solar project area, the project is situated along a north-south-trending ridgeline - the Grand Wash Cliffs - that could concentrate migrating raptors, including eagles, in the fall. The Nelson Solar project area contains an extensive prairie dog colony that could similarly attract migrating (and breeding) raptors. There are no specific physical characteristics of the project areas that would concentrate migrating songbirds or other avian species.

Golden eagle nesting habitat was delineated before SWCA conducted an aerial raptor nest inventory survey in May-June 2012. Golden eagle nesting habitat was considered to be analogous to nesting habitat for most other nesting raptor species. The aerial inventory raptor nest survey was habitat driven, rather than transect driven, focusing on identification of golden eagle nest structures; nests of other raptor species were also identified. We located 93 unique nest structures within 3 miles of the proposed project areas and two additional nest structures just outside (on the border of) on the 3-mile-radius survey areas. Eleven golden eagle nests and 19 possible golden eagle nests were identified.

We recommend early consultation with Arizona Game and Fish Department, U.S. Fish and Wildlife Service, and Bureau of Land Management regarding additional pre-construction avian species surveys that are likely warranted to further inform project infrastructure design, as well as Bird and Bat Conservation Strategy/Eagle Conservation Strategy and National Environmental Policy Act considerations. 



\subsection{INTRODUCTION}

\subsection{Project Overview}

The Hualapai Tribal Nation's Planning Department has identified six project areas for potential renewable energy development in Mohave and Coconino Counties, Arizona. The six project areas are known collectively in this report as the Hualapai Renewable Energy Development Project (HREDP). For survey and reporting purposes, two noncontiguous project areas of the HREDP have been identified (the Grand Wash Cliffs and the Nelson survey areas) (Figure 1). The Grand Wash Cliffs survey area includes the Grand Canyon West Wind Energy, Clay Springs Wind Energy, Bureau of Land Management (BLM) Wind North, BLM Wind South, and Hualapai Solar project areas. The Nelson survey area includes six relatively small (each less than one Section of land) noncontiguous project areas. The entire HREDP is composed of approximately 42,436 acres located on Hualapai Tribal, BLM, private, and state lands.

SWCA Environmental Consultants (SWCA) was retained by the Hualapai Tribe in April 2012 to conduct a Critical Issues Analysis (CIA) for the proposed HREDP. This report presents the avian use assessment study and results used to inform the CIA (see Critical Issues Analysis of Renewable Energy Development Project Areas [SWCA 2012]). This document reports findings of the avian use assessment specific to the HREDP, including occurrence or potential occurrence and/or use of the following species, avian assemblages, and habitats:

- Listed and special-status avian species

- California condor (Gymnogyps californianus)

- Avian migration corridors and flyways

- Raptor nesting habitat

- Golden eagle (Aquila chrysaetos) and bald eagle (Haliaeetus leucocephalus)

The findings in this report are based on desktop reviews/analyses, literature searches, communication with and data requests from local and regional experts, and an aerial raptor nest inventory survey conducted in May-June 2012.

\subsection{Relevant Regulations}

The regulatory framework for protecting birds includes the Endangered Species Act (ESA) of 1973, as amended, the Migratory Bird Treaty Act (MBTA) of 1918, as amended, the Bald and Golden Eagle Protection Act (BGEPA) of 1940, as amended, and Executive Order 13186.

All migratory birds are covered under the MBTA, while the BGEPA specifically protects golden and bald eagles. The BGEPA prohibits anyone without a permit from "taking" golden and bald eagles and their parts, eggs, or nests. "Take" is defined by the BGEPA as "to pursue, shoot, shoot at, poison, wound, kill, capture, trap, collect, molest, or disturb;" it differs from the ESA in that it does not include habitat destruction or alteration, unless such damage "disturbs" an eagle. "Disturb" is defined as "to agitate or bother to a degree that causes, or is likely to cause, based on the best scientific information available, 1) injury to an eagle, 2) a decrease in its productivity, by substantially interfering with normal breeding, feeding, or sheltering behavior, or 3) nest abandonment, by substantially interfering with normal breeding, feeding, or sheltering behavior." The MBTA prohibits incidental "take" of migratory birdsmore than 1,000 species (Federal Register; 50 Code of Federal Regulations [CFR] 10 and 21), including golden and bald eagle and their parts, eggs, or nests "at any time, by any means." "Take" is defined by the 


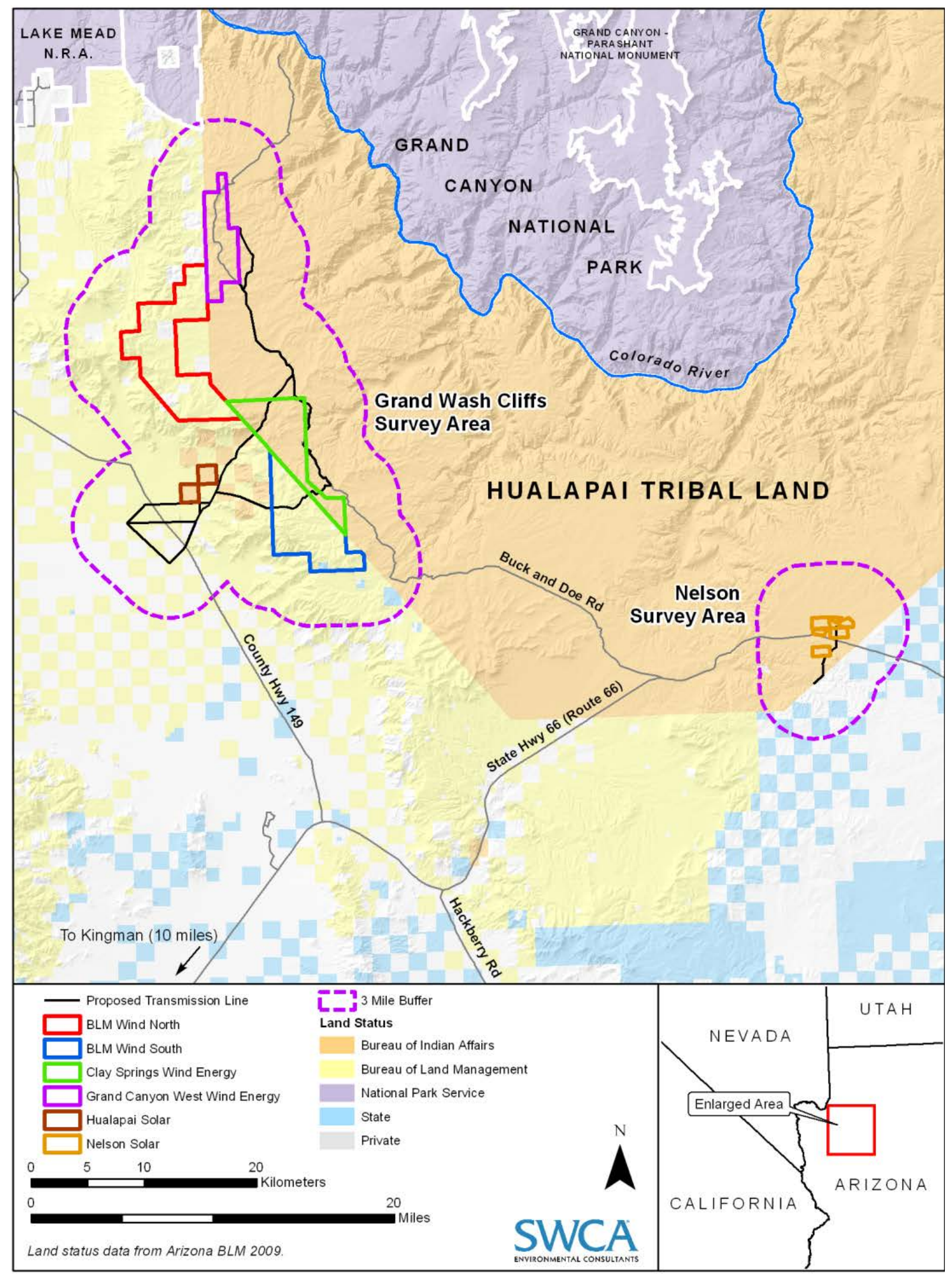

Figure 1. The two study areas, including the six project areas and 3-mile-radius aerial raptor nest inventory survey areas. 
MBTA as "to pursue, hunt, shoot, wound, kill, trap, capture, or collect, or any attempt to carry out these activities." A "take" does not include habitat destruction or alteration, as long as it does not involve a known direct taking of birds, nests, or eggs.

On September 11, 2009 (Federal Register 2009; 50 CFR 13 and 22), the U.S. Fish and Wildlife Service (USFWS) set in place rules establishing two new permit types under the BGEPA: 1) take of golden and bald eagles that is associated with, but not the purpose of, the activity; and 2) purposeful take of an active or inactive nest where necessary to alleviate a safety emergency; an inactive eagle nest when the removal is necessary to ensure public health and safety; an inactive nest that is built on a human-engineered structure and creates a functional hazard that renders the structure inoperable for its intended use; or an inactive nest, provided the take is necessary to protect an interest in a particular locality and the activity necessitating the take or the mitigation for the take will, with reasonable certainty, provide a clear and substantial benefit to eagles. USFWS received the first application from a proposed wind project for a take permit under BGEPA earlier this year (2012). USFWS recommends wind energy project proponents prepare a Bird and Bat Conservation Strategy (BBCS) as well as an Eagle Conservation Strategy (ECS), in collaboration with USFWS, to avoid, minimize, and otherwise mitigate project-related impact to birds and bats, and specifically to bald and golden eagles to ensure no net loss to eagle populations. The proposed project is subject to all relevant federal, state, and local statutes, regulations, and plans.

\subsection{Environmental Setting}

The Grand Wash Cliffs survey area ranges from approximately 2,700 to 6,400 feet above mean sea level and is generally bounded by Quartermaster Canyon to the northeast, West Water Canyon to the southeast, Red Lake to the southwest, and Garnet Mountain to the west; the Grand Wash Cliffs are situated along the western edge of the wind development project areas. The Nelson survey area ranges from approximately 5,000 to 5,800 feet above mean sea level and is generally bounded by Faught and Nelson Canyons to the south, Blue Mountain to the northeast, and western boundary of the Aubrey Valley to the east.

Vegetation within the Grand Wash Cliffs survey area is dominated by two vegetation communities: Colorado Plateau Pinyon-Juniper Woodland and Mohave Mid-Elevation Mixed Desert Scrub, with lesser amounts of Apacherian-Chihuahuan Mesquite Upland Scrub, Colorado Plateau Blackbrush-Mormon-tea Shrubland, Colorado Plateau Mixed Bedrock Canyon and Tableland, Great Basin Conifer Woodland, Inter-Mountain Basins Big Sagebrush Shrubland, Inter-Mountain Basins Juniper Savanna, Inter-Mountain Basins Mixed Salt Desert Scrub, Inter-Mountain Basins Semi-Desert Grassland, Inter-Mountain Basins Semi-Desert Shrub Steppe, Mogollon Chaparral, Sonora-Mohave Creosotebush-White Bursage Desert Scrub, and Sonoran Mid-Elevation Desert Scrub (U.S. Geological Survey [USGS] 2004). Vegetation within the Nelson survey area is dominated by Colorado Plateau Pinyon-Juniper Woodland, InterMountain Basins Semi-Desert Grassland, and Inter-Mountain Basins Semi-Desert Shrub Steppe, with lesser amounts of Colorado Plateau Mixed Bedrock Canyon and Tableland, Inter-Mountain Basins Big Sagebrush Shrubland, Inter-Mountain Basins Salt Desert Scrub, and Invasive Annual and Biennial Forbland (USGS 2004). These vegetation community descriptions and composition by project area are detailed in SWCA (2012). 


\subsection{METHODS}

\subsection{Listed and Special-Status Avian Species Occurrence}

Special-status avian species include any species that is listed, or proposed for listing, as threatened or endangered by the USFWS under the provisions of the ESA; any species designated by the USFWS as a candidate or species of concern, and any species listed by the Arizona Game and Fish Department (AGFD). Potential occurrence of federal or state special-status avian species was evaluated using publicly available data sources, including topographic and aerial maps and land cover data, the AGFD Heritage Database Management System (HDMS), the USFWS Critical Habitat Portal, special-status species lists by county, the Birds of North America Online species accounts, and communication with local and regional experts.

\subsection{California Condor Occurrence}

To evaluate potential use of the project areas by the California condor, we initiated communication with and requested species' use data from the Peregrine Fund, which has been conducting condor studies in northern Arizona since 1996.

\subsection{Migration Corridors and Flyways}

To evaluate potential use of the project areas by migrating raptors, we conducted a literature review and initiated communication with and requested data from Hawk Watch International, which has been conducting raptor migration studies in northern Arizona since 1997. We also opportunistically recorded Gunnison's prairie dog [Cynomys gunnisoni]) colonies during the aerial raptor nest survey (see Section 2.5 below), as a high density of small-mammal prey has been considered to correlate with high raptor use of an area (National Academy of Sciences 2007). We describe potential use of the project areas by songbirds and shorebirds/wading birds in the context of available wetlands, playas, reservoirs, lakes, and/or riparian corridors and other features of the landscape that could concentrate these birds during migratory periods.

\subsection{Raptor Nesting Habitat Delineation}

Prior to conducting the raptor nest inventory survey (see Section 2.5 below), raptor nesting habitat (e.g., headwalls, ridgelines, rock faces/outcrops, large trees and snags, and transmission towers), with a focus on golden and bald eagle, was delineated out to a 3-mile radius of both the Grand Wash Cliffs and Nelson survey areas (Figure 1). A geographic information system (GIS) specialist and avian ecologist used a combination of aerial imagery (National Agriculture Imagery Program) and USGS topographic maps in ArcGIS to manually digitize/delineate potentially suitable raptor nesting habitat. The delineation included a conservative estimate for golden eagle nesting habitat as well as habitats most suitable for other nesting raptor species.

\subsection{Raptor Nest Inventory Survey}

The raptor nest inventory survey was conducted by two observers (one GIS specialist and one avian ecologist) and one pilot in May-June 2012. Surveys were conducted out to a 3-mile radius of both the Grand Wash Cliffs and Nelson survey areas (Figure 1). The survey was habitat driven, rather than transect driven. Based on target areas provided by SWCA observers, the pilot typically flew contours for full coverage within all potentially suitable raptor habitats identified via nesting habitat delineation (see 
Section 2.4 above). While in the air, surveyors limited and/or discontinued surveys in areas identified via the desktop habitat delineation that did not exhibit appropriate raptor nesting characteristics once observed from the air.

Survey methods generally followed Pagel et al. (2010) and USFWS (2011). The survey was sensitive to local nesting chronologies and disturbance at nests and was conducted during weather conditions favorable for aerial surveys. A Bell 206 BIII "Jet Ranger" helicopter was used, which allowed for close approach, relative to fixed-wing aircraft, to accurately determine nest contents (Phillips et al. 1984). The GIS specialist used a Panasonic Toughbook laptop and TopCon GRS-1 global positioning system (GPS) unit to record survey tracks and nest locations. SWCA observers used a Garmin Aera navigational GPS, which recorded backup survey tracks and aided in ensuring full survey coverage. During refuel and nightly breaks, the surveyors and pilot evaluated survey coverage and logistics for completing survey objectives.

For each nest found, surveyors recorded the date and time of observation, a nest identification number, species 4-letter alpha code (in accordance with the methods of the Institute for Bird Populations 2012) or "undetermined species," nest substrate (e.g., cliff, tree, transmission tower), and nest condition/contents. "Undetermined species" nests included any nests that were too deteriorated to confidently identify species or exhibited qualities characteristic of several species; for these nests, surveyors recorded an informed opinion regarding which species was most likely to use the nest based on nest structure and placement (e.g., "undetermined: resembles Buteo spp. or golden eagle," or "undetermined: resembles Buteo spp. or common raven [Corvus corax]"). Non-raptor species (e.g., common raven) nests also were recorded incidentally when identified. The following nest conditions/contents were recorded: 1) sticks-intact, 2) sticks-deteriorating, 3) greenery/ornamentation, 4) adult in incubation/brooding posture, and 5) number of egg(s)/nestling(s). Surveyors photographed nests as conditions allowed using a digital SLR with a 100$500 \mathrm{~mm}$ lens and noted specific nest structure characteristics and proximity of nearby nests.

Terms used to describe nest occupancy, nest productivity, and golden eagle territories in the results below include the terms discussed below.

Occupied nest: A nest in which one or more of the following occurred: 1) young were raised, 2) eggs were laid, 3) an adult was observed sitting, presumably in incubation or brooding posture, in the nest, 4) two adults were observed perched on or near the nest, 5) an adult and a bird in immature plumage were observed on or near the nest, if mating behavior was observed (e.g., display flights, copulation), and/or 6) recent repairs (e.g., fresh greenery, sticks with fresh breaks), mute, or feathers were visible at or near the nest (Driscoll 2010; Postupalsky 1974; Steenhof and Newton 2007).

Active nest: A nest in which an egg or eggs were laid and/or young were raised (Driscoll 2010; Postupalsky 1974).

Successful nest: A nest in which at least one young reached the minimum acceptable age for assessing success (typically $80 \%$ of first-flight age for raptor species); a nestling observed to be $\geq 52$ days old is indicative of success for golden eagle (Steenhof and Newton 2007).

Alternate, or supernumerary, nest: Some eagle (and other large raptor) pairs use the same nest every year but may repair and add material to alternate nests situated within the same territory until eggs are laid, and some switch nest sites from year to year regardless of previous year's success (Boeker and Ray 1971; McGahan 1968). Golden eagles use an average of two to three alternate nests, with some territories containing only one nest (range: 0-14 alternate nests [Kochert et al. 2002]). Golden eagle alternate nests can be placed $<3$ feet or $>3$ miles $(<1 \mathrm{~m}$ or $>4.8 \mathrm{~km})$ apart (McGahan 1968). 
Productivity: The number of young that reach the minimum acceptable age for assessing success, reported as the number of young produced per occupied nesting territory in a particular year (Steenhof and Newton 2007; USFWS 2011).

Territory: An area that contains, or historically contained, one or more nests within the home range of a mated pair: a confined locality where nests are found, usually in successive years, and where no more than one pair is known to have bred at any one time. A territory can be further defined as "regular" (in use every, or almost every, year) or "irregular" (in use in certain years out of many) (Steenhof and Newton 2007). A territory can also be further defined as "current:" selected by eagles for "use" in the current nesting season or "historical." Unique golden eagle territories were distinguished by observation of simultaneously "active" nests/nest clusters and disparate nests or nest clusters spaced $>1.9$ miles $(3.1 \mathrm{~km})$ apart (Phillips et al. 1984); number of unique territories can be refined over multiple years of survey.

Occupied nesting territory: A territory containing an occupied nest or nests; equivalent to "occupied breeding area" presented by Postupalsky (1974) and Driscoll (2010). Distinguishing the number of occupied nesting territories within a survey area can be problematic when relying on observation of recent nest repairs alone. For example, within the same golden eagle territory, alternate nests can be spaced $>3$ miles apart (McGahan 1968), while nearest neighbors can be spaced 0.5 to 10.0 miles $(0.8-16.0 \mathrm{~km})$ apart (Kochert 1972). Some non-eagle species (e.g., red-tailed hawks [Buteo jamaicensis]) present a second problem because they are known to add fresh greenery to unoccupied eagle nests (Driscoll 2010; personal communication, D. Driscoll, American Eagle Research Institute, July 21, 2011); this can result in overestimation of occupied eagle nesting territories.

Following the survey, photographs of each nest were thoroughly examined to confirm species determinations and record evidence of occupancy and specific nest contents that may have been overlooked or were unclear during surveys. Nestlings were aged to 5- to 10-day increments using Driscoll (2010) and Watson (2010). In accordance with Pagel et al. (2010), all recommended data fields for each nest found (e.g., USFWS naming convention, territory status, nest elevation, hatch date, fledge date) were entered into an Access database.

\subsection{RESULTS}

\subsection{Listed and Special-Status Avian Species Occurrence}

The HDMS indicates that 17 special-status avian species are known to occur within Mohave and Coconino Counties (Table 1). Adapted from Table 9 in SWCA (2012), Table 1 also includes an evaluation of occurrence potential within the proposed project areas.

Table 1. Special-Status Avian Species Known to Occur in Mohave and Coconino Counties

\begin{tabular}{llccccc}
\hline Common Name & Scientific Name & County* $^{\dagger}$ & $\begin{array}{c}\text { USFWS } \\
\text { Status }^{\ddagger}\end{array}$ & $\begin{array}{c}\text { BLM } \\
\text { Status }^{\S}\end{array}$ & $\begin{array}{c}\text { State } \\
\text { Status }^{\pi}\end{array}$ & $\begin{array}{c}\text { Potential for } \\
\text { Occurrence within }^{\text {the HREDP }}\end{array}$ \\
\hline American peregrine falcon & Falco peregrinus anatum & C, M & SC, D & S & WSC & H \\
\hline Bald eagle & Haliaeetus leucocephalus & C, M & SC, D & S & WSC & M \\
\hline California black rail & $\begin{array}{l}\text { Laterallus jamaicensis } \\
\text { coturniculus }\end{array}$ & M & SC & S & WSC & L \\
\hline California condor & Gymnogyps californicus & C, M & LE, XN & & WSC & L \\
\hline California least tern & Sterna atillarum browni & M & LE & & L
\end{tabular}


Table 1. Special-Status Avian Species Known to Occur in Mohave and Coconino Counties (Continued)

\begin{tabular}{|c|c|c|c|c|c|c|}
\hline Common Name & Scientific Name & County* ${ }^{\dagger}$ & $\begin{array}{l}\text { USFWS } \\
\text { Status }^{\ddagger}\end{array}$ & $\begin{array}{l}\text { BLM } \\
\text { Status }^{\S}\end{array}$ & $\begin{array}{l}\text { State } \\
\text { Status }\end{array}$ & $\begin{array}{l}\text { Potential for } \\
\text { Occurrence within } \\
\text { the HREDP }\end{array}$ \\
\hline Clark's grebe & Aechmophorus clarkii & M & & $S$ & WSC & L \\
\hline Common black-hawk & Buteogallus anthracinus & M & & $S$ & WSC & L \\
\hline Ferruginous hawk & Buteo regalis & $\mathrm{C}, \mathrm{M}$ & SC & S & WSC & $\mathrm{H}$ \\
\hline Golden eagle & Aquila chrysaetos & $\mathrm{C}, \mathrm{M}$ & & S & & $\mathrm{H}$ \\
\hline Least bittern & Ixobrychus exilis & M & & & WSC & $\mathrm{L}$ \\
\hline Mexican spotted owl & Strix occidentalis lucida & $\mathrm{C}, \mathrm{M}$ & LT & & WSC & L \\
\hline Northern goshawk & Accipiter gentilis & $\mathrm{C}, \mathrm{M}$ & SC & $S$ & WSC & L \\
\hline $\begin{array}{l}\text { Southwestern willow } \\
\text { flycatcher }\end{array}$ & Empidonax traillii extimus & $\mathrm{C}, \mathrm{M}$ & LE & & WSC & $\mathrm{L}$ \\
\hline Western burrowing owl & $\begin{array}{l}\text { Athene cunicularia } \\
\text { hypugaea }\end{array}$ & $\mathrm{C}, \mathrm{M}$ & SC & S & & M \\
\hline White-faced ibis & Plegadis chihi & C & SC & & & $\mathrm{L}$ \\
\hline $\begin{array}{l}\text { Yellow-billed cuckoo } \\
\text { (Western U.S. DPS }{ }^{\star \star} \text { ) }\end{array}$ & Coccyzus americanus & M & C & & WSC & L \\
\hline Yuma clapper rail & $\begin{array}{l}\text { Rallus longirostris } \\
\text { yumanensis }\end{array}$ & M & LE & S & WSC & L \\
\hline
\end{tabular}

Source: AGFD (2012b).

* Status data taken from the HDMS (AGFD 2012a, 2012b).

${ }^{\dagger} \mathrm{C}=$ Coconino County; $\mathrm{M}=$ Mojave County.

${ }^{\ddagger} \mathrm{C}=$ Candidate for Listing; $\mathrm{D}=$ Delisted LE = Listed Endangered; $\mathrm{LT}=$ Listed Threatened; $\mathrm{SC}=$ Species of Concern; $\mathrm{XN}=$ Experimental Nonessential population.

${ }^{\S} \mathrm{S}=$ BLM Special Status (BLM State Office).

"WSC = Wildlife of Special Concern in Arizona

${ }^{\#} \mathrm{~L}$ = Low (unlikely to occur; outside of species range, and/or habitat is not present); $M=$ Moderate (habitat is limited, may be used for foraging or passing through); $\mathrm{H}=$ High (observed on site or habitat is widespread, likely nests and forages on site).

Golden eagle and ferruginous hawk (Buteo regalis) nesting was confirmed within or in close proximity to project areas (see Section 3.5 below). Peregrine falcon (Falco peregrinus) may also nest within or in close proximity to project areas (see Section 3.5 below), as high-quality nesting habitat is present throughout the Grand Wash Cliffs. Although bald eagle nesting habitat (nesting substrate in close proximity to water bodies that support fish) is not present within the survey areas, the species occurs widely in northern Arizona during winter months and may occur during the winter within the project areas. Although habitat for burrowing owl (Athene cunicularia hypugaea) is limited throughout the project areas, prairie dog burrows (used for nesting by burrowing owls) were recorded within the Grand Canyon West and in close proximity to the Nelson project areas (see Section 3.3 below). For all other species listed in Table 1, potential for occurrence is low, as suitable habitats are not present. The project areas do not contain critical habitat for any federally listed avian species, and no avian species protected by the ESA are likely to occur. The project areas are not located within or do not contain a specially designated state or federal management area, Important Bird Area, Western Hemisphere Shorebird Reserve Network site, or Ramsar Convention site.

\subsection{California Condor Occurrence}

Both the Grand Wash Cliffs and Nelson study areas are located within an experimental California condor (Gymnogyps californianus) population, which is established as a "non-essential experimental population" 
under Section 10(j) of the federal ESA. Outside the designated 10(j) areas, this species is considered endangered.

With respect to the California condor, range-wide movement studies conducted from 1996 to 2006 (Hunt et al. 2007; Southwest Condor Review Team 2007) indicate that species occurrence within the study area would be low. Recent (2009) condor movement data collected via satellite telemetry from 12 individuals were obtained from the Peregrine Fund (Figure 2). The 2009 Peregrine Fund data are consistent with those from 1996 to 2006, with no locations recorded near the study areas. Furthermore, 2007 to 2010 movement data indicate that it is unlikely condors will occur near the study areas at this time, as the species is using higher-quality habitat from Grand Canyon northwest to southern Utah (personal communication, Chris Parrish, the Peregrine Fund, September 3, 2010). However, although current telemetry studies show that condors do not use the study areas, they are a wide-ranging species that can travel long distances and may expand beyond their current range over time. Therefore, the potential exists for condors to occur in the study areas in the future.

\subsection{Migration Corridors and Flyways}

Raptor migration has been a well-studied phenomenon for decades, and it is well known that migrating raptors concentrate along long-established routes or corridors. Hawkwatch International has identified a major raptor migration area at Yaki and Lipan Points, within Grand Canyon National Park (approximately 110 miles east of the Grand Wash Cliffs). AGFD studies currently being conducted at the nearby Aubrey Cliffs (approximately 55 miles east of the Grand Wash Cliffs) indicate that the Aubrey Cliffs are a major raptor migration area, and certain topographic features of the site (e.g., north-south ridgelines) concentrate migrant raptors in northern Arizona. The Grand Wash Cliffs comprise somewhat prominent north-southtrending ridgelines that could potentially concentrate raptor migration during fall, similar to the Aubrey Cliffs.

During the May-June 2012 raptor nest inventory survey, prairie dog colonies were recorded. One colony was observed in the Grand Canyon West Wind Energy project area, consisting of a handful of burrows, while the Nelson Solar project area colony was large, encompassing much of the project area. Prairie dog activity (i.e., live animals) was not observed during the aerial survey; therefore, it is not known whether these colonies are viable or to what extent migrating raptors may use these areas for foraging.

There are no riparian corridors, wetlands, playas, reservoirs, or lakes within the project areas. Therefore, it is unlikely that songbirds, shorebirds, or wading birds would concentrate during fall and spring migration. During the 2012 raptor nest inventory survey, some scattered cottonwoods and tamarisk were noted on the southwestern and easternmost extremes of the Grand Wash Cliffs survey area; however, these were sparsely distributed and did not form substantive riparian corridors.

\subsection{Raptor Nesting Habitat Delineation}

A total of 125,527 acres $\left(508 \mathrm{~km}^{2}\right)$ of potentially suitable golden eagle nesting habitat was delineated within a 3-mile radius of the Grand Wash Cliffs and Nelson survey areas (Figure 3), with potential golden eagle habitat considered analogous to nesting habitat for most other local raptor species. The most dominant raptor nesting habitat within the project areas consists of cliff faces, ridgelines, and headwalls associated with the Grand Wash Cliffs, Quartermaster Canyon, smaller canyons (e.g., Grapevine Canyon, Meriwhitica Canyon), rocky outcrops (e.g., Garnet Mountain, Iron Mountain), and transmission towers. 


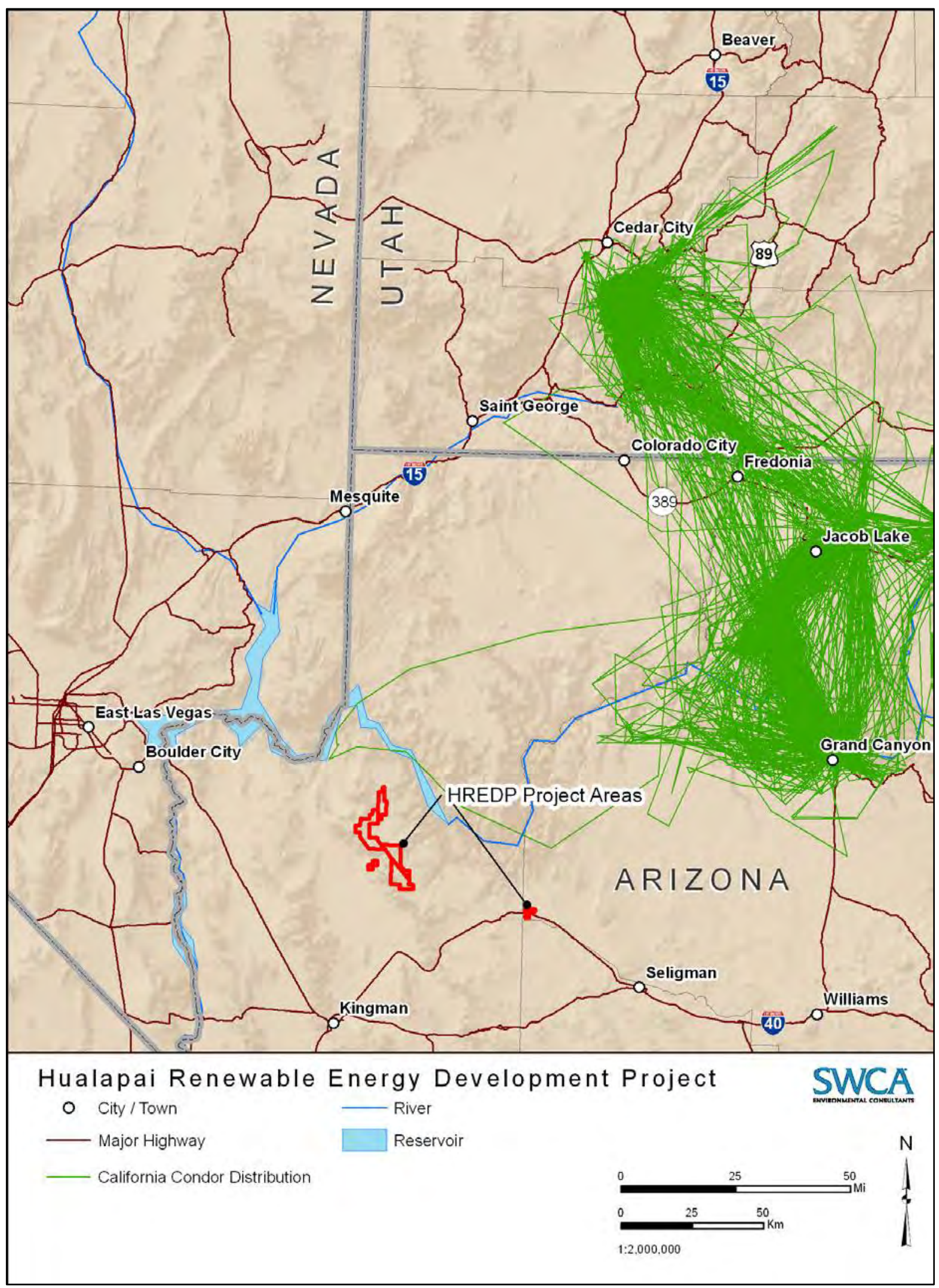

Figure 2. Draft 2009 California condor movement/location data obtained via satellite telemetry studies (data obtained from the Peregrine Fund, October 1, 2010). 


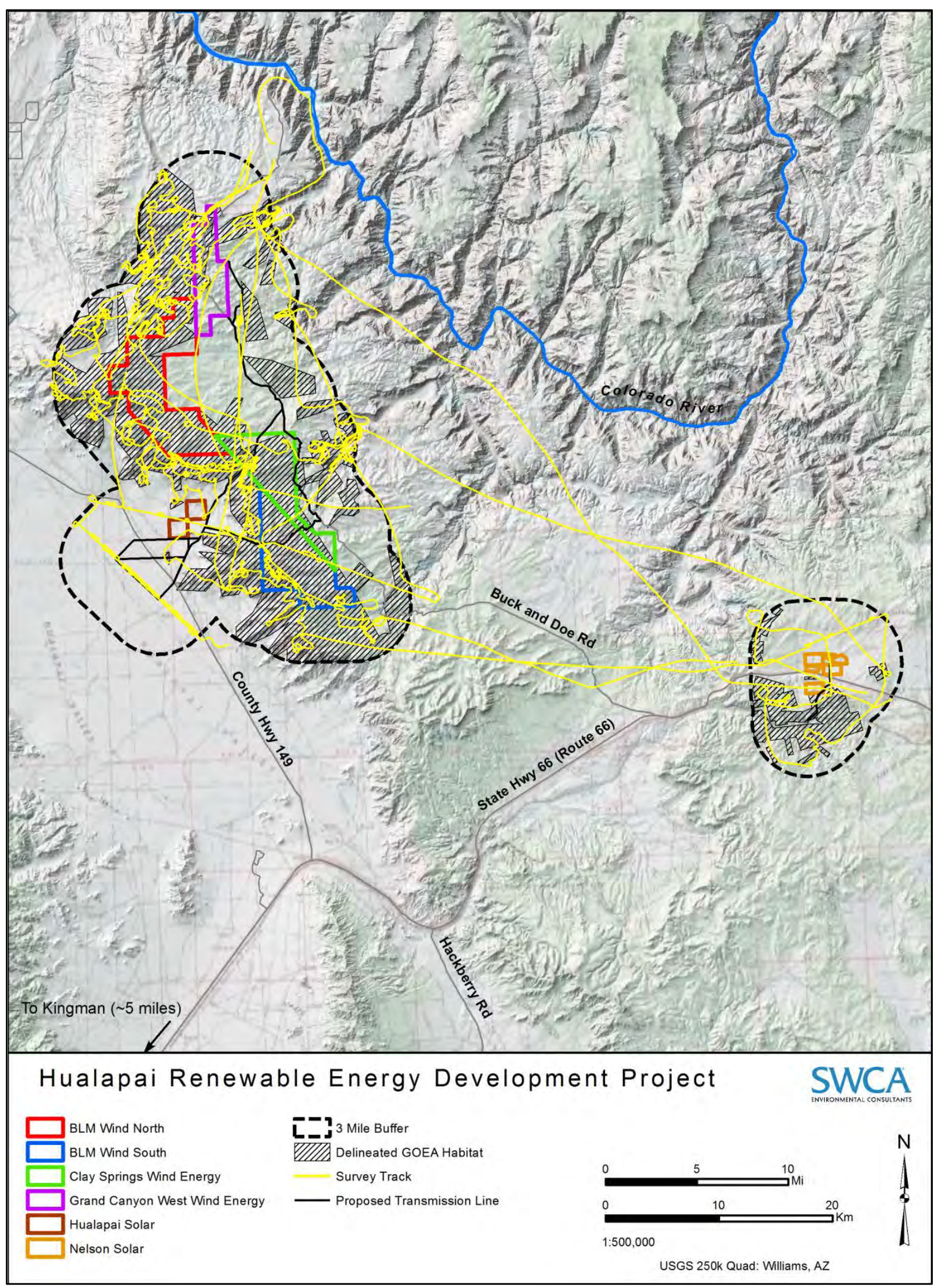

Figure 3. Potential golden eagle nesting habitat delineated for the HREDP survey areas and MayJune 2012 raptor nest inventory survey tracks. 


\subsection{Raptor Nest Inventory Survey}

From May 31 to June 2, 2012, we spent a total of 15.5 flight hours conducting the aerial raptor nest inventory survey. The survey covered all habitat delineated within a 3-mile radius of the project areas and approximately 34.7 miles $(89.9 \mathrm{~km}$ ) of transmission line (see Figure 3 ).

We located 93 unique raptor nest structures: 21 nests were located in the proposed project areas, and 72 nests were located in the area between the project areas and out to 3 miles (Table 2, Appendix Table A-1, Figures 4-6). Two additional nests were located just outside the survey areas. Including these two nests, 11 golden eagle nests and 19 possible golden eagle nests (i.e., "unknown: resembles Buteo spp. of golden eagle) were located. The remaining 65 nests were identified as common raven (9), ferruginous hawk (1), peregrine falcon/prairie falcon (Falco mexicanus) (1), red-tailed hawk (12), "undermined: resembles Buteo spp." (11), "undetermined: resembles Buteo spp. or common raven" (22), "undetermined: resembles Buteo spp. or ferruginous hawk" (2), "undetermined: resembles common raven" (6), and "undetermined: resembles common raven or packrat" (1).

Table 2. Nests Found within the HREDP 3-Mile-Radius Survey Areas, May-June 2012

\begin{tabular}{lcc}
\hline Species & $\begin{array}{c}\text { Within Grand Wash Cliffs and } \\
\text { Nelson Survey Areas } \\
\text { Combined }\end{array}$ & $\begin{array}{c}\text { Opportunistically } \\
\text { Found, Outside } \\
\text { Survey Areas }\end{array}$ \\
\hline Common Raven (Corvus corax) & 9 & 1 \\
\hline Ferruginous Hawk (Buteo regalis) & 1 & -- \\
\hline Golden Eagle (Aquila chrysaetos) & 10 & 1 \\
\hline Peregrine Falcon (Falco peregrinus)/Prairie Falcon (Falco mexicanus) & 1 & -- \\
\hline Red-tailed Hawk (Buteo jamaicensis) & 12 & -- \\
\hline Undetermined: resembles Buteo spp. & 11 & -- \\
\hline Undetermined: resembles Buteo spp. or Common Raven & 24 & -- \\
\hline Undetermined: resembles Buteo spp. or Ferruginous Hawk & 2 & -- \\
\hline Undetermined: resembles Buteo spp. or Golden Eagle & 19 & -- \\
\hline Undetermined: resembles Common Raven & 6 & -- \\
\hline Undetermined: resembles Common Raven or packrat & 1 & -- \\
\hline Total & $\mathbf{9 3}$ & $\mathbf{2}$ \\
\hline
\end{tabular}

Although the 2012 raptor nest inventory survey was conducted late in the breeding season, we identified 25 occupied nests: 2 golden eagle (both active; each containing one nestling), 5 common raven (each active containing at least one nestling), 1 ferruginous hawk (active; containing $3+$ nestlings), 1 peregrine falcon/prairie falcon (active; containing 5 nestlings), 10 red-tailed hawk (each active; 2 containing adults with nest contents unclear; 8 containing at least one nestling), 2 "undetermined: resembles Buteo spp." (both occupied; containing greenery/ornamentation), and 4 "undetermined: resembles Buteo spp. or common raven (each occupied; containing greenery/ornamentation). The two active golden eagle nests were both considered to be successful (nestlings were $\geq 52$ days old). 


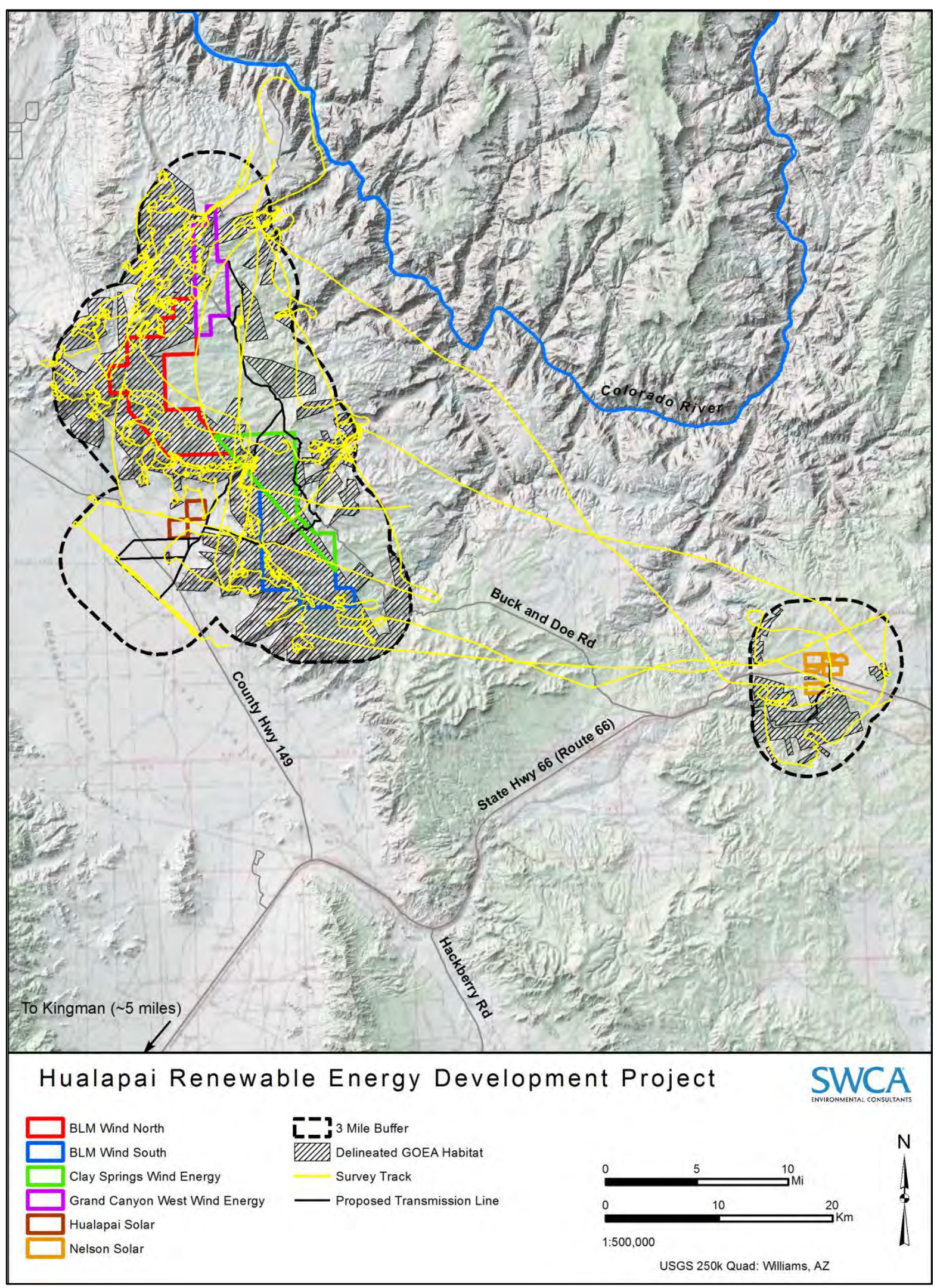

Figure 4. Golden eagle and possible golden eagle nests found within the HREDP survey areas, May-June 2012. 


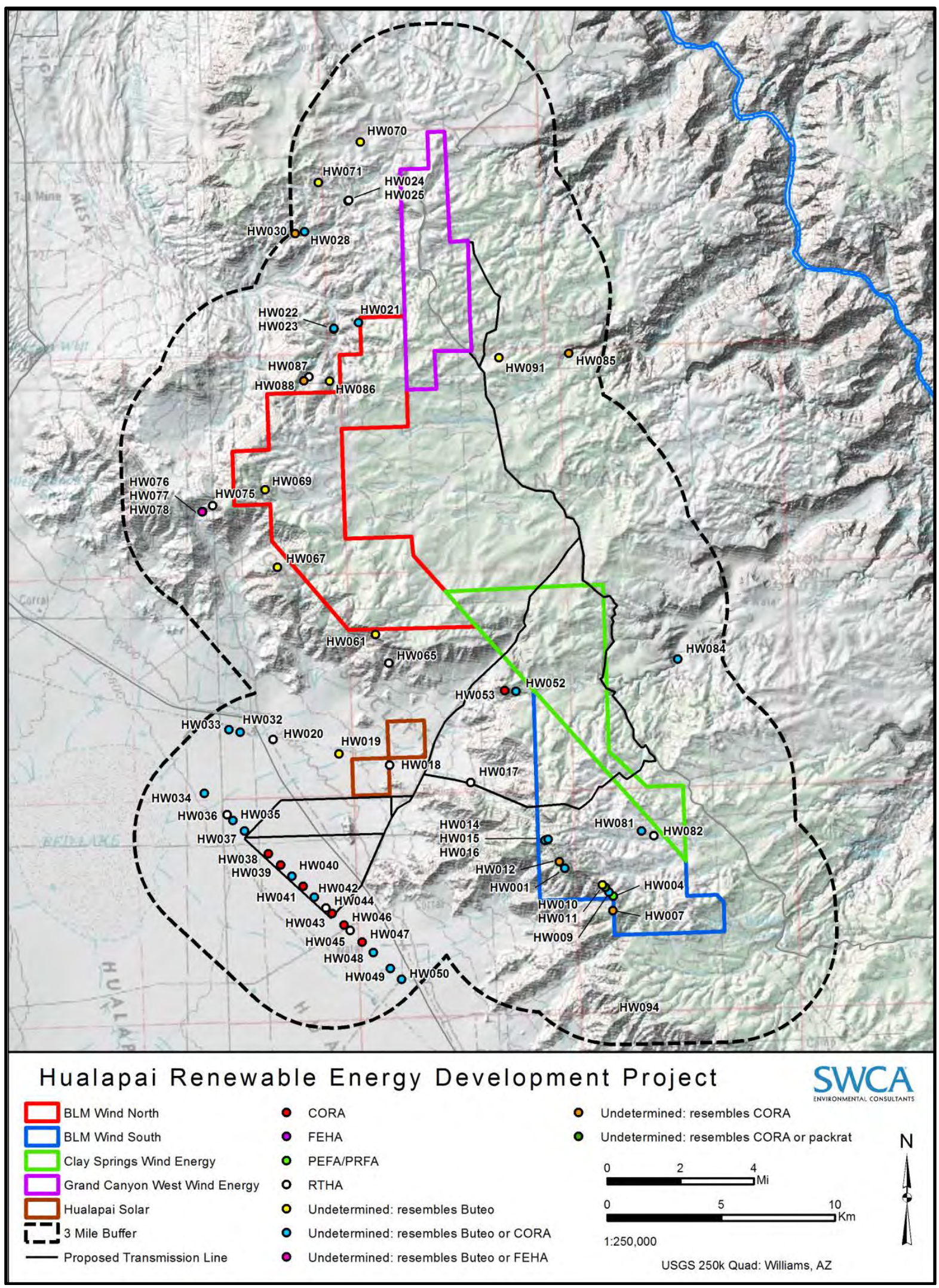

Figure 5. Raptor nests, excluding golden eagle and possible golden eagle, found within Grand Wash Cliffs survey area, 2012. 


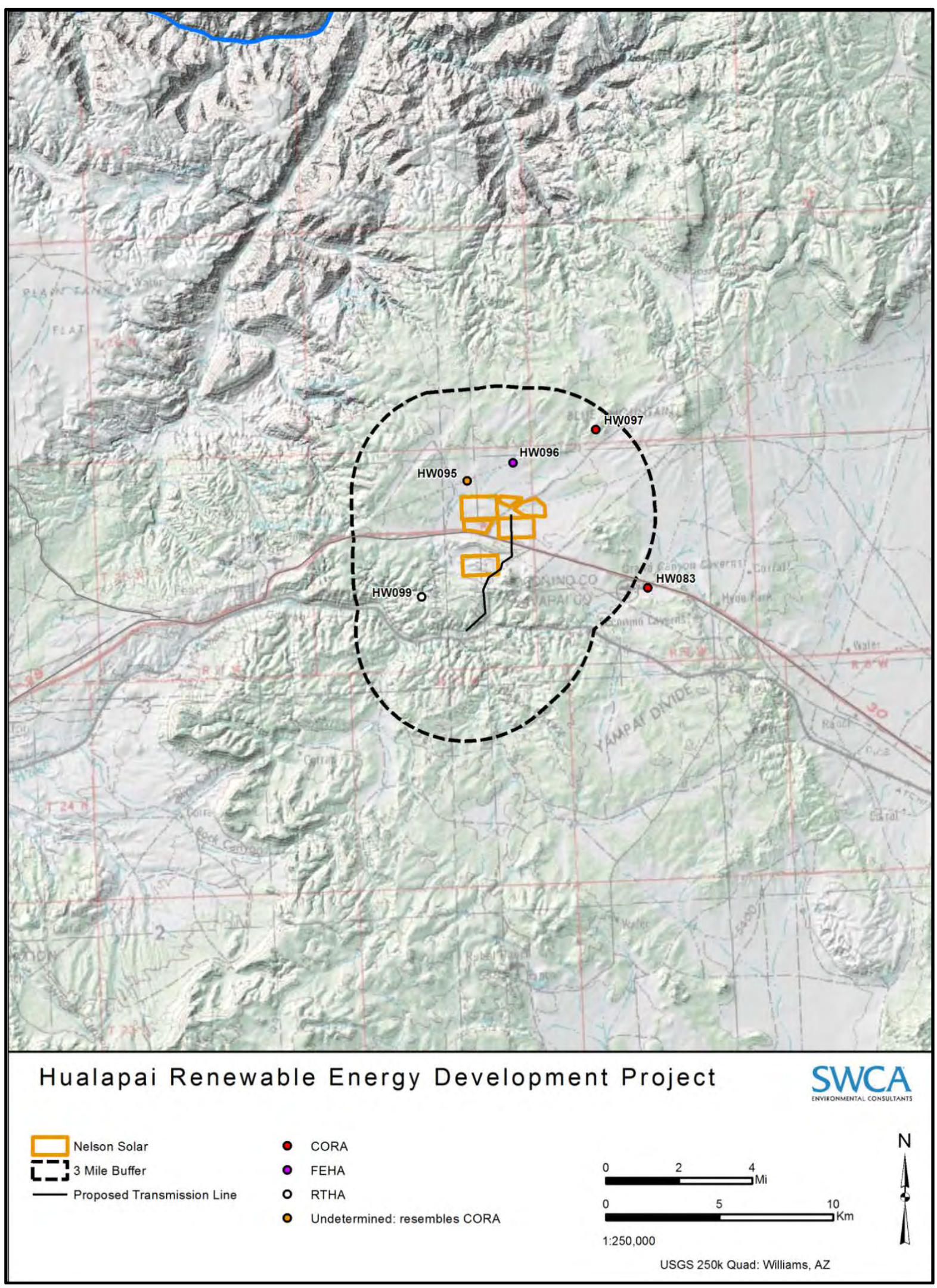

Figure 6. Raptor nests, excluding golden eagle and possible golden eagle, found within the Nelson survey area, May-June 2012. 


\subsection{DISCUSSION}

\subsection{Potential Renewable Energy Development Impacts to Birds}

Little scientific information exists on the impacts of renewable energy projects to wildlife (Kevlesky et al. 2007); the focus has primarily been on the effects of wind energy development on birds and bats because of their vulnerability to turbine strike (Drewitt and Langston 2006; Kunz et al. 2007). Impacts of solar energy development generally cited include habitat modification and related barriers to movement and genetic exchange in populations of ungulates and tortoises (Lovich and Ennen 2011). It has also been speculated that wastewater associated with solar development could also attract, and be harmful to, birds (Herbst 2006; Lovich and Ennen 2011). With regard to golden eagle, large-scale habitat loss/modification could result in "disturbance" (as defined by the BGEPA) to breeding, feeding, or sheltering behavior to specific individuals/territories.

Early controversy with respect to impacts to avian species from wind-energy projects stemmed largely from post-construction surveys in the early 1990s at the Altamont Pass Wind Resource Area (APWRA) in California, which documented substantial golden eagle mortality (40-70 eagles per year), especially among sub-adult and non-breeding "floating" adults (Fielding et al. 2006; Whitfield et al. 2008). There are some key findings from the Altamont Pass case study with regard to project design: mortality was greater in areas with older 'type-13' turbines and where turbines were situated in canyons, on ridge tops, or in areas with abundant prey (Hunt 2002; Thelander and Smallwood 2007). The APWRA contains over 5,000 older and smaller turbines and high raptor use relative to other wind energy developments where fatality studies have been conducted (National Academy of Sciences 2007). Studies at other western United States sites have not recorded comparable levels of golden eagle mortality - with the exception of Pine Tree Wind Farm in the Tehachapi Mountains in California ${ }^{1}$ - which may reflect improved siting and new technologies with turbine design in relation to eagle activity (Young and Poulton 2004). The Foote Creek Rim wind project in Wyoming is estimated to have golden eagle use similar to APWRA, and as of 2002, it had the highest estimated mortality for golden eagles using new technology turbines (except for Pine Tree), with an estimated 0.3 golden eagle fatality $/ 100,000 \mathrm{~m}^{2}$ rotor-swept area (RSA), or approximately 3 to 7 times lower than old technology used at APWRA (Erikson et al. 2002).

Despite the focus on wind development impacts to raptors - because raptors are slow to recover from anthropogenic impacts as a result of their long lifespan, long time required to reach sexual maturity, and low reproductive rate relative to other bird species - songbird collisions account for roughly $75 \%$ of bird casualties at U.S. wind facilities (Erickson et al. 2001; Johnson et al. 2002). Migrating songbirds appear to be especially vulnerable during poor weather conditions that force them to lower altitudes and where wind facilities are situated in close proximity to stopover sites (Erickson et al. 2001; Johnson et al. 2002).

The HREDP proposed wind-energy development would be a new-generation development, which uses fewer, larger, slower-moving turbines, tubular towers, smooth nacelle surfaces, and underground electrical collection systems, which all contribute to reduced avian mortality levels, compared with older regeneration facilities (Erickson et al. 2002; National Academy of Sciences 2007). Newer turbine and tower designs eliminate potential perch sites for raptors, and the larger rotors turns at slower speeds that may be more visible to birds (Erickson et al. 2002).

\footnotetext{
${ }^{1}$ The Pine Tree Wind Farm appears to have been poorly sited in an area of southern California in which concentrations of subadult golden eagles winter; USFWS is currently investigating eagle mortalities at this location (Sahagun 2012).
} 


\subsection{Pre-construction Avian Assessment Summary and Recommendations}

As related to the HREDP, in this report we presented:

- Listed and special-status avian species occurrence

- California condor (Gymnogyps californianus) occurrence

- Avian migration corridors and flyways

- Raptor nesting habitat

- Raptor nest inventory survey results

Three special-status species have a high potential to occur in the proposed project areas: American peregrine falcon, golden eagle, and ferruginous hawk. Golden eagle and ferruginous hawk individuals were observed during the aerial raptor nest inventory survey, and nests for these species were recorded. Western burrowing owl was identified as having a moderate potential for occurrence. No avian species protected by the ESA are likely to occur, and no critical habitat is present within the proposed project.

The project is within an experimental California condor population, which is established as a "nonessential experimental population" under Section 10(j) of the ESA. Telemetry data obtained from the Peregrine Fund show that telemetered individuals use habitat within the Grand Canyon and point northwest into Utah. It is possible that individuals could use the project areas in the future, as condors are wide-ranging and the species has the potential to expand with recovery efforts continuing.

With the exception of the Nelson Solar project area, the project is situated along a north-south-trending ridgeline - the Grand Wash Cliffs - that could concentrate migrating raptors, including eagles. The Nelson Solar project area contains an extensive prairie dog colony that could similarly attract migrating (and breeding) raptors. There are no specific characteristics of the project that would concentrate migrating songbirds, shorebirds, wading birds, or other avian species.

We delineated golden eagle nesting habitat prior to conducting an aerial raptor nest inventory survey. Eagle nesting habitat was considered to be analogous to nesting habitat for most other local nesting raptor species. The aerial inventory raptor nest survey was habitat driven, rather than transect driven, focusing on identification of golden eagle nest structures; other raptor species nests were identified. We located 93 unique nest structures within 3 miles of the project areas and 2 additional nest structures just outside (on the border of) the 3-mile-radius survey areas. Eleven golden eagle nests and 19 possible golden eagle nests were identified.

We recommend early consultation with AGFD, USFWS, and BLM regarding specific species concerns and additional pre-construction avian species surveys (e.g., raptor migration surveys) that are warranted to further inform project infrastructure design, as well as BBCS/ECS and National Environmental Policy Act considerations.

Pre-construction evaluation can reduce environmental project impacts. Although there is not currently a strong linkage between pre-construction avian use assessments and post-construction fatalities (Ferrer et al. 2011), wildlife agency consultation can identify types of pre-construction data to collect and the duration and intensity of the pre-construction studies, streamlining permitting. Additional surveys that would supplement this initial pre-construction avian assessment include raptor migration surveys; avian year-round use counts; and aerial raptor nest inventory surveys for all raptor nesting habitat within a 10 -mile radius of proposed project areas. It is likely that a BBCS/ECS will need to be prepared for the HREDP that includes all practical up-front avoidance and minimization measures and adaptive management, which may include both compensatory and operational mitigation. 


\subsection{LITERATURE CITED}

Arizona Game and Fish Department (AGFD). 2012a. Heritage Data Management System — special status species by county, taxon, scientific name. Available at: http://www.azgfd.gov/w_c/edits/ hdms_abstracts.shtml. Accessed May 30, 2012.

2012b. Heritage Data Management System — special status species by county, taxon, scientific name. Available at: http://www.azgfd.gov/w_c/edits/documents/allspecies_bycounty_005.pdf. Accessed May 2012.

Boeker, E.L., and T.D. Ray. 1971. Golden eagle population studies in the southwest. Condor 73:463-467.

Drewitt, A.L., and R.H.W. Langston. 2006. Assessing the impacts of wind farms on birds. Ibis 148: $29-42$.

Driscoll, D.E. 2010. Protocol for Golden Eagle Occupancy, Reproduction, and Prey Population Assessment. Apache Junction, Arizona: American Eagle Research Institute.

Erickson, W.P., G. Johnson, M.D. Strickland, D.P. Young, Jr., K.J. Sernka, and R.E. Good. 2001. Avian Collisions with Wind Turbines: A Summary of Existing Studies and Comparisons to Other Sources of Avian Collision Mortality in the United States. National Wind Coordinating Committee Resource Document. Cheyenne, Wyoming: Western EcoSystems Technology, Inc.

Erickson, W.P., G. Johnson, M.D. Strickland, D.P. Young, Jr., K.J. Sernka, M. Bourassa, K. Bay, and R.E. Good. 2002. Synthesis and Comparison of Baseline Avian and Bat Use, Raptor Nesting and Mortality Information from Proposed and Existing Wind Developments. Prepared for Bonneville Power Administration. Cheyenne, Wyoming: Western Ecosystems Technology, Inc.

Federal Register. 2009. Eagle permits; take necessary to protect interests in particular localities. Federal Register 74(175):46836-46879.

Ferrer, M., M. de Lucas, G.F.E. Janss, E. Casado, A.R. Muñoz, M.J. Bechard, and C.P. Calabuig. 2011. Weak relationship between risk assessment studies and recorded mortality in wind farms. Journal of Applied Ecology.

Fielding, A.H., D.P. Whitfield, and D.R.A. McCleod. 2006. Spatial association as an indicator of the potential for future interactions between wind energy developments and golden eagles Aquila chrysaetos in Scotland. Biological Conservation 131:359-369.

Herbst, D.B. 2006. Salinity controls on trophic interactions among invertebrates and algae of solar evaporation ponds in the Mohave Desert and relation to shorebird foraging and selenium risk. Wetlands 26:475-485.

Hunt, W.G. 2002. Golden Eagles in a Perilous Landscape: Predicting the Effects of Mitigation for Wind Turbine Blade-strike Mortality. University of California, Santa Cruz. Contract No. 500-97-4033. Prepared for PIER - Environmental Area.

Hunt, W.G., C.N. Parrish, S.C. Farry, T.G. Lord, and R. Seig. 2007. Movements of introduced California Condors in Arizona in relation to lead exposure. In California Condors in the 21st Century. Series in Ornithology, No. 2. 
Institute for Bird Populations. 2012. English and Scientific Alpha Codes for North American Birds through the 52nd AOU Supplement (2011). Available at:

http://www.birdpop.org/AlphaCodes.htm. Accessed July 2012.

Johnson, G.D., W.P. Erickson, M.D. Strickland, M.F. Sheperd, D.A. Sheperd, and S.A. Sarappo. 2002. Collision mortality of local and migrant birds at a large-scale wind-power development on Buffalo Ridge, Minnesota. Wildlife Society Bulletin 30:879-887.

Kevlesky, W.P., Jr., L.A. Brennan, M.L. Morrison, K.K. Boydston, B.M. Ballard, and F.C. Bryant. 2007. Wind energy development and wildlife conservation: challenges and opportunities. Journal Wildlife Management 71:2487-2498.

Kochert, M.N. 1972. Population status and chemical contamination in golden eagles in southwestern Idaho. M.S. thesis, University of Idaho, Moscow, Idaho.

Kochert, M.N., M.N., K. Steenhof, C.L. Mcintyre, and E.H. Craig. 2002. Golden eagle (Aquila chrysaetos). The Birds of North America Online, edited by A. Poole. Cornell Lab of Ornithology, Ithaca, New York, and American Ornithologists Union. Available at: http//bna.birds.cornell.edu/bna/species/ 684. Accessed July 2011.

Kunz, T.H., E.B. Arnett, W.P. Erickson, A.R. Hoar, G.D. Johnson, R.P. Larkin, M.D. Strickland, R.W. Thresher, and M.D. Tuttle. 2007. Ecological impacts of wind energy development on bats: questions, research needs, and hypotheses. Frontiers in Ecology and the Environment 5:315-324.

Lovich, J.E., and J.R. Ennen. Wildlife conservation and solar energy development in the desert southwest, United States. BioScience 61:982-992.

McGahan, J. 1968. Ecology of the golden eagle. Auk 85:1-12.

National Academy of Sciences. 2007. Environmental Impacts of Wind Energy Projects. Washington, D.C.: National Academies Press.

Pagel, J.E., D.M. Whittington, and G.T. Allen. 2010. Interim Golden Eagle Technical Guidance: Inventory and Monitoring Protocols; and Other Recommendations in Support of Eagle Management and Permit Issuance. Division of Migratory Bird Management, U.S. Fish and Wildlife Service.

Phillips, R.L., T.P. McEneaney, and A.E. Beske. 1984. Population densities of breeding golden eagles in Wyoming. Wildlife Society Bulletin 12:269-273.

Postupalsky, S. 1974. Raptor reproductive success: some problems with methods, criteria, and terminology. In Raptor Research Report No. 2: Management of Raptors, edited by F.N. Hamerstrom, Jr., B.E. Harrell, and R.R. Olendorff. pp. 21-31. Vermillion, South Dakota: Raptor Research Foundation.

Sahagun, L. 2012. U.S. probes golden eagles' deaths at DWP wind farm. Los Angeles Times article, February 16, 2012.

Southwest Condor Review Team. 2007. A Review of the Second Five Years of the California Condor Reintroduction Program in the Southwest. Prepared for the California Condor Recovery Team and U.S. Fish and Wildlife Service, California/Nevada Operations Office, Sacramento, California. 
Steenhof, K., and I. Newton. 2007. Assessing nest success and productivity. In Raptor Research and Management Techniques, edited by D.M. Bird and K.L. Bildstein, pp. 181-191. B.C. Canada: Hancock House Publishers, Raptor Research Foundation.

SWCA Environmental Consultants (SWCA). 2012. Critical Issues Analysis of Renewable Energy Development Projects. Report prepared for Hualapai Tribal Nation Planning Department. Las Vegas, Nevada: SWCA.

Thelander, C.G., and K.S. Smallwood. 2007. The Altamont Pass Wind Resource Area's effects on birds: a case history. In Birds and Wind Farms: Risk Assessment and Mitigation, edited by M. De Lucas, G.F.E. Janss, and M. Ferrer, pp. 25-46. Quercus, Madrid.

U.S. Fish and Wildlife Service (USFWS). 2011. Draft Eagle Conservation Plan Guidance. January 2011. Available at: http://www.fws.gov/windenergy/docs/ECP_draft_guidance_2_10_final_ clean_omb.pdf. Accessed July 2012.

U.S. Geological Survey (USGS). 2004. Provisional Digital Land Cover Map for the Southwestern United States. Version 1.0. RS/GIS Laboratory, College of Natural Resources, Utah State University.

Watson, J. 2010. The Golden Eagle. 2nd ed. New Haven, Connecticut: Yale University Press.

Whitfield, D.P., A.H. Fielding, D.R.A. McCleod, and P.F. Haworth. 2008. A Conservation Framework for the Golden Eagle: Implications for Conservation and Management in Scotland. Scottish Natural Heritage Commissioned Report No. 193. Battleby: Scottish Natural Heritage.

Young, D.P., and V. Poulton. 2004. Evaluation of Biological Resource Issues Associated with the Sunshine Wind Park, Coconino County, Arizona: Phase I Biological Report. Prepared for Foresight Wind Energy. 



\section{APPENDIX A}

\section{Raptor Nest Data}





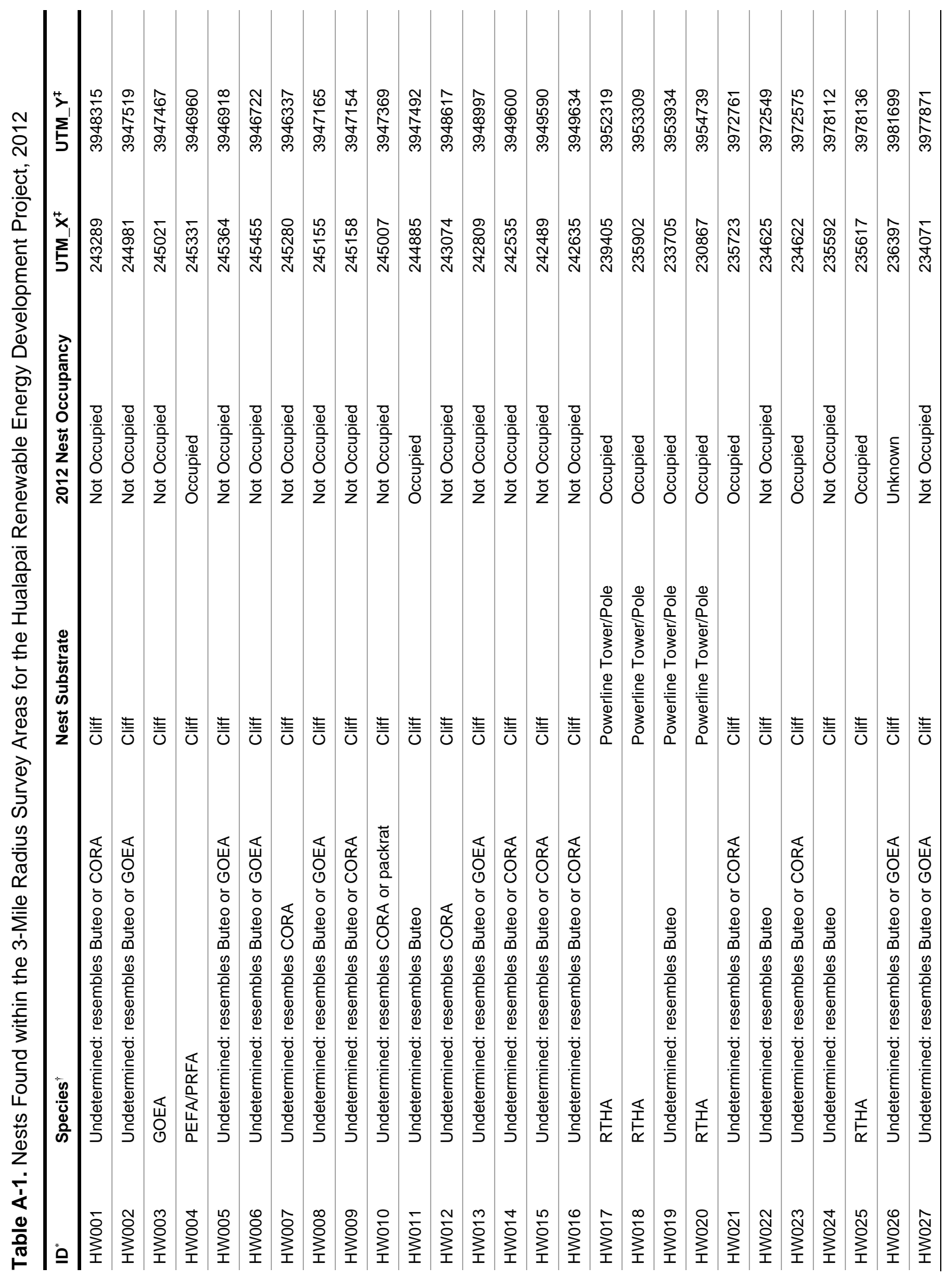




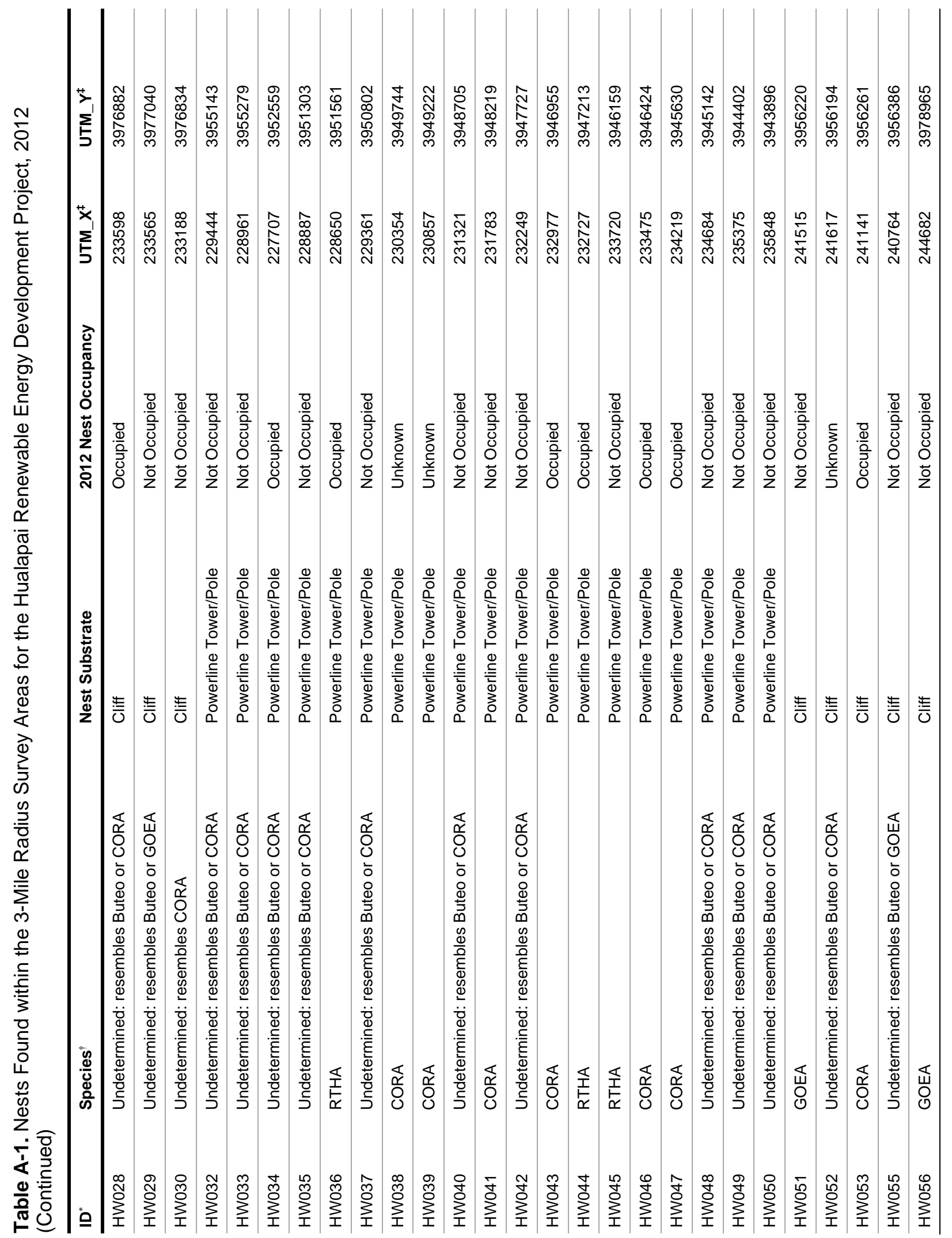




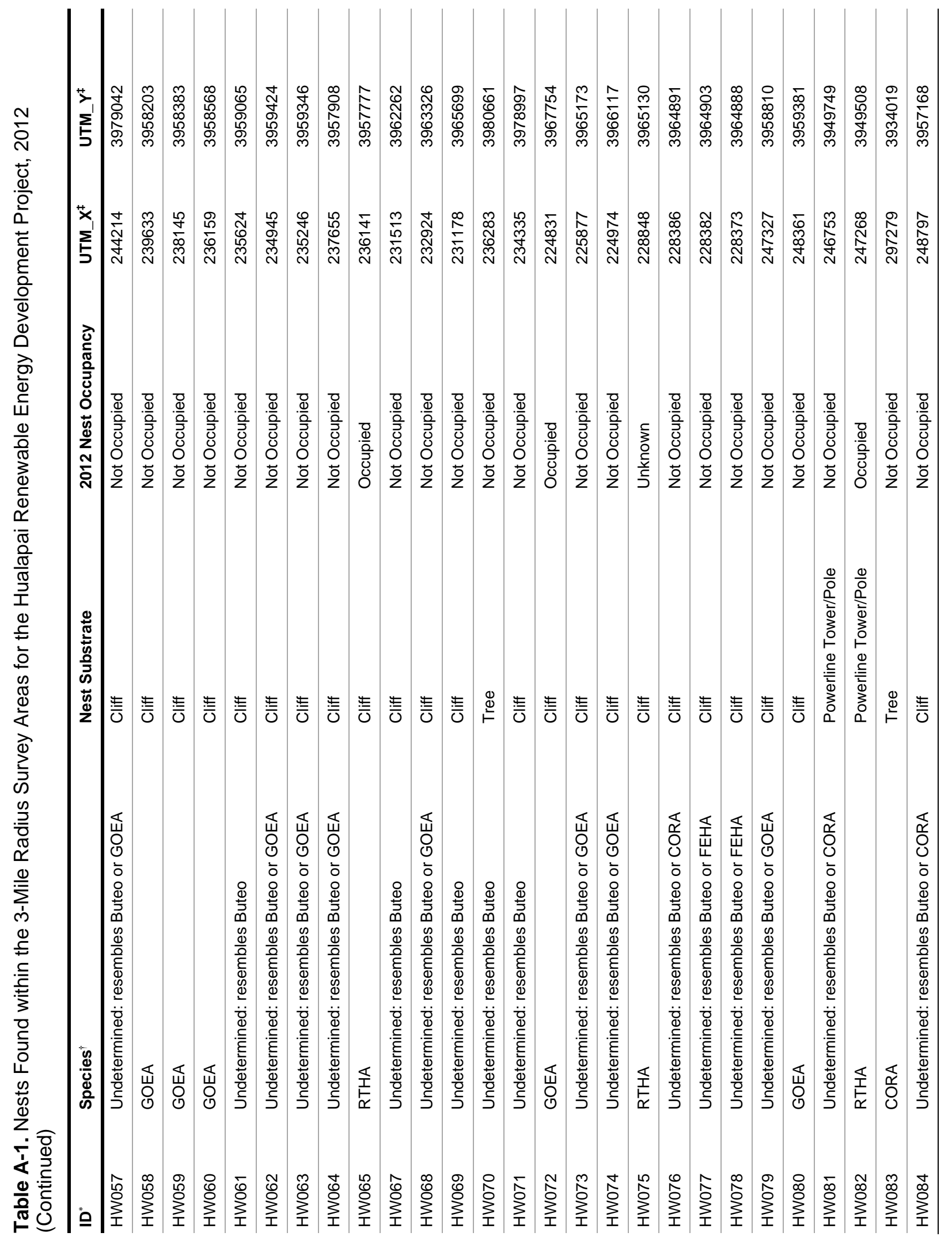




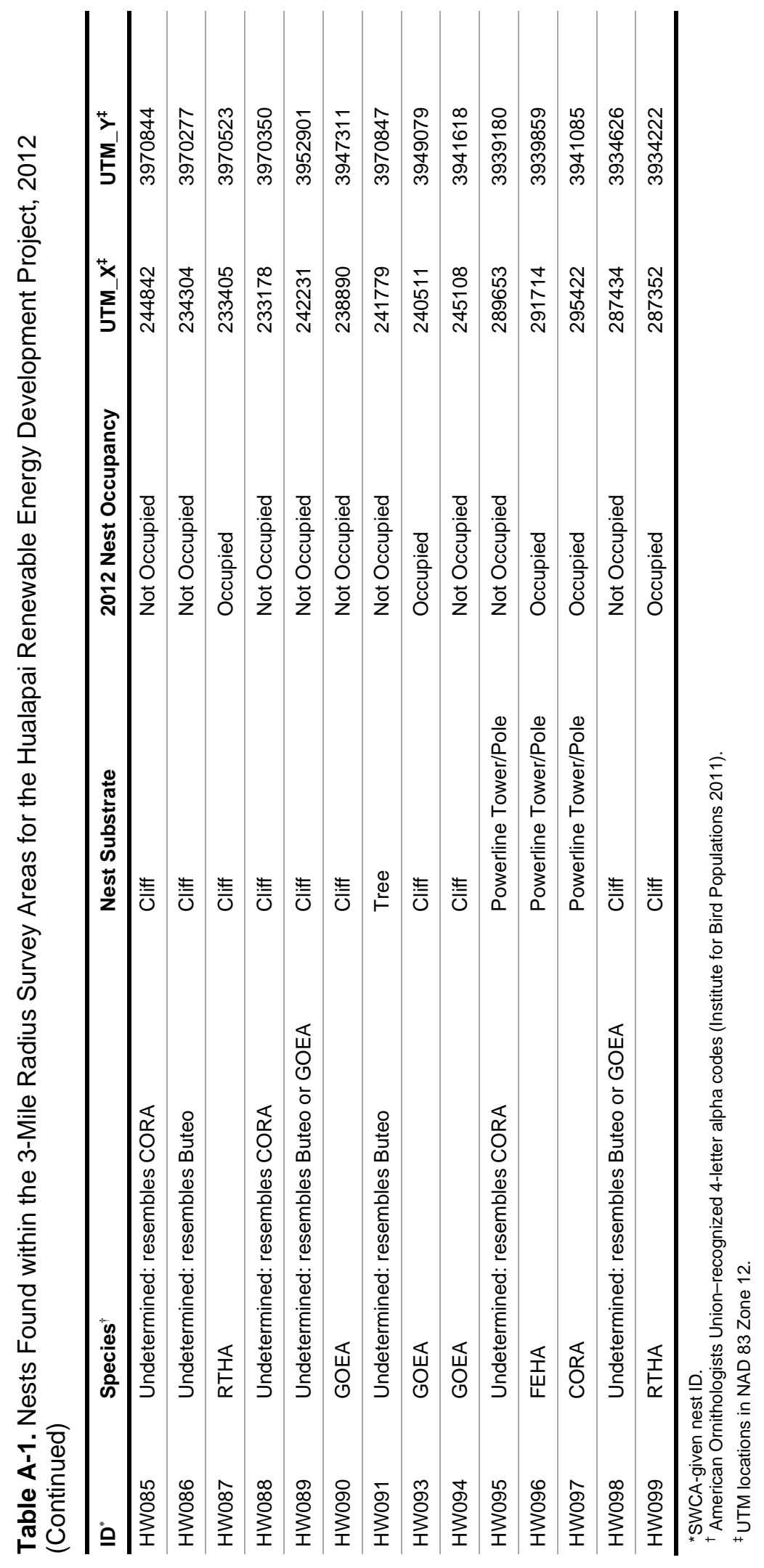




\section{Exhibit J}

Fall Raptor Migration Study

(SWCA Environmental) 
Final Results of 2012 Fall Raptor Migration Study for Potential Wind Energy Development on Hualapai Tribal Lands

Prepared for

Hualapai Tribal Nation Planning Department

Prepared by

SWCA Environmental Consultants

November 2012 


\title{
FINAL RESULTS OF 2012 FALL RAPTOR MIGRATION STUDY FOR POTENTIAL WIND ENERGY DEVELOPMENT ON HUALAPAI TRIBAL LANDS
}

\author{
Prepared for \\ Hualapai Tribal Nation Planning Department \\ P.O. Box 179 \\ 941 Hualapai Way \\ Peach Springs, Arizona 86434
}

\author{
Prepared by \\ SWCA Environmental Consultants \\ 114 North San Francisco Street \\ Flagstaff, Arizona 86001 \\ www.swca.com
}

SWCA Project No. 24482

November 2012 


\section{CONTENTS}

EXECUTIVE SUMMARY iii

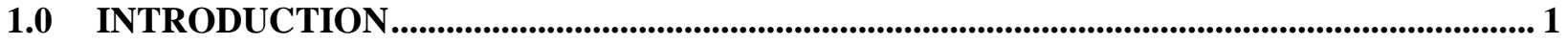

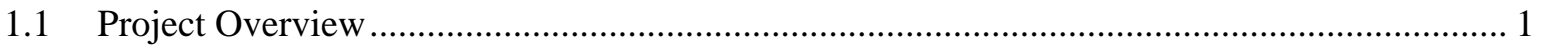

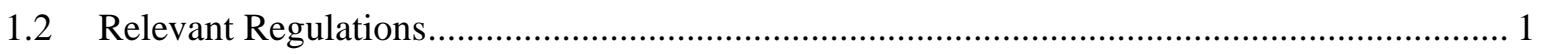

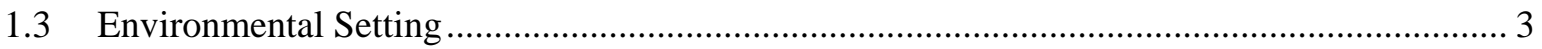

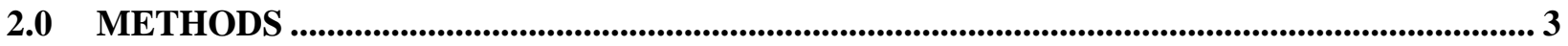

2.1 Literature and Data Search—Raptor Migration .................................................................... 3

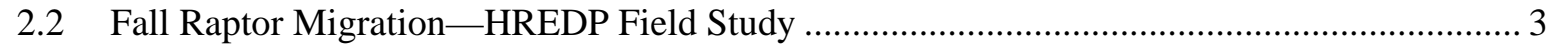

2.3 Fall Migrant Raptor Risk Analyses_HREDP ............................................................. 6

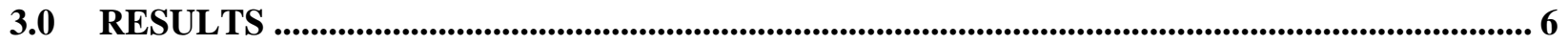

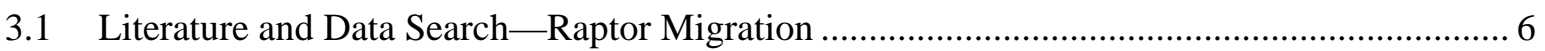

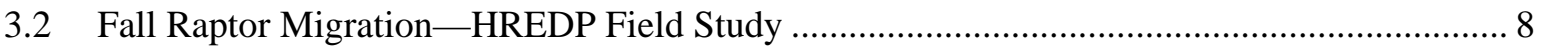

3.3 Fall Migrant Raptor Risk Analyses_-HREDP ................................................................ 10

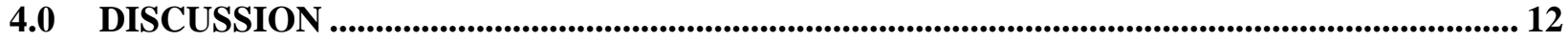

4.1 Literature and Data Search—Raptor Migration ................................................................ 12

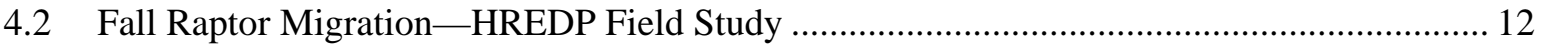

4.3 Fall Migrant Raptor Risk Analyses_-HREDP ............................................................... 13

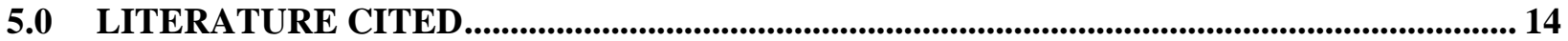

\section{Figures}

1. HREDP study areas and location of observation points used for conducting 2012 fall raptor

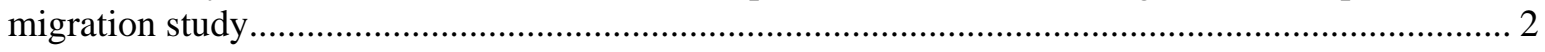

2. Viewshed of the Grand Point observation point used for conducting 2012 fall raptor migration study; view facing north.

3. Viewshed of the East observation point used for conducting 2012 fall raptor migration study; view facing north.

4. Viewshed of the MET observation point used for conducting 2012 fall raptor migration study; view facing north.....

5. Number of raptor observations recorded by flight height category, HREDP, 25 September-4 October 2012.

6. Risk indices for species recorded within the RSA during fall raptor migration studies, HREDP, 25 September-4 October 2012. 


\section{Tables}

1. Fall Raptor Migration Count Data from Yaki Point at South Rim, Grand Canyon National Park,

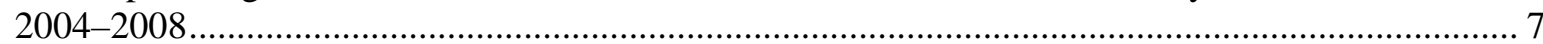

2. Fall Raptor Migration Count Data from Yaki and Lipan Points at South Rim, Grand Canyon National Park, 25 September through 4 October 2008

3. Fall Raptor Migration Count Data from the HREDP, 25 September-4 October 2012 ..................... 9

4. Observations and Risk Indices of Fall Migrating Raptors Recorded within the RSA, HREDP, 25 September-4 October 2012. 


\section{EXECUTIVE SUMMARY}

The Hualapai Tribal Nation's Planning Department retained SWCA Environmental Consultants (SWCA) to conduct a fall raptor migration study to assess risk to migrant raptors in relation to potential renewable energy development on Hualapai Tribal lands in Mohave and Coconino Counties, Arizona. The project areas (six total: four wind and two solar) are known collectively in this report as the Hualapai Renewable Energy Development Project. The entire Hualapai Renewable Energy Development Project encompasses approximately 42,436 acres. The main objective of the study was to determine the degree to which migrating raptors use the area designated for potential wind energy development (four project areas) and to identify the potential for any significant risks to migrating raptors.

To evaluate use of the four project areas by migrating raptors, SWCA first conducted a literature and data search and initiated communications with HawkWatch International (HWI), which has been conducting raptor migration studies in Grand Canyon since 1997. All pertinent Grand Canyon HWI data were summarized, and data collected during this study were compared with those of HWI to in order to ascertain the degree to which migrating raptors use the project areas.

SWCA conducted a field reconnaissance and designated three observation points (stations) where field counts of migrating raptors were conducted. Fall raptor migration studies were conducted for a 10-field day period. One observer per station conducted field observations simultaneously daily; field personnel consisted of two SWCA avian ecologists and one avian ecologist from the Hualapai Department of Natural Resources. Count days were conducted back to back for 10 consecutive days, with observations starting at 1000 hours for a 6-hour period for each of the 10 days. Migration counts assessed species composition and relative abundance, assessed raptor use within the wind turbine rotor-swept area (RSA), and determined the magnitude (raptors detected per hour) of migrant raptor use in the project area. A risk analysis determined from estimates of exposure was completed by assessing avian use and developing risk metrics for each species. A risk index was completed to determine which species would have a higher risk of collisions with turbine blades.

HWI fall raptor migration count data proved useful in interpreting the magnitude of fall raptor migration in the project areas. HWI annual and date-specific data were summarized, with an annual average of 7.3 raptors detected per hour and a date-specific average of 9.9 raptors detected per hour at the HWI study sites.

A comparison of raptor migration magnitude of the project areas with the HWI data shows that the greater project area is an area that concentrates raptors during fall migration, with a magnitude of moderate to low across the three observation stations (3.58 Grand Point; 1.47 East; 2.02 MET). Although the greater project area is an area that concentrates raptors during fall migration, the core migration flyway appears to be on the western boundaries of the greater project area along the Grand Wash Cliffs proper. Siting wind turbines as far as possible off of the western ridgelines should decrease negative impacts to migrating raptors.

The greater project area is also an area that concentrates turkey vultures during fall migration, with large kettles of migrating individuals recorded. However, the core turkey vulture migration flyway appears to be west of western boundaries of the greater project area along the Hualapai Valley.

Approximately one-fifth of the recorded species were observed in the RSA, with American kestrel, redtailed hawk, Cooper's hawk, and sharp-shinned hawk having the highest Risk Indices. American kestrel, red-tailed hawk, Cooper's hawk, and sharp-shinned hawk were also the most abundant species of raptors recorded during fall raptor migration surveys. Although it is predicted that the project may have some impact on these species, it is not anticipated that construction and operation of the facility alone would be detrimental to these species' long-term persistence. 


\subsection{INTRODUCTION}

\subsection{Project Overview}

The Hualapai Tribal Nation's Planning Department retained SWCA Environmental Consultants (SWCA) in September 2012 to conduct a fall raptor migration study to assess potential risk to migrant raptors in relation to potential renewable energy development on Hualapai Tribal lands in Mohave and Coconino Counties, Arizona. The project areas (six total: four wind and two solar) are known collectively in this report as the Hualapai Renewable Energy Development Project (HREDP) (Figure 1). The entire HREDP encompasses approximately 42,436 acres. The main objective of the study was to determine the degree to which migrating raptors use the project areas designated for potential wind energy development (four project areas) and to identify the potential for any significant risks to migrating raptors.

\subsection{Relevant Regulations}

The regulatory framework for protecting birds includes the Endangered Species Act (ESA) of 1973, as amended, the Migratory Bird Treaty Act (MBTA) of 1918, as amended, the Bald and Golden Eagle Protection Act (BGEPA) of 1940, as amended, and Executive Order 13186.

All migratory birds are covered under the MBTA, while the BGEPA specifically protects golden and bald eagles. The BGEPA prohibits anyone without a permit from "taking" golden and bald eagles and their parts, eggs, or nests. "Take" is defined by the BGEPA as "to pursue, shoot, shoot at, poison, wound, kill, capture, trap, collect, molest, or disturb;" it differs from the ESA in that it does not include habitat destruction or alteration, unless such damage "disturbs" an eagle. "Disturb" is defined as "to agitate or bother to a degree that causes, or is likely to cause, based on the best scientific information available, 1) injury to an eagle, 2) a decrease in its productivity, by substantially interfering with normal breeding, feeding, or sheltering behavior, or 3) nest abandonment, by substantially interfering with normal breeding, feeding, or sheltering behavior.” The MBTA prohibits incidental "take” of migratory birdsmore than 1,000 species (Federal Register; 50 Code of Federal Regulations [CFR] 10 and 21), including golden and bald eagle and their parts, eggs, or nests "at any time, by any means." "Take” is defined by the MBTA as "to pursue, hunt, shoot, wound, kill, trap, capture, or collect, or any attempt to carry out these activities." A "take” does not include habitat destruction or alteration, as long as it does not involve a known direct taking of birds, nests, or eggs.

On September 11, 2009 (Federal Register 2009; 50 CFR 13 and 22), the U.S. Fish and Wildlife Service (USFWS) set in place rules establishing two new permit types under the BGEPA: 1) take of golden and bald eagles that is associated with, but not the purpose of, the activity; and 2) purposeful take of an active or inactive nest where necessary to alleviate a safety emergency; an inactive eagle nest when the removal is necessary to ensure public health and safety; an inactive nest that is built on a human-engineered structure and creates a functional hazard that renders the structure inoperable for its intended use; or an inactive nest, provided the take is necessary to protect an interest in a particular locality and the activity necessitating the take or the mitigation for the take will, with reasonable certainty, provide a clear and substantial benefit to eagles. USFWS received the first application from a proposed wind project for a take permit under BGEPA earlier this year (2012). USFWS recommends wind energy project proponents prepare a Bird and Bat Conservation Strategy and an Eagle Conservation Strategy, in collaboration with USFWS, to avoid, minimize, and otherwise mitigate project-related impacts to birds and bats, and specifically to bald and golden eagles to ensure no net loss to eagle populations. The proposed HREDP is subject to all relevant federal, state, and local statutes, regulations, and plans. 


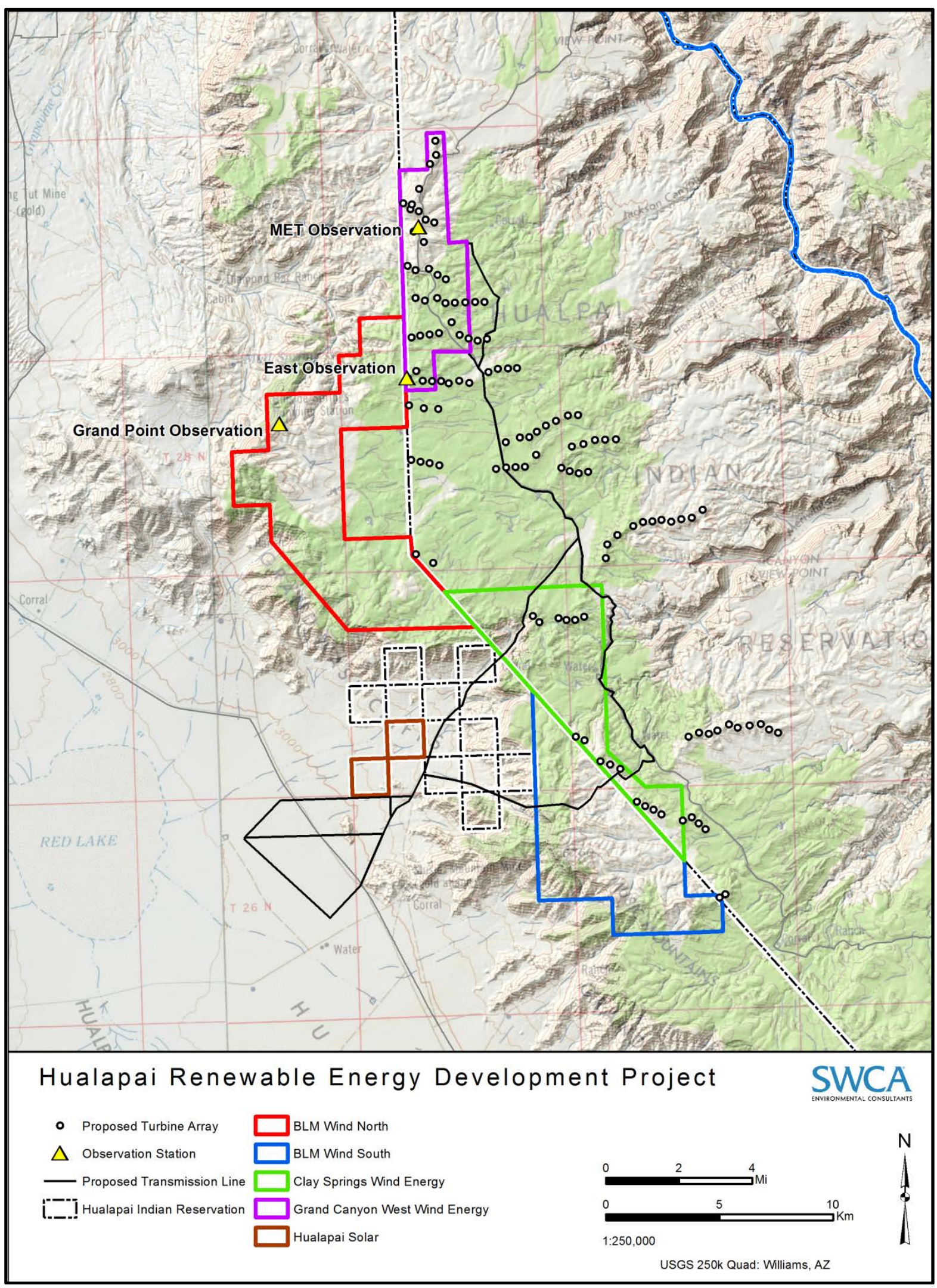

Figure 1. HREDP study areas and location of observation points used for conducting 2012 fall raptor migration study. 


\subsection{Environmental Setting}

The HREDP area ranges from approximately 2,700 to 6,400 feet above mean sea level and is generally bounded by Quartermaster Canyon to the northeast, West Water Canyon to the southeast, Red Lake to the southwest, and Garnet Mountain to the west; the Grand Wash Cliffs are situated along the western edge of the wind development project areas. The most prominent land features of the HREDP are the Grand Wash Cliffs and Music Mountains, which comprise prominent north-south-trending ridgelines, cliff faces, headwalls, and canyons.

Vegetation within the HREDP is dominated by two vegetation communities: Colorado Plateau PinyonJuniper Woodland and Mohave Mid-Elevation Mixed Desert Scrub, with lesser amounts of ApacherianChihuahuan Mesquite Upland Scrub, Colorado Plateau Blackbrush-Mormon-tea Shrubland, Colorado Plateau Mixed Bedrock Canyon and Tableland, Great Basin Conifer Woodland, Inter-Mountain Basins Big Sagebrush Shrubland, Inter-Mountain Basins Juniper Savanna, Inter-Mountain Basins Mixed Salt Desert Scrub, Inter-Mountain Basins Semi-Desert Grassland, Inter-Mountain Basins Semi-Desert Shrub Steppe, Mogollon Chaparral, Sonora-Mohave Creosotebush-White Bursage Desert Scrub, and Sonoran Mid-Elevation Desert Scrub (U.S. Geological Survey [USGS] 2004). Vegetation within the Nelson survey area is dominated by Colorado Plateau Pinyon-Juniper Woodland, Inter-Mountain Basins SemiDesert Grassland, and Inter-Mountain Basins Semi-Desert Shrub Steppe, with lesser amounts of Colorado Plateau Mixed Bedrock Canyon and Tableland, Inter-Mountain Basins Big Sagebrush Shrubland, InterMountain Basins Salt Desert Scrub, and Invasive Annual and Biennial Forbland (USGS 2004). These vegetation community descriptions and composition by project area are detailed in SWCA (2012).

\subsection{METHODS}

\subsection{Literature and Data Search—Raptor Migration}

To evaluate use of the HREDP area by migrating raptors, SWCA first conducted a literature and data search and initiated communications with HawkWatch International (HWI), which has been conducting raptor migration studies in Grand Canyon since 1997. The HWI Grand Canyon study location lies approximately 100 miles east of the HREDP study area. All pertinent Grand Canyon HWI data were summarized, and data collected during this study were compared with those of HWI to in order to ascertain the degree to which migrating raptors use the four project areas.

\subsection{Fall Raptor Migration-HREDP Field Study}

We first conducted a 2-day field reconnaissance of the greater project area to locate three observation points (stations) where field counts of migrating raptors were conducted. The three observation points/stations were located essentially on an east-west axis within the greater project area and offered wide fields of view near topographic features that serve to concentrate migrant raptors (Figures 2-4; see Figure 1). Observation points were also spaced sufficiently far apart to detect all migrant raptors crossing an "east-west axis count boundary." Although the MET observation point was located north/northeast of the East observation station (see Figure 1), it was determined necessary, as this area contains prominent land features that potentially can concentrate migrating raptors (prominent north-south-trending ridgelines, cliff faces, headwalls, and canyons; see Figure 4) within the area deemed suitable for wind energy development. 


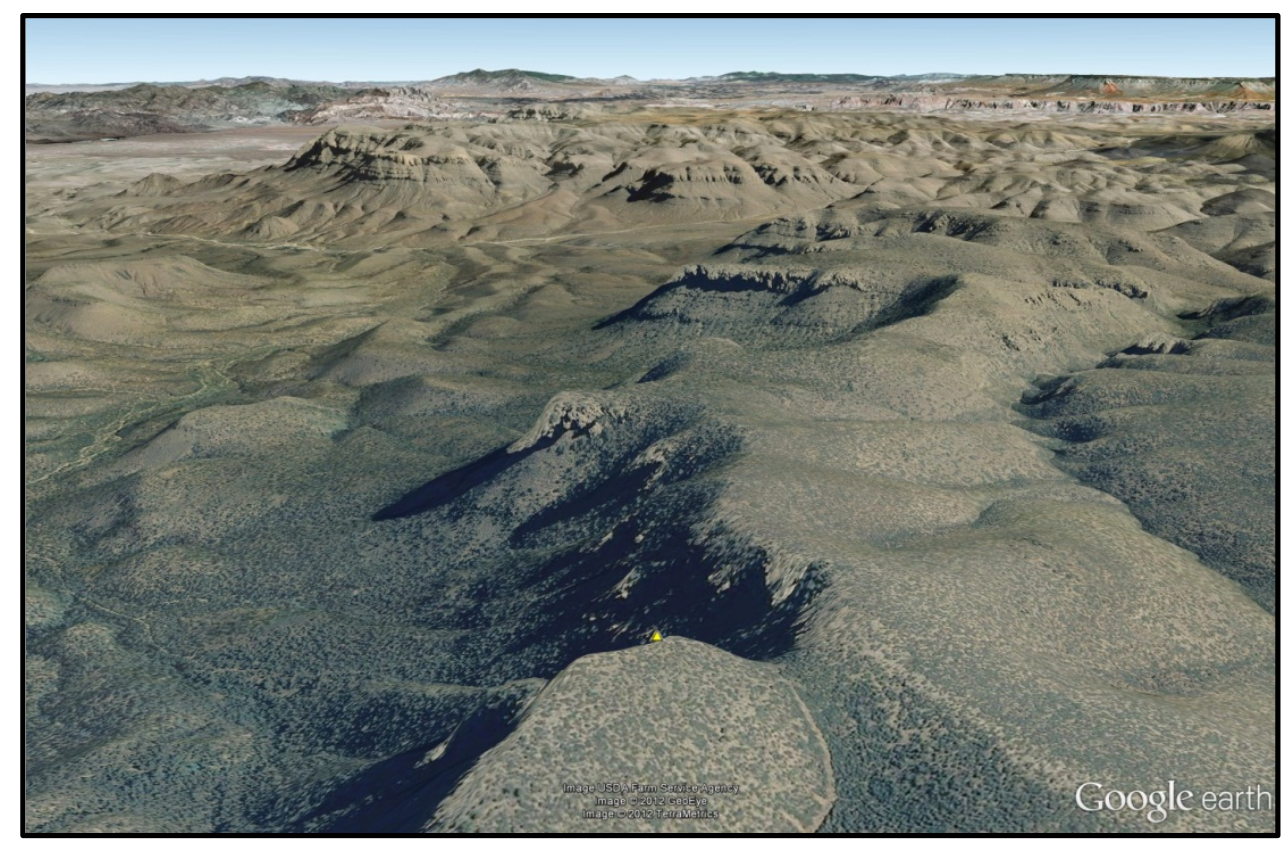

Figure 2. Viewshed of the Grand Point observation point used for conducting 2012 fall raptor migration study; view facing north.

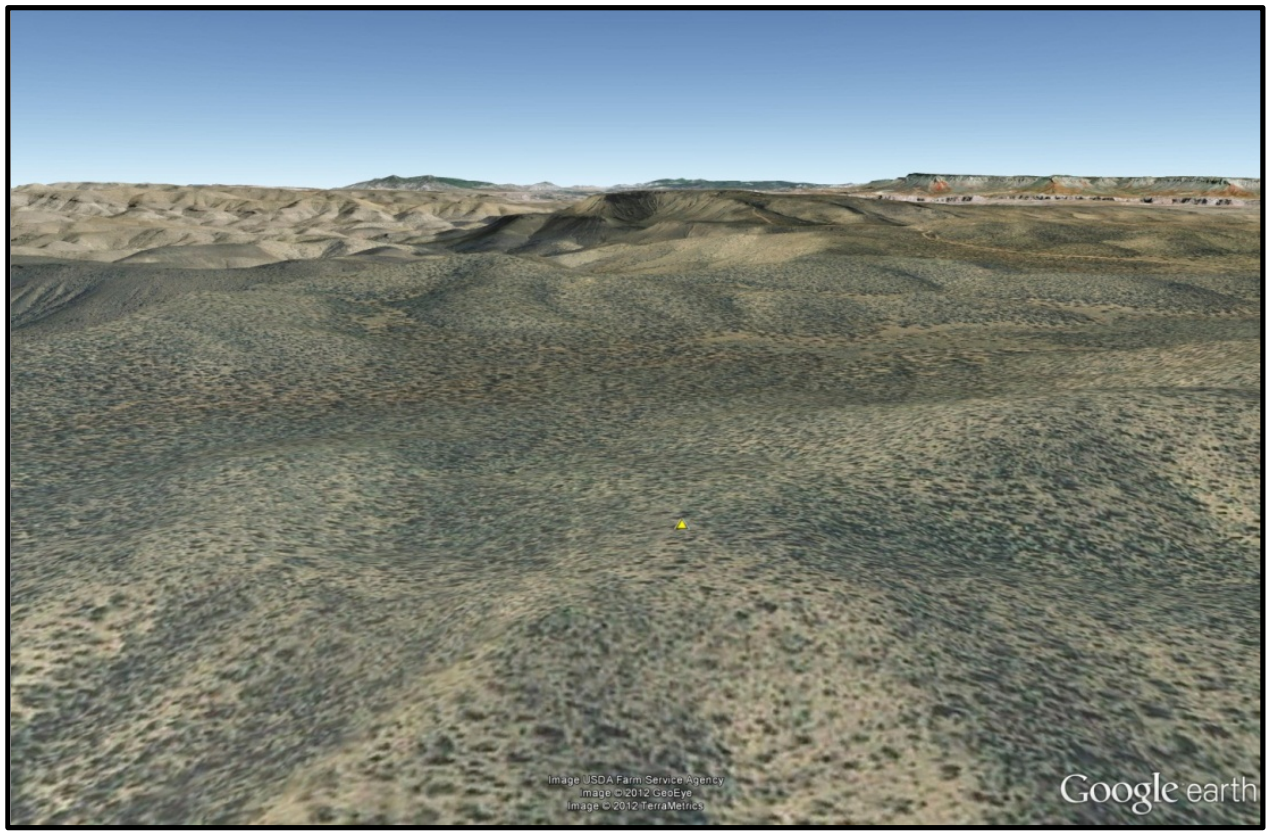

Figure 3. Viewshed of the East observation point used for conducting 2012 fall raptor migration study; view facing north. 


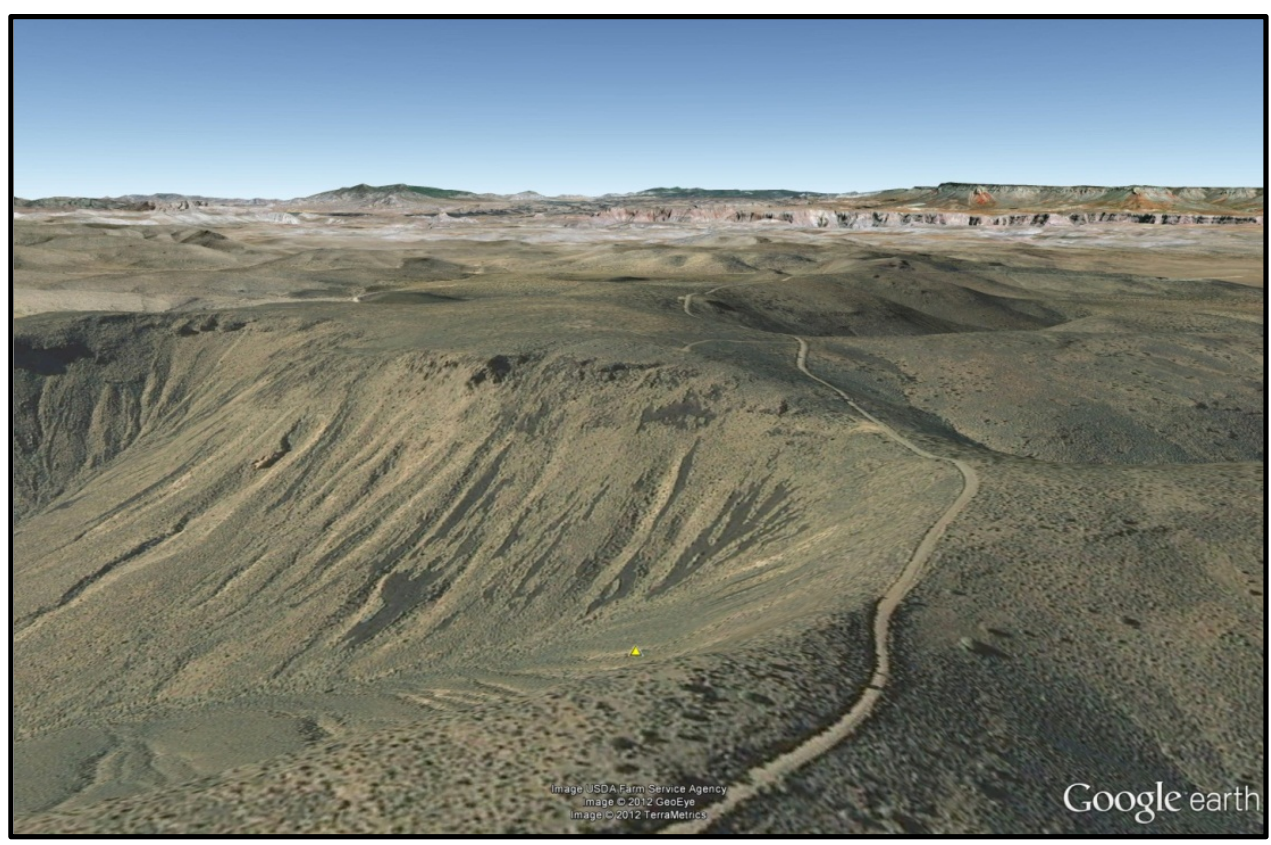

Figure 4. Viewshed of the MET observation point used for conducting 2012 fall raptor migration study; view facing north.

Although current Arizona Game and Fish Department (AGFD) draft guidelines for wind energy development recommend preconstruction raptor migration surveys to be conducted for 2 days per week from mid-September to the end of October (6 weeks), in recent communication (July 25, 2012) with SWCA, Kenneth Jacobson, AGFD Eagle Management Coordinator, stated the AGFD guidelines will be changing. Therefore, fall raptor migration studies were conducted for a 10-field day period from 20 September to 5 October. One observer per station (for a total of three observers), conducted field observations simultaneously daily; field personnel consisted of two SWCA avian ecologists and one avian ecologist from the Hualapai Department of Natural Resources. Count days were conducted back to back for 10 consecutive days, with observations starting at 1000 hours for a 6-hour period for each of the 10 days. Migration counts assessed species composition and relative abundance, assessed raptor use within the wind turbine rotor-swept area (RSA), and determined the magnitude (number of raptors counted per hour) of migrant raptor use in the four project areas. Data recorded included the following:

- Time

- Species

- Number of individuals by species

- Behavior

- Flight direction

- Estimated flight height

Weather and environmental data recorded during each count included the following:

- Temperature

- Wind speed and direction

- Cloud cover

- Precipitation 
Field methods, metrics, and analyses are based largely on those used by HWI to allow results to be compared with existing data from HWI. HWI has been conducting fall migration counts on the South Rim of Grand Canyon for over 10 years, and results from these studies were compared with those obtained from the HREDP. In order to compare project site data to HWI data, turkey vulture (Cathartes aura) was excluded from fall migrant raptor species assemblage data but was included in the flight height and risk index (RI) analysis.

As the East observation point was located due south/southwest of the MET observation point, the possibility of double counting migrating raptors and/or turkey vultures at the East station had to be taken into consideration. Therefore, species detection and temporal data were compared to ascertain whether migrating birds recorded at the MET observation point were double counted at the East observation point.

\subsection{Fall Migrant Raptor Risk Analyses-HREDP}

A risk analysis determined from estimates of exposure was completed by assessing avian use and developing risk metrics for each species. For consistency in comparing use within the project areas, bird use was recorded as the number of instances a bird species was recorded within the 6-hour observation period. These metrics were used to analyze bird use within core wind turbine areas, at some height above ground, and within the RSA. Flight heights and distance from observer were recorded to analyze potential risk of collisions with the anticipated wind turbines to be used at this facility.

Flight heights of birds are often used to assess risk when analyzing potential impacts at wind energy facilities. Birds flying within the RSA are considered to be at high risk because of potential collisions with turbine blades. For this facility, the RSA is anticipated to be between approximately 41 and $121 \mathrm{~m}$ above ground level. An RI was calculated by multiplying the percentage of instances in the RSA by the frequency of observations. Percentage of observations in the RSA alone tends to give higher RIs to species that were observed on only a few occasions. Therefore, the frequency of observation, or percentage of surveys during which a species was observed, also factors into its RI. Using these two factors, the following equation was used to determine the RI of bird species:

$$
\mathrm{RI}=(\text { Frequency of Observations } \times \% \text { Observations in RSA }) / 100
$$

As shown in the equation above, the product of these two factors was divided by 100 to place the estimate between 0 and 100 for ease of interpretation.

This RI establishes which species would have a higher risk of collisions with turbine blades. RIs derived from the formula assess a particular species' potential risk of collision with a turbine blade based on observed flight height, behavior, and the frequency with which the species was observed in the project area. This RI establishes which species would have a higher risk of collisions with turbine blades relative to other birds recorded.

\subsection{RESULTS}

\subsection{Literature and Data Search-Raptor Migration}

HWI fall raptor migration count data (excluding turkey vultures) were obtained from studies conducted at South Rim, Grand Canyon National Park, which lies approximately 100 miles east of the survey area. The first data set summarizes annual fall (approximately 1 Sept through 5 Nov) raptor migration data from 2004 to 2008 (Table 1). Results show that, on average, at one of two observation stations, 4,014 raptors were counted annually during an approximately 2.5-month-long sampling period from 2004 to 2008. From 2004 to 2008, respectively, HWI recorded 9.32, 5.56. 7.71, 7.48, and 6.45 raptors detected 
per hour at the Yaki observation point. This equates to an annual average of 7.3 raptors detected per hour from 2004 to 2005. Given the large number of individuals recorded annually across years for all raptor groups except for golden eagle, this area is a major fall raptor migration corridor (Smith and Neal 2009).

The second HWI data set from 2008 summarizes raptors detected per hour at two observation stations (Yaki and Lipan) across the same 10-day sampling period conducted at the HREDP (25 September through 4 October). HWI results show that, on average, 8.3 and 9.7 raptors per hour were recorded at Yaki and Lipan, respectively (Table 2). It is important to note that, on average, 7.3 to 7.6 hours of sampling per day were conducted at Yaki and Lipan, respectively, whereas for the HREDP study, only 6 hours per day were sampled at each observation station.

Table 1. Fall Raptor Migration Count Data from Yaki Point at South Rim, Grand Canyon National Park, 2004-2008

\begin{tabular}{|c|c|c|c|c|c|}
\hline Year & 2004 & 2005 & 2006 & 2007 & 2008 \\
\hline Start date & 27 Aug & 27 Aug & 27 Aug & 27 Aug & 1 Sep \\
\hline End date & $5 \mathrm{Nov}$ & $5 \mathrm{Nov}$ & $4 \mathrm{Nov}$ & $5 \mathrm{Nov}$ & $5 \mathrm{Nov}$ \\
\hline Days of observation & 68 & 70 & 70 & 71 & 66 \\
\hline Hours of observation & 559.40 & 570.48 & 533.33 & 566.76 & 514.09 \\
\hline Raptors / hour & 9.32 & 5.56 & 7.71 & 7.50 & 6.45 \\
\hline Species & \multicolumn{5}{|c|}{ Raptor Counts } \\
\hline Year & 2004 & 2005 & 2006 & 2007 & 2008 \\
\hline Osprey & 42 & 31 & 37 & 29 & 30 \\
\hline Northern harrier & 29 & 38 & 45 & 38 & 35 \\
\hline Sharp-shinned hawk & 1,743 & 1,008 & 1,627 & 1,417 & 1,417 \\
\hline Cooper's hawk & 855 & 516 & 695 & 761 & 417 \\
\hline Northern goshawk & 7 & 2 & 14 & 12 & 18 \\
\hline Unknown small accipiter & 122 & 108 & 118 & 298 & 112 \\
\hline Unknown large accipiter & 1 & 4 & 4 & 79 & 29 \\
\hline Unknown accipiter & 125 & 15 & 14 & 88 & 51 \\
\hline Total Accipiters, Osprey, Harrier & 2,924 & 1,722 & 2,554 & 2,722 & 2,109 \\
\hline Broad-winged hawk & 19 & 2 & 12 & 18 & 3 \\
\hline Swainson's hawk & 80 & 32 & 30 & 9 & 10 \\
\hline Red-tailed hawk & 1,169 & 765 & 995 & 903 & 641 \\
\hline Ferruginous hawk & 1 & 6 & 6 & 3 & 3 \\
\hline Rough-legged hawk & 0 & 0 & 1 & 0 & 0 \\
\hline Zone-tailed hawk & 0 & 0 & 0 & 0 & 5 \\
\hline Unidentified buteo & 17 & 24 & 48 & 36 & 34 \\
\hline Total Buteos & 1,286 & 829 & 1,092 & 969 & 696 \\
\hline Golden eagle & 4 & 5 & 1 & 5 & 4 \\
\hline Bald eagle & 10 & 22 & 18 & 30 & 20 \\
\hline Unidentified eagle & 0 & 0 & 0 & 3 & 0 \\
\hline Total Eagles & 14 & 27 & 19 & 38 & 24 \\
\hline
\end{tabular}


Table 1. Fall Raptor Migration Count Data from Yaki Point at South Rim, Grand Canyon National Park, 2004-2008 (Continued)

\begin{tabular}{|c|c|c|c|c|c|}
\hline \multirow{2}{*}{$\frac{\text { Species }}{\text { Year }}$} & \multicolumn{5}{|c|}{ Raptor Counts } \\
\hline & 2004 & 2005 & 2006 & 2007 & 2008 \\
\hline American kestrel & 930 & 555 & 384 & 475 & 395 \\
\hline Merlin & 9 & 9 & 9 & 12 & 18 \\
\hline Prairie falcon & 7 & 2 & 9 & 2 & 8 \\
\hline Peregrine falcon & 6 & 11 & 13 & 19 & 12 \\
\hline Unknown small falcon & 0 & 1 & 15 & 1 & 16 \\
\hline Unknown large falcon & 0 & 2 & 10 & 2 & 4 \\
\hline Unknown falcon & 4 & 3 & 2 & 1 & 5 \\
\hline Total Falcons & 956 & 583 & 442 & 512 & 458 \\
\hline Unidentified raptor & 36 & 12 & 6 & 8 & 31 \\
\hline Total & 5,216 & 3,173 & 4,113 & 4,249 & 3,318 \\
\hline
\end{tabular}

Source: Adapted from Smith and Neal (2009).

Table 2. Fall Raptor Migration Count Data from Yaki and Lipan Points at South Rim, Grand Canyon National Park, 25 September through 4 October 2008

\begin{tabular}{|c|c|c|c|c|c|}
\hline \multicolumn{3}{|c|}{ Yaki Point 2008} & \multicolumn{3}{|c|}{ Lipan Point 2008} \\
\hline Date & Obs. Hours & Raptors/Hour & Date & Obs. Hours & Raptors/Hour \\
\hline 25 Sep & 8 & 9.3 & 25 Sep & 8 & 14 \\
\hline 26 Sep & 8.25 & 8.4 & 26 Sep & 8 & 5.4 \\
\hline 27 Sep & 7 & 5 & 27 Sep & 8 & 11.4 \\
\hline 28 Sep & 8 & 7.1 & 28 Sep & 8 & 3.4 \\
\hline 29 Sep & 8 & 5.6 & 29 Sep & 8 & 5.5 \\
\hline 30 Sep & 8 & 12.4 & 30 Sep & 8 & 6.5 \\
\hline 1 Oct & 8 & 26.9 & $1 \mathrm{Oct}$ & 8 & 12 \\
\hline 2 Oct & 8 & 3.1 & $2 \mathrm{Oct}$ & 8.5 & 32.6 \\
\hline 3 Oct & 8 & 2.9 & 3 Oct & 7.83 & 6.4 \\
\hline 4 Oct & 1.67 & 1.8 & 4 Oct & 3.25 & 0 \\
\hline Average & 7.292 & 8.25 & Average & 7.558 & 9.72 \\
\hline
\end{tabular}

Source: Adapted from Smith and Neal (2009).

\subsection{Fall Raptor Migration-HREDP Field Study}

As the East observation point was located due south/southwest of the MET observation point (see Figure 1), the possibility of double counting migrating raptors and/or turkey vultures at the East station had to be taken into consideration. Species detection and temporal data were compared, and results indicated that migrating birds recorded at the MET observation point were not double counted at the East observation point.

From 25 September to 4 October 2012, observers tallied a total of 424 migrating raptors (excluding turkey vultures), comprising 12 species over 179 hours (30 person count days) of observation across the 
three observation points (Table 3). This equates to 2.37 raptors detected per hour across the three observation points. Red-tailed hawk (Buteo jamaicensis) and sharp-shinned hawk (Accipiter striatus) were the most common migrant species recorded, with the species assemblage composed of $45 \%$ accipiters, $22 \%$ buteos, $15 \%$ falcons, 3\% eagles, $2 \%$ harriers, $2 \%$ ospreys, and $11 \%$ unidentified raptors. Sharp-shinned hawks (19\%), red-tailed hawks (17\%), Cooper’s hawks (Accipiter cooperii) (11\%), and American kestrels (Falco sparverius) (10\%) were the most abundant species detected.

Across the sampling period, 215 (3.58 raptors detected per hour), 88 (1.47 raptors detected per hour), and 121 (2.02 raptors detected per hour) migrating raptors were recorded at the Grand Point, East, and MET observation stations (see Figure 2), respectively. A comparison of the HREDP and HWI raptor migration magnitude shows that the greater project area is an area that concentrates raptors during fall migration, with a moderate to low magnitude across the four project areas. Specifically, analysis of the total number of raptors recorded by observation point shows that the majority of migrating raptors (79\%) were recorded at the Grand Point and MET observation stations, which are located directly on major headwalls and ridgelines of the Grand Wash Cliffs proper (see Figures 1, 2, and 4). Therefore, although the greater project area is an area that concentrates raptors during fall migration, the results of this study showed that the core migration corridor appears to be on the western boundaries of the project area along the Grand Wash Cliffs proper.

Although turkey vulture was excluded from fall migrant raptor species assemblage data, across the sampling period, 501 migrating individuals were recorded across observation stations, with 387, 74, and 40 migrating individuals recorded at the Grand Point, East, and MET observation stations, respectively (see Figure 1). Kettles (groups of migrating individuals following the same route during migration to take advantage of the best thermals and/or other wind currents to aid flight) accounted for up to 175 individuals. All kettles observed remained in groups/formation, with no individuals observed breaking out of the group. Of the 387 turkey vultures recorded at the Grand Point observation station, 84\% were detected from 1,000 to 5,000 m west of the observation point/project area; all of the turkey vultures recorded at MET were detected from 500 to $900 \mathrm{~m}$ west of the observation point/project area. Therefore, during this study, areas west of the western boundary of project area concentrated turkey vultures.

Table 3. Fall Raptor Migration Count Data from the HREDP, 25 September-4 October 2012

\begin{tabular}{lc}
\hline Year & $\mathbf{2 0 1 2}$ \\
\hline Start date & 25 Sept \\
\hline End date & 4 Oct \\
\hline Days of observation & 10 \\
\hline Hours of observation & 179 \\
\hline Raptors / 100 hours & \\
\hline Species & Count \\
\hline Osprey & 8 \\
\hline Northern harrier & 89 \\
\hline Sharp-shinned hawk & 53 \\
\hline Cooper's hawk & 48 \\
\hline Unidentified accipiter & 207 \\
\hline Total Accipiter, Osprey, Harrier & \\
\hline
\end{tabular}


Table 3. Fall Raptor Migration Count Data from the HREDP, 25 September-4 October 2012

(Continued)

\begin{tabular}{lr}
\hline Species & Count \\
\hline Swainson's hawk & 1 \\
\hline Red-tailed hawk & 81 \\
\hline Zone-tailed hawk & 1 \\
Unidentified Buteo & 12 \\
\hline Total Buteo & 95 \\
\hline Golden eagle & 9 \\
\hline Bald eagle & 1 \\
\hline Unidentified eagle & 1 \\
\hline Total Eagle & 11 \\
\hline American kestrel & 48 \\
\hline Prairie falcon & 3 \\
\hline Peregrine falcon & 7 \\
\hline Unknown falcon & 5 \\
\hline Total Falcon & 63 \\
\hline Unidentified raptor & 48 \\
\hline Total & $\mathbf{4 2 4}$ \\
\hline
\end{tabular}

\subsection{Fall Migrant Raptor Risk Analyses-HREDP}

Approximately one-fifth of the recorded species were observed in the RSA (9\% below the RSA, 20\% within the RSA, 71\% above the RSA). Figure 5 depicts the number of all observations recorded by flight height category.

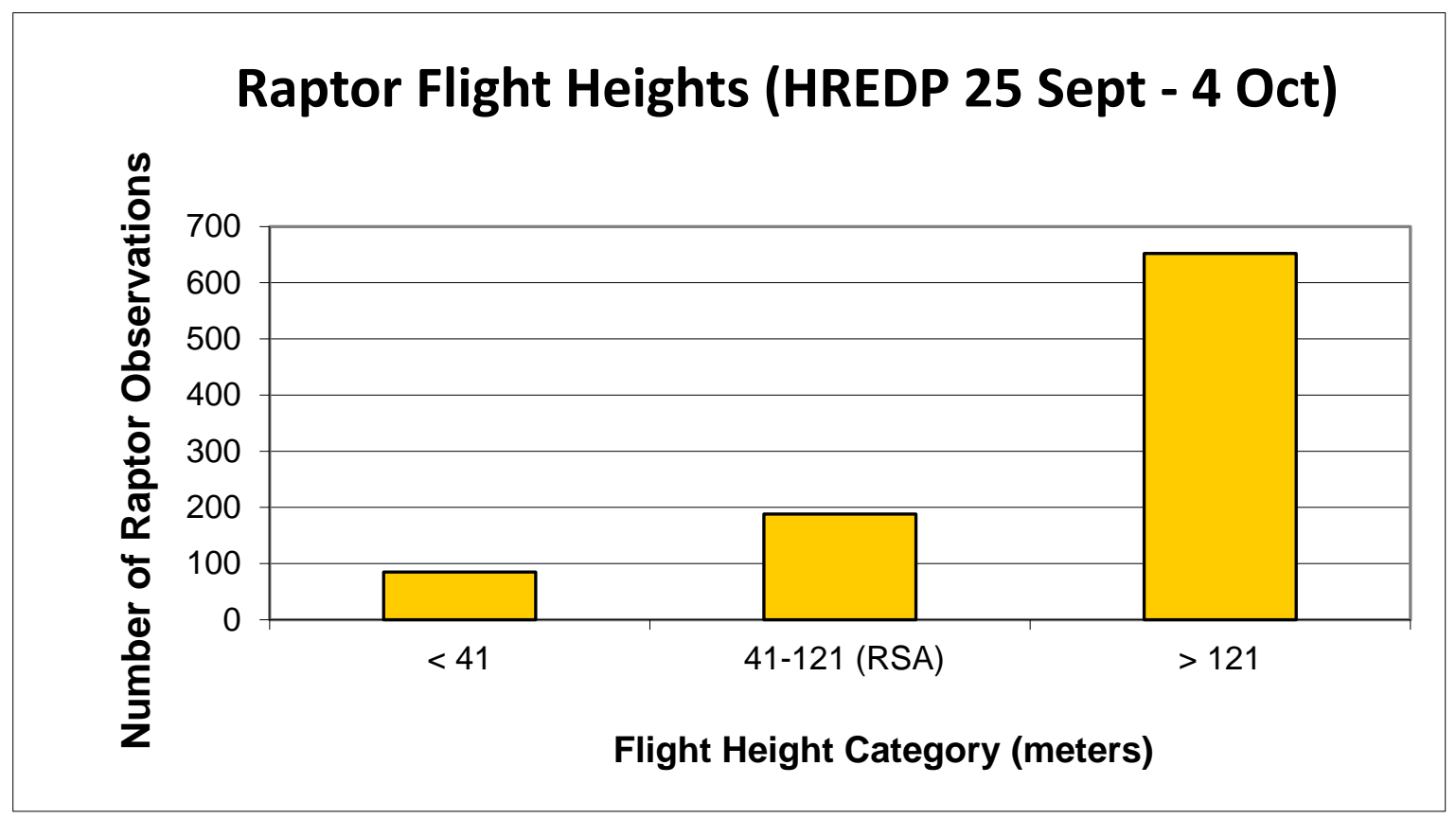

Figure 5. Number of raptor observations recorded by flight height category, HREDP, 25 September-4 October 2012. 
Table 4 shows the RIs for the 11 species recorded within the RSA. American kestrel, red-tailed hawk, Cooper's hawk, and sharp-shinned hawk had the highest RIs-43.1, 25.7, 24.1 and 21.7, respectively, followed by peregrine falcon $(\mathrm{RI}=9.5)$, golden eagle $(\mathrm{RI}=8.9)$, northern harrier ( $\mathrm{RI}=5.9)$, Swainson's hawk $(\mathrm{RI}=3.3)$, bald eagle $(\mathrm{RI}=3.3)$, turkey vulture $(\mathrm{RI}=2.9)$, and osprey $(\mathrm{RI}=2.5)$. Figure 6 depicts the RIs for species recorded during fall migration studies.

Table 4. Observations and Risk Indices of Fall Migrating Raptors Recorded within the RSA, HREDP, 25 September-4 October 2012

\begin{tabular}{llllll}
\hline Species & $\begin{array}{l}\text { No. of } \\
\text { Observations }\end{array}$ & $\begin{array}{l}\text { Frequency } \\
\text { (\% Surveys } \\
\text { Observed) }\end{array}$ & $\begin{array}{l}\text { Total No. of } \\
\text { Observations } \\
\text { in the RSA }\end{array}$ & $\begin{array}{l}\text { \% Observations } \\
\text { in RSA }\end{array}$ & RI \\
\hline American kestrel & 48 & 66.7 & 31 & 64.6 & 43.1 \\
\hline Red-tailed hawk & 81 & 83.3 & 25 & 24 & 25.7 \\
\hline Cooper's hawk & 53 & 53.3 & 66.7 & 45.3 & 24.1 \\
\hline Sharp-shinned hawk & 89 & 16.7 & 4 & 32.6 & 21.7 \\
\hline Peregrine falcon & 7 & 20 & 4 & 57.1 & 9.5 \\
\hline Golden eagle & 9 & 26.7 & 2 & 44.4 & 8.9 \\
\hline Northern harrier & 9 & 3.3 & 1 & 100 & 5.9 \\
\hline Swainson's hawk & 1 & 3.3 & 1 & 100 & 3.3 \\
\hline Bald eagle & 1 & 60 & 23 & 4.6 & 3.3 \\
\hline Turkey vulture & 501 & 20 & 1 & 12.5 & 2.9 \\
\hline Osprey & 8 & & & 2.5 \\
\hline
\end{tabular}

${ }^{*}$ Risk Index $=($ Frequency $\times \%$ of Observations in RSA $) / 100$

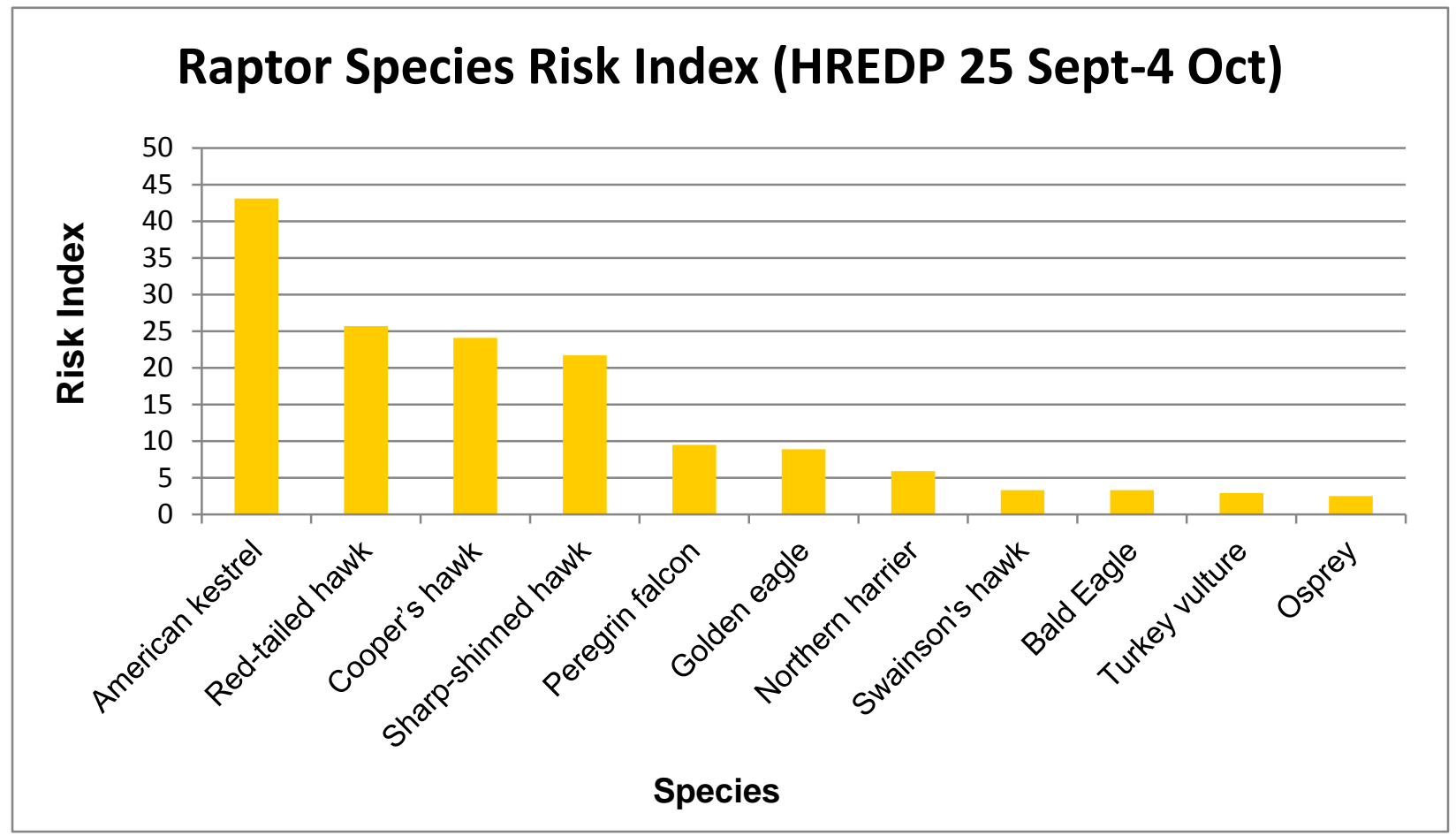

Figure 6. Risk indices for species recorded within the RSA during fall raptor migration studies, HREDP, 25 September-4 October 2012. 


\subsection{DISCUSSION}

\subsection{Literature and Data Search-Raptor Migration}

HWI fall raptor migration count data obtained from studies conducted at South Rim, Grand Canyon National Park, proved useful in interpreting the magnitude of fall raptor migration at the HREDP. Annual and date-specific (i.e., the same sampling periods) data were summarized for the Grand Canyon sites, with an annual average of 7.3 raptors detected per hour and a date-specific average of 9.9 raptors detected per hour, respectively. Absent the HWI data, interpretation of the HREDP data would be highly subjective.

\subsection{Fall Raptor Migration-HREDP Field Study}

Although observation points were spaced sufficiently far apart to detect all migrant raptors crossing an "east-west axis count boundary," out of necessity, the MET observation point was located north/northeast of the East observation station, presenting the possibility of double counting migrating birds. However, species detection and temporal data were compared, and the results indicated that migrating birds recorded at the MET observation point were not double counted at the East observation point. A likely explanation is that migrating birds heading south toward the project area either follow the prominent ridgelines of the Grand Wash Cliffs and quickly break to the southwest, or they break southeast because the topography and elevation from the MET observation station southward quickly trend southeast toward Horse Flat and Meriwhitica Canyons. If this migration behavior is exhibited, with birds breaking southwest and southeast as they approach the project area from the north, the topography of the site may direct the majority of migrating birds to the outer boundaries of the greater project area. Further study would be required to substantiate this observation.

A comparison of raptor migration magnitude of the HREDP with the HWI data shows that the greater project area is an area that concentrates raptors during fall migration, with a magnitude (raptors detected per hour) of moderate to low across the three observation stations (3.58 Grand Point; 1.47 East; 2.02 MET). Importantly, results show that the majority of migrating raptors (79\%) were recorded at the Grand Point and MET observation stations, which are located directly on major headwalls and ridgelines of the Grand Wash Cliffs proper. Therefore, although the greater project area is an area that concentrates raptors during fall migration, during this study, the core migration flyway appeared to be on the western boundaries of the project area along the Grand Wash Cliffs proper. Raptor migration has been a wellstudied phenomenon for decades, and it is well known that migrating raptors concentrate along longestablished routes or corridors characterized by prominent north-south-trending ridgelines and mountains, such as the Grand Wash Cliffs. Therefore, it may be that the raptor migration flyway patterns observed during this study are exhibited annually through time; additional studies are needed to support these results. For wind energy development in the project area, siting wind turbines as far as possible off of the western ridgelines should decrease negative impacts to migrating raptors.

Similar to the results of raptor migration magnitude, the greater project area is also an area that concentrates turkey vultures during fall migration, with large kettles of migrating individuals recorded. However, during this study, the core turkey vulture migration flyway appeared to be west of the western boundaries of the project area, as $84 \%$ of individuals were detected between 1,000 and 5,000 m west of the Grand Point observation station, and all of the turkey vultures recorded at the MET observation station were detected between 500 and 900 m west of the observation station. Unlike raptors, migrating turkey vultures rely almost exclusively on thermal lift, as opposed to raptors, which also use declivity wind currents/updrafts formed off of ridgelines and headwalls. As related to the HREDP, during this study, the core turkey vulture migration flyway was recorded west of the western boundaries of the 
project area, along the north-south-trending Hualapai Valley, which provides excellent thermal lift conditions during mid-day when temperatures are high on and above the desert floor. Therefore, it may be that the turkey vulture migration flyway patterns observed during this study are exhibited annually through time; additional studies are needed to support these results. As related to wind energy development in the project areas, the Hualapai Valley is likely to attract and concentrate migrating turkey vultures much more so than the project areas.

\subsection{Fall Migrant Raptor Risk Analyses-HREDP}

During this study, only approximately one-fifth of the recorded species were observed in the RSA, with American kestrel, red-tailed hawk, Cooper's hawk, and sharp-shinned hawk having the highest RIs43.1, 25.7, 24.1, and 21.7, respectively. However, all of species are quite capable of flying at a wide range of heights above the ground, including within the anticipated RSA for the proposed wind energy facility.

American kestrel, red-tailed hawk, Cooper's hawk, and sharp-shinned hawk were also the most abundant raptor species recorded during fall raptor migration surveys. These species are frequently observed mortalities at other wind energy facilities throughout the western United States (Erickson et al. 2002; Kingsley and Whittam 2005). As described by Orloff and Flannery (1992) at the Altamont Pass Wind Resource Area, American kestrels and red-tailed hawks are killed disproportionately, compared with turkey vultures, based on their abundance at the site. And based on this information, as Young et al. (2007) hypothesized, we would expect American kestrel, red-tailed hawk, Cooper's hawk, and sharpshinned hawk to account for most of the raptor mortalities from wind energy infrastructure during the fall migration period. However, these species are ubiquitous throughout the western United States and inhabit a variety of habitats and landscapes, and although it is predicted that the project may have some impact on these species, it is not anticipated that construction and operation of the facility alone would be detrimental to these species’ long-term persistence. 


\subsection{LITERATURE CITED}

Erickson, W.P., G. Johnson, D. Young, D. Strickland, R. Good, M. Bourassa, K. Bay, and K. Sernka. 2002. Synthesis and Comparison of Baseline Avian and Bat Use, Raptor Nesting and Mortality Information from Proposed and Existing Wind Developments. Prepared for Bonneville Power Administration. Prepared by Western EcoSystems Technology, Inc., Cheyenne, Wyoming.

Kingsley, A., and B. Whittam. 2005. Wind Turbines and Birds - A Background Review for Environmental Assessment. Prepared for Environment Canada/Canadian Wildlife Service.

Orloff, S., and A. Flannery. 1992. Wind Turbine Effects on Avian Activity, Habitat Use, and Mortality in Altamont Pass and Solano County Wind Resource Areas, 1989-1991. Final report to Alameda, Contra Costa and Solano Counties and the California Energy Commission. Tiburon, California: Biosystems Analysis, Inc.

Smith, J.P., and M.C. Neal. 2009. Fall 2008 Raptor Migration Studies in the Grand Canyon of Arizona. Project coordinated by HawkWatch International, Inc.

SWCA Environmental Consultants (SWCA). 2012. Critical Issues Analysis of Renewable Energy Development Projects. Report prepared for Hualapai Tribal Nation Planning Department. Las Vegas, Nevada: SWCA Environmental Consultants.

U.S. Geological Survey (USGS). 2004. Provisional Digital Land Cover Map for the Southwestern United States. Version 1.0. RS/GIS Laboratory, College of Natural Resources, Utah State University.

Young, D.P., Jr., G.D. Johnson, V.K. Poulton, and K. Bay. 2007. Ecological Baseline Studies for the Hatchet Ridge Wind Energy Project, Shasta County, California. Technical report for Hatchet Ridge Wind, LLC, Portland, Oregon. Western EcoSystems Technology, Inc., Cheyenne, Wyoming. 


\title{
Exhibit K
}

\section{Typical Development Costs Spreadsheet}

\author{
(MJH Consulting)
}




\section{TYPICAL DEVELOPMENT COST BREAKDOWNS FOR UTILITY-SCALE WIND FARMS}

Typical development Costs for Utility-Scale Wind Power Projects run from about $1 \%$ to $3 \%$ of the total wind project cost. Utility-Scale wind projects are typically from about 50MW to $300 \mathrm{MW}$ in size with larger projects having lower percentages of the total wind project cost. So typically a 300MW project will have development costs running at about $1 \%$ of the total The following development costs are for typical Utility-Scale wind projects from about 50MW to 300MW in size.

Note that development costs can very significantly by wind project depending on the location and many other factors.

PHASE

I: Feasibility Studies

Typical Cost (\$) Project Size of.. Hualapai Project

\begin{tabular}{|c|c|c|c|c|}
\hline \multirow[t]{4}{*}{ I: Feasibility Studies } & Power Sale & 50,000 & 50,000 & 50,000 \\
\hline & Identify Site & 40,000 & 50,000 & 40,000 \\
\hline & Wind Resource & 170,000 & 300,000 & 170,000 \\
\hline & Permitting Assessment & 50,000 & 75,000 & 50,000 \\
\hline \multirow[t]{7}{*}{ II: Reality Factors } & Lease/Option Site & 75,000 & 125,000 & 115,000 \\
\hline & Preliminary Engineering & 200,000 & 300,000 & 270,000 \\
\hline & Wind Energy Studies & 125,000 & 250,000 & 200,000 \\
\hline & Environmental Studies & 200,000 & 300,000 & 280,000 \\
\hline & Permitting Strategy & 110,000 & 125,000 & 120,000 \\
\hline & Bid/Negotiate PPA & 125,000 & 150,000 & 145,000 \\
\hline & Electrical Interconnect & 115,000 & 125,000 & 125,000 \\
\hline III: Select Development & In-House & 20,000 & 20,000 & 20,000 \\
\hline Team & Outside Consultants & 60,000 & 80,000 & 80,000 \\
\hline IV: Financial Partner & Select Finance Partner & 50,000 & 100,000 & 100,000 \\
\hline V: Execute Commerical & Site Leases & 90,000 & 100,000 & 100,000 \\
\hline \multirow[t]{6}{*}{ Documents } & EPC Agreement & 90,000 & 100,000 & 100,000 \\
\hline & WTG PSA Agreement & 90,000 & 100,000 & 100,000 \\
\hline & Wind Energy to Finance & 90,000 & 100,000 & 100,000 \\
\hline & Submit Permits \& Fees & 90,000 & 100,000 & 100,000 \\
\hline & Power Agreement (PPA) & 90,000 & 100,000 & 100,000 \\
\hline & Interconnect Agreement & 90,000 & 100,000 & 100,000 \\
\hline \multirow[t]{2}{*}{ VI: Construct-Operate } & Process Management & 80,000 & 100,000 & 100,000 \\
\hline & Contingency & 200,000 & 900,000 & 800,000 \\
\hline Total Phases I-VI: & & $2,300,000$ & $3,750,000$ & $3,365,000$ \\
\hline \multirow[t]{2}{*}{ Project Capital Cost } & Installed Cost $(\$ / \mathrm{kW})$ & $\$ 1,800$ & $\$ 1,450$ & $\$ 1,600$ \\
\hline & Project Total & $\$ 90,000,000$ & $\$ 435,000,000$ & $\$ 320,000,000$ \\
\hline
\end{tabular}

Development Costs as Percent of Total Project Cost: 


\title{
Exhibit L
}

\section{Estimates for Project Construction Costs}

\author{
(MJH Consulting)
}


This schedule is a high-level calculation of the various components of total installed costs per Kw of capacity for the Hualapai 200MW Wind Project. The wind turbine cost assumptions are the largest component of the project's total cost, typically representing about $75-80 \%$ of the total cost of a wind project. The assumption for turbines below is based on a recent non-binding quote from the manufacturer of the GE-1.6xle/82.5. The GE $1.7 / 100$ meter rotor diameter turbine is the best performing turbine for the Hualapai site. I use the quote on the GE- 1.6/82.5 meter turbine as the basis for my calculated assumed cost for the GE 1.7/100 meter turbine. Most of the BOP costs are assessed as a percentage of total project costs with the exception of foundations. Foundation \& access road construction for the Hualapai Wind Project are two of the more costly components of the project, therefore, MJH Power has estimated the likely cost of foundations and the roads for 80 meter hub height GE-1.7/100 turbines based on a site visit and the existing geotech investigation of the site for a 31 mile 6 inch water line.

\section{Proforma Hualapai Capacity}

Per Updated WAPA IA - Feasibility Study

\section{Turbine Cost -}

GE-1.6xle/82.5, 80 meter hub

Preliminary quote from GE rep to $\mathrm{MJH}$

Est market discount available off of quote Incremental cost - 1.7MW 100 meter rotor Estimated installed cost for GE-1.7/100m Total turbines for Hualapai capacity

Transmission/Interconnect Inc Costs Pending WAPA Feasibiity Study Results Inc costs from typical-WAPA Interconnect Inc higher 69/345KV Interconnect costs

\section{BALANCE OF PLANT:}

\section{Foundations -}

Per turbine preliminary estimate - typical Potential range of costs - Hualapai site

(higher cost-remote, hilly access, rocky site)

Assumed cost of Hualapai site foundations

Access Road Costs remote/hilly/rocky

\section{Balance of Plant -}

Assumed BOP $\%$ at $1.7 / 100 /$ turbine pricing Plus Inc BOP components specified above Incremental foundation costs @ \$15K/WTG Incremental access road costs (15 miles) Inc Envir \& Cultural Const Monitoring based on Perrin Ranch Inc-Avian\&Bat-Bald\&Golden Eagle Fund bases Perrin Ranch

TOTAL INSTALLED COSTS - Hualapai Wind Project

TOTAL INSTALLED COSTS - \$ per Kw

Development Fee and Financing Costs -

Development fee

Financing/placement costs

\section{\$ / Kw Installed}

\section{MW}

\begin{tabular}{ccc} 
& $1.6 \mathrm{Mw}$ \\
& 1,100 & \\
& $(165)$ & $15 \%$ \\
& 125 \\
& $\$ 1,060$ \\
\hline \hline
\end{tabular}

$118 \$ 212,000,000$

\begin{tabular}{|c|c|c|}
\hline \multicolumn{3}{|c|}{$\underline{\text { PE's est design } \$}$} \\
\hline$\$$ & $4,000,000$ & \\
\hline$\$$ & $7,400,000$ & $\$$ \\
\hline$\$$ & 180,000 & per $\mathrm{MJH}$ experience \\
\hline
\end{tabular}

$\$ 11,400,000$

TOTAL COSTS THRU FINANCING- Hualapai Wind Project

$\$ 195,000$

estimated 55 miles $X \$ 24 / \mathrm{ft}$
Est. Installed Cost

\section{TOTAL COSTS THRU FINANCING- \$ per Kw}

\begin{tabular}{rrr}
$22.5 \%$ & $\$$ & $61,548,387$ \\
& & \\
& $\$$ & $1,770,000$ \\
& $\$$ & $1,900,800$ \\
Ranch & $\$$ & 800,000 \\
Ranch & $\$$ & 500,000 \\
\hline
\end{tabular}

$66,519,187$
$22,941,176$

$6,969,600$
$\$ 45,000$ per MW
$\$ 289,919,187$

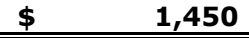

$9,000,000$

$26,902,727$ 


\title{
Exhibit M
}

\section{Preliminary Project Pro Forma}

\author{
(MJH Consulting)
}




\section{Hualapai 200 Wind Project}

\section{Project Proforma Financial Model}

NOTE: Refer to Model Inputs sheet and other supporting schedules for Capital Costs, Depreciation, O\&M Costs and Debt Service for underlying assumptions and detail.

\section{REVENUES}

$\mathrm{kWh} / \mathrm{yr}$
PPA Rate (\$/kWh)

Pectricity Sales Revenue per PPA

REC/Green Tag kWh/yr contracted

REC/Green Tag Sales Revenue

Total Annual Revenues

\section{EXPENSES}

Landowner Royalty

Operations \& Maintenance

Service/maint.

insurance

Land maint.

BOP

Admin
Bird Mitigation

cheduled maint/repair

Total Expenses Total Expenses- $\$ / k W h$
Expenses - \% of Revenues

\begin{tabular}{|c|c|c|c|c|c|c|c|c|}
\hline YEAR & YEAR & YEAR & YEAR & YEAR & YEAR & YEAR & YEAR & YEAR \\
$\mathbf{1}$ & $\mathbf{2}$ & $\mathbf{3}$ & $\mathbf{4}$ & $\mathbf{5}$ & $\mathbf{6}$ & $\mathbf{7}$ & \\
\hline
\end{tabular}

Property Taxes
Property Tax Abatements

Net Property Taxes

Project Management Fee

Admin/Financial/Legal Management

EBITDA

Interest Expense

Net Income(Loss) Before Taxes
Necion and Amortizan

Taxes Benefit(Expense)

State Tax
Federal Income Tax

Total Taxes on Incom

Net Tax Benefit(Expense)

Net Income(Loss)

Calculation of Net Cash Flow -

Net Income(Loss)
Add: Depreciation and Interest

row

Less: Debt Service

Interest Expense

$\begin{array}{ccccccccc}475513474 & 475513474 & 475513474 & 475513474 & 475513474 & 475513474 & 475513474 & 475513474 & 475513474 \\ \$ 0.0074 & \$ 0.074 & \$ 0.075 & \$ 0.077 & \$ 0.078 & \$ 0.080\end{array}$ $\begin{array}{rrrrrrrrr}\$ 28,530,808 & \$ 29,101,425 & \$ 30,556,496 & \$ 32,695,451 & \$ 34,984,132 & \$ 35,683,815 & \$ 36,397,491 & \$ 37,125,441 & \$ 37,867,950\end{array}$

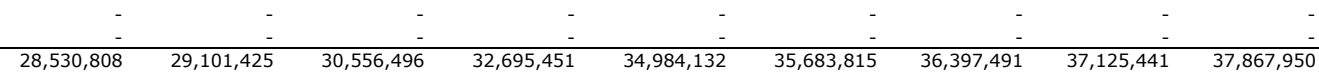

\begin{tabular}{|c|c|c|c|c|c|c|c|c|}
\hline $1,141,232$ & $1,164,057$ & $1,222,260$ & $1,307,818$ & $1,399,365$ & $1,427,353$ & $1,455,900$ & $1,485,018$ & $1,514,718$ \\
\hline $2,478,000$ & $2,527,560$ & $2,578,111$ & $2,629,673$ & $2,682,267$ & $2,714,000$ & $2,768,280$ & $2,823,646$ & 2,880 \\
\hline $2,242,000$ & $2,286,840$ & $2,332,577$ & $2,379,228$ & $2,426,813$ & 472,000 & 481,440 & 491,069 & 500,890 \\
\hline $\begin{array}{l}826,000 \\
354\end{array}$ & 842,520 & $\begin{array}{l}859,370 \\
368\end{array}$ & $\begin{array}{l}876,558 \\
337668\end{array}$ & $\begin{array}{l}894,089 \\
383\end{array}$ & 826,000 & 842,520 & $\begin{array}{l}859,370 \\
368\end{array}$ & $\begin{array}{r}876,558 \\
376668\end{array}$ \\
\hline 354,000 & 361,080 & 368,302 & $\begin{array}{l}375,668 \\
438,270\end{array}$ & 383,181 & 354,000 & $\begin{array}{l}361,080 \\
421260\end{array}$ & $\begin{array}{l}368,302 \\
429685\end{array}$ & $\begin{array}{l}375,668 \\
438279\end{array}$ \\
\hline 413,000 & 421,260 & 429,685 & $\begin{array}{l}438,279 \\
438,270\end{array}$ & 447,044 & 413,000 & $\begin{array}{l}421,260 \\
421260\end{array}$ & $\begin{array}{l}429,685 \\
429685\end{array}$ & $\begin{array}{l}438,2 \\
438,2\end{array}$ \\
\hline 413,000 & 421,260 & 429,685 & 438,279 & 447,044 & 413,000 & 421,260 & 429,685 & 438,2 \\
\hline 200,000 & 204,000 & 208,080 & & & 2360,000 & $2,407,200$ & $2,455,344$ & \\
\hline $6,926,0$ & $7,064,5$ & $7,205,8$ & $7,137,685$ & $7,280,4$ & $7,552,000$ & $7,703,040$ & $7,857,101$ & $\frac{2,504,451}{8,014,243}$ \\
\hline$\$ 0.0$ & & $\$ 0.0$ & $\$ 0.0$ & $\$ 0.015$ & $\$ 0.016$ & $\$ 0.016$ & $\$ 0.017$ & \\
\hline $24.3 \%$ & $24.3 \%$ & $23.6 \%$ & $21.8 \%$ & $20.8 \%$ & $21.2 \%$ & $21.2 \%$ & $21.2 \%$ & $1.2 \mathrm{C}$ \\
\hline
\end{tabular}

\begin{tabular}{rrrrrrrrr}
- & - & - & - & - & - & - & - & - \\
\hline 180,000 & 183,600 & - & - & - & - & - & - \\
\hline & 187,272 & 191,017 & 194,838 & 198,735 & 202,709 & 206,763 & $210,899^{-}$
\end{tabular}
\begin{tabular}{rrrrrrrrr}
180,000 & 183,600 & 187,272 & 191,017 & 194,838 & 198,735 & 202,709 & 206,763 & 210,899 \\
120,000 & 122,400 & 124,848 & 127,345 & 129,892 & 132,490 & 135,139 & 137,842 & 140,599 \\
\hline $8,367,232$ & $8,534,577$ & $8,740,190$ & $8,763,865$ & $9,004,534$ & $9,310,577$ & $9,496,788$ & $9,686,724$ & $9,880,459$
\end{tabular}

$\begin{array}{llllllllll}\$ 20,163,576 & \$ 20,566,848 & \$ 21,816,306 & \$ 23,931,585 & \$ 25,979,598 & \$ 26,373,238 & \$ 26,900,703 & \$ 27,438,717 & \$ 27,987,491\end{array}$

\begin{tabular}{llllllllll}
$7.5 \%$ & $16,570,641$ & $15,145,210$ & $13,719,778$ & $12,294,347$ & $10,868,915$ & $9,443,484$ & $8,018,052$ & $6,592,621$ & $5,167,189$ \\
\hline
\end{tabular} \begin{tabular}{rrrrrrrrr}
$15,153,700$ & $91,862,559$ & $58,774,929$ & $38,699,203$ & $32,243,072$ & $28,782,099$ & $8,054,386$ & $7,989,608$ & $7,814,464$ \\
\hline$\$(11,560,765)$ & $\$(86,440,921)$ & $\$(50,678,401)$ & $\$(27,061,965)$ & $\$(17,132,388)$ & $\$(11,852,345)$ & $\$ 10,828,264$ & $\$ 12,856,488$ & $\$ 15,005,838$
\end{tabular}

\begin{tabular}{rrrrrrrrr}
$4,046,268$ & $30,254,322$ & $17,737,441$ & $9,471,688$ & $5,996,336^{-}$ & $4,148,321$ & $(3,789,893)$ & $(4,499,771)$ & $(5,252,043)$ \\
\hline $4,046,268$ & $30,254,322$ & $17,737,441$ & $9,471,688$ & $5,996,336$ & $4,148,321$ & $(3,789,893)$ & $(4,499,771)$ & $(5,252,043)$ \\
\hline
\end{tabular}

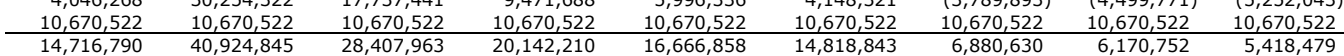
\begin{tabular}{llllllllll}
$\$ 3,156,025$ & $\$(45,516,076)$ & $\$(22,270,439)$ & $\$(6,919,755)$ & $\$$ & $(465,530)$ & $\$ 2,966,498$ & $\$ 17,708,894$ & $\$ 19,027,239$ & $\$ 20,424,317$ \\
\hline \hline
\end{tabular}

$\$ 3,156,025 \quad \$(45,516,076) \$(22,270,439) \$(6,919,755) \$(465,530) \$ 2,966,498 \quad \$ 17,708,894 \quad \$ 19,027,239 \quad \$ 20,424,317$

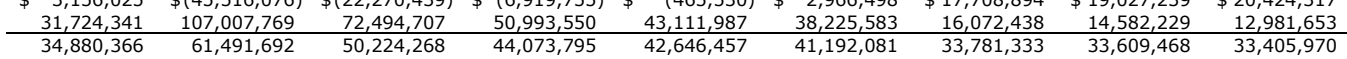
\begin{tabular}{rrrrrrrrr}
$19,005,753$ & $19,005,753$ & $19,005,753$ & $19,005,753$ & $19,005,753$ & $19,005,753$ & $19,005,753$ & $19,005,753$ & $19,005,753$ \\
$16,570,641$ & $15,145,210$ & $13,719,778$ & $12,294,347$ & $10,868,915$ & $9,443,484$ & $8,018,052$ & $6,592,621$ & $5,167,189$ \\
\hline $35,576,394$ & $34,150,963$ & $32,725,531$ & $31,300,100$ & $29,874,668$ & $28,449,237$ & $27,023,805$ & $25,598,374$ & $24,172,942$ \\
\hline
\end{tabular}

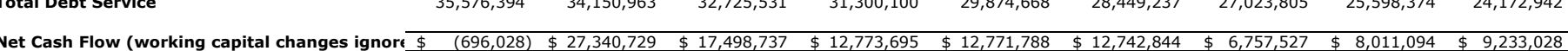
MEMO: Outstanding Balance on Debt \begin{tabular}{lllllllll}
$\$ 209,063,286$ & $\$ 190,057,533$ & $\$ 171,051,780$ & $\$ 152,046,027$ & $\$ 133,040,273$ & $\$ 114,034,520$ & $\$ 95,028,767$ & $\$ 76,023,013$ & $\$ 57,017,260$ \\
\hline \hline
\end{tabular} Operating Cash Flow as \% of Debt Service

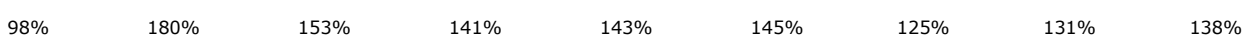




\section{Hualapai 200 Wind Project}

Project Proforma Financial Model

NOTE: Refer to Model Inputs sheet and other :

\section{REVENUES}

$\mathrm{kWh} / \mathrm{yr}$
PPA Rate (\$/kWh)

(lectricity Sales Revenue per PPA

REC/Green Tag kWh/yr contracted

EC/Green Tag Sales Revenue

Total Annual Revenues

\section{EXPENSES}

Jevdowner Royalty

Operations \& Maintenance

Service/maint
Warranty

insurance

Land maint.

BOP

Bird Mitigation

cheduled maint/repair

Total Expenses

Total Expenses- $\$ / k W h$
Total Expenses - \% of Revenues

Property Taxes

Property Tax Abatements

Project Management

Admin/Financial/Legal Management

EBITDA

Interest Expense

Wection and Amortization

Before Taxes

Taxes Benefit(Expense)

State Tax
Federal Income Tax

Total Taxes on Incom

Net Tax Benefit(Expense)

Net Income(Loss)

Calculation of Net Cash Flow -

Net Income(Loss)
Add: Depreciation and Interest

Less: Debt Service

Interest Expense

Debt Service

MEMO: Outstanding Balance on Debt

Operating Cash Flow as \% of Debt Service

\begin{tabular}{|c|c|c|c|c|c|c|c|c|c|c|}
\hline YEAR & YEAR & YEAR & YEAR & YEAR & YEAR & YEAR & YEAR & YEAR & $\begin{array}{c}\text { YEAR } \\
10\end{array}$ & $\begin{array}{c}\text { YEAR } \\
10\end{array}$ \\
\hline
\end{tabular}

$\begin{array}{lllllllllll}475513474 & 475513474 & 475513474 & 475513474 & 475513474 & 475513474 & 475513474 & 475513474 & 475513474 & 475513474 & 475513474\end{array}$

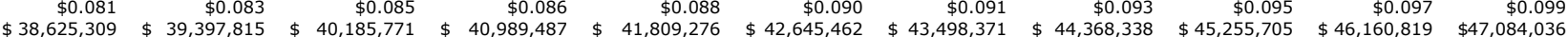

\begin{tabular}{|c|c|c|c|c|c|c|c|c|c|c|}
\hline $38,625,309$ & $39,397,815$ & $40,185,771$ & $40,989,487$ & $41,809,276$ & $42,645,462$ & $43,498,371$ & $44,368,338$ & $45,255,705$ & $46,160,819$ & $47,084,036$ \\
\hline $1,545,012$ & $1,969,891$ & $2,009,289$ & $2,049,474$ & $2,090,464$ & $2,132,273$ & $2,174,919$ & $2,218,417$ & $2,262,785$ & $2,308,041$ & $2,354,202$ \\
\hline $2,937,721$ & $2,996,475$ & $3,056,405$ & $3,117,533$ & $3,179,884$ & 3,2 & $3,308,351$ & $3,374,518$ & $3,442,008$ & $3,510,848$ & $3,581,065$ \\
\hline 510,908 & 521,126 & 531,5 & 54 & 553,023 & 564,084 & 575,365 & 586,873 & 598,610 & 610,582 & 622,794 \\
\hline 894,089 & 911,971 & 930,210 & 94 & 967,791 & 987,146 & $1,006,889$ & $1,027,027$ & 1,047 & $1,068,519$ & $1,089,889$ \\
\hline 383,18 & & & & & & & & & & 467,095 \\
\hline 44 & 45 & & & 483,895 & 493, & 50 & 513,514 & 523, & 534,260 & 544,945 \\
\hline 447,044 & 455,985 & 465,105 & 474,407 & 483,895 & 493,573 & 503,445 & 513,514 & 523,784 & 534,260 & 544,945 \\
\hline $2,554,5$ & 5,631 & $2,657,743$ & 8 & 55,116 & 2, & 27 & 663 & 51 & & \\
\hline $8,174,5$ & $8,338,018$ & $8,504,7$ & $8,674,874$ & $8,848,372$ & 9,025, & $9,205,846$ & 9,389, & 9,577 & $9,769,317$ & 964 \\
\hline$\$ 0.017$ & $\$ 0.018$ & $\$ 0.018$ & $\$ 0.018$ & $\$ 0.019$ & $\$ 0.019$ & $\$ 0.019$ & $\$ 0.020$ & $\$ 0.020$ & $\$ 0.021$ & $\$ 0.021$ \\
\hline & & & & & & & & & & \\
\hline
\end{tabular}

\begin{tabular}{rrrrrrrrrrr}
\hline & & & & \\
215,117 & 219,419 & 223,807 & $228,28 \overline{1}$ & 232,849 & $237,50 \overline{6}$ & $242,25 \overline{6}$ & $247,10 \overline{1}$ & 252,043 & $257,08 \overline{-}$ & 262,226 \\
143,411 & 146,279 & 149,205 & 152,189 & 155,233 & 158,337 & 161,504 & 164,734 & 168,029 & 171,390 & 174,817 \\
\hline $10,078,068$ & $10,673,607$ & $10,887,079$ & $11,104,821$ & $11,326,917$ & $11,553,456$ & $11,784,525$ & $12,020,215$ & $12,260,620$ & $12,505,832$ & $12,755,949$
\end{tabular}

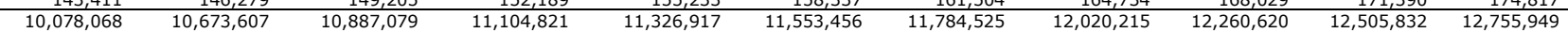

$\begin{array}{llllllllllllll}\$ 28,547,241 & \$ 28,724,208 & \$ 29,298,692 & \$ 29,884,666 & \$ & 30,482,359 & \$ 31,092,006 & \$ 31,713,846 & \$ 32,348,123 & \$ 32,995,086 & \$ 33,654,987 & \$ 34,328,087\end{array}$ \begin{tabular}{rrrrrrrrrrrr}
$7.5 \%$ & $3,741,758$ & $2,316,326$ & 890,895 & $(0)$ & $(0)$ & $(0)$ & - & - & - & - \\
\hline $7,820,871$ & $4,324,191$ & $4,330,598$ & $4,324,191$ & $4,330,598$ & $4,324,191$ & $3,856,486$ & 544,109 & 544,223 & 544,109 & 544,223 \\
\hline$\$ 16,984,612$ & $\$ 22,083,690$ & $\$ 24,077,199$ & $\$ 25,560,474$ & $\$ 26,151,760$ & $\$ 26,767,815$ & $\$ 27,857,360$ & $\$ 31,804,014$ & $\$ 32,450,862$ & $\$ 33,110,878$ & $\$ 33,783,864$
\end{tabular} \begin{tabular}{lllllllllll}
$(5,944,614)$ & $(7,729,291)$ & $(8,427,020)$ & $(8,946,166)$ & $(9,153,116)$ & $(9,368,735)$ & $(9,750,076)$ & $(11,131,405)$ & $(11,357,802)$ & $(11,588,807)$ & $(11,824,352)$ \\
\hline$(5,944,614)$ & $(7,729,291)$ & $(8,427,020)$ & $(8,946,166)$ & $(9,153,116)$ & $(9,368,735)$ & $(9,750,076)$ & $(11,131,405)$ & $(11,357,802)$ & $(11,588,807)$ & $(11,824,352)$
\end{tabular}

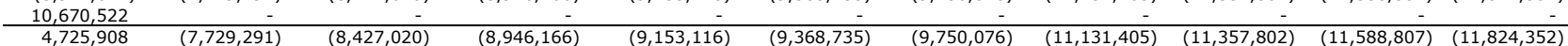
\begin{tabular}{llllllllllllll}
$\$ 21,710,520$ & $\$ 14,354,398$ & $\$ 15,650,179$ & $\$ 16,614,308$ & $\$ 16,998,644$ & $\$ 17,399,079$ & $\$ 18,107,284$ & $\$ 20,672,609$ & $\$ 21,093,060$ & $\$ 21,522,071$ & $\$ 21,959,511$ \\
\hline \hline
\end{tabular}

$\$ 21,710,520 \quad \$ 14,354,398 \quad \$ 15,650,179 \quad \$ 16,614,308 \quad \$ 16,998,644 \quad \$ 17,399,079 \quad \$ 18,107,284 \quad \$ 20,672,609 \quad \$ 21,093,060 \quad \$ 21,522,071 \quad \$ 21,959,511$

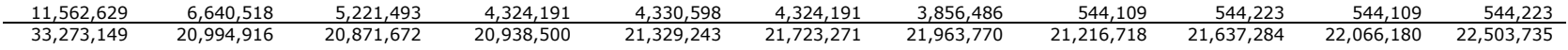
\begin{tabular}{rrrrrr}
$19,005,753$ & $19,005,753$ & $19,005,753$ & - & - & $(0)$ \\
$3,71,758$ & $2,316,326$ & 890,895 & $(0)$ & $(0)$ & $(0)$ \\
\hline $22,747,511$ & $21,322,080$ & $19,896,648$ & $(0)$ & $(0)$ & $(0)$
\end{tabular}

\begin{tabular}{llllllllllllll}
$\$ 10,525,638$ & $\$$ & $(327,163)$ & $\$$ & 975,024 & $\$ 20,938,500$ & $\$ 21,329,243$ & $\$ 21,723,271$ & $\$ 21,963,770$ & $\$ 21,216,718$ & $\$ 21,637,284$ & $\$ 22,066,180$ & $\$ 22,503,735$ \\
\hline \hline
\end{tabular}

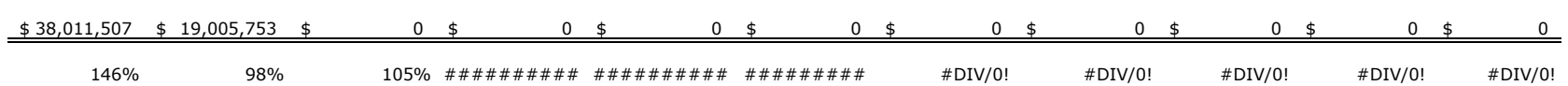


Hualapai, AZ - 200MW Wind Project

Summary of Installed Cost Assumptions

General Methodology

This schedule is a high-level calculation of the various components of total installed costs per Kw of capacity for the Hualapai 200MW Wind Project. The wind turbine cost assumptions are the largest component of the project's total cost, typically representing about $75-80 \%$ of the total cost of a wind project. The assumption for turbines below is based on a recent non-binding quote from the manufacturer of the GE-1.6xle/82.5. The GE $1.7 / 100$ meter rotor diameter turbine is the best performing turbine for the Hualapai site. I use the quote on the GE- 1.6/82.5 meter turbine as the basis for my calculated assumed cost for the GE $1.7 / 100$ meter turbine. Most of the BOP costs are assessed as a percentage of total project costs with the exception of foundations. Foundation \& access road construction for the Hualapai Wind Project are two of the more costly components of the project, therefore, $\mathrm{MJH}$ Power has estimated the likely cost of foundations and the roads for 80 meter hub height GE-1.7/100 turbines based on a site visit and the existing geotech investigation of the site for a 31 mile 6 inch water line.

Proforma Hualapai Capacity

Per Updated WAPA IA - Feasibility Study

\section{Turbine Cost -}

GE-1.6xle/82.5, 80 meter hub Preliminary quote from GE rep to $\mathrm{MJH}$ Est market discount available off of quote Incremental cost - 1.7MW 100 meter rotor Estimated installed cost for GE-1.7/100m Total turbines for Hualapai capacity

Transmission/Interconnect Inc Costs Pending WAPA Feasibiity Study Results Inc costs from typical-WAPA Interconnect Inc higher 69/345KV Interconnect costs

\section{BALANCE OF PLANT:}

Foundations -

Per turbine preliminary estimate - typical Potential range of costs - Hualapai site

(higher cost-remote, hilly access, rocky site)

Assumed cost of Hualapai site foundations

Access Road Costs remote/hilly/rocky

Balance of Plant -

Assumed BOP $\%$ at 1.7/100/turbine pricing Plus Inc BOP components specified above Incremental foundation costs @ \$15K/WTG Incremental access road costs (15 miles)

Inc Envir \& Cultural Const Monitoring based on Perrin Ranch

Inc-Avian\&Bat-Bald\&Golden Eagle Fund based Perrin Ranch

TOTAL INSTALLed costs - Hualapai Wind Project

TOTAL INSTALLED COSTS - \$ per Kw

\begin{tabular}{cc} 
PE's est design $\$$ \\
\hline$\$$ & $4,000,000$ \\
$\$$ & $7,400,000$ \\
\hline
\end{tabular}

118

$212,000,000$

$\$ \quad 180,000$ per MJH experience

$\$ 190$ to 200K per MJH experience

$\$ \quad 195,000$

$22,941,176$

estimated 55 miles $X \$ 24 / \mathrm{ft}$

$6,969,600$
$22.5 \% \$ 61,548,387$

$\$ \quad 1,770,000$

$\$ 1,900,800$

$\$ \quad 500,000$
$\$ 800,000$

Development Fee and Financing Costs -

Development fee

Financing/placement costs

$\$ 45,000$ per $M W$

$9.0 \%$

$9,000,000$

$26,902,727$

TOTAL costs thru finANCING- Hualapai Wind Project

TOTAL COSTS THRU FINANCING- \$ per Kw

$\$ \quad 1,629$


Depreciation \& Amortization

Wind Turbine and Equipment
Organization Cost Organization Cost
Raod Claering and Grading
Developer L Lender Fees
Land Improvements

Trensmission Linesilnterconnection
Lease
Buidings

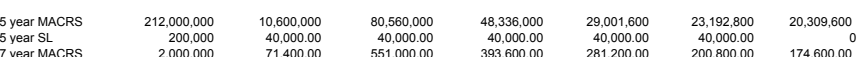

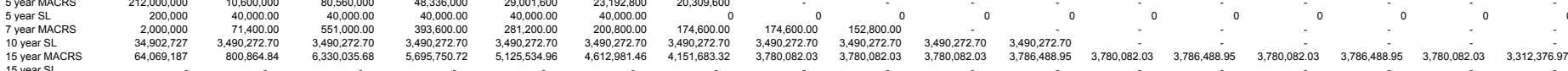

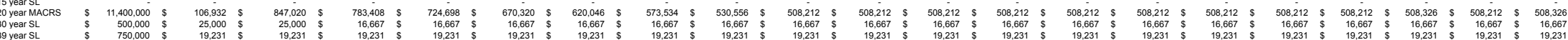

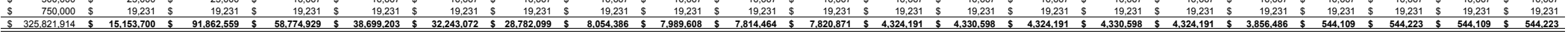

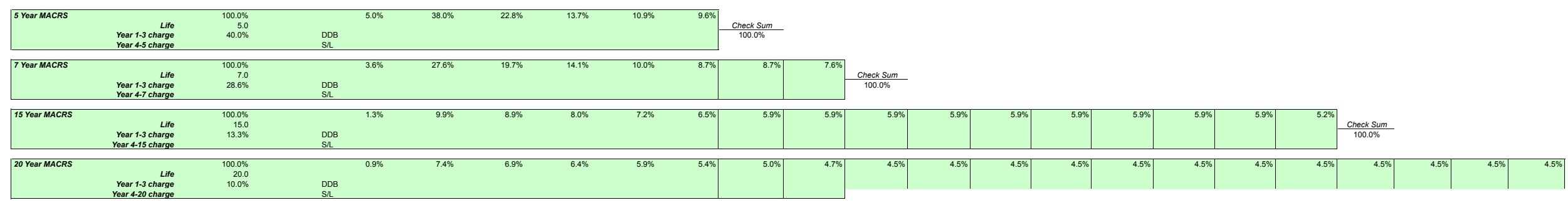




\section{Hualapai Wind Project - 200MW \\ Debt Service Schedule}

For Hualapai Proforma

Assumptions -

Total Cost of Project

Capitalization Structure

Debt: Amortization (Yrs)

Payments -

Equity:

$\$ 325,812,914$ Installed Cost Assumptions D63

\begin{tabular}{rrr} 
& \multicolumn{3}{c}{ Tax Equity Financing } \\
12 & $70 \%$ & $228,069,040$ \\
Quarterly & Rate - & $7.5 \%$ \\
& $30 \%$ & $97,743,874$
\end{tabular}

\begin{tabular}{|c|c|c|c|c|c|c|c|}
\hline \multicolumn{2}{|c|}{ Amortization Table } & 48 & \multicolumn{2}{|l|}{ Total Payments } & \multicolumn{3}{|c|}{ Annual Totals } \\
\hline Payment \# & Debt Balance & Interest & Principal & Total Payment & Interest & Principal & Total Payment \\
\hline Beginning & $228,069,040$ & & & & & & \\
\hline 1 & $223,317,601$ & $4,276,294$ & $4,751,438$ & $9,027,733$ & & & \\
\hline 2 & $218,566,163$ & $4,187,205$ & $4,751,438$ & $8,938,643$ & & & \\
\hline 3 & $213,814,725$ & $4,098,116$ & $4,751,438$ & $8,849,554$ & & & \\
\hline 4 & $209,063,286$ & $4,009,026$ & $4,751,438$ & $8,760,464$ & $16,570,641$ & $19,005,753$ & $35,576,394$ \\
\hline 5 & $204,311,848$ & $3,919,937$ & $4,751,438$ & $8,671,375$ & & & \\
\hline 6 & $199,560,410$ & $3,830,847$ & $4,751,438$ & $8,582,285$ & & & \\
\hline 7 & $194,808,971$ & $3,741,758$ & $4,751,438$ & $8,493,196$ & & & \\
\hline 8 & $190,057,533$ & $3,652,668$ & $4,751,438$ & $8,404,107$ & $15,145,210$ & $19,005,753$ & $34,150,963$ \\
\hline 9 & $185,306,095$ & $3,563,579$ & $4,751,438$ & $8,315,017$ & & & \\
\hline 10 & $180,554,657$ & $3,474,489$ & $4,751,438$ & $8,225,928$ & & & \\
\hline 11 & $175,803,218$ & $3,385,400$ & $4,751,438$ & $8,136,838$ & & & \\
\hline 12 & $171,051,780$ & $3,296,310$ & $4,751,438$ & $8,047,749$ & $13,719,778$ & $19,005,753$ & $32,725,531$ \\
\hline 13 & $166,300,342$ & $3,207,221$ & $4,751,438$ & $7,958,659$ & & & \\
\hline 14 & $161,548,903$ & $3,118,131$ & $4,751,438$ & $7,869,570$ & & & \\
\hline 15 & $156,797,465$ & $3,029,042$ & $4,751,438$ & $7,780,480$ & & & \\
\hline 16 & $152,046,027$ & $2,939,952$ & $4,751,438$ & $7,691,391$ & $12,294,347$ & $19,005,753$ & $31,300,100$ \\
\hline 17 & $147,294,588$ & $2,850,863$ & $4,751,438$ & $7,602,301$ & & & \\
\hline 18 & $142,543,150$ & $2,761,774$ & $4,751,438$ & $7,513,212$ & & & \\
\hline 19 & $137,791,712$ & $2,672,684$ & $4,751,438$ & $7,424,122$ & & & \\
\hline 20 & $133,040,273$ & $2,583,595$ & $4,751,438$ & $7,335,033$ & $10,868,915$ & $19,005,753$ & $29,874,668$ \\
\hline 21 & $128,288,835$ & $2,494,505$ & $4,751,438$ & $7,245,943$ & & & \\
\hline 22 & $123,537,397$ & $2,405,416$ & $4,751,438$ & $7,156,854$ & & & \\
\hline 23 & $118,785,958$ & $2,316,326$ & $4,751,438$ & $7,067,765$ & & & \\
\hline 24 & $114,034,520$ & $2,227,237$ & $4,751,438$ & $6,978,675$ & $9,443,484$ & $19,005,753$ & $28,449,237$ \\
\hline 25 & $109,283,082$ & $2,138,147$ & $4,751,438$ & $6,889,586$ & & & \\
\hline 26 & $104,531,643$ & $2,049,058$ & $4,751,438$ & $6,800,496$ & & & \\
\hline 27 & $99,780,205$ & $1,959,968$ & $4,751,438$ & $6,711,407$ & & & \\
\hline 28 & $95,028,767$ & $1,870,879$ & $4,751,438$ & $6,622,317$ & $8,018,052$ & $19,005,753$ & $27,023,805$ \\
\hline 29 & $90,277,328$ & $1,781,789$ & $4,751,438$ & $6,533,228$ & & & \\
\hline 30 & $85,525,890$ & $1,692,700$ & $4,751,438$ & $6,444,138$ & & & \\
\hline 31 & $80,774,452$ & $1,603,610$ & $4,751,438$ & $6,355,049$ & & & \\
\hline 32 & $76,023,013$ & $1,514,521$ & $4,751,438$ & $6,265,959$ & $6,592,621$ & $19,005,753$ & $25,598,374$ \\
\hline 33 & $71,271,575$ & $1,425,431$ & $4,751,438$ & $6,176,870$ & & & \\
\hline 34 & $66,520,137$ & $1,336,342$ & $4,751,438$ & $6,087,780$ & & & \\
\hline 35 & $61,768,698$ & $1,247,253$ & $4,751,438$ & $5,998,691$ & & & \\
\hline 36 & $57,017,260$ & $1,158,163$ & $4,751,438$ & $5,909,601$ & $5,167,189$ & $19,005,753$ & $24,172,942$ \\
\hline 37 & $52,265,822$ & $1,069,074$ & $4,751,438$ & $5,820,512$ & & & \\
\hline 38 & $47,514,383$ & 979,984 & $4,751,438$ & $5,731,422$ & & & \\
\hline 39 & $42,762,945$ & 890,895 & $4,751,438$ & $5,642,333$ & & & \\
\hline 40 & $38,011,507$ & 801,805 & $4,751,438$ & $5,553,244$ & $3,741,758$ & $19,005,753$ & $22,747,511$ \\
\hline 41 & $33,260,068$ & 712,716 & $4,751,438$ & $5,464,154$ & & & \\
\hline 42 & $28,508,630$ & 623,626 & $4,751,438$ & $5,375,065$ & & & \\
\hline 43 & $23,757,192$ & 534,537 & $4,751,438$ & $5,285,975$ & & & \\
\hline 44 & $19,005,753$ & 445,447 & $4,751,438$ & $5,196,886$ & $2,316,326$ & $19,005,753$ & $21,322,080$ \\
\hline 45 & $14,254,315$ & 356,358 & $4,751,438$ & $5,107,796$ & & & \\
\hline 46 & $9,502,877$ & 267,268 & $4,751,438$ & $5,018,707$ & & & \\
\hline 47 & $4,751,438$ & 178,179 & $4,751,438$ & $4,929,617$ & & & \\
\hline 48 & (0) & 89,089 & $4,751,438$ & $4,840,528$ & 890,895 & $19,005,753$ & $19,896,648$ \\
\hline 49 & (0) & (0) & - & (0) & & & \\
\hline 50 & (0) & (0) & - & (0) & & & \\
\hline 51 & (0) & (0) & - & (0) & & & \\
\hline 52 & (0) & (0) & - & (0) & (0) & - & (0) \\
\hline 53 & (0) & (0) & - & (0) & & & \\
\hline 54 & (0) & (0) & - & (0) & & & \\
\hline 55 & (0) & (0) & - & (0) & & & \\
\hline 56 & (0) & (0) & - & (0) & (0) & - & (0) \\
\hline 57 & (0) & (0) & - & (0) & & & \\
\hline 58 & (0) & (0) & - & (0) & & & \\
\hline 59 & (0) & (0) & - & (0) & & & \\
\hline 60 & (0) & (0) & - & (0) & (0) & - & (0) \\
\hline & & $104,769,215$ & $228,069,040$ & $332,838,255$ & $104,769,215$ & $228,069,040$ & $332,838,255$ \\
\hline
\end{tabular}


Haulapai Wind Project 200MW

O\&M and Post Warranty Expenses

For Hualapai Project Proforma Financial Model

General Methodology

This schedule provides support for and the calculation of the proforma O\&M expenses and post warranty costs associated with the service, maintenance and repairs of the installed turbines. Most manufacturers offer 2-3 year warranties and require 5 year service/maintenance agreements as part of the purchase of the turbines. For an additional cost, the buyer can take out a full 5 year warranty. Additionally, the OEM will offer various postwarranty services that can be contracted at time of purchase, such as condition monitoring, extended service/maintenance, preferred parts pricing, availablity insurance, etc. For purposes of this proforma, the OEM approach to post-warranty operations is assumed. The fixed cost associated with this approach is generally expected to be slightly higher than an independent service provider or internally servicing the turbines. Howeve the OEM approach also tends to have fewer variable and non-controlled costs which provideds increased certainty in the future cost of service, maintenance and repairs. It is anticipated that this approach by having greater certainty in the modelling of the Hualapai 200MW Wind Project will be more attractive to potential financing partners.

Proforma Hualapai Capacity

(per WAPA Feasibility Study request)

Turbine Cost - GE-1.6xle/82.5, 80m hub height

Preliminary quote from GE rep to MJH

Est market discount available off of quote $-15 \%$

Incremental cost - $1.7 \mathrm{MW} 100$ meter rotor

Estimated installed cost for GE-1.7/100m

Total turbines for Hualapai capacity

Net Capacity Factor - GE-1.7/100 80 m hub height

$\$ /$ Kw Installed

\begin{tabular}{cc} 
& 1,100 \\
$\$$ & $(165)$ \\
& 125 \\
\hline$\$$ & 1,060 \\
\hline
\end{tabular}

200 MW

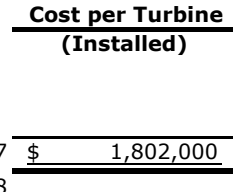

118

$27.06 \%$

\section{INPUTS FOR OPEX COMPONENTS -}

Primary sources:

(1) National Renewable Energy Laboratory - Cost Trend Study (NREL/CP-500-46180 12/09)

(2) Roeper, U. (President, ORTECH Power) presentation - Capex, Opex, Feasibility - 11/07

(3) O\&M detail from comparable models by Marion Horna, PE \& Principal of MJH Power

Total OPEX as \% of revenues - industry data

$20 \%-25 \%$ levelized

Components of O\&M (source (2) as reference) Service/maint.

Warranty - standard manufacturer's 2 yr.

Taxes and insurance

Land maint.

BOP

Admin

Reserve for unscheduled maint/repair

Total unescalated \$ / WTG

OPEX \$/kWh for GE-1.7/100 spec'd above

$\%$ of total Year 1 revenue rate (costs not escalated

\begin{tabular}{|c|c|c|}
\hline $\begin{array}{l}\text { Warranty Period } \\
\$ / \text { WTG per Year }\end{array}$ & \begin{tabular}{|l|} 
Post-Warranty \\
\$ / WTG per Yead
\end{tabular} & $\begin{array}{c}\text { Post-Reserve } \\
\text { \$ / WTG per Year }\end{array}$ \\
\hline$($ (Yrs $1 \& 2)$ & $(\operatorname{Yr} 3-20)$ & $(\operatorname{Yr} 21-25)$ \\
\hline 25,000 & 30,000 & 30,000 \\
\hline 15,000 & - & - \\
\hline 16,000 & 16,000 & 16,000 \\
\hline 5,000 & 5,000 & 5,000 \\
\hline 5,000 & 5,000 & 5,000 \\
\hline 5,000 & 5,000 & 5,000 \\
\hline- & 20,000 & - \\
\hline 71,000 & 81,000 & 61,000 \\
\hline$\$ 0.0000$ & $\$ 0.0000$ & $\$ 0.0000$ \\
\hline $0.0 \%$ & $0.1 \%$ & $0.0 \%$ \\
\hline
\end{tabular}

Approximate range of recent vintage WTG's

(Source (1) above, Figures 4 and 5)

$\$ .008$ to $\$ .016 / \mathrm{kWh}$

Conclusion:

For the Hualapai Wind Project an OPEX cost build-up has been prepared based on the historical OPEX data available as noted above. Total levelized OPEX $\$ / \mathrm{kWh}$ will fit within the ranges articulated recognizing this project's lower net capacity factor increases the cost/kWh.

\section{Hualapai Wind Project OPEX Assumptions}

Components of O\&M (source (2) as reference) Service/maint.

Warranty and OEM services - 5 yr warranty Insurance (property taxes stated separately)

Land maint.

BOP

Admin

Bird mitigation system

Reserve for unscheduled maint/repair

OPEX $\$ / k W h$ for GE-1.7/100 spec'd above $\%$ of total Year 1 revenue rate (costs not escalated

\begin{tabular}{|c|c|c|}
\hline \multicolumn{3}{|c|}{ Current Costs before Escalation for CPI } \\
\hline $\begin{array}{c}\text { Warranty Period } \\
\text { \$ / WTG per Year }\end{array}$ & $\begin{array}{c}\text { Post-Warranty } \\
\text { \$ / WTG per Year }\end{array}$ & $\begin{array}{c}\text { Post-Reserve } \\
\text { \$ WTG per Year }\end{array}$ \\
\hline (Yrs 1 - 5) & (Yr 6 - 20) & $($ Yr 21 - 25) \\
21,000 & 23,000 & 28,000 \\
19,000 & 4,000 & - \\
7,000 & 7,000 & 7,000 \\
3,000 & 3,000 & 3,000 \\
3,500 & 5,500 & 5,000 \\
3,500 & 3,500 & 3,500 \\
2,500 & 2,500 & 2,500 \\
- & 20,000 & 10,000 \\
\hline 59,500 & 68,500 & 59,000 \\
\hline \hline & & \\
$\$ 0.0146$ & $\$ 0.0168$ & $24.1 \%$ \\
$24.3 \%$ & $28.0 \%$ & 0.0144 \\
\hline
\end{tabular}




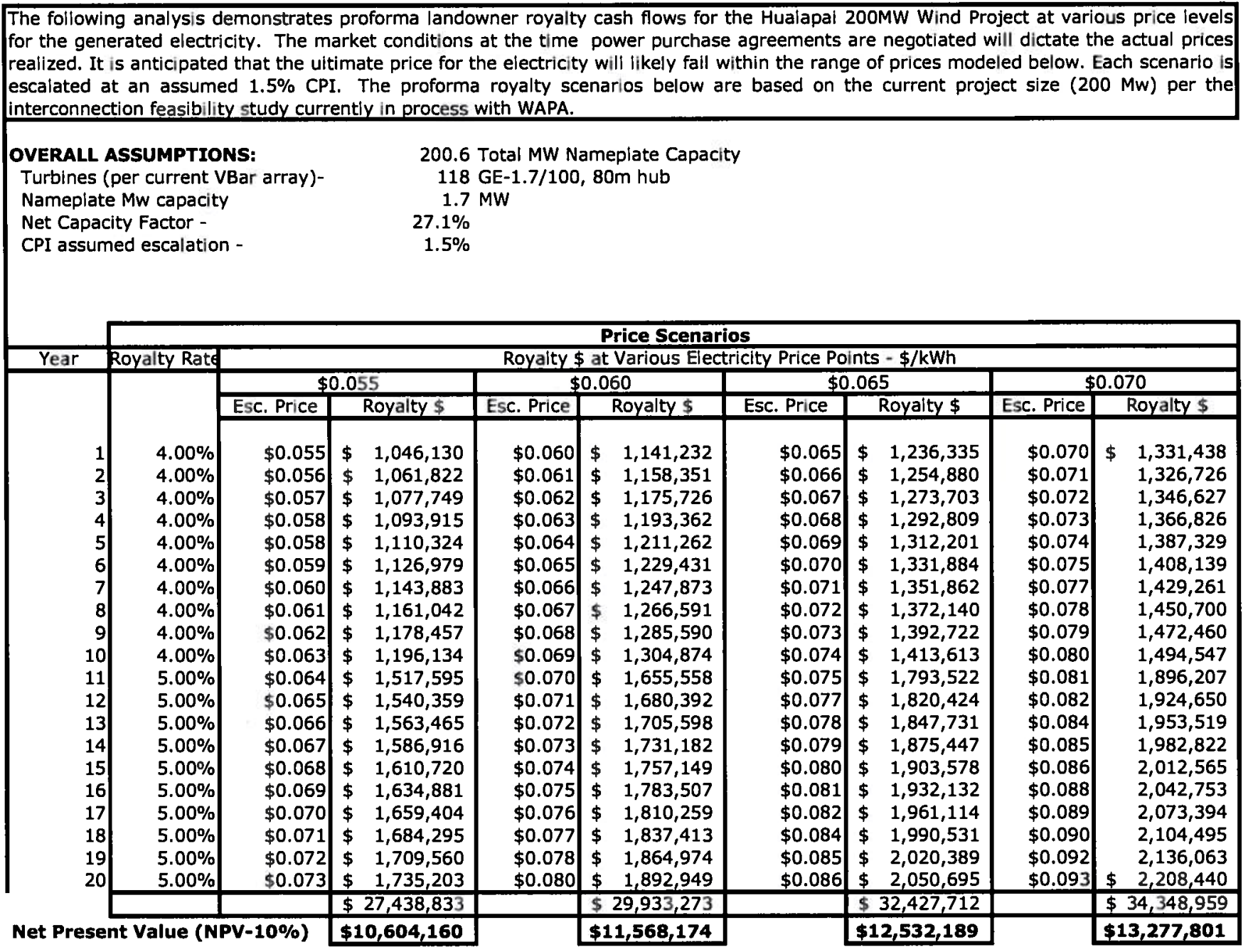




\section{Exhibit N}

Introduction to Financing for Project

(Squire Sanders Dempsey) 


\section{Finance Introduction}

Consultants for the Hualapai Tribe ("Tribe") have considered a number of potential arrangements for renewable energy development by the Tribe; these sites are located on tribal lands and BLM lands. Consultants for the Tribe considered both wind and solar projects and evaluated the feasibility of developing renewable energy resources at each site by studying interconnection, transmission, biological resources, environmental impacts, viewshed impacts, and other related factors such as federal and local regulations. These factors have led the Tribe and its consultants to narrow the list to four options and ultimately to recommend a single $200 \mathrm{MW}$ wind project with a $50 \mathrm{MW}$ solar project located entirely within the Reservation. This section discusses some of the typical financing arrangements for the development of renewable energy projects and highlights some important considerations for the Tribe.

\section{a. Types of Financing Commonly Used to Develop Renewable Energy and Applicability}

Parties developing renewable energy projects currently use a range of mechanisms to finance each project. Financial institutions, developers, and attorneys will typically select a mechanism that meets the specific needs of each client and each project based on a number of factors described below. It is too early to know which of the following mechanisms will best serve the needs of the Tribe-that decision will need to be made by the Tribe's financial advisors and the Tribal Council once the specifics of the project and the Tribe's financial resources are better defined-but these are the most common alternatives and it is likely that one or more of these can be used to provide financing for the selected project.

\section{i. Non-recourse Project Financing}

Non-recourse financing is perhaps the most common form of project financing and consists of borrowing money against the future anticipated revenue of a financed project. This is a possible financing mechanism for any project that has a predicted stream of reliable revenue. A non-recourse loan would be especially attractive to a lender if the borrower has secured a Power Purchase Agreement ("PPA") as such an agreement can be used to determine the base level of revenue that can be expected as well as the period of time over which the revenue will generated. The revenue stream and the project itself serve as collateral for the loan. The Lender is guaranteed payment by the revenues generated by the project, and in the event that the borrower stops making payments on the loans, the lender can take steps to ensure performance of the PPA and repayment of the loan. These steps can include seizure of the asset. Any project that would be involve selling power to a third party such as a utility would be suitable candidates for non-recourse financing.

If the project is developed to provide power for tribal use rather than for sale to a third party, then non-recourse financing may not make sense because there would be no anticipated revenue stream on which to rely for loan repayment. For example, at times the Tribe has discussed developing wind power in the Grand Canyon West area for tribal use. Financial institutions may be understandably reluctant to loan money against a non-existent revenue stream. In the event that the Tribe chooses to develop a project solely to meet its own power needs, non-recourse financing many not be the best choice for that aspect of the project.

From a financing perspective, a project designed to provide power on the Reservation could be made more attractive from the lender's perspective if the Tribe were to form a properly capitalized 
Tribal Utility Authority. In that scenario, the entity developing the project and the Tribal Utility Authority could enter into a PPA setting rates and terms between the corporate entities that develop the wind project and the tribal utility company.

\section{ii. Corporate Financing}

For projects that are not designed to sell power to third parties, but are intended to provide power to the Tribe, corporate financing may make sense. The Tribe can look to borrow against the strength of its own balance sheet in order to fund a small-scale project designed to provide power for tribal use. The advantage of corporate financing is that it can obviate the need for a revenue stream from the financed project and may afford the Tribe better terms than would otherwise be available. This method of financing is however, contingent upon the financial health of tribally controlled entities and the Tribe's ability to post suitable collateral for the loan.

In the event that the Tribe decides to explore corporate financing, it may wish to consider creating a special purpose corporate entity for financing, this could be a Tribal Utility Authority, or it could be a separate corporate entity that is meant to exist solely for the financing and construction of the project. In either instance, creation of a properly organized and capitalized entity can serve to protect tribal interests and resources. Final recommendations on any corporate financing, as well as a complete analysis of tribal financial resources and associated risks should involve the Tribe, legal counsel, and the Tribe's financial advisor.

\section{iii. Tax Financing}

Tax financing involves leveraging current tax subsidies offered by the federal government over the long term to gain access to capital in the short term. Tribal corporations organized under section 17 of the Indian Reorganization Act of 1934 are not subject to federal income tax on its income. ${ }^{1}$ As the Tribe derives no direct benefit from federal tax incentives for the development of renewable energy, it can effectively sell those benefits to a partner who is subject to federal taxes. There are a number of current tax subsidies available, but the list changes from year to year depending on the economic and political climate.

\section{Existing Tax Incentives}

There are a number of tax incentives available to help finance renewable energy developments, though many are set to expire on December 31, 2012. Below is a discussion of federal ${ }^{2}$ options:

\footnotetext{
${ }^{1}$ Rev. Rul. 94-16, 1994-1 C.B. 19.

${ }^{2}$ There is one significant program run by the State of Arizona, the Renewable Energy Business Tax Incentives. This Program is designed for projects that are subject to local property taxes and state income taxes. These incentives could be useful for a tribal partner who wishes to develop manufacturing capabilities within Arizona. Businesses can qualify for up to a $10 \%$ reduction in state income tax and a $75 \%$ reduction in property tax if certain criteria are met. To generate tax credits a renewable energy manufacturing companies must create 1.5 new full-time jobs for every $\$ 500,000$ of capital investment. Businesses making new qualifying investment of $\$ 25$ million or more in Arizona in manufacturing and/or headquarter operations in renewable energy industries are eligible for: (1) 10 years of property tax savings, if the company pays at least $51 \%$ of the net new full-time employment positions between $125 \%$ and $199 \%$ of the annual median wage (currently $\$ 33,051$ ) or (2) 15 years of property tax savings, if the company pays at least $51 \%$ of the net new full-time employment positions $200 \%$ or more of the annual median
} 
- Business Energy Investment Tax Credit. The ITC is a corporate tax credit (30\% for solar, fuel cells, and small wind, and $10 \%$ for geothermal, micro turbines, and combined heat and power (CHP) systems. This tax credit expires for large-scale wind projects not in production by December 31, 2012, but remains in place for solar development through 2016. A U.S. Senate budget bill included a one-year extension of the wind credits but has yet to move forward..$^{3}$

- Renewable Electricity Production Tax Credit. The PTC is a corporate tax credit for wind energy projects $(\$ .022 / \mathrm{kWh}$ for the first 10 years of operation). This tax credit also only applies to projects in service by December 31, 2012 though a one-year extension has been proposed.

\section{Tax Equity Financing}

Tax equity financing is a relatively new method of financing renewable energy projects and is dependent on federal or state renewable energy policies that provide tax credits and tax benefits for renewable energy development, as described above in subsection c. Federal tax credits and other tax benefits are some of the most used tools to incentivize development of renewable resources. Examples of tax benefits include:

- A 30\% investment tax credit, available through 2016 for solar and through 2012 for wind,

- A production tax credit of $2.2 \mathrm{c} / \mathrm{kWh}$ for wind and $1.1 \mathrm{c} / \mathrm{kWh}$ for solar, this is only available to those who do not use the investment tax credit, and

- Accelerated depreciation which can be deducted against taxable income. ${ }^{4}$

These tax credits and other benefits are most valuable to those who have enough taxable income to pay income taxes. As a result, developers of new projects, such as the Tribe, may not be able to reap the full benefit of the renewable energy tax incentives. The developer can transfer the tax benefits to other parties who can realize the full range of the tax benefits in exchange for funds. This transfer of the developer's tax benefits and incentives in exchange for funds-essentially the sale of the tax benefits to a third-party-is known as tax equity financing.

The tax benefit can be taken up front as a $30 \%$ investment tax credit, useful for raising a significant amount of capital up front. This is an attractive scenario to investors facing an immediate tax and need to offset current earnings. The tax benefit can be spread out over time as a production credit. The production tax credit runs over the course of ten years, this makes it especially attractive to investors who have a predictable and steady stream of revenue over the long term. But if an investor believes it only has sufficient taxable income to benefit from the credit in six of the ten years, the value of the investment drops.

Tax equity financing does have limitations. Tax equity financing is relatively rare (but becoming more common), transaction costs can be high, and it can make additional financing difficult. Regardless of the

wage. Both the real and personal property can be reclassified to Class 6 property for both primary and secondary property tax purposes. \$139M in tax credits remain for 2012 and $\$ 70 \mathrm{M}$ for 2013.

${ }^{3}$ Updates periodically available at http://thomas.loc.gov/cgi-bin/bdquery/z?d112:s.3521:, and

http://www.dsireusa.org/incentives/incentive.cfm?Incentive_Code=USO2F.

${ }^{4}$ American Recovery and Reinvestment Tax Act of 2009, Sec. 1101-1102. 
class of tax credit used as equity, there are significant costs associated with such a financing. Tax equity financings are complex and must be tailored to the needs of each project. For example, the Tribe must first undertake a complicated legal analysis to determine which credits the proposed project is eligible for and then seek out a qualified investor. Perhaps the most significant complication for the Tribe is that tax equity financing would make the overall project somewhat inconsistent with subsequent project level debt. Project level debt, such as a non-recourse loan, would introduce a senior claim on the project's collateral. In the event that the Tribe's project encounters difficulty, the debt holders, not the tax equity investors, have first claim on project asset. Many tax equity investors will not invest in a project where there is also a lender providing debt that would have senior claim. This risk could be mitigated, but not eliminated, by the presence of a 20-year PPA if the amount of the senior project level debt is small relative to the value of the 20-year PPA. The tax equity investor may ask for a premium on its investment in exchange for the added risk of senior project level debt. The Tribe could pursue other forms of financing, such as a corporate loan, that provide alternative forms of collateral, other than the renewable energy project, to secure the loan.

Within the world of tax equity financing, there are three common scenarios, the partnership flip, the lease pass through, and the sale leaseback. Each of these options uses the available tax credit to induce the investment of a development partner who has available capital in exchange for the tax credit.

In the partnership flip, the Tribe and a tax investor form a partnership and own the project jointly. The tax investor makes a disproportionately larger initial investment. In exchange, the tax investor receives a larger portion of the income generated (frequently 99\%) from the project via both power sales and the aforementioned federal incentives. This arrangement continues until a previously-agreed upon rate of return for the tax investor is reached-typically about six years. At that time, the allocation of the project's income stream "flips," and the Tribe-as the developer-then earns the majority of the proceeds from the project. Partnership flips are often constructed to allow for the developer/Tribe to purchase the entire project post flip. In any case, the investor's continuing stake in the project is typically small and is agreed upon by the parties.

In a lease pass through the Tribe builds the project and leases it to the tenant (which will include the tax equity investor) and elects to pass the tax credit through to the tenant. In this scenario the investor (as the lessee) sells the power and pays rent to the Tribe on the land. Rent is in the neighborhood of $\$ 4,000$ to $\$ 6,000$ annually per megawatt of installed tower capacity for fixed-compensation arrangements. ${ }^{5}$ Rent under royalty-based leases is typically between three and five percent of gross revenue from electricity sales. ${ }^{6}$ A lease pass through structure saves the Tribe the difficulty of operating the project on a day-to-day basis.

In terms of ownership, a sale leaseback is the opposite of the lease pass through. In a leaseback, the Tribe finances and builds the project, but sells it to the tax equity investor. After the sale, the Tribe then leases the project back from the tax equity investor. The tax equity investor legally owns the asset and continues to enjoy the tax incentives, but the Tribe would run the project and sell the power, through an entity such as a Tribal Utility Authority. These different financing options allow for variety of options in

\footnotetext{
${ }^{5}$ Aakre and Haugen, Wind Turbine Lease Considerations for Owners, EC-1394 February 2009, available at http://www.ag.ndsu.edu/pubs/agecon/market/ec1394.htm; Wind Energy Easements and Leases: Compensation Packages, Windustry, June 2009, available at http://www.windustry.org/sites/windustry.org/files/Compensation2009-07-06.pdf.

${ }^{6} / d$.
} 
terms of project ownership, the responsibility of day-to-day operations, and project construction responsibility.

\section{b. Government Grants and Assistance}

There are a few federal government grants available at this time that focus primarily on renewable energy development in tribal communities or on renewable energy development in general. It is possible that this may change after the federal budget cycle. Grants to note for future consideration include:

- $\quad$ DEMD. The Department of Interior's Division of Energy and Mineral Development ("DEMD") program provides scientific and technical assistance to tribes developing renewable energy for investors and energy providers. Assistance available includes opportunity assessment, feasibility studies, business planning, marketing, negotiation assistance, access to capital, capacity building, community planning, and sustainable economic development.

- Energy and Mineral Development Program ("EMDP"). Administered by DEMD, the EMDP solicits proposals from Tribes and using a competitive review system selects qualified projects for funding. DEMD staff will also monitor those projects to ensure that the best possible product is obtained for the funds allocated. EMDP projects may include such activities as performing initial exploration and defining potential targets for development; performing market analyses to establish production/demand for a given commodity; provide outreach and education to Tribes concerning energy or mineral development issues; perform economic evaluation and analyses of the resource; and promote completed projects at industry conferences and to prospective partners or investors. The 2013 solicitation for applications is expected to be released in December of 2012 with a deadline in March 2013 (based on the 2012 application cycle).

- Tribal Energy Program. The Department of Energy's Tribal Energy Program provides financial and technical assistance that enables tribes to evaluate and develop their renewable resources. No solicitations are available at this time. The Department will accept unsolicited applications on a case-by-case basis if the project meets specific criteria.

- Strategic Technical Assistance Response Team ("START") Program. The US Department of Energy Office of Indian Energy Policy and Programs started this initiative in 2011 to provide technical expertise to support development of next-generation energy projects in Indian County. The Hualapai Renewable Energy Development Program was selected by DOE-OIEPP to receive direct START technical assistance. START assistance is non-monetary assistance targeted at assisting the Tribe to quickly implement its renewable energy objectives.

\section{Impact of Project Location on Financing Options}

From the initial list of project sites, the Tribe's consultants narrowed the possible projects down to two $200 \mathrm{MW}$ arrays located entirely within the Reservation. Each array would also include an additional $50 \mathrm{MW}$ solar component. The combined solar and wind components would be combined for a $250 \mathrm{MW}$ interconnection with WAPA's Peacock to Mead 345KV transmission line. The Tribe's consultants recommend the $200 \mathrm{MW}$ array of GE 1.7/100 turbines on tribal lands due to their greater efficiency and productivity. 
There has also been some discussion of constructing of a $250 \mathrm{~kW}$ or $500 \mathrm{~kW}$ solar project with utility grid connection and no storage located near the airport terminal and built for the exclusive use of the Tribe. This solar array would connect to a future $20.8 \mathrm{kV}$ distribution system designed to serve Grand Canyon West. Once constructed, this distribution system would be powered by diesel generators in the short term. In the long-term, the distribution system is designed to be connected to the UniSource power grid and the generators will be used for emergency backup. When this occurs, the solar array may be directly metered to UniSource.

The location of the project plays an important, but not necessarily determinative, role in determining tribal jurisdiction over any disputes between the Tribe and its partners. To exercise tribal jurisdiction over non-Indians, tribal courts must meet the framework of Montana $v$. United States, 450 U.S. 544, (1981). ${ }^{7}$ In Montana, the Supreme Court held that absent congressional authorization, Indian tribes lack civil authority over the conduct of non-members within a Reservation, subject to two exceptions: the first exception relates to nonmembers who enter consensual relationships with the tribe or its members; the second concerns activity that directly affects the tribe's political integrity, economic security, health, or welfare. ${ }^{8}$ A consensual relationship with a tribe includes: commercial dealing, contracts, leases, or other arrangements. ${ }^{9}$

By entering into a project financing agreement with the Tribe, lenders and investors enter into a consensual relationship and therefore would likely be subject to tribal jurisdiction with respect to any dispute arising from the agreement. For those projects located within the Hualapai Reservation, the Tribe would also exercise almost exclusive control over the project site and therefore the revenue stream and the collateral. As the Tribe approaches lenders and investors, it should be sensitive to concerns that parties may have both with respect to being subject to tribal jurisdiction as a tribal partner and due to the extensive control the Tribe will have over the asset itself. The recent developments in the disagreement between the Tribe and the developer of the Skywalk illustrate the need for project developers on tribal lands to obtain limited, concrete, waivers of sovereign immunity from the Tribe. The Tribe should expect to provide a limited waiver of sovereign immunity to any project partner and to agree in writing to jurisdiction and choice of law if disagreements arise and the Tribe or its partner wish to seek resolution in court.

\section{Next Steps}

To date the Tribe has applied, through its consultants, for a variety of federal programs such as START (which it successfully won) through the Department of Energy. These programs provide access to federal funds to develop the earliest stages of renewable energy projects as well as access to experts who can assist the Tribe with project development and market feasibility. The Tribe should continue to take advantage of these programs to further its efforts to develop a wind facility at the selected site by developing additional wind data to reduce the uncertainties in the current wind resource projections as suggested by V-BAR. As the Tribe collects additional data showing that the project is feasible, it will be easier to raise the necessary capital.

It may be useful to the Tribe to prioritize its goals with respect to project development. Does the Tribe want to sell power to earn a profit? Or does the Tribe want to develop renewable resources solely

\footnotetext{
${ }^{7}$ Smith v. Salish Kootenai College, 378 F. 3d 1048, 1051 (9th Cir. 2004).

${ }^{8}$ Montana, 450 U.S. at 565-66.

${ }^{9}$ Id.
} 
for consumption on the Reservation? The answers to these questions will help the Tribe determine its priorities as it seeks out potential project partners and explores financing options. As the Tribe determines which course to take, where the power will be consumed, and what type of power will be generated, it can begin to more closely examine available financing options. Recent meetings indicate a desire to develop power for local consumption on the Reservation at Peach Springs and Grand Canyon West. As it negotiates with project partners, the Tribe may wish to secure some percentage of the power for tribal consumption. In the alternative, revenues from the wind project can be used to fund power development for tribal consumption at a later date. In order to secure third-party funding however, the Tribe will most likely need to agree to sell most of the power, at least initially.

Now that the Tribe's consultants have identified initial goals and selected a promising project site, the Tribe needs to consult with a financial advisor to discuss the costs of financing the project. The financing questions will depend on the details of the financed project, the Tribe's financial situation, and on the energy needs of the Tribe. A financial advisor can evaluate the Tribe's current and projected financial situation, evaluate available financing options and advise the Tribe as to the best options. A financial advisor can also advise the Tribe regarding the economic cost of each financing option and help select the one that is best for the Tribe. Once the Tribe and its financial advisor select a financing option-which will likely affect the ownership structure of the project-the Tribe's attorneys can create the legal documents that will govern financing and ownership.

Next steps for the Tribe include:

- Determining tribal priorities with respect to:

- Providing power to the Reservation, and

- Generating revenue;

- Creating a Tribal Utility Authority or other special purpose corporate entities to develop the project;

- Consulting with a financial advisor regarding likely financing scenarios, the costs of each, and selection of the best financing option;

- Negotiating financing based on project details and tribal needs; and

- Working with attorneys and consultants to structure the financing and ownership arrangements for the selected project. 


\section{Appendix $\mathrm{O}$}

Revised Financial Model

Ater Wynne, LLP \& First

American Financial

Advisors 


\title{
ATERWYNNE
}

ATTORNEYS AT LAW

\section{Financial Model Presentation}

\author{
WORKING WITH A \\ FINANCIAL ADVISOR \\ FOR \\ HUALAPAI'S \\ NEW WIND FARM
}
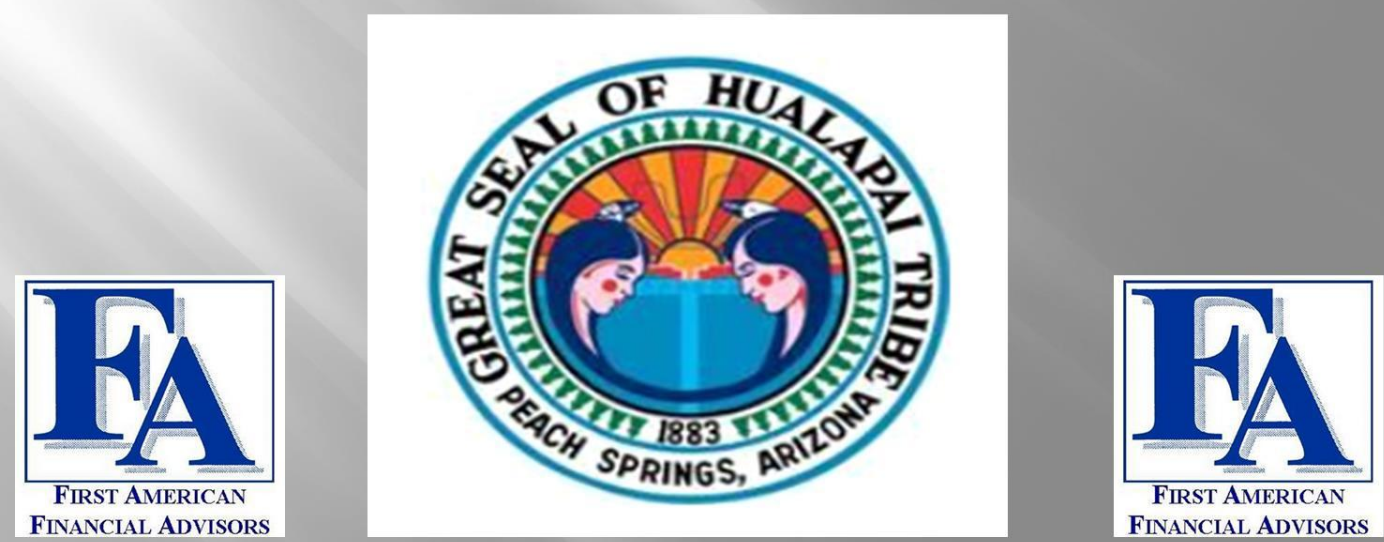


\section{ATERWYNNE}

ATTORNEYS AT LAW

\section{Assumptions to Model}

\section{HUALAPAI WIND FARM MODEL AND COST ASSUMPTIONS}

June 29, 2013

1. Interest rates will rise over the next two years

2. It will take two years to complete EIS and other site development issues

3. Accelerated depreciation for renewable energy projects will remain unaffected by Congress

4. Investment tax credits for renewable energy projects will remain unaffected by Congress

5. Only $4.5 \%$ of the funding will come from New Market Tax Credits

6. Equity investors will receive a required minimum distribution of $\$ 750,000$ per year

7. The Hualapai Tribe will receive a minimum operating lease rate, beginning at $\$ 1,141,232$ per year

8. Inflation, lease and $\mathrm{O} \& \mathrm{M}$ escalators are computed using a 3\% annual rate

9. Operating and Maintenance expenses are $\$ 6,926,000$ for the first year

10. PPA annual sales rates do not come from Pro-forma prepared by Marion J. Horna, dated 11/25/12

11. Interest rate on debt service is $6 \%$

12. Debt Service Reserve Fund is $\$ 12,200,000$ (roughly equal to one year debt service)

13. Construction period interest is equal to $60 \%$ of the loan outstanding for 1 year at $6 \%$ interest

14. PPA is for 25 years

15. Construction costs are from Marion J. Horna \& MJH Power Consulting Inc.

16. A new cost item titled "Contigency Costs" has been added

17. Soft costs have become more detailed

18. Construction period interest during construction has been added to soft costs

19. A lease payment to the Hualapai Tribe during construction has been added, in the amount of $\$ 450,000$. 


\section{ATERWYNNE LLP}

ATTORNEYS AT LAW

\section{Sources of Capital for Project}

\begin{tabular}{|l|r|r|}
\hline SOURCES: & & \\
\hline USDA Loan & $\$ 162,753,098$ & $46.85 \%$ \\
\hline Capitalized Investment Tax Credit & $\$ 90,910,390$ & $26.17 \%$ \\
\hline Capitalized Depreciation & $\$ 93,729,640$ & $26.98 \%$ \\
\hline \hline & & \\
\hline Total Sources & $\mathbf{\$ 3 4 7 , 3 9 3 , 1 2 8}$ & $\mathbf{1 0 0 . 0 0 \%}$ \\
\hline
\end{tabular}

Sources of Funds
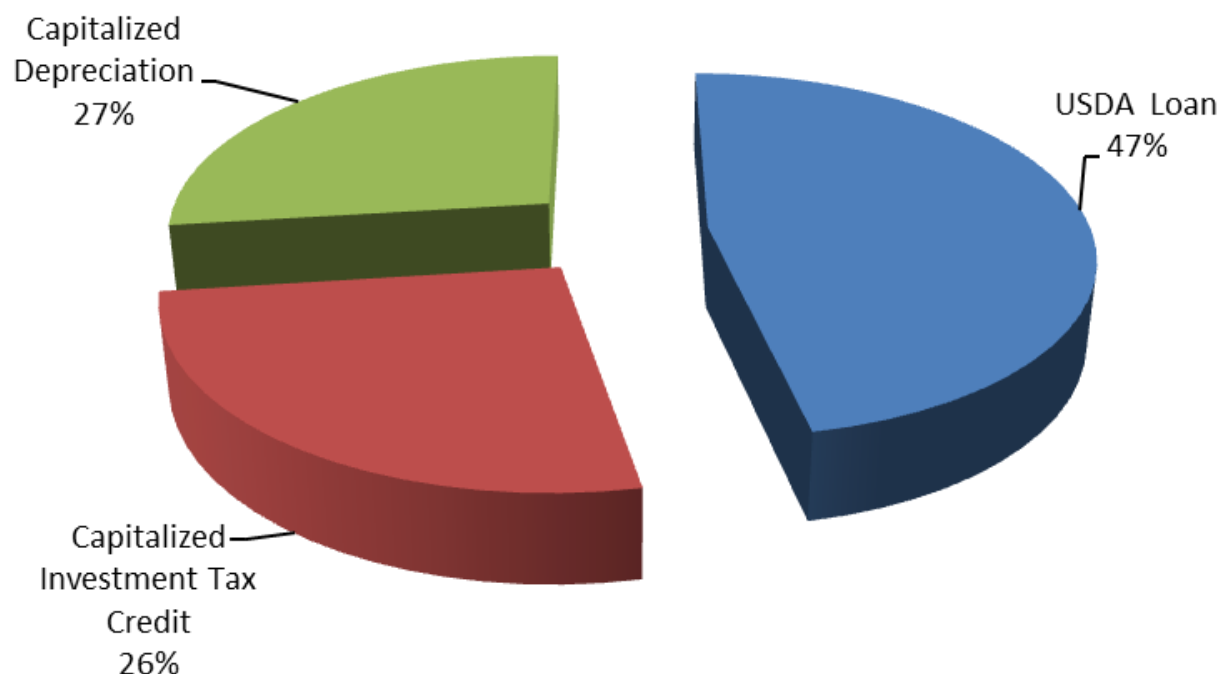


\section{ATERWYNNE}

ATTORNEYS AT LAW

\section{Uses of Capital}

\section{USES:}

Equipment Costs

$\$ 212,000,000$

$61.03 \%$

Transmission Costs

$\$ 11,000,000$

$3.17 \%$

Construction Costs

$\$ 96,429,963$

$27.76 \%$

Soft Costs

$\$ 24,963,165$

$7.19 \%$

Contingency Costs

$\$ 3,000,000$

$0.86 \%$

Total Uses

$\$ 347,393,128$

$100.00 \%$

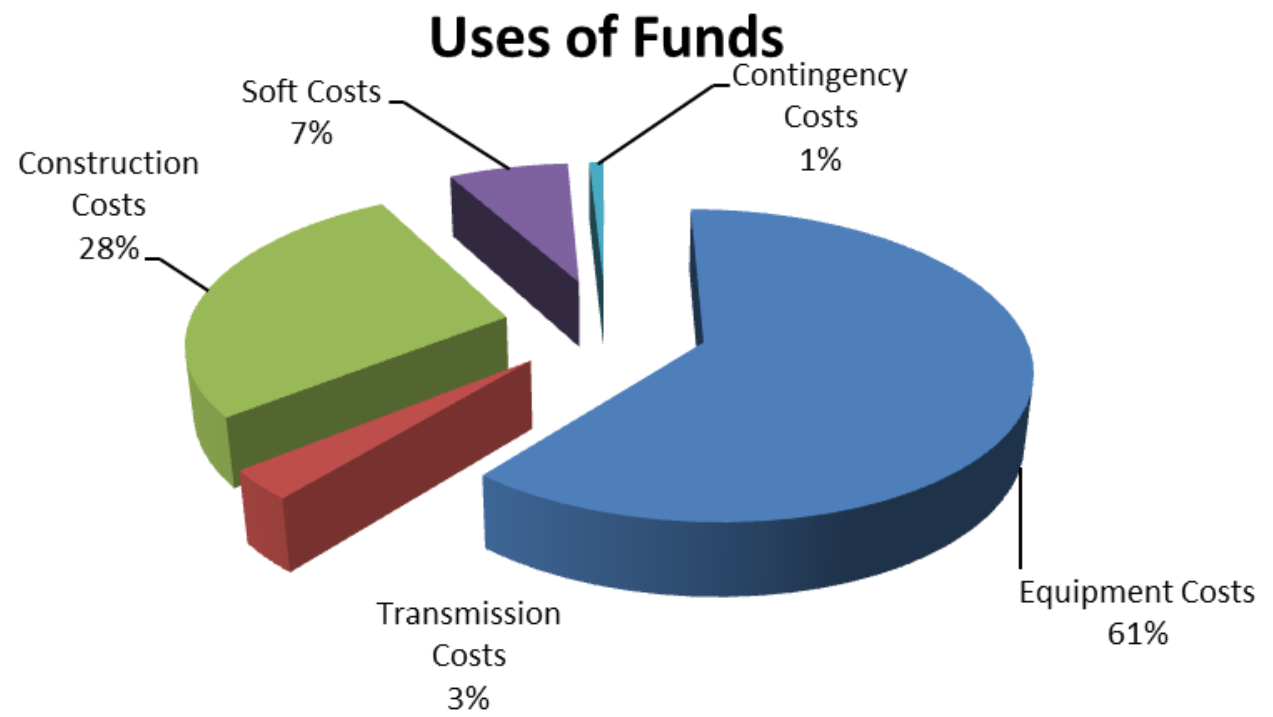




\section{ATERWYNNE}

ATTORNEYS AT LAW

\section{Equipment \& Construction Costs}

\section{Equipment \& Construction Costs}

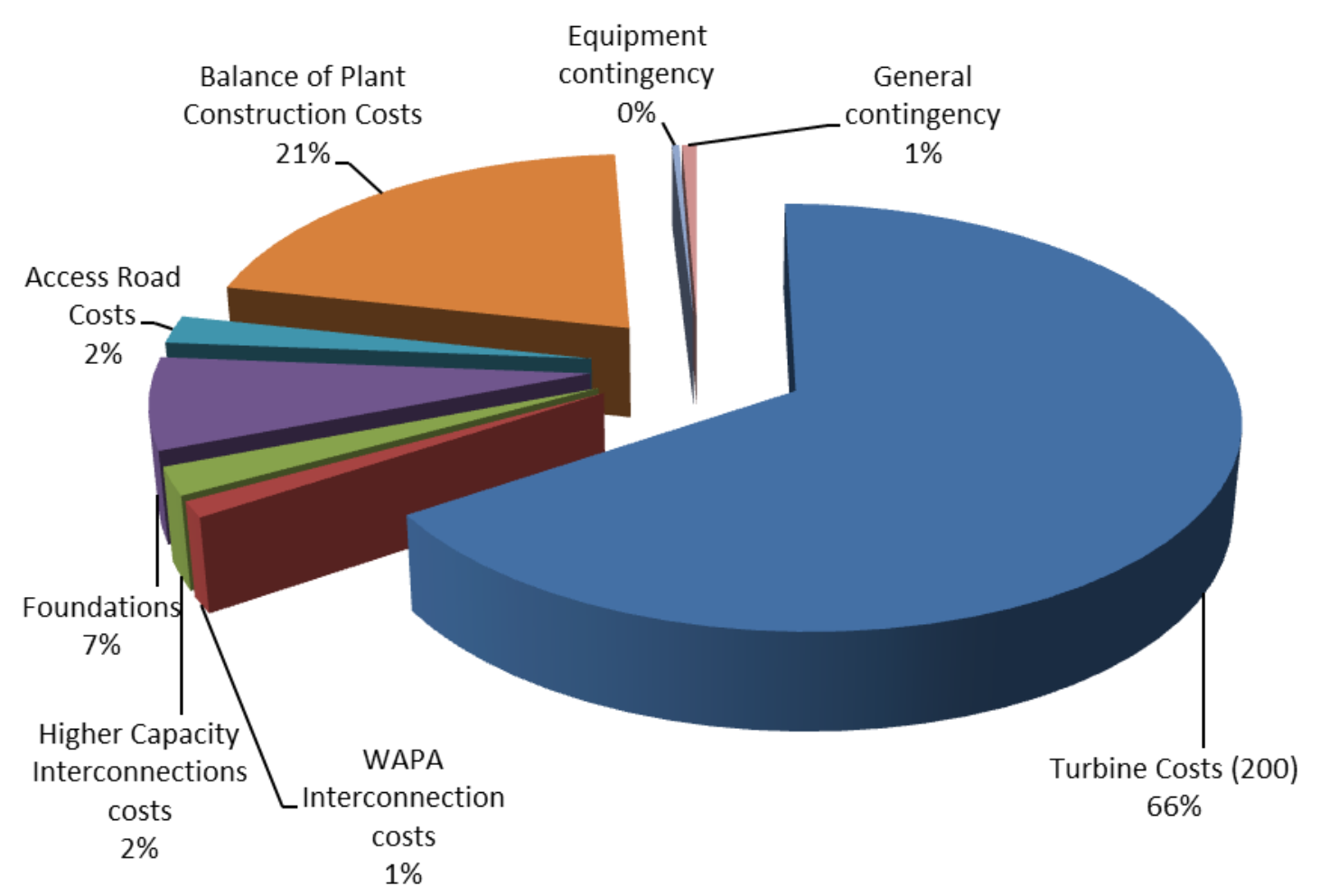




\section{ATERWYNNE}

ATTORNEYS AT LAW

\section{Soft Costs}

\section{Soft Costs}

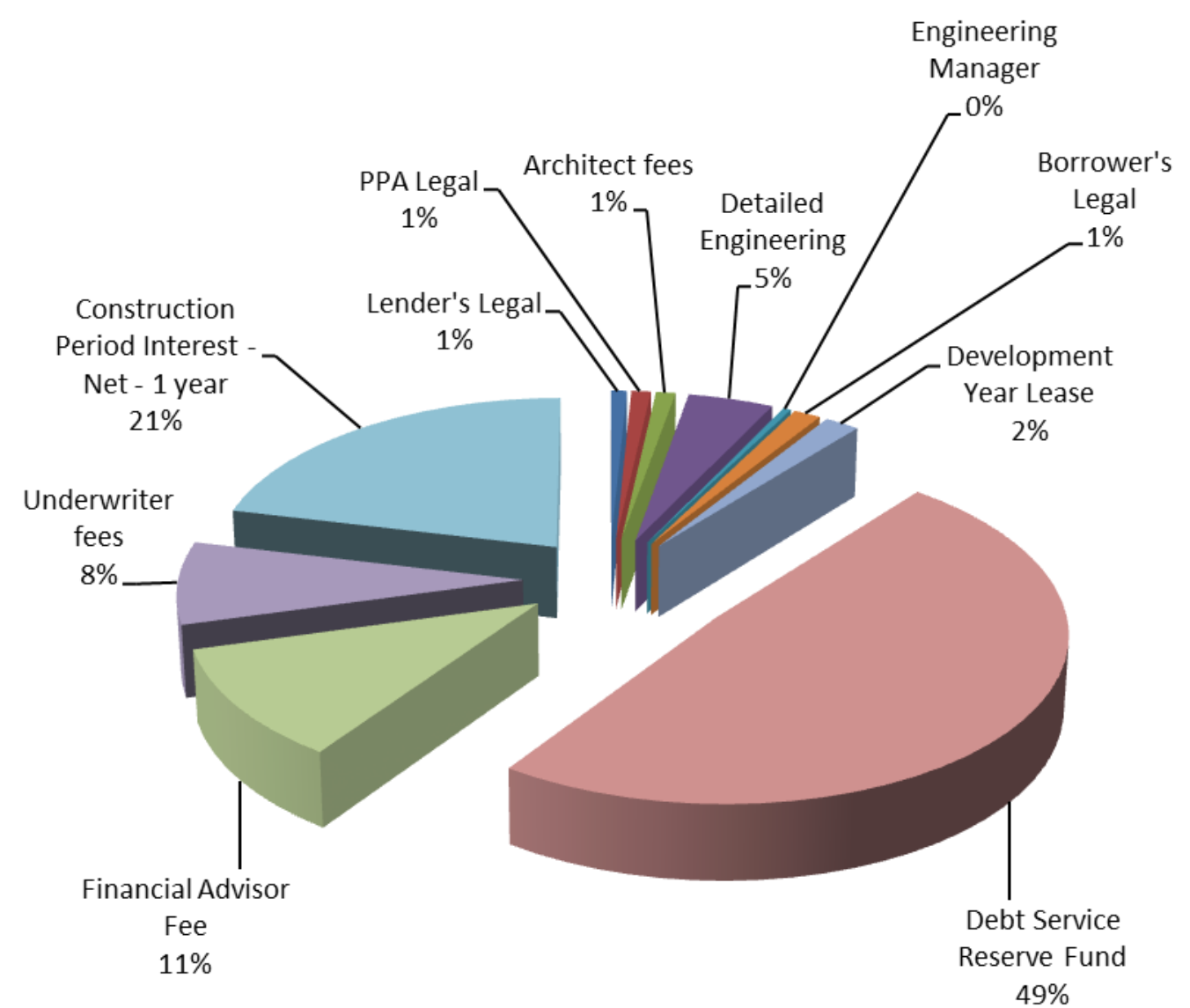




\section{ATERWYNNE}

ATTORNEYS AT LAW

\section{What is a Financial Model?}

\begin{tabular}{|c|c|c|c|c|c|c|c|c|c|c|}
\hline $\begin{array}{l}\text { Hualapa } \\
\text { Model is sut }\end{array}$ & W/nd Farm & $\begin{array}{l}\text { roject - } \\
\text { verification }\end{array}$ & June 29 & 2013 & & & $\begin{array}{l}\text { repared by Fi } \\
\text { NP) }\end{array}$ & st American Finan & visors, Inc. & \\
\hline $\begin{array}{l}\text { Total Const } \\
\text { RR for Tax } \\
\text { Investor }\end{array}$ & $\begin{array}{l}\text { truction Cost (in } \\
\text { Equity }\end{array}$ & ludes construct & iction interest) & $\begin{array}{r}347,393,128 \\
10.00 \%\end{array}$ & Sources \& & Jses of Funds for & \begin{tabular}{|l|} 
Loan \\
$\$ 347,393,128$ \\
\end{tabular} & & $\begin{array}{r}\text { Annual Loa } \\
\$ 14,082 \\
\end{array}$ & $\begin{array}{l}\text { Service } \\
2.09 \\
\end{array}$ \\
\hline $\begin{array}{l}\text { Loan Rate } \\
\text { Investor Ta }\end{array}$ & & & & $\begin{array}{r}6.00 \% \\
33.00 \%\end{array}$ & Uses & & & & & \\
\hline Sources of & Funds & & & & Construction Fund (Incl & | road) & $\$ 319,429,963$ & & & \\
\hline & & & & & Soft Costs (incl DSR ar & nd Const. Int) & $\$ 24,963,165$ & & & \\
\hline Inv. Tax Cre & edits & $90,910,390$ & $26.17 \%$ & & Contingency & & $\$ 3,000,00 \mathrm{~d}$ & & & \\
\hline pepreciation & $n$ (tax equity) & $93,729,640$ & $26.98 \%$ & & & & $\$ 347,393,128$ & & & \\
\hline Loan Debt & 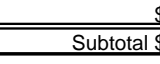 & $\begin{array}{r}162,753,098 \\
347,393,128 \\
\end{array}$ & $\begin{array}{r}46.85 \% \\
100.00 \% \\
\end{array}$ & & & & & & & \\
\hline $\begin{array}{c}\text { Year of } \\
\text { Operation }\end{array}$ & $\begin{array}{c}\text { Annual kwh's } \\
\text { Produced }\end{array}$ & $\begin{array}{l}\text { Lease Pay } \\
\text { to HT }\end{array}$ & $\begin{array}{l}\text { Repair \& Main. } \\
\text { Reserve Acct }\end{array}$ & $\begin{array}{c}\text { Annual } \\
\text { O\&M }\end{array}$ & $\begin{array}{c}\text { Annual Debt } \\
\text { Service }\end{array}$ & $\begin{array}{c}\text { Total Annual } \\
\text { Expenses }\end{array}$ & $\begin{array}{c}\text { All-in } \\
\text { cents/kwh }\end{array}$ & $\begin{array}{c}\text { Cost } \\
\text { Per Kwh } \\
\end{array}$ & $\begin{array}{l}\text { Gross } \\
\text { Income }\end{array}$ & $\begin{array}{c}\text { Net } \\
\text { Income }\end{array}$ \\
\hline 0 & & $\$ 450,000$ & & $\$ 0$ & $\$ 0$ & $\$ 0$ & $\$ 0.000$ & $\$ 0.000$ & $\$ 0$ & $\$ 0$ \\
\hline 1 & $475,513,474$ & $\$ 1,141,232$ & $\$ 750,000$ & $\$ 6,926,000$ & $\$ 14,082,172 \$$ & $22,899,404$ & $\$ 0.048$ & $\begin{array}{l}\$ 0.051 \\
\text { IRR of Tax Equity }\end{array}$ & $\$ 24,251,187 \$$ & $1,351,783$ \\
\hline 2 & $475,513,474$ & $\$ 1,175,469$ & $\$ 750,000$ & $\$ 7,133,780$ & $\$ 14,082,172: \$$ & $23,141,421$ & $\$ 0.049$ & Center $\$ 0.052$ & $\$ 24,726,701 \$$ & $1,585,280$ \\
\hline 3 & $475,513,474$ & $\$ 1,210,733$ & $\$ 750,000$ & $\$ 7,347,793$ & $\$ 14,082,172 \$ \$$ & $23,390,699$ & $\$ 0.049$ & $\$ 0.053$ & $\$ 25,202,214 \$$ & $1,811,516$ \\
\hline 4 & $475,513,474$ & $\$ 1,247,055$ & $\$ 750,000$ & $\$ 7,568,227:$ & $\$ 14,082,172: \$$ & $23,647,454$ & $\$ 0.050$ & $\$ 0.054$ & $\$ 25,677,728 \$$ & $2,030,273$ \\
\hline 5 & $475,513,474$ & $\$ 1,284,467$ & $\$ 750,000$ & $\$ 7,795,274$ & $\$ 14,082,172: \$$ & $23,911,913$ & $\$ 0.050$ & $\$ 0.055$ & $\$ 26,153,241 \$$ & $2,241,328$ \\
\hline 6 & $475,513,474$ & $\$ 1,323,001$ & $\$ 1,100,000$ & $\$ 8,029,132$ & $\$ 14,082,172: \$$ & $24,534,305$ & $\$ 0.052$ & $\$ 0.056$ & $\$ 26,628,755 \$$ & $2,094,450$ \\
\hline 7 & $475,513,474$ & $\$ 1,362,691$ & $\$ 1,100,000$ & $\$ 8,270,006$ & $\$ 14,082,172 \$$ & $24,814,869$ & $\$ 0.052$ & $\$ 0.057$ & $\$ 27,104,268 \$$ & $2,289,399$ \\
\hline 8 & $475,513,474$ & $\$ 1,403,571$ & $\$ 350,000$ & $\$ 8,518,106$ & $\$ 14,082,172: \$$ & $24,353,850$ & $\$ 0.051$ & $\$ 0.058$ & $\$ 27,579,781 \$$ & $3,225,932$ \\
\hline 9 & $475,513,474$ & $\$ 1,445,679$ & $\$ 350,000$ & $\$ 8,773,650$ & $\$ 14,082,172 ; \$$ & $24,651,500$ & $\$ 0.052$ & $\$ 0.059$ & $\$ 28,055,295 \$$ & $3,403,795$ \\
\hline 10 & $475,513,474$ & $\$ 1,489,049$ & $\$ 350,000$ & $\$ 9,036,859$ & $\$ 14,082,172 \$$ & $24,958,080$ & $\$ 0.052$ & $\$ 0.060$ & $\$ 28,530,808 \$$ & $3,572,728$ \\
\hline 11 & $475,513,474$ & $\$ 1,533,720$ & $\$ 350,000$ & $\$ 9,307,965$ & $\$ 14,082,172 \$$ & $25,273,857$ & $\$ 0.053$ & $\$ 0.061$ & $\$ 29,006,322 \$$ & $3,732,465$ \\
\hline 12 & $475,513,474$ & $\$ 1,579,732$ & 350,000 & $\$ 9,587,204$ & $\$ 14,082,172: \$$ & $25,599,108$ & $\$ 0.054$ & $\$ 0.062$ & $\$ 29,481,835 \$$ & $3,882,728$ \\
\hline 13 & $475,513,474$ & $\$ 1,627,124$ & $\$ 350,000$ & $\$ 9,874,820$ & $\$ 14,082,172 \$ \$$ & $25,934,116$ & $\$ 0.055$ & $\$ 0.063$ & $\$ 29,957,349 \$$ & $4,023,233$ \\
\hline 14 & $475,513,474$ & $\$ 1,675,938$ & $\$ 350,000$ & $\$ 10,171,064$ & $\$ 14,082,172 \$$ & $26,279,174$ & $\$ 0.055$ & $\$ 0.064$ & $\$ 30,432,862 \$$ & $4,153,688$ \\
\hline 15 & $475,513,474$ & $\$ 1,726,216$ & $\$ 350,000$ & $\$ 10,476,196$ & $\$ 14,082,172: \$$ & $26,634,584$ & $\$ 0.056$ & $\$ 0.065$ & $\$ 30,908,376 \$$ & $4,273,791$ \\
\hline 16 & $475,513,474$ & $\$ 1,778,002$ & $\$ 350,000$ & $\$ 10,790,482$ & $\$ 14,082,172 \$$ & $27,000,657$ & $\$ 0.057$ & $\$ 0.066$ & $\$ 31,383,889 \$$ & $4,383,233$ \\
\hline 17 & $475,513,474$ & $\$ 1,831,342$ & $\$ 350,000$ & $\$ 11,114,197$ & $\$ 14,082,172 \$$ & $27,377,711$ & $\$ 0.058$ & $\$ 0.067$ & $\$ 31,859,403 \$$ & $4,481,692$ \\
\hline 18 & $475,513,474$ & $\$ 1,886,283$ & $\$ 350,000$ & $\$ 11,447,623$ & $\$ 14,082,172: \$$ & $27,766,077$ & $\$ 0.058$ & $\$ 0.068$ & $\$ 32,334,916 \$$ & $4,568,839$ \\
\hline 19 & $475,513,474$ & $\$ 1,942,871$ & $\$ 350,000$ & $\$ 11,791,051:$ & $\$ 14,082,172: \$$ & $28,166,095$ & $\$ 0.059$ & $\$ 0.069$ & $\$ 32,810,430 \$$ & $4,644,335$ \\
\hline 20 & $475,513,474$ & $\$ 2,001,157$ & $\$ 350,000$ & $\$ 12,144,783:$ & $\$ 14,082,172: \$$ & $28,578,112$ & $\$ 0.060$ & $\$ 0.070$ & $\$ 33,285,943 \$$ & $4,707,831$ \\
\hline 21 & $475,513,474$ & $\$ 2,061,192$ & $\$ 350,000$ & $\$ 12,509,126$ & $\$ 0 \$$ & $14,920,318$ & $\$ 0.031$ & $\$ 0.071$ & $\$ 33,761,457 \$$ & $18,841,138$ \\
\hline 22 & $475,513,474$ & $\$ 2,123,028$ & $\$ 350,000$ & $\$ 12,884,400$ & $\$ 0: \$$ & $15,357,428$ & $\$ 0.032$ & $\$ 0.072$ & $\$ 34,236,970 \$$ & $18,879,542$ \\
\hline 23 & $475,513,474$ & $\$ 2,186,719$ & $\$ 350,000$ & $\$ 13,270,932$ & $\$ 0: \$$ & $15,807,651$ & $\$ 0.033$ & $\$ 0.073$ & $\$ 34,712,484 \$$ & $18,904,833$ \\
\hline 24 & $475,513,474$ & $\$ 2,252,320$ & $\$ 350,000$ & $\$ 13,669,060$ & $\$ 0: \$$ & $16,271,380$ & $\$ 0.034$ & $\$ 0.074$ & $\$ 35,187,997 \$$ & $18,916,617$ \\
\hline 25 & $475,513,474$ & $\$ 2,319,890$ & $\$ 350,000$ & $\$ 14,079,132$ & $\$ 0: \$$ & $\begin{array}{l}16,749,022 \\
\end{array}$ & $\$ 0.035$ & $\$ 0.075$ & $\$ 35,663,511 \$$ & $18,914,489$ \\
\hline
\end{tabular}

$\begin{array}{ll}\text { Hualapai Tribe Revenue } & \$ \\ \text { T202,523,415 }\end{array}$

$\begin{array}{cr}\text { Tax Equity investor Required Distributions } & \$ 750,000.00 \\ \text { Reserve and Replacement Annual Expense } \quad \$ 350,000.00\end{array}$ 


\section{ATERWYNNE}

ATTORNEYS AT LAW

\section{Loan and Investor Rates}

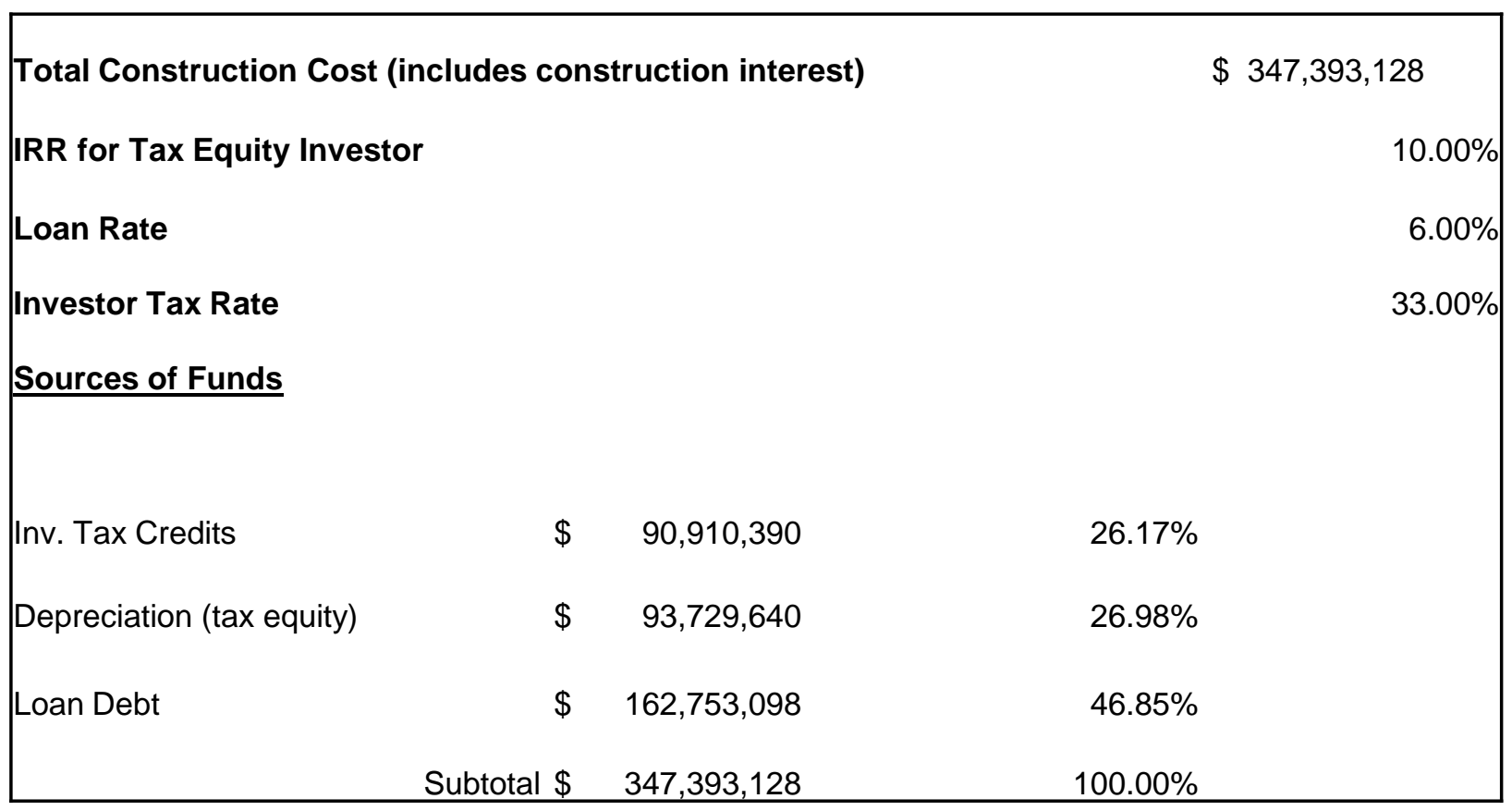

Annual Loan Service

$\$ 14,082,172.09$ 


\section{ATERWYNNE}

ATTORNEYS AT LAW

\section{Income and Expenses}

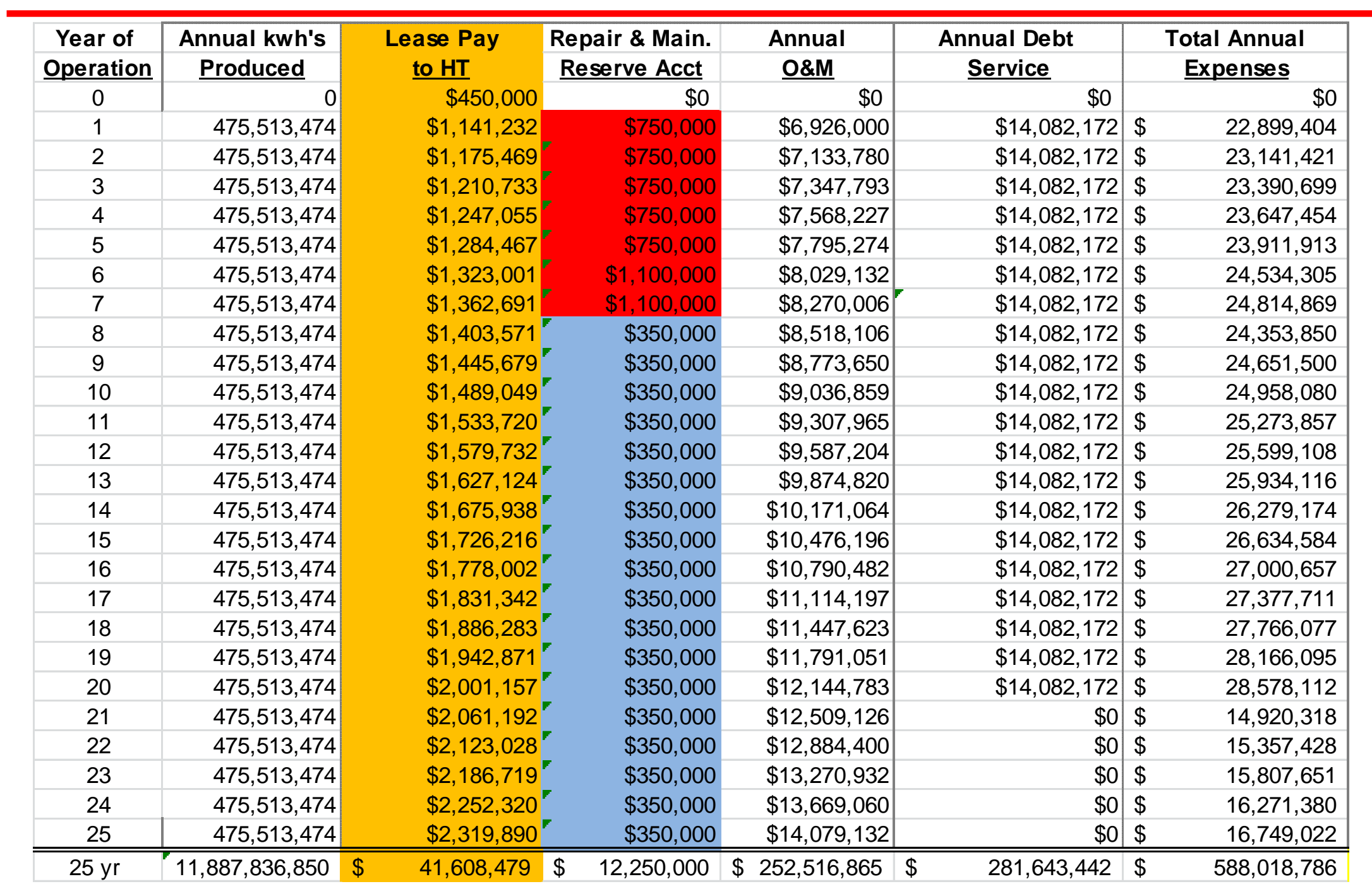




\section{Exhibit C}

Geotechnical Report for Water Pipeline

(Foree \& Vann) 


\title{
Attachment -1
}

\section{Renewable Energy Development in Indian Country:}

\author{
A Handbook for Tribes
}

\author{
(Ater Wynne, LLP)
}

